\title{
Three Essays in Labour Economics: An Application of Mixed Methods Research to Understanding of the Employment Status of Aboriginal Workers in Canada
}

\author{
by
}

Senada Delic

A thesis submitted to the Faculty of Graduate Studies and Postdoctoral Affairs in partial fulfilment of the requirements for the degree of

Doctor of Philosophy

in

Public Policy

Carleton University

Faculty of Public Affairs

School of Public Policy and Administration

Ottawa, Ontario, Canada

Copyright (C) 2012

Senada Delic 
Library and Archives

Canada

Published Heritage

Branch

395 Wellington Street

Ottawa ON K1A ON4

Canada
Bibliothèque et

Archives Canada

Direction du

Patrimoine de l'édition

395 , rue Wellington

Ottawa ON K1A ON4

Canada
Your file Votre référence

ISBN: 978-0-494-94217-8

Our file Notre référence

ISBN: $978-0-494-94217-8$
NOTICE:

The author has granted a nonexclusive license allowing Library and Archives Canada to reproduce, publish, archive, preserve, conserve, communicate to the public by telecommunication or on the Internet, loan, distrbute and sell theses worldwide, for commercial or noncommercial purposes, in microform, paper, electronic and/or any other formats.

The author retains copyright ownership and moral rights in this thesis. Neither the thesis nor substantial extracts from it may be printed or otherwise reproduced without the author's permission.
AVIS:

L'auteur a accordé une licence non exclusive permettant à la Bibliothèque et Archives Canada de reproduire, publier, archiver, sauvegarder, conserver, transmettre au public par télécommunication ou par l'Internet, prêter, distribuer et vendre des thèses partout dans le monde, à des fins commerciales ou autres, sur support microforme, papier, électronique et/ou autres formats.

L'auteur conserve la propriété du droit d'auteur et des droits moraux qui protege cette thèse. $\mathrm{Ni}$ la thèse ni des extraits substantiels de celle-ci ne doivent être imprimés ou autrement reproduits sans son autorisation.
In compliance with the Canadian Privacy Act some supporting forms may have been removed from this thesis.

While these forms may be included in the document page count, their removal does not represent any loss of content from the thesis.
Conformément à la loi canadienne sur la protection de la vie privée, quelques formulaires secondaires ont été enlevés de cette thèse.

Bien que ces formulaires aient inclus dans la pagination, il n'y aura aucun contenu manquant. 


\begin{abstract}
This thesis consists of three self-contained essays examining the link between Aboriginal identity and economic success in the Canadian labour market. The analytical approach encompasses mixed methods research, with two empirical tests and one qualitative inquiry. Both the neoclassical and institutional economics approaches to labour market analysis are considered, as discussed in the introduction to the thesis.

Essay I employs monthly Labour Force Survey data and examines the difference in the impact of the 2008-2011 economic downturn on Aboriginal and non-Aboriginal workers. The main findings in this essay indicate that the economic downturn had a markedly more negative impact on Aboriginal than on non-Aboriginal workers. For one subgroup of Aboriginal workers - Métis - a large portion of the difference in the impact can be attributed to the difference in endowments while for the other subgroup of Aboriginal workers - North American Indian - only a small portion of the difference in the impact can be attributed to the difference in endowments, much remains unexplained.

Essay II employs the 2006 Census and Aboriginal Peoples Survey data and examines the relative impact of various sources of capital — human, social and cultural - on the employment success of Aboriginal labour force participants living on-reserve, offreserve, in urban and in rural areas across Canada. The single most important finding in this essay is that the predictions of human capital theory do not hold up when the associated empirical models are applied to different Aboriginal groups. Another important finding is that for some subgroups of Aboriginal population social capital and cultural factors are potentially important omitted variables in the associated equations.

Essay III employs primary data collected from knowledgeable key informants working in the area of Aboriginal labour force development and investigates the role institutional and other constraints, not observable in the statistical information, play in the labour market experience of Aboriginal people. Findings here suggest that many paradoxes and puzzles that persist in the empirical literature can be better understood once the institutional arrangements related to the investment in human and other capital, the legacy of historical disadvantage, and the experience of workplace discrimination are taken into account.
\end{abstract}

JEL classification: B41, B52, C21, C23, I24, J21, J24, J64, J78

Keywords: Aboriginal workers, employment, human capital, social capital, institutional economics, recession, labour market discrimination, mixed methods research design 


\section{Acknowledgments}

The work presented in this doctoral dissertation would not have been possible without my close association and collaboration with many people who have contributed greatly to my personal and intellectual growth.

First and foremost, I would like to extend my deep and sincere respect and appreciation to my dissertation supervisor, Professor Dr. Saul Schwartz, for his dedicated support and encouragement throughout my dissertation writing days and for his rigorous academic guidance. I attribute much of the credit that this dissertation received from the examiners to his adherence to the scientific rigor and to clarity of expression, which he insisted on transferring on me. Thank you Saul! I sincerely respected your thorough and perceptive feedback, your patience with me, and your continued effort to keep me on the right track.

I also wish to extend my sincere gratitude to my co-supervisor for the qualitative part of this dissertation, Professor Dr. Frances Abele, for her invaluable guidance and for her continued support. Thank you Frances! I am deeply indebted to you for instilling in me the confidence needed to pursue this research and for giving me plenty of opportunities to work with you and thus expand my understanding of the policy issues in this topic area.

Professor Dr. Gustave Goldmann was officially a committee member on my doctoral dissertation board but our scholarly collaboration went far beyond my dissertation work. Gustave, I take this opportunity to express my sincere gratitude for the invaluable guidance and support I received from you at all levels of our collaborations. Thank you!

I also wish to convey my sincere gratitude to Professor Dr. Paul Maxim, Department of Economics, Wilfred Laurier University, for taking his valuable time to serve as my external examiner. The same appreciation I extend to Professor Dr. Christopher Worswick, Department of Economics, Carleton University, who served as my internalexternal examiner. I appreciated your meticulous reading of my dissertation. Your critical evaluation of my work was very helpful and your feedback was very reassuring.

I wish to acknowledge the importance of contributions made by the representatives of Aboriginal organizations and of other governmental and non-governmental organizations who participated in the qualitative component of the research in this dissertation. It is with sincere gratitude that I acknowledge their willingness to set aside a generous amount of their valuable time and to share with me their professional insights on the topic.

Special acknowledgement goes to the Canadian Research Data Centre Network (CRCDN) for granting me access to the confidential master files at the Carleton, Ottawa, Outaouais Local Research Data Centre (COOL RDC). It is with immense gratitude that I 
here acknowledge the generous support that Dr. Jean-Michel Billette, then the Statistics Canada's analyst at the COOL RDC, offered to me during my work at the COOL RDC.

I would also like to acknowledge the help I received from other researchers while working on my dissertation at the COOL RDC. These include: Professor Dr. Pierre Brochu, Department of Economics, University of Ottawa; Professor Dr. Marcel-Cristian Voia, Department of Economics, Carleton University; Dr. Winnie Chan, an economist at Statistics Canada, Analytical Studies Branch; Heather Tait and Nancy Zukewich, senior research analysts at Statistics Canada, Social and Aboriginal Statistical Division.

Dr. Sacha Sénécal, a research manager at the Aboriginal Affairs and Northern Development Canada, also provided useful sources and constructive comments on some of the findings presented in this dissertation. I extend my special gratitude to him for that.

I am indebted to Dwight Powless, an Aboriginal community liaison officer at the Algonquin College Access Program, for his sincere effort to expand my understanding of the issues in this research area and to enable me to grasp its rich complexity. Through his meticulous and very logical questioning of my research questions, Dwight brought me closer to the reality that I initially perceived through my research and gave me a valuable lesson in critical thought upon which I will always draw. Thank you Dwight!

I wish to acknowledge the helpful feedback on my doctoral research and guidance that I received from the faculty in the School of Public Policy and Administration at Carleton University. My special thanks go to Professor Jennifer Stewart, Professor Jose Galdo, and to Professor Graeme Auld. I also benefited from conversations with my fellow colleagues and I wish to acknowledge the insights I gained from my conversations with Dr. Tamara Krawchenko and with Dmitry Lysenko. The administrative staff in the School of Public Policy and Administration was very helpful and I would like to convey my special thanks to Meghan Innes, Nicole Enouy, Mary Au and Tabbatha Malouin.

I also wish to acknowledge the support and encouragement I received at the outset of this pursuance from the faculty in the School of Public Policy at Simon Fraser University. My sincere gratitude goes to Professor Dough McArthur, my Master's Thesis supervisor who originally inspired my research interests in Aboriginal policy. I am also deeply grateful to Professor Nancy Olewiler, the School's Director, and to Professor Jonathan Kesselman for inspiring and supporting my pursuit of this degree at Carleton University.

Funding I received from different organizations facilitated the timely completion of my doctoral studies and I gratefully acknowledge here Carleton University, Statistics Canada, the Social Science and Humanities Research Council of Canada, and Government of Ontario. 
A portion of the empirical work presented in this dissertation was completed while I held the Tom Symons Research Fellowship at Statistics Canada. My special thanks goes to Statistics Canada for giving me that honor and for generous financial support. Research results and conclusions drawn here are solely mine and do not indicate concurrence by Statistics Canada or by any other aforementioned individual or organization.

In closing, I would like to extend my utmost gratitude to two very special friends and great humanitarians, Munib Dzumhur and Anisa Dzumhur from Niagara Falls, for providing me and my then-young family with refuge from our war-torn country and thus enabling me to continue pursuing my intellectual pursuits. I would not have contemplated this road if not for their selfless generosity and loving kindness. Hvala Hadzija!

I extend my warm thanks to my siblings in Germany, my sister Sedina Durakovic-Dzubur and my brother Senad Durakovic, and in Sweden, my brother Seid Durakovic, for their steadfast reminders that I am not alone. Thank you! Your cheery words of encouragement and love on my defence day have been heartfelt and much appreciated. I was not alone!

Finally, this dissertation marks the fulfillment of the silent promise I gave to my father in response to his constant reminders of his belief in me. Hvala Babo! My greatest aspiration is to keep the sparkles in the eyes of my daughters - Lejla and Emina - shining bright as you and mama kept mine. The words of gratitude I wish to convey to my husband - who left his important job in Vancouver at the pick of his success so that I can pursue this degree here in Ottawa - simply do not exist. I dedicate this doctoral work to him and to our daughters and to my parents, with deepest love.

Senada Delic, Ph.D.

Ottawa 


\section{Dedication}

To

my father

Rifet Durakovic

my mother

Hanifa Durakovic

and

To

my daughters

Lejla Delic and Emina Delic

and

To

my loving husband

Zijad Delic 


\section{Table of Contents}

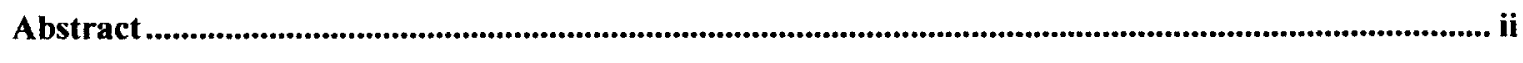

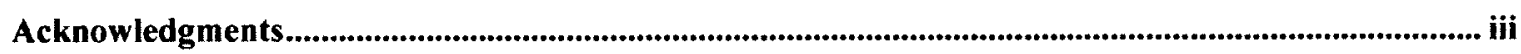

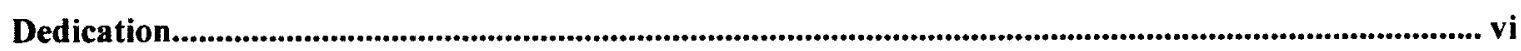

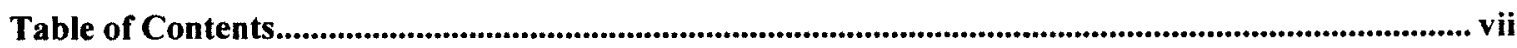

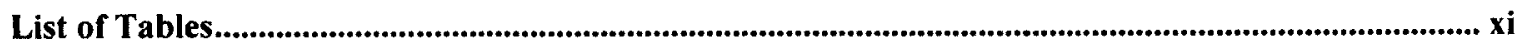

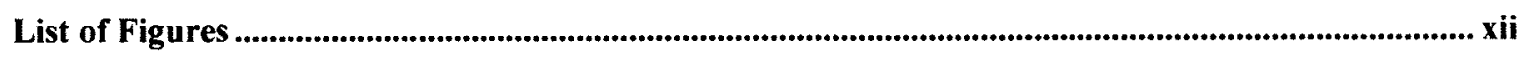

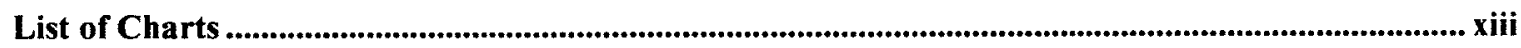

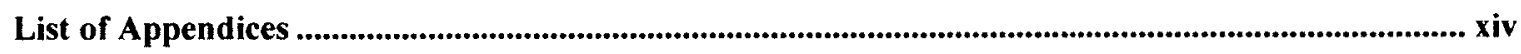

Introduction: Background and Theoretical Foundations ................................................................... 1

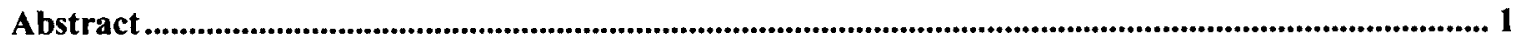

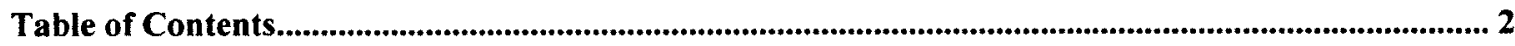

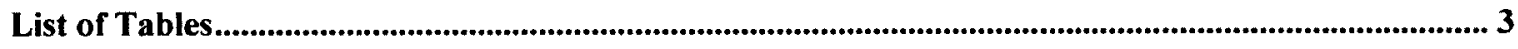

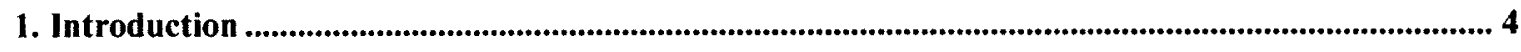

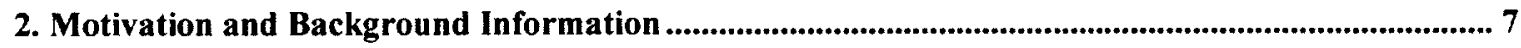

3. The Neoclassical Economics Approach to Labour Market..............................................................13

4. The Institutional Economics Approach to Labour Market ...........................................................19

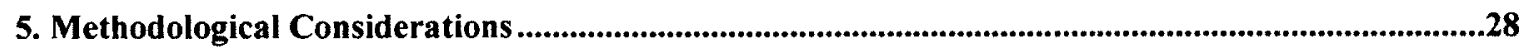

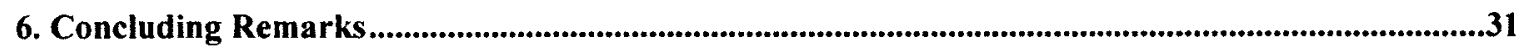

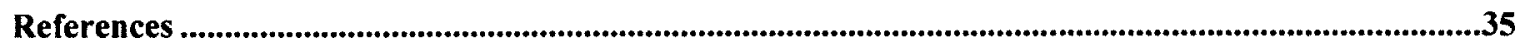

Essay I: Economic Downturn and Aboriginal Workers: Evidence from the 2008-2011 Recession .....47

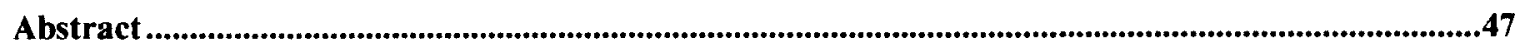

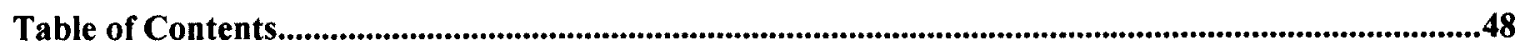

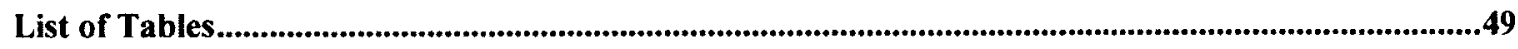

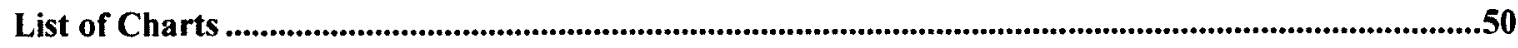


List of Figures ............................................................................................................................................................51

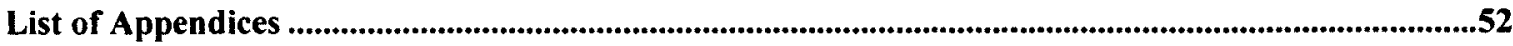

1. Introduction ..............................................................................................................................................................53

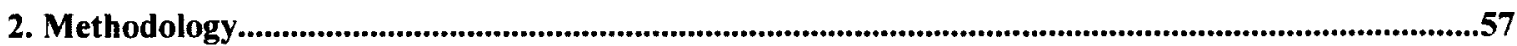

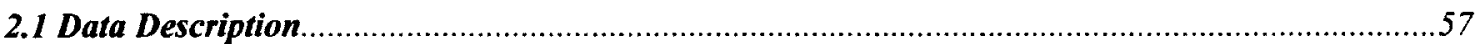

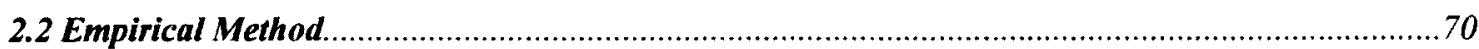

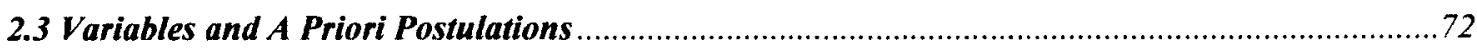

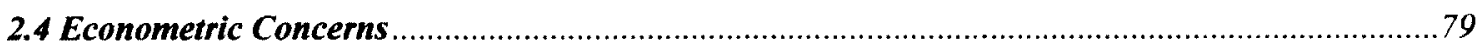

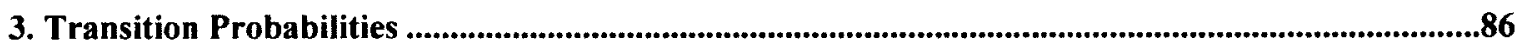

3.1 Estimated Probabilities of Layoff and of Reemployment .......................................................91

3.2 Steady State Employment and Durations of Unemployment ......................................................96

4. Blinder-Oaxaca Decomposition ......................................................................................................................99

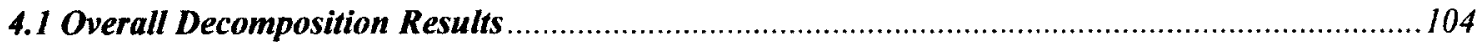

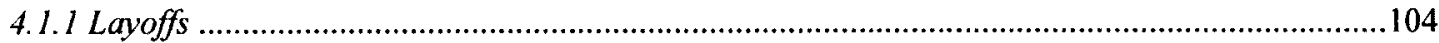

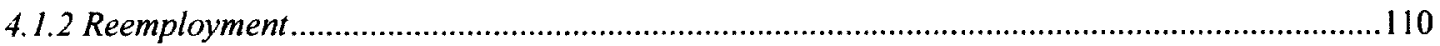

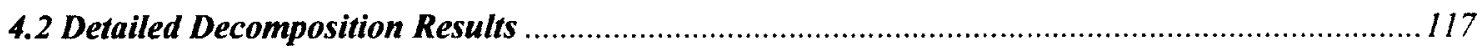

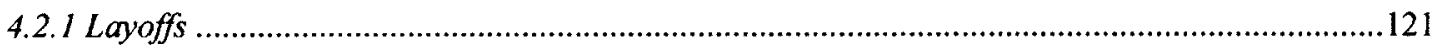

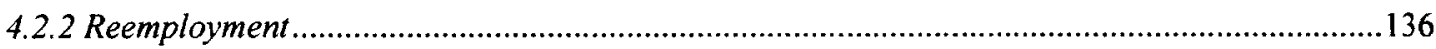

5. Concluding Remarks.......................................................................................................................................143

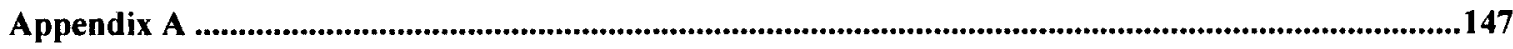

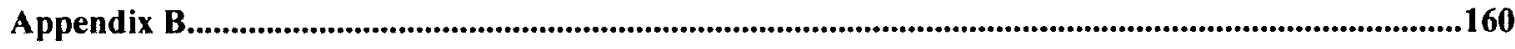

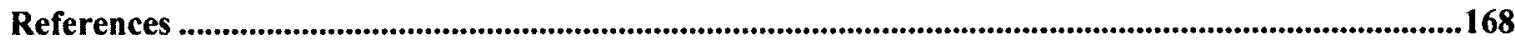

Essay II: The Determinants of the Employment Status of Aboriginal Peoples in Canada ....................177

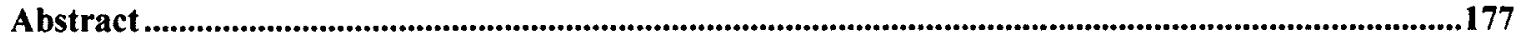

Table of Contents.............................................................................................................................................178

List of Tables...........................................................................................................................................179

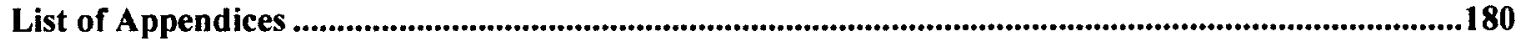

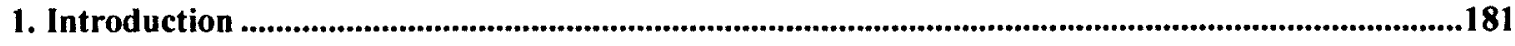

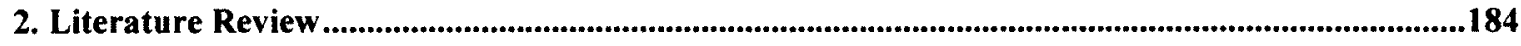

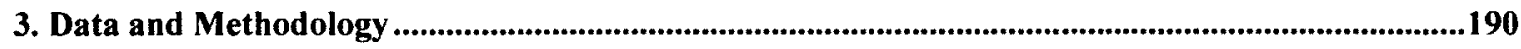




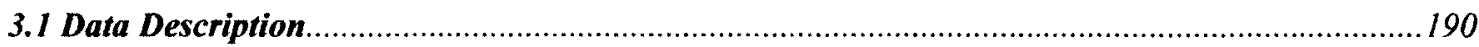

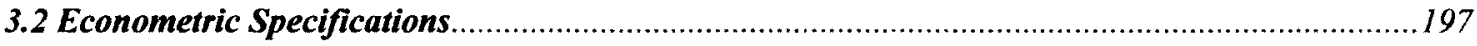

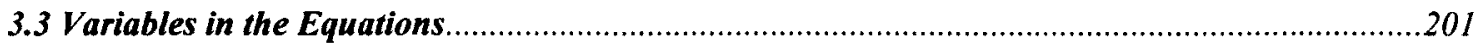

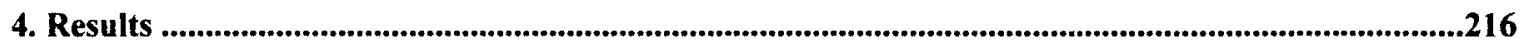

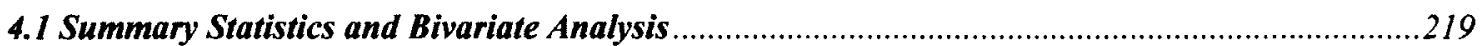

4.2 Multivariate Probit Regression Results for NAI Population ................................................227

4.3 Multivariate Probit Regression Results for Metis Population ..................................................242

4.4 Multivariate Probit Regression Results for Inuit Population ...................................................247

5. Robustness Checks ...............................................................................................................................................252

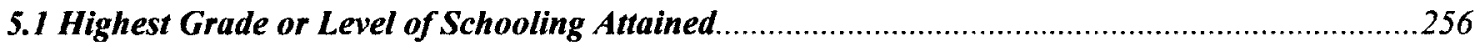

5.2 Highest Accreditation Attained ...............................................................................................261

5.3 High School Certification and Highest Accreditation Attained ….............................................264

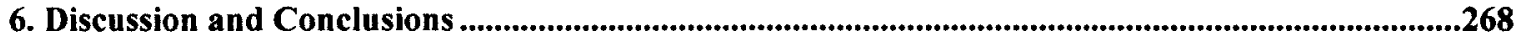

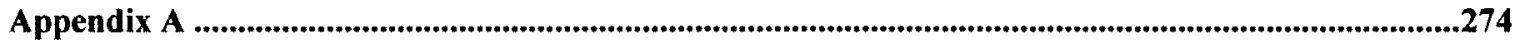

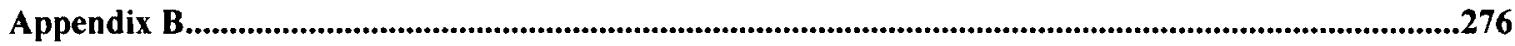

Appendix C .....................................................................................................................................................283

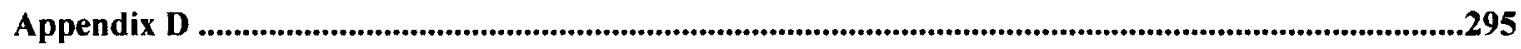

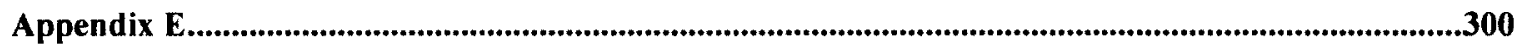

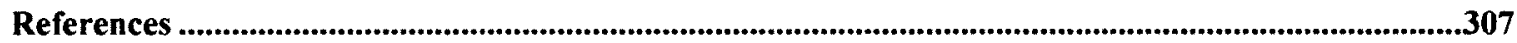

Essay III: Enhancing our Understanding of Empirical Findings on Labour Market Outcomes of

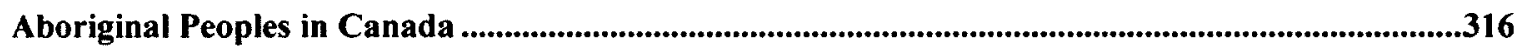

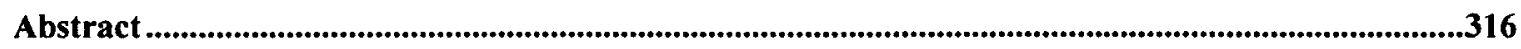

Table of Contents....................................................................................................................................................317

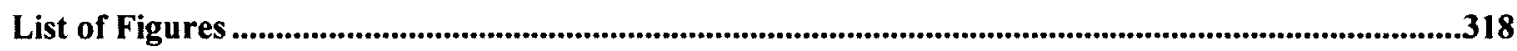

List of Appendices ..................................................................................................................................................319

1. Introduction ..........................................................................................................................................................320

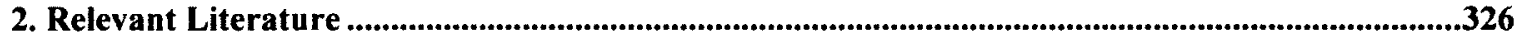

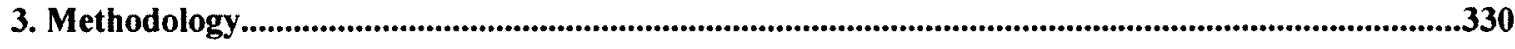

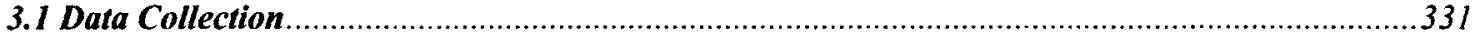




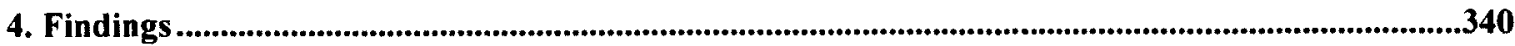

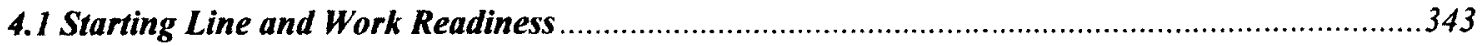

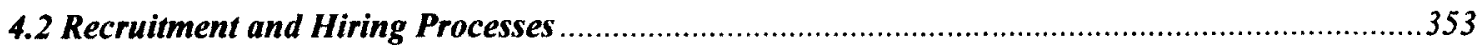

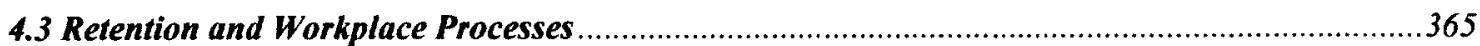

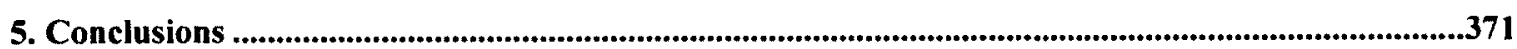

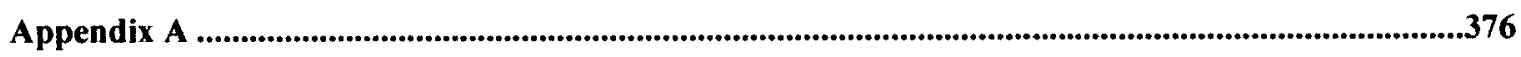

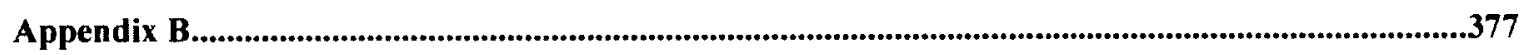

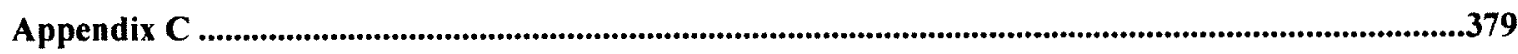

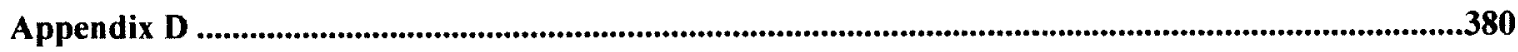

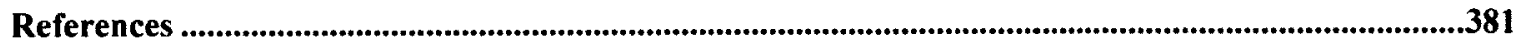

Thesis Conclusions: Findings Summary and Policy Implications.........................................................388

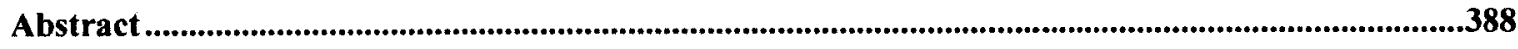

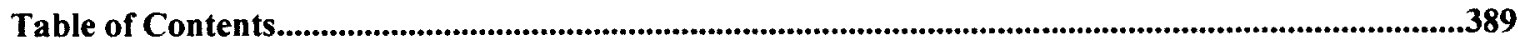

1. Summary of Findings .............................................................................................................................390

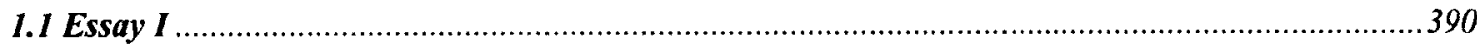

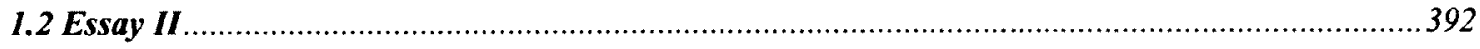

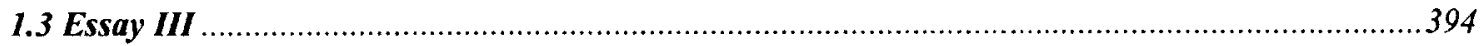

2. Policy Implications ….....................................................................................................................................397

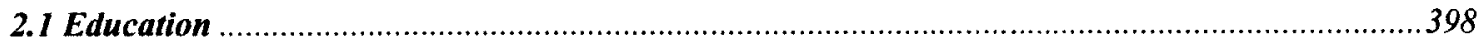

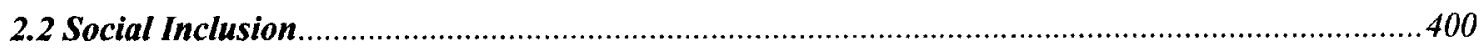

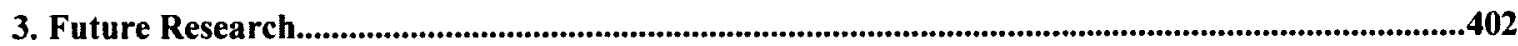




\section{List of Tables}

Table 1: The population counts and the labour force profile of the Aboriginal and non-Aboriginal identity population in Canada, Census $1996-2006$.

Table 1: Sample sizes and means of selected sample characteristics, by identity

Table 2: The equilibrium level of employment and average first duration of unemployment, by identity. .96

Table 3: Overall decomposition of the difference in predicted probability of layoff, main sample ...106 Table 4: Overall decomposition of the difference in predicted probability of layoff, restricted sample

Table 5: Overall decomposition of the difference in predicted probability of reemployment, main sample

Table 6: Overall decomposition of the difference in predicted probability of reemployment, restricted sample

Table 7: Detailed decomposition of the difference in the predicted probability of layoff for men ......122

Table 8: Detailed decomposition of the difference in the predicted probability of layoff for women 128

Table 9: Detailed decomposition of the difference in the predicted probability of reemployment ....137

Table 1: The proportions of employed in the North American Indian, Métis and Inuit samples ......196

Table 2: Selected estimates from probit employment function for urban NAI, APS 2006 ..................228

Table 3: Selected estimates from probit employment function for rural NAl, APS 2006 ..................232

Table 4: Selected estimates from probit employment function for registered NAI, APS 2006...........235

Table 5: Selected estimates from probit employment function for non-registered NAI, APS 2006 ....239

Table 6: Selected estimates from probit employment function for Métis, APS $2006 \ldots \ldots \ldots \ldots \ldots \ldots \ldots \ldots . . . . .243$

Table 7: Selected estimates from probit employment function for Inuit, APS $2006 \ldots \ldots \ldots \ldots \ldots \ldots \ldots \ldots \ldots . .248$

Table 8: Robustness checks for highest grade and highest level of schooling attained, APS 2006 ....258

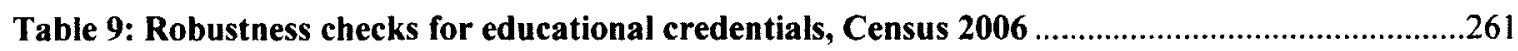

Table 10: Robustness checks for educational certification and credentials, Census $2006 \ldots \ldots \ldots \ldots \ldots \ldots . . .265$ 


\section{List of Figures}

Figure 1: Two State Markov-like Model of Labour Force Transitions

.86

Figure 1: The stages and processes from potential labour pool to successful employment ................341

Figure 2: Job application and interviewing selection processes .360 


\section{List of Charts}

Chart A: Monthly layoff rates for Aboriginal and non-Aboriginal identity groups, the 2008-

2011 recession

Chart B: Monthly layoff rates for Aboriginal and non-Aboriginal identity workers, the 2008-

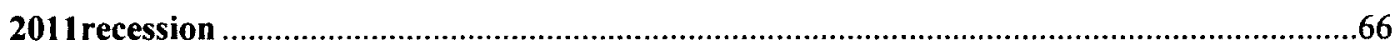




\section{List of Appendices}

\section{Appendix A}

Appendix A 1: A Summary of Definitions of Independent Variables.

Appendix A 2: Mean Characteristics of Paid Employees in the Main Samples, by Identity 148

Appendix A 3: Mean Characteristics of Paid Employees in the Restricted Samples, by Identity. .151

Appendix A 4: Mean Characteristics of Laid-Off Workers in the Main Samples, by Identity .154

Appendix A 5: Mean Characteristics of Laid-Off Workers in the Restricted Samples, by Identity...157

Appendix A 1: Definitions of explanatory variables in the probit employment models, APS $2006 \ldots 274$

Appendix A 2: Definitions of explanatory variables in the robustness checks, Census 2006. .275

Appendix A 1: In-Depth Interview Theme Guide .376

\section{Appendix B}

Appendix B 1: Probit estimates used in decompositions of difference in layoffs for men .160

Appendix B 2: Probit estimates used in decompositions of difference in layoffs for women 163

Appendix B 3: Probit estimates used in reemployment decompositions, Aboriginal identity groups 166

Appendix B 1: Sample means of all explanatory variables in the probit employment models, APS 2006 .

Appendix B 1: Letter of Information (standard)

Appendix B 2: Letter of Information (personalized) .378 


\section{Appendix C}

Appendix C 1: Detailed results from probit models predicting employment for urban NAI, APS 2006

Appendix C 2: Detailed results from probit models predicting employment for rural NAI, APS 2006 .285

Appendix C 3: Detailed results from probit models predicting employment for registered NAI, APS 2006 .

Appendix C 4: Detailed results from probit models predicting employment for non-registered $\mathbf{N A I}$, APS 2006

Appendix C 5: Detailed results from probit models predicting employment for Métis, APS 2006 ...291

Appendix C 6: Detailed results from probit models predicting employment for Inuit, APS 2006....293

Appendix C 1: A Copy of the Informed Consent Form .379

\section{Appendix D}

Appendix D 1: Detailed results from robustness checks for highest grade and highest level of schooling attained, APS 2006

Appendix D 1: List of Participating Organizations

\section{Appendix E}

Appendix E 1: Sample means of all explanatory variables in the probit employment models, Census 2006. .300

Appendix E 2: Detailed results from robustness checks for educational credentials, Census $2006 \ldots 303$ Appendix E 3: Detailed results from robustness checks for certification and credentials, Census 2006 


\title{
Introduction: Background and Theoretical Foundations
}

\begin{abstract}
Literature identifying and explaining the distribution of labour market disadvantage among Aboriginal population groups in Canada is relatively scarce. What exists is based primarily on neoclassical economics, as set forth in human capital theory. As an introduction to the proposed thesis, this chapter challenges the notion that the economic integration and distribution of labour market disadvantage among Aboriginal populations is solely the responsibility of individual economic agents and highlights the reasons for the need to consider views expressed in institutional economics, as set forth in segmented labour market theory. After providing a brief critical assessment of the relative effectiveness of the two approaches in explaining the issue at hand, the chapter concludes by introducing the adopted mixed methods research design and an argument that multiple approaches are required for a proper assessment of labour market issues that are as multifaceted as those facing different Aboriginal population groups in Canada.
\end{abstract}

Key words: Aboriginal workers, labour market disadvantage, human capital theory, social capital theory, institutional economics, mixed methods research design 


\section{Table of Contents}

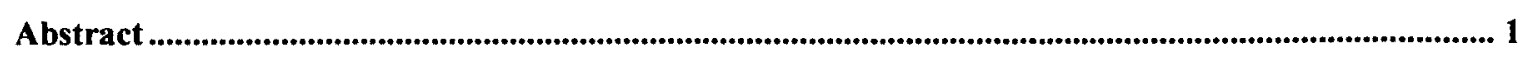

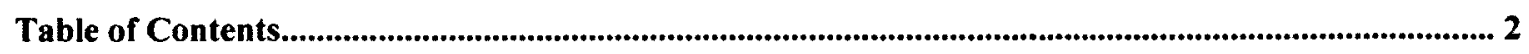

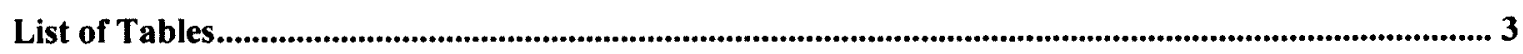

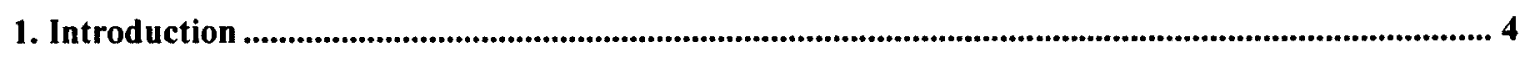

2. Motivation and Background Information ............................................................................................... 7

3. The Neoclassical Economics Approach to Labour Market.................................................................13

4. The Institutional Economics Approach to Labour Market ......................................................................19

5. Methodological Considerations ...........................................................................................................28

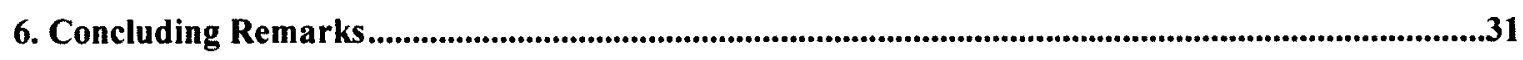

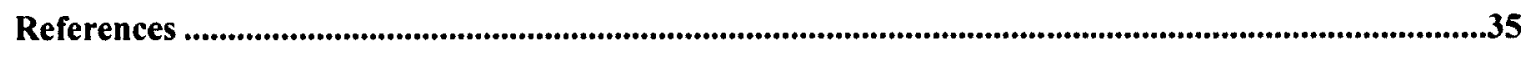




\section{List of Tables}

Table 1: The population counts and the labour force profile of the Aboriginal and non-Aboriginal

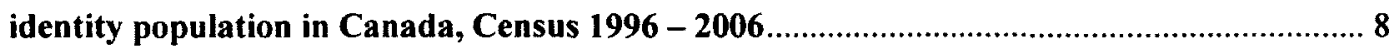




\section{Introduction}

In recent years, a number of Canadian economists and sociologists have allocated a significant amount of attention to labour market integration issues of different population groups in Canada (Baker \& Benjamin, 1994; Pendakur \& Pendakur, 1998, 2002, 2007, 2011; Hum \& Simpson, 1999; White, Maxim \& Gyimah, 2003; Walters, White \& Maxim, 2004; Boudarbat, Lemieux \& Riddell, 2006; Gee, Kobayashi, \& Prus, 2006; Maxim \& White, 2006). To differing degrees, all of these studies report poorer labour market outcomes for designated group members (such as Aboriginal people) when compared to the general population. ${ }^{1}$ A common theoretical approach endorsed in such studies, and especially in studies done by mainstream economists, is based on the view that wages and employment outcomes depend on workers' productivity, which, in turn, depends primarily on workers' education, skills, and work experience as well as on other factors such as health, mobility, and labour market information. This neoclassical economics view is generally known as the human capital view, and is often associated with the theoretical and empirical work of Gary Becker who, apart from playing a key role in the development of the human capital model, used the model to evaluate the effects of discrimination on the employment outcomes of visible minorities in the United States (Becker, 1993).

A rich and diverse alternative view to human capital, broadly identified as institutional economics, has evolved over time, offering theories that are directed

\footnotetext{
1 "Aboriginal people" in Canada is a broad collective term commonly applied in reference to all Indigenous Peoples of North America and their descendants. The three distinct Aboriginal groups that are recognized by the Canadian Constitution (the Constitution Act, 1982) include North American Indian, Inuit and Metis (Statistics Canada, 2007). This thesis, for the most part, focuses exclusively on these three Aboriginal identity groups. Each essay provides more details on the definition of Aboriginal population groups considered in the pertinent analysis.
} 
primarily at explaining the substantive impacts that institutional arrangements have on either furthering or preventing economic success and progress of different groups in a society (Samuels, 1995; Boyer \& Smith, 2001; Kaufman, 2004; Gray \& Chapman, 2004). Associated with more heterodox economists and some sociologists (Commons, 1931; Piore, 1975; North, 1991; Arrow, 1987; Williamson, 2000; Kinnear, 2004; Richter, 2005) the institutional approach to labour economics deemphasizes the power of market pressures in favour of political pressures, asserting that institutional arrangements reflecting majority wishes play a crucial role in the labour market outcomes of certain segments in a society. Under this perspective, offered wages, as well as other work conditions, depend largely on the structure of labour markets and the institutions governing them. This view is shared by all labour market institutionalists, regardless of the degree to which they actively study these institutional arrangements and regardless of whether or not they explicitly identify themselves as institutionalists (Kaufman, 2004).

The research presented in this thesis draws both on the neoclassical and on the institutional economics framework and explores the labour market issues facing different groups of Aboriginal populations in Canada in an attempt to better capture some of the complexities involved in the factors determining their labour market outcomes. The purpose of this introductory chapter is to provide the motivation for the approach taken here and to discuss the theoretical postulations and their relevance to the examination of the topic at hand. The chapter is structured as follows: the next section presents some relevant background information on Aboriginal populations in Canada. Section 3 discusses the key postulations of human and social capital theories. The key postulations put forth by segmentation theories are discussed in Section 4 while Section 5 briefly 
addresses the methodological divide and recent development in methodological convergences. Section 6 concludes the chapter by introducing the adopted mixed methods research design. 


\section{Motivation and Background Information}

The labour market behaviour of Aboriginal Canadians and the determinants of their labour market outcomes are important empirical issues with potentially significant policy consequences. Amid growing concerns about potential negative effects of projected future systemic labour shortages caused by low fertility rates and the rapid aging of non-Aboriginal Canadian population, the demographic dynamics and the composition of Aboriginal populations have attracted a significant amount of attention among labour market analysts and policy-makers. In fact, aside from new immigrants, labour force projections list Aboriginal youth as the only significant potential source of new labour supply for years to come (Guimond, 2003; Statistics Canada, 2007a; Human Resources and Social Development Canada, 2007; Government of Canada, 2008; Hull, 2008; Anonson, Desjarlais, Nixon, Whiteman, \& Bird, 2008; Perrin, Thorau \& Associates Ltd., 2009; Sharpe, Arsenault, Lapointe \& Cowan, 2009). ${ }^{2}$

Over the past few decades, the total Aboriginal population in Canada has experienced considerable growth. The current average annual growth rate of the Aboriginal population is 1.8 percent, which is almost twice the growth rate of the total population of Canada (INAC, 2007). In the last decade alone, as illustrated in Table 1, the total population reporting a single Aboriginal identity has increased by 47 percent, compared to an 8 percent increase of Canada's non-Aboriginal identity population. ${ }^{3}$

\footnotetext{
${ }^{2}$ In its 2008 discussions about a new economic development framework, the federal government presented estimates which showed that "over 600,000 Aboriginal youth will enter the labour market between 2001 and 2026" (Government of Canada, 2008, p.3).

${ }^{3}$ Possibly, some of this increase could be due to Aboriginal individuals changing their ethnic affiliation on the Census questionnaire, a phenomenon coined "ethnic mobility" (Goldmann \& Siggner, 1995; Guimond, 2003; Goldmann, 2007; Goldmann \& Delic, in press).
} 
Table 1: The population counts and the labour force profile of the Aboriginal and non-Aboriginal identity population in Canada, Census 1996 - 2006

\begin{tabular}{|c|c|c|c|}
\hline \multirow{2}{*}{ Total Canadian Population } & \multicolumn{3}{|c|}{ Census Year } \\
\hline & 1996 & 2001 & 2006 \\
\hline $\begin{array}{l}\text { Non-Aboriginal Identity } \\
\text { Pooulation counts }\end{array}$ & & & \\
\hline Population counts & $27,729,115$ & $29,030,793$ & $30,068,240$ \\
\hline Median age & 35.4 & 37.7 & 40 \\
\hline Working age population (age 15+) & $21,857,910$ & $23,247,850$ & $24,840,255$ \\
\hline Population in the labour force & $14,326,185$ & $15,470,800$ & $16,626,830$ \\
\hline Less-than-high school education (\%) & 35.0 & 31.0 & 23.1 \\
\hline Completed any university education (\%) & 16.0 & 15.7 & 18.5 \\
\hline Participation rate (\%) & 66.0 & 66.5 & 66.9 \\
\hline Employment rate (\%) & 59.0 & 61.8 & 62.7 \\
\hline Unemployment rate $(\%)$ & 10.0 & 7.1 & 6.3 \\
\hline Full- time full-year workers (\%) & - & 53.2 & 51.2 \\
\hline Average Earnings: full- time full- year (\$) & - & 48,773 & 51,505 \\
\hline Median Earnings: full-time full- year (\$) & - & 40,819 & 41,592 \\
\hline $\begin{array}{l}\text { Aboriginal Identity } \\
\text { Population counts }\end{array}$ & 799,010 & 976,305 & $1,172,790$ \\
\hline Median age & 25.5 & 24.7 & 27 \\
\hline Working age population (age 15+) & 458,730 & 648,650 & 819,855 \\
\hline Population in the labour force & 264,530 & 398,280 & 517,375 \\
\hline Less-than-high school education (\%) & 54.0 & 48.0 & 43.6 \\
\hline Completed any university education (\%) & 4.5 & 4.4 & 5.8 \\
\hline Participation rate $(\%)$ & 57.7 & 61.4 & 63.1 \\
\hline Employment rate (\%) & 44.7 & 49.7 & 53.8 \\
\hline Unemployment rate (\%) & 24.2 & 19.1 & 14.8 \\
\hline Full-time full-year workers (\%) & - & 39.7 & 42.3 \\
\hline Average Earnings: full-time full- year (\$) & - & 37,496 & 39,980 \\
\hline Median Earnings: full-time full-year $(\$)$ & - & 33,666 & 34,960 \\
\hline
\end{tabular}

Sources: The 2001 and the 2006 Census figures are taken from the Statistics Canada, topic based tabulations. The 1996 Census figures are taken from INAC (2000) and Statistics Canada (1998). All of the figures exclude information on incompletely enumerated reserves. Participation rate refers to the labour force in the week prior to Census Day expressed as a percentage of the population 15 years of age and older. Employment rate refers to the number of persons employed in the week prior to Census Day expressed as a percentage of the total population 15 years of age and over. Unemployment rate refers to the unemployed expressed as a percentage of the labour force in the week prior to Census Day. The percentage of full-time full-year workers and the educational attainment figures are calculations by the author, based on the relevant census tables; the other figures are taken directly from the referenced sources.

Note: The Aboriginal Identity population refers to all individuals reporting a single Aboriginal identity, that is North American Indian only, Métis only, or Inuit only.

Assuming moderate declines in fertility levels and moderate improvements in life expectancy among Aboriginal people, Statistics Canada projects a continuation of this

trend well into the future. Thus, by 2017, the total number of Canadians who identify 
themselves as Aboriginal is projected to increase to between $1,390,200$ and $1,431,800$ (Statistics Canada, 2005), and to reach 1,566,900 in 2026 (INAC, 2007). The former estimate would constitute another 47 percent increase above the 2001 level. In contrast, for Canada as a whole, projections are that natural population increase will become negative in the medium and long term and increase in immigration will become the only source of population growth in 2046 (Statistics Canada, 2005a).

The projected entry of the young and growing Aboriginal population into the Canadian labour market over the coming years presents an important opportunity for Canada to offset some of the demographic pressures in its shrinking non-Aboriginal workforce, but it also raises serious concerns about this potential workforce's prospects in the Canadian labour market. It is by now well established that Aboriginal people in Canada have historically had drastically different labour market experiences characterized by substantially lower labour force participation rates, lower employment rates and lower earnings — than any other segment of Canadian population (Drost, 1994; George \& Kuhn, 1994; Bernier, 1997; De Silva, 1999; Kuhn \& Sweetman, 2002; White et al., 2003; Maxim, White \& Beavon, 2003; Pendakur \& Pendakur, 1989, 2002, 2011).

As shown in Table 1, despite overall improvements in the past decade, the participation rates and especially the employment rates of the total Aboriginal identity population are still well below the rates of the non-Aboriginal identity population. In 2006, the difference in the labour force participation rates between the two populations amounted to 3.8 percentage points, while the discrepancy in the employment rates was almost 9 percentage points. In the same way, the unemployment rates of the Aboriginal identity population have historically exceeded the national rates, and, as indicated in 
Table 1, despite a steady decline since 1996, the 2006 unemployment rate for the total Aboriginal identity population, 14.8 percent, remains more than twice the 6.3 percent unemployment rate of the non-Aboriginal identity population in Canada.

In observing these differentials in labour market outcomes, public policy discourses have long emphasized the importance of narrowing the gaps and encouraging greater economic participation of Aboriginal Canadians through education and targeted labour market programs (Sloane-Seale, Wallace \& Levin, 2000; Mendelson, 2006; Government of Canada, 2008, 2009). ${ }^{4}$ Although some improvements in the educational attainment of the Aboriginal people have occurred in recent years, the general levels of literacy and education of the Aboriginal population in Canada are still considerably lower than those of the non-Aboriginal population (Mendelson, 2006; Biswal, 2008). As indicated in Table 1, the proportion of the total Aboriginal identity population with lessthan-high school education has decreased from 54 percent in 1996 to 43.6 percent in 2006 while the proportion with university credentials has increased from 4.5 percent to 5.8 percent in the same time period. ${ }^{5}$ Even so, these rates do not even come close to the rates of the non-Aboriginal population. In 2006, 18.5 percent of non-Aboriginal people had a university education and only about 23 percent had less-than-high school education. In the face of stronger gains among the non-Aboriginal population in 2006, one can observe in Table 1 that the education gaps have in fact widened. skills training programs targeted at Aboriginal community members

\footnotetext{
${ }^{4}$ These labour market programs are specific skills training programs targeted at Aboriginal community members. More details about these programs are presented in Essay III of this thesis.

${ }^{5}$ The "university credentials" here refer to completed university certificate, diploma or degree as reported by the respondents on Census questionnaire (Statistics Canada, 2008a).
} 
This growing disparity in educational attainment, as some of the existing literature suggests, partly explains the observed large income and earnings gaps between the two groups. As shown in Table 1, even looking only at those with full-time full-year employment, the 2006 average and median earnings of the Aboriginal identity population were lower, by $\$ 11,525$ and $\$ 6,632$, respectively, than those of the non-Aboriginal identity population. The proportion of full-time full-year workers among the nonAboriginal identity workers is also significantly larger, although the gap has narrowed over the past two census-taking periods.

Disaggregating the Aboriginal identity population by gender and place of residence accentuates the relatively poor labour market outcomes, particularly for some groups. Not shown here, the labour market statistics for Inuit men, for instance, are much worse than those presented in Table 1 for the total Aboriginal identity population. ${ }^{6}$ North American Indian men living on reserve, who have historically endured a disproportionate share of socioeconomic disadvantage in the Canadian labour market, also have far worse labour market statistics when compared to the overall Aboriginal identity population (Drost 1994; George \& Kuhn, 1994; Bernier, 1997; De Silva, 1999; Kuhn \& Sweetman 2002; Maxim et al., 2003; Pendakur \& Pendakur 1998, 2002, 2011).

Moreover, while the unemployment rates of all other Aboriginal identity groups, including the on-reserve North American Indian group, declined steadily over the 19962006 period, the unemployment rate of Inuit people actually went up slightly between 1996 and 2001 and remained around 20 percent in 2006, a rate that is more than triple the rate of the non-Aboriginal Canadians in the same year. The statistics for Métis men and

\footnotetext{
${ }^{6}$ These figures are available, upon request.
} 
women, on the other hand, are very similar to those of the non-Aboriginal identity Canadians, especially for those residing in large urban centers (INAC, 2000; Statistics Canada, 2003, 2008, 2008a, 2008b).

This brief overview raises two perplexing questions: (1) what accounts for such an uneven distribution of disadvantage between Aboriginal and non-Aboriginal populations existing within what appears to be the same labour market?; and (2) why is labour market disadvantage especially pronounced among certain Aboriginal identity groups existing within apparently the same labour market? In the general literature, a number of explanations have been proposed and they are commonly rooted either in theories of neoclassical economics, where the distribution of disadvantage is explained in terms of human capital theory and utility maximization, or in institutional economics, where labour market segmentation and segregation of workers in different segments of the labour market are held responsible for the uneven distribution of disadvantage. The following two sections present a brief overview of the two theoretical approaches and assess their relative strengths in explaining labour market disadvantage in the context of Aboriginal population in Canada. 


\section{The Neoclassical Economics Approach to Labour Market}

The neoclassical approach to labour market analysis is based on the postulations of human capital theory. In the most simplistic terms and in relation to distribution of labour market disadvantage, human capital theory holds that some individuals fare worse than others in the labour market because they invest less in education and skills development. Most of the empirical literature focuses on assessing the effect of these factors using different empirical models, some focusing on educational quantity while others trying to take difference in educational quality into account. This section gives a broad brush review of human capital theory as it relates to the present analyses.

The essence of the human capital view of the determinants of labour market outcomes lies in that individuals knowingly decide, by carefully weighing the benefits and costs associated with each choice, on the amount and the type of human capital investment that will optimize their on-the-job productivity and therefore maximize future gains in lifetime earnings. The benefits are not limited to increases in earnings but may also include political, cultural and other non-monetary gains. Similarly, the costs may pertain to monetary costs, non-monetary costs and the opportunity costs associated with the next best alternative foregone (Becker, 1996).

The original conceptualization of the human capital model was characterized by methodological individualism and was an extension of standard microeconomic consumer theory in which the decision-making problem facing a utility-maximizing individual consists of two parts: the first part containing a depiction of what the individual would like to do (preferences) and the second part listing the choices available 
to the individual (constraints). The assumptions of perfect rationality and perfect selfcontrol are then used to link the two parts together, thus producing a unique description of the given optimum choice as a function of those preferences and market constraints (Benjamin, Gunderson, \& Riddell, 2002).

The intended scope of the theoretical model of investment in human capital is very broad and encompassing in the sense that it rests on utility maximization and thus "helps in understanding a large and varied class of behaviour not only in the Western world, but also in developing countries and countries with [multiple and] very different cultures" (Becker, 1996, p. 17) such as Canada. The model presumes that individuals' behaviour is driven by an intricate set of values and preferences that extend beyond mere selfishness or material gain and that "individuals maximize welfare as they conceive it" (Becker, 1996, p. 2, emphasis in original). Although there is an assumption about consistent, forward-looking behaviour, it is acknowledged that this forward-looking behaviour "may still be rooted in the past, for the past can exert a long shadow on attitudes and values" of an individual (Becker, 1996, p. 2).

In relation to the functioning of the paid labour markets, the human capital approach holds that differentials in labour market outcomes between otherwise equally productive workers are inconsistent with competitive equilibrium, in situations in which rationality and optimization govern the behaviour of all economic agents, regardless of whether they are households, firms, or governments. The approach, however, recognizes that individuals' decisions about investment in human capital "may reflect rational choice but may also reflect discrimination as well as rational choice subject to discriminatory constraints" (Benjamin et al., 2002, p. 353). This discrimination, however, is not 
expected to persist in the long run because the costs incurred by the discriminating agents would make the decision to discriminate economically detrimental to them and thus discourage the practice of discrimination over time (Becker, 1993). Hence, to achieve greater equality in labour market outcomes between various social, racial or ethnic groups within a society, the human capital approach advocates policies that "equalize" individual human capital endowments and let the markets clear (Schultz, 1961). ${ }^{7}$

While intensely opposed in the early 1960 s (Bowles \& Gintis, 1975), the human capital theory is nowadays deemed "uncontroversial" (Becker, 1996, p. 10), enjoying the "strongest acceptance amongst those who formulate public policy and those that advise them" (Maglen, 1990, p. 282). Empirical examinations of the theory, however, continue producing inconclusive evidence, particularly in relation to the link between education, productivity and earnings. The innate ability measurement problem is the most frequently cited ground for this incongruity. For instance, "signalling" theorists have argued that more highly educated individuals are also more likely to have higher abilities, selfdiscipline, and motivation, all of which contribute to the higher productivity and the observed evidence of higher earnings (Spence, 2002). These factors, however, are difficult to measure in empirical models. Such individuals tend to do well in the labour market not only because of their education, but because of other, unmeasured positive

\footnotetext{
${ }^{7}$ Market-clearing, the idea underlying most of neoclassical economics, in the context of wage disadvantage implies that wages in markets with homogeneous workforce (homogenous in terms of their productive characteristics) and homogenous jobs (homogenous in terms of how desirable they are to workers) will be equalized across workers, in the long run. That is, in the long run, markets will clear - firms would not pay above the going market wage because they can employ as much labour as desired at the going wage rate and workers would not accept wages below the market wage because there are equally satisfactory jobs available at the going wage rate. At this equilibrium point, solutions to both individual (utility) and firm (profit) maximization problems are reached. The same competitive market forces are assumed to be at work to determine job allocations and training opportunities so that in the long run, full employment is reached.
} 
traits. Their education only signals the presence of those traits that are highly valued and highly rewarded in the competitive labour markets (Sobel, 1982).

A more serious measurement problem is the lack of direct evidence demonstrating a consistent match of earnings differences to productivity differences (Maglen, 1990; Livingstone, 1997; Gray \& Chapman, 2004). In re-examining the existing research on the links between education and productivity and between earnings and productivity, Maglen (1990), for instance, finds that "evidence is fragmentary and inconclusive. That which is affirming applies mainly to lower levels of education, to the performance of workers in the agricultural sector, and mostly to developing countries" (p. 291). In advanced market economies, as Livingstone (1997) summarizes it, "the school enrolment rates [since 1970s] have continued to increase while average incomes have stagnated, unemployment rates have worsened and underemployment of highly schooled people has been recognized as a social problem" (p. 9).

In response to this incongruity, some proponents of human capital theory have shifted their emphases from levels of formal schooling to informal life-long, job-related learning (Senge, 1990; Reich, 1992). It has been recognized that, by measuring education in terms of years of formal schooling, the empirical models based on this theory do not take account of the possible differences in educational quality or job market relevance. These two factors are potentially more revealing than the mere quantity of formal education and training, as postulated in the standard human capital theory (Sobel, 1982; Livingstone, 1997), yet again the difficulty remains here in delineating how much these two factors could be related to the innate ability and how much to the institutional factors such as social class or family background (Card, 2001; Smith, 2003). 
However measured, the key productivity-related characteristics emphasized by human capital theory are routinely accounted for in empirical work and included in the basic human capital model. In recent years, other (related) characteristics from social capital theory have been recognized as important and analyses analogous to those of investment in human capital have been done (Glaeser, Laibson \& Sacerdote, 2002). The acceptance of this view among neoclassical economists, however, is not wide-spread, primarily due to the measurement difficulties that arise in applying this theory.

Social capital, however, is often treated as a subset of human capital because of its potential to expand an individual's human capital by enhancing his or her abilities to identify viable opportunities and gain access to valuable resources through positive interpersonal networks (Becker \& Murphy, 2000). Looked at from an economic point of view, social capital can enhance the benefits of investment in both physical and human capital and thus affect economic outcomes in a positive way. For instance, social networks can reduce costs by lowering the amount of time an individual spends searching for and gathering relevant information. Mutual co-operation in turn can increase the level of essential knowledge available for an economic activity, thus facilitating better decisions and innovations (Becker \& Murphy, 2000; Hunter, 2000; Gomez \& Santor, 2001; Glaeser, et al., 2002; Annen, 2007; Van Staveren \& Knorringa, 2007).

In terms of its measurement, however, social capital is still a contested concept, with an ongoing dispute over what might constitute a proper measure of its existence and strength (Foley \& Edwards, 1999; Sobel, 2002; Durlauf, 2002; Brough et al., 2006; Van Staveren \& Knorringa, 2007; Knorringa \& Van Staveren, 2007). In the existing empirical work, social capital is generally defined as a set of formal and informal social relations 
that facilitate individuals' actions (Gomez \& Santor, 2001) and is measured in a number of ways, ranging from "strong" social ties, which are generally observed in close relations with family, friends and people of similar identities and values to "weak" social ties, which individuals develop through memberships in different voluntary, business or political organizations and associations (Foley \& Edwards, 1999; Nelson, Kaboolin \& Carver, 2003; Mignone, 2009).

To distinguish between the two categories of "ties" is important because studies have shown that strong ties, more formally defined as internal networks between family members and ethnic groups, seem to add very little in terms of economic value (Gomez \& Santor, 2001; Glaeser, et al., 2002; Annen, 2007). It is the weak ties - the networks that extend across ethnic groups and different social and political classes - that seems to benefit participants the most (Nelson et al., 2003; Mognone, 2009). ${ }^{8}$

\footnotetext{
${ }^{8}$ On a community level, social capital is assessed along similar lines as (1) bonding social capital, reflecting intra-community ties, (2) bridging social capital, reflecting inter-community ties and, (3) linking social capital, referring to relations with formal institutions beyond the community (Mignone, 2009).
} 


\section{The Institutional Economics Approach to Labour Market}

Unlike neoclassical economics, the institutional economics approach to labour market assumes imperfect competition, thus implying that disparities and "nonmarketclearing" outcomes are a basic characteristic of the labour market (Wang \& Dobbs, 2008). Institutionalists oppose the notion that economic agents maximize utility, as proposed by neoclassical economists. Instead, they endorse a theory of motivation where imperfect information and bounded rationality dictate "satisficing" as the highest attainment - in other words, individuals pursue a course of action only until they reach a minimum satisfactory level, the "good enough level," even if that is not the best they can do (Kaufman, 2004).

Focusing primarily on the social context in which labour market outcomes occur, the institutional approach examines how both individual employers and individual employees form subjective perceptions of marginal benefits and marginal costs associated with a particular decision and how institutions affect those perceptions under uncertainty. This line of thinking drew from Kenneth Arrow's path-breaking work on the economics of information, in which he put forth a conceptual formulation of mutual interdependence in which individuals both shape institutions and are shaped by them. This conception diverges from the orthodox economic presumption of methodological individualism as Arrow maintained that rationality is not a property of the individual alone because the "perceptions of others and, in particular, of their rationality become part of one's own rationality" (Arrow, 1987, p. 387). This mutual interdependence argument was well received by those whose observations of "real" life experiences have 
led them to conclude that human capital acquisition is a social product and not an individual investment decision at all (Tomaskovic-Devey, Thomas, \& Johnson, 2005).

Institutions have been defined differently by different scholars, but the most encompassing description of institutions, their origin and the way they operate, is given by Douglass North, who defines them as:

humanly devised constraints that structure political, economic and social interaction. They consist of both informal constraints (sanctions, taboos, customs, traditions, and codes of conduct), and formal rules (constitutions, laws, property rights). Throughout history, institutions have been devised by human beings to create order and reduce uncertainty in exchange. Together with the standard constraints of economics they define the choice set and therefore determine transaction and production costs and hence the profitability and feasibility of engaging in economic activity. (North, 1991, p. 98, emphases added)

The first attempt to articulate the institutionalists' postulations in relation to labour markets was done through the "dual labour market theory," and later through the more complex "segmented labour market theory" (Gray \& Chapman, 2004). At the same time, a number of scholars influenced by varieties of Marxist theory were also describing their own perceptions of the "fragmented" or "split' nature of labour markets, following, to different degrees, the classic Marxist class struggle reasoning, "divide-and-conquer" (Bonacich, 1972; Bowles \& Gintis, 1975; Roemer, 1979; Reich, 1981).

Regardless of the perceived degree of segmentation, the institutional labour market approach sees labour markets as divided into two or more separate sectors in which wages and work conditions depend as much on the jobs and the sectors as on the amount of human capital individual workers bring to a given job in a given sector (Stiglitz, 1973; Marshall, 1974; Leontaridi, 1998; Kinnear, 2004). Formal credentials, from the segmented labour market point of view, are merely "screening devices" serving to "open the door" to upward mobility to the more advantaged population "within the 
internal labour markets, which enables even greater enhancement of [their] human capital through the opportunity to acquire specific training and/or costless learning-by-doing experience" (Sobel, 1982, p. 262). What follows is a brief overview of the existing literature, starting with earliest conceptions of this approach by its founders to the more contemporary applications of the theory.

The origin of the modern theories of labour market segmentation dates back to Clark Kerr's early 1950s work in which he developed the concept of "institutional" labour markets that are governed by formal and informal rules rather than by economic variables (Boyer \& Smith, 2001, p. 213). In this work, Kerr depicted his observations of "balkanization" of labour markets into internal and external components. These two components were connected only at the entry job level while "the remainder of jobs above the entry level [were] filled from within by institutional rules delineating access to particular jobs and determining the pay structure" (Sobel, 1982, p. 262).

Building on Kerr's work, John Dunlop observed that segmented labour markets were not only filled with various entry barriers, but that they were fundamentally different from other markets. His work extended Kerr's internal labour market theory by distinguishing between what he referred to as the "political" theory of wages in the internal labour market and the "economic" theory of wages in the external labour market, asserting that both theories must be studied to fully understand the wage determination process. But in doing so, Dunlop cautioned that one should not put too much emphasis on the distinction between the two but rather analyze each as closely and as detailed as possible (Kinnear, 2004, p. 109). 
The economic mobility issues facing disadvantaged workers in segmented labour markets and institutional factors perpetuating those issues were first captured by Michael Piore in his attempt to formally construct a segmented labour market theory. In mid1970s, Piore theorized that the labour market was not a homogeneous entity, but, instead, was composed of two independent sub-markets, reflecting a dichotomy that has developed over time between a high-wage primary sector and a low-wage secondary sector. These two sub-markets were characterized not only by earnings differentials, but also by "qualitative differences in working conditions, processes of skill acquisition, job mobility patterns, and organizational rules" (Piore, 1975, p. 126).

As Piore observed, the primary sector, then largely filled with prime-age white workers, offered generally favourable working conditions in the sense that there was steady employment and job security, and that the rules that governed the organization of employment were well defined and equitable. This sector was further subdivided into upper and lower tier, with formal educational credentials being required only for employment in the upper tier. Formal education played no role in the secondary sector, where general work conditions were less favourable, as revealed by a lack of job security and high turnover rates. This sector offered a few opportunities for training or career progress, the work tended to be menial and repetitive, and the jobs were typically filled with those whose attachment to paid employment had traditionally been weak (Piore, 1975).

Matching this duality in the characteristics of jobs was the duality within the industry, where primary (core) and secondary (periphery) industrial sectors coexisted. Firms existing in the primary sectors were generally large-scale production firms 
operating in both national and global product markets and making an extensive use of capital-intensive methods. These firms generally had a monopoly power and a strong trade union representation. In contrast, firms in the periphery sector were small, nonunionized firms operating in competitive local product markets and employing labourintensive methods of production. Piore thought that the two dualities were closely related in the sense that one could easily observe an ample overlap between the primary jobs and primary sector industries, and between secondary jobs and secondary sector industries, with specific institutions in place that kept the dualities intact (Piore, 1975).

Others have also observed that the prevailing social and institutional arrangements served to reinforce the dual labour market structures, thus allowing persistence of labour market discrimination, as reflected in the different treatment of otherwise equally productive workers. Marshall (1974), for instance, argued that the formal employment services served to perpetuate secondary employment conditions by referring workers with prescribed personal characteristics to secondary jobs. According to Marshall, it was not these workers' lack of job skills that barred them from the primary sector but rather the perception that they would work irregularly and would be unreliable. They then get trapped in the periphery industry where the vicious cycle continues. Unions, on the other hand, which operate mainly in the primary sector, contribute to the perpetuation of better wages and job security for the incumbent workers in the primary sector (Marshall, 1974). In his theoretical formulation, Piore provided an extensive explanation of how mobility between the two main segments is severely restricted, arguing that it reflects institutional and social barriers in the labour market more than a lack of productive capacities among lower segment workers. As he concluded, "socioeconomic movement 
in our society is not random, but tends to occur in more or less regular channels [as] people hold jobs in some regular order or sequence [that can be conceptualized as] "mobility chains sequence" (Piore, 1975, p. 128). While admitting that his "mobility chains" typology applied more to the male than female jobs and more to urban, capitalist market settings than to rural, non-capitalist market settings, Piore maintained that a reflection on segmental differences and the constraints they place on all disadvantaged workers is essential for understanding the true nature of discrimination and marginalization they are exposed to in the labour market (Piore, 1975).

Piore was describing segmentation in the 1970s labour market, but his concept of "internal labour market" and his general depiction of how the labour market discrimination operates remains relevant to date (Piore, 2002; Agocs, 2002; Flecker, 2007; Schmidt, 2010). Even though the old concept of internal labour markets, dominated by large bureaucratic organizations, appears increasingly narrow and less relevant to the new system of information-driven economy, characterized by new and rapidly changing forms of economic organization (Chun, 2008),

the world into which we are moving is not a world in which the pricing and allocation of labour will be wholly governed by market forces, unmediated by administrative rules and social customs. We will, in other words, continue to have internal labour markets in the broad sense of the term. But the particular forms these internal labour markets will take are extremely varied. No single form will be dominant in the way in which the bureaucratic organization was dominant earlier in the post-war period. (Piore, 2002, p. 273)

As in the rest of post-industrial world, starting in late 1990s, a surge of fundamental changes have occurred in Canada, creating a steady demand for labour flexibility (Agocs, 2002). The very nature of knowledge, skills, and experience have been redefined as have the ways of interactions between workers and employers. To 
accommodate this flexibility, the legal structures in which business operates have also been changed. But, as Piore (2002) observes it,

the structures which have emerged in their place are no less internal labour markets, albeit internal labour markets whose borders tend to be socially defined (as opposed to coincident with the boundaries of a formal organization). They are not flexible in the strict sense of the term, but they appear to be compatible with the kind of flexibility required by the current economic environment. They seem, in other words, to be consistent with economic efficiency, growth, and prosperity but they are likely to pose their own problems for policy and for research. (p. 277)

The main problem the new internal labour markets pose for policy is the problem of social equity (Agocs, 2002; Piore, 2002) and the problem is likely to arise because "the social groups which are emerging within professional and occupational associations are formed along precisely the lines of sex, race and ethnicity which have been the concern of equal employment opportunity policy for the last fifty years" (Piore, 2002, p. 277). Employment equity policy in Canada is based on an assumption of long-term jobs with somewhat specialized job descriptions, not the "flexible" and contingent jobs favoured by many employers today" (Agocs, 2002, p. 274).

While acknowledge that these new arrangements and social networks can be utilized by the disadvantaged group members as mediums to gain access to better positions in the labour market, Piore (2002) warns that,

their reach could easily extend beyond the remedial to create privilege; in many ways, these new groups are like the social organizations that maintained white, male dominance earlier. It was in part that dominance which the bureaucratic rules of the large corporation were designed to control, and a good deal of our equal employment policy operated through these rules, purging them of bias. It will be harder to identify the source of bias and to control it without a set of explicit rules upon which to focus. Hence, for research, it seems to me, the critical question is to understand the relationship between economic and social processes. (p. 277) 
Gaining an understanding of the relationship between economic and social processes and demonstrating the existence of segmentation in the labour market, however, has proven to be rather a daunting task and has been subject serious criticisms. As the existing segmentation literature reveals, the examined outcome depends very much on the choice of methodology, and in particular on the way segments are defined. Although several approaches exist, there is little consensus on which method is the most appropriate. Following the earlier studies on dual labour market, most researchers have used industry as the basis of analysis. Industry, however, is deemed an imperfect starting point for describing segmentation because one can easily observe that almost all industries have both primary and secondary elements (Dickens \& Lang, 1988).

Starting in the early 1970 s, a number of studies have looked at different aspects of the segmented labour market theory by examining the hiring and compensation practices of individual firms as well as exploring the reasons for and the consequences of the existence of internal labour markets on the distribution of earnings and on the difference in economic opportunity among different groups of populations (Sobel, 1982; Boyer \& Smith, 2001; Gray \& Chapman, 2004). Making generalizations from these studies, however, has proven to be rather difficult due to the nature of the competing segmented labour market theories used, each with a somewhat different approach and specifications of the segmented sectors, and the nature of the empirical data employed. As Sobel (1982) observed, lacking a unified, clearly specified model and theoretical base, the segmented labour market researchers work with their own theories and their own specifications that best fit the topic under investigation. Before concluding this chapter, the following 
section provides a brief discussion on the issue of methodological clashes and congruencies between the two approaches. 


\section{Methodological Considerations}

As originally conceptualized, the research methods of the segmented labour market theory fell clearly outside those of the mainstream economic theory as they dictated a strictly descriptive, historical, case-study approach to labour market research (Piore, 1979; 1983; Dugger, 1979; Dickens \& Lang, 1988). The four key features of this approach, as summarized in Kaufman (2004), included: "the emphasis on fact-gathering, the importance of realism of assumptions, the virtues of a "go and see" participant/observer method of investigation, and the necessity of an interdisciplinary approach to theory-construction" (p. 16-17).

With these four features highlighted, the institutional economics research approach appeared to be in direct opposition to the neoclassical research standards, and as current literature reveals, this antithetical notion is still strongly present. However, there is no consensus among the scholars on either side. While some institutional economists consider their research approach to be mutually exclusive with neoclassicism (Gray \& Chapman, 2004), others consider the approaches of institutionalism and neoclassicism to be supplementary (Sobel, 1982; Samuels, 1995). Likewise, as Kaufman (2004) points out, there are many economists who do not self-identify as institutionalists, yet important aspects of their work "are broadly institutional in method and/or theory" (p. 25).

The visions of antithetical relations between the institutional and neoclassical proponents are likely to diminish further in the face of constant evolution of the institutional labour market approach (Samuels, 1995; Piore, 2002a) and the growing 
interest among neoclassical labour economists in relevant public policy issues (Boyer \& Smith, 2001). As Samuels (1995) has observed,

during the last twenty-five years there has been a considerable renaissance of institutionalism, as well as of other heterodox approaches to economics. Institutionalism today is rich, complex and diverse. Although certain strains of it have been myopic, backward looking and in conflict, institutionalism is, quite possibly, on the threshold of a great transformation, one which will build on foundations laid by Thorstain Veblen, John R. Commons and others, but which will strike out on its own, giving effect to the pragmatic methodological promise of institutionalism in the past. (p. 570)

Similar tendencies are being observed on the other side. Neoclassical labor economists, as Boyer and Smith (2001) have observed,

are now - at long last, some would say — addressing some of the issues that had so consumed the interest of neoinstitutionalists, and they have turned out to be similar to their neoinstitutionalists predecessors in the fundamental desire to understand how employers and employees really behave. (p. 218)

Indeed, the existing literature indicates steady progress toward what seems to be 'blending' of the two approaches (Piore, 2002a), albeit still strongly adhering to their core empirical analysis methods. Strictly following the institutional methodological guidelines, the work of the earlier generations of the labour market institutionalists remained descriptive, presenting mainly observational studies, interviews, and historical and institutional analysis (Piore, 1983; Gray \& Chapman, 2004). Later generations of institutionalists maintained the institutionalists' data collection guidelines but "began to develop discernible causal arguments about the origin and maintenance of segmentation" (Gray \& Chapman, 2004, p. 118).

Similarly, the neoclassical economists have begun incorporating institutional postulations into their conventional methods of analysis. Viewing dualism as a useful simplification of labour markets, in late 1980s some neoclassical economists have 
attempted applying the standard econometrics techniques to study wages by jointly estimating two log-wage equations, one for each sector of the segmented labour market, with a "switching" equation, allowing them to determine in which sector individuals were employed (Dickens \& Lang, 1988). Their conclusion was that "the dual labor market model not only outperforms an equally complex single labor market model, but it provides a succinct description of the wage distribution" (Dickens \& Lang, 1988, p. 131). 


\section{Concluding Remarks}

The rise of these blending tendencies is encouraging in the sense that it can alter the long-held view about the fundamental differences between the institutional and neoclassical approaches to labour economics (Wang \& Dobbs, 2008). One of these differences has sprung from the fact that, historically, the institutionalists have emphasized the word "labour" while the neoclassical economists have emphasized the word "economics" when approaching labour economics issues (Boyer \& Smith, 2001). It seems now that both groups have come to recognize the importance of the other group's main postulations, education and experience on the human capital side and the institutional arrangements on the institutional side. However, as pointed out earlier, each group continues ignoring the other group's variables in its empirical work and relies mainly on its established empirical tools (Piore, 2002a).

While the differences in empirical orientation toward labour market research between the two approaches remain appreciable, they do not necessarily make the two approaches antithetical. As Samuels (1995) points out "notwithstanding their different methodological and philosophical foundations and practices, institutional and neoclassical economics are supplementary [and] one does not have summarily to reject that with which one tends to disagree; one can learn from the other" (p. 570). Furthermore, some research topics, such as the one being examined in this thesis, requires some sort of blending of the two approaches for a fuller understanding of the issues at hand. Indeed, the present research topic requires an investigation of both "socio-political" and "economic" components if one is to make an earnest attempt to understand the 
determination of the labour market outcomes for this segment of Canadian population. As John Dunlop suggested, "one can accomplish this by analyzing each component according to its own characterizations, without imposing on them any predetermined model" (Kinnear, 2004, p. 109).

This thesis is designed to expand the line of work that explores the determinants of labour market outcomes of designated social groups in Canada, namely Aboriginal groups, by applying both classical econometric and institutional non-econometric methods of inquiry. Given the limitations of the neoclassical human capital approach, the more pragmatic perspective of institutional economics can aid in better understanding the labour market motivations and outcomes of Aboriginal workers, particularly of those residing on reserves and in northern parts of Canada. As summarized above, the neoclassical approach holds that inequality exists in the labour market primarily because some workers are more productive than others. The less-productive workers earn less than others and fare worse in the labour market because they invest less in productivityenhancing characteristics and because they exhibit a lesser degree of commitment to the labour market. It follows then that, aside from innate abilities, if all workers invested the same amount of resources in productivity-enhancing characteristics, the distribution of earnings would be exactly the same.

The observed persistence of earnings and employment differentials between seemingly equally productive Aboriginal and non-Aboriginal workers over the past three decades is a clear sign of the weakness of the neoclassical approach in explaining the real issue. Indeed, the static nature of this approach is incapable of explaining the long-term dynamics of the processes and the more complex characteristics of the labour market 
segmentation and marginalization of Aboriginal workers. As Wilber and Harrison (1978) pointed out, "formal [neoclassical economic] models simply cannot handle the range of variables, the specificity of institutions, and the nongenerality of behavior" that can be observed in natural settings and conveyed by those affected by it (p.72).

A strict application of neoclassical theory to labour market also offers no support for those who strongly believe that an active anti-discrimination policy is essential (Agocs, 2002; Flecker, 2007). The neoclassical approach only emphasizes the need to promote competition in the product and labour markets, and the need to break down informational barriers that act against Aboriginal workers. The theory suggests that such policies, in the long run, would promote a greater choice and provide the right environment for eradicating discrimination through market forces (Schultz, 1961).

In response to this, one cannot help but ask, how long is the long run? As Drost and Richards (2003, p. 1) point out "aboriginal poverty is probably Canada's most serious social policy blight" and as such requires immediate and continuous policy responses. The fact that the young Aboriginals are the fastest-growing labour pool in Canada only adds to this urgency. As discussed earlier in this chapter, although some gains in educational attainment have been realized, nationally this 'potential' labour pool of workforce is still less educated than the general population. This implies that this potential workforce will need significant improvements in the productivity-enhancing characteristics but it may also need more effective protection from labour market discrimination. This thesis tries to provide some timely insights into the needs and concerns of Aboriginal workers to assist policy-makers in receiving this new wave of 
(potential) workforce and in helping them integrate more easily (and more successfully) than their predecessors in the Canadian labour market.

The thesis adopts a mixed methods research approach, "the explanatory sequential design," in which elements of both quantitative and qualitative research procedures are combined, as specified in Creswell and Plano Clark (2007). This particular design is a two-phase mixed methods research design, known as an "unusual blend" of the "followup explanation model" in which emphasis is placed on the quantitative methods. The first phase involves an analysis of quantitative data, which is followed by the subsequent collection and analysis of qualitative data. The purpose of this design is to use qualitative data to help explain quantitative results. The adopted assumption is that in combination, the quantitative and the qualitative methods will serve to provide a better understanding of the issue at hand than would either method alone (Creswell \& Plano Clark, 2007). 


\section{References}

Agocs, C. (2002). Canada's employment equity legislation and policy, 1987-2000: The gap between policy and practice. International Journal of Manpower, 23(3), 256276.

Anonson, J., Desjarlais, J., Nixon, J. Whiteman, L., \& Bird, A. (2008). Strategies to support recruitment and retention of First Nations youth in baccalaureate nursing programs in Saskatchewan, Canada. Journal of Transcultural Nursing, 19(3), 274-283.

Annen, K. (2007). Economic returns to social capital in the urban informal sector in developing countries: Micro evidence from small textile producers in Bolivia. Unpublished Manuscript. University of Guelph, Department of Economics. Retrieved March 2009: http://www.economics.uoguelph.ca/kannen/ Informal\%20Firms.pdf

Arrow, K. J. (1987). Rationality of self and others in an economic system. In R. Hogarth \& M. Reder (Eds.), Rational choice: The contrast between economics and psychology (pp. 201-215). Chicago: University of Chicago Press.

Baker, M. \& Benjamin, D. (1994). The performance of immigrants in the Canadian labor market. Journal of Labor Economics, 12(3), 369-346.

Becker, G. S. (1996). The economic way of looking at behavior: The Nobel lecture. Essays in Public Policy, No. 69, Stanford University: Hoover Institution on War, Revolution and Peace.

Becker, G. S. (1993). The human capital: A theoretical and empirical analysis, with special reference to education $\left(3^{\text {rd }}\right.$ ed.). Chicago: University of Chicago Press. 
Becker, G. S. \& Murphy, K. M. (2000). Social economics: Market behavior in a social environment. Cambridge: The Balknap Press of Harvard University Press.

Benjamin, D., Gunderson, M., \& Riddell, C. W. (2002). Labour market economics: Theory, evidence and policy in Canada ( $5^{\text {th }}$ ed.). Toronto: McGraw-Hill Ryerson Limited.

Bernier, R. (1997). The dimensions of wage inequality among Aboriginal Peoples. Research Paper No. 109, Statistics Canada, Analytical Studies Branch - Research Paper Series. ISSN: 1200-5223, No. 11F0019MPE No. 109.

Biswal, B. (2008). Literacy performance of working-age Aboriginal people in Canada: Findings based on the International Adult Literacy and Skills Survey (IALSS) 2003. Research report prepared for the Human Resources and Social Development Canada, Learning Policy Directorate, Strategic Policy and Research, July 2008, Catalogue no.: HS28-147/2008E-PDF.

Bonacich, E. M. (1972). A theory of ethnic antagonism: The split labor market. American Sociological Review, 37(5), 547-559.

Boudarbat, B., Lemieux, T., \& Riddell, C. (2006). Recent trends in wage inequality and the wage structure in Canada. In D. A. Green \& J. R. Kesselman (Eds.), Dimensions of inequality in Canada (pp. 274-306). Vancouver: UBC Press.

Bowles, S. \& Gintis, H. (1975). The problem with human capital theory - A Marxian critique. The American Economic Review, 65(2), 74-82.

Boyer, G. R. \& Smith, R. S. (2001). The development of the neoclassical tradition in labor economics. Industrial and Labor Relations Review, 54(2), 199-223. 
Brough, M., Bond, C., Hunt, J., Jenkins, D. Shannon, S., \& Schubert, L. (2006). Social capital meets identity: Aboriginality in an urban setting. Journal of Sociology, $42(4), 396-411$.

Card, D. (2001). Estimating the return to schooling: Progress on some persistent econometric problems. Econometrica, 69(5), 1127-1160.

Chun, J. J. (2008). The limits of labor exclusion: Redifining the politics of split labor markets under globalization. Critical Sociology, 34(3), 433-452.

Commons, J. R. (1931). Institutional economics. American Economic Review, 21(3), 648657.

Creswell, J. W. \& Plano Clark, V. L. (2007). Designing and conducting mixed methods research. Thousand Oaks, CA: Sage.

De Silva, A. (1999). Wage discrimination against natives. Canadian Public Policy, 25(1), $65-85$.

Dickens, W. T. \& Lang, K. (1988). The re-emergence of segmented labor market theory. American Economic Review, 78(2), 129-134.

Drost, H. (1994). Schooling, vocational training and unemployment: The case of Canadian Aboriginals. Canadian Public Policy, 20(1), 52-65.

Drost, H. \& Richards, J. (2003). Income on-and-off reserve: How Aboriginals are faring. C.D. Howe Institute, Commentary No.175. Toronto: C. D. Howe Institute.

Dugger, W. (1979). Methodological differences between institutional and neoclassical economics. Journal of Economic Issues, 13(4), 899-909.

Durlauf, N. S. (2002). On the empirics of social capital. The Economic Journal, $112(483), 459-479$. 
Flecker, K. (2007). Racism remains a problem: Why are people of colour having trouble getting good jobs? Canadian Centre for Policy Alternatives Monitor, October 1, 2007, p. 32-33.

Foley, W. M. \& Edwards, B. (1999). Is it time to disinvest in social capital? Journal of Public Policy, 19(2),141-73.

George, P. \& Kuhn, P. (1994). The size and structure of native-white wage differentials in Canada. The Canadian Journal of Economics, 27(1), 20-42.

Gee, E., Kobayashi, K. \& Prus, S. (2006). Ethnic inequality in Canada: Economic and health dimensions. In D. A. Green \& J. R. Kesselman (Eds.), Dimensions of inequality in Canada (pp. 249-271). Vancouver: University of British Columbia Press.

Glaeser, E. L., Laibson, D., \& Sacerdote, B. (2002). The economic approach to social capital. The Economic Journal, 112(483), 437-458.

Goldmann, G. \& Delic, S. (in press). Counting Aboriginal Peoples in Canada. In F. Trovato \& A. Romaniuk (Eds.), Aboriginal populations: Social, demographic, and epidemiological dimensions. Edmonton: University of Alberta Press.

Goldmann, G. (2007). The Aboriginal population and the census: 135 years of information - 1871 to 2006. Paper presented at the 2007 Annual Meetings of the Population Association of America, New York, March 31, 2007.

Goldmann, G. \& Siggner, A. (1995). Statistical concepts of Aboriginal people and factors affecting the counts in the Census and the Aboriginal Peoples Survey. Paper presented to the 1995 Symposium of the Federation of Canadian Demographers, October 23-25. 
Gomez, R. \& Santor, E. (2001). Membership has its privileges: The effect of social capital and neighbourhood characteristics on the earnings of microfinance borrowers. Canadian Journal of Economics, 34(4), 943-966.

Government of Canada (2009). Federal framework for Aboriginal economic development. Published under the authority of Minister of Indian Affairs and Northern Development and Federal Interlocutor for Métis and Non-Status Indians. Ottawa: Public Works and Government Services Canada. ISBN: 978-1-10050004-1.

Government of Canada (2008). Toward a new federal framework for Aboriginal economic development: Discussion guide. Published under the authority of Minister of Indian Affairs and Northern Development and Federal Interlocutor for Métis and Non-Status Indians. Ottawa: Public Works and Government Services Canada. ISBN: 978-1-100-10324-2.

Gray, J. \& R. Chapman (2004). The significance of segmentation for institutionalist theory and public policy. In D. P. Champlin \& J. T. Knoedler (Eds.), The institutionalist tradition in labor economics (pp. 117-130). Armonk: M.E. Sharpe. Guimond, E. (2003). Fuzzy definitions and population explosion: Changing identities of Aboriginal groups in Canada. In D. Newhouse \& E. Peters (Eds.), Not strangers in these parts: Urban Aboriginal Peoples (pp. 35-50). Ottawa: Policy Research Initiative.

Hull, J. (2008). Aboriginal youth in the Canadian labour market. Research Brief, Policy Research Initiative. Horizons, 10(1), 40-44. 
Hum, D. \& Simpson, W. (1999). Wage opportunities for visible minorities in Canada. Canadian Public Policy, 25(3), 379-394.

Human Resources and Social Development Canada (2007). Looking-ahead: A 10-year outlook for the Canadian labour market (2006-2015) report. Human Resources and Social Development Canada, Strategic Policy Research Directorate, Labour Market and Skills Forecasting and Analysis Unit. SP-615-10-06E

Hunter, B. H. (2000). Social exclusion, social capital and Indigenous Australians: Measuring the social costs of unemployment. Canberra: Centre for Aboriginal Economic Policy Research, Australian National University. Discussion Paper No. 204.

INAC (2007). Aboriginal demography: Population, household and family projections, 2001-2026. Indian and Northern Affairs of Canada, Strategic Research and Analysis Directorate, Policy and Research Division. Catalogue no. R3-63/2007.

INAC (2000). Aboriginal labour force characteristics from the 1996 Census. Indian and Northern Affairs of Canada, First Nations and Northern Statistics Corporate Information Management Directorate, Information Management Branch. Catalogue no. R21152000.

Kaufman, B. E. (2004). The institutional and neoclassical schools in labor economics. In D. P. Champlin \& J. T. Knoedler (Eds.), The institutionalist tradition in labor economics (pp. 13-38). Armonk: M.E. Sharpe.

Kinnear, D. (2004). Two sides of the same coin: Institutionalist theories of wage rates and wage differentials. In D. P. Champlin \& J. T. Knoedler (Eds.), The institutionalist tradition in labor economics (pp. 105-116). Armonk: M.E. Sharpe. 
Knorringa, P. \& Van Staveren, I. (2007). Beyond social capital: A critical approach. Review of Social Economy, 65(1), 1-9.

Kuhn, P. \& Sweetman, A. (2002). Aboriginals as unwilling immigrants; Contact, assimilation and labour market outcomes. Journal of Population Economics, 15(1), 331-355.

Leontaridi, M. R. (1998). Segmented labour markets: Theory and evidence. Journal of Economic Surveys, 12(1), 63-101.

Livingstone, D. W. (1997). The limits of human capital theory: Expanding knowledge, informal learning and underemployment. Policy Options, 18(6), 9-13.

Maglen, L. R. (1990). Challenging the human capital orthodoxy: The educationproductivity link re-examined. The Economic Record, 66, 281-294.

Marshall, R. (1974). The economics of racial discrimination: A survey. Journal of Economic Literature, 12(3), 849-871.

Maxim, P. \& White, J. (2006). School completion and workforce transition among urban Aboriginal youth. in White, J. P., S. Wingert, D. Beavon and P. Maxim (Eds.). Aboriginal Policy Research: Moving Forward, Making a Difference. Volume 3, (pp. 33-52). Toronto: Thompson Educational Publishing.

Maxim, P. S., White, J. P. \& Beavon, D. (2003). Dispersion and polarization of income among Aboriginal and non-Aboriginal Canadians. In J. P. White, P. S. Maxim, \& D. Beavon (Eds.), Aboriginal conditions: Research as a foundation for public policy (pp. 222-247). Vancouver, British Columbia: UBC Press.

Mendelson, M. (2006). Aboriginal Peoples and postsecondary education in Canada. Ottawa: Caledon Institute of Social Policy. 
Mignone, J. (2009). Social capital and Aboriginal communities: A critical assessment. Journal of Aboriginal Health, 5(3), 100-147.

Nelson, B. J., Kaboolian, L. \& Carver, K. A. (2003). The concord handbook: How to build social capital across communities. The Concord Project, UCLA School of Public Policy and Social Research, University of California, Los Angeles.

North, D. (1991). Institutions. Journal of Economic Perspectives, 5(1), 97-112.

Pendakur, K. \& Pendakur, R. (2011). Aboriginal income disparity in Canada. Canadian Public Policy, 37(1), 61-83.

Pendakur, K. \& Pendakur, R. (2007). Minority earnings across the distribution. Canadian Public Policy, 33(1), 41-62.

Pendakur, K. \& Pendakur, R. (2002). Colour my world: Have earnings gaps for Canadian-born ethnic minorities changed over time? Canadian Public Policy, 28(4), 489-512.

Pendakur, K. \& Pendakur, R. (1998). The colour of money: Earnings differentials among ethnic groups in Canada. Canadian Journal of Economics, 31(3), 518-548.

Perrin, Thorau \& Associates Ltd. (2009). First Nations and economic prosperity in the coming decade. A paper prepared for the Business Council of British Columbia Outlook 2020 project.

Piore, M. J. (1975). Notes for a theory of labor market stratification. In R. C. Edwards, M. Reich, \& D. M. Gordon (Eds.), Labor market segmentation (pp. 125-150). Lexington: D. C. Health and Company.

Piore, M. J. (1979). Qualitative research techniques in Economics. Administrative Science Quarterly, 24(4), 560-569. 
Piore, M. J. (1983). Labour market segmentation: To what paradigm does it belong? American Economic Review, 73(2), 249-253.

Piore, M. J. (2002). Thirty years later: Internal labour markets, flexibility and the new economy. Journal of Management and Governance, 6(4), 271-279.

Piore, M. J. (2002a). Economics and Sociology. Revue Economique, 53(2), 291-300.

Reich, M. (1981). Racial inequality: A political-economic analysis. Princeton: Princeton University Press.

Reich, R. B. (1992). The work of nations: Preparing ourselves for $21^{\text {st }}$ Century capitalism. New York: Vintage Books.

Richter, R. (2005). The new institutional economics - Its start, its meaning, its prospects. European Business Organization Law Review, 6(2), 161-200.

Roemer, J. (1979). Divide and conquer: Microfoundations of a Marxian theory of wage discrimination. The Bell Journal of Economics, 10(2), 695-705.

Samuels, W. J. (1995). The present state of institutional economics. Cambridge Journal of Economics, 19, 569-590.

Schmidt, C. (2010). Systemic discrimination as a barrier for immigrant teachers. Diaspora, Indigenous, and Minority Education, 4(1), 235-252.

Schultz, T. W. (1961). Investment in human capital. American Economic Review, 5l(1), $1-17$.

Senge, P. M. (1990). The fifth discipline: The art and practice of the learning organization. New York: Doubleday Currency.

Sharpe, A., Arsenault, J. F., Lapointe, S. \& Cowan, F. (2009). The effect of increasing Aboriginal educational attainment on the labour force, output and fiscal balance. 
Centre for the Study of Living Standards, CSLS Research Report 2009-3, prepared for the Educational Branch of Indian and Northern Affairs Canada.

Sloane-Seale, A., Wallace, L. \& Levin, B. (2000). Educational achievements and labour market outcomes of students in the University of Manitoba Access Program. The Canadian Journal of Native Studies, 20(2), 347-370.

Smith, S. (2003). Labour Economics ( $2^{\text {nd }}$ ed.). London: Routledge.

Sobel, J. (2002). Can we trust social capital? Journal of Economic Literature, 40(1), 139154.

Sobel, I. (1982). Human capital and institutional theories of the labor market: Rivals or complements? Journal of Economic Issues, 16(1), 255-272.

Spence, M. (2002). Signaling in retrospect and the informational structure of markets. American Economic Review, 92(3), 434-459.

Statistics Canada (2008). Selected Labour Force Characteristics (50), Aboriginal Identity (8), Age Groups (5A), Sex (3) and Area of Residence (7) for Population 15 Years and Over, for Canada, Provinces and Territories, 2001 Census - 20\% Sample Data. Ottawa: Statistics Canada. Census 2001 and Census 2006. Release date : December 9, 2008. Catalogue number: 97F0011XCB2001044.

Statistics Canada (2008a). Employment Income Statistics (4) in Constant (2005) Dollars, Work Activity in the Reference Year (3), Aboriginal Identity, Registered Indian Status and Aboriginal Ancestry (21), Age Groups (5A), Highest Certificate, Diploma or Degree (5) and Sex (3) for the Population 15 Years and Over With Employment Income of Canada, Provinces, Territories, 2000 and $2005-20 \%$ 
Sample Data. Ottawa: Statistics Canada. Census 2001 and Census 2006. Release date: December 9, 2008. Catalogue number: 97-563-XCB2006061.

Statistics Canada (2008b). Aboriginal Identity (8), Highest Certificate, Diploma or Degree (14), Major Field of Study - Classification of Instructional Programs, 2000 (14), Area of Residence (6), Age Groups (10A) and Sex (3) for the Population 15 Years and Over of Canada, Provinces and Territories, 2006 Census - 20\% Sample Data. Ottawa: Statistics Canada. Census 2006. Release date :

March 4, 2008. Catalogue number: 97-560-XCB2006028.

Statistics Canada (2007). How Statistics Canada identifies Aboriginal Peoples. Ottawa, Minister of Industry, Catalogue No. 12-592-XIE.

Statistics Canada (2007a). Labour force projections for Canada, 2006-2031. Canadian Economic Observer, Catalogue Number: 11-010.

Statistics Canada (2005). Projections of the Aboriginal populations, Canada, provinces and territories, 2001-2017. Ottawa: Minister of Industry, June, Catalogue no. 911547-XIE.

Statistics Canada (2005a). Population projections for Canada, provinces and territories, 2005-2031. Ottawa: Minister of Industry, December, Catalogue no. 91-520-XIE. Statistics Canada (2003). Selected Educational Characteristics (29), Aboriginal Identity (8), Age Groups (5A), Sex (3) and Area of Residence (7) for Population 15 Years and Over, for Canada, Provinces and Territories, 2001 Census - 20\% Sample Data. Ottawa: Statistics Canada. Census 2001. Catalogue number: 97F0011XCB2001042. 
Stiglitz, J. (1973). Approaches to the economics of discrimination. American Economic Review, 63(2), 287-295.

Tomaskovic-Devey, D. Thomas, M. \& Johnson, K. (2005). Race and the accumulation of human capital across the career: A theoretical model and fixed-effects application. American Journal of Sociology, 111(1), 58-89.

Van Staveren, I. \& Knorringa, P. (2007). Unpacking social capital in economic development: How social relations matter. Review of Social Economy, 65(1), 107135.

Walters, D., White, J., \& Maxim, P. (2004). Does postsecondary education benefit Aboriginal Canadians? An examination of earnings and employment outcomes of recent Aboriginal graduates. Canadian Public Policy, 30(3), 283-301.

Wang, G. G. \& Dobbs, R. L. (2008). Institutional economics and human resource development. Advances in Developing Human Resources, 10(6), 770-787.

White, J., Maxim, P., \& Gyimah, S. O. (2003). Labour force activity of women in Canada: A comparative analysis of Aboriginal and non-Aboriginal women. The Canadian Review of Sociology and Anthropology, 40(4), 391-415.

Wilber, C. \& Harrison, C. K. (1978). The methodological basis of institutional economics: Pattern model, storytelling, and holism. Journal of Economic Issues, $12(1), 61-89$.

Williamson, O. (2000). The new institutional economics: Taking stock, looking ahead. Journal of Economic Literature, 38(3), 595-613. 


\title{
Essay I: Economic Downturn and Aboriginal Workers: Evidence from the 2008-2011 Recession
}

\begin{abstract}
Empirical literature examining the dynamics in the Canadian labour market under general economic conditions unanimously reports persistent disparities in labour market outcomes between Aboriginal and non-Aboriginal identity workers. This essay examines the relationship between Aboriginal identity and economic success in the Canadian labour market in the context of an economic downturn. Using matched individual-level monthly data from the Labour Force Survey, September 2008 - May 2011, the essay examines the differences in the impact of the latest recession on Aboriginal and nonAboriginal identity workers in the form of probabilities of layoff and reemployment. Comparisons of transition probabilities reveal that Aboriginal workers were much more likely to be laid off than were non-Aboriginal workers but they were also more likely to be reemployed. Blinder-Oaxaca decompositions suggest that for one subgroup of Aboriginal workers - Métis - a large portion of the difference in the probability of layoff can be attributed to the difference in endowments while for the other subgroup of Aboriginal workers - North American Indian - only a small portion of the difference can be attributed to the difference in endowments, much remains unexplained.
\end{abstract}

Key words: Aboriginal workers, non-Aboriginal workers, recession, layoffs, reemployment, Blinder-Oaxaca decomposition for non-linear models 


\section{Table of Contents}

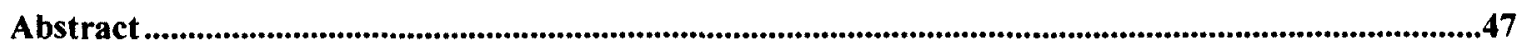

Table of Contents.................................................................................................................................................48

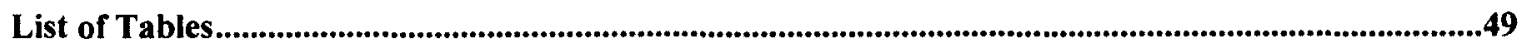

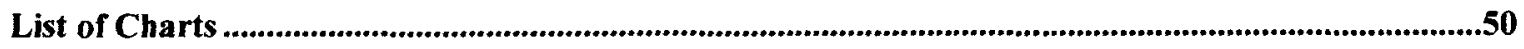

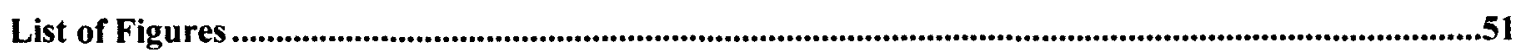

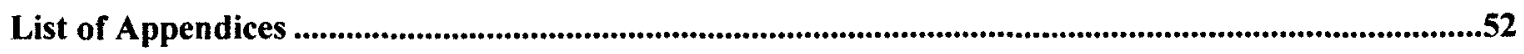

1. Introduction

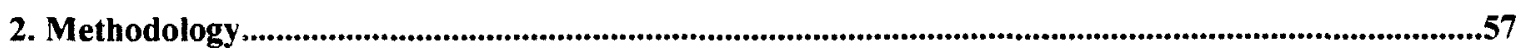

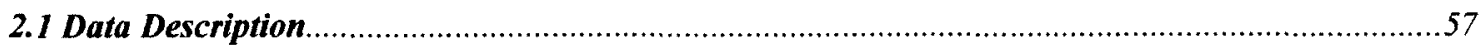

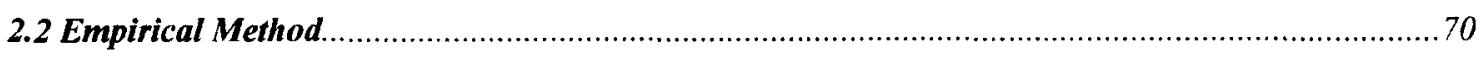

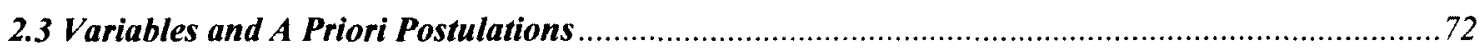

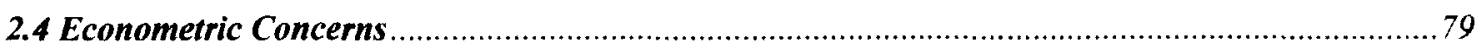

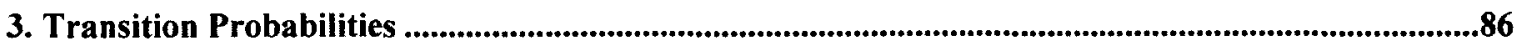

3.1 Estimated Probabilities of Layoff and of Reemployment …......................................................91

3.2 Steady State Employment and Durations of Unemployment .....................................................96

4. Blinder-Oaxaca Decomposition ......................................................................................................................99

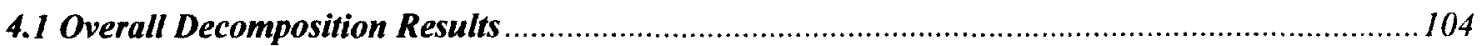

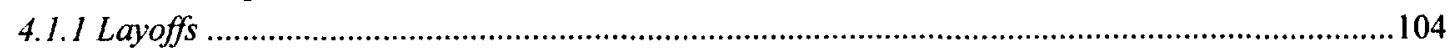

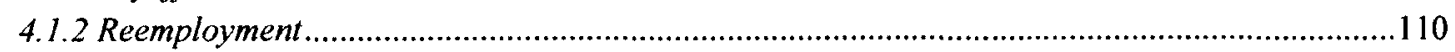

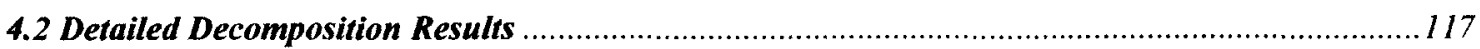

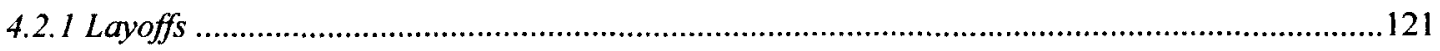

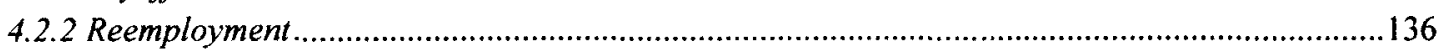

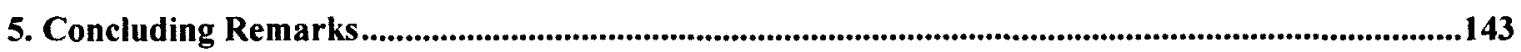

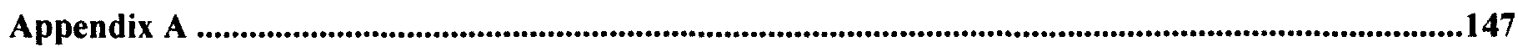

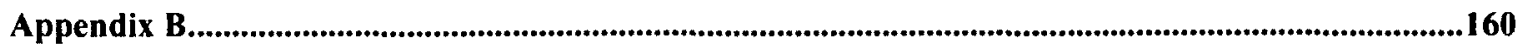

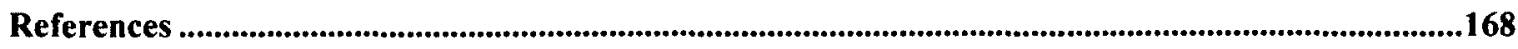




\section{List of Tables}

Table 1: Sample sizes and means of selected sample characteristics, by identity

Table 2: The equilibrium level of employment and average first duration of unemployment, by identity. 96

Table 3: Overall decomposition of the difference in predicted probability of layoff, main sample ... 106

Table 4: Overall decomposition of the difference in predicted probability of layoff, restricted sample

Table 5: Overall decomposition of the difference in predicted probability of reemployment, main sample.

Table 6: Overall decomposition of the difference in predicted probability of reemployment, restricted sample

Table 7: Detailed decomposition of the difference in the predicted probability of layoff for men .....122

Table 8: Detailed decomposition of the difference in the predicted probability of layoff for women 128

Table 9: Detailed decomposition of the difference in the predicted probability of reemployment ....137 


\section{List of Charts}

Chart A: Monthly layoff rates for Aboriginal and non-Aboriginal identity groups, the 2008-

2011 recession.

Chart B: Monthly layoff rates for Aboriginal and non-Aboriginal identity workers, the 2008-

2011 recession 


\section{List of Figures}

Figure 1: Two State Markov-like Model of Labour Force Transitions 


\section{List of Appendices}

Appendix A 1: A Summary of Definitions of Independent Variables.....................................147

Appendix A 2: Mean Characteristics of Paid Employees in the Main Samples, by Identity .............148

Appendix A 3: Mean Characteristics of Paid Employees in the Restricted Samples, by Identity.......151

Appendix A 4: Mean Characteristics of Laid-Off Workers in the Main Samples, by Identity ..........154

Appendix A 5: Mean Characteristics of Laid-Off Workers in the Restricted Samples, by Identity...157

Appendix B 1: Probit estimates used in decompositions of difference in layoffs for men................160

Appendix B 2: Probit estimates used in decompositions of difference in layoffs for women ............163

Appendix B 3: Probit estimates used in reemployment decompositions, Aboriginal identity groups 166 


\section{Introduction}

The economic consequences of Aboriginal identity in the Canadian labour market have been well documented in the academic literature and government reports. Over the past few decades, a number of studies have consistently reported sizable gaps between Aboriginal and non-Aboriginal Canadians in labour market outcomes. Some of these studies have attempted to identify the sources behind the persistence of the disparities, albeit most in the context of general economic growth. Drawing mainly on crosssectional Canadian Census data, the majority of the studies focused on the Aboriginal disadvantage in the form of earnings and income gaps (Kuo, 1976; Patrinos \& Sakellariou; 1992; George \& Kuhn, 1994; De Silva, 1999; Kuhn \& Sweetman, 2002; Walters, White \& Maxim, 2004; Pendakur \& Pendakur, 1998; 2002; 2011) though some have examined the disparities in the labour force indicators such as employment and unemployment rates (Drost, 1994; White, Maxim \& Gyimah, 2003).

Until recently, little was known about the extent and the nature of disadvantages facing Aboriginal identity workers in recessionary periods, largely due to a lack of suitable panel data (Delic, 2009). Taking advantage of a recent amendment (Statistics Canada, 2007) made to the method of collection of monthly data in the Labour Force Survey (LFS), which allowed identification of Aboriginal identity workers, two recent studies (Delic \& Abele, 2010; Usalcas, 2011) have provided some evidence on the impact of the latest recession on Aboriginal identity workers, though these analyses were largely descriptive in nature. To date, no empirical model has been applied to the Aboriginal panel data now available, although the LFS has been recently used to assess the impact of 
the past three recessions on the population as a whole (Chan, Morissette \& Frenette, 2011).

This essay attempts to fill this gap in the literature by presenting findings from an empirical examination of the relationship between Aboriginal identity and economic success in the Canadian labour market in the context of an economic downturn. Drawing on the longitudinal nature of the monthly individual-level data from the LFS, the essay explores the impact of the 2008-2011 recession on Aboriginal identity workers, focusing on two specific aspects that convey the relative degree of vulnerability and the extent of hardship experienced in the adjustment process: the probability of layoff and the likelihood of reemployment following the layoff. The estimated results for layoffs are presented and discussed in a comparative Aboriginal-to-non-Aboriginal identity format, as per the definition of the Constitution Act, $1982 .^{9}$

Although in essence the layoffs resulting from an economic downturn, relative to other types of layoffs, could be viewed as neutral in the sense that an employer does not necessarily apply strategic steps in choosing whom to lay off, especially not when closing a plant (Gibbons \& Katz, 1991; Elvira \& Zatzick, 2002; Wilson, 2005), the existing research suggests that recession layoffs can be discriminatory (Ornati \& Giblin, 1975) and that the racial economic inequality is in fact reproduced through this process of economic change (Mar \& Ong, 1994). Historically, all past economic downturns have

\footnotetext{
${ }^{9}$ The concept of Aboriginal identity population in the LFS pertains to the members of the three distinct groups of Aboriginal people (North American Indians, Métis, and Inuit) that have been recognized by Canadian Constitution (the Constitution Act, 1982). Although not identical, the concept is very similar to the one derived from Canadian Census data in the sense that it includes only individuals who self-identify as belonging to at least one of the three recognized Aboriginal identity groups (Statistics Canada, 2007). When mentioned in an aggregate sense, the concept 'Aboriginal identity workers' in this essay pertains to the members of all three Aboriginal identity groups living off reserve. Disaggregated analyses, however, are carried only for Métis and North American Indian groups as the observations on people of Inuit identity in this data source were too small for a reliable separate analysis. The concept of non-Aboriginal identity workers pertains to the rest of the Canadian working age population in the labour force.
} 
had substantially more negative effects on ethnic and racial minorities than on the mainstream population (Hu \& Taber, 2005). Studies from the United States, for instance, report a much higher probability of a layoff (Hamermesh, 1989; Singh \& Reid, 1998; Elsby, Hobijn \& Sahin, 2010) and a much lower likelihood of reemployment for designated group members (Kletzer, 1991; Moore, 1992; Mar \& Ong, 1994; Fairlie \& Kletzer, 1998). The existing descriptive evidence from Canada also suggests that the recent economic downturn had a more adverse impact on Aboriginal workers than on the mainstream population, as reflected in large increases in already sizable gaps in labour force participation, employment and unemployment rates between the two groups (Delic \& Abele, 2010; Usalcas, 2011).

This very observation begs some obvious questions: Why is this happening in Canada? What underlies the apparently disproportionate impact of recession on two groups that exist in the same labour market? To what extent is the difference in the impact the result of the differences in the observable characteristics of Aboriginal and non-Aboriginal workers and the jobs they hold and to what extent it is the result of employer discrimination in favour of non-Aboriginal workers? These questions are of essential importance to the informed development of policy in this area and this essay seeks to shed some light on these questions by examining the determinants of differences in transition probabilities of layoffs and reemployment for Aboriginal and non-Aboriginal workers and by examining the decomposed sources of differences in outcomes using the standard Blinder-Oaxaca decomposition procedure that was previously applied only in the context of Aboriginal-to-non-Aboriginal earnings differentials examinations. Considering that Aboriginal people rank much lower on all post-secondary educational 
attainment indicators (Hull 2000, 2009) the key hypothesis here is that the differences in observable personal and employment-related characteristics of workers would likely explain much of the difference in the impact of recession, though systemic discriminatory behavioural practices of employers could play a role as well, given the existing stereotypes about relatively lower productive abilities and weaker attachment to the labour market of Aboriginal workers (Kleinfeld \& Kruse, 1982; Merrill, Bruce \& Marlin, 2010).

The essay is structured as follows: the following section outlines the key methodological considerations. It describes the data source and the construction of the analytical samples used to measure the patterns in transitions between the employed and unemployed labour force states for Aboriginal and non-Aboriginal labour force participants for the time period commencing in September 2008 and ending in May 2011. It then briefly explains the choice of the empirical methods used to estimate the results, and the variables included in the analysis. The section also presents descriptive evidence and discusses potential econometric issues associated with the empirical analysis. The estimation results from the comparison of transition probabilities and the decomposition of the differences in probabilities of layoffs and reemployment are presented in Section 3 and Section 4, respectively. The results are presented both for the aggregated (all three Aboriginal identity groups combined) and for disaggregated (Métis and NAI) Aboriginal identity groups that underlie the policy debate, and comparisons are done for layoffs within and for reemployment across gender. Section 5 concludes the essay. 


\section{Methodology}

The impact of the latest recession on Aboriginal and non-Aboriginal identity workers is analyzed in several stages in this essay. This section outlines the pertinent methodological considerations. After describing the data source and the methodology for constructing the working samples, the section presents some descriptive evidence of the impact and introduces the methods underlying the empirical estimation. It then describes the variables included in the empirical models and discusses the potential econometric problems associated with the empirical models.

\subsection{Data Description}

Examining transitions between labour force states requires individual-level longitudinal data. The data employed in this essay are drawn from the national master file of the Canadian Labour Force Survey (LFS), which is a monthly household survey that samples dwellings and collects information on labour market activity from a representative sample of civilian, non-institutionalized population aged 15 and over living off-reserve in the ten provinces. ${ }^{10}$ To date, the LFS has not collected any information on labour market activities of the population living on Indian reserves and settlements. Hence, the present analysis pertains only to Aboriginal Identity population living off-reserve in the ten provinces.

\footnotetext{
${ }^{10}$ Although collected, the information on labour market activities of population living in the territories is not included in the national files of the LFS used in this study but is published separately in a territorial file (Statistics Canada, 2011), which is currently not made available to researchers outside of Statistics Canada's Labour Statistics Division. As of Census 2006, the total of 593,000 of Aboriginal identity people, aged 15 years and over, live off-reserve in the ten provinces; 269,000 North American Indian, 286,000 Métis, and 14,000 Inuit (Zietsma, 2010, p. 10).
} 
While the LFS was not explicitly designed to be used longitudinally, its monthly records of household members can be reorganized into longitudinal records that allow following individuals over time and as such has been used for analysis of transitions between labour force states (Jones \& Riddell, 2006; Rowe \& Nguyen, 2004). The survey uses a rotating panel sample of around 54,000 households, keeping each household in the sample for six consecutive months. This rotation group structure creates a $5 / 6$ month-tomonth sample overlap, which is what allows the analysis of month-to-month changes in layoffs and reemployment (Statistics Canada, 2011).

To make use of this longitudinal component in the present analysis, individuals were matched across the recession that spanned the period September 2008 - May 2011, employing a matching procedure similar to the one developed by Chan, Morissette and Frenette (2011). ${ }^{11}$ The matching procedure entailed several steps: first, a pseudo individual identifier was created by combining several variables from the survey. ${ }^{12}$ This identifier was then used to identify individual records in each month and match-merge them across months to create panels. This resulted in total of 28 panels, which were then

\footnotetext{
"November 2008 was the month when employment began to drop and January 2011 when it returned to its initial level; hence, officially October 2008 is taken as the month marking the onset and January 2011 the end of the latest recession (Gilmore \& La Rochbelle, 2011; Statistics Canada, 2011 la). The data for September 2008 are included in this analysis to allow capturing layoffs that occurred in the first month of the recession. Similarly, several months beyond January 2011 are retained to ensure individuals who entered the rotation in the last month of the recession could be followed for their full stay in the panel to observe their likelihood of reemployment.

${ }^{12}$ Because the LFS data set used in this analysis contained no unique individual identifier that is required to match records across months, a pseudo individual identifier was created by combining these ten variables: the one-digit province code ( PROV1), the pseudo unit identification code for region (PSEUDOUI), the region frame identifier (FRAME), the stratum frame identifier (STRATFRAM), an identifier for sample design type (TYPE), the cluster id (CLUST), the rotation number identifier (ROTATION), a sequential number assigned to every dwelling within a cluster (LISTLINE), a code used to identify structures that have more than one dwelling (MULT), a sequential number that uniquely identifies a person within a household (LINE), the survey month (SMTH) and the survey year (SYEAR) (Statistics Canada, 2007a).
} 
appended to create the final database for the analysis. ${ }^{13}$ Several restrictions were placed to ensure each individual was followed for only six consecutive months. ${ }^{14}$ No restrictions were placed on the age of respondents since the information about the labour market activity was solicited only from respondents aged 15 and over (Statistics Canada, 2011).

The resulting sample in this constructed longitudinal database contained six monthly records for 260,480 individuals aged 15 and over, 11,434 of whom selfidentified as having an Aboriginal identity. ${ }^{15}$ For analytical purposes, the classification of Aboriginal identity was based on an individual's consistency in reporting an Aboriginal identity across the time of stay in the survey. ${ }^{16}$ The total sample size of Aboriginal identity workers was about evenly split between North American Indians $(5,659)$ and Métis $(5,608)$. The counts for Inuit identity workers are retained in the total Aboriginal identity sample only as they were too small to allow Inuit workers to be considered separately. These total samples were used to select the analytical samples for this study. Table 1 presents main sample sizes, disaggregated by gender, for all considered groups.

\footnotetext{
${ }^{13}$ The author is grateful to Dr. Winnie Chan, Statistics Canada, for providing guidance on selection of variable for creation of the pseudo individual identifier, to Dr. Pierre Brochu, University of Ottawa, Department of Economics, for providing a Stata program that was used to create the pseudo individual identifier in the selected monthly database and especially to Dr. Jean-Michel Billette, then the Statistics Canada's analyst at the COOL RDC, for helping to construct the final database used in the analysis.

${ }^{14}$ Only completed cases were retained in the panels. Observations were dropped for those that were in the outgoing group as of period 1 of the panel and incoming group in period 2. Observations were also dropped for those that were new in the second year of the mini panel, joining existing and ongoing rotations. Finally, observations of those that were in the survey in period 1 but disappeared in the second period were dropped as were observations of those who suddenly appeared in the period 2 of the panel.

${ }^{15}$ Many observations were lost in the first four panels due to unusual inconsistencies in the data construct in the master file that involved unexplained duplicates. These observations were not unique to identity and they were deleted from the panels. Nevertheless, the analysis pertaining to those four panels should be taken with caution.

${ }^{16}$ An attempt was made to maximize the sample size by relaxing this definition of Aboriginal identity in such a way as to allow any reporting to be counted in. Because this provision produced a total gain of only four counts, the consistent reporting of the same identity was retained as the criterion for Aboriginal identity classification. Overall, 99.97 percent of the total sample was consistent in reporting the same selfidentity every month.
} 
Table 1: Sample sizes and means of selected sample characteristics, by identity

\begin{tabular}{|c|c|c|c|c|c|c|c|c|}
\hline \multirow[t]{2}{*}{ Characteristics } & \multicolumn{2}{|c|}{$\begin{array}{l}\text { Non-Aboriginal } \\
\text { Identity }\end{array}$} & \multicolumn{2}{|c|}{$\begin{array}{l}\text { Aboriginal } \\
\text { Identity }\end{array}$} & \multicolumn{2}{|c|}{ Métis } & \multicolumn{2}{|c|}{$\begin{array}{c}\text { North American } \\
\text { Indian }\end{array}$} \\
\hline & Men & Women & Men & Women & Men & Women & Men & Women \\
\hline \multicolumn{9}{|l|}{ Paid employees } \\
\hline $\begin{array}{l}\text { main sample size } \\
\text { weighted counts }\end{array}$ & $\begin{array}{c}82587 \\
21571478\end{array}$ & $\begin{array}{c}83724 \\
21676813\end{array}$ & $\begin{array}{c}3179 \\
543838\end{array}$ & $\begin{array}{c}3542 \\
570845\end{array}$ & $\begin{array}{c}1727 \\
290371\end{array}$ & $\begin{array}{c}1754 \\
288047\end{array}$ & $\begin{array}{c}1404 \\
243761\end{array}$ & $\begin{array}{c}1733 \\
274087\end{array}$ \\
\hline Mean hourly wage & $\begin{array}{l}25.7 \\
(12.8)\end{array}$ & $\begin{array}{c}22.0 \\
(10.9)\end{array}$ & $\begin{array}{c}23.1 \\
(11.2)\end{array}$ & $\begin{array}{l}19.3 \\
(9.4)\end{array}$ & $\begin{array}{c}24.1 \\
(11.5)\end{array}$ & $\begin{array}{r}19.3 \\
(9.2)\end{array}$ & $\begin{array}{c}22.1 \\
(10.8)\end{array}$ & $\begin{array}{l}19.3 \\
(9.6)\end{array}$ \\
\hline Mean wks absence & $\begin{array}{c}9.6 \\
(31.6)\end{array}$ & $\begin{array}{c}17.3 \\
(37.6)\end{array}$ & $\begin{array}{c}7.2 \\
(12.8)\end{array}$ & $\begin{array}{c}18.8 \\
(61.2)\end{array}$ & $\begin{array}{c}7.1 \\
(14.0)\end{array}$ & $\begin{array}{c}23.9 \\
(82.3)\end{array}$ & $\begin{array}{c}7.0 \\
(11.0)\end{array}$ & $\begin{array}{c}14.4 \\
(25.9)\end{array}$ \\
\hline $\begin{array}{l}\text { Laid off } \\
\text { (main sample) }\end{array}$ & $\begin{array}{c}0.16 \\
(0.37)\end{array}$ & $\begin{array}{c}0.11 \\
(0.31)\end{array}$ & $\begin{array}{c}0.24 \\
(0.42)\end{array}$ & $\begin{array}{c}0.15 \\
(0.36)\end{array}$ & $\begin{array}{c}0.21 \\
(0.40)\end{array}$ & $\begin{array}{c}0.14 \\
(0.35)\end{array}$ & $\begin{array}{c}0.27 \\
(0.44)\end{array}$ & $\begin{array}{c}0.16 \\
(0.37)\end{array}$ \\
\hline $\begin{array}{l}\text { Laid off } \\
\text { (restricted sample) }\end{array}$ & $\begin{array}{c}0.15 \\
(0.35)\end{array}$ & $\begin{array}{c}0.10 \\
(0.30)\end{array}$ & $\begin{array}{c}0.23 \\
(0.42)\end{array}$ & $\begin{array}{c}0.15 \\
(0.35)\end{array}$ & $\begin{array}{c}0.20 \\
(0.40)\end{array}$ & $\begin{array}{c}0.14 \\
(0.35)\end{array}$ & $\begin{array}{c}0.26 \\
(0.44)\end{array}$ & $\begin{array}{c}0.15 \\
(0.36)\end{array}$ \\
\hline $\begin{array}{l}\text { Laid-off workers } \\
\text { main sample size } \\
\text { weighted counts }\end{array}$ & $\begin{array}{c}15007 \\
3504808\end{array}$ & $\begin{array}{c}10334 \\
2421173\end{array}$ & $\begin{array}{c}789 \\
129422\end{array}$ & $\begin{array}{c}569 \\
86822\end{array}$ & $\begin{array}{c}378 \\
60372\end{array}$ & $\begin{array}{c}255 \\
40890\end{array}$ & $\begin{array}{c}395 \\
65980\end{array}$ & $\begin{array}{c}301 \\
44631\end{array}$ \\
\hline $\begin{array}{l}\text { Reemployed } \\
\text { (main sample) }\end{array}$ & $\begin{array}{c}0.28 \\
(0.45)\end{array}$ & $\begin{array}{c}0.22 \\
(0.41)\end{array}$ & $\begin{array}{c}0.31 \\
(0.46)\end{array}$ & $\begin{array}{c}0.24 \\
(0.43)\end{array}$ & $\begin{array}{c}0.33 \\
(0.47)\end{array}$ & $\begin{array}{c}0.24 \\
(0.43)\end{array}$ & $\begin{array}{c}0.30 \\
(0.46)\end{array}$ & $\begin{array}{c}0.24 \\
(0.43)\end{array}$ \\
\hline $\begin{array}{l}\text { Reemployed } \\
\text { (restricted sample) }\end{array}$ & $\begin{array}{c}0.36 \\
(0.48)\end{array}$ & $\begin{array}{c}0.28 \\
(0.45)\end{array}$ & $\begin{array}{c}0.39 \\
(0.49)\end{array}$ & $\begin{array}{c}0.27 \\
(0.45)\end{array}$ & $\begin{array}{c}0.42 \\
(0.49)\end{array}$ & $\begin{array}{c}0.26 \\
(0.44)\end{array}$ & $\begin{array}{c}0.36 \\
(0.48)\end{array}$ & $\begin{array}{c}0.29 \\
(0.45)\end{array}$ \\
\hline
\end{tabular}

Source: The Labour Force Survey, national monthly master file, September 2008 - May 2011.

Note: Main sample includes all paid employees, aged between 15 and 64, who are not full-time students. Restricted sample includes only permanent paid employees, aged between 15 and 64, who are single-job holders and who are not students. Survey sampling weights (abowt) are applied in all analyses to account for complex survey design. The counts represent pooled populations for the recession time period. Standard deviations are reported in parentheses.

For the analysis of transitions from paid employment into unemployment due to layoff, the analytical samples for each group were restricted to paid employees who were aged between 15 and 64 in the month preceding the layoff and who were not full-time students. Any observations with missing values were excluded from the samples. The resulting, gender combined, sample of paid employees was 166,311 for non-Aboriginal identity workers and 6,721 for Aboriginal identity workers. The main samples for Métis contained 3,481 and for North American Indian 3,137 paid employees. 
To examine the probability of reemployment, these main samples of paid employees in each group were further restricted to individuals who were laid off at any point between the second and fifth month in the rotation. Under this restriction, the total main sample for non-Aboriginal identity workers contained 25,341 individuals while the total main Aboriginal identity sample contained 1,358 individuals. In this reemployment probability analysis the main sample of North American Indian (696) was slightly larger from that of Métis (633), as illustrated in Table 1.

In acknowledging that the results in the main analysis might be affected by the presence of seasonal workers, multiple-job holders, and part-time students, the empirical analysis of layoffs and reemployment for each group is repeated using a more restricted sample that included only permanent paid employees, aged between 15 and 64, who are single-job holders and who are not students. This additional restriction resulted in a reduction of about 17 percent of the main sample size for non-Aboriginal identity workers and about 18 percent reduction of the main sample size for Aboriginal identity workers in the analysis of layoffs. The reductions in the sample sizes in the analysis of reemployment amounted to about 26 percent for non-Aboriginal identity workers and about 21 percent for Aboriginal identity workers.

Tables presented in Appendix A (Appendix A1 through Appendix A5) contain definitions for all independent variables as well as the mean characteristics for all groups considered in the main samples and in the more-restricted samples of paid employees at risk of being laid off and of laid-off workers. Here, only the main features are discussed, 
mostly from the main samples. Sampling weights were applied in the analyses to account for complex survey design (Statistics Canada, 2011). ${ }^{17}$

As illustrated in Table 1, the mean hourly earnings of Aboriginal identity workers are quite a bit lower than those of the non-Aboriginal identity workers, with the greatest difference being for NAI workers. As indicated in the second row of Table 1, the gap for NAI men (3.6) is larger both from the gap for Métis and for NAI women (2.7) and from the gap for Métis men (1.6). This point does not really come as a surprise as it is consistent with findings in the existing literature on the topic.

The new information on job absence, measured by the number of weeks an individual was continually absent from work, is rather unanticipated, except for Métis women and for the total Aboriginal identity group of women. ${ }^{18}$ As shown in Table 1, the mean number of weeks of absence from work for NAI men (7.0) is clearly below the mean for non-Aboriginal identity men (9.6). The same holds true when the comparison is made between NAI women (14.4) and non-Aboriginal identity women (17.3).

The new information on layoffs seems to lean in the same direction as the information on earnings for all three Aboriginal identity groups. As shown in Table 1, both NAI men and NAI women were much more likely to be laid off than were their nonAboriginal identity counterparts. The observed difference, both in the main sample and in the more restricted sample, amounts to 5 percentage points for women and 11 percentage points for men. The same pattern appears when comparing the layoff figures for the other

\footnotetext{
${ }^{17}$ Since the LFS samples dwellings, not individuals, sampling weights must be applied to obtain the correct standard errors. The LFS master file does not provide bootstrap weights but instead provides several weight variables that account for the complex survey design in that they control for clustering and the stratification of the survey design as well as for the different probabilities of selection into the sample, non-response and coverage issues. The weight variable included in the present analyses is Aboriginal-specific weight, abowt.

${ }^{18}$ Potentially, job absence, as it will be explained later on in the methodology section, can play an important role for employers making decisions about which workers to lay off.
} 
two Aboriginal identity groups, Métis and the total Aboriginal identity groups, although the magnitude of the difference appears relatively smaller in comparison to that observed for NAI group. The following two charts (Chart A and Chart B) illustrate this difference in monthly layoff patterns more clearly for the aggregated (all three Aboriginal identities together) and for the disaggregated (Métis and NAI identity separately) groups. 
Chart A: Monthly layoff rates for Aboriginal and non-Aboriginal identity groups, the 2008-2011 recession

\section{Aggregated identity groups}

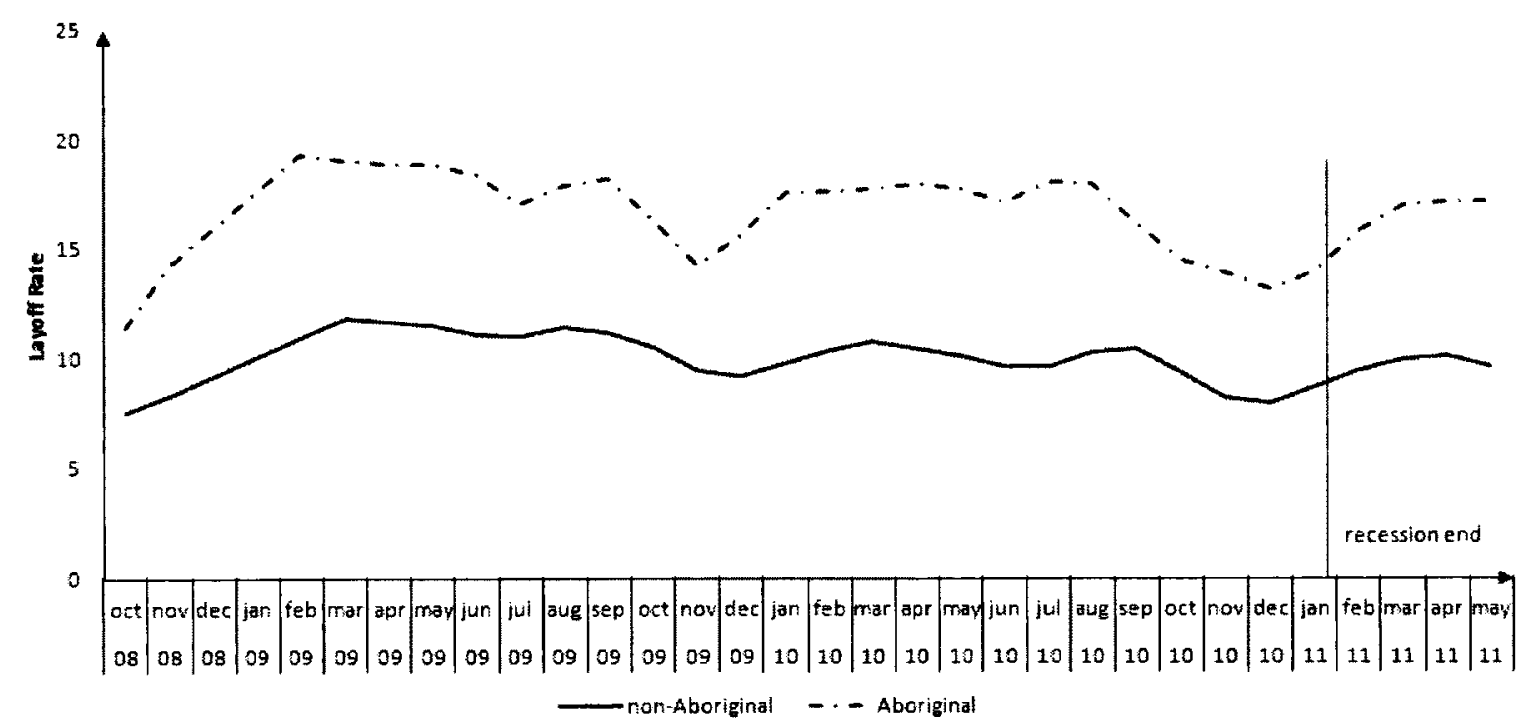

\section{Disaggregated identity groups}

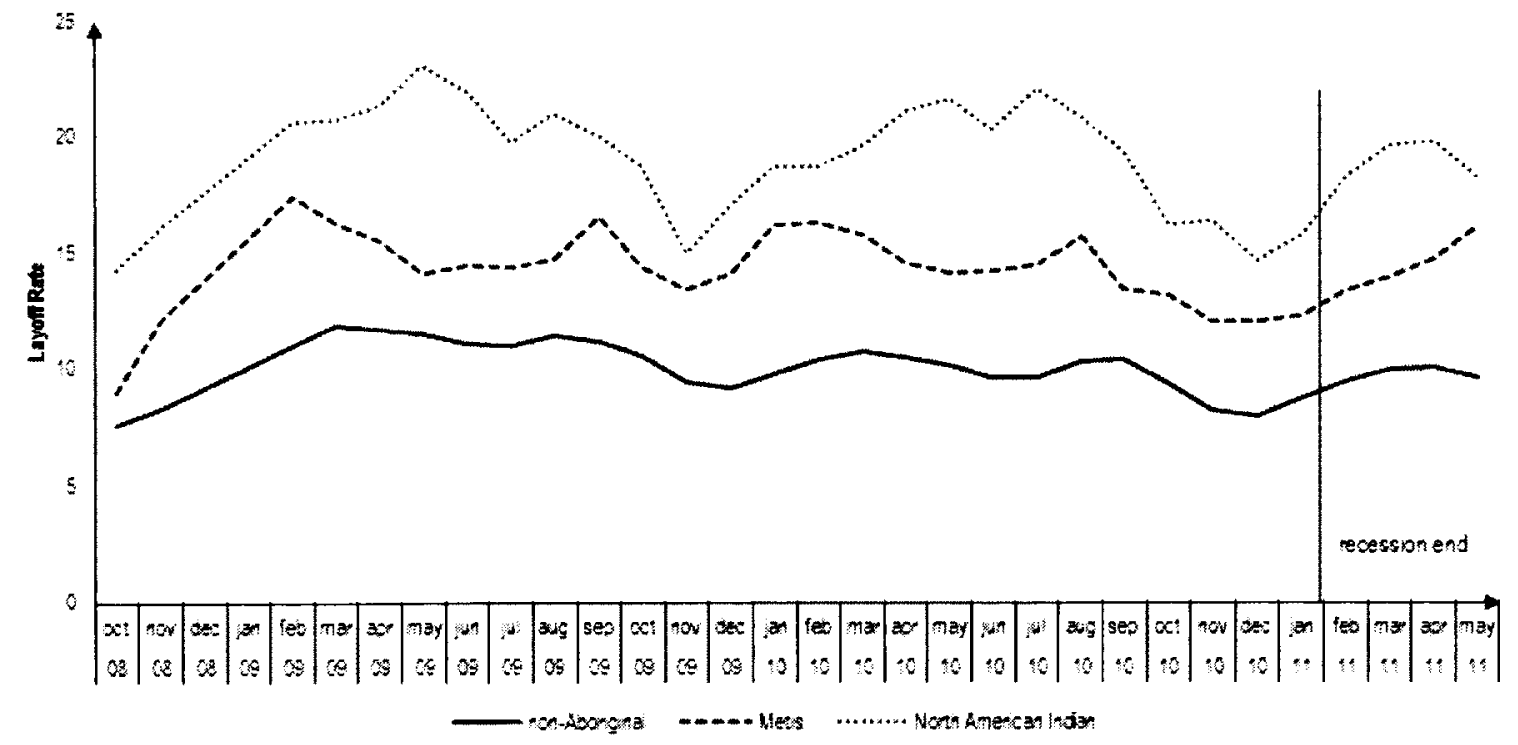

Source: Labour Force Survey, weighted monthly data, layoff rates are seasonally adjusted 3-month moving average 
The monthly, seasonally-adjusted, layoff rates in the presented Charts are based on the main sample restrictions (paid employed, who were aged between 15 and 64 in the month preceding the layoff, and who were not full-time students) and they capture monthly transitions from paid employment into unemployment due to layoffs. ${ }^{19}$ Starting from September 2008, for each pair of subsequent months, weighted inflows into layoffs between month $t-l$ and month $t$ are divided by the weighted number of paid employees in month $t-1$. The rates for the aggregated groups are presented in Chart A while the rates for the disaggregated groups are presented in Chart B.

As plotted in the top portion of Chart A, monthly seasonally-adjusted layoff rates for the aggregated identity groups were consistently higher for Aboriginal than for nonAboriginal identity workers over the recession time period. This aggregate gap, however, conceals the more drastic difference that exists when the comparison is made between NAI and non-Aboriginal identity workers. As illustrated in the lower portion of Chart A, while the layoff rates of Métis workers are higher than those of non-Aboriginal identity workers, they are still much lower than those of NAI workers. Disaggregated rates by gender, presented in Chart B, provide more insight into this difference

\footnotetext{
${ }^{19}$ Since the dataset used in the present analysis covers the time span shorter than 3 years, seasonal adjustment was done using the standard 3-month moving average method (Bilodeau, 1997). The recommended, more advanced seasonal adjustment method, ARIMA (Auto-Regressive Integrated Moving Average), was not applicable because the method requires a minimum of 5-7 years of time series to properly identify and estimate the seasonal and calendar effects (Statistics Canada, 2009). The series presented in Chart A and Chart B are calculated using the weighted counts and the seasonal effects for each group were filtered out, leaving in only the trend-cycle and the irregular components for each group. The corresponding raw series can be made available to interested readers, upon request.
} 


\section{Chart B: Monthly layoff rates for Aboriginal and non-A boriginal identity workers, the 2008-2011recession}

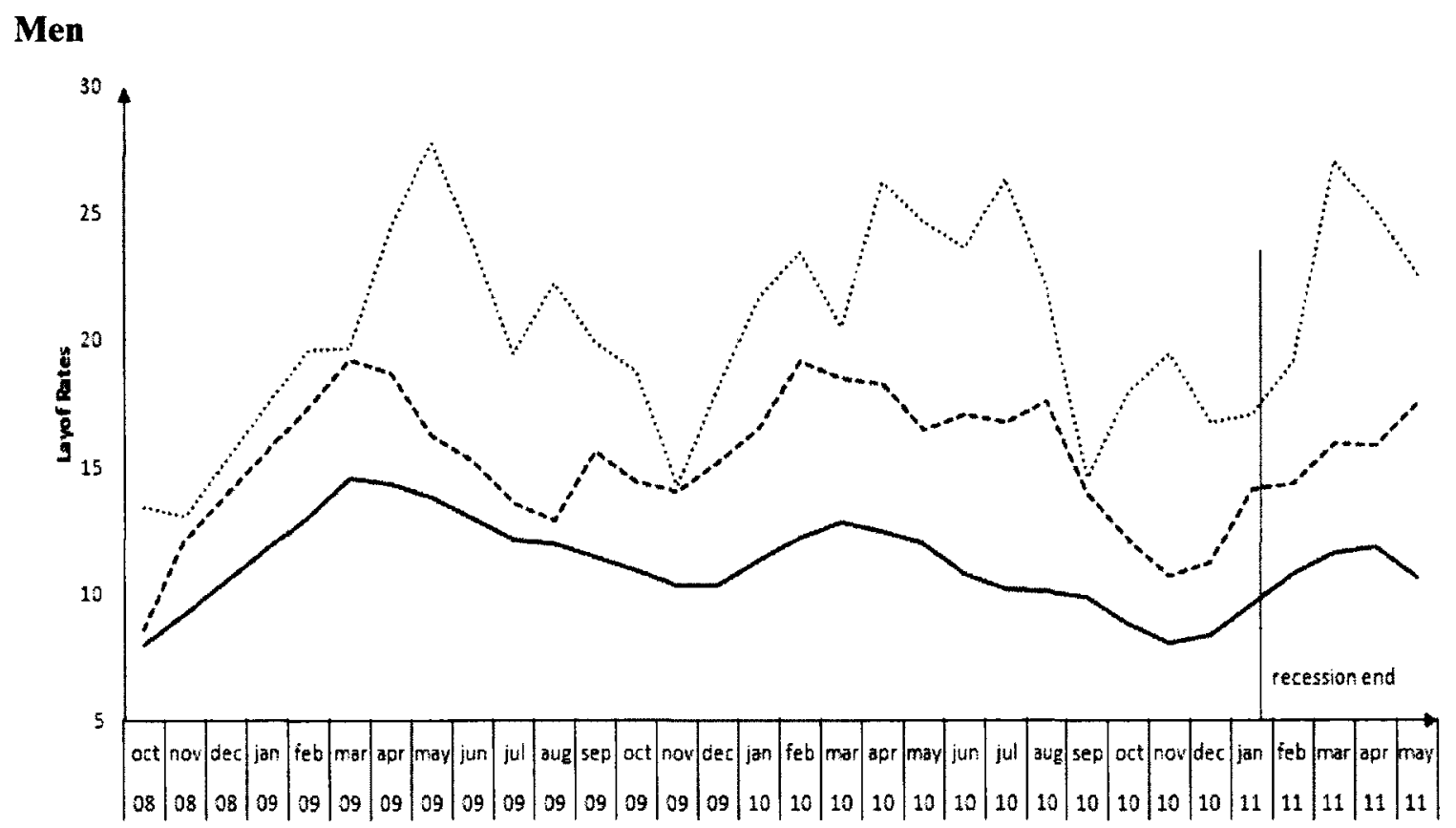

\section{Women}

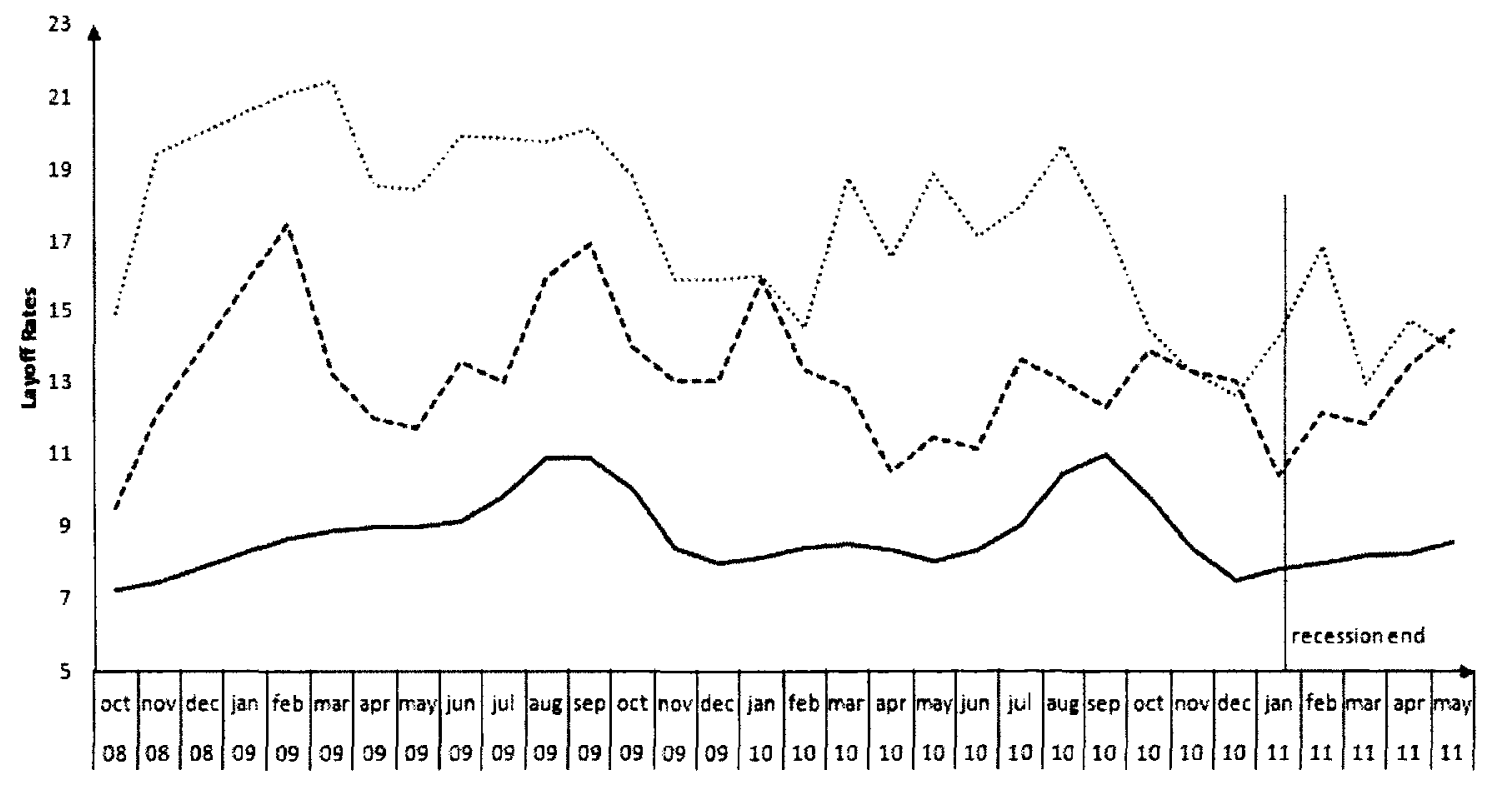

Source: Labour Force Survey, weighted monthly data, layoff rates are seasonally adjusted 3-month moving average 
As shown in Chart B, the patterns over the recession period and the patterns across gender are quite similar to those of the aggregated identity groups presented above, though the trends for men and women are somewhat different. While fluctuations are notable, it appears that the gap in layoff rates between Aboriginal and non-Aboriginal identity men has widened substantially over most of the recession time period while the gap in layoff rates between Aboriginal and non-Aboriginal identity women followed more of a narrowing down trend, albeit the difference did not diminish. In fact, as illustrated in Chart B, the gap in layoff rates at the end of the recession period for NAI women was very close to the gap at the onset of the recession and for Métis women the gap was larger from that at the onset of the recession. The erratic patterns in the rates of NAI identity group also stand out in this chart, as they do in the previous chart, possibly indicating that more complex processes underlie the layoff outcomes of this group of Aboriginal identity workers. This cannot be attributed to the seasonal effect since these trends were smoothed by the 3-month averaging, as explained above.

In regard to prospects of reemployment shortly following the layoff, which could be as little as one month after the layoff and at most five months after the layoff, Table 1 reveals somewhat unexpected outcomes. It appears that, while a greater proportion of Aboriginal than non-Aboriginal identity workers was laid off at some point during their stay in the survey, a greater proportion of Aboriginal workers was also successful in obtaining subsequent paid employment before exiting the survey, relative to nonAboriginal workers. This fact comes as a surprise given the overwhelmingly dismal picture of the employment prospects for Aboriginal identity workers reported in the existing literature on the topic. 
However, as illustrated in Table 1, significant differences exist within gender, with males both in Aboriginal and in non-Aboriginal identity groups having greater proportions of reemployed, relative to their female counterparts. In the main sample, the gap in the proportion of reemployed ranges from 6 percentage points for NAI and for non-Aboriginal identity groups to 9 percentage points for Métis. Notably, as observed in the more restricted sample, these gaps appear slightly larger for NAI and for nonAboriginal identity groups and substantially larger for Métis group, suggesting that Métis men were the most likely to secure paid employment shortly after their layoff.

These simple descriptions of the recession outcomes do not adjust for the differences in characteristics between the groups, several of which deserve mention here. As shown in Appendix A2 and in Appendix A3, the age distribution among Aboriginal and non-Aboriginal identity workers at risk of being laid off appears very different as does the distribution of the level of educational attainment. In regard to age, Aboriginal identity samples contain larger proportions of youth, workers aged between 15 and 24 , and smaller proportions of mature prime-age workers, workers aged between 45 and 54 . In regard to the level of educational attainment, Aboriginal identity samples contain substantially larger proportions of workers with less than a high school education and substantially smaller proportions of university-educated workers, compared to nonAboriginal identity samples. These important differences might explain the greater layoff probabilities but not the greater reemployment probabilities of Aboriginal workers.

The Aboriginal identity samples also contain much larger proportions of workers at risk of being laid off with job tenure of less than 24 months and much smaller proportions of workers with job tenure over 20 years, relative to the non-Aboriginal 
identity samples. In regard to industry, while non-Aboriginal identity men at risk of being laid off were about evenly distributed across industry sectors, Aboriginal identity men were concentrated in primary industry and Aboriginal identity women, like nonAboriginal identity women, were concentrated in public services and retail sector. The same patterns in regard to industry appear in the samples of laid-off workers, as illustrated in Appendix A4 and Appendix A5. In the samples of laid off workers, however, non-Aboriginal identity men were also concentrated in the primary industry and they were proportionately younger and less educated than non-Aboriginal identity men at risk of being laid off, which might account for their lower reemployment probabilities.

In each identity sample, larger proportions of women than men were employed part-time. Union membership was most present in the sample of Métis women and in the sample of non-Aboriginal identity women while the sample of NAI men had the smallest proportions of union members. The proportion of permanent job-holders was also smaller in the sample of NAI men, relative to that of other groups at risk of being laid off. The proportion of seasonal job-holders was largest in the sample of NAI men at risk of being laid off while the proportions of casual/contract job-holders and of multiple-job holders were largest in the samples of Métis women at risk of being laid off. Finally, greater proportions of Aboriginal identity employees at risk of being laid off worked in smallsized workplaces, relative to non-Aboriginal identity employees. Also, greater proportions of Aboriginal than non-Aboriginal identity employees worked for minimum or below minimum wages, particularly women.

All of these differences could be contributing to the differences in the patterns of layoff outcomes depicted in Chart A and Chart B above. Previous research has shown 
that variations in the distributions of important characteristics, and particularly of educational attainment, have obvious implications for the labour market outcomes of individuals. The following section outlines the empirical framework for more detailed analyses of the differences in the impact of recession on different groups in the forms of the probability of layoff and the probability of reemployment, respectively.

\subsection{Empirical Method}

The econometric analysis of the layoffs and reemployment in this essay is divided into two parts. In the first part, panel tools are applied to estimate: (a) the probabilities of transitions from paid employment into unemployment due to layoff and; (b) the probabilities of reemployment, conditional on being laid off. The analysis is done for four groups, male and female Aboriginal and non-Aboriginal identity workers, and makes use of a simple two-state Markov-like model of labour force transitions (Eaton, 1970). That model assumes, unrealistically, that the probabilities of layoff and subsequent reemployment are fixed parameters that do not vary with time or personal characteristics. This simple model is used only to set the stage for the second part of the analysis.

In that second part, a recent extension of the Blinder-Oaxaca decomposition technique for nonlinear functions (Powers, Yoshioka \& Yun, 2011) is applied to multivariate probit estimates of the probability of layoff and subsequent reemployment in order to identify the underlying causes of differences in those probabilities. In this part, pooled samples are examined for Aboriginal and non-Aboriginal workers and the estimated differences are decomposed into characteristics effects and coefficients effects, both at an aggregate (all variables included) and at an individual variable level. 
Originally developed by Blinder (1973) and Oaxaca (1973) and applied extensively in the literature on racial and gender wage differentials (Ashenfelter \& Oaxaca, 1987), the Blinder-Oaxaca decomposition method has undergone various forms of modifications and extensions (Oaxaca \& Ransom, 1988; Oaxaca, 1994, 1999; Yun, 2000, 2004, 2005, 2008; Fairlie, 2005; Bauer \& Sinning, 2008; Sinning, Hahn \& Bauer, 2008; Powers, Yoshioka \& Yun, 2011). Until recently, however, the Blinder-Oaxaca decomposition method and its various generalizations have mainly been applied to linear regression models (Ben, 2008), though the interest in application to nonlinear models such as probit (Gomulka \& Stern, 1990; Even \& Macpherson, 1993; Yun, 2000; Gang, Sen \& Yun, 2002) and logit (Nielsen, 1998; Fairlie, 1999, 2005; Powers, Yoshioka \& Yun, 2011) has grown rapidly over the last two decades.

The practical feature of the Blinder-Oaxaca regression decomposition method that attracts attention of researchers studying socioeconomic inequality is that it permits the difference in mean outcomes between two groups to be decomposed into two distinct parts: the first part is generally termed the "explained" part that reflects the differences in the observed characteristics (often called "endowments") of the individuals in the two groups. The second part is termed the "unexplained" part that reflects the portion of the differential that is attributable to the differences in the estimated coefficients. This "unexplained" part may be due to discrimination or to any other unobserved differences between the groups.

To assess the relative contribution of each variable to each of the two components of the difference, a detailed decomposition is carried out. In that decomposition, both the endowment and the coefficient components of the difference in means are themselves 
split into a number of components, each of which represents the unique contribution of each variable. This detailed part of the decomposition method is of particular value to the present analysis because it can shed some light on the question posed in the introduction to the essay; that is, the detailed decomposition can help in assessing how much of the difference in the mean outcomes between the two groups can be explained by differences in particular endowments (e. g. educational attainment) and how much by the differences in behavioural response associated with that endowment (e. g. the coefficient on educational attainment).

\subsection{Variables and A Priori Postulations}

The dependent variable in the layoffs model is a dichotomous dummy variable, coded one if a paid employee experienced a layoff at any time during his or her sixmonth stay in the survey, and zero otherwise. The dependent variable in the reemployment model is a dichotomous dummy variable coded one if the laid-off worker obtained paid employment prior to exiting the survey, and zero otherwise. The layoff and reemployment concepts used to measure these two outcomes are adopted from Chan, Morissette and Frenette (2011) whose study employed the same data source. These concepts are explained below; the explanatory variables included in the analysis are defined in Appendix A1.

Much of the existing literature on layoffs in Canada makes a strict distinction between permanent and temporary layoffs, in an acknowledgment of the differences in economic consequences the two experiences may have on individuals and on economy as a whole (Picot, 1992; Picot, Lin \& Pyper, 1997, 1998; Fairlie \& Kletzer, 1998; Galarneau \& Stratychuk, 2001; Bernard \& Galarneau, 2010). Making this distinction in the LFS 
data, however, is inexact at best because the LFS does not contain sufficient information to make an accurate distinction between official definitions of temporary and permanent layoffs. ${ }^{20}$ Since an approximation is not essential to the current analysis, a choice was made to adopt the more inclusive concept of layoffs whose key feature rests on the fact that the process was initiated by the employer. Thus the concept of layoffs here covers not only individuals who were laid off either temporarily or permanently but it also covers cases of individuals who may have experienced multiple layoffs. These layoffs may be seasonal or non-seasonal. However, the same analysis is repeated applying the more restricted sample, as explained earlier.

The concept of reemployment is also inclusive in the sense that it does not differentiate between part-time and full-time reemployment, though this distinction is more relevant to the current analysis. ${ }^{21}$ Reemployed individuals in the present analysis are defined as those who were laid off at some point in their six-month stay in the survey and who obtained subsequent employment before exiting the survey. This conditional

\footnotetext{
${ }^{20}$ While the LFS does collect information about recall expectations from temporarily laid-off respondents, it does not collect any information about whether or not those respondents in fact return to their employer, which is one of the other criteria that must be satisfied in order to make accurate conclusions about temporary layoffs (Statistics Canada, 2011; Chan, Morissette \& Frenette, 2011). The studies mentioned here used longitudinal data sources such as the Longitudinal Worker File (LWF) and the Survey of Labour and Income Dynamics (SLID) that allowed the distinction to be made precisely. In the present analysis, the distinction would be arbitrary as the classification ultimately depends on the perceptions of respondents about the nature of their layoff. Moreover, as Blau and Kahn (1981) note, temporary layoffs become permanent by definition if, instead of returning to the same employer, the individual finds a job at another employment place.

${ }^{21}$ Arguably, the pressures from continuing financial obligations and a likely excess supply of labour in the market during the recessionary times may induce some laid-off workers to take either temporary or otherwise substandard jobs; hence, accounting somehow for the 'quality' of reemployment might be informative for the present analysis. It is possible that someone who is laid off from a full-time position is then rehired into a part time position, a scenario which has serious implications with respect to the impact of being laid off. The fact that some individuals regain employment would no longer be sufficient indicator. It is important to know whether they regain full-time employment. Migration is another aspect worth examining because the reemployment may not be with the same employer nor necessarily in the same region. Consideration of these issues will be carried out post-dissertation.
} 
probability of reemployment is used as a measure of an individual's short-term adaption to employment shocks caused by the economic downturn.

The LFS contains a wide range of conventional explanatory variables that have been found to play an important role in influencing the probabilities of layoff and reemployment. These variables will be included in the multivariate probit analyses that follow the estimation of simple transition rates below. In addition to the standard demographic and human capital variables, the LFS offers a number of job-related variables such as a measure of job tenure, work absence, the degree of coverage by a collective agreement, the size of the employment place, type of work, job permanency, hourly earnings, economic sector, industry and occupational status. The great majority of these job-related variables have never been included in empirical analyses of labour market outcomes of Aboriginal people in Canada.

The selection of the explanatory variables here is based on previous research on the topic and the variables are classified in three basic groups: (1) those that relate to personal characteristics; (2) employment-related characteristics; and (3) other variables. Of primary interest are the personal and employment-related groups of variables, as classified in this section; the other variables are included only as controls.

The key variable of interest in the personal characteristics group is educational attainment. Although the existing literature generally reports the expected association between individuals' educational attainment and their probability of layoff and reemployment (Blau \& Kahn, 1981; Cornfield, 1982; Hamermesh, 1989; Singh \& Reid, 1998; Galarneau \& Stratychuk, 2001; Elsby, Hobijn \& Sahin, 2010; Chan, Morissette \& Frenette, 2011) it is unclear what to expect for Aboriginal workers. While it is reasonable 
to expect, based on human capital theory, that less-educated workers, regardless of identity, would be more likely to be laid off and less likely to be reemployed in the shortterm following the layoff, the same reasoning cannot be applied to the expectations for educated Aboriginal workers, particularly not for male Aboriginal workers. Drost (1994), for instance, has found that economic returns to postsecondary schooling for Aboriginal men become positive only at the university level. Walters et al. (2004), on the other hand, found that "Aboriginals with a university degree are having difficulty acquiring even part-time employment" (p. 292). A detailed classification of educational attainment variable is included here in order to assess the impact of different levels of educational attainment on the outcome variables. ${ }^{22}$

The expected effect of job tenure (or seniority) is unclear as well. In the past, a general finding was that job tenure is inversely related to the probability of layoff (Blau \& Kahn, 1981). The LFS does not measure job tenure (seniority) directly. Instead, it measures seniority as the duration of the most recent period of uninterrupted work. Using that measure, Chan, Morissette and Frenette (2011) report that although in the most recent recession lower seniority workers were more likely to be laid off, higher seniority workers were also affected, relative to their counterparts in the previous two recessions. Seniority is expected to have a positive association with the probability of reemployment following the layoff (Mar \& Ong, 1994) and in this case it is proxied by age variable. ${ }^{23}$

\footnotetext{
${ }^{22}$ Of special interest here are trade programs. Some of these programs offered through apprenticeship, vocational schools or private trade schools do not require high school graduation yet these programs are classified under postsecondary education category in the LFS (Statistics Canada, 2011, p.12).

${ }^{23}$ The information on "job tenure" in the LFS pertains to the most recent period of uninterrupted work reported by respondents. In the guide to the LFS, this means "the number of consecutive months or years a person has worked for the current (or, if employed within the previous twelve months, the most recent) employer" (Statistics Canada, 2011, p.13). However, the information on job tenure is solicited from "currently employed only" (Statistics Canada, 2007a, p. 46); hence, the age category of the laid-off respondents is used to proxy seniority in the models examining the probability of reemployment.
} 
Job absence, a possible indicator of job commitment, has not been assessed in the existing literature on recession layoffs. ${ }^{24}$ However, in the general literature, job absence has been found to be positively related to the probability of layoff in the United States, and more so for African American workers than for white American workers (Wilson, 2005). Search theory offers some insights on this phenomenon in terms of employers' perceptions about workers' quit probability (Blau \& Kahn, 1981) which is highly relevant to the present analysis. As indicated earlier, even a mild work discipline behaviour that would otherwise be tolerated for non-Aboriginal workers could be judged more seriously for Aboriginal workers, simply because of the prevalence of stereotypes about weak job commitment among Aboriginal workers. If this is the case then Aboriginal workers with a given number of weeks of continuous absence from work would be more likely to be laid off than would non-Aboriginal workers with the same number of weeks of continuous absence from work, thus giving us a possible measure of discrimination. In regard to age distribution, the recent literature unanimously reports greater probabilities of layoff for young workers, aged less than 25 (Picot, 1992; Singh \& Reid, 1998; Galarneau \& Stratychuk, 2001; Elsby, Hobijn \& Sahin, 2010; Chan, Morissette \& Frenette, 2011). This relationship does not, however, carry over to reemployment models. Mar and Ong (1994) find no significant relation between age and the probability of reemployment, even after accounting for correlation with seniority variables. This insight is potentially more important for Aboriginal than for non-Aboriginal groups of workers

\footnotetext{
${ }^{24}$ Job absence is measured by the number of weeks of continual absence from work at current job. For employees "reasons for absence include: own illness or disability, personal or family responsibilities, maternity or parental leave, vacation, weather, labour dispute, job started or ended during reference week, holiday, working short time, and other reasons" (Statistics Canada, 201, p.10)
} 
because of the growing difference in the age structure between the two populations in Canada. $^{25}$

All of the employment-related variables listed above are of interest, given that the impact of many of them has not been previously assessed in analyses of Aboriginal workers. Economic sector, occupational status, and industry have all been found to play a role in the probability of layoff (Kletzer, 1991; Picot, 1992; Fairlie \& Kletzer, 1998; Galarneau \& Stratychuk, 2001; Chan, Morissette and Frenette, 2011; Wilkins \& Wooden, 2011). ${ }^{26}$ The size of the workers' employer has also been identified as important, with workers in small-sized workplaces experiencing a much higher probability of layoff, relative to workers in medium-sized and in large-sized workplaces (Picot, 1992; Picot, Lin \& Pyper,1998; Singh \& Reid, 1998; Galarneau \& Stratychuk, 2001; Bernard \& Galarneau, 2010). ${ }^{27}$ Studies that controlled for wage rates have found that low-wage workers are more likely to be laid off (Picot, 1992; Galarneau \& Stratychuk, 2001).

Whether the job is covered by a collective agreement is also an important variable as it may reveal some insights into possible systemic discrimination that naturally occurs as unionized firms adhere to the seniority criterion in their layoff procedures (Ornati \& Giblin, 1975). Given that, on average, the members of employment equity designated

\footnotetext{
${ }^{25}$ The Aboriginal population of Canada is growing much faster and has a much younger age structure than the non-Aboriginal population. According to the 2006 Census, the median age for total Aboriginal population is 27 , compared to median age of 40 for non-Aboriginal population. Among the three Aboriginal identity groups, Inuit have the youngest age structure with median age of 22 , followed by North American Indian (25) and Métis (30) (Delic \& Abele, 2010).

${ }^{26}$ The five occupational categories were based on the NOC-S 2001 codes and the six industry categories were based on NAICS 2002 codes (Statistics Canada, 2007b; 2007c). The specifics of these categories, as well as the specifics of categories pertaining to all other variables, are summarized in Appendix A1.

${ }^{27}$ To measure the size of the workplace, the LFS asked the respondents about the number of workers $a t$ their place of employment. Three categories were included in the present analysis: small size workplace, containing 99 or less workers; medium size workplace, containing between 100 and 500 workers; and large size workplace, containing over 500 workers. This measure is different from the firm size measure, which pertains to the number of workers at all locations of the employer (Statistics Canada, 201 1, p.12).
} 
groups such as Aboriginal people in Canada tend to have less seniority, they would consequently be disproportionately affected by the unionized firms' adherence to the procedural rules. ${ }^{28}$ The existing literature, however, does not find this to be the case. In the United States, for instance, Blau and Kahn (1981) find that union coverage increases the probability of layoff only for white men while for the other groups, blacks and women, it lowers the layoff probability. Similarly, in examining layoff rates separately in unionized and non-unionized sectors in Canada, Singh and Reid (1998) find the relationship between the probability of layoff and designated group status weaker at unionized workplaces than at non-unionized workplaces. ${ }^{29}$ In general, the literature finds greater probabilities of layoff among workers not covered by collective agreements (Galarneau \& Stratychuk, 2001; Wilson, 2005).

The main analyses below also control for type of work, job status and job permanency, student status and family structure as a proxy for the perceived quit probabilities (Blau \& Kahn, 1981) and for region. The existing studies from Canada report a great regional variation in the impact of recession, with Atlantic and Quebec residence being strongly positively associated with the probability of layoff. With one exception (Singh \& Reid, 1998) all of the reviewed studies report higher probabilities of layoff for men than for women. Based on the insights derived from the descriptive evidence, the comparison of the layoff estimates is done within gender (Aboriginal versus non-Aboriginal) while the comparison of the reemployment estimates is done across gender (men versus women) within Aboriginal identity groups.

\footnotetext{
${ }^{28}$ This apparent conflict of objectives has not been addressed in the Employment Equity Act (Government of Canada, 2012).

${ }^{29}$ Singh and Reid (1998) did not include Aboriginal people in their examination of designated group members.
} 


\subsection{Econometric Concerns}

The attempt to measure the pure Aboriginal-to-non-Aboriginal differentials in the probability of layoff and the probability of reemployment in this study entails a number of interrelated issues, the most important being those concerning the possibility or the choice to fully control for important layoff-determining and reemployment-determining factors. This section provides a brief discussion of two sets of potentially important econometric issues that arise in assessing the estimates of the probability of layoff and the probability of reemployment presented in this study. Although they are related, the discussion is organized around the idea that the first set of issues arises from the construction of the analytical samples and the second set of issues arises from the specification of the models.

The first set of econometric issues arises from the fact that the layoff estimates are based on a sample of individuals who were already employed and the reemployment estimates are based on a subsample of already-employed workers who became unemployed due to layoff. To some extent, both samples may be self-selected, and that may result in a sample selection bias. For example, the already-employed individuals may have unobserved characteristics that make them less layoff-prone, compared to those who were not employed. In regard to reemployment, the construction of the subsample of workers unemployed due to layoff does not take into account the possibility that some of them may have stopped searching for a job and returned to school or become discouraged workers as a consequence of this layoff. The fact that these samples are observed only for a relatively short period of time (six months) exacerbates these issues. 
Another possible source of sample selection bias is induced by the endogeneity of firms' decisions on whom to hire in the first place, whom to lay off, and whom to rehire. Structural variations across firms and industries, in particular, may confound layoff analysis; what makes a particular firm make a particular decision depends on a number of factors, a taste for discrimination not excluded, and subsequently the hiring, like the layoff decision outcomes cannot be attributed to purely random factors.

A related possible source of selection bias arises from the endogeneity of workers' decision to seek reemployment following the layoff; what induces an individual to seek reemployment shortly after the layoff and to accept a job depends on a number of factors, some of which are unobserved. For instance, in constructing the subsample used to estimate the probability of reemployment in this study, it was not possible to take account of potential differences in individuals" "sense of urgency" to accept a job. As indicated earlier in footnote 20 , this subsample might disproportionately consist of workers forced by financial pressures to seek reemployment and to accept any available job. That is, individuals accepting jobs shortly after layoff might differ because of unobservable factors, which necessarily biases the comparison of the estimated effects.

Since firms' decisions about hiring and layoffs, and individuals' propensities to accept work, are likely nonrandom, the analysis of the layoffs and reemployment here may be subject to possible sample selection bias, threatening both the internal and the external validity. If being already-employed is systemically correlated with unobservable factors that affect the probability of layoff, including only the already-employed people would render the sample biased and would produce inaccurate estimators in the layoff 
equation; inferences derived from a biased sample would likely not be generalizable to the population of concern. The same applies to the reemployment equation.

To address the issue of sample selectivity, studies generally apply maximum likelihood methods that allow for correlation among the structural equations underlying the estimated model (Wooldridge, 2010). The importance of controlling for sample selection bias in the existing literature on the topic, however, is not clear. Most of the studies reviewed here do not even discuss the issue and those that do generally report inconclusive evidence; while some report no striking changes in the size and precision of the estimated coefficients (Blau \& Kahn, 1981) others report opposing effects (Miller, 1987; George \& Kuhn, 1994).

In examining the wage gap between Canadian men and Canadian women, Miller (1987), for instance, has found that correcting for sample selection bias made the estimated discrimination problem more pronounced in that it increased the estimate of gender gap in the offered wage distribution. Looking at the wage differentials between Aboriginal and non-Aboriginal Canadians, George and Kuhn (1994), on the other hand, have found that correcting for sample selection bias reduced the size of estimated differentials, albeit it did not change the pattern of gaps. In view of this incongruity, some researchers have chosen not to correct for the sample selection bias, arguing that the procedure makes not much practical difference when attempting to assess possible discrimination in the labour market (Pendakur \& Pendakur, 1998).

The methods for testing and correcting for sample selection bias are based on certain sets of assumptions (Wooldridge, 2010) and they perform well when those assumptions are strong and when they hold in the used dataset. Weaker assumptions 
generally produce different results and when the assumptions are necessarily false or when there is uncertainty as to the source of bias, the sample selection correction methods generally do not work (Winship \& Mare, 1992; Jung \& Pirog, 2011). In some contexts, sample selection correction worsens rather than improves the biased estimates (Stolzenberg \& Relles, 1997). Following Pendakur and Pendakur (1998), no attempt is made in this study to deal with the potential sample selection bias. Arguably, if the bias is present, its magnitude would likely be small since most of the important variables affecting layoffs and reemployment are indeed included in the estimated models.

The second set of econometric issues arises from the specification of the models and involves the treatment of the conventionally-important control variables that relate to job characteristics such as occupation, industry, or collective agreement coverage. These variables are routinely included in the examinations of labour market outcomes in a general context; in the context of examining the sources of differentials in outcomes among groups, however, the value of their inclusion is subject to debate. The primary issue here is the sorting of workers across industries and sectors. The two most obvious cases are sorting by measured human capital (education and experience) and by unmeasured human capital (ability). The unobservable ability is generally unknown to researchers but possibly known to employers who have screening procedures in place, feedback from which they can use to decide which workers to hire or to lay off.

Another related issue is the nonrandom sorting of workers into sectors with different workplace conditions and different turnover rates. If employers have a taste for discrimination based on identity so that non-Aboriginal workers are segregated in largefirm sectors that are less sensitive to economic shocks and that provide ample 
opportunities for enrichment of human capital while Aboriginal workers are clustered in small-firm bust-sensitive sectors of the economy with little or no opportunities for human capital development, then this very segregation of workers by identity across sectors becomes responsible to a large extent for differential outcomes. If this unobserved factor (the employer's taste for discrimination) influences the outcome and the selection processes, then the estimated impacts of union, industry, and occupation are biased.

To estimate pure Aboriginal-to-non-Aboriginal layoff and reemployment differentials, it is important to control for other outcome-determining factors, possibly accounting for the joint determination of a factor and its impact on the examined outcome. For example, in order to be able to attribute the observed differential purely to the union status, it is important to control for factors that jointly determine the union status and the impact union membership has on the examined outcome. The same holds for all other employment-related factors; the issue of the selection process determining which workers enter the union or industry sector, for example, need to be addressed. As mentioned in the preceding section, the existing literature reports quite a concentration of permanent layoffs in certain industries and in the small-firm sector (Picot, 1992; Picot, Lin \& Pyper,1998; Galarneau \& Stratychuk, 2001; Bernard \& Galarneau, 2010), implying that inclusion of these job-related variables may confound the estimates in the current study. In fact, to assess if the labour market discrimination is an issue in the present analysis, the potential nonrandom sorting issues imply exclusion from the layoff equations most of the job-related variables; if occupational distribution, for example, is the result of discrimination then controlling for occupation will likely reduce the possibility of finding discrimination effect in the layoffs estimates. 
Indeed, it appears that this practice of exclusion of job-related characteristics is becoming a standard in the wage discrimination literature. In their recent assessment of earnings differentials between ethnic groups, Pendakur and Pendakur (2002), for instance, deliberately exclude all job-related variables from their models, arguing that job characteristics of workers "are at least as susceptible to ethnic discrimination as the wages paid to the workers" (492) due to prevalence of ethnic segregation of workforce across occupational categories and industry sectors. ${ }^{30}$ Gunderson, Hyatt and Riddell (2000) provide a similar rationale for not controlling for occupational distribution and union coverage in their assessment of wage differentials between employees in public and private sectors. They, however, suggest that

it is appropriate to control for such occupational differences if they are indicative of what is necessary to do the job. However, if some of the higher occupational status in the public sector were simply a mechanism whereby higher wages were paid, then it is not appropriate to control for such occupational differences since they in fact are the source of the public sector rents. This is especially the case if underlying factors such as education are already controlled for. In such circumstances, it may be appropriate not to include any occupational categories in the wage equations. (p. 9-10, emphasis in the original)

They offer a similar rationale for the choice of controlling or not controlling for differences in the degree of collective agreement coverage but note that there is no end to which the application of the rationale ceases; even the personal characteristics of workers such as age and educational attainment can be regarded as being not essential factors for doing the job but rather as means of "legitimizing the payment of a public sector wage premium," in which case it would be appropriate not to control for those variables in the estimation models (Gunderson, Hyatt \& Riddell, 2000, p. 11).

\footnotetext{
${ }^{30}$ In their other work on the same topic, they included controls for all job-related characteristics and found that the gap in earnings differentials narrowed but did not disappear (Pendakur \& Pendakur, 1998; 2007).
} 
To account for these issues in some way, the layoff models with omitted employment-related variables were run, thus restricting attention to a sample designed to minimize the importance of possible negative selection. For comparison purposes, the estimates from that analysis are presented along with the estimates from models that included all job-related characteristics of workers. ${ }^{31}$ Also, an attempt was made to account for the potential segregation issue in the full models by constructing broader levels of occupational classification, as opposed to the finer levels, as suggested by Gunderson, Hyatt and Riddell (2000).

${ }^{31}$ It was instructive to observe changes in the gaps when the layoff models were estimated with omitted occupational status variable. For policy discussion purposes, following the wage literature, the larger gap can be regarded as an upper bound on the pure outcome differential because it is the gap that includes the effect of occupational segregation as well as pure outcome differential in the same occupation (Gunderson, Hyatt \& Riddell, 2000). 


\section{Transition Probabilities}

This section presents estimated monthly probabilities of transitions from paid employment into unemployment due to layoff and the probabilities of reemployment conditional on being laid off, separately for male and for female Aboriginal and nonAboriginal workers. In contrast to the discussion in Section 2.3, where the individual and job characteristics that affect layoff and reemployment were discussed, this part of the analysis assumes that, within each sample, there is only one transition probability for a layoff and one for reemployment. Using these parametric transition probabilities, the associated measures for the steady state level of employment and the average first duration of unemployment for each group are computed. The transition probabilities are derived using a simple descriptive technique that is in some ways similar to the two-state Markov model of labour force transitions (Eaton, 1970). The model, however, does not exhibit all Markov properties, as will be discussed below, and is therefore referred to here as a "Markov-like" model. Figure 1 captures the basic idea of the model.

Figure 1: Two State Markov-like Model of Labour Force Transitions

\begin{tabular}{|c|c|c|}
\hline Employed & $P_{x}^{10}$ & Unemployed \\
\hline as paid employee & & due to layoff \\
\hline (State 0$)$ & $P_{x}{ }^{01}$ & (State 1) \\
\hline
\end{tabular}

In the empirical framework of this simple model, the two states in Figure 1 are: (1) State 0, labeled as employed (E); and (2) State 1, labeled as unemployed (U). These 
two states are mutually exclusive and exhaustive classifications of an individual's labour force status. At time zero, all individuals are paid employees (E) and in each of the subsequent six months, some of them will become unemployed (U) due to layoff. The assumed to be constant probability of layoff will be denoted by the Greek letter $\alpha$; the probability of remaining employed is then $1-\alpha{ }^{32}$

Immediately following a layoff, laid-off workers are assumed to be unemployed; that is, the laid-off individuals have moved from $\mathrm{E}$ to $\mathrm{U}$, as opposed to moving out of the labour force. With one month being the unit of time in the LFS, the assumption is that during any month of the stay in the survey, the individual who became unemployed due to layoff will become reemployed with probability $\beta$ and will remain unemployed with the probability $1-\beta$. These probabilities, $\beta$ and $1-\beta$, describe the initial absorption process for an individual who was unemployed due to layoff.

The second significant process accounts for cases of multiple layoffs whereby the reemployed individual becomes re-unemployed, that is, moves from state $\mathrm{E}$ to state $\mathrm{U}$. Here again the assumption is that during any month of the stay in the survey, a laid-off individual who was reemployed will become unemployed again due to layoff with probability $\alpha$ and will remain reemployed with probability $1-\alpha$. This second set of probabilities, $\alpha$ and $1-\alpha$, describes the disemployment process. The state transition matrix is then

\footnotetext{
${ }^{32}$ Adopting the notation $\mathrm{p}_{i j}$ to denote the conditional probability of a one-step transition to state $i$ from state $j$, that is $p_{i j}=\mathrm{P}\{X(m+1)=j \mid X(m)=i\}$, for $i, j=0,1$, and for any $m=1,2,3, \ldots$, then $p_{01}=\mathrm{P}\{X(m+1)=$ $1 \mid X(m)=0\}=\alpha$. Arranged in a $2 \times 2$ matrix, these probabilities $p_{i j}$ are $\mathrm{P}=\left[\begin{array}{ll}p_{00} & p_{01} \\ p_{10} & p_{11}\end{array}\right]=\left[\begin{array}{cc}1-\alpha & \alpha \\ \beta & 1-\beta\end{array}\right]$ with rows summing to 1 .
} 


$$
P=\frac{E}{U}\left[\begin{array}{cc}
E & U \\
\beta & 1-\alpha
\end{array}\right]
$$

where the current states are listed on the left of the transition matrix, and the future states in the next month time period are listed across the top.

Looked at in this way, $\beta$ and $\alpha$ describe the reemployment process as a first-order regular Markov chain, common to all individuals in the identity group. As noted above, the assumption here is that $\beta$ and $\alpha$ are the same for each individual in the group and that they are constant over time. ${ }^{33}$ Nevertheless, this examination is informative even if treated only as a descriptive initial look into the average transition dynamics for the groups examined here. ${ }^{34}$

Using the initial distribution vector $S_{0}=\left[\begin{array}{ll}0 & 1\end{array}\right]$, the resultant transition matrix, the time path of reemployment in period 1 is written as

$$
S_{1}=S_{0} \times P=\left[\begin{array}{ll}
0 & 1
\end{array}\right]\left[\begin{array}{cc}
1-\alpha & \alpha \\
\beta & 1-\beta
\end{array}\right]=\left[\begin{array}{ll}
\beta & 1-\beta
\end{array}\right] \text {. }
$$

${ }^{33}$ The equality assumption applies within the ethnic identity and gender groups considered here.

${ }^{34}$ The transition probabilities reported here are estimated using the Stata's xttrans command, along with the prescribed special procedure for generating Markov probabilities. The Stata manual suggests that the transition probabilities obtained using the command on its own cannot be regarded as transition probabilities in a true Markov sense, unless missing data is clearly a non issue. Otherwise, the manual suggests applying a special procedure to ensure the estimated probabilities are indeed Markov probabilities. This procedure was applied in the computation of the probabilities presented in this section. To be more specific, in estimating the Markov transition matrix for each identity group in this study, the reshaped long form unbalanced data were first normalized (rectangulirized) using fillin command to account for missing observations and missing periods at the end of each panel. According to Stata's manual, this 'normalization' is necessary because the xttrans command counts transitions from each observation to the next once the observations have been put in $t$ order within $i$ and if there are missing values, the command produces inefficient estimates of transition probabilities. With all this said, there seems to be no consensus among researchers on whether or not the command is in fact doing what the manual claims to be doing. It is possible that the estimated probabilities are simple means and hence they are treated here as simple basic descriptive evidence. The author is grateful to Dr. Marcel-Cristian Voia, Carleton University, Department of Economics, for providing consultations and guidance on this matter. 
The vector $S_{1}$ here suggests that $\beta$ of the entire group of laid-off workers will become reemployed in the first period after they are laid off and $1-\beta$ will remain unemployed.

In period 2, the status distribution vector $S_{2}$ is

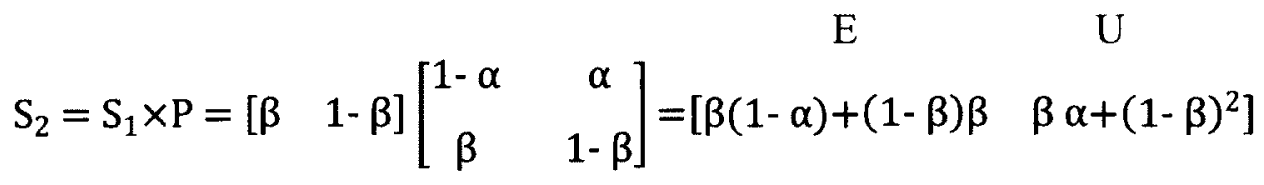

suggesting that $\beta(1-\alpha)+(1-\beta) \beta$ of the layoff group will become reemployed in period 2 . In this sum, the first term $[\beta(1-\alpha)]$ represents the individuals who became reemployed in period 1 and who remained employed in period 2 and second term (1- $\beta) \beta$ represents the individuals who remained unemployed in period 1 but who became reemployed in period 2. In more general terms, the status distribution vector $S_{n}$ is described as

$$
S_{n}=S_{0} \times P^{n}=\left[\begin{array}{ll}
0 & 1
\end{array}\right]\left[\begin{array}{cc}
1-\alpha & \alpha \\
\beta & 1-\beta
\end{array}\right]^{n}
$$

where $\beta$ is the rate per month at which the laid-off workers become reemployed (rate of absorption) and $\alpha$ is the rate at which the reemployed laid-off workers become unemployed again (rate of disemployment). Since these rates in the present analysis are averaged over the time period, then

$$
n^{-1} \sum_{m=1}^{n} P^{m}(1,0)
$$


is the probability of being in state 1 averaged over $n$ consecutive steps, six in this case. These averaged rates are then used to derive the measures for equilibrium level of employment

$$
S_{E}=\left[\begin{array}{cc}
E & \mathrm{U} \\
\frac{\beta}{\beta+\alpha} & \frac{\alpha}{\beta+\alpha}
\end{array}\right]
$$

as well as the average duration of unemployment from layoff to first job (average first duration) $(1 / \beta)$ and its associated variance $\left[(1-\beta) / \beta^{2}\right]$. The results are presented next. 


\subsection{Estimated Probabilities of Layoff and of Reemployment}

The estimated transition probability matrix for each identity group is presented below, separately for men and women, followed by the tabulated measures of the equilibrium level of employment and the average first duration of unemployment. A simple example is given here to illustrate how these probabilities are calculated.

Suppose a sample consists of only three workers, all of whom are paid employees in period 1 . There is no other information for any of the three workers but their employment status is known for each of their six months in the survey. To calculate the transition probabilities, no information other than the $0-1$ labour force status variables is needed. The balanced panel data matrix would be: ${ }^{35}$

\begin{tabular}{ccccccc} 
ID & Month 1 & Month 2 & Month 3 & Month 4 & Month 5 & Month 6 \\
\hline 1 & 0 & 1 & 1 & 1 & 1 & 1 \\
2 & 0 & 1 & 0 & 0 & 0 & 0 \\
3 & 0 & 0 & 0 & 0 & 0 & 0
\end{tabular}

As shown in this matrix, Person \#1 was laid off at the beginning of month 2 of the survey and was never reemployed. Person $\# 2$ was laid off at the beginning of month 2 but was reemployed at the beginning of month 3 . Person \#3 remained employed for all six months of the survey. Note that each person has five possible transitions during the six-

\footnotetext{
${ }^{35}$ For space considerations, the data matrix here is presented in a wide form. As indicated earlier, in the calculations of the actual probabilities, the data were reshaped into long form where each observation was individual-time $(i, t)$ pair. Presenting this simple example here in a long form would expand these three observations from the wide form to 18 in the long form, since each individual had six 0-1 labour force status figures. A balanced panel means that each of the units, $i=1, \ldots, \mathrm{G}$, is observed in every period, $t=$ $1, \ldots, T$. As indicated earlier, this was achieved by using the Stata's fillin command.
} 
month stay in the survey, between months 1 and 2, 2 and 3,3 and 4, 4 and 5 and, finally, between months 5 and 6 .

In the above data, there are thus 15 possible transitions. Ten of the fifteen are possible transitions from 0 to 1 while the remaining five are from 1 to 0 . Of the ten possible 0-1 transitions, two actually occur (for Person \#1 and Person \#2 between months 1 and 2); of the five possible 1-0 transitions, only one occurs (for Person \#2 between months 2 and 3). Therefore, using the method of this section, the probability of being laid off - moving from 0 to 1 - would be $2 / 10$ and the probability of reemployment, conditional on layoff, would be $1 / 5$. The resulting transition matrix would be:

$$
P=\left[\begin{array}{ccc}
\mathbf{E} & \mathbf{E} & \mathbf{U} \\
\mathbf{U} & 0.8 & 0.2 \\
& 0.2 & 0.8
\end{array}\right]
$$

In the actual analysis presented in this section, there were, of course, far more than three workers and missing values had to be taken into account (see Note 33) but the basic calculation was similar.

Survey weights have not been applied in deriving any of the estimates presented in this section. ${ }^{36}$ The rows in the matrices that follow for each identity group reflect the initial values at time $t-l$ and the columns reflect the final values at time $t$, in a percentage form. The discussion starts with the comparison of the results for aggregated groups and continues with the comparison of the results for disaggregated groups, for each gender.

The key finding arising from the comparison of the average monthly transition probabilities between non-Aboriginal $\left(P_{\text {naid }}\right)$ and the aggregate Aboriginal $\left(P_{\text {aid }}\right)$

\footnotetext{
${ }^{36}$ The Stata's xttrans command, used to derive the transition probabilities, does not support weights.
} 
identity groups of men confirms the weighted results discussed in Section 2.1 of the essay. As shown in the following matrices, Aboriginal men were much more likely to be laid off in the 2008-2011 recession than were non-Aboriginal men (8.7 compared to 5.4) but they were also more likely to have obtained paid employment following the layoff than were non-Aboriginal men (2.4 compared to 2.1$)$. The difference in the transition probabilities of reemployment between the two groups, however, is not as pronounced as it is in the case of layoffs.

$$
\begin{aligned}
& P_{\text {naid }_{m}}=\left[\begin{array}{ccc}
\mathbf{E} & \mathbf{E} & \mathbf{U} \\
\mathbf{U} & 2.1 & 5.4 \\
& 97.9
\end{array}\right] \quad P_{\text {aid }_{m}}=\left[\begin{array}{ccc}
\mathbf{E} & \mathbf{E} & \mathbf{U} \\
\mathbf{U} & 2.4 & 9.7 \\
& & 97.6
\end{array}\right] \\
& P_{\text {aidmet }_{m}}=\left[\begin{array}{ccc}
\mathbf{E} & \mathbf{E} & \mathbf{U} \\
\mathbf{U} & 2.8 & 7.2 \\
97.8
\end{array}\right] \\
& P_{\text {aidnai }_{m}}=\left[\begin{array}{ccc}
\mathbf{E} & \mathbf{E} & \mathbf{U} \\
\mathbf{U} & 2.7 & 10.3 \\
& 2.7 & 97.3
\end{array}\right]
\end{aligned}
$$

This finding becomes clearer at the disaggregate level of grouping for Aboriginal identity men, revealing that the observed difference is most drastic in the comparison of non-Aboriginal identity and NAI groups of men. As revealed in the matrix of NAI men $\left(P_{\text {aidnai }}\right)$, the probability of layoff for NAI men (10.3) was almost twice the probability of layoff for non-Aboriginal identity men (5.4). The difference in the probability of layoff between non-Aboriginal men and Métis men, as revealed in the matrix for Métis men $\left(P_{\text {aidmet }}\right)$, is also notable (7.2 compared to 5.4).

In terms of transition probabilities of reemployment, the disaggregate groups comparison suggests that Métis men were only slightly more likely to have obtained paid 
employment following the layoff than were non-Aboriginal identity men (2.2 compared to 2.1) while the transition probability for NAI men (2.7) was highest among the groups. This evidence is not in agreement with the descriptive evidence presented earlier in Section 2.1 of the essay and the difference in the application of the survey weights could, possibly, account for some of this incongruity.

The transition matrices for women, presented next, reveal similar patterns to those of men, although the differences in the transition probabilities for reemployment are more evident and to a degree surprising. As shown in the following set of matrices, Aboriginal women were not only more likely to have obtained paid employment following the layoff than were non-Aboriginal women (4.4 compared to 2.7 ), they were also much more likely to have obtained paid employment (after a layoff) relative to Aboriginal men - a finding which does not coincide with the descriptive evidence presented in Section 2.1.

$$
\begin{aligned}
& P_{\text {naid }_{w}}=\left[\begin{array}{ccc}
\mathbf{E} & \mathbf{E} & \mathbf{U} \\
\mathbf{U} & 2.7 & 9.5 \\
& 97.3
\end{array}\right] \quad P_{\text {aid }}=\left[\begin{array}{ccc}
\mathbf{E} & \mathbf{E} & \mathbf{U} \\
\mathbf{U} & 4.4 & 4.8 \\
& & 95.6
\end{array}\right] \\
& P_{\text {aidmet }_{w}}=\left[\begin{array}{ccc}
\mathbf{E} & \mathbf{E} & \mathbf{U} \\
\mathbf{U} & 3.6 & 4.4 \\
& 96.2
\end{array}\right] \\
& P_{\text {aidnai }_{w}}=\left[\begin{array}{ccc}
\mathbf{E} & \mathbf{E} & \mathbf{U} \\
\mathbf{U} & 4.8 & 5.2 \\
& 95.3
\end{array}\right]
\end{aligned}
$$

In terms of layoffs, the comparison of transition probabilities between nonAboriginal women $\left(P_{n a i d_{w}}\right)$ and the aggregate group of Aboriginal women $\left(P_{a i d_{w}}\right)$ indicates that the transition probability of layoff for Aboriginal women (4.8) was higher 
than the transition probability of layoff for non-Aboriginal women (3.5). This difference, however, is slightly less pronounced than is the difference in the transition probabilities of reemployment.

As it was the case of men, the disaggregated matrices of women suggest that the greatest differences in the transition probabilities are between non-Aboriginal and NAI women. As shown in the NAI women matrix $\left(P_{\text {aidnai }}\right)$ the probability of transition from paid employment to unemployment due to layoff for NAI women was much higher than the probability for non-Aboriginal women (5.2 compared to 3.5). However, the probability for NAI women obtaining paid employment after the layoff (4.7) was also significantly above that of non-Aboriginal women (2.7). The matrix for Métis women $\left(P_{\text {aidmet }}\right)$ shows the same patterns although the magnitudes of the differences in the transition probabilities here are smaller than those of NAI women. The transition probabilities of Métis women were 4.4 and 3.8 for layoff and reemployment, respectively.

Overall, the above comparisons of the monthly transition probabilities suggest that Aboriginal identity workers were more likely to be laid off but they were also more likely to be reemployed following the layoff, relative to non-Aboriginal identity workers. In terms of gender, men, in each group, were more likely to be laid off than women, with NAI men showing the highest probability of moving from paid employment to unemployment due to layoff. Women, on the other hand, in each group were more likely to have secured paid employment shortly after the layoff, with NAI women showing the highest rate of this transition probability. 


\subsection{Steady State Employment and Durations of Unemployment}

While informative, the comparisons of the estimated transition probabilities presented above give no insights into the differences in the duration of employment and unemployment among the groups. That information could be insightful in terms of gauging the extent of hardship experienced by each group. Table 2 displays the equilibrium levels of employment, under the assumption of parametric probabilities, along with average first durations of unemployment, measured in months, for each group.

Table 2: The equilibrium level of employment and average first duration of unemployment, by identity

\begin{tabular}{|c|c|c|c|c|c|c|c|c|}
\hline \multirow{2}{*}{ Measures } & \multicolumn{2}{|c|}{$\begin{array}{c}\text { Non-Aboriginal } \\
\text { Identity }\end{array}$} & \multicolumn{2}{|c|}{$\begin{array}{l}\text { Aboriginal } \\
\text { Identity }\end{array}$} & \multicolumn{2}{|c|}{ Métis } & \multicolumn{2}{|c|}{$\begin{array}{c}\text { North American } \\
\text { Indian }\end{array}$} \\
\hline & Men & Women & Men & Women & Men & Women & Men & Women \\
\hline Steady state employment & 0.28 & 0.43 & 0.22 & 0.48 & 0.23 & 0.46 & 0.21 & 0.47 \\
\hline $\begin{array}{l}\text { Average first duration of } \\
\text { unemployment }\end{array}$ & $\begin{array}{l}0.476 \\
(4.71)\end{array}$ & $\begin{array}{l}0.370 \\
(3.65)\end{array}$ & $\begin{array}{l}0.416 \\
(4.12)\end{array}$ & $\begin{array}{l}0.227 \\
(2.22)\end{array}$ & $\begin{array}{l}0.454 \\
(4.49)\end{array}$ & $\begin{array}{l}0.263 \\
(2.58)\end{array}$ & $\begin{array}{l}0.370 \\
(3.65)\end{array}$ & $\begin{array}{l}0.213 \\
(2.08)\end{array}$ \\
\hline
\end{tabular}

Note: The author's calculations from the relevant matrices. Standard deviations in parentheses.

The first row in Table 2 lists equilibrium levels of employment for each group by gender. This measure provides some comparative insight into the steady state where the tendency for the employment and unemployment states to change is diminished. This does not mean that the groups will stay in one state for they will continue to move from state to state in the future time periods; however, the average probabilities of moving from state to state for all periods will remain constant, in the long run. ${ }^{37}$ That is, if $\frac{\beta}{(\beta+\alpha)}$

\footnotetext{
${ }^{37}$ A qualifying note is warranted here to emphasize that the 'long run' here is less meaningful since the data used to study this phenomenon are seriously censored and the behaviour of individuals is observed for only six months. Nevertheless, possible insights can be revealing, especially if consistent with other methods.
} 
of the group were employed in period $m$, than we would expect to find the same proportion employed in the following periods, $m+n,(n \geq 1)$. The average time listed in the following row of Table 2 does not pertain to the time it takes to reach this steady state but rather to the average first duration of unemployment (from layoff to first job) for each group for their time of stay in the survey, which is six months.

As shown in Table 2, the equilibrium levels of employment for Aboriginal men are lower than the equilibrium level of employment for non-Aboriginal men. In the case of women, the opposite appears to be true; the equilibrium levels of employment for Aboriginal women are higher than those of non-Aboriginal women, which coincides with the preceding discussion. As expected, the average first duration of unemployment is higher among men in each group than it is among women. Among groups, NAI display the lowest average first duration of unemployment; for NAI men, the average first duration of unemployment is 0.370 , which is comparable to that of non-Aboriginal identity women, while for NAI women the average first duration of unemployment is 0.213 . The associated standard deviations in each case, however, are relatively large in comparison to the estimated values, suggesting that many observations differ substantially from the average.

The key conclusion arising from this analysis of the average transition dynamics of labour force states during the 2008-2011 recession is that the patterns of Aboriginal identity workers were quite different from those of non-Aboriginal identity workers. Aboriginal identity workers, and in particular NAI men, were more likely to be laid off than were non-Aboriginal identity workers but they were also more likely to be 
reemployed, and in particular NAI women, than were non-Aboriginal identity workers. The average first duration of unemployment is in fact lowest in the case of NAI women. The estimates presented in this section, however, pool individuals who differ on many levels. As outlined in Section 2.3, many factors contribute to an individual's likelihood to be in one state or the other; it is therefore important to control for that heterogeneity. Also, as mentioned in Section 2.1, applying survey weights is important, given the complex survey design. The following section reports results from the decompositions of the mean differences in estimates derived from binomial probit models of the determinants of probability of layoff and the probability of reemployment for each group separately. Survey sampling weights are applied in all of the analyses that follow to account for complex survey design. 


\section{Blinder-Oaxaca Decomposition}

This section presents the results from the main analyses of this essay whereby multivariate models of layoffs and reemployment are estimated in an attempt to gain some insights into the research questions posed in the introduction section: What underlies the disproportionate impact of recession on the two groups? To what extent is the difference in the impact the result of the differences in the observable characteristics among Aboriginal and non-Aboriginal workers and the jobs they hold and to what extent it is the result of employer discrimination in favour of non-Aboriginal workers? To gain some insights, pooled samples of Aboriginal and non-Aboriginal workers are examined and the estimated differences are decomposed into characteristics effects and coefficients effects, both at an aggregate (all variables included) and at detailed (individual variables) level. Some discussion on this method of analysis was offered earlier in Section 2.2; here more details are provided in each pertinent subsection, before presenting the results.

To determine the sources of the difference in the probability of layoff and the probability of reemployment between Aboriginal and non-Aboriginal identity workers, an econometric technique developed over time by Yun $(2000,2004,2008)$ and summarized in Powers, Yoshioka and Yun (2011) is employed. This technique is the most recent extension of the Blinder-Oaxaca decomposition method to nonlinear models and it provides the aggregate and the detailed decomposition and standard errors both for the explained (characteristics or endowments effects) and for the unexplained (coefficients effects) components of the differences. It also provides solutions to two problems inherent in the nonlinear detailed decomposition: (1) path dependency and (2) the 
identification problem associated with the choice of reference category when binary indicators are included in the list of predictors, as is the case in the present analysis.

The results from the overall and from the detailed decomposition are presented separately in the following two subsections. The decomposition is based on the output from a multivariate binomial probit model (Wooldridge, 2010) estimated separately for each group by maximum likelihood, specifications of which are summarized below. ${ }^{38}$

The analysis is based on a sample of $\mathrm{N}$ observations $i=1 \ldots, \mathrm{N}$, assumed to be independently and identically distributed, of the dependent binary response variable $y_{\mathrm{i}}$ and $\mathrm{a}(\mathrm{K}+1)$-dimensional vector $x_{i}^{\prime}$ of explanatory variables. The probability that the dependent variable (either layoff or reemployment) takes value of 1 is modeled as $P\left(y_{i}=1 \mid x_{i}\right)=F\left(z_{i}\right)=F\left(x_{i}^{\prime} \beta\right)$

where $\beta$ is a $(\mathrm{K}+1)$-dimensional column vector of unknown parameters and $z_{i}=x_{i}^{\prime} \beta$ is a single linear index. The transformation function $\mathrm{F}$ maps the single index into the $[0,1]$ interval; in the probit model $\mathrm{F}$ is assumed to be cumulative density function (cdf) of the standard normal distribution. The response probabilities are then

$P\left(y_{i}=1 \mid x_{i}\right)=\Phi\left(x_{i}^{\prime} \beta\right)=\int_{-\infty}^{x_{i}^{\prime} \beta} \phi(t) d t=\int_{-\infty}^{x_{i}^{\prime} \beta} \frac{1}{\sqrt{2 \pi}} e^{-\frac{1}{2} t^{2} d t}$

where $\phi($.$) is the pdf and \Phi($.$) the cdf of the standard normal distribution.$

${ }^{38}$ The underlying probit estimates are not discussed here but they are made available to interested readers in Appendix B (Appendix B1, Appendix B2, and Appendix B3). 
Following Yun, the difference between $\bar{Y}_{A}$ and $\bar{Y}_{B}$ at the first moment, that is the overall decomposition of the mean difference in $y_{\mathrm{i}}$ between Aboriginal groups (A) and non-Aboriginal group (B) in a probit decomposition equation is written as,

$\bar{Y}_{A}-\bar{Y}_{B}=\overbrace{\left[\overline{\Phi\left(X_{A} \beta_{A}\right)}-\overline{\Phi\left(X_{B} \beta_{A}\right)}\right]}^{E}+\overbrace{\left[\overline{\Phi\left(X_{B} \beta_{A}\right)}-\overline{\Phi\left(X_{B} \beta_{B}\right)}\right]}^{C}$,

where "over bar" represents the value of the sample's average and the components labeled $E$ and $C$ represent the overall difference in observable characteristics or endowments (the explained difference) and overall difference in coefficients (the unexplained difference), respectively.

Since in this equation, group A is the comparison group (Aboriginal) and group B is the reference group (non-Aboriginal), the component $E$ reflects the expected difference in average predicted outcomes if group A were given group B's distribution of covariates; in other words, the component $E$ is the part of the differential attributable to differences in characteristics between Aboriginal and non-Aboriginal workers, at the aggregate level of variables. The component $\mathrm{C}$ reflects the expected difference in average predicted outcomes if group B experienced group A's behavioural responses to $\mathrm{X}$, at the aggregate level of variables. In a univariate regression model, for instance, if only the level of educational attainment is considered and the two groups had different distributions on this variable, the component $E$ would tell by how much the probability of layoff (or of reemployment) would change if the distribution on this variable were equalized; no change would be an indication that educational attainment is not an important determinant of the probability of layoff (or the probability of reemployment). 
While the interpretation of the explained component of the decomposition is straightforward, the interpretation of the unexplained component demands more care as it involves considerations of differences in the returns to endowment and behavioural responses associated with those endowments that are tied not only to the individuals in the groups but also to the institutional context in which the individuals exist. For instance, the differences in the coefficients between the two groups can be interpreted as differences in the average behavioural response by individuals to individual characteristics if the choice is made of the individual's own free will, or as discrimination if the choice is made by others. The general trend, especially in the wage literature, has been to interpret the unexplained part of the decomposition as a measure of labour market discrimination, provided no reason exists to expect unobserved differences in productivity (Blinder, 1973; Oaxaca, 1973, 1994; Nielsen, 1998; Fairlie, 1999; Ben, 2008). The existing studies that applied Blinder-Oaxaca decomposition methods in examining the labour market disadvantage of Aboriginal people in Canada in the form of income and earnings have followed this trend as well (Pendakur \& Pendakur, 1998; De Silva, 1999; Kuhn \& Sweetman, 2002).

In the present analysis, this approach to interpretation of the unexplained component of the differences in outcomes is adopted only in the layoffs models, with the rationale that decision- making process here involves only employers; that is, the concerned individuals are either not contributing to that decision or at least not contributing in any observable and measurable way. The reemployment models not only involve different dynamics in terms of decision-making (reemployment is the outcome of decisions made both by individuals and by employers) but the comparison of the 
differentials here is made across gender within Aboriginal identity groups only. The examination of the difference that exists between non-Aboriginal men and women is not an objective of the analysis in this essay. ${ }^{39}$

The following section presents estimated results from the overall Aboriginal-tonon-Aboriginal decomposition separately for men and women in relation to layoffs and from the overall Aboriginal men to Aboriginal women decomposition in relation to reemployment. Specifications of the detailed decomposition method and the pertinent results of the unique contribution of each variable to each component of the difference are then presented in Section 4.2.

${ }^{39}$ This examination, however, was performed and the results will be discussed in a separate paper, postdissertation. The results can be made available to interested readers, upon request. 


\subsection{Overall Decomposition Results}

This section presents estimated results from the overall decomposition of the mean difference in the probability of layoff (and the probability of reemployment) between Aboriginal identity groups and non-Aboriginal identity groups (and between Aboriginal identity men and women) as observed in the main and in the more restricted samples, respectively. As explained in Section 2.1, the main samples include all paid employees who were aged between 15 and 64 and who were not full-time students; in the more restricted samples, all students are excluded along with seasonal workers and multiple-job holders, leaving in only permanent paid employees, aged between 15 and 65 , who were single job-holders. In each sample, two separate models for each group were run, one accounting only for the basic personal characteristics and the other adding in the job-related characteristics, discussed in Section 2.3. In the pertinent tables, the results from the former are labeled Model 1 while the results from the latter are labeled Model 2. The discussion starts with the presentation of the findings observed in the main samples and continues to those observed in the more restricted samples for men and women, respectively. When appropriate, attention is drawn to the size of the gaps presented in Sections 2.1 and 3.1.

\subsubsection{Layoffs}

The overall decomposition of the probit estimates of the mean difference in the predicted probability of layoff for men and for women, observed in the main sample, is presented in Table 3 below. Two key points need to be highlighted here: (1) the apparent change in the magnitude of the estimated raw gaps in the probability of layoff; and (2) the 
apparent dissimilarity in the contribution of the endowment and of the coefficient components across identity groups. Discussion here centers on these two points.

In regard to the first point, it appears that when the considered explanatory variables are included, both in Model 1 and in Model 2, the raw gaps in the predicted probability of layoffs between Aboriginal and non-Aboriginal identity men, as shown in the upper portion of Table 3, appear larger, relative to the gaps estimated in the previous analysis of transition probabilities in Section 3.1 but smaller from those reported in Section 2.1. As shown in Table 3, the estimated 4.1 percentage points gap in the probability of layoff between the aggregate Aboriginal identity group of men and nonAboriginal identity group of men is larger than the 3.3 percentage points gap estimated in the previous analysis in Section 3.1 but substantially smaller than the eight percentage points gap reported in Section 2.1. Similarly, the estimated 2.9 percentage points gap for Métis men presented in Table 3 is larger than the 1.8 percentage points gap estimated in Section 3.1 but smaller than the 5 percentage points gap presented in Section 2.1. As discussed in Section 2.1, NAI men had the largest probability of layoff. This fact remains true in the multivariate analysis; however, the size of the gap here, 5.5 percentage points, is significantly smaller than the 11 percentage points gap reported in Section 2.1 and slightly larger than the 4.9 percentage points gap estimated in the simple non-weighted analysis reported in Section 3.1. The application of the weights and the inclusion of other factors in the multivariate analysis account for these discrepancies. 
Table 3: Overall decomposition of the difference in predicted probability of layoff, main sample

\begin{tabular}{|c|c|c|c|c|c|c|}
\hline \multirow[t]{2}{*}{$\begin{array}{l}\text { Overall decomposition - layoffs } \\
\text { (difference in the first moment) } \\
\text { MAIN SAMPLE }\end{array}$} & \multicolumn{2}{|c|}{$\begin{array}{l}\text { Aboriginal } \\
\text { vs. } \\
\text { non-Aboriginal } \\
\text { Identity }\end{array}$} & \multicolumn{2}{|c|}{$\begin{array}{c}\text { Métis } \\
\text { vs. } \\
\text { non-Aboriginal } \\
\text { Identity }\end{array}$} & \multicolumn{2}{|c|}{$\begin{array}{l}\text { North American Indian } \\
\text { vs. } \\
\text { non-Aboriginal } \\
\text { Identity }\end{array}$} \\
\hline & Model 1 & Model 2 & Model 1 & Model 2 & Model 1 & Model 2 \\
\hline $\begin{array}{l}\text { Men ( } N=85766) \\
\text { Raw differential estimates } \\
\text { Due to endowments effect (E) }\end{array}$ & $\begin{array}{c}0.0414 \\
(0.009)^{4 t}\end{array}$ & $\begin{array}{c}0.0416 \\
(0.008)^{* \star * \star}\end{array}$ & $\begin{array}{c}0.0293 \\
(0.011)^{* *}\end{array}$ & $\begin{array}{l}0.0297 \\
(0.010)^{*}\end{array}$ & $\begin{array}{c}0.0551 \\
(0.014)^{* \star \star *}\end{array}$ & $\begin{array}{c}0.0542 \\
(0.013)^{*+* * t}\end{array}$ \\
\hline Estimate & $\begin{array}{c}0.004 \\
(0.009)\end{array}$ & $\begin{array}{c}0.012 \\
(0.009)\end{array}$ & $\begin{array}{c}0.012 \\
(0.010)\end{array}$ & $\begin{array}{l}0.016 \\
(0.011)\end{array}$ & $\begin{array}{l}-0.003 \\
(0.017)\end{array}$ & $\begin{array}{l}0.006 \\
(0.013)\end{array}$ \\
\hline Share (\%) & 10.2 & 28.3 & 41.3 & 54.3 & -6.5 & 11.2 \\
\hline $\begin{array}{l}\text { Due to coefficients effect (C) } \\
\text { Estimate }\end{array}$ & $\begin{array}{c}0.037 \\
(0.014)^{* *}\end{array}$ & $\begin{array}{c}0.030 \\
(0.012)^{*}\end{array}$ & $\begin{array}{c}0.017 \\
(0.015)\end{array}$ & $\begin{array}{c}0.013 \\
(0.014)\end{array}$ & $\begin{array}{c}0.059 \\
(0.024)^{\star}\end{array}$ & $\begin{array}{l}0.048 \\
(0.02)^{*}\end{array}$ \\
\hline Share (\%) & 89.8 & 71.7 & 58.6 & 45.7 & 106.5 & 88.8 \\
\hline $\begin{array}{l}\text { Women }(N=87267) \\
\text { Raw differential estimates } \\
\text { Due to endowments effect }(E)\end{array}$ & $\begin{array}{c}0.0152 \\
(0.006)^{*}\end{array}$ & $\begin{array}{l}0.0148 \\
(0.006)^{*}\end{array}$ & $\begin{array}{l}0.007 \\
(0.008)\end{array}$ & $\begin{array}{c}0.007 \\
(0.008)\end{array}$ & $\begin{array}{c}0.021 \\
(0.008)^{*}\end{array}$ & $\begin{array}{c}0.020 \\
(0.008)^{*}\end{array}$ \\
\hline Estimate & $\begin{array}{l}0.006 \\
(0.006)\end{array}$ & $\begin{array}{c}0.007 \\
(0.005)\end{array}$ & $\begin{array}{r}-0.005 \\
(0.011)\end{array}$ & $\begin{array}{r}-0.002 \\
(0.009)\end{array}$ & $\begin{array}{c}0.013 \\
(0.006)^{\star}\end{array}$ & $\begin{array}{c}0.014 \\
(0.005)^{\text {t* }}\end{array}$ \\
\hline Share $(\%)$ & 41.8 & 44.8 & -79.8 & -23.6 & 62.8 & 72.1 \\
\hline $\begin{array}{l}\text { Due to coefficients effect (C) } \\
\text { Estimate }\end{array}$ & $\begin{array}{c}0.009 \\
(0.009)\end{array}$ & $\begin{array}{c}0.008 \\
(0.009)\end{array}$ & $\begin{array}{c}0.011 \\
(0.016)\end{array}$ & $\begin{array}{c}0.008 \\
(0.013)\end{array}$ & $\begin{array}{c}0.008 \\
(0.010)\end{array}$ & $\begin{array}{c}0.006 \\
(0.009)\end{array}$ \\
\hline Share (\%) & 58.1 & 55.2 & 179.8 & 123.6 & 37.7 & 27.9 \\
\hline
\end{tabular}

Source: The Labour Force Survey, national monthly master file, September 2008 - May 2011.

Note: Decomposition refers to equation (1) components $E$ and $C$. Share is percentage share of predicted difference in the probability of being laid-off. Model 1 does not include any of the job-related characteristics. Model 2 includes all characteristics. Main samples include all paid employees, aged between 15 and 64, who are not full-time students. Survey weights in effect. Standard errors reported in parentheses. Statistical significance ${ }^{* * *} p<.001 ;{ }^{* *} p<.01 ;{ }^{*} p<.05$.

For women, the pattern appears similar, except in the case of Métis women. As shown in the lower portion of Table 3 , for the aggregate Aboriginal identity group of women and for NAI women, the estimated raw gaps (1.5\% and $2.1 \%$ respectively) are slightly larger than the gaps reported in Section 3.1 (1.3\% and $1.7 \%$ respectively) but much smaller than the gaps reported in Section 2.1 (4\% and 5\% respectively). For Métis women, however, the relatively small raw gap estimated here, 0.7 percentage points, is 
smaller than the gaps reported both in Section $3.1(0.9 \%)$ and in Section $2.1(3 \%)$. Thus in this case, only the coefficient estimates for the aggregate group of Aboriginal identity women and for NAI women are statistically significant.

In regard to the second point, it appears that when only basic characteristics are considered (Model 1) the endowment effect accounts for 41.3 percent of the mean difference in the predicted probability of layoff for Métis men — for NAI men, however, all of the difference is attributed to the difference in coefficients. When employmentrelated characteristics are included (Model 2) the explained component for Métis men rises to 54.3 percent but for NAI men to only 11.2 percent - suggesting that most of the difference still remains unexplained and, following the previous literature on the topic, is attributable to discrimination. The differences in endowments between non-Aboriginal identity women and NAI women appear to explain relatively large portion of the predicted gap in the probability of layoff, 62.8 percent to 72.1 percent, depending on whether or not the employment-related characteristics are considered in the model. These findings for the other groups of Aboriginal women are not statistically significant.

Table 4 presents the results from the same analysis, done using the more restricted samples in which seasonal workers and multiple-job holders as well as students are excluded. This analysis was done to check if the results from the main samples are affected by the presence of individuals (especially in the NAI samples) whose labour force activity is likely driven or constrained by other factors. For instance, it is possible that the layoffs associated with workers who hold seasonal jobs may not be related to the recession layoffs and multiple-job holders may be laid off from one job but remain employed at their other job(s). Eliminating these individuals from the samples should 
thus improve the precision of the findings reported in this study and provide clearer insights on the impact of recession, especially for NAI men.

As shown in the top row of Table 4, the estimated raw gaps in the probability of layoff for each group of men are smaller in comparison to those estimated in the main samples. The same appears to be the case for NAI women, suggesting that some of the layoffs in the main sample were likely related to seasonality. For Métis women, however, the small and statistically insignificant gap reported in the previous table appears relatively large in this table (1.7\%) and statistically significant in Model 2, which accounts for all personal as well as for all job-related characteristics. This is potentially an important insight; as indicated in Section 2.1, of all groups examined here, Métis women had the greatest proportion of multiple-job holders and they were disproportionately represented in casual or contract jobs. This finding suggests that the layoffs that occurred among Métis women were in fact layoffs from permanent jobs and might be related to sorting of Métis women into the layoff-prone industries or sectors.

Another unusual finding here is that, unlike in the main samples of men, in the restricted samples of men the size of the endowment effect declines slightly from Model 1 (where only basic personal characteristics are considered) to Model 2 (where jobrelated characteristics are included). This decline is relatively small for NAI men (it goes from $26.5 \%$ to $26.1 \%$ ) but not so small for Métis men (the decline goes from 47.6 to $35.0 \%)$. Nevertheless, the explained components remain relatively small, particularly for $\mathrm{NAI}$ men, thus maintaining the conclusion reached in the main samples - that for NAI much of the difference remains unexplained. 
Table 4: Overall decomposition of the difference in predicted probability of layoff, restricted sample

\begin{tabular}{|c|c|c|c|c|c|c|}
\hline \multirow[t]{2}{*}{$\begin{array}{l}\text { Overall decomposition - layoffs } \\
\text { (difference in the first moment) } \\
\text { RESTRICTED SAMPLE }\end{array}$} & \multicolumn{2}{|c|}{$\begin{array}{l}\text { Aboriginal } \\
\text { vs. } \\
\text { non-Aboriginal } \\
\text { Identity }\end{array}$} & \multicolumn{2}{|c|}{$\begin{array}{c}\text { Métis } \\
\text { vs. } \\
\text { non-Aboriginal } \\
\text { Identity }\end{array}$} & \multicolumn{2}{|c|}{$\begin{array}{l}\text { North American Indian } \\
\text { vs. } \\
\text { non-Aboriginal } \\
\text { Identity }\end{array}$} \\
\hline & Model 1 & Model 2 & Model 1 & Model 2 & Model 1 & Model 2 \\
\hline $\begin{array}{l}\text { Men }(N=72531) \\
\text { Raw differential estimates } \\
\text { Due to endowments effect (E) }\end{array}$ & $\begin{array}{c}0.0324 \\
(0.008)^{* t}\end{array}$ & $\begin{array}{c}0.0334 \\
(0.008)^{\star \star}\end{array}$ & $\begin{array}{c}0.0249 \\
(0.010)^{*}\end{array}$ & $\begin{array}{c}0.0262 \\
(0.009)^{* *}\end{array}$ & $\begin{array}{c}0.0439 \\
(0.014)^{* *}\end{array}$ & $\begin{array}{l}0.0427 \\
(0.013)^{* *}\end{array}$ \\
\hline Estimate & $\begin{array}{c}0.009 \\
(0.009)\end{array}$ & $\begin{array}{c}0.007 \\
(0.009)\end{array}$ & $\begin{array}{c}0.012 \\
(0.009)\end{array}$ & $\begin{array}{l}0.009 \\
(0.009)\end{array}$ & $\begin{array}{c}0.012 \\
(0.017)\end{array}$ & $\begin{array}{c}0.011 \\
(0.015)\end{array}$ \\
\hline Share (\%) & 26.9 & 21.5 & 47.6 & 35.0 & 26.5 & 26.1 \\
\hline $\begin{array}{l}\text { Due to coefficients effect (C) } \\
\text { Estimate }\end{array}$ & $\begin{array}{c}0.024 \\
(0.012)\end{array}$ & $\begin{array}{c}0.026 \\
(0.013)^{*}\end{array}$ & $\begin{array}{c}0.013 \\
(0.013)\end{array}$ & $\begin{array}{l}0.017 \\
(0.009)\end{array}$ & $\begin{array}{c}0.032 \\
(0.021)^{*}\end{array}$ & $\begin{array}{c}0.031 \\
(0.018)^{*}\end{array}$ \\
\hline Share (\%) & 73.1 & 78.5 & 52.4 & 65.0 & 73.5 & 73.9 \\
\hline $\begin{array}{l}\text { Women }(N=71498) \\
\text { Raw differential estimates }\end{array}$ & & & & & & \\
\hline $\begin{array}{l}\text { Raw differential estimates } \\
\text { Due to endowments effect }(\mathbf{E})\end{array}$ & $\begin{array}{c}0.0164 \\
(0.006)^{\star \star}\end{array}$ & $\begin{array}{c}0.0165 \\
(0.006)^{* *}\end{array}$ & $\begin{array}{l}0.0164 \\
(0.008)\end{array}$ & $\begin{array}{c}0.0172 \\
(0.007)^{\star}\end{array}$ & $\begin{array}{l}0.0146 \\
(0.008)\end{array}$ & $\begin{array}{l}0.0145 \\
(0.008)\end{array}$ \\
\hline Estimate & $\begin{array}{c}0.002 \\
(0.007)\end{array}$ & $\begin{array}{c}0.004 \\
(0.006)\end{array}$ & $\begin{array}{l}-0.002 \\
(0.012)\end{array}$ & $\begin{array}{c}0.007 \\
(0.007)\end{array}$ & $\begin{array}{c}0.008 \\
(0.005)\end{array}$ & $\begin{array}{c}0.009 \\
(0.005)\end{array}$ \\
\hline Share $(\%)$ & 14.3 & 25.4 & -12.8 & 41.3 & 57.93 & 64.3 \\
\hline Due to coefficients effect (C) & & & & & & \\
\hline Estimate & $\begin{array}{c}0.014 \\
(0.011)\end{array}$ & $\begin{array}{c}0.012 \\
(0.009)\end{array}$ & $\begin{array}{c}0.018 \\
(0.017)\end{array}$ & $\begin{array}{c}0.010 \\
(0.011)\end{array}$ & $\begin{array}{c}0.006 \\
(0.009)\end{array}$ & $\begin{array}{c}0.005 \\
(0.009)\end{array}$ \\
\hline Share $(\%)$ & 85.7 & 74.6 & 112.8 & 58.6 & 42.0 & 35.6 \\
\hline
\end{tabular}

Source: The Labour Force Survey, national monthly master file, September 2008 - May 2011.

Note: Decomposition refers to equation (1) components $E$ and $C$. Share is percentage share of predicted difference in the probability of being laid-off. Model 1 does not include any of the job-related characteristics. Model 2 includes all characteristics. Restricted samples include only permanent paid employees, aged between 15 and 64 , who are singlejob holders and who are not students. Survey weights in effect. Standard errors reported in parentheses. Statistical significance ${ }^{* * *} p<.001 ;{ }^{* *} p<.01 ;{ }^{*} p<.05$.

Interestingly, this decline is not evident in the case of women. As shown in Table 4 , the percentage share of the predicted mean difference in the probability of being laid off that can be attributed to the difference in endowments increases from Modell to Model 2 for all three groups of women. However, the estimated raw gap for NAI women is not statistically significant in this restricted sample, suggesting that the layoffs that occurred among NAI women were related to the factors excluded from this sample. The 
results from the overall decomposition of the mean difference in the probability of reemployment between Aboriginal men and Aboriginal women are discussed next.

\subsubsection{Reemployment}

The results from the overall decomposition of the mean difference in the predicted probability of reemployment among the Aboriginal identity groups, across gender, are presented in Table 5 and in Table 6 for the main and for the restricted samples, respectively. ${ }^{40}$ The discussion here centers on the magnitude of the estimated raw gaps, as compared to those reported in Section 2.1 and on the variation in the contribution of the endowment and of the coefficient components across identity groups. Also, the assessment is done using both models, as in the preceding discussion of layoffs.

\footnotetext{
${ }^{40}$ As indicated earlier in the discussion of the weighted descriptive evidence in Section 2.1, Aboriginal identity workers, and in particular Aboriginal identity men, have been more successful in securing paid employment following layoff than were their non-Aboriginal counterparts. Since this essay is concerned primarily with the impact of the 2008-2011 recession on Aboriginal identity workers, the reemployment decomposition results here are reported only for the three Aboriginal identity groups; for the aggregate group (NAI, Inuit and Métis together) and for NAI and Métis separately. In the two tables presented in this section, full results from the overall decomposition for all three Aboriginal identity groups are presented. For practical reasons, only findings relating to the endowment effect from the detailed decompositions are presented for the pertinent groups. Full detailed decomposition results, however, is available, upon request.
} 
Table 5: Overall decomposition of the difference in predicted probability of reemployment, main sample

\begin{tabular}{|c|c|c|c|c|c|c|}
\hline \multirow[t]{2}{*}{$\begin{array}{l}\text { Overall decomposition - reemployment } \\
\text { (the difference in the first moment) } \\
\text { MAIN SAMPLE }\end{array}$} & \multicolumn{2}{|c|}{$\begin{array}{l}\text { Aboriginal } \\
\text { Women } \\
\text { vs. } \\
\text { Aboriginal } \\
\text { Men }\end{array}$} & \multicolumn{2}{|c|}{$\begin{array}{l}\text { Métis } \\
\text { Women } \\
\text { vs. } \\
\text { Métis } \\
\text { Men }\end{array}$} & \multicolumn{2}{|c|}{$\begin{array}{c}\begin{array}{c}\text { North American Indian } \\
\text { Women } \\
\text { vs. } \\
\text { North American Indian } \\
\text { Men }\end{array}\end{array}$} \\
\hline & Model 1 & Model 2 & Model 1 & Model 2 & Model 1 & Model 2 \\
\hline Raw differential estimates & $\begin{array}{c}0.0718 \\
(0.031)^{\star}\end{array}$ & $\begin{array}{c}0.079 \\
(0.041)\end{array}$ & $\begin{array}{c}0.0864 \\
(0.041)^{*}\end{array}$ & $\begin{array}{c}0.1383 \\
(0.047)^{\star *}\end{array}$ & $\begin{array}{l}0.0573 \\
(0.042)\end{array}$ & $\begin{array}{l}0.0121 \\
(0.050)\end{array}$ \\
\hline \multicolumn{7}{|l|}{ Due to endowments effect $(E)$} \\
\hline Estimate & $\begin{array}{l}0.0034 \\
(0.014)\end{array}$ & $\begin{array}{c}0.106 \\
(0.043)^{*}\end{array}$ & $\begin{array}{c}0.022 \\
(0.021)\end{array}$ & $\begin{array}{c}0.175 \\
(0.053)^{\star \star}\end{array}$ & $\begin{array}{c}0.024 \\
(0.019)\end{array}$ & $\begin{array}{c}0.164 \\
(0.050)^{\star \star}\end{array}$ \\
\hline Share $(\%)$ & 4.8 & 133.9 & 25.7 & 126.4 & 42.2 & 1349.8 \\
\hline $\begin{array}{l}\text { Due to coefficients effect (C) } \\
\text { Estimate }\end{array}$ & $\begin{array}{c}0.068 \\
(0.034)^{*}\end{array}$ & $\begin{array}{l}-0.0269 \\
(0.043)\end{array}$ & $\begin{array}{c}0.064 \\
(0.045)\end{array}$ & $\begin{array}{l}-0.036 \\
(0.070)\end{array}$ & $\begin{array}{c}0.033 \\
(0.045)\end{array}$ & $\begin{array}{c}-0.152 \\
(0.063)^{*}\end{array}$ \\
\hline Share $(\%)$ & 95.2 & -33.9 & 74.3 & -26.4 & 57.8 & -1249.8 \\
\hline Decomposition sample & \multicolumn{2}{|c|}{$(N=1358)$} & \multicolumn{2}{|c|}{$(N=633)$} & \multicolumn{2}{|c|}{$(N=696)$} \\
\hline
\end{tabular}

Source: The Labour Force Survey, national monthly master file, September 2008 - May 2011.

Note: Decomposition refers to equation (1) components $E$ and $C$. Share is percentage share of predicted difference in the probability of being reemployed. Model 1 does not include any of the job-related characteristics. Model 2 includes all characteristics. Main samples include all paid employees, aged between 15 and 64, who are not full-time students. Survey weights in effect. Standard errors reported in parentheses. Statistical significance ${ }^{* * *} p<.001 ;{ }^{* *} p<.01 ;{ }^{*} p<.05$.

As in the assessment of the overall decomposition results for layoffs, the first point that stands out in the assessment of the overall decomposition results for reemployment is the discrepancies in the sizes of the predicted gaps for each group in the main samples. As shown in Table 5, the sizes of the gaps in the predicted probability of reemployment for all three groups of Aboriginal identity people are different from those that can be discerned from Table 1 presented in Section 2.1; the magnitude of the gaps here is either smaller or larger, depending on whether they are observed in the model with only basic characteristics or in the model with all characteristics. The variation between the two models is particularly notable for Métis and for NAI group.

As shown in Table 5, decomposed probit estimates suggest that the raw gap in the predicted probability of reemployment for Métis is 8.6 percentage points if only basic 
personal characteristics of workers are considered (Model 1) and 13.8 percentage points if all other characteristics are accounted for (Model 2). In Section 2.1, the difference between predicted probabilities of reemployment between Métis men and Métis women was 9 percentage points. Similarly, Table 5 shows that the estimated raw gap in the probability of reemployment for NAI group in Model $1(5.7 \%)$ is slightly smaller from the gap presented in Section $2.1(6 \%)$; however, when all characteristics of workers are considered (Model 2), the gap is reduced to 1.2 percentage points.

Another point that stands out in Table 5 is the variation in the percentage share of the predicted difference in the probability of reemployment that can be attributed to the endowment effect across groups. As observed in Model 1, the size of the endowment effect is relatively large in the case of NAI (42.2\%) while for Métis and for the aggregate Aboriginal identity group, the endowment effect accounts for 25.7 percent and for 4.8 percent of the mean difference of the probability of reemployment, respectively. However, when all considered characteristics of workers are included in the model (Model 2), it becomes clear that the endowment effect accounts fully for the difference in the predicted probability of reemployment for all three Aboriginal identity groups. ${ }^{41}$

Table 6 presents estimated results from the same analysis of the overall decomposition of the mean difference in the predicted probability of reemployment for Aboriginal groups, based on the more restricted samples. The results derived from the

\footnotetext{
${ }^{41}$ The reviewed literature does not say anything about what could be causing the unrealistically large shares such as those reported in Table 5 for NAI group. In the context of the present analysis, it is reasonable to speculate that relatively small sample sizes are one of the possible reasons for this; as indicated earlier in Section 2.1, the necessary restrictions applied in the construction of the samples used to analyze the difference in the probability of reemployment left in relatively small numbers of individuals of Aboriginal identity. The effect of this is particularly notable in the detailed decomposition, as will be shown in Section 4.2 , possibly suggesting that some cells on some variables contained a very small number of observations. Thus, although survey weights were applied in the analysis, important differences were likely missed.
} 
more restricted samples reveal roughly the same patterns as those derived from the main samples just described. However, the difference in the magnitude of the estimated gaps is different in different models and for different groups.

Table 6: Overall decomposition of the difference in predicted probability of reemployment, restricted sample

\begin{tabular}{|c|c|c|c|c|c|c|}
\hline \multirow[t]{2}{*}{$\begin{array}{l}\text { Overall decomposition - reemployment } \\
\text { (the difference in the first moment) } \\
\text { RESTRICTED SAMPLE }\end{array}$} & \multicolumn{2}{|c|}{$\begin{array}{l}\text { Aboriginal } \\
\text { Women } \\
\text { vs. } \\
\text { Aboriginal } \\
\text { Men }\end{array}$} & \multicolumn{2}{|c|}{$\begin{array}{l}\text { Métis } \\
\text { Women } \\
\text { vs. } \\
\text { Métis } \\
\text { Men }\end{array}$} & \multicolumn{2}{|c|}{$\begin{array}{c}\text { North American Indian } \\
\text { Women } \\
\text { vs. } \\
\text { North American Indian } \\
\text { Men }\end{array}$} \\
\hline & Model 1 & Model 2 & Model 1 & Model 2 & Model 1 & Model 2 \\
\hline Raw differential estimates & $\begin{array}{c}0.1109 \\
(0.036)^{* *}\end{array}$ & $\begin{array}{c}0.1064 \\
(0.042)^{\star *}\end{array}$ & $\begin{array}{c}0.1577 \\
(0.047)^{\star \star}\end{array}$ & $\begin{array}{c}0.1969 \\
(0.047)^{\star \star}\end{array}$ & $\begin{array}{l}0.0700 \\
(0.047)\end{array}$ & $\begin{array}{l}0.0342 \\
(0.049)\end{array}$ \\
\hline \multicolumn{7}{|l|}{ Due to endowments effect (E) } \\
\hline Estimate & $\begin{array}{l}0.0045 \\
(0.017)\end{array}$ & $\begin{array}{c}0.1467 \\
(0.039)^{\star \star}\end{array}$ & $\begin{array}{c}0.084 \\
(0.029)\end{array}$ & $\begin{array}{c}0.202 \\
(0.056)^{*}\end{array}$ & $\begin{array}{c}0.054 \\
(0.023)^{*}\end{array}$ & $\begin{array}{c}0.2045 \\
(0.050)^{\star \star \star}\end{array}$ \\
\hline Share $(\%)$ & 4.1 & 137.9 & 5.3 & 102.6 & 77.5 & 598.1 \\
\hline $\begin{array}{l}\text { Due to coefficients effect (C) } \\
\text { Estimate }\end{array}$ & $\begin{array}{c}0.1064 \\
(0.039)^{\star \star}\end{array}$ & $\begin{array}{l}-0.0403 \\
(0.057)\end{array}$ & $\begin{array}{c}0.1493 \\
(0.051)^{\star \star}\end{array}$ & $\begin{array}{r}-0.0051 \\
(0.073)\end{array}$ & $\begin{array}{l}0.0157 \\
(0.049)\end{array}$ & $\begin{array}{l}-0.1703 \\
(0.061)^{\star \star}\end{array}$ \\
\hline Share $(\%)$ & 95.9 & -37.9 & 94.7 & -2.6 & 22.5 & -498.1 \\
\hline Decomposition sample & \multicolumn{2}{|c|}{$(N=1066)$} & \multicolumn{2}{|c|}{$(N=503)$} & \multicolumn{2}{|c|}{$(N=543)$} \\
\hline
\end{tabular}

Source: The Labour Force Survey, national monthly master file, September 2008 - May 2011.

Note: Decomposition refers to equation (1) components $E$ and $C$. Share is percentage share of predicted difference in the probability of being reemployed. Model 1 does not include any of the job-related characteristics. Model 2 includes all characteristics. Restricted samples include only permanent paid employees, aged between 15 and 64, who are single-job holders and who are not students. Survey weights in effect. Standard errors reported in parentheses. Statistical significance ${ }^{* * *} p<.001 ;{ }^{* *} p<.01 ;{ }^{*} p<.05$.

As shown in Table 6, the sizes of the estimated raw gaps appear larger in each of the more restricted samples, relative to those estimated using the main samples, and in one instance (Métis Model 2) larger from those reported in Section 2.1, suggesting that the characteristics of excluded workers in these more restricted samples (student status and the non-permanent nature of employment) carry some weight in whether or not reemployment is observed for Aboriginal people; in other words, the larger gaps in the 
more restricted samples suggest that the observed greater probability of reemployment for Aboriginal men, relative to Aboriginal women, was possibly not related to contract or casual types of employment but rather to permanent employment.

As discussed in Section 2.1, the mean differences in the probability of reemployment for Aboriginal identity groups, as observed in the more restricted samples, ranged from 7 percentage points for NAI to 16 percentage points for Métis. As shown in Table 6, the 7 percentage points gap for NAI remains in this multivariate analysis in Model 1 but in Model 2, where all observable characteristics are taken into account, the gap drops down to only 3.4 percentage points. The estimated raw gap for Métis in Model 1 also remains close to the 16 percentage points reported in Section 2.1, but in Model 2 it raises to close to 20 percentage points. Possibly, this finding could be an indication of positive sorting of Métis men across industries or sectors.

It is interesting to note that the endowment effect, as observed in Model 1 for Métis and for the aggregate Aboriginal identity group, accounts for relatively small percentage share of the predicted difference in their probability of reemployment $(5.3 \%$ and $4.1 \%$ respectively), in comparison to that of NAI group $(77.5 \%)$. However, when all considered characteristics of workers are included in the model (Model 2), the endowment effect accounts fully for the difference in the predicted probability of reemployment for all three Aboriginal identity groups.

In summary, the evidence from the overall decompositions presented here suggests that Aboriginal identity workers were more negatively affected by the 20082011 recession in that they were more likely to be laid off than were non-Aboriginal workers. Among the examined groups, NAI men had the greatest probability of being 
laid off. The most distressing fact about this is that most of the mean difference in the probability of being laid off between NAI men and non-Aboriginal men is unexplained, and thus possibly attributable to discrimination in the labour market - the percentage share of the predicted difference in the probability of being laid off that can be attributed to the differences in endowments between NAI men and non-Aboriginal men differs depending on whether or not the analytical sample includes students, seasonal workers and multiple job holders and whether or not the estimated model controls for the employment-related characteristics of workers, but even the largest percentage share (26.5\%) estimated in the more restricted sample of NAI men with no control for employment-related characteristics is a cause for concern as it is relatively small. Another potential cause for concern revealed in this analysis of layoffs relates to the overall decomposition results for women, and in particular for Métis women. As discussed above, the main sample yields a relatively small and statistically insignificant gap in the probability of layoff between Métis women and non-Aboriginal identity women. However, when the sample is restricted to workers who hold single and permanent jobs and to non-students, the gap becomes statistically significant and relatively large. This finding could be an indication that the layoffs that occurred among Métis women were in fact layoffs from permanent jobs and might be related to sorting of Métis women into the layoff-prone industries or sectors. For NAI women, the indication is that the layoffs that occurred were related to the factors excluded from this more restricted sample (multiple and seasonal jobs and student status).

In relation to the examination of reemployment, the evidence from the overall decomposition suggests that the estimated greater probability of reemployment for 
Aboriginal men, and in particular for Métis men, relative to Aboriginal women, was possibly not related to contract or casual employment but rather to permanent employment. For Métis men, this finding can be taken as an indication of positive sorting across industries or sectors. Also, in the reemployment decomposition results, the percentage share of the predicted difference in the probability of reemployment among Aboriginal gender groups that can be attributed to the differences in the observable characteristics differs across groups when observed in a model that includes only basic personal characteristics; however, when all considered characteristics of workers are included in the model, the endowment effect accounts fully for the difference in the predicted probability of reemployment for all three Aboriginal identity groups.

The results from the overall decomposition presented here are informative to an extent but they raise a question about which of the considered characteristics play a more important role in determining the observed outcomes. This question is examined in the following section using a detailed decomposition method. As in this section, the discussion in the following section centers on the statistically significant findings and the results are presented separately for layoffs and for reemployment. 


\subsection{Detailed Decomposition Results}

The overall decomposition results for layoffs and for reemployment presented in Section 4.1 provide some useful insights into the overall picture of the difference in the impact of latest recession on Aboriginal and non-Aboriginal workers but do not provide any indication as to the relative importance of the included variables in determining the observed outcomes. Given that the Aboriginal labour force development has received a significant amount of policy attention in the past two decades or so, it would be useful to know how much of the explained gap in the outcomes examined in this essay is due to group differences in educational attainment, for example, and how much is due to differences in work experience, occupational affiliation or any other characteristic of interest. It might also be informative to learn how much of the unexplained gap can be ascribed to differing returns to educational attainment and how much to differing returns to work experience or the other characteristics of interest. If the decomposition shows that one of these characteristics, occupational status for example, accounts for a large proportion of the gap in the observed outcomes then this would warrant exploring in more detail how different Aboriginal groups choose their fields of study or occupation.

This section presents the results from a detailed decomposition where an attempt was made to determine the contribution share of each considered variable to each of the two components of the difference. In this detailed decomposition, the components $E$ and $C$ are divided into segments, $\mathrm{E}_{k}$ and $\mathrm{C}_{k}(k=1, \ldots, \mathrm{K})$, that represent the unique contribution of the $k$ th predictor. This method of analysis was briefly addressed in Section 2.2 and in this section more details are provided along with the results. 
In the past literature, the general approach to finding out contributions of individual variables has been to use a sequential replacement of the values associated with one of the groups with the corresponding values of the other group. Applying this approach to nonlinear models, however, creates a problem, known as path dependency, because a nonlinear decomposition is sensitive to the order in which the independent variables are entered into decomposition. Yun (2004) has provided a solution to this problem by proposing a "weights" method in which an approximation of the weights is obtained by evaluating the value of the function using mean characteristics and then applying a first-order Taylor expansion to linearize the endowments and coefficients effects around $\bar{X}_{A} \beta_{A}$ and $\bar{X}_{B} \beta_{B}$, respectively, thus yielding the detailed decomposition that is free from path dependency. The equation for this detailed decomposition is specified as,

$$
\bar{Y}_{A}-\bar{Y}_{B}=\overbrace{\left\{\sum_{k=1}^{K} W_{\Delta x}^{k} \overline{\left[\Phi\left(X_{A} \beta_{A}\right)\right.}-\overline{\left.\Phi\left(X_{B} \beta_{A}\right)\right]}\right\}}^{E}+\overbrace{\left\{\sum_{k=1}^{K} W_{\Delta \beta}^{k} \overline{\left[\Phi\left(X_{B} \beta_{A}\right)\right.}-\overline{\left.\Phi\left(X_{B} \beta_{B}\right)\right]}\right\}}^{c}
$$

where weight components for $\mathrm{E}$ and $\mathrm{C}$ are

$$
W_{\Delta x}^{k}=\frac{\left(\bar{X}_{A}^{k}-\bar{X}_{B}^{k}\right) \beta_{A}^{k}}{\left(\bar{X}_{A}-\bar{X}_{B}\right) \beta_{A}}, W_{\Delta \beta}^{k}=\frac{\bar{X}_{B}^{k}\left(\beta_{A}^{k}-\beta_{B}^{k}\right)}{\bar{X}_{B}\left(\beta_{A}-\beta_{B}\right)}, \text { and } \sum_{k=1}^{K} W_{\Delta x}^{k}=\sum_{k=1}^{K} W_{\Delta \beta}^{k}=1
$$

In this equation, the endowment weights $W_{\Delta x}^{k}$ reflect the contribution of the $k$ th predictor to the linearization of $\mathrm{E}$ as determined by the magnitude of the group difference in means $\left(\bar{X}_{A}^{k}-\bar{X}_{B}^{k}\right)$ weighted by the reference group's effect $\beta_{A}^{k}$. Likewise, the coefficient weights $W_{\Delta \beta}^{k}$ reflect predictor k's contribution to the linearization of $\mathrm{C}$ as 
determined by the magnitude of the group difference in the effects $\left(\beta_{A}^{k}-\beta_{B}^{k}\right)$ weighted by the comparison group's mean $\bar{X}_{B}^{k}$. Since these weights are invariant to change in the scale of the predictors, the raw difference $\bar{Y}_{A}-\bar{Y}_{B}$ can be expressed in terms of the overall components as a sum of weighted sums of the unique contributions; that is, $\bar{Y}_{A}-\bar{Y}_{B}=E+C=E \sum_{k=1}^{K} W_{\Delta X k}+C \sum_{k=1}^{K} W_{\Delta \beta k}=\sum_{k=1}^{K} E_{k}+\sum_{k=1}^{K} C_{k}$ where $\mathrm{E}$ and $\mathrm{C}$ preceding the capital sigma notations are from the overall decomposition.

While the detailed decomposition equation (2) solves the problem of path dependency it does not address the identification problem that arises from adopting a different reference category in a set of dummy variables, as demonstrated in Oaxaca (1999). To resolve this problem, Yun (2005) suggests averaging the coefficients effects of a set of dummy variables while changing the order of the reference groups, which is equivalent to computing a normalized equation that can identify the intercept and coefficients of all dummy variables, including the reference groups, by averaging estimates obtained and then using these estimates to perform the decomposition. ${ }^{42}$ The probit estimates in the present analysis were decomposed using this method and the findings are reported below, separately for layoffs and for reemployment. ${ }^{43}$ The reported findings are based on the probit estimates from the main samples.

\footnotetext{
${ }^{42}$ The algorithm for constructing this normalized equation, as specified in $Y$ un (2008), required the use of Stata's oaxaca command in conjunction with the $m v d c m p$ command where normalization was offered as an option in the $m v d c m p$ command. This option was applied to derive the reported decompositions in each model. In deriving standard errors of the detailed contribution and tests for statistical significance of the endowments and coefficients effects in equation (2), the delta method incorporated in the $m v d c m p$ command, as summarized in Yun (2005), was used.

${ }^{43}$ This choice was made in order to assess the impact of the employment-related characteristics such as seasonal type of job that were excluded from the more restricted sample. The output from detailed decomposition using the more restricted samples, however, can be made available, upon request.
} 
Results from the detailed decomposition of the mean differences in the predicted probability of layoff, estimated from equation (2) separately for Aboriginal and for nonAboriginal identity men and women, are presented in Table 7 and Table 8, respectively. This is followed by the presentation of the detailed decomposition results of the predicted probability of reemployment for Aboriginal identity men and women in Table 9. In the reemployment table, only the decomposition of the endowment effects and the associated percentage shares are presented for all three Aboriginal identity groups. In the two tables presenting detailed decomposition results for layoffs, both the endowment and the coefficient effects are presented, along with the associated percentage shares.

In the endowments column of each table, the $\mathrm{E}$ coefficient indicates the expected difference in average predicted outcomes if the individuals in the comparison group (group A from equation 2) were given the distribution of covariates of the individuals in the reference group (group B from equation 2). In the coefficients column, the C coefficient indicates the expected difference in average predicted outcomes if the individuals in the reference group experienced behavioural responses of the individuals in the comparison group. The associated columns "share" list the ratios of the contribution of each factor to the predicted difference, in percentage terms. 


\subsubsection{Layoffs}

This section presents the results from detailed decomposition of the difference in the predicted probability of layoff for Aboriginal and non-Aboriginal identity men and women. The results for men, presented in Table 7, are discussed first. In the second and in the sixth column of Table 7, a positive $\mathrm{E}$ coefficient indicates the expected reduction in the Aboriginal to non-Aboriginal predicted layoff probability gap if Aboriginal men (Métis and NAI men) were made equal to non-Aboriginal men on the distribution of the pertinent explanatory variable. The adjacent columns on the right list the ratios of the contribution of each factor to the predicted difference, in percentage terms. Based on the same reasoning, in the fourth and in the eight column of Table 7 , a negative $C$ coefficient indicates the expected increase in the Aboriginal to non-Aboriginal gap if Aboriginal men had the same behavioural responses as non-Aboriginal men, with the share columns indicating the percentage share of the contribution of each examined factor.

As revealed in the results of the overall decomposition (Model 2) presented earlier in Table 3, more than half $(54.3 \%)$ of the raw difference in the predicted probability of layoff between Métis men and non-Aboriginal identity men can be attributed to the differences in the endowments between the two groups. According to the results of the detailed decomposition presented in Table 7, the difference in educational attainment between Métis men and non-Aboriginal identity men does not seem to be one of those contributing factors; as shown in Table 7, none of the estimates for educational attainment categories are statistically different from zero, implying that differences in educational attainment between the two groups of men play no significant role in this. 
Table 7: Detailed decomposition of the difference in the predicted probability of layoff for men

\begin{tabular}{|c|c|c|c|c|c|c|c|c|}
\hline \multirow{3}{*}{$\begin{array}{l}\text { Detailed } \\
\text { decomposition } \\
\text { for layoffs } \\
\text { MAIN SAMPLE }\end{array}$} & \multicolumn{4}{|c|}{$\begin{array}{c}\text { Métis Men } \\
\text { vs. } \\
\text { non-Aboriginal Identity Men }\end{array}$} & \multicolumn{4}{|c|}{$\begin{array}{l}\text { North American Indian Men } \\
\text { vs. } \\
\text { non-Aboriginal Identity Men }\end{array}$} \\
\hline & \multicolumn{2}{|c|}{$\begin{array}{l}\text { Endowments Effect } \\
\text { (E) }\end{array}$} & \multicolumn{2}{|c|}{$\begin{array}{l}\text { Coefficients Effect } \\
\text { (C) }\end{array}$} & \multicolumn{2}{|c|}{$\begin{array}{c}\text { Endowments Effect } \\
\text { (E) }\end{array}$} & \multicolumn{2}{|c|}{$\begin{array}{l}\text { Coefficients Effect } \\
\text { (C) }\end{array}$} \\
\hline & Estimate & $\begin{array}{c}\text { Share } \\
(\%)\end{array}$ & Estimate & $\begin{array}{c}\text { Share } \\
(\%)\end{array}$ & Estimate & $\begin{array}{c}\text { Share } \\
(\%)\end{array}$ & Estimate & Share $(\%)$ \\
\hline $\begin{array}{l}\text { Less than } \\
\text { high school diploma }\end{array}$ & $\begin{array}{l}0.0013 \\
(0.001)\end{array}$ & 4.4 & $\begin{array}{r}0.0010 \\
(0.004)\end{array}$ & 3.42 & $\begin{array}{l}0.0017 \\
(0.003)\end{array}$ & 3.20 & $\begin{array}{l}0.0025 \\
(0.009)\end{array}$ & 4.64 \\
\hline $\begin{array}{l}\text { Completed } \\
\text { high school diploma }\end{array}$ & $\begin{array}{l}0.00015 \\
(0.0002)\end{array}$ & 0.50 & $\begin{array}{r}-0.0014 \\
(0.005)\end{array}$ & -4.63 & $\begin{array}{l}0.0015 \\
(0.002)\end{array}$ & 2.74 & $\begin{array}{l}0.0396 \\
(0.04)\end{array}$ & 72.98 \\
\hline $\begin{array}{l}\text { Incomplete } \\
\text { postsecondary }\end{array}$ & $\begin{array}{c}-0.00007 \\
(0.0004)\end{array}$ & -0.24 & $\begin{array}{l}0.00057 \\
(0.003)\end{array}$ & 1.93 & $\begin{array}{l}-0.0002 \\
(0.0004)\end{array}$ & -0.36 & $\begin{array}{c}-0.0050 \\
(0.008)\end{array}$ & -9.23 \\
\hline $\begin{array}{l}\text { Completed } \\
\text { trade certificate }\end{array}$ & $\begin{array}{l}0.00058 \\
(0.0005)\end{array}$ & 1.95 & $\begin{array}{c}-0.0043 \\
(0.01)\end{array}$ & -14.52 & $\begin{array}{c}-0.00007 \\
(0.0001)\end{array}$ & -0.14 & $\begin{array}{l}0.0058 \\
(0.02)\end{array}$ & 10.68 \\
\hline $\begin{array}{l}\text { Completed } \\
\text { college diploma }\end{array}$ & $\begin{array}{c}-0.00010 \\
(0.001)\end{array}$ & -0.34 & $\begin{array}{c}-0.0047 \\
(0.01)\end{array}$ & -15.98 & $\begin{array}{l}-0.0003 \\
(0.0005)\end{array}$ & -0.61 & $\begin{array}{l}0.0345 \\
(0.04)\end{array}$ & 63.54 \\
\hline $\begin{array}{l}\text { Completed } \\
\text { university degree }\end{array}$ & $\begin{array}{l}0.00427 \\
(0.003)\end{array}$ & 14.35 & $\begin{array}{l}0.0074 \\
(0.02)\end{array}$ & 25.03 & $\begin{array}{l}0.0078 \\
(0.01)\end{array}$ & 14.31 & $\begin{array}{c}-0.068 \\
(0.09)\end{array}$ & -125.7 \\
\hline Tenure $<2$ years & $\begin{array}{c}0.00328 \\
(0.001)^{\star \star}\end{array}$ & 11.02 & $\begin{array}{l}-0.0008 \\
(0.006)\end{array}$ & -2.79 & $\begin{array}{r}0.0032 \\
(0.005)\end{array}$ & 5.91 & $\begin{array}{l}0.0214 \\
(0.032)\end{array}$ & 39.47 \\
\hline Tenure $2-5$ years & $\begin{array}{l}-0.00002 \\
(0.00009)\end{array}$ & -0.07 & $\begin{array}{c}0.00428 \\
(0.01)\end{array}$ & 14.39 & $\begin{array}{l}0.00023 \\
(0.0005)\end{array}$ & 0.42 & $\begin{array}{c}-0.0008 \\
(0.01)\end{array}$ & -1.57 \\
\hline Tenure $5-10$ years & $\begin{array}{l}0.00012 \\
(0.0003)\end{array}$ & 0.40 & $\begin{array}{l}0.00077 \\
(0.005)\end{array}$ & 2.58 & $\begin{array}{c}9.0365 \\
(0.0002)\end{array}$ & 0.02 & $\begin{array}{c}0.00198 \\
(0.01)\end{array}$ & 3.66 \\
\hline Tenure $10-20$ years & $\begin{array}{l}0.00008 \\
(0.0006)\end{array}$ & 0.29 & $\begin{array}{l}-0.0027 \\
(0.008)\end{array}$ & -9.20 & $\begin{array}{l}0.00017 \\
(0.0005)\end{array}$ & 0.31 & $\begin{array}{c}0.0049 \\
(0.01)\end{array}$ & 9.08 \\
\hline Tenure $>20$ years & $\begin{array}{l}0.00126 \\
(0.0009)\end{array}$ & 4.23 & $\begin{array}{r}-0.0010 \\
(0.006)\end{array}$ & -3.45 & $\begin{array}{l}0.0021 \\
(0.003)\end{array}$ & 3.91 & $\begin{array}{c}-0.0173 \\
(0.03)\end{array}$ & -31.92 \\
\hline No work absence & $\begin{array}{r}-0.00027 \\
(0.0004)\end{array}$ & -0.91 & $\begin{array}{c}-0.0217 \\
(0.06)\end{array}$ & -73.01 & $\begin{array}{c}-0.0001 \\
(0.0003)\end{array}$ & -0.026 & $\begin{array}{l}0.0829 \\
(0.12)\end{array}$ & 152.77 \\
\hline Absent 1 wk & $\begin{array}{c}-2.9788 \\
(2.88)\end{array}$ & -0.001 & $\begin{array}{c}0.00009 \\
(0.001)\end{array}$ & 0.301 & $\begin{array}{c}-0.00006 \\
(0.0002)\end{array}$ & -0.11 & $\begin{array}{c}-0.0013 \\
(0.05)\end{array}$ & -2.40 \\
\hline Absent 2 wks & $\begin{array}{l}0.00015 \\
(0.0002)\end{array}$ & 0.51 & $\begin{array}{c}-0.00051 \\
(0.001)\end{array}$ & -1.70 & - & - & - & - \\
\hline Absent 3 wks & $\begin{array}{l}0.00003 \\
(0.0001)\end{array}$ & 0.15 & $\begin{array}{c}-0.00028 \\
(0.0008)\end{array}$ & -0.94 & $\begin{array}{r}-9.2656 \\
(0.0001)\end{array}$ & -0.02 & $\begin{array}{l}0.0015 \\
(0.001)\end{array}$ & 0.29 \\
\hline Absent $4-8$ wks & $\begin{array}{c}-5.6165 \\
(0.00005)^{\star \star}\end{array}$ & -0.02 & $\begin{array}{c}0.00037 \\
(0.0009)\end{array}$ & 1.24 & $\begin{array}{l}0.00004 \\
(0.0002)\end{array}$ & 0.08 & $\begin{array}{c}-0.00043 \\
(0.002)\end{array}$ & -0.80 \\
\hline Absent over 8 wks & $\begin{array}{l}-0.0001 \\
(0.0001)\end{array}$ & -0.38 & $\begin{array}{l}0.00088 \\
(0.002)\end{array}$ & 2.97 & $\begin{array}{l}-0.00008 \\
(0.0004)\end{array}$ & -0.14 & $\begin{array}{c}-0.00027 \\
(0.003)\end{array}$ & -0.49 \\
\hline
\end{tabular}




\begin{tabular}{|c|c|c|c|c|c|c|c|c|}
\hline \multirow{3}{*}{$\begin{array}{l}\text { Table } 7 \\
\text { (continued) }\end{array}$} & \multicolumn{4}{|c|}{$\begin{array}{c}\text { Métis Men } \\
\text { Vs. } \\
\text { non-Aboriginal Identity Men }\end{array}$} & \multicolumn{4}{|c|}{$\begin{array}{l}\text { North American Indian Men } \\
\text { vs. } \\
\text { non-Aboriginal Identity Men }\end{array}$} \\
\hline & \multicolumn{2}{|c|}{$\begin{array}{l}\text { Endowments Effect } \\
\text { (E) }\end{array}$} & \multicolumn{2}{|c|}{$\begin{array}{l}\text { Coefficients Effect } \\
\text { (C) }\end{array}$} & \multicolumn{2}{|c|}{$\begin{array}{l}\text { Endowments Effect } \\
\text { (E) }\end{array}$} & \multicolumn{2}{|c|}{$\begin{array}{l}\text { Coefficients Effect } \\
\text { (C) }\end{array}$} \\
\hline & Estimate & $\begin{array}{c}\text { Share } \\
(\%) \\
\end{array}$ & Estimate & $\begin{array}{c}\text { Share } \\
(\%)\end{array}$ & Estimate & $\begin{array}{c}\text { Share } \\
(\%)\end{array}$ & Estimate & Share $(\%)$ \\
\hline Age $15-24$ & $\begin{array}{l}0.00064 \\
(0.0004)\end{array}$ & 2.14 & $\begin{array}{c}-0.0069 \\
(0.01)\end{array}$ & -23.08 & $\begin{array}{l}-0.00036 \\
(0.0006)\end{array}$ & -0.67 & $\begin{array}{c}-0.0074 \\
(0.01)\end{array}$ & -13.66 \\
\hline Age $25-34$ & $\begin{array}{c}6.9658 \\
(0.0004)\end{array}$ & 0.023 & $\begin{array}{c}-0.00075 \\
(0.006)\end{array}$ & -2.51 & $\begin{array}{l}0.00036 \\
(0.0006)\end{array}$ & 0.68 & $\begin{array}{l}0.0269 \\
(0.03)\end{array}$ & 49.61 \\
\hline Age $35-44$ & $\begin{array}{l}-4.1129 \\
(7.689)\end{array}$ & -0.014 & $\begin{array}{l}0.00130 \\
(0.006)\end{array}$ & 4.37 & $\begin{array}{r}-0.00004 \\
(0.0003)\end{array}$ & -0.08 & $\begin{array}{c}0.00269 \\
(0.02)\end{array}$ & 4.95 \\
\hline Age $45-54$ & $\begin{array}{c}0.00085 \\
(0.0004)^{\star}\end{array}$ & 2.85 & $\begin{array}{l}0.0160 \\
(0.035)\end{array}$ & 53.86 & $\begin{array}{c}-0.00006 \\
(0.0005)\end{array}$ & -0.11 & $\begin{array}{c}0.00288 \\
(0.02)\end{array}$ & 5.31 \\
\hline Age $55-64$ & $\begin{array}{c}-0.00074 \\
(0.0008)\end{array}$ & -2.50 & $\begin{array}{c}-0.0012 \\
(0.005)\end{array}$ & -4.19 & $\begin{array}{l}0.00010 \\
(0.0004)\end{array}$ & 0.18 & $\begin{array}{c}-0.0108 \\
(0.02)\end{array}$ & -19.89 \\
\hline Married & $\begin{array}{l}0.00039 \\
(0.0003)\end{array}$ & 1.31 & $\begin{array}{l}0.0137 \\
(0.03)\end{array}$ & 46.13 & $\begin{array}{l}0.0012 \\
(0.002)\end{array}$ & 2.21 & $\begin{array}{c}-0.0508 \\
(0.07)\end{array}$ & -93.74 \\
\hline $\begin{array}{l}\text { Widowed, } \\
\text { divorced, } \\
\text { separated }\end{array}$ & $\begin{array}{r}-0.00001 \\
(0.0001)\end{array}$ & -0.035 & $\begin{array}{r}0.0004 \\
(0.002)\end{array}$ & 1.49 & $\begin{array}{c}0.00079 \\
(0.001)\end{array}$ & 1.47 & $\begin{array}{l}0.00571 \\
(0.009)\end{array}$ & 10.52 \\
\hline Single & $\begin{array}{c}0.00004 \\
(0.00003)\end{array}$ & 0.15 & $\begin{array}{l}-0.008 \\
(0.02)\end{array}$ & -27.11 & $\begin{array}{r}-0.00005 \\
(0.0005)\end{array}$ & -0.09 & $\begin{array}{c}-0.0051 \\
(0.02)\end{array}$ & -9.41 \\
\hline No children present & $\begin{array}{c}0.00009 \\
(0.00005)\end{array}$ & 0.29 & $\begin{array}{l}0.2653 \\
(0.06)\end{array}$ & 89.13 & $\begin{array}{r}-0.00014 \\
(0.0004)\end{array}$ & -0.26 & $\begin{array}{l}-0.023 \\
(0.04)\end{array}$ & 43.31 \\
\hline Children < age 6 & $\begin{array}{l}-0.00033 \\
(0.0005)\end{array}$ & -1.12 & $\begin{array}{l}0.0022 \\
(0.006)\end{array}$ & 7.41 & $\begin{array}{c}-0.00002 \\
(0.0001)\end{array}$ & -0.03 & $\begin{array}{l}0.0044 \\
(0.01)\end{array}$ & 8.04 \\
\hline Children, 6-14 & $\begin{array}{l}-0.00013 \\
(0.0003)\end{array}$ & -0.43 & $\begin{array}{l}-0.0016 \\
(0.006)\end{array}$ & -5.45 & $\begin{array}{r}-0.00003 \\
(0.0002)\end{array}$ & -0.05 & $\begin{array}{c}-0.0017 \\
(0.01)\end{array}$ & -3.24 \\
\hline Children, 14-24 & $\begin{array}{r}-0.00045 \\
(0.0003)\end{array}$ & -1.52 & $\begin{array}{c}-0.0055 \\
(0.01)\end{array}$ & -18.63 & $\begin{array}{r}-0.00009 \\
(0.0005)\end{array}$ & -0.17 & $\begin{array}{l}0.0025 \\
(0.01)\end{array}$ & 4.56 \\
\hline Allantic provinces & $\begin{array}{l}-0.0007 \\
(0.0006)\end{array}$ & -2.54 & $\begin{array}{c}0.00031 \\
(0.003)\end{array}$ & 1.03 & $\begin{array}{l}-3.5984 \\
(5.5043)\end{array}$ & -0.007 & $\begin{array}{l}0.0074 \\
(0.01)\end{array}$ & 13.71 \\
\hline Quebec & $\begin{array}{l}-0.0096 \\
(0.005)^{*}\end{array}$ & -32.26 & $\begin{array}{c}-0.01348 \\
(0.04)\end{array}$ & -45.29 & $\begin{array}{c}-0.0097 \\
(0.01)\end{array}$ & -17.94 & $\begin{array}{l}0.0609 \\
(0.06)\end{array}$ & 112.3 \\
\hline Ontario & $\begin{array}{c}0.00455 \\
(0.003)\end{array}$ & 15.3 & $\begin{array}{l}0.0171 \\
(0.04)\end{array}$ & 57.53 & $\begin{array}{l}-0.00002 \\
(0.00009)\end{array}$ & -0.04 & $\begin{array}{c}-0.0047 \\
(0.02)\end{array}$ & -8.63 \\
\hline Manitoba & $\begin{array}{c}-0.00176 \\
(0.002)\end{array}$ & -5.92 & $\begin{array}{r}-0.00044 \\
(0.0009)\end{array}$ & -1.47 & $\begin{array}{c}-0.00192 \\
(0.003)\end{array}$ & -3.54 & $\begin{array}{r}-0.0033 \\
(0.004)\end{array}$ & -6.20 \\
\hline Saskatchewan & $\begin{array}{c}-0.00130 \\
(0.002)\end{array}$ & -4.37 & $\begin{array}{c}-0.0004 \\
(0.0007)\end{array}$ & -0.122 & $\begin{array}{c}-0.00006 \\
(0.0006)\end{array}$ & -0.10 & $\begin{array}{l}0.0017 \\
(0.003)\end{array}$ & 3.13 \\
\hline Alberta & $\begin{array}{l}0.00250 \\
(0.002)\end{array}$ & 8.40 & $\begin{array}{l}-0.0049 \\
(0.01)\end{array}$ & -16.52 & $\begin{array}{l}-0.0015 \\
(0.002)\end{array}$ & -2.86 & $\begin{array}{c}-0.0155 \\
(0.02)\end{array}$ & -28.50 \\
\hline
\end{tabular}




\begin{tabular}{|c|c|c|c|c|c|c|c|c|}
\hline \multirow{3}{*}{$\begin{array}{l}\text { Table } 7 \\
\text { (continued) }\end{array}$} & \multicolumn{4}{|c|}{$\begin{array}{c}\text { Métis Men } \\
\text { vs. } \\
\text { non-Aboriginal Identity Men } \\
\end{array}$} & \multicolumn{4}{|c|}{$\begin{array}{l}\text { North American Indian Men } \\
\text { vs. } \\
\text { non-Aboriginal Identity Men }\end{array}$} \\
\hline & \multicolumn{2}{|c|}{$\begin{array}{l}\text { Endowments Effect } \\
\text { (E) }\end{array}$} & \multicolumn{2}{|c|}{$\begin{array}{l}\text { Coefficients Effect } \\
\text { (C) }\end{array}$} & \multicolumn{2}{|c|}{$\begin{array}{c}\text { Endowments Effect } \\
(E)\end{array}$} & \multicolumn{2}{|c|}{$\begin{array}{l}\text { Coefficients Effect } \\
\text { (C) }\end{array}$} \\
\hline & Estimate & Share $(\%)$ & Estimate & Share $(\%)$ & Estimate & Share $(\%)$ & Estimate & Share $(\%)$ \\
\hline British Columbia & $\begin{array}{c}-0.00036 \\
(0.0002)\end{array}$ & -1.20 & $\begin{array}{l}0.0054 \\
(0.01)\end{array}$ & 18.24 & $\begin{array}{c}-0.0015 \\
(0.002)\end{array}$ & -2.86 & $\begin{array}{c}-0.0111 \\
(0.01)\end{array}$ & -20.40 \\
\hline Primary industries & $\begin{array}{c}0.0047 \\
(0.002)^{*}\end{array}$ & 15.84 & $\begin{array}{c}-0.0068 \\
(0.01)\end{array}$ & -22.95 & $\begin{array}{l}0.0014 \\
(0.002)\end{array}$ & 2.58 & $\begin{array}{l}0.0043 \\
(0.01)\end{array}$ & 8.05 \\
\hline Manufacturing & $\begin{array}{c}-0.00158 \\
(0.001)\end{array}$ & -5.31 & $\begin{array}{c}-0.0061 \\
(0.01)\end{array}$ & -20.47 & $\begin{array}{c}-0.0009 \\
(0.001)\end{array}$ & -1.76 & $\begin{array}{l}0.0246 \\
(0.03)\end{array}$ & 45.41 \\
\hline Retail & $\begin{array}{c}0.00012 \\
(0.00007)\end{array}$ & 0.41 & $\begin{array}{l}0.0054 \\
(0.01)\end{array}$ & 18.02 & $\begin{array}{c}-0.00011 \\
(0.0002)\end{array}$ & -0.20 & $\begin{array}{c}-0.0077 \\
(0.02)\end{array}$ & -14.21 \\
\hline High skill services & $\begin{array}{l}0.00040 \\
(0.0007)\end{array}$ & 1.35 & $\begin{array}{c}0.00021 \\
(0.004)\end{array}$ & 0.715 & $\begin{array}{c}-0.0006 \\
(0.001)\end{array}$ & -1.08 & $\begin{array}{l}0.0168 \\
(0.02)\end{array}$ & 30.97 \\
\hline Public services & $\begin{array}{c}-0.00042 \\
(0.0006)\end{array}$ & -1.40 & $\begin{array}{l}0.0053 \\
(0.01)\end{array}$ & 17.96 & $\begin{array}{c}-0.0006 \\
(0.001)\end{array}$ & -1.19 & $\begin{array}{c}-0.0226 \\
(0.03)\end{array}$ & -41.71 \\
\hline Other services & $\begin{array}{l}0.00039 \\
(0.0005)\end{array}$ & 1.31 & $\begin{array}{c}0.00141 \\
(0.005)\end{array}$ & 4.73 & $\begin{array}{l}0.00021 \\
(0.0003)\end{array}$ & 0.38 & $\begin{array}{c}-0.0193 \\
(0.02)\end{array}$ & -35.53 \\
\hline Management & $\begin{array}{c}-0.00066 \\
(0.0007)\end{array}$ & -2.22 & $\begin{array}{c}-0.0056 \\
(0.01)\end{array}$ & -18.75 & $\begin{array}{l}0.0009 \\
(0.002)\end{array}$ & 1.67 & $\begin{array}{c}-0.0070 \\
(0.02)\end{array}$ & -12.92 \\
\hline Professionals & $\begin{array}{c}0.00154 \\
(0.002)\end{array}$ & 5.19 & $\begin{array}{c}0.00459 \\
(0.01)\end{array}$ & 15.44 & $\begin{array}{c}-0.00114 \\
(0.002)\end{array}$ & -2.10 & $\begin{array}{l}0.0184 \\
(0.03)\end{array}$ & 33.98 \\
\hline Semi-professionals & $\begin{array}{c}0.00004 \\
(0.00005)\end{array}$ & 0.13 & $\begin{array}{l}0.00318 \\
(0.008)\end{array}$ & 10.70 & $\begin{array}{c}8.4923 \\
(0.00001)\end{array}$ & 0.01 & $\begin{array}{l}0.0111 \\
(0.01)\end{array}$ & 20.47 \\
\hline Sales services & $\begin{array}{c}0.000066 \\
(0.0001)\end{array}$ & 0.22 & $\begin{array}{c}-0.0094 \\
(0.02)\end{array}$ & -13.70 & $\begin{array}{r}-0.00017 \\
(0.0002)\end{array}$ & -0.31 & $\begin{array}{c}-0.0379 \\
(0.04)\end{array}$ & -69.96 \\
\hline Manual workers & $\begin{array}{c}0.00064 \\
(0.001)\end{array}$ & 2.14 & $\begin{array}{l}0.0090 \\
(0.021)\end{array}$ & 30.38 & $\begin{array}{c}0.00086 \\
(0.002)\end{array}$ & 1.58 & $\begin{array}{c}-0.0215 \\
(0.04)\end{array}$ & -39.71 \\
\hline Union member & $\begin{array}{l}0.00028 \\
(0.0003)\end{array}$ & 0.95 & $\begin{array}{c}-0.0020 \\
(0.01)\end{array}$ & -6.82 & $\begin{array}{c}-0.00006 \\
(0.0006)\end{array}$ & -0.11 & $\begin{array}{c}-0.0136 \\
(0.03)\end{array}$ & -25.17 \\
\hline Union contract & $\begin{array}{c}-0.00029 \\
(0.0003)\end{array}$ & -0.98 & $\begin{array}{l}0.0010 \\
(0.002)\end{array}$ & 3.46 & $\begin{array}{l}0.00005 \\
(0.0002)\end{array}$ & 0.10 & $\begin{array}{c}0.00151 \\
(0.003)\end{array}$ & 2.78 \\
\hline Non-unionized & $\begin{array}{c}-0.00070 \\
(0.0006)\end{array}$ & -2.37 & $\begin{array}{c}-0.0251 \\
(0.06)\end{array}$ & -84.32 & $\begin{array}{c}-0.00036 \\
(0.0008)\end{array}$ & -0.66 & $\begin{array}{c}-0.0149 \\
(0.05)\end{array}$ & -27.56 \\
\hline Small workplace & $\begin{array}{c}0.00067 \\
(0.0003)^{*}\end{array}$ & 2.35 & $\begin{array}{c}0.001587 \\
(0.007)\end{array}$ & -43.70 & $\begin{array}{c}0.00232 \\
(0.003)\end{array}$ & 4.28 & $\begin{array}{l}0.0847 \\
(0.01)\end{array}$ & 156.19 \\
\hline Medium workplace & $\begin{array}{l}0.000250 \\
(0.00015)\end{array}$ & 0.84 & $\begin{array}{c}0.0056 \\
(0.01)\end{array}$ & 18.73 & $\begin{array}{c}0.0004 \\
(0.0006)\end{array}$ & 0.73 & $\begin{array}{c}-0.0201 \\
(0.02)\end{array}$ & -37.07 \\
\hline Large workplace & $\begin{array}{l}0.000014 \\
(0.0004)\end{array}$ & 0.48 & $\begin{array}{l}-0.0006 \\
(0.004)\end{array}$ & -2.10 & $\begin{array}{l}0.00054 \\
(0.001)\end{array}$ & 1.00 & $\begin{array}{c}-0.0053 \\
(0.01)\end{array}$ & -9.71 \\
\hline
\end{tabular}




\begin{tabular}{|c|c|c|c|c|c|c|c|c|}
\hline \multirow{3}{*}{$\begin{array}{l}\text { Table } 7 \\
\text { (continued) }\end{array}$} & \multicolumn{4}{|c|}{$\begin{array}{c}\text { Métis Men } \\
\text { vs. } \\
\text { non-Aboriginal Identity Men }\end{array}$} & \multicolumn{4}{|c|}{$\begin{array}{l}\text { North American Indian Men } \\
\text { vs. } \\
\text { non-Aboriginal Identity Men }\end{array}$} \\
\hline & \multicolumn{2}{|c|}{$\begin{array}{l}\text { Endowments Effect } \\
\text { (E) }\end{array}$} & \multicolumn{2}{|c|}{$\begin{array}{l}\text { Coefficients Effect } \\
\text { (C) }\end{array}$} & \multicolumn{2}{|c|}{$\begin{array}{c}\text { Endowments Effect } \\
\text { (E) }\end{array}$} & \multicolumn{2}{|c|}{$\begin{array}{l}\text { Coefficients Effect } \\
\text { (C) }\end{array}$} \\
\hline & Estimate & Share $(\%)$ & Estimate & Share (\%) & Estimate & Share $(\%)$ & Estimate & Share (\%) \\
\hline Public sector & $\begin{array}{l}-0.00005 \\
(0.0004)^{*}\end{array}$ & -0.16 & $\begin{array}{c}-0.0044 \\
(0.01)\end{array}$ & -14.79 & $\begin{array}{c}-0.00016 \\
(0.0004)\end{array}$ & -0.30 & $\begin{array}{l}0.0059 \\
(0.01)\end{array}$ & 10.84 \\
\hline Private sector & $\begin{array}{l}-0.00006 \\
(0.0005)\end{array}$ & -0.20 & $\begin{array}{l}0.0172 \\
(0.04)\end{array}$ & 57.93 & $\begin{array}{c}-0.00008 \\
(0.0002)\end{array}$ & -0.144 & $\begin{array}{l}-0.023 \\
(0.05)\end{array}$ & -42.39 \\
\hline Permanent job & $\begin{array}{c}0.00304 \\
(0.0009)^{* *}\end{array}$ & 10.20 & $\begin{array}{c}0.00259 \\
(0.02)\end{array}$ & 8.70 & $\begin{array}{l}0.00012 \\
(0.002)\end{array}$ & 2.15 & $\begin{array}{l}0.1171 \\
(0.12)\end{array}$ & 215.88 \\
\hline Seasonal job & $\begin{array}{c}0.00159 \\
(0.0005)^{\star *}\end{array}$ & 5.35 & $\begin{array}{l}-0.0011 \\
(0.002)\end{array}$ & -3.69 & $\begin{array}{c}0.00074 \\
(0.001)\end{array}$ & 1.37 & $\begin{array}{l}-0.0043 \\
(0.005)\end{array}$ & -7.90 \\
\hline $\begin{array}{l}\text { Temporary, } \\
\text { contract, casual job }\end{array}$ & $\begin{array}{c}-0.00003 \\
(0.0002)\end{array}$ & -0.11 & $\begin{array}{l}0.0017 \\
(0.004)\end{array}$ & 5.72 & $\begin{array}{l}0.00015 \\
(0.0003)\end{array}$ & 0.27 & $\begin{array}{l}-0.0009 \\
(0.005)\end{array}$ & -1.75 \\
\hline Single job & $\begin{array}{c}-0.00051 \\
(0.0004)\end{array}$ & -1.73 & $\begin{array}{l}-0.0188 \\
(0.04)\end{array}$ & -63.07 & $\begin{array}{l}-0.00003 \\
(0.00005)\end{array}$ & 0.05 & $\begin{array}{c}-0.0783 \\
(0.10)\end{array}$ & -144.35 \\
\hline Multiple jobs & $\begin{array}{l}-0.0003 \\
(0.0002)\end{array}$ & -1.09 & $\begin{array}{c}0.00098 \\
(0.002)\end{array}$ & 3.30 & $\begin{array}{l}0.00007 \\
(0.0001)\end{array}$ & 0.13 & $\begin{array}{l}0.0041 \\
(0.005)\end{array}$ & 7.56 \\
\hline Full-time job & $\begin{array}{c}0.00053 \\
(0.0003)^{\star}\end{array}$ & 1.77 & $\begin{array}{c}0.02783 \\
(0.06)\end{array}$ & 93.53 & $\begin{array}{l}-0.0001 \\
(0.0001)\end{array}$ & -0.18 & $\begin{array}{c}-0.0880 \\
(0.10)\end{array}$ & -162.25 \\
\hline Part-time job & $\begin{array}{c}0.00024 \\
(0.0001)^{\star}\end{array}$ & 0.80 & $\begin{array}{r}-0.0019 \\
(0.004)\end{array}$ & -6.52 & $\begin{array}{l}0.00023 \\
(0.0003)\end{array}$ & 0.42 & $\begin{array}{c}0.00613 \\
(0.007)\end{array}$ & 11.31 \\
\hline Wage below min & $\begin{array}{l}0.00045 \\
(0.0004)\end{array}$ & 1.54 & $\begin{array}{c}-0.00059 \\
(0.001)\end{array}$ & -1.98 & $\begin{array}{l}0.00007 \\
(0.0001)\end{array}$ & 0.13 & $\begin{array}{l}0.0008 \\
(0.001)\end{array}$ & 1.54 \\
\hline Wage minimum & $\begin{array}{c}-0.00016 \\
(0.0003)\end{array}$ & -0.53 & $\begin{array}{l}0.0020 \\
(0.004)\end{array}$ & 6.76 & $\begin{array}{c}-0.00038 \\
(0.0009)\end{array}$ & -0.71 & $\begin{array}{l}-0.0054 \\
(0.009)\end{array}$ & -9.94 \\
\hline Wage median & $\begin{array}{c}-0.00080 \\
(0.0005)\end{array}$ & -2.69 & $\begin{array}{l}0.0086 \\
(0.02)\end{array}$ & 28.79 & $\begin{array}{r}-0.0007 \\
(0.001)\end{array}$ & -1.38 & $\begin{array}{c}-0.0168 \\
(0.02)\end{array}$ & -31.03 \\
\hline Wage average & $\begin{array}{l}0.000014 \\
(0.00007)\end{array}$ & 0.047 & $\begin{array}{c}0.00411 \\
(0.01)\end{array}$ & 13.81 & $\begin{array}{l}0.00018 \\
(0.0006)\end{array}$ & 0.33 & $\begin{array}{l}0.0053 \\
(0.03)\end{array}$ & 9.77 \\
\hline Wage high & $\begin{array}{c}0.00118 \\
(0.001)\end{array}$ & 3.97 & $\begin{array}{c}0.01186 \\
(0.03)\end{array}$ & 39.84 & $\begin{array}{c}0.00047 \\
(0.002)\end{array}$ & 0.87 & $\begin{array}{l}0.0048 \\
(0.04)\end{array}$ & 8.87 \\
\hline Constant & & & $\begin{array}{l}-0.0216 \\
(0.08)\end{array}$ & -72.46 & & & $\begin{array}{c}-0.11994 \\
(0.28)\end{array}$ & -221.07 \\
\hline
\end{tabular}

Source: The Labour Force Survey, national monthly master file, September 2008 - May 2011.

Note: Decomposition refers to equation (2) components $E$ and $C$. Share is percentage share of predicted difference in the probability of being laid-off. Decomposition is based on Model 2, with all characteristics included, estimated using the main samples, which include all paid employees, aged between 15 and 64, who are not full-time students. Survey weights in effect. Standard errors reported in parentheses. Statistical significance ${ }^{* * *} p<.001 ;{ }^{* *} p<.01 ;{ }^{*} p<.05$. 
Among personal characteristics considered in the model, job tenure (less than 24 months) appears to be the largest contributing factor. As shown in the second column of Table 7, if Métis men were made equal to non-Aboriginal men in relation to this category of job tenure, the predicted gap in the probability of layoff would be reduced by about 11 percentage points. Among other personal characteristics, there is also a small positive contribution of age (category 45-54) and a small negative contribution of job absence (48 weeks category). Also, somewhat related, Quebec as the place of residence has a large negative effect (32\%) on the probability of layoff for Métis men. However, most of the explained difference seems to be related to job-related factors, and in particular to the industry and the type of job they hold, possibly suggesting sorting of Métis men across industry and sectors that are sensitive to economic shocks.

As shown in Table 7, if the distribution of Métis men in the primary industries were made equal to that of non-Aboriginal identity men, the predicted gap in the probability of layoff between the two groups of men would be reduced by about 16 percentage points. Similarly, if the distributions of permanent job-holders and seasonal workers were equalized across the two groups of men, the predicted gap in the probability of layoff between the two groups of men would be decreased by about 10 percentage points and by about 5 percentage points, respectively. Although statistically significant, the effects of the other job-related variables (workplace size, economic sector and full-time/part-time status) are relatively small, as shown in Table 7.

The results of the detailed decomposition for NAI men, presented at the right hand side portion of Table 7, appear completely uninformative - the 11.2 percentage points of the mean difference in the probability of layoff that was identified in the overall 
decomposition in Table 3 as being attributable to the differences in endowments between NAI men and non-Aboriginal identity men is not captured in the list of variables included in the present analysis; as shown in the last two columns of Table 7, while estimated contribution of some of the important variables such as completed university degree is relatively large (14.31\%), none of the variables included in the detailed decomposition here is statistically significant. The column listing the coefficient effects also shows some indication of large-sized contributors but none of them is statistically different from zero.

Table 8 presents the results from detailed decomposition of the mean difference in the predicted probability of layoff for Aboriginal and non-Aboriginal identity women. The decomposition here is carried only for the aggregate group of Aboriginal women (NAI, Inuit, and Métis) and for NAI women. As pointed out earlier, the predicted relatively small-sized gap (0.07\%) in the probability of layoff between Métis women and non-Aboriginal identity women, estimated using the main samples and presented in Table 3 , is not statistically different from zero. More restricted samples are not considered here.

As revealed in the overall decomposition results presented in Table 3 , a relatively large portion (44.8\%) of the mean difference in the predicted probability of layoff between the aggregate group of Aboriginal women and non-Aboriginal identity women can be attributed to the differences in endowments between the two groups of women. For NAI women, more than two thirds (72.1\%) of the predicted raw gap in the probability of layoff can be explained by the differences in observable characteristics between NAI women and non-Aboriginal identity women. However, the unexplained portions of the difference, possibly attributable to discrimination, are quite large as well (55.2\% for the aggregate group of Aboriginal women and $27.9 \%$ for NAI women alone). 
Table 8: Detailed decomposition of the difference in the predicted probability of layoff for women

\begin{tabular}{|c|c|c|c|c|c|c|c|c|}
\hline \multirow{3}{*}{$\begin{array}{l}\text { Detailed } \\
\text { decomposition } \\
\text { for layoffs } \\
\text { MAIN SAMPLE }\end{array}$} & \multicolumn{4}{|c|}{$\begin{array}{l}\text { Aboriginal Identity Women } \\
\text { vs. } \\
\text { non-Aboriginal Identity Women }\end{array}$} & \multicolumn{4}{|c|}{$\begin{array}{l}\text { North American Indian Women } \\
\text { vs. } \\
\text { non-Aboriginal ldentity Women }\end{array}$} \\
\hline & \multicolumn{2}{|c|}{$\begin{array}{l}\text { Endowments Effect } \\
\text { (E) }\end{array}$} & \multicolumn{2}{|c|}{$\begin{array}{l}\text { Coefficients Effect } \\
\text { (C) }\end{array}$} & \multicolumn{2}{|c|}{$\begin{array}{l}\text { Endowments Effect } \\
\text { (E) }\end{array}$} & \multicolumn{2}{|c|}{$\begin{array}{l}\text { Coefficients Effect } \\
\text { (C) }\end{array}$} \\
\hline & Estimate & $\begin{array}{c}\text { Share } \\
(\%)\end{array}$ & Estimate & $\begin{array}{c}\text { Share } \\
(\%)\end{array}$ & Estimate & $\begin{array}{c}\text { Share } \\
(\%)\end{array}$ & Estimate & Share (\%) \\
\hline $\begin{array}{l}\text { Less than } \\
\text { high school diploma }\end{array}$ & $\begin{array}{l}-0.0003 \\
(0.0005)\end{array}$ & -2.16 & $\begin{array}{c}-0.0037 \\
(0.007)\end{array}$ & -25.3 & $\begin{array}{c}0.0010 \\
(0.0007)\end{array}$ & 4.98 & $\begin{array}{l}-0.0004 \\
(0.002)\end{array}$ & -1.91 \\
\hline $\begin{array}{l}\text { Completed } \\
\text { high school diploma }\end{array}$ & $\begin{array}{r}-0.00007 \\
(0.0001)\end{array}$ & -0.47 & $\begin{array}{c}-0.0056 \\
(0.011)\end{array}$ & -37.9 & $\begin{array}{l}-0.00003 \\
(0.00005)\end{array}$ & -0.13 & $\begin{array}{r}-0.0005 \\
(0.003)\end{array}$ & -2.69 \\
\hline $\begin{array}{l}\text { Incomplete } \\
\text { postsecondary }\end{array}$ & $\begin{array}{c}-0.00012 \\
(0.0003)\end{array}$ & -0.82 & $\begin{array}{l}0.0008 \\
(0.003)\end{array}$ & 5.75 & $\begin{array}{l}-0.0003 \\
(0.0005)\end{array}$ & -1.65 & $\begin{array}{l}0.0001 \\
(0.001)\end{array}$ & 0.70 \\
\hline $\begin{array}{l}\text { Completed } \\
\text { trade certificate }\end{array}$ & $\begin{array}{c}0.0001 \\
(0.0002)\end{array}$ & 0.73 & $\begin{array}{l}0.00134 \\
(0.004)\end{array}$ & 9.05 & $\begin{array}{c}0.0005 \\
(0.0003)\end{array}$ & 0.24 & $\begin{array}{l}-0.00008 \\
(0.001)\end{array}$ & -0.37 \\
\hline $\begin{array}{l}\text { Completed } \\
\text { college diploma }\end{array}$ & $\begin{array}{c}6.5741 \\
(0.00004)\end{array}$ & 0.04 & $\begin{array}{l}0.00284 \\
(0.009)\end{array}$ & 19.21 & $\begin{array}{l}0.00001 \\
(6.994)\end{array}$ & 0.054 & $\begin{array}{l}0.00413 \\
(0.012)\end{array}$ & 20.32 \\
\hline $\begin{array}{l}\text { Completed } \\
\text { university degree }\end{array}$ & $\begin{array}{l}-0.0012 \\
(0.001)\end{array}$ & -0.80 & $\begin{array}{c}0.01165 \\
(0.02)\end{array}$ & 78.70 & $\begin{array}{c}-0.0004 \\
(0.001)\end{array}$ & -1.94 & $\begin{array}{l}-0.0016 \\
(0.006)\end{array}$ & -7.08 \\
\hline Tenure $<2$ years & $\begin{array}{c}0.0016 \\
(0.0008)^{\star}\end{array}$ & 11.0 & $\begin{array}{l}-0.0059 \\
(0.012)\end{array}$ & -40.45 & $\begin{array}{c}0.0031 \\
(0.001)^{*}\end{array}$ & 15.17 & $\begin{array}{l}-0.0014 \\
(0.005)\end{array}$ & -7.07 \\
\hline Tenure 2-5 years & $\begin{array}{r}-0.00005 \\
(0.0002)\end{array}$ & -0.32 & $\begin{array}{l}-0.0033 \\
(0.008)\end{array}$ & -22.82 & $\begin{array}{l}-0.00001 \\
(0.0002)\end{array}$ & -0.07 & $\begin{array}{l}0.0008 \\
(0.003)\end{array}$ & 3.97 \\
\hline Tenure $5-10$ years & $\begin{array}{l}-0.0001 \\
(0.0003)\end{array}$ & -0.78 & $\begin{array}{l}0.0040 \\
(0.009)\end{array}$ & 26.29 & $\begin{array}{l}-0.0002 \\
(0.0003)\end{array}$ & -0.88 & $\begin{array}{l}-0.0019 \\
(0.006)\end{array}$ & -9.55 \\
\hline Tenure $10-20$ years & $\begin{array}{c}0.0002 \\
(0.0004)\end{array}$ & 1.62 & $\begin{array}{l}-0.0013 \\
(0.006)\end{array}$ & -8.86 & $\begin{array}{c}0.0007 \\
(0.0005)\end{array}$ & 3.67 & $\begin{array}{l}0.0026 \\
(0.008)\end{array}$ & 12.67 \\
\hline Tenure $>20$ years & $\begin{array}{l}0.0008 \\
(0.001)\end{array}$ & 5.36 & $\begin{array}{l}0.0035 \\
(0.008)\end{array}$ & 23.70 & $\begin{array}{l}0.00113 \\
(0.001)\end{array}$ & 5.59 & $\begin{array}{l}-0.0004 \\
(0003)\end{array}$ & -1.82 \\
\hline No work absence & $\begin{array}{l}0.00001 \\
(0.00003)\end{array}$ & 0.07 & $\begin{array}{l}0.0291 \\
(0.06)\end{array}$ & 196.99 & $\begin{array}{l}-0.00007 \\
(0.0001)\end{array}$ & -0.36 & $\begin{array}{l}0.0023 \\
(0.013)\end{array}$ & 11.19 \\
\hline Absent 1 wk & $\begin{array}{c}0.00008 \\
(0.00003)^{*}\end{array}$ & 0.54 & $\begin{array}{l}0.0043 \\
(0.008)\end{array}$ & 29.02 & $\begin{array}{c}0.00009 \\
(0.00004)^{*}\end{array}$ & -0.48 & $\begin{array}{l}-0.0015 \\
(0.004)\end{array}$ & -7.48 \\
\hline Absent 2 wks & $\begin{array}{c}-0.0001 \\
(0.00005)^{*}\end{array}$ & -0.70 & $\begin{array}{l}0.0025 \\
(0.005)\end{array}$ & 16.80 & $\begin{array}{l}-0.00003 \\
(0.00007)\end{array}$ & 0.13 & $\begin{array}{l}-0.00002 \\
(0.0006)\end{array}$ & -0.87 \\
\hline Absent 3 wks & $\begin{array}{c}0.0001 \\
(0.00006)\end{array}$ & 0.74 & $\begin{array}{l}-0.0020 \\
(0.004)\end{array}$ & -13.68 & $\begin{array}{l}-0.00009 \\
(0.00005)\end{array}$ & 0.44 & $\begin{array}{l}0.0005 \\
(0.002)\end{array}$ & 2.61 \\
\hline Absent $4-8$ wks & $\begin{array}{c}0.00012 \\
(0.00006)^{*}\end{array}$ & 0.82 & $\begin{array}{l}-0.002 \\
(0.004)\end{array}$ & -15.60 & $\begin{array}{l}0.00005 \\
(0.0001)\end{array}$ & 0.24 & $\begin{array}{c}0.0009 \\
(0.0005)\end{array}$ & 0.44 \\
\hline Absent over 8 wks & $\begin{array}{l}-0.00002 \\
(0.0001)\end{array}$ & -0.16 & $\begin{array}{l}0.0039 \\
(0.007)\end{array}$ & 26.51 & $\begin{array}{l}-0.00003 \\
(0.0003)\end{array}$ & -0.13 & $\begin{array}{l}-0.0010 \\
(0.004)\end{array}$ & -5.15 \\
\hline
\end{tabular}




\begin{tabular}{|c|c|c|c|c|c|c|c|c|}
\hline \multirow{3}{*}{$\begin{array}{l}\text { Table } 8 \\
\text { (continued) }\end{array}$} & \multicolumn{4}{|c|}{$\begin{array}{l}\text { Aboriginal Identity Women } \\
\text { vs. } \\
\text { non-Aboriginal Identity Women }\end{array}$} & \multicolumn{4}{|c|}{$\begin{array}{l}\text { North American Indian Women } \\
\text { vs. } \\
\text { non-Aboriginal Identity Women }\end{array}$} \\
\hline & \multicolumn{2}{|c|}{$\begin{array}{l}\text { Endowments Effect } \\
\text { (E) }\end{array}$} & \multicolumn{2}{|c|}{$\begin{array}{l}\text { Coefficients Effect } \\
\text { (C) }\end{array}$} & \multicolumn{2}{|c|}{$\begin{array}{l}\text { Endowments Effect } \\
\text { (E) }\end{array}$} & \multicolumn{2}{|c|}{$\begin{array}{l}\text { Coefficients Effect } \\
\text { (C) }\end{array}$} \\
\hline & Estimate & $\begin{array}{c}\text { Share } \\
(\%)\end{array}$ & Estimate & $\begin{array}{c}\text { Share } \\
(\%)\end{array}$ & Estimate & $\begin{array}{c}\text { Share } \\
(\%)\end{array}$ & Estimate & Share $(\%)$ \\
\hline Age 15-24 & $\begin{array}{r}-0.00007 \\
(0.0002)\end{array}$ & -0.48 & $\begin{array}{l}0.0034 \\
(0.007)\end{array}$ & 23.05 & $\begin{array}{l}-0.0001 \\
(0.0001)\end{array}$ & -0.50 & $\begin{array}{c}-0.0002 \\
(0.001)\end{array}$ & -0.91 \\
\hline Age 25-34 & $\begin{array}{c}2.6434 \\
(0.00002)\end{array}$ & 0.02 & $\begin{array}{c}0.002 \\
(0.003)\end{array}$ & 35.48 & $\begin{array}{c}4.4521 \\
(0.0001)\end{array}$ & 0.02 & $\begin{array}{l}-0.0011 \\
(0.004)\end{array}$ & -5.52 \\
\hline Age $35-44$ & $\begin{array}{l}-0.0003 \\
(0.0003)\end{array}$ & -2.0 & $\begin{array}{l}-0.0082 \\
(0.002)\end{array}$ & -55.31 & $\begin{array}{c}0.0003 \\
(0.0007)\end{array}$ & 1.61 & $\begin{array}{l}-0.0008 \\
(0.003)\end{array}$ & -4.14 \\
\hline Age $45-54$ & $\begin{array}{l}-0.0004 \\
(0.0003)\end{array}$ & -2.5 & $\begin{array}{l}0.0038 \\
(0.010)\end{array}$ & 25.92 & $\begin{array}{l}-0.0002 \\
(0.0003)\end{array}$ & -0.96 & $\begin{array}{l}-0.0084 \\
(0.004)\end{array}$ & -4.13 \\
\hline Age 55-64 & $\begin{array}{r}-0.00004 \\
(0.0003)\end{array}$ & -0.28 & $\begin{array}{l}-0.0045 \\
(0.009)\end{array}$ & -30.20 & $\begin{array}{c}0.0001 \\
(0.0005)\end{array}$ & 0.67 & $\begin{array}{l}0.0019 \\
(0.006)\end{array}$ & 9.17 \\
\hline Married & $\begin{array}{l}-0.0006 \\
(0.0003)\end{array}$ & -4.03 & $\begin{array}{l}0.0271 \\
(0.05)\end{array}$ & 183.35 & $\begin{array}{l}-0.0006 \\
(0.0007)\end{array}$ & -2.88 & $\begin{array}{l}-0.0049 \\
(0.014)\end{array}$ & -24.12 \\
\hline $\begin{array}{l}\text { Widowed, } \\
\text { divorced, separated }\end{array}$ & $\begin{array}{l}-0.0001 \\
(0.0001)\end{array}$ & -1.06 & $\begin{array}{l}-0.0035 \\
(0.008)\end{array}$ & -24.08 & $\begin{array}{l}-0.00008 \\
(0.0003)\end{array}$ & -0.39 & $\begin{array}{l}0.0046 \\
(0.001)\end{array}$ & 2.27 \\
\hline Single & $\begin{array}{l}-0.00006 \\
(0.0002)\end{array}$ & -0.43 & $\begin{array}{l}-0.0018 \\
(0.006)\end{array}$ & -12.28 & $\begin{array}{r}-0.0002 \\
(0.0006)\end{array}$ & -0.97 & $\begin{array}{l}0.0007 \\
(0.003)\end{array}$ & 3.51 \\
\hline No children present & $\begin{array}{l}0.00016 \\
(0.0002)\end{array}$ & 1.07 & $\begin{array}{l}-0.0068 \\
(0.02)\end{array}$ & -46.02 & $\begin{array}{l}0.00003 \\
(0.0004)\end{array}$ & 0.14 & $\begin{array}{l}-0.0001 \\
(0.005)\end{array}$ & -0.50 \\
\hline Children < age 6 & $\begin{array}{l}-0.00007 \\
(0.00006)\end{array}$ & -0.50 & $\begin{array}{c}-0.00569 \\
(0.011)\end{array}$ & -38.46 & $\begin{array}{l}-0.0007 \\
(0.0001)\end{array}$ & -0.34 & $\begin{array}{l}0.0010 \\
(0.004)\end{array}$ & 5.21 \\
\hline Children, 6-14 & $\begin{array}{l}0.00014 \\
(0.0003)\end{array}$ & 0.94 & $\begin{array}{l}0.00188 \\
(0.005)\end{array}$ & 12.68 & $\begin{array}{c}0.0004 \\
(0.0005)\end{array}$ & 1.93 & $\begin{array}{c}-0.00132 \\
(0.004)\end{array}$ & -6.50 \\
\hline Children, 14-24 & $\begin{array}{l}-0.00025 \\
(0.0001)\end{array}$ & -1.69 & $\begin{array}{l}0.00596 \\
(0.012)\end{array}$ & 40.29 & $\begin{array}{c}6.5308 \\
(0.0001)\end{array}$ & 0.03 & $\begin{array}{l}0.00009 \\
(0.002)\end{array}$ & 0.45 \\
\hline Aflantic provinces & $\begin{array}{c}-0.00026 \\
(0.0001)\end{array}$ & -1.75 & $\begin{array}{l}0.00378 \\
(0.008)\end{array}$ & 25.57 & $\begin{array}{l}-0.00007 \\
(0.00009)\end{array}$ & -0.37 & $\begin{array}{l}-0.0005 \\
(0.001)\end{array}$ & -2.28 \\
\hline Quebec & $\begin{array}{l}0.0001 \\
(0.003)\end{array}$ & 6.56 & $\begin{array}{l}-0.0015 \\
(0.02)\end{array}$ & -10.10 & $\begin{array}{l}0.0040 \\
(0.004)\end{array}$ & 19.81 & $\begin{array}{l}0.0037 \\
(0.009)\end{array}$ & 18.39 \\
\hline Ontario & $\begin{array}{c}-0.00007 \\
(0.001)\end{array}$ & 0.48 & $\begin{array}{l}0.0002 \\
(0.01)\end{array}$ & -1.74 & $\begin{array}{l}-0.0010 \\
(0.0006)\end{array}$ & -4.77 & $\begin{array}{l}-0.0059 \\
(0.02)\end{array}$ & -28.87 \\
\hline Manitoba & $\begin{array}{l}0.0007 \\
(0.001)\end{array}$ & 4.72 & $\begin{array}{l}0.0008 \\
(0.002)\end{array}$ & 5.46 & $\begin{array}{l}0.0002 \\
(0.001)\end{array}$ & 1.23 & $\begin{array}{c}-0.0001 \\
(0.0005)\end{array}$ & -0.69 \\
\hline Saskatchewan & $\begin{array}{c}0.0004 \\
(0.0008)\end{array}$ & 2.68 & $\begin{array}{l}0.00036 \\
(0.001)\end{array}$ & 2.44 & $\begin{array}{r}-0.0014 \\
(0.001)\end{array}$ & -7.05 & $\begin{array}{l}0.0006 \\
(0.002)\end{array}$ & 3.01 \\
\hline Alberta & $\begin{array}{l}-0.0008 \\
(0.0008)\end{array}$ & -5.59 & $\begin{array}{c}-0.0037 \\
(0.007)\end{array}$ & -24.73 & $\begin{array}{l}0.00005 \\
(0.0004)\end{array}$ & 2.58 & $\begin{array}{l}-0.0018 \\
(0.005)\end{array}$ & -9.01 \\
\hline
\end{tabular}




\begin{tabular}{|c|c|c|c|c|c|c|c|c|}
\hline \multirow{3}{*}{$\begin{array}{l}\text { Table } 8 \\
\text { (continued) }\end{array}$} & \multicolumn{4}{|c|}{$\begin{array}{l}\text { Aboriginal Identity Women } \\
\text { vs. } \\
\text { non-Aboriginal ldentity Women }\end{array}$} & \multicolumn{4}{|c|}{$\begin{array}{l}\text { North American Indian Women } \\
\text { vs. } \\
\text { non-Aboriginal Identity Women }\end{array}$} \\
\hline & \multicolumn{2}{|c|}{$\begin{array}{l}\text { Endowments Effect } \\
\text { (E) }\end{array}$} & \multicolumn{2}{|c|}{$\begin{array}{l}\text { Coefficients Effect } \\
\text { (C) }\end{array}$} & \multicolumn{2}{|c|}{$\begin{array}{l}\text { Endowments Effect } \\
\text { (E) }\end{array}$} & \multicolumn{2}{|c|}{$\begin{array}{l}\text { Coefficients Effect } \\
\text { (C) }\end{array}$} \\
\hline & Estimate & $\begin{array}{c}\text { Share } \\
(\%)\end{array}$ & Estimate & $\begin{array}{c}\text { Share } \\
(\%)\end{array}$ & Estimate & $\begin{array}{c}\text { Share } \\
(\%)\end{array}$ & Estimate & Share (\%) \\
\hline British Columbia & $\begin{array}{c}-0.00056 \\
(0.0004)\end{array}$ & -3.77 & $\begin{array}{c}-0.0037 \\
(0.007)\end{array}$ & -25.1 & $\begin{array}{c}-0.0014 \\
(0.001)\end{array}$ & -6.94 & $\begin{array}{l}0.0013 \\
(0.004)\end{array}$ & 6.41 \\
\hline Primary industries & $\begin{array}{c}0.000103 \\
(0.00004)^{\star}\end{array}$ & 0.69 & $\begin{array}{l}0.0047 \\
(0.009)\end{array}$ & 31.7 & $\begin{array}{c}-0.0002 \\
(0.0001)^{*}\end{array}$ & -1.20 & $\begin{array}{l}-0.0012 \\
(0.003)\end{array}$ & -5.74 \\
\hline Manufacturing & $\begin{array}{l}-0.00066 \\
(0.0008)\end{array}$ & -4.49 & $\begin{array}{l}0.0014 \\
(0.006)\end{array}$ & 9.45 & $\begin{array}{l}-0.0002 \\
(0.001)\end{array}$ & -1.10 & $\begin{array}{l}0.0003 \\
(0.002)\end{array}$ & 1.61 \\
\hline Retail & $\begin{array}{l}-0.0003 \\
(0.0005)\end{array}$ & -2.17 & $\begin{array}{l}0.0018 \\
(0.009)\end{array}$ & 12.35 & $\begin{array}{l}0.00004 \\
(0.0008)\end{array}$ & 0.20 & $\begin{array}{l}-0.0021 \\
(0.007)\end{array}$ & -10.31 \\
\hline High skill services & $\begin{array}{c}0.0009 \\
(0.0006)\end{array}$ & 5.81 & $\begin{array}{c}-0.0062 \\
(0.013)\end{array}$ & -42.01 & $\begin{array}{c}0.0002 \\
(0.0007)\end{array}$ & 1.03 & $\begin{array}{c}-0.00004 \\
(0.002)\end{array}$ & -0.19 \\
\hline Public services & $\begin{array}{l}-0.0006 \\
(0.0003)^{*}\end{array}$ & -4.28 & $\begin{array}{c}-0.0256 \\
(0.05)\end{array}$ & -173.03 & $\begin{array}{l}-0.0003 \\
(0.0007)\end{array}$ & -1.29 & $\begin{array}{l}0.0018 \\
(0.009)\end{array}$ & 9.13 \\
\hline Other services & $\begin{array}{c}0.00004 \\
(0.00002)\end{array}$ & 0.26 & $\begin{array}{r}-0.0064 \\
(0.013)\end{array}$ & -43.50 & $\begin{array}{c}0.0006 \\
(0.0003)\end{array}$ & 2.87 & $\begin{array}{l}0.0032 \\
(0.010)\end{array}$ & 15.70 \\
\hline Management & $\begin{array}{c}0.00072 \\
(0.0002)^{\star \star *}\end{array}$ & 4.90 & $\begin{array}{c}-0.00963 \\
(0.02)\end{array}$ & -65.13 & $\begin{array}{c}0.0005 \\
(0.0005)\end{array}$ & 1.38 & $\begin{array}{l}0.0006 \\
(0.002)\end{array}$ & 3.07 \\
\hline Professionals & $\begin{array}{l}-0.0007 \\
(0.0007)\end{array}$ & -5.13 & $\begin{array}{l}0.0061 \\
(0.01)\end{array}$ & 41.34 & $\begin{array}{l}-0.0003 \\
(0.003)\end{array}$ & -1.06 & $\begin{array}{c}-0.00006 \\
(0.003)\end{array}$ & -0.31 \\
\hline Semi-professionals & $\begin{array}{l}0.00014 \\
(0.0001)\end{array}$ & 0.97 & $\begin{array}{c}0.0086 \\
(0.02)\end{array}$ & 58.46 & $\begin{array}{c}0.0003 \\
(0.0003)\end{array}$ & 1.47 & $\begin{array}{l}-0.0024 \\
(0.007)\end{array}$ & -11.83 \\
\hline Sales services & $\begin{array}{l}-0.00012 \\
(0.0005)\end{array}$ & -0.84 & $\begin{array}{l}0.0128 \\
(0.03)\end{array}$ & 86.51 & $\begin{array}{l}-0.0012 \\
(0.0007)\end{array}$ & -6.17 & $\begin{array}{l}0.0051 \\
(0.015)\end{array}$ & 25.22 \\
\hline Manual workers & $\begin{array}{c}0.00007 \\
(0.00004)\end{array}$ & 0.45 & $\begin{array}{l}0.00002 \\
(0.003)\end{array}$ & 1.52 & $\begin{array}{l}-0.0003 \\
(0.0003)\end{array}$ & -1.46 & $\begin{array}{l}0.0003 \\
(0.001)\end{array}$ & 1.36 \\
\hline Union member & $\begin{array}{l}0.00020 \\
(0.0001)\end{array}$ & 1.32 & $\begin{array}{l}-0.0250 \\
(0.05)\end{array}$ & -168.24 & $\begin{array}{c}0.0073 \\
(0.0004)^{*}\end{array}$ & 3.60 & $\begin{array}{l}0.0103 \\
(0.030)\end{array}$ & 50.88 \\
\hline Union contract & $\begin{array}{l}-0.00006 \\
(0.0001)\end{array}$ & -0.41 & $\begin{array}{l}0.0001 \\
(0.002)\end{array}$ & 6.76 & $\begin{array}{c}-0.0005 \\
(0.0002)^{*}\end{array}$ & -2.35 & $\begin{array}{l}-0.0010 \\
(0.003)\end{array}$ & -5.02 \\
\hline Non-unionized & $\begin{array}{l}0.00007 \\
(0.0001)\end{array}$ & 0.50 & $\begin{array}{c}0.01389 \\
(0.03)\end{array}$ & 93.85 & $\begin{array}{l}-0.0007 \\
(0.0004)\end{array}$ & -3.45 & $\begin{array}{l}0.0132 \\
(0.04)\end{array}$ & 65.04 \\
\hline Small workplace & $\begin{array}{c}0.0009 \\
(0.0004)^{*}\end{array}$ & 6.42 & $\begin{array}{c}-0.0013 \\
(0.01)\end{array}$ & -8.60 & $\begin{array}{l}0.00013 \\
(0.0006)\end{array}$ & 0.62 & $\begin{array}{l}0.0079 \\
(0.02)\end{array}$ & 38.79 \\
\hline Medium workplace & $\begin{array}{l}0.00008 \\
(0.0001)\end{array}$ & 0.56 & $\begin{array}{l}-0.0029 \\
(0.007)\end{array}$ & -19.56 & $\begin{array}{l}-0.0006 \\
(0.0001)\end{array}$ & -0.56 & $\begin{array}{l}-0.0020 \\
(0.006)\end{array}$ & -10.09 \\
\hline Large workplace & $\begin{array}{l}0.00049 \\
(0.0006)\end{array}$ & 3.29 & $\begin{array}{l}0.00227 \\
(0.006)\end{array}$ & 15.31 & $\begin{array}{l}0.0006 \\
(0.008)\end{array}$ & 2.84 & $\begin{array}{l}-0.0002 \\
(0.002)\end{array}$ & -1.21 \\
\hline
\end{tabular}




\begin{tabular}{|c|c|c|c|c|c|c|c|c|}
\hline \multirow{3}{*}{$\begin{array}{l}\text { Table } 8 \\
\text { (continued) }\end{array}$} & \multicolumn{4}{|c|}{ 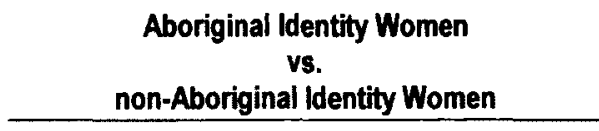 } & \multicolumn{4}{|c|}{$\begin{array}{l}\text { North American Indian Women } \\
\text { vs. } \\
\text { non-Aboriginal Identity Women }\end{array}$} \\
\hline & \multicolumn{2}{|c|}{$\begin{array}{l}\text { Endowments Effect } \\
\text { (E) }\end{array}$} & \multicolumn{2}{|c|}{$\begin{array}{l}\text { Coefficients Effect } \\
\text { (C) }\end{array}$} & \multicolumn{2}{|c|}{$\begin{array}{l}\text { Endowments Effect } \\
\text { (E) }\end{array}$} & \multicolumn{2}{|c|}{$\begin{array}{l}\text { Coefficients Effect } \\
\text { (C) }\end{array}$} \\
\hline & Estimate & $\begin{array}{c}\text { Share } \\
(\%)\end{array}$ & Estimate & $\begin{array}{c}\text { Share } \\
(\%)\end{array}$ & Estimate & $\begin{array}{c}\text { Share } \\
(\%)\end{array}$ & Estimate & $\begin{array}{c}\text { Share } \\
(\%)\end{array}$ \\
\hline Public sector & $\begin{array}{c}0.00022 \\
(0.0001)^{\star}\end{array}$ & 1.49 & $\begin{array}{l}0.0071 \\
(0.01)\end{array}$ & 47.92 & $\begin{array}{c}0.0003 \\
(0.0002)\end{array}$ & 1.60 & $\begin{array}{l}-0.0010 \\
(0.003)\end{array}$ & -5.02 \\
\hline Private sector & $\begin{array}{c}0.00032 \\
(0.0001)^{*}\end{array}$ & 2.13 & $\begin{array}{c}-0.0135 \\
(0.03)\end{array}$ & -91.62 & $\begin{array}{c}0.0004 \\
(0.0003)\end{array}$ & 1.82 & $\begin{array}{l}0.0019 \\
(0.007)\end{array}$ & 9.59 \\
\hline Permanent job & $\begin{array}{c}0.00112 \\
(0.0003)^{*}\end{array}$ & 7.57 & $\begin{array}{l}0.0417 \\
(0.07)\end{array}$ & 281.96 & $\begin{array}{c}0.0012 \\
(0.0003)^{*}\end{array}$ & 6.00 & $\begin{array}{l}0.0074 \\
(0.024)\end{array}$ & 36.52 \\
\hline Seasonal job & $\begin{array}{c}0.00020 \\
(0.00006)^{\star \star}\end{array}$ & 1.33 & $\begin{array}{r}0.00031 \\
(0.001)\end{array}$ & 2.10 & $\begin{array}{c}0.00024 \\
(0.0007)^{\star * *}\end{array}$ & 1.20 & $\begin{array}{l}-0.0007 \\
(0.002)\end{array}$ & -3.34 \\
\hline $\begin{array}{l}\text { Temporary, } \\
\text { contract, casual job }\end{array}$ & $\begin{array}{c}-0.00014 \\
(0.0002)\end{array}$ & -0.95 & $\begin{array}{c}-0.0054 \\
(0.01)\end{array}$ & -36.40 & $\begin{array}{c}-0.00022 \\
(0.0001)\end{array}$ & -1.09 & $\begin{array}{l}0.0023 \\
(0.007)\end{array}$ & 11.39 \\
\hline Single job & $\begin{array}{l}-0.0002 \\
(0.0001)\end{array}$ & -1.39 & $\begin{array}{c}-0.0091 \\
(0.03)\end{array}$ & -61.48 & $\begin{array}{l}0.00005 \\
(0.0002)\end{array}$ & 0.26 & $\begin{array}{l}0.0117 \\
(0.035)\end{array}$ & 57.52 \\
\hline Multiple jobs & $\begin{array}{c}-0.00014 \\
(0.0001)\end{array}$ & -0.99 & $\begin{array}{l}0.0007 \\
(0.002)\end{array}$ & 4.74 & $\begin{array}{l}0.00004 \\
(0.0001)\end{array}$ & 0.20 & $\begin{array}{r}-0.0009 \\
(0.003)\end{array}$ & -4.45 \\
\hline Full-time job & $\begin{array}{c}-0.00008 \\
(0.00002)^{*}\end{array}$ & -0.527 & $\begin{array}{c}-0.0126 \\
(0.03)\end{array}$ & -85.54 & $\begin{array}{l}-0.00003 \\
(0.00002)\end{array}$ & -0.13 & $\begin{array}{r}-0.0031 \\
(0.010)\end{array}$ & -15.14 \\
\hline Part-time job & $\begin{array}{c}-0.00019 \\
(0.00006)^{\star \star}\end{array}$ & -1.30 & $\begin{array}{l}0.0034 \\
(0.007)\end{array}$ & 22.72 & $\begin{array}{l}-0.00005 \\
(0.00005)\end{array}$ & -0.28 & $\begin{array}{l}0.0008 \\
(0.003)\end{array}$ & 4.01 \\
\hline Wage below min & $\begin{array}{l}-0.00007 \\
(0.00005)\end{array}$ & -0.49 & $\begin{array}{r}-0.0007 \\
(0.001)\end{array}$ & -4.89 & $\begin{array}{l}-0.00007 \\
(0.0001)\end{array}$ & -0.34 & $\begin{array}{l}0.00015 \\
(0.0005)\end{array}$ & 0.73 \\
\hline Wage minimum & $\begin{array}{c}0.0017 \\
(0.0006)^{\star \star}\end{array}$ & $\$ 1.61$ & $\begin{array}{l}0.0099 \\
(0.02)\end{array}$ & 67.09 & $\begin{array}{c}0.0033 \\
(0.001)^{\star \star}\end{array}$ & 16.48 & $\begin{array}{r}-0.0041 \\
(0.011)\end{array}$ & -20.07 \\
\hline Wage median & $\begin{array}{c}0.00054 \\
(0.0004)\end{array}$ & 3.63 & $\begin{array}{c}0.0039 \\
(0.008)^{*}\end{array}$ & 26.39 & $\begin{array}{c}0.0005 \\
(0.0005)\end{array}$ & 2.57 & $\begin{array}{r}-0.0011 \\
(0.004)\end{array}$ & -5.53 \\
\hline Wage average & $\begin{array}{c}-8.374 \\
(0.00004)\end{array}$ & -0.006 & $\begin{array}{l}0.0062 \\
(0.01)\end{array}$ & 41.89 & $\begin{array}{c}0.000014 \\
(0.0001)\end{array}$ & 0.07 & $\begin{array}{c}-0.00133 \\
(0.006)\end{array}$ & -6.58 \\
\hline Wage high & $\begin{array}{l}0.0014 \\
(0.001)\end{array}$ & 9.39 & $\begin{array}{l}0.0079 \\
(0.02)\end{array}$ & 53.21 & $\begin{array}{c}0.0038 \\
(0.001)^{\star}\end{array}$ & 18.69 & $\begin{array}{l}0.0040 \\
(0.012)\end{array}$ & 19.76 \\
\hline Constant & & & $\begin{array}{c}-0.0810 \\
(0.17)\end{array}$ & -547.15 & & & $\begin{array}{l}-0.0260 \\
(0.087)\end{array}$ & -127.84 \\
\hline
\end{tabular}

Source: The Labour Force Survey, national monthly master file, September 2008 - May 2011.

Note: Decomposition refers to equation (2) components $E$ and $C$. Share is percentage share of predicted difference in the probability of being laid-off. Decomposition is based on Model 2, with all characteristics included, estimated using the main samples, which include all paid employees, aged between 15 and 64, who are not full-time students. Survey weights in effect. Standard errors reported in parentheses. Statistical significance ${ }^{* * *} p<.001 ;{ }^{* *} p<.01 ;{ }^{*} p<.05$. 
The results from the detailed decomposition presented in Table 8 suggest that, in regard to the endowment effects, the differences in the level of educational attainment between Aboriginal and non-Aboriginal identity women do not play a significant role in the difference of their probability of layoff. As shown in Table 8, the estimates for educational attainment and the associated percentage shares are extremely small, with a slight exception of the first educational attainment category in the decomposition of the difference for NAI women; as it was the case for men, none of the estimates for the education categories of women in the detailed decomposition is statistically significant.

Among all personal characteristics included in the detailed decomposition of the difference in the probability of layoff between Aboriginal and non-Aboriginal identity women, only the differences in the distribution of low job tenure (less than 24 months) seem to make a large, statistically significant and positive contribution to the observed outcome. As shown in Table 8, if the distribution of the low job tenure among the aggregate group of Aboriginal identity women were comparable to that of nonAboriginal identity women, the predicted gap in the probability of layoff would be reduced by 11 percentage points. Similarly, the reduction in the predicted gap of the probability of layoff between NAI and non-Aboriginal identity women would amount to about 15 percentage points if the distributions of women with job tenure less than two years were equalized across the two groups of women. Job absence (at different levels) appears to have an ambiguous, though a very small impact on the observed outcome.

Among the employment-related characteristics, minimum wage (\$8.00-\$10.99) and permanent job (as opposed to seasonal, contract or casual job) seem to have the greatest positive impact. As shown in Table 8, equalizing the distribution of minimum 
wage earners would be expected to reduce the predicted gap in the probability of layoff between NAI women and non-Aboriginal women by 16.5 percentage points. The reduction in the predicted gap between the aggregate group of Aboriginal identity women and non-Aboriginal identity women would amount 11.6 percentage points if the distribution of minimum wage earners were equalized across the two groups of women. Similarly, if the distributions of women holding permanent jobs were equalized across the groups, the expected gap in the probability of layoff would be reduced by 7.6 percentage points for the aggregate group of Aboriginal identity women and by 6 percentage points for NAI women.

Union membership also seems to make a positive contribution to the reduction of the predicted gap in the probability of layoff between NAI and non-Aboriginal identity women. As shown in Table 8, the predicted gap in the probability of layoff between NAI and non-Aboriginal identity women would be reduced by 3.6 percentage points if the distribution of union membership were equalized across the two groups. Interestingly, the effect is opposite for union contract, suggesting that the predicted gap in the probability of layoff between NAI women and non-Aboriginal identity women would increase by 2.3 percentage points if the distribution of union contract holders were equalized across the two groups of women.

The indicator capturing seasonal job is a positive contributing factor for both groups of Aboriginal women examined here, although the percentage share is very small, just above one percentage points in each model. Similarly, the economic sector makes a positive, although relatively small contribution to the reduction in the gap of the probability of layoff between the aggregate group of Aboriginal identity women and non- 
Aboriginal identity women. Small size workplace, on the other hand, is a positive contributing factor with much larger percentage share, suggesting that the predicted gap in the probability of layoff between the aggregate group of Aboriginal identity women and non-Aboriginal identity women would be reduced by 6 percentage points if the distribution of women working in small-size workplaces (with 99 or less employees) were equalized across the two groups.

Finally, if the distribution of Aboriginal and non-Aboriginal identity women in the management occupation were equalized across the two groups, the gap in the probability of layoff between the aggregate group of Aboriginal identity women and nonAboriginal identity women would be reduced by 4.9 percentage points. Equalizing the distribution of Aboriginal and non-Aboriginal women in the primary industry also has a positive, though extremely small $(0.69 \%)$, effect on the reduction of the gap in the probability of layoff between the two groups of women. The effect in the NAI women model, however, is opposite and slightly larger (-1.2\%), implying that the predicted gap in the probability of layoff between NAI women and non-Aboriginal identity women would increase by 1.2 percentage points if the distributions of them working in the primary industries were equalized.

As shown in Tables 7 and 8 , no statistical significance is evident in relation to the coefficient effects for any of the groups examined here. This is interesting and worth further examination because the estimates for the coefficient effects in Table 3 are statistically significant for NAI men and for Aggregate group of Aboriginal men. The next section presents the results from the detailed decomposition of the mean difference 
in the probability of reemployment for Aboriginal identity groups, decomposed by gander. 


\subsubsection{Reemployment}

This section presents the results from the detailed decomposition of the mean difference in the probability of reemployment between each of the three groups of Aboriginal identity men and Aboriginal identity women: the aggregate Aboriginal identity group (which includes NAI, Inuit, and Métis), NAI and Métis. As mentioned earlier, only the endowment effects are discussed here. Also, as in the previous discussions, the discussion here centers on the effect of statistically significant findings.

As pointed out earlier in the discussion of the overall decomposition results for reemployment in Section 4.1.2, the endowment effect accounts fully for the mean difference in the predicted probability of reemployment between Aboriginal identity men and Aboriginal identity women. However, as was the case in the preceding discussions of the results from the detailed decomposition concerning layoffs, the results from the detailed decomposition concerning reemployment suggest that the differences in the level of education do not play a significant role in creating difference in the probability of reemployment between any of the three examined groups of Aboriginal identity men and Aboriginal identity women. As shown in Table 9, although the contribution share in relation to some categories of educational attainment for some groups (NAI group) are quite large, none of the effects for educational attainment is statistically significant.

In fact, the results from this detailed decomposition provide a very few insights into the difference in the estimated gaps in the probability of reemployment of the examined groups. As mentioned earlier, rigorous limitations were imposed in the constriction of these samples; hence the statistically significant results presented next should be treated with added caution. 
Table 9: Detailed decomposition of the difference in the predicted probability of reemployment

\begin{tabular}{|c|c|c|c|c|c|c|}
\hline \multirow{3}{*}{$\begin{array}{l}\text { Detailed } \\
\text { decomposition } \\
\text { for reemployment } \\
\text { MAIN SAMPLE }\end{array}$} & \multicolumn{6}{|c|}{ Endowment Effect (E) } \\
\hline & \multicolumn{2}{|c|}{$\begin{array}{l}\text { Total Aboriginal identity } \\
\text { Men vs. Women }\end{array}$} & \multicolumn{2}{|c|}{$\begin{array}{c}\text { Métis } \\
\text { Men vs. Women }\end{array}$} & \multicolumn{2}{|c|}{$\begin{array}{l}\text { North American Indian } \\
\text { Men vs. Women }\end{array}$} \\
\hline & Estimate & Share $(\%)$ & Estimate & Share $(\%)$ & Estimate & Share $(\%)$ \\
\hline $\begin{array}{l}\text { Less than } \\
\text { high school diploma }\end{array}$ & $\begin{array}{r}0.00037 \\
(0.0004)\end{array}$ & 0.47 & $\begin{array}{c}-0.0001 \\
(0.003)\end{array}$ & -0.10 & $\begin{array}{r}-0.0023 \\
(0.005)\end{array}$ & -19.33 \\
\hline $\begin{array}{l}\text { Completed } \\
\text { high school diploma }\end{array}$ & $\begin{array}{l}0.00004 \\
(0.0001)\end{array}$ & 0.05 & $\begin{array}{l}0.0011 \\
(0.001)\end{array}$ & 0.77 & $\begin{array}{l}0.0003 \\
(0.001)\end{array}$ & 2.47 \\
\hline $\begin{array}{l}\text { Incomplete } \\
\text { postsecondary }\end{array}$ & $\begin{array}{l}-0.0001 \\
(0.001)\end{array}$ & -0.13 & $\begin{array}{l}-0.0009 \\
(0.004)\end{array}$ & -0.69 & $\begin{array}{c}0.0006 \\
(0.0007)\end{array}$ & 5.06 \\
\hline $\begin{array}{l}\text { Completed } \\
\text { trade certificate }\end{array}$ & $\begin{array}{l}0.0047 \\
(0.003)\end{array}$ & 5.88 & $\begin{array}{l}-0.0003 \\
(0.004)\end{array}$ & -0.24 & $\begin{array}{l}0.0033 \\
(0.003)\end{array}$ & 27.26 \\
\hline $\begin{array}{l}\text { Completed } \\
\text { college diploma }\end{array}$ & $\begin{array}{r}0.00437 \\
(0.006)\end{array}$ & 5.52 & $\begin{array}{l}0.0059 \\
(0.006)\end{array}$ & 4.30 & $\begin{array}{l}0.0081 \\
(0.009)\end{array}$ & 66.89 \\
\hline $\begin{array}{l}\text { Completed } \\
\text { university degree }\end{array}$ & $\begin{array}{l}0.00047 \\
(0.002)\end{array}$ & 0.59 & $\begin{array}{r}-0.0007 \\
(0.006)\end{array}$ & -0.48 & - & - \\
\hline Age 15-24 & $\begin{array}{l}0.0051 \\
(0.002)^{\star}\end{array}$ & 6.48 & $\begin{array}{c}0.0001 \\
(0.0002)\end{array}$ & 0.09 & $\begin{array}{c}0.0178 \\
(0.005)^{\star \star}\end{array}$ & 146.97 \\
\hline Age $25-34$ & $\begin{array}{l}0.00034 \\
(0.0005)\end{array}$ & 0.42 & $\begin{array}{r}-0.0022 \\
(0.005)\end{array}$ & -1.62 & $\begin{array}{l}0.0010 \\
(0.003)\end{array}$ & 8.52 \\
\hline Age $35-44$ & $\begin{array}{c}-0.00003 \\
(0.004)\end{array}$ & 0.04 & $\begin{array}{l}-0.0010 \\
(0.005)\end{array}$ & -0.70 & $\begin{array}{l}0.0008 \\
(0.006)\end{array}$ & 7.05 \\
\hline Age $45-54$ & $\begin{array}{l}0.00105 \\
(0.0008)\end{array}$ & 1.33 & $\begin{array}{r}-0.0007 \\
(0.002)\end{array}$ & -0.49 & $\begin{array}{l}-0.00174 \\
(0.0007)^{\star}\end{array}$ & -14.35 \\
\hline Age $55-64$ & $\begin{array}{l}0.00904 \\
(0.004)^{\star}\end{array}$ & 11.41 & $\begin{array}{l}-0.00002 \\
(0.00003)\end{array}$ & -0.02 & $\begin{array}{c}0.0429 \\
(0.010)^{\star * *}\end{array}$ & 353.65 \\
\hline Married & $\begin{array}{c}-0.00678 \\
(0.005)\end{array}$ & -7.29 & $\begin{array}{l}-0.00656 \\
(0.003)^{*}\end{array}$ & -4.74 & $\begin{array}{c}-0.00135 \\
(0.010)\end{array}$ & -11.17 \\
\hline $\begin{array}{l}\text { Widowed, } \\
\text { divorced, separated }\end{array}$ & $\begin{array}{l}0.00222 \\
(0.002)\end{array}$ & 2.80 & $\begin{array}{c}0.00383 \\
(0.003)\end{array}$ & 2.77 & $\begin{array}{r}-0.0003 \\
(0.001)\end{array}$ & -2.42 \\
\hline Single & $\begin{array}{c}0.00140 \\
(0.003)\end{array}$ & 1.76 & $\begin{array}{c}0.00002 \\
(0.00005)\end{array}$ & 0.01 & $\begin{array}{l}-0.0028 \\
(0.006)\end{array}$ & -23.07 \\
\hline No children present & $\begin{array}{c}0.000155 \\
(0.003)\end{array}$ & 0.19 & $\begin{array}{l}0.0005 \\
(0.003)\end{array}$ & 0.38 & $\begin{array}{l}0.0147 \\
(0.009)\end{array}$ & 121.47 \\
\hline Children < age 6 & $\begin{array}{l}0.00269 \\
(0.001)^{\star}\end{array}$ & 3.39 & $\begin{array}{c}0.0091 \\
(0.002)^{* *}\end{array}$ & 6.59 & $\begin{array}{l}0.00012 \\
(0.0004)\end{array}$ & 1.02 \\
\hline Children aged 6-14 & $\begin{array}{l}-0.0085 \\
(0.005)\end{array}$ & -10.71 & $\begin{array}{c}-0.0005 \\
(0.0002)^{*}\end{array}$ & -0.35 & $\begin{array}{l}0.0024 \\
(0.010)\end{array}$ & 20.09 \\
\hline & & & & & & ...continued \\
\hline
\end{tabular}


Endowment Effect (E)

Table 9

(continued)

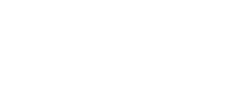

Children aged 14-24

Atlantic provinces

Quebec

Ontario

Manitoba

Saskatchewan

Alberta

British Columbia

Held full time job

Held part time job

Primary industries

Manufacturing

Retail

High skill services

Public services

Other services

Management

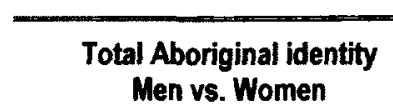

Men vs. Women

Estimate Share (\%)

$-0.0011$

(0.004)

$-1.39$

0.00007

(0.001)

0.00074

$(0.0007)$

$-0.00518$

$(0.003)$

0.00002

$(0.00001)$

$-0.00025$

$(0.0006)$

$-0.00209$

$(0.003)$

$-0.000124$

$(0.00008)$

0.00270

$(0.001)$

0.00986

$(0.005)$

0.0043

(0.01)

0.00079

$(0.0005)$

0.01027

(0.01)

$-0.00236$

$(0.001)^{\star}$

0.01862

(0.02)

0.00281

$(0.002)$

0.0009

(0.004)

0.94

$-6.53$

0.03

$-0.32$

$-2.63$

$-0.16$

3.41

12.44

5.46

$-2.97$

1.15

23.49

3.54
Métis

Men vs. Women

Estimate

Share (\%)

$-0.87 \quad 0.0053$

(0.008)

43.52

$-0.34$

0.0017

(0.001)

13.70

$0.10 \quad 0.00064$

0.00013

$(0.001)$

$-0.0002$

$(0.0001)$

$-0.14$

0.00004

$(0.0001)$

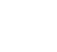

0.00022

$(0.0004)$

$-0.01332$

$(0.006)^{*}$

0.00032

(0.0003)

0.0020

$(0.003)$

0.00689

$(0.009)$

0.0020

(0.016)

$-0.0001$

(0.002)

0.0100

(0.021)

$-0.0076$

$(0.002)^{\star *}$

0.0339

$(0.031)$

$-0.0011$

$(0.005)$

Men vs. Women

$(0.002)$

5.33

4.64

13.29

0.16

$-9.52$

0.0040

$(0.003)$

32.88

$-0.0003$

$(0.0003)$

$-2.89$

1.48

0.0007

$(0.0004)$

5.98

0.0076

$(0.004)$

63.08

0.0002

(0.020)

1.77

$-0.11$

0.0087

$(0.003)^{\star \star *}$

72.15

7.24

0.0057

(0.011)

47.30

$-0.00032$

$(0.0004)$

$-2.61$

.5 .51

$-0.0003$

24.51

$(0.030)$

$-2.25$

$-0.84$

0.00167

$(0.0007)^{*}$

13.72

0.00044

$(0.001)$ 
Endowment Effect (E)

\begin{tabular}{|c|c|c|c|c|c|c|}
\hline \multirow[t]{2}{*}{$\begin{array}{l}\text { Table 9 } \\
\text { (continued) }\end{array}$} & \multicolumn{2}{|c|}{$\begin{array}{l}\text { Total Aboriginal identity } \\
\text { Men vs. Women }\end{array}$} & \multicolumn{2}{|c|}{$\begin{array}{c}\text { Métis } \\
\text { Men vs. Women }\end{array}$} & \multicolumn{2}{|c|}{$\begin{array}{l}\text { North American Indian } \\
\text { Men vs. Women }\end{array}$} \\
\hline & Estimate & Share $(\%)$ & Estimate & Share (\%) & Estimate & Share $(\%)$ \\
\hline Professionals & $\begin{array}{l}-0.0015 \\
(0.005)\end{array}$ & -1.96 & $\begin{array}{l}0.0002 \\
(0.003)\end{array}$ & 0.18 & $\begin{array}{l}-0.0084 \\
(0.006)\end{array}$ & -69.60 \\
\hline Semi-professionals & $\begin{array}{c}-0.00089 \\
(0.001)\end{array}$ & -1.12 & $\begin{array}{l}0.00139 \\
(0.002)\end{array}$ & 1.01 & $\begin{array}{l}0.00012 \\
(0.0001)\end{array}$ & -1.04 \\
\hline Sales services & $\begin{array}{l}0.0434 \\
(0.03)\end{array}$ & 54.76 & $\begin{array}{l}0.0408 \\
(0.043)\end{array}$ & 29.51 & $\begin{array}{c}0.070 \\
(0.03)^{\star}\end{array}$ & 580.38 \\
\hline Manual workers & $\begin{array}{l}0.00424 \\
(0.03)\end{array}$ & 5.35 & $\begin{array}{l}0.0815 \\
(0.04)^{*}\end{array}$ & 58.93 & $\begin{array}{l}-0.0265 \\
(0.03)\end{array}$ & -218.54 \\
\hline Public sector & $\begin{array}{c}-0.00119 \\
(0.005)\end{array}$ & -1.49 & $\begin{array}{l}-0.0007 \\
(0.005)\end{array}$ & -0.53 & $\begin{array}{l}0.0020 \\
(0.01)\end{array}$ & 16.55 \\
\hline Private sector & $\begin{array}{c}-0.000175 \\
(0.0008)\end{array}$ & -0.22 & $\begin{array}{l}0.00022 \\
(0.002)\end{array}$ & 0.16 & $\begin{array}{l}0.0007 \\
(0.004)\end{array}$ & 6.06 \\
\hline
\end{tabular}

Source: The Labour Force Survey, national monthly master file, September 2008 - May 2011.

Note: Decomposition refers to equation (2) components $E$ only. Share is percentage share of predicted difference in the probability of being reemployed. Decomposition is based on Model 2, with all characteristics included, estimated using the main samples, which include all paid employees, aged between 15 and 64, who are not full-time students. Survey weights in effect. Standard errors reported in parentheses. Statistical significance ${ }^{* * *} p<.001 ;{ }^{* *} p<.01 ;{ }^{*} p<.05$.

The results from the detailed decompositions of the mean differences in the predicted probability of reemployment presented in Table 9 suggest that the differences in the age structure play a significant role in the reemployment gap for the aggregate Aboriginal identity group and for NAI, while for Métis, the differences in the family structure play a significant role. As shown in Table 9, the differences in the age structure is one of the largest contributing factors of the mean difference in the probability of reemployment between NAI men and NAI women; if the distribution of age (in the 15-24 category and in the 55-64 category) were equal across NAI gender groups, the expected reduction in the reemployment gap would be considerably large (147\% and 353\%, respectively), provided these shares are not inflated due to sample size issues. For the 4554 age category, however, the effect for NAI group appears negative, suggesting that the 
predicted gap in the probability of reemployment between NAI men and NAI women would increase by 14.3 percentage points if the age distribution in this category were equalized across gender. For the aggregate Aboriginal identity group, the effect is positive and relatively large for two age categories: the 15-24 category (6.5\%) and for the $55-64$ category (11.4\%).

In regard to personal characteristics of Métis, a positive effect is evident only for the presence of dependent children (aged 6 or less) variable, suggesting that the gap in the probability of reemployment between Métis men and Métis women would be decreased by 6.6 percentage points if young dependent children were equally present across groups. The same positive effect of this variable appears in the detailed decomposition results of the aggregate Aboriginal identity group, though the contribution share here is about as half as small (3.4\%). Interestingly, the variables capturing marital status (married) and the presence of school-aged children (aged 6-14) have a negative effect, though the magnitude of the share of the latter $(-0.35 \%)$ is rather negligible. The magnitude of the share of the former, however, is not negligible (-4.7\%), suggesting that the predicted gap in the mean difference of the probability of reemployment between Métis men and Métis women would increase by 4.7 percentage points if the distribution of this personal characteristic were equalized across gender.

In regard to job-related factors, the results from the detailed decomposition in Table 9 suggest that the differences in the distribution of workers across occupational categories and across industry play a significant role, both for Métis and for NAI. As shown in Table 9, for Métis, the effect is quite large and positive in regard to the difference in the distribution of manual workers but relatively small and negative in 
regard to the difference in the distribution of workers in high-skill service sector; equalizing the distribution of Métis men and Métis women in manual work occupations would reduce the expected gap in reemployment by about 59 percentage points but would increase the gap by 5.5 percentage points if their distribution in the high skill service sector were equalized. For NAI, the difference in the distribution in the sales service occupations appears to be the single largest positive effect, accounting for an unrealistic share $(580 \%)$ of the predicted difference in the probability of reemployment between NAI men and NAI women. The differences in their distribution across industry sectors manufacturing and other services) also account for a significant portions of the difference in the predicted probability of reemployment ( $72.1 \%$ and $13.7 \%$, respectively).

Finally, the effect of place of residence (provinces) is evident in the Métis decomposition (-9.5\% for residence in Alberta) and in the NAI decomposition (13.3\% for residence in Manitoba). For Métis, this negative effect suggests that the gap in the probability of reemployment between Métis men and Métis women would increase by 9.5 percentage points if the distribution of their residence in Alberta were equalized. For NAI, this positive effect suggests that the gap in the probability of reemployment between NAI men and NAI women would decrease by 13.3 percentage points if the distribution of their residence in Manitoba were equalized.

In summary, with some exceptions, the detailed decomposition results presented in this section do not add much to our understanding as to the factors underlying the difference in the impact of recession on Aboriginal and non-Aboriginal identity workers. In the explained component of the difference, some of the individual variables effects are implausibly large and in the unexplained component there appears no story worth telling. 
The most surprising finding here is that the differences in educational attainment between Aboriginal and non-Aboriginal (and among Aboriginal) workers play no significant role in explaining the difference in their probabilities of layoff (and the probabilities of reemployment). Low job tenure (less than 24 months) however, appears important in the detailed decompositions for layoffs, both for men and for women. Among other personal characteristics, there is a small positive contribution of the prime age category (age 45-54) and a small negative contribution of job absence (4-8 weeks category) in the detailed decomposition of layoffs, but only for Métis men. For each group examined here, most of the explained difference seems to be related to various jobrelated factors, especially industry and occupation, possibly suggesting sorting of workers across industry and sectors that are sensitive to economic shocks.

In the detailed decomposition of the difference in the probability of reemployment, personal factors such as family structure (for Métis) and age distribution (for the other two groups of Aboriginal identity workers) play some role in the observed differences of the predicted gaps in the probability of reemployment between the examined gender groups. However, as in the layoffs detailed decomposition, job-related variables (industry and occupation) and province of residence account for a greater portion of the explained difference. Finally, some variation in the impact of these factors exists among groups. 


\section{Concluding Remarks}

This essay examined the relationship between Aboriginal identity and economic success in the Canadian labour market in the context of an economic downturn. Using monthly data from the 2008-2011 LFS, the essay analyzed the difference in the impact of the latest recession on Aboriginal and on non-Aboriginal identity workers, focusing on two specific aspects that convey the relative degree of vulnerability and the extent of hardship experienced in the adjustment process: the probability of layoff and the probability of reemployment following the layoff. The essay was inspired by recent descriptive evidence that suggested that the impact of the latest recession on Aboriginal workers, relative to non-Aboriginal workers, was disproportionately negative. The key research question that guided this analysis asked: What underlies the disproportionate impact of recession on the two groups of Canadian workers? To what extent is the difference in the impact the result of the differences in the observable characteristics among Aboriginal and non-Aboriginal workers and the jobs they hold and to what extent it is the result of employer discrimination in favour of non-Aboriginal workers?

The results derived from this analysis are briefly summarized here along the two guiding aspects: (1) the degree of vulnerability; and (2) the extent of hardship. In regards to the first aspect, the findings indicate that Aboriginal identity workers, and particularly NAI men, experienced a greater degree of vulnerability to the changes in the labour market brought about by the 2008-2011 recession than were non-Aboriginal identity workers, as measured by their probabilities of being laid off. In regards to the second aspect, the findings suggest that Aboriginal identity workers, and particularly Métis men, 
experienced a lesser degree of hardship in the adjustment process, as measured by their probability of being reemployed following the layoff. The following few paragraphs provide more details on each of the measured outcomes for each of the examined groups. As revealed in the descriptive as well as in the empirical parts of the analysis, both Aboriginal men and Aboriginal women were much more likely to be laid off than were their non-Aboriginal counterparts. Among Aboriginal identity groups, NAI men were disproportionately affected. As revealed in the overall decomposition, the predicted gaps in the probability of layoff between NAI men and non-Aboriginal identity men appear largest, both in the main and in the more restricted samples, with a very small share of the differences being attributable to the differences in the observed characteristics between the two groups of men - much remains unexplained and, possibly, attributable to discrimination in the labour market against NAI men.

The impact of the 2008-2011 recession on Métis workers was less severe, in comparison to that of NAI workers. Moreover, as revealed in the overall decomposition, much of the gap in the probability of layoff between Métis workers and non-Aboriginal identity workers can be explained by the differences in the observable characteristics between the two groups of workers. Also, in the case of Métis workers at risk of being laid off, there seems to be some indication of sorting across industry and sectors that were relatively more susceptible to this economic change.

In regard to the unique contributions of the factors examined in the detailed decomposition of the difference in the probability of layoff, the conclusions reached here are rather tentative - the method was certainly worth a try but the results turned out to be not very informative, particularly not for shedding some light on the outcomes of NAI 
men. For the other groups of Aboriginal identity workers, this detailed decomposition method revealed some insights but they do not have much policy relevance. The most policy-relevant factor examined in this decomposition (educational attainment) turned out to be not significant in creating gaps in the probability of layoff. This finding, however, might not be due to the limitation of the method; as discussed earlier in Section 2.3, the existing literature consistently reports inconclusive evidence on the role education plays in the labour market outcomes of Aboriginal people. Further investigation of this question, using this method but different data sets, could provide more clarity on this. Finally, the findings pertaining to the examination of the differences in the probability of reemployment following the layoff suggest different stories for different identity groups, and particularly for different genders. Although the evidence based on the weighted data suggested that, relative to non-Aboriginal identity workers, both Aboriginal men and Aboriginal women were more likely to have secured paid employment shortly after the layoff, Aboriginal men, and in particular Métis men, seem to have been the most successful. The examination of the same question using the nonweighted data, however, suggested that Aboriginal women were the most successful in obtaining paid employment shortly after the layoff and that the duration of their unemployment was much shorter in comparison to that of the other groups.

The results from the overall decomposition of the difference in the probability of reemployment suggested that, when all considered factors are included in the model, the mean difference in the probability of reemployment between Aboriginal gender groups is fully explained by the differences in those observable factors among them. As with the analysis of layoffs, the insights derived from the detailed decomposition as to the relative 
importance of those factors in affecting the gaps in reemployment for the examined groups were not very informative, aside from suggesting that there might be some positive sorting (particularly in the case of Métis men) across occupational affiliations and industry sectors. Data limitations and the econometric issues discussed earlier in this essay, however, need to be taken into account when interpreting these findings, particularly these pertaining to the differences in the probability of reemployment. 


\section{Appendix A}

Appendix A 1: A Summary of Definitions of Independent Variables

\begin{tabular}{|c|c|}
\hline Variables & Definitions \\
\hline Educational attainment & $\begin{array}{l}\text { Six dummy indicators capturing highest level of schooling completed: less than high } \\
\text { school diploma, completed high school diploma, incomplete postsecondary education, } \\
\text { completed trade certificate or diploma from a vocational or apprenticeship training, } \\
\text { completed community college certificate or diploma, and university degree or higher. }\end{array}$ \\
\hline Job tenure & $\begin{array}{l}\text { Five dummy indicators relating the most recent period of uninterrupted work: two years or } \\
\text { less, } 2-5 \text { years, } 5-10 \text { years, } 10-20 \text { years, and } 20 \text { years or more. }\end{array}$ \\
\hline Work absence & $\begin{array}{l}\text { Dummies indicating the number of weeks of continual absence from work: one or no } \\
\text { weeks absent, two weeks or less, three weeks or less, four weeks or less. }\end{array}$ \\
\hline Age & $\begin{array}{l}\text { Five dummies depicting age groups: youth } 15-24 \text {, young workers } 25-34 \text {, younger prime- } \\
\text { age workers } 35-44 \text {, mature prime-age workers } 45-54 \text {, and older workers } 55-64 \text {. }\end{array}$ \\
\hline Industry & $\begin{array}{l}\text { Six dummy indicators, classified using the NAICS } 2002 \text { codes: primary industries and } \\
\text { construction, manufacturing, retail trade accommodation and food service, high-skill } \\
\text { services, public services, and other service-producing industries. }\end{array}$ \\
\hline Occupation & $\begin{array}{l}\text { Five dummies for occupational status, classified using the NOC-S } 2001 \text { codes: } \\
\text { management, professionals, semi-professionals, clerical sales and service personnel, and } \\
\text { manual workers and trade personnel. }\end{array}$ \\
\hline Union coverage & $\begin{array}{l}\text { Three dummies for union status: union member, not a member but covered by a union } \\
\text { contract, and non-unionized. }\end{array}$ \\
\hline Workplace size & $\begin{array}{l}\text { Three dummies describing the size of the workplace establishment: small } 99 \text { or less } \\
\text { employees, medium } 100-500 \text { employees, and large over } 500 \text { employees. }\end{array}$ \\
\hline Economic sector & A dummy indicating if a worker was employed in government or private sector. \\
\hline Job permanency & Three dummies depicting permanent, seasonal and contract/casual jobs. \\
\hline Number of jobs & A dummy indicating if the worker has a single or multiple jobs. \\
\hline Type of work & A dummy for full-time (part-time) work schedule, 30 hours or more (less) per week. \\
\hline Wage level & $\begin{array}{l}\text { Five dummies for hourly wages, including tips and commissions, before taxes and other } \\
\text { deductions: below minimum wage } \$ 8.00 \text { or less, minimum wage } \$ 8.00-\$ 10.99 \text {, median } \\
\text { wage } \$ 11.00-\$ 14.99 \text {, above average wage } \$ 15.00-\$ 24.99 \text {, and high wage } \$ 25 \text { or higher. }\end{array}$ \\
\hline Region & $\begin{array}{l}\text { Seven dummies for provinces: Atlantic provinces, Quebec, Ontario, Manitoba, } \\
\text { Saskatchewan, Alberta and British Columbia. }\end{array}$ \\
\hline Marital status & Three dummies for marital status: single, married, and widowed/divorced/separated \\
\hline Presence of children & $\begin{array}{l}\text { Four dummies indicating presence of children: no children present, dependent children } \\
\text { younger than } 6 \text {, school-aged children aged } 6-14 \text {, and adult children aged } 14-24 \text { present. }\end{array}$ \\
\hline
\end{tabular}

Note: Each dummy is coded one if the condition holds and zero otherwise. Reference categories are in italics. 
Appendix A 2: Mean Characteristics of Paid Employees in the Main Samples, by Identity

\begin{tabular}{|c|c|c|c|c|c|c|c|c|}
\hline \multirow[t]{2}{*}{ Characteristics } & \multicolumn{2}{|c|}{$\begin{array}{c}\text { Non-Aboriginal } \\
\text { Identity }\end{array}$} & \multicolumn{2}{|c|}{$\begin{array}{c}\text { Aboriginal } \\
\text { Identity }\end{array}$} & \multicolumn{2}{|c|}{ Métis } & \multicolumn{2}{|c|}{ North American Indian } \\
\hline & Men & Women & Men & Women & Men & Women & Men & Women \\
\hline Less than h-school & $\begin{array}{c}0.12 \\
(0.33)\end{array}$ & $\begin{array}{c}0.08 \\
(0.27)\end{array}$ & $\begin{array}{c}0.22 \\
(0.41)\end{array}$ & $\begin{array}{c}0.14 \\
(0.35)\end{array}$ & $\begin{array}{c}0.21 \\
(0.41)\end{array}$ & $\begin{array}{c}0.14 \\
(0.35)\end{array}$ & $\begin{array}{c}0.23 \\
(0.42)\end{array}$ & $\begin{array}{c}0.15 \\
(0.35)\end{array}$ \\
\hline High school & $\begin{array}{c}0.21 \\
(0.41)\end{array}$ & $\begin{array}{c}0.20 \\
(0.40)\end{array}$ & $\begin{array}{c}0.24 \\
(0.43)\end{array}$ & $\begin{array}{c}0.22 \\
(0.41)\end{array}$ & $\begin{array}{c}0.23 \\
(0.42)\end{array}$ & $\begin{array}{c}0.23 \\
(0.42)\end{array}$ & $\begin{array}{c}0.25 \\
(0.43)\end{array}$ & $\begin{array}{c}0.20 \\
(0.40)\end{array}$ \\
\hline Incomplete pse & $\begin{array}{c}0.08 \\
(0.27)\end{array}$ & $\begin{array}{c}0.07 \\
(0.26)\end{array}$ & $\begin{array}{c}0.09 \\
(0.29)\end{array}$ & $\begin{array}{c}0.10 \\
(0.30)\end{array}$ & $\begin{array}{c}0.09 \\
(0.29)\end{array}$ & $\begin{array}{c}0.10 \\
(0.30)\end{array}$ & $\begin{array}{c}0.09 \\
(0.29)\end{array}$ & $\begin{array}{c}0.10 \\
(0.30)\end{array}$ \\
\hline Completed trade & $\begin{array}{c}0.17 \\
(0.38)\end{array}$ & $\begin{array}{c}0.09 \\
(0.28)\end{array}$ & $\begin{array}{c}0.18 \\
(0.39)\end{array}$ & $\begin{array}{c}0.11 \\
(0.31)\end{array}$ & $\begin{array}{c}0.20 \\
(0.40)\end{array}$ & $\begin{array}{c}0.11 \\
(0.31)\end{array}$ & $\begin{array}{l}0.10 \\
(0.36)\end{array}$ & $\begin{array}{c}0.10 \\
(0.31)\end{array}$ \\
\hline Completed college & $\begin{array}{c}0.21 \\
(0.41)\end{array}$ & $\begin{array}{c}0.30 \\
(0.46)\end{array}$ & $\begin{array}{c}0.17 \\
(0.38)\end{array}$ & $\begin{array}{c}0.29 \\
(0.46)\end{array}$ & $\begin{array}{c}0.15 \\
(0.36)\end{array}$ & $\begin{array}{c}0.29 \\
(0.45)\end{array}$ & $\begin{array}{c}0.19 \\
(0.39)\end{array}$ & $\begin{array}{c}0.30 \\
(0.46)\end{array}$ \\
\hline University degree & $\begin{array}{c}0.19 \\
(0.39)\end{array}$ & $\begin{array}{c}0.25 \\
(0.43)\end{array}$ & $\begin{array}{c}0.09 \\
(0.28)\end{array}$ & $\begin{array}{c}0.13 \\
(0.34)\end{array}$ & $\begin{array}{c}0.10 \\
(0.30)\end{array}$ & $\begin{array}{c}0.13 \\
(0.34)\end{array}$ & $\begin{array}{c}0.08 \\
(0.26)\end{array}$ & $\begin{array}{c}0.14 \\
(0.35)\end{array}$ \\
\hline Tenure $<2$ years & $\begin{array}{c}0.29 \\
(0.45)\end{array}$ & $\begin{array}{c}0.27 \\
(0.44)\end{array}$ & $\begin{array}{c}0.36 \\
(0.48)\end{array}$ & $\begin{array}{c}0.37 \\
(0.48)\end{array}$ & $\begin{array}{c}0.36 \\
(0.48)\end{array}$ & $\begin{array}{c}0.37 \\
(0.48)\end{array}$ & $\begin{array}{c}0.36 \\
(0.48)\end{array}$ & $\begin{array}{c}0.37 \\
(0.48)\end{array}$ \\
\hline Tenure $2-5$ years & $\begin{array}{c}0.20 \\
(0.40)\end{array}$ & $\begin{array}{c}0.21 \\
(0.41)\end{array}$ & $\begin{array}{c}0.22 \\
(0.41)\end{array}$ & $\begin{array}{c}0.24 \\
(0.43)\end{array}$ & $\begin{array}{c}0.21 \\
(0.41)\end{array}$ & $\begin{array}{c}0.25 \\
(0.44)\end{array}$ & $\begin{array}{c}0.23 \\
(0.42)\end{array}$ & $\begin{array}{c}0.23 \\
(0.42)\end{array}$ \\
\hline Tenure $5-10$ years & $\begin{array}{c}0.18 \\
(0.38)\end{array}$ & $\begin{array}{c}0.19 \\
(0.39)\end{array}$ & $\begin{array}{c}0.16 \\
(0.37)\end{array}$ & $\begin{array}{c}0.16 \\
(0.37)\end{array}$ & $\begin{array}{c}0.16 \\
(0.37)\end{array}$ & $\begin{array}{c}0.16 \\
(0.36)\end{array}$ & $\begin{array}{c}0.16 \\
(0.37)\end{array}$ & $\begin{array}{c}0.18 \\
(0.38)\end{array}$ \\
\hline Tenure $10-20$ years & $\begin{array}{c}0.17 \\
(0.38)\end{array}$ & $\begin{array}{c}0.18 \\
(0.38)\end{array}$ & $\begin{array}{c}0.14 \\
(0.35)\end{array}$ & $\begin{array}{c}0.15 \\
(0.35)\end{array}$ & $\begin{array}{c}0.15 \\
(0.35)\end{array}$ & $\begin{array}{c}0.15 \\
(0.35)\end{array}$ & $\begin{array}{c}0.14 \\
(0.34)\end{array}$ & $\begin{array}{c}0.15 \\
(0.36)\end{array}$ \\
\hline Tenure $>20$ years & $\begin{array}{c}0.16 \\
(0.36)\end{array}$ & $\begin{array}{c}0.14 \\
(0.35)\end{array}$ & $\begin{array}{c}0.11 \\
(0.32)\end{array}$ & $\begin{array}{c}0.07 \\
(0.26)\end{array}$ & $\begin{array}{c}0.12 \\
(0.32)\end{array}$ & $\begin{array}{c}0.07 \\
(0.26)\end{array}$ & $\begin{array}{c}0.11 \\
(0.31)\end{array}$ & $\begin{array}{c}0.08 \\
(0.27)\end{array}$ \\
\hline Absent & $\begin{array}{c}0.06 \\
(0.23)\end{array}$ & $\begin{array}{c}0.10 \\
(0.30)\end{array}$ & $\begin{array}{c}0.07 \\
(0.25)\end{array}$ & $\begin{array}{c}0.09 \\
(0.28)\end{array}$ & $\begin{array}{c}0.06 \\
(0.25)\end{array}$ & $\begin{array}{c}0.09 \\
(0.28)\end{array}$ & $\begin{array}{c}0.07 \\
(0.25)\end{array}$ & $\begin{array}{c}0.08 \\
(0.28)\end{array}$ \\
\hline Absent 1 week & $\begin{array}{c}0.02 \\
(0.16)\end{array}$ & $\begin{array}{c}0.03 \\
(0.17)\end{array}$ & $\begin{array}{c}0.03 \\
(0.16)\end{array}$ & $\begin{array}{c}0.03 \\
(0.17)\end{array}$ & $\begin{array}{c}0.02 \\
(0.16)\end{array}$ & $\begin{array}{c}0.03 \\
(0.17)\end{array}$ & $\begin{array}{c}0.03 \\
(0.16)\end{array}$ & $\begin{array}{c}0.03 \\
(0.16)\end{array}$ \\
\hline Absent $>8$ weeks & $\begin{array}{c}0.01 \\
(0.10)\end{array}$ & $\begin{array}{c}0.04 \\
(0.19)\end{array}$ & $\begin{array}{c}0.01 \\
(0.12)\end{array}$ & $\begin{array}{c}0.03 \\
(0.18)\end{array}$ & $\begin{array}{c}0.01 \\
(0.11)\end{array}$ & $\begin{array}{c}0.02 \\
(0.18)\end{array}$ & $\begin{array}{c}0.02 \\
(0.13)\end{array}$ & $\begin{array}{c}0.03 \\
(0.17)\end{array}$ \\
\hline Youth $15-24$ & $\begin{array}{c}0.14 \\
(0.35)\end{array}$ & $\begin{array}{c}0.12 \\
(0.32)\end{array}$ & $\begin{array}{c}0.18 \\
(0.38)\end{array}$ & $\begin{array}{c}0.15 \\
(0.36)\end{array}$ & $\begin{array}{c}0.17 \\
(0.37)\end{array}$ & $\begin{array}{c}0.16 \\
(0.36)\end{array}$ & $\begin{array}{c}0.18 \\
(0.39)\end{array}$ & $\begin{array}{c}0.14 \\
(0.34)\end{array}$ \\
\hline Young 25-34 & $\begin{array}{c}0.23 \\
(0.42)\end{array}$ & $\begin{array}{c}0.23 \\
(0.42)\end{array}$ & $\begin{array}{c}0.25 \\
(0.43)\end{array}$ & $\begin{array}{c}0.24 \\
(0.42)\end{array}$ & $\begin{array}{c}0.25 \\
(0.43)\end{array}$ & $\begin{array}{c}0.25 \\
(0.43)\end{array}$ & $\begin{array}{c}0.25 \\
(0.43)\end{array}$ & $\begin{array}{c}0.22 \\
(0.41)\end{array}$ \\
\hline Young prime $35-44$ & $\begin{array}{c}0.22 \\
(0.42)\end{array}$ & $\begin{array}{c}0.22 \\
(0.42)\end{array}$ & $\begin{array}{c}0.23 \\
(0.42)\end{array}$ & $\begin{array}{c}0.26 \\
(0.44)\end{array}$ & $\begin{array}{c}0.23 \\
(0.42)\end{array}$ & $\begin{array}{c}0.24 \\
(0.43)\end{array}$ & $\begin{array}{c}0.24 \\
(0.43)\end{array}$ & $\begin{array}{c}0.28 \\
(0.45)\end{array}$ \\
\hline Mature prime $45-54$ & $\begin{array}{c}0.26 \\
(0.44)\end{array}$ & $\begin{array}{c}0.28 \\
(0.45)\end{array}$ & $\begin{array}{c}0.22 \\
(0.42)\end{array}$ & $\begin{array}{c}0.23 \\
(0.42)\end{array}$ & $\begin{array}{c}0.24 \\
(0.43)\end{array}$ & $\begin{array}{c}0.23 \\
(0.42)\end{array}$ & $\begin{array}{c}0.21 \\
(0.41)\end{array}$ & $\begin{array}{c}0.25 \\
(0.43)\end{array}$ \\
\hline Older worker 55-64 & $\begin{array}{c}0.14 \\
(0.35)\end{array}$ & $\begin{array}{c}0.15 \\
(0.36)\end{array}$ & $\begin{array}{c}0.11 \\
(0.31)\end{array}$ & $\begin{array}{c}0.12 \\
(0.33)\end{array}$ & $\begin{array}{c}0.11 \\
(0.31)\end{array}$ & $\begin{array}{c}0.12 \\
(0.33)\end{array}$ & $\begin{array}{c}0.11 \\
(0.31)\end{array}$ & $\begin{array}{c}0.12 \\
(0.32)\end{array}$ \\
\hline Married & $\begin{array}{c}0.64 \\
(0.48)\end{array}$ & $\begin{array}{c}0.66 \\
(0.47)\end{array}$ & $\begin{array}{c}0.59 \\
(0.49)\end{array}$ & $\begin{array}{c}0.60 \\
(0.49)\end{array}$ & $\begin{array}{c}0.61 \\
(0.49)\end{array}$ & $\begin{array}{c}0.62 \\
(0.48)\end{array}$ & $\begin{array}{c}0.55 \\
(0.50)\end{array}$ & $\begin{array}{c}0.58 \\
(0.49)\end{array}$ \\
\hline Widowed div/sep & $\begin{array}{c}0.06 \\
(0.24)\end{array}$ & $\begin{array}{c}0.11 \\
(0.31)\end{array}$ & $\begin{array}{c}0.08 \\
(0.27)\end{array}$ & $\begin{array}{c}0.12 \\
(033)\end{array}$ & $\begin{array}{c}0.07 \\
(0.26)\end{array}$ & $\begin{array}{c}0.12 \\
(0.33)\end{array}$ & $\begin{array}{c}0.09 \\
(0.29)\end{array}$ & $\begin{array}{c}0.12 \\
(0.33)\end{array}$ \\
\hline
\end{tabular}




\begin{tabular}{|c|c|c|c|c|c|c|c|c|}
\hline \multirow{2}{*}{$\begin{array}{l}\text { Appendix A2 } \\
\text { (continued) }\end{array}$} & \multicolumn{2}{|c|}{$\begin{array}{c}\text { Non-Aboriginal } \\
\text { Identity }\end{array}$} & \multicolumn{2}{|c|}{$\begin{array}{l}\text { Aboriginal } \\
\text { Identity }\end{array}$} & \multicolumn{2}{|c|}{ Métis } & \multicolumn{2}{|c|}{ North American Indian } \\
\hline & Men & Women & Men & Women & Men & Women & Men & Women \\
\hline Single & $\begin{array}{c}0.30 \\
(0.46)\end{array}$ & $\begin{array}{c}0.23 \\
(0.42)\end{array}$ & $\begin{array}{c}0.33 \\
(0.47)\end{array}$ & $\begin{array}{c}0.27 \\
(0.45)\end{array}$ & $\begin{array}{c}0.31 \\
(0.46)\end{array}$ & $\begin{array}{c}0.25 \\
(0.43)\end{array}$ & $\begin{array}{c}0.35 \\
(0.48)\end{array}$ & $\begin{array}{c}0.29 \\
(0.45)\end{array}$ \\
\hline No children present & $\begin{array}{c}0.62 \\
(0.49)\end{array}$ & $\begin{array}{c}0.56 \\
(0.49)\end{array}$ & $\begin{array}{c}0.63 \\
(0.48)\end{array}$ & $\begin{array}{c}0.53 \\
(0.50)\end{array}$ & $\begin{array}{c}0.62 \\
(0.49)\end{array}$ & $\begin{array}{c}0.54 \\
(0.50)\end{array}$ & $\begin{array}{c}0.64 \\
(0.48)\end{array}$ & $\begin{array}{c}0.51 \\
(0.50)\end{array}$ \\
\hline Children $<6$ & $\begin{array}{c}0.14 \\
(0.34)\end{array}$ & $\begin{array}{c}0.14 \\
(0.34)\end{array}$ & $\begin{array}{c}0.15 \\
(0.36)\end{array}$ & $\begin{array}{c}0.15 \\
(0.36)\end{array}$ & $\begin{array}{c}0.16 \\
(0.37)\end{array}$ & $\begin{array}{c}0.15 \\
(0.36)\end{array}$ & $\begin{array}{c}0.13 \\
(0.34)\end{array}$ & $\begin{array}{c}0.15 \\
(0.36)\end{array}$ \\
\hline Children 6-14 & $\begin{array}{c}0.13 \\
(0.34)\end{array}$ & $\begin{array}{c}0.16 \\
(0.36)\end{array}$ & $\begin{array}{c}0.13 \\
(0.34)\end{array}$ & $\begin{array}{c}0.20 \\
(0.40)\end{array}$ & $\begin{array}{c}0.12 \\
(0.33)\end{array}$ & $\begin{array}{c}0.19 \\
(0.39)\end{array}$ & $\begin{array}{c}0.14 \\
(0.34)\end{array}$ & $\begin{array}{c}0.21 \\
(0.40)\end{array}$ \\
\hline Children 14-24 & $\begin{array}{c}0.11 \\
(0.31)\end{array}$ & $\begin{array}{c}0.14 \\
(0.34)\end{array}$ & $\begin{array}{c}0.09 \\
(0.28)\end{array}$ & $\begin{array}{c}0.12 \\
(0.33)\end{array}$ & $\begin{array}{c}0.10 \\
(0.30)\end{array}$ & $\begin{array}{c}0.11 \\
(0.32)\end{array}$ & $\begin{array}{c}0.08 \\
(0.29)\end{array}$ & $\begin{array}{c}0.13 \\
(0.34)\end{array}$ \\
\hline Atlantic provinces & $\begin{array}{c}0.09 \\
(0.29)\end{array}$ & $\begin{array}{c}0.10 \\
(0.30)\end{array}$ & $\begin{array}{l}0.08 \\
(0.28)\end{array}$ & $\begin{array}{c}0.08 \\
(0.27)\end{array}$ & $\begin{array}{l}0.06 \\
(0.24)\end{array}$ & $\begin{array}{c}0.06 \\
(0.23)\end{array}$ & $\begin{array}{c}0.09 \\
(0.29)\end{array}$ & $\begin{array}{c}0.09 \\
(0.28)\end{array}$ \\
\hline Quebec & $\begin{array}{c}0.29 \\
(0.45)\end{array}$ & $\begin{array}{c}0.29 \\
(0.45)\end{array}$ & $\begin{array}{c}0.08 \\
(0.28)\end{array}$ & $\begin{array}{c}0.09 \\
(0.29)\end{array}$ & $\begin{array}{c}0.10 \\
(0.30)\end{array}$ & $\begin{array}{c}0.09 \\
(0.29)\end{array}$ & $\begin{array}{c}0.07 \\
(0.26)\end{array}$ & $\begin{array}{c}0.09 \\
(0.28)\end{array}$ \\
\hline Ontario & $\begin{array}{c}0.34 \\
(0.47)\end{array}$ & $\begin{array}{c}0.34 \\
(0.47)\end{array}$ & $\begin{array}{c}0.24 \\
(0.43)\end{array}$ & $\begin{array}{c}0.23 \\
(0.42)\end{array}$ & $\begin{array}{c}0.18 \\
(0.39)\end{array}$ & $\begin{array}{c}0.18 \\
(0.38)\end{array}$ & $\begin{array}{c}0.31 \\
(0.46)\end{array}$ & $\begin{array}{c}0.29 \\
(0.45)\end{array}$ \\
\hline Manitoba & $\begin{array}{c}0.03 \\
(0.17)\end{array}$ & $\begin{array}{c}0.03 \\
(0.17)\end{array}$ & $\begin{array}{c}0.14 \\
(0.35)\end{array}$ & $\begin{array}{c}0.15 \\
(0.36)\end{array}$ & $\begin{array}{c}0.18 \\
(0.39)\end{array}$ & $\begin{array}{c}0.19 \\
(0.39)\end{array}$ & $\begin{array}{c}0.10 \\
(0.30)\end{array}$ & $\begin{array}{c}0.11 \\
(031)\end{array}$ \\
\hline Saskatchewan & $\begin{array}{c}0.03 \\
(0.17)\end{array}$ & $\begin{array}{c}0.03 \\
(0.17)\end{array}$ & $\begin{array}{c}0.09 \\
(0.29)\end{array}$ & $\begin{array}{c}0.10 \\
(0.30)\end{array}$ & $\begin{array}{c}0.11 \\
(0.32)\end{array}$ & $\begin{array}{c}0.12 \\
(0.32)\end{array}$ & $\begin{array}{c}0.07 \\
(0.26)\end{array}$ & $\begin{array}{c}0.09 \\
(0.28)\end{array}$ \\
\hline Alberta & $\begin{array}{c}0.11 \\
(0.31)\end{array}$ & $\begin{array}{c}0.10 \\
(0.30)\end{array}$ & $\begin{array}{c}0.20 \\
(0.40)\end{array}$ & $\begin{array}{c}0.18 \\
(0.39)\end{array}$ & $\begin{array}{c}0.24 \\
(0.42)\end{array}$ & $\begin{array}{c}0.23 \\
(0.42)\end{array}$ & $\begin{array}{c}0.16 \\
(0.37)\end{array}$ & $\begin{array}{c}0.13 \\
(0.34)\end{array}$ \\
\hline British Columbia & $\begin{array}{c}0.11 \\
(0.31)\end{array}$ & $\begin{array}{c}0.11 \\
(0.31)\end{array}$ & $\begin{array}{c}0.15 \\
(0.36)\end{array}$ & $\begin{array}{c}0.16 \\
(0.37)\end{array}$ & $\begin{array}{c}0.12 \\
(0.32)\end{array}$ & $\begin{array}{c}0.12 \\
(0.33)\end{array}$ & $\begin{array}{c}0.19 \\
(0.39)\end{array}$ & $\begin{array}{c}0.20 \\
(0.40)\end{array}$ \\
\hline Primary industries & $\begin{array}{c}0.19 \\
(0.39)\end{array}$ & $\begin{array}{c}0.04 \\
(0.19)\end{array}$ & $\begin{array}{c}0.28 \\
(0.45)\end{array}$ & $\begin{array}{c}0.04 \\
(0.20)\end{array}$ & $\begin{array}{c}0.28 \\
(0.45)\end{array}$ & $\begin{array}{c}0.05 \\
(0.22)\end{array}$ & $\begin{array}{c}0.27 \\
(0.44)\end{array}$ & $\begin{array}{c}0.03 \\
(0.18)\end{array}$ \\
\hline Manufacturing & $\begin{array}{c}0.17 \\
(0.38)\end{array}$ & $\begin{array}{c}0.06 \\
(0.25)\end{array}$ & $\begin{array}{c}0.12 \\
(0.33)\end{array}$ & $\begin{array}{c}0.04 \\
(0.19)\end{array}$ & $\begin{array}{c}0.12 \\
(0.32)\end{array}$ & $\begin{array}{c}0.03 \\
(0.18)\end{array}$ & $\begin{array}{c}0.13 \\
(0.33)\end{array}$ & $\begin{array}{c}0.04 \\
(0.19)\end{array}$ \\
\hline Retail & $\begin{array}{c}0.17 \\
(0.38)\end{array}$ & $\begin{array}{c}0.23 \\
(0.42)\end{array}$ & $\begin{array}{c}0.17 \\
(0.38)\end{array}$ & $\begin{array}{c}0.29 \\
(0.45)\end{array}$ & $\begin{array}{c}0.17 \\
(0.38)\end{array}$ & $\begin{array}{c}0.29 \\
(0.45)\end{array}$ & $\begin{array}{c}0.17 \\
(0.38)\end{array}$ & $\begin{array}{c}0.29 \\
(0.45)\end{array}$ \\
\hline High skill services & $\begin{array}{c}0.13 \\
(0.34)\end{array}$ & $\begin{array}{c}0.16 \\
(0.37)\end{array}$ & $\begin{array}{c}0.10 \\
(0.30)\end{array}$ & $\begin{array}{c}0.11 \\
(0.32)\end{array}$ & $\begin{array}{c}0.10 \\
(0.31)\end{array}$ & $\begin{array}{c}0.11 \\
(0.31)\end{array}$ & $\begin{array}{c}0.10 \\
(0.30)\end{array}$ & $\begin{array}{c}0.12 \\
(0.33)\end{array}$ \\
\hline Public services & $\begin{array}{c}0.16 \\
(0.36)\end{array}$ & $\begin{array}{c}0.41 \\
(0.49)\end{array}$ & $\begin{array}{c}0.17 \\
(0.37)\end{array}$ & $\begin{array}{c}0.42 \\
(0.49)\end{array}$ & $\begin{array}{c}0.17 \\
(0.38)\end{array}$ & $\begin{array}{c}0.42 \\
(0.49)\end{array}$ & $\begin{array}{c}0.17 \\
(0.37)\end{array}$ & $\begin{array}{c}0.43 \\
(0.49)\end{array}$ \\
\hline Other services & $\begin{array}{c}0.17 \\
(0.38)\end{array}$ & $\begin{array}{c}0.09 \\
(0.29)\end{array}$ & $\begin{array}{c}0.15 \\
(0.36)\end{array}$ & $\begin{array}{c}0.09 \\
(0.29)\end{array}$ & $\begin{array}{c}0.15 \\
(0.36)\end{array}$ & $\begin{array}{c}0.09 \\
(0.29)\end{array}$ & $\begin{array}{c}0.15 \\
(0.36)\end{array}$ & $\begin{array}{c}0.08 \\
(0.28)\end{array}$ \\
\hline Management occup & $\begin{array}{c}0.09 \\
(0.28)\end{array}$ & $\begin{array}{c}0.06 \\
(0.25)\end{array}$ & $\begin{array}{c}0.05 \\
(0.22)\end{array}$ & $\begin{array}{c}0.05 \\
(0.22)\end{array}$ & $\begin{array}{c}0.06 \\
(0.24)\end{array}$ & $\begin{array}{c}0.05 \\
(0.22)\end{array}$ & $\begin{array}{c}0.05 \\
(0.21)\end{array}$ & $\begin{array}{c}0.05 \\
(0.22)\end{array}$ \\
\hline Professionals & $\begin{array}{c}0.13 \\
(0.34)\end{array}$ & $\begin{array}{c}0.20 \\
(0.40)\end{array}$ & $\begin{array}{c}0.07 \\
(0.26)\end{array}$ & $\begin{array}{c}0.14 \\
(0.34)\end{array}$ & $\begin{array}{c}0.08 \\
(0.27)\end{array}$ & $\begin{array}{c}0.13 \\
(0.34)\end{array}$ & $\begin{array}{c}0.07 \\
(0.26)\end{array}$ & $\begin{array}{c}0.14 \\
(0.35)\end{array}$ \\
\hline Semi-professionals & $\begin{array}{c}0.08 \\
(0.27)\end{array}$ & $\begin{array}{c}0.10 \\
(0.30)\end{array}$ & $\begin{array}{c}0.07 \\
(0.26)\end{array}$ & $\begin{array}{c}0.10 \\
(0.30)\end{array}$ & $\begin{array}{c}0.07 \\
(0.26)\end{array}$ & $\begin{array}{c}0.10 \\
(0.30)\end{array}$ & $\begin{array}{c}0.07 \\
(0.26)\end{array}$ & $\begin{array}{c}0.11 \\
(0.31)\end{array}$ \\
\hline Sales personnel & $\begin{array}{c}0.27 \\
(0.44)\end{array}$ & $\begin{array}{c}0.56 \\
(0.50)\end{array}$ & $\begin{array}{c}0.27 \\
(0.45)\end{array}$ & $\begin{array}{c}0.62 \\
(0.48)\end{array}$ & $\begin{array}{c}0.27 \\
(0.45)\end{array}$ & $\begin{array}{c}0.63 \\
(0.48)\end{array}$ & $\begin{array}{c}0.27 \\
(0.44)\end{array}$ & $\begin{array}{c}0.62 \\
(0.48)\end{array}$ \\
\hline
\end{tabular}




\begin{tabular}{|c|c|c|c|c|c|c|c|c|}
\hline \multirow{2}{*}{$\begin{array}{l}\text { Appendix A2 } \\
\text { (continued) }\end{array}$} & \multicolumn{2}{|c|}{$\begin{array}{c}\text { Non-Aboriginal } \\
\text { Identity }\end{array}$} & \multicolumn{2}{|c|}{$\begin{array}{l}\text { Aboriginal } \\
\text { Identity }\end{array}$} & \multicolumn{2}{|c|}{ Métis } & \multicolumn{2}{|c|}{ North American Indian } \\
\hline & Men & Women & Men & Women & Men & Women & Men & Women \\
\hline Manual workers & $\begin{array}{c}0.42 \\
(0.49)\end{array}$ & $\begin{array}{c}0.05 \\
(0.23)\end{array}$ & $\begin{array}{c}0.51 \\
(0.50)\end{array}$ & $\begin{array}{c}0.06 \\
(0.24)\end{array}$ & $\begin{array}{c}0.50 \\
(0.50)\end{array}$ & $\begin{array}{c}0.07 \\
(0.26)\end{array}$ & $\begin{array}{c}0.53 \\
(0.50)\end{array}$ & $\begin{array}{c}0.06 \\
(0.23)\end{array}$ \\
\hline Union & $\begin{array}{c}0.34 \\
(0.47)\end{array}$ & $\begin{array}{c}0.37 \\
(0.48)\end{array}$ & $\begin{array}{c}0.34 \\
(0.47)\end{array}$ & $\begin{array}{c}0.35 \\
(0.48)\end{array}$ & $\begin{array}{c}0.37 \\
(0.48)\end{array}$ & $\begin{array}{c}0.37 \\
(0.48)\end{array}$ & $\begin{array}{c}0.31 \\
(0.46)\end{array}$ & $\begin{array}{c}0.34 \\
(0.47)\end{array}$ \\
\hline Small workplace & $\begin{array}{c}0.65 \\
(0.48)\end{array}$ & $\begin{array}{c}0.66 \\
(0.47)\end{array}$ & $\begin{array}{c}0.69 \\
(0.46)\end{array}$ & $\begin{array}{c}0.72 \\
(0.45)\end{array}$ & $\begin{array}{c}0.68 \\
(0.47)\end{array}$ & $\begin{array}{c}0.73 \\
(0.44)\end{array}$ & $\begin{array}{c}0.71 \\
(0.45)\end{array}$ & $\begin{array}{c}0.71 \\
(0.45)\end{array}$ \\
\hline $\begin{array}{l}\text { Medium } \\
\text { workplace }\end{array}$ & $\begin{array}{c}0.22 \\
(0.42)\end{array}$ & $\begin{array}{c}0.20 \\
(0.40)\end{array}$ & $\begin{array}{c}0.21 \\
(0.40)\end{array}$ & $\begin{array}{c}0.19 \\
(0.39)\end{array}$ & $\begin{array}{c}0.21 \\
(0.41)\end{array}$ & $\begin{array}{c}0.19 \\
(0.39)\end{array}$ & $\begin{array}{c}0.20 \\
(0.40)\end{array}$ & $\begin{array}{c}0.19 \\
(0.39)\end{array}$ \\
\hline Large workplace & $\begin{array}{c}0.13 \\
(0.33)\end{array}$ & $\begin{array}{c}0.14 \\
(0.35)\end{array}$ & $\begin{array}{c}0.10 \\
(0.30)\end{array}$ & $\begin{array}{c}0.08 \\
(0.28)\end{array}$ & $\begin{array}{c}0.11 \\
(0.31)\end{array}$ & $\begin{array}{c}0.08 \\
(0.27)\end{array}$ & $\begin{array}{c}0.09 \\
(0.28)\end{array}$ & $\begin{array}{c}0.09 \\
(0.29)\end{array}$ \\
\hline Private sector & $\begin{array}{c}0.81 \\
(0.39)\end{array}$ & $\begin{array}{c}0.66 \\
(0.47)\end{array}$ & $\begin{array}{c}0.79 \\
(0.40)\end{array}$ & $\begin{array}{c}0.66 \\
(0.47)\end{array}$ & $\begin{array}{c}0.78 \\
(0.41)\end{array}$ & $\begin{array}{c}0.67 \\
(0.47)\end{array}$ & $\begin{array}{c}0.80 \\
(0.40)\end{array}$ & $\begin{array}{c}0.65 \\
(0.48)\end{array}$ \\
\hline Permanent job & $\begin{array}{c}0.90 \\
(0.30)\end{array}$ & $\begin{array}{c}0.89 \\
(0.31)\end{array}$ & $\begin{array}{c}0.86 \\
(0.34)\end{array}$ & $\begin{array}{c}0.88 \\
(0.33)\end{array}$ & $\begin{array}{c}0.87 \\
(0.33)\end{array}$ & $\begin{array}{c}0.88 \\
(0.33)\end{array}$ & $\begin{array}{c}0.85 \\
(0.35)\end{array}$ & $\begin{array}{c}0.88 \\
(0.32)\end{array}$ \\
\hline Seasonal job & $\begin{array}{c}0.04 \\
(0.19)\end{array}$ & $\begin{array}{c}0.02 \\
(0.14)\end{array}$ & $\begin{array}{c}0.06 \\
(0.23)\end{array}$ & $\begin{array}{c}0.02 \\
(0.15)\end{array}$ & $\begin{array}{c}0.05 \\
(0.23)\end{array}$ & $\begin{array}{c}0.02 \\
(0.14)\end{array}$ & $\begin{array}{c}0.07 \\
(0.25)\end{array}$ & $\begin{array}{c}0.02 \\
(0.14)\end{array}$ \\
\hline $\begin{array}{l}\text { Contract/casual } \\
\text { job }\end{array}$ & $\begin{array}{c}0.06 \\
(0.24)\end{array}$ & $\begin{array}{c}0.08 \\
(0.28)\end{array}$ & $\begin{array}{c}0.08 \\
(0.27)\end{array}$ & $\begin{array}{c}0.10 \\
(0.28)\end{array}$ & $\begin{array}{c}0.07 \\
(0.26)\end{array}$ & $\begin{array}{c}0.10 \\
(0.30)\end{array}$ & $\begin{array}{c}0.08 \\
(0.27)\end{array}$ & $\begin{array}{c}0.09 \\
(0.29)\end{array}$ \\
\hline Multiple jobs & $\begin{array}{c}0.05 \\
(0.22)\end{array}$ & $\begin{array}{c}0.07 \\
(0.26)\end{array}$ & $\begin{array}{c}0.06 \\
(0.24)\end{array}$ & $\begin{array}{c}0.09 \\
(0.28)\end{array}$ & $\begin{array}{c}0.06 \\
(0.24)\end{array}$ & $\begin{array}{c}0.10 \\
(0.30)\end{array}$ & $\begin{array}{c}0.06 \\
(0.23)\end{array}$ & $\begin{array}{c}0.08 \\
(0.28)\end{array}$ \\
\hline Full-time job & $\begin{array}{c}0.93 \\
(0.25)\end{array}$ & $\begin{array}{c}0.79 \\
(0.41)\end{array}$ & $\begin{array}{c}0.93 \\
(0.26)\end{array}$ & $\begin{array}{c}0.80 \\
(0.40)\end{array}$ & $\begin{array}{c}0.93 \\
(0.26)\end{array}$ & $\begin{array}{c}0.80 \\
(0.40)\end{array}$ & $\begin{array}{c}0.93 \\
(0.39)\end{array}$ & $\begin{array}{c}0.80 \\
(0.40)\end{array}$ \\
\hline Part-time student & $\begin{array}{c}0.02 \\
(0.14)\end{array}$ & $\begin{array}{c}0.03 \\
(0.18)\end{array}$ & $\begin{array}{c}0.02 \\
(0.13)\end{array}$ & $\begin{array}{c}0.03 \\
(0.18)\end{array}$ & $\begin{array}{c}0.02 \\
(0.13)\end{array}$ & $\begin{array}{c}0.03 \\
(0.18)\end{array}$ & $\begin{array}{c}0.02 \\
(0.13)\end{array}$ & $\begin{array}{c}0.03 \\
(0.18)\end{array}$ \\
\hline Wage minimum & $\begin{array}{c}0.06 \\
(0.24)\end{array}$ & $\begin{array}{c}0.11 \\
(0.32)\end{array}$ & $\begin{array}{c}0.08 \\
(0.27)\end{array}$ & $\begin{array}{c}0.15 \\
(0.35)\end{array}$ & $\begin{array}{c}0.07 \\
(0.25)\end{array}$ & $\begin{array}{c}0.13 \\
(0.34)\end{array}$ & $\begin{array}{c}0.09 \\
(0.28)\end{array}$ & $\begin{array}{c}0.16 \\
(0.36)\end{array}$ \\
\hline Wage median & $\begin{array}{c}0.10 \\
(0.30)\end{array}$ & $\begin{array}{c}0.15 \\
(0.35)\end{array}$ & $\begin{array}{c}0.11 \\
(0.32)\end{array}$ & $\begin{array}{c}0.17 \\
(0.38)\end{array}$ & $\begin{array}{c}0.11 \\
(0.31)\end{array}$ & $\begin{array}{c}0.18 \\
(0.38)\end{array}$ & $\begin{array}{c}0.12 \\
(0.32)\end{array}$ & $\begin{array}{c}0.16 \\
(0.37)\end{array}$ \\
\hline Wage average & $\begin{array}{c}0.32 \\
(0.47)\end{array}$ & $\begin{array}{c}0.36 \\
(0.48)\end{array}$ & $\begin{array}{c}0.31 \\
(0.46)\end{array}$ & $\begin{array}{c}0.34 \\
(0.47)\end{array}$ & $\begin{array}{c}0.31 \\
(0.46)\end{array}$ & $\begin{array}{c}0.36 \\
(0.48)\end{array}$ & $\begin{array}{c}0.31 \\
(0.46)\end{array}$ & $\begin{array}{c}0.33 \\
(0.47)\end{array}$ \\
\hline Wage high & $\begin{array}{c}0.49 \\
(0.50)\end{array}$ & $\begin{array}{c}0.36 \\
(0.48)\end{array}$ & $\begin{array}{c}0.46 \\
(0.50)\end{array}$ & $\begin{array}{c}0.32 \\
(0.47)\end{array}$ & $\begin{array}{c}0.48 \\
(0.50)\end{array}$ & $\begin{array}{c}0.31 \\
(0.46)\end{array}$ & $\begin{array}{c}0.45 \\
(0.50)\end{array}$ & $\begin{array}{c}0.33 \\
(0.47)\end{array}$ \\
\hline Sample Size & 82587 & 83724 & 3179 & 3542 & 1727 & 1754 & 1404 & 1733 \\
\hline Weighted counts & 21571478 & 21676813 & 543838 & 570845 & 290371 & 288047 & 243761 & 274087 \\
\hline
\end{tabular}

Source: The Labour Force Survey, national monthly master file, September 2008 - May 2011.

Note: Main samples include all paid employees, aged between 15 and 64, who are not full-time students. Survey sampling weights (abowt) applied to account for complex survey design: stratification, clustering and non-response. The counts represent pooled populations for the recession time period. Standard deviations reported in parentheses. 
Appendix A 3: Mean Characteristics of Paid Employees in the Restricted Samples, by Identity

\begin{tabular}{|c|c|c|c|c|c|c|c|c|}
\hline \multirow[t]{2}{*}{ Characteristics } & \multicolumn{2}{|c|}{$\begin{array}{c}\text { Non-Aboriginal } \\
\text { Identity }\end{array}$} & \multicolumn{2}{|c|}{$\begin{array}{c}\text { Aboriginal } \\
\text { Identity }\end{array}$} & \multicolumn{2}{|c|}{ Métis } & \multicolumn{2}{|c|}{ North American Indian } \\
\hline & Men & Women & Men & Women & Men & Women & Men & Women \\
\hline Less than h-school & $\begin{array}{c}0.12 \\
(0.33)\end{array}$ & $\begin{array}{c}0.08 \\
(0.27)\end{array}$ & $\begin{array}{c}0.22 \\
(0.41)\end{array}$ & $\begin{array}{c}0.15 \\
(0.35)\end{array}$ & $\begin{array}{c}0.21 \\
(0.41)\end{array}$ & $\begin{array}{c}0.15 \\
(0.36)\end{array}$ & $\begin{array}{c}0.23 \\
(0.42)\end{array}$ & $\begin{array}{c}0.14 \\
(0.35)\end{array}$ \\
\hline High school & $\begin{array}{c}0.22 \\
(0.41)\end{array}$ & $\begin{array}{c}0.21 \\
(0.41)\end{array}$ & $\begin{array}{c}0.25 \\
(0.43)\end{array}$ & $\begin{array}{c}0.22 \\
(0.41)\end{array}$ & $\begin{array}{c}0.23 \\
(0.42)\end{array}$ & $\begin{array}{c}0.23 \\
(0.42)\end{array}$ & $\begin{array}{c}0.27 \\
(0.44)\end{array}$ & $\begin{array}{c}0.21 \\
(0.40)\end{array}$ \\
\hline Incomplete pse & $\begin{array}{l}0.07 \\
(0.26)\end{array}$ & $\begin{array}{c}0.07 \\
(0.25)\end{array}$ & $\begin{array}{c}0.08 \\
(0.28)\end{array}$ & $\begin{array}{c}0.10 \\
(0.29)\end{array}$ & $\begin{array}{c}0.09 \\
(0.29)\end{array}$ & $\begin{array}{c}0.09 \\
(0.29)\end{array}$ & $\begin{array}{c}0.08 \\
(0.28)\end{array}$ & $\begin{array}{c}0.10 \\
(0.30)\end{array}$ \\
\hline Completed trade & $\begin{array}{c}0.18 \\
(0.38)\end{array}$ & $\begin{array}{c}0.09 \\
(0.29)\end{array}$ & $\begin{array}{c}0.18 \\
(0.39)\end{array}$ & $\begin{array}{c}0.11 \\
(0.32)\end{array}$ & $\begin{array}{c}0.21 \\
(0.41\rangle\end{array}$ & $\begin{array}{c}0.11 \\
(0.32)\end{array}$ & $\begin{array}{c}0.15 \\
(0.35)\end{array}$ & $\begin{array}{c}0.11 \\
(0.31)\end{array}$ \\
\hline Completed college & $\begin{array}{c}0.22 \\
(0.41)\end{array}$ & $\begin{array}{c}0.30 \\
(0.46)\end{array}$ & $\begin{array}{c}0.17 \\
(0.38)\end{array}$ & $\begin{array}{c}0.30 \\
(0.46)\end{array}$ & $\begin{array}{c}0.15 \\
(0.36)\end{array}$ & $\begin{array}{c}0.28 \\
(0.45)\end{array}$ & $\begin{array}{c}0.19 \\
(0.40)\end{array}$ & $\begin{array}{c}0.31 \\
(0.46)\end{array}$ \\
\hline University degree & $\begin{array}{c}0.19 \\
(0.39)\end{array}$ & $\begin{array}{c}0.24 \\
(0.43)\end{array}$ & $\begin{array}{c}0.08 \\
(0.28)\end{array}$ & $\begin{array}{c}0.13 \\
(0.33)\end{array}$ & $\begin{array}{c}0.10 \\
(0.29)\end{array}$ & $\begin{array}{c}0.12 \\
(0.32)\end{array}$ & $\begin{array}{c}0.07 \\
(0.26)\end{array}$ & $\begin{array}{c}0.13 \\
(0.33)\end{array}$ \\
\hline Tenure $<2$ years & $\begin{array}{c}0.24 \\
(0.43)\end{array}$ & $\begin{array}{c}0.22 \\
(0.42)\end{array}$ & $\begin{array}{c}0.30 \\
(0.46)\end{array}$ & $\begin{array}{c}0.31 \\
(0.46)\end{array}$ & $\begin{array}{c}0.31 \\
(0.46)\end{array}$ & $\begin{array}{c}0.31 \\
(0.46)\end{array}$ & $\begin{array}{c}0.29 \\
(0.45)\end{array}$ & $\begin{array}{c}0.31 \\
(0.46)\end{array}$ \\
\hline Tenure $2-5$ years & $\begin{array}{c}0.21 \\
(0.40)\end{array}$ & $\begin{array}{c}0.21 \\
(0.41)\end{array}$ & $\begin{array}{c}0.23 \\
(0.42)\end{array}$ & $\begin{array}{c}0.25 \\
(0.43)\end{array}$ & $\begin{array}{c}0.22 \\
(0.41)\end{array}$ & $\begin{array}{c}0.26 \\
(0.44)\end{array}$ & $\begin{array}{c}0.25 \\
(0.43)\end{array}$ & $\begin{array}{c}0.24 \\
(0.42)\end{array}$ \\
\hline Tenure $5-10$ years & $\begin{array}{c}0.19 \\
(0.39)\end{array}$ & $\begin{array}{c}0.20 \\
(0.40)\end{array}$ & $\begin{array}{l}0.17 \\
(0.38)\end{array}$ & $\begin{array}{c}0.18 \\
(0.39)\end{array}$ & $\begin{array}{l}0.17 \\
(0.37)\end{array}$ & $\begin{array}{c}0.17 \\
(0.38)\end{array}$ & $\begin{array}{c}0.17 \\
(0.38)\end{array}$ & $\begin{array}{c}0.19 \\
(0.39)\end{array}$ \\
\hline Tenure $10-20$ years & $\begin{array}{c}0.19 \\
(0.39)\end{array}$ & $\begin{array}{c}0.20 \\
(0.40)\end{array}$ & $\begin{array}{c}0.16 \\
(0.38)\end{array}$ & $\begin{array}{c}0.16 \\
(0.37)\end{array}$ & $\begin{array}{l}0.16 \\
(0.37)\end{array}$ & $\begin{array}{c}0.17 \\
(0.37)\end{array}$ & $\begin{array}{c}0.15 \\
(0.36)\end{array}$ & $\begin{array}{c}0.16 \\
(0.37)\end{array}$ \\
\hline Tenure $>20$ years & $\begin{array}{c}0.17 \\
(0.38)\end{array}$ & $\begin{array}{c}0.16 \\
(0.37)\end{array}$ & $\begin{array}{c}0.13 \\
(0.34)\end{array}$ & $\begin{array}{c}0.09 \\
(0.28)\end{array}$ & $\begin{array}{c}0.14 \\
(0.35)\end{array}$ & $\begin{array}{c}0.08 \\
(0.27)\end{array}$ & $\begin{array}{c}0.13 \\
(0.33)\end{array}$ & $\begin{array}{c}0.09 \\
(0.29)\end{array}$ \\
\hline Absent & $\begin{array}{c}0.06 \\
(0.24)\end{array}$ & $\begin{array}{c}0.11 \\
(0.31)\end{array}$ & $\begin{array}{l}0.07 \\
(0.25)\end{array}$ & $\begin{array}{c}0.08 \\
(0.28)\end{array}$ & $\begin{array}{c}0.06 \\
(0.25)\end{array}$ & $\begin{array}{c}0.08 \\
(0.28)\end{array}$ & $\begin{array}{c}0.07 \\
(0.26)\end{array}$ & $\begin{array}{c}0.08 \\
(0.28)\end{array}$ \\
\hline Absent 1 week & $\begin{array}{c}0.03 \\
(0.16)\end{array}$ & $\begin{array}{c}0.03 \\
(0.17)\end{array}$ & $\begin{array}{c}0.03 \\
(0.16)\end{array}$ & $\begin{array}{c}0.03 \\
(0.16)\end{array}$ & $\begin{array}{c}0.03 \\
(0.16)\end{array}$ & $\begin{array}{c}0.02 \\
(0.16)\end{array}$ & $\begin{array}{c}0.03 \\
(0.16)\end{array}$ & $\begin{array}{c}0.02 \\
(0.16)\end{array}$ \\
\hline Absent $>8$ weeks & $\begin{array}{c}0.01 \\
(0.10)\end{array}$ & $\begin{array}{c}0.04 \\
(0.20)\end{array}$ & $\begin{array}{c}0.01 \\
(0.12)\end{array}$ & $\begin{array}{c}0.03 \\
(0.18)\end{array}$ & $\begin{array}{c}0.01 \\
(0.10)\end{array}$ & $\begin{array}{c}0.04 \\
(0.19)\end{array}$ & $\begin{array}{c}0.02 \\
(0.13)\end{array}$ & $\begin{array}{c}0.03 \\
(0.18)\end{array}$ \\
\hline Youth 15-24 & $\begin{array}{c}0.12 \\
(0.32)\end{array}$ & $\begin{array}{c}0.09 \\
(0.29)\end{array}$ & $\begin{array}{c}0.16 \\
(0.37)\end{array}$ & $\begin{array}{c}0.13 \\
(0.33)\end{array}$ & $\begin{array}{c}0.14 \\
(0.35)\end{array}$ & $\begin{array}{c}0.13 \\
(0.34)\end{array}$ & $\begin{array}{c}0.18 \\
(0.39)\end{array}$ & $\begin{array}{c}0.12 \\
(0.33)\end{array}$ \\
\hline Young 25-34 & $\begin{array}{c}0.23 \\
(0.42)\end{array}$ & $\begin{array}{c}0.22 \\
(0.41)\end{array}$ & $\begin{array}{c}0.25 \\
(0.43)\end{array}$ & $\begin{array}{c}0.23 \\
(0.42)\end{array}$ & $\begin{array}{c}0.25 \\
(0.43)\end{array}$ & $\begin{array}{c}0.25 \\
(0.43)\end{array}$ & $\begin{array}{c}0.25 \\
(0.43)\end{array}$ & $\begin{array}{c}0.21 \\
(0.41)\end{array}$ \\
\hline Young prime $35-44$ & $\begin{array}{c}0.23 \\
(0.42)\end{array}$ & $\begin{array}{c}0.23 \\
(0.42)\end{array}$ & $\begin{array}{c}0.24 \\
(0.42)\end{array}$ & $\begin{array}{c}0.26 \\
(0.44)\end{array}$ & $\begin{array}{c}0.23 \\
(0.42)\end{array}$ & $\begin{array}{c}0.25 \\
(0.43)\end{array}$ & $\begin{array}{c}0.24 \\
(0.43)\end{array}$ & $\begin{array}{c}0.28 \\
(0.45)\end{array}$ \\
\hline Mature prime 45-54 & $\begin{array}{c}0.27 \\
(0.44)\end{array}$ & $\begin{array}{c}0.29 \\
(0.45)\end{array}$ & $\begin{array}{c}0.23 \\
(0.42)\end{array}$ & $\begin{array}{c}0.25 \\
(0.43)\end{array}$ & $\begin{array}{c}0.25 \\
(0.43)\end{array}$ & $\begin{array}{c}0.24 \\
(0.43)\end{array}$ & $\begin{array}{c}0.22 \\
(0.42)\end{array}$ & $\begin{array}{c}0.25 \\
(0.44)\end{array}$ \\
\hline Older worker 55-64 & $\begin{array}{c}0.15 \\
(0.35)\end{array}$ & $\begin{array}{c}0.16 \\
(0.36)\end{array}$ & $\begin{array}{c}0.11 \\
(0.31)\end{array}$ & $\begin{array}{c}0.13 \\
(0.34)\end{array}$ & $\begin{array}{c}0.12 \\
(0.32)\end{array}$ & $\begin{array}{c}0.13 \\
(0.33)\end{array}$ & $\begin{array}{c}0.10 \\
(0.30)\end{array}$ & $\begin{array}{c}0.13 \\
(0.34)\end{array}$ \\
\hline Married & $\begin{array}{c}0.66 \\
(0.47)\end{array}$ & $\begin{array}{c}0.68 \\
(0.46)\end{array}$ & $\begin{array}{c}0.60 \\
(0.49)\end{array}$ & $\begin{array}{c}0.62 \\
(0.48)\end{array}$ & $\begin{array}{c}0.63 \\
(0.48)\end{array}$ & $\begin{array}{c}0.64 \\
(0.48)\end{array}$ & $\begin{array}{c}0.56 \\
(0.50)\end{array}$ & $\begin{array}{c}0.60 \\
(0.49)\end{array}$ \\
\hline
\end{tabular}




\begin{tabular}{|c|c|c|c|c|c|c|c|c|}
\hline \multirow{2}{*}{$\begin{array}{l}\text { Appendix A3 } \\
\text { (continued) }\end{array}$} & \multicolumn{2}{|c|}{$\begin{array}{c}\text { Non-Aboriginal } \\
\text { Identity }\end{array}$} & \multicolumn{2}{|c|}{$\begin{array}{l}\text { Aboriginal } \\
\text { Identity }\end{array}$} & \multicolumn{2}{|c|}{ Métis } & \multicolumn{2}{|c|}{ North American Indian } \\
\hline & Men & Women & Men & Women & Men & Women & Men & Women \\
\hline Wid/divorced & $\begin{array}{c}0.06 \\
(0.24)\end{array}$ & $\begin{array}{c}0.11 \\
(0.31)\end{array}$ & $\begin{array}{c}0.08 \\
(0.27)\end{array}$ & $\begin{array}{c}0.12 \\
(0.33)\end{array}$ & $\begin{array}{c}0.07 \\
(0.26)\end{array}$ & $\begin{array}{c}0.12 \\
(0.33)\end{array}$ & $\begin{array}{c}0.10 \\
(0.29)\end{array}$ & $\begin{array}{c}0.13 \\
(0.33)\end{array}$ \\
\hline Single & $\begin{array}{c}0.28 \\
(0.45)\end{array}$ & $\begin{array}{c}0.21 \\
(0.40)\end{array}$ & $\begin{array}{c}0.32 \\
(0.46)\end{array}$ & $\begin{array}{c}0.25 \\
(0.43)\end{array}$ & $\begin{array}{c}0.29 \\
(0.45)\end{array}$ & $\begin{array}{c}0.23 \\
(0.42)\end{array}$ & $\begin{array}{c}0.35 \\
(0.47)\end{array}$ & $\begin{array}{c}0.27 \\
(0.45)\end{array}$ \\
\hline No children present & $\begin{array}{c}0.60 \\
(0.49)\end{array}$ & $\begin{array}{c}0.55 \\
(0.50)\end{array}$ & $\begin{array}{c}0.62 \\
(0.48)\end{array}$ & $\begin{array}{c}0.51 \\
(0.50)\end{array}$ & $\begin{array}{c}0.60 \\
(0.49)\end{array}$ & $\begin{array}{c}0.53 \\
(0.50)\end{array}$ & $\begin{array}{c}0.64 \\
(0.48)\end{array}$ & $\begin{array}{c}0.50 \\
(0.50)\end{array}$ \\
\hline Children $<6$ & $\begin{array}{c}0.14 \\
(0.35)\end{array}$ & $\begin{array}{c}0.14 \\
(0.35)\end{array}$ & $\begin{array}{c}0.16 \\
(0.36)\end{array}$ & $\begin{array}{c}0.15 \\
(0.36)\end{array}$ & $\begin{array}{c}0.17 \\
(0.38)\end{array}$ & $\begin{array}{c}0.16 \\
(0.37)\end{array}$ & $\begin{array}{c}0.14 \\
(0.35)\end{array}$ & $\begin{array}{c}0.14 \\
(0.35)\end{array}$ \\
\hline Children 6-14 & $\begin{array}{c}0.14 \\
(0.35)\end{array}$ & $\begin{array}{c}0.16 \\
(0.37)\end{array}$ & $\begin{array}{c}0.13 \\
(0.34)\end{array}$ & $\begin{array}{c}0.21 \\
(0.40)\end{array}$ & $\begin{array}{c}0.12 \\
(0.33)\end{array}$ & $\begin{array}{c}0.20 \\
(0.40)\end{array}$ & $\begin{array}{c}0.14 \\
(0.35)\end{array}$ & $\begin{array}{c}0.22 \\
(0.41)\end{array}$ \\
\hline Children 14-24 & $\begin{array}{c}0.11 \\
(0.32)\end{array}$ & $\begin{array}{c}0.14 \\
(0.35)\end{array}$ & $\begin{array}{c}0.09 \\
(0.29)\end{array}$ & $\begin{array}{c}0.13 \\
(0.33)\end{array}$ & $\begin{array}{c}0.10 \\
(0.30)\end{array}$ & $\begin{array}{c}0.12 \\
(0.32)\end{array}$ & $\begin{array}{c}0.08 \\
(0.27)\end{array}$ & $\begin{array}{c}0.14 \\
(0.34)\end{array}$ \\
\hline Atlantic provinces & $\begin{array}{c}0.09 \\
(0.28)\end{array}$ & $\begin{array}{c}0.10 \\
(0.29)\end{array}$ & $\begin{array}{c}0.08 \\
(0.28)\end{array}$ & $\begin{array}{c}0.08 \\
(0.26)\end{array}$ & $\begin{array}{c}0.06 \\
(0.24)\end{array}$ & $\begin{array}{c}0.06 \\
(0.24)\end{array}$ & $\begin{array}{c}0.09 \\
(0.28)\end{array}$ & $\begin{array}{c}0.08 \\
(0.27)\end{array}$ \\
\hline Quebec & $\begin{array}{c}0.29 \\
(0.45)\end{array}$ & $\begin{array}{c}0.29 \\
(0.45)\end{array}$ & $\begin{array}{c}0.08 \\
(0.27)\end{array}$ & $\begin{array}{c}0.09 \\
(0.28)\end{array}$ & $\begin{array}{c}0.10 \\
(0.30)\end{array}$ & $\begin{array}{c}0.09 \\
(0.29)\end{array}$ & $\begin{array}{c}0.06 \\
(0.24)\end{array}$ & $\begin{array}{c}0.09 \\
(0.28)\end{array}$ \\
\hline Ontario & $\begin{array}{c}0.34 \\
(0.47)\end{array}$ & $\begin{array}{c}0.34 \\
(0.47)\end{array}$ & $\begin{array}{c}0.24 \\
(0.43)\end{array}$ & $\begin{array}{c}0.23 \\
(0.42)\end{array}$ & $\begin{array}{c}0.18 \\
(0.38)\end{array}$ & $\begin{array}{c}0.17 \\
(0.37)\end{array}$ & $\begin{array}{c}0.31 \\
(0.46)\end{array}$ & $\begin{array}{c}0.29 \\
(0.45)\end{array}$ \\
\hline Manitoba & $\begin{array}{c}0.03 \\
(0.17)\end{array}$ & $\begin{array}{c}0.03 \\
(0.17)\end{array}$ & $\begin{array}{c}0.14 \\
(0.35)\end{array}$ & $\begin{array}{c}0.15 \\
(0.35)\end{array}$ & $\begin{array}{c}0.18 \\
(0.39)\end{array}$ & $\begin{array}{c}0.19 \\
(0.40)\end{array}$ & $\begin{array}{c}0.10 \\
(0.30)\end{array}$ & $\begin{array}{c}0.11 \\
(0.31)\end{array}$ \\
\hline Saskatchewan & $\begin{array}{c}0.03 \\
(0.17)\end{array}$ & $\begin{array}{c}0.03 \\
(0.17)\end{array}$ & $\begin{array}{c}0.09 \\
(0.29)\end{array}$ & $\begin{array}{c}0.10 \\
(0.30)\end{array}$ & $\begin{array}{c}0.11 \\
(0.31)\end{array}$ & $\begin{array}{c}0.12 \\
(0.32)\end{array}$ & $\begin{array}{c}0.07 \\
(0.26)\end{array}$ & $\begin{array}{c}0.09 \\
(0.28)\end{array}$ \\
\hline Alberta & $\begin{array}{c}0.11 \\
(0.31)\end{array}$ & $\begin{array}{c}0.10 \\
(0.30)\end{array}$ & $\begin{array}{c}0.21 \\
(0.41)\end{array}$ & $\begin{array}{c}0.19 \\
(0.39)\end{array}$ & $\begin{array}{c}0.25 \\
(0.43)\end{array}$ & $\begin{array}{c}0.23 \\
(0.42)\end{array}$ & $\begin{array}{c}0.17 \\
(0.38)\end{array}$ & $\begin{array}{c}0.14 \\
(0.35)\end{array}$ \\
\hline British Columbia & $\begin{array}{c}0.11 \\
(0.31)\end{array}$ & $\begin{array}{c}0.11 \\
(0.31)\end{array}$ & $\begin{array}{c}0.15 \\
(0.35)\end{array}$ & $\begin{array}{c}0.17 \\
(0.37)\end{array}$ & $\begin{array}{c}0.11 \\
(0.32)\end{array}$ & $\begin{array}{c}0.13 \\
(0.34)\end{array}$ & $\begin{array}{c}0.19 \\
(0.39)\end{array}$ & $\begin{array}{c}0.20 \\
(0.40)\end{array}$ \\
\hline Primary industries & $\begin{array}{c}0.18 \\
(0.39)\end{array}$ & $\begin{array}{c}0.03 \\
(0.18)\end{array}$ & $\begin{array}{c}0.27 \\
(0.44)\end{array}$ & $\begin{array}{c}0.04 \\
(0.20)\end{array}$ & $\begin{array}{c}0.27 \\
(0.45)\end{array}$ & $\begin{array}{c}0.05 \\
(0.21)\end{array}$ & $\begin{array}{c}0.26 \\
(0.44)\end{array}$ & $\begin{array}{c}0.03 \\
(0.18)\end{array}$ \\
\hline Manufacturing & $\begin{array}{c}0.19 \\
(0.39)\end{array}$ & $\begin{array}{c}0.07 \\
(0.26)\end{array}$ & $\begin{array}{c}0.13 \\
(0.34)\end{array}$ & $\begin{array}{c}0.04 \\
(0.19)\end{array}$ & $\begin{array}{c}0.14 \\
(0.34)\end{array}$ & $\begin{array}{c}0.03 \\
(0.18)\end{array}$ & $\begin{array}{c}0.13 \\
(0.33)\end{array}$ & $\begin{array}{c}0.04 \\
(0.20)\end{array}$ \\
\hline Retail & $\begin{array}{c}0.17 \\
(0.38)\end{array}$ & $\begin{array}{c}0.23 \\
(0.42)\end{array}$ & $\begin{array}{c}0.18 \\
(0.38)\end{array}$ & $\begin{array}{c}0.29 \\
(0.46)\end{array}$ & $\begin{array}{c}0.17 \\
(0.38)\end{array}$ & $\begin{array}{c}0.30 \\
(0.46)\end{array}$ & $\begin{array}{c}0.18 \\
(0.39)\end{array}$ & $\begin{array}{c}0.29 \\
(0.45)\end{array}$ \\
\hline High skill services & $\begin{array}{c}0.13 \\
(0.34)\end{array}$ & $\begin{array}{c}0.17 \\
(0.37)\end{array}$ & $\begin{array}{c}0.10 \\
(0.30)\end{array}$ & $\begin{array}{c}0.12 \\
(0.33)\end{array}$ & $\begin{array}{c}0.11 \\
(0.31)\end{array}$ & $\begin{array}{c}0.12 \\
(0.32)\end{array}$ & $\begin{array}{c}0.10 \\
(0.30)\end{array}$ & $\begin{array}{c}0.13 \\
(0.33)\end{array}$ \\
\hline Public services & $\begin{array}{c}0.15 \\
(0.35)\end{array}$ & $\begin{array}{c}0.39 \\
(0.49)\end{array}$ & $\begin{array}{c}0.15 \\
(0.36)\end{array}$ & $\begin{array}{c}0.42 \\
(0.49)\end{array}$ & $\begin{array}{c}0.15 \\
(0.36)\end{array}$ & $\begin{array}{c}0.41 \\
(0.49)\end{array}$ & $\begin{array}{c}0.16 \\
(0.37)\end{array}$ & $\begin{array}{c}0.43 \\
(0.49)\end{array}$ \\
\hline Other services & $\begin{array}{c}0.17 \\
(0.38)\end{array}$ & $\begin{array}{c}0.09 \\
(0.29)\end{array}$ & $\begin{array}{c}0.16 \\
(0.37)\end{array}$ & $\begin{array}{c}0.08 \\
(0.28)\end{array}$ & $\begin{array}{c}0.16 \\
(0.37)\end{array}$ & $\begin{array}{c}0.09 \\
(0.28)\end{array}$ & $\begin{array}{c}0.16 \\
(0.37)\end{array}$ & $\begin{array}{c}0.08 \\
(0.27)\end{array}$ \\
\hline Management occup & $\begin{array}{c}0.09 \\
(0.29)\end{array}$ & $\begin{array}{c}0.07 \\
(0.26)\end{array}$ & $\begin{array}{c}0.06 \\
(0.24)\end{array}$ & $\begin{array}{c}0.06 \\
(0.24)\end{array}$ & $\begin{array}{c}0.07 \\
(0.25)\end{array}$ & $\begin{array}{c}0.06 \\
(0.24)\end{array}$ & $\begin{array}{c}0.05 \\
(0.22)\end{array}$ & $\begin{array}{c}0.05 \\
(0.23)\end{array}$ \\
\hline Professionals & $\begin{array}{c}0.13 \\
(0.34)\end{array}$ & $\begin{array}{c}0.20 \\
(0.40)\end{array}$ & $\begin{array}{c}0.06 \\
(0.24)\end{array}$ & $\begin{array}{c}0.13 \\
(0.34)\end{array}$ & $\begin{array}{c}0.07 \\
(0.25)\end{array}$ & $\begin{array}{c}0.13 \\
(0.33)\end{array}$ & $\begin{array}{c}0.06 \\
(0.24)\end{array}$ & $\begin{array}{c}0.14 \\
(0.35)\end{array}$ \\
\hline
\end{tabular}




\begin{tabular}{|c|c|c|c|c|c|c|c|c|}
\hline \multirow{2}{*}{$\begin{array}{l}\text { Appendix A3 } \\
\text { (continued) }\end{array}$} & \multicolumn{2}{|c|}{$\begin{array}{c}\text { Non-Aboriginal } \\
\text { Identity }\end{array}$} & \multicolumn{2}{|c|}{$\begin{array}{l}\text { Aboriginal } \\
\text { Identity }\end{array}$} & \multicolumn{2}{|c|}{ Métis } & \multicolumn{2}{|c|}{ North American Indian } \\
\hline & Men & Women & Men & Women & Men & Women & Men & Women \\
\hline $\begin{array}{l}\text { Semi- } \\
\text { professionals }\end{array}$ & $\begin{array}{c}0.07 \\
(0.26)\end{array}$ & $\begin{array}{c}0.09 \\
(0.29)\end{array}$ & $\begin{array}{c}0.07 \\
(0.26)\end{array}$ & $\begin{array}{c}0.10 \\
(0.30)\end{array}$ & $\begin{array}{c}0.06 \\
(0.25)\end{array}$ & $\begin{array}{c}0.09 \\
(0.29)\end{array}$ & $\begin{array}{c}0.08 \\
(0.27)\end{array}$ & $\begin{array}{c}0.11 \\
(0.31)\end{array}$ \\
\hline Sales personnel & $\begin{array}{c}0.27 \\
(0.44)\end{array}$ & $\begin{array}{c}0.56 \\
(0.49)\end{array}$ & $\begin{array}{c}0.28 \\
(0.45)\end{array}$ & $\begin{array}{c}0.62 \\
(0.48)\end{array}$ & $\begin{array}{c}0.27 \\
(0.44)\end{array}$ & $\begin{array}{c}0.63 \\
(0.48)\end{array}$ & $\begin{array}{c}0.28 \\
(0.45)\end{array}$ & $\begin{array}{c}0.62 \\
(0.48)\end{array}$ \\
\hline Manual workers & $\begin{array}{c}0.42 \\
(0.49)\end{array}$ & $\begin{array}{c}0.05 \\
(0.23)\end{array}$ & $\begin{array}{c}0.52 \\
(0.50)\end{array}$ & $\begin{array}{c}0.06 \\
(0.24)\end{array}$ & $\begin{array}{c}0.51 \\
(0.50)\end{array}$ & $\begin{array}{c}0.07 \\
(0.25)\end{array}$ & $\begin{array}{c}0.52 \\
(0.50)\end{array}$ & $\begin{array}{c}0.06 \\
(0.23)\end{array}$ \\
\hline Union & $\begin{array}{c}0.35 \\
(0.47)\end{array}$ & $\begin{array}{c}0.37 \\
(0.48)\end{array}$ & $\begin{array}{c}0.35 \\
(0.48)\end{array}$ & $\begin{array}{c}0.36 \\
(0.48)\end{array}$ & $\begin{array}{c}0.36 \\
(0.48)\end{array}$ & $\begin{array}{c}0.38 \\
(0.48)\end{array}$ & $\begin{array}{c}0.32 \\
(0.46)\end{array}$ & $\begin{array}{c}0.34 \\
(0.47)\end{array}$ \\
\hline Small workplace & $\begin{array}{c}0.63 \\
(0.48)\end{array}$ & $\begin{array}{c}0.65 \\
(0.48)\end{array}$ & $\begin{array}{c}0.68 \\
(0.47)\end{array}$ & $\begin{array}{c}0.71 \\
(0.45)\end{array}$ & $\begin{array}{c}0.65 \\
(0.48)\end{array}$ & $\begin{array}{c}0.73 \\
(0.44)\end{array}$ & $\begin{array}{c}0.71 \\
(0.45)\end{array}$ & $\begin{array}{c}0.70 \\
(0.45)\end{array}$ \\
\hline $\begin{array}{l}\text { Medium } \\
\text { workplace }\end{array}$ & $\begin{array}{c}0.23 \\
(0.42)\end{array}$ & $\begin{array}{c}0.21 \\
(0.40)\end{array}$ & $\begin{array}{c}0.22 \\
(0.41)\end{array}$ & $\begin{array}{c}0.20 \\
(0.40)\end{array}$ & $\begin{array}{c}0.23 \\
(0.42)\end{array}$ & $\begin{array}{c}0.20 \\
(0.40)\end{array}$ & $\begin{array}{c}0.20 \\
(0.40)\end{array}$ & $\begin{array}{c}0.20 \\
(0.40)\end{array}$ \\
\hline Large workplace & $\begin{array}{c}0.13 \\
(0.34)\end{array}$ & $\begin{array}{c}0.14 \\
(0.35)\end{array}$ & $\begin{array}{c}0.10 \\
(0.30)\end{array}$ & $\begin{array}{c}0.08 \\
(0.28)\end{array}$ & $\begin{array}{c}0.11 \\
(0.32)\end{array}$ & $\begin{array}{c}0.07 \\
(0.26)\end{array}$ & $\begin{array}{c}0.09 \\
(0.29)\end{array}$ & $\begin{array}{c}0.10 \\
(0.30)\end{array}$ \\
\hline Private sector & $\begin{array}{c}0.81 \\
(0.39)\end{array}$ & $\begin{array}{c}0.68 \\
(0.47)\end{array}$ & $\begin{array}{c}0.80 \\
(0.40)\end{array}$ & $\begin{array}{c}0.67 \\
(0.47)\end{array}$ & $\begin{array}{c}0.80 \\
(0.40)\end{array}$ & $\begin{array}{c}0.68 \\
(0.47)\end{array}$ & $\begin{array}{c}0.80 \\
(0.40)\end{array}$ & $\begin{array}{c}0.66 \\
(0.47)\end{array}$ \\
\hline Full-time job & $\begin{array}{c}0.96 \\
(0.20)\end{array}$ & $\begin{array}{c}0.83 \\
(0.37)\end{array}$ & $\begin{array}{c}0.95 \\
(0.22)\end{array}$ & $\begin{array}{c}0.84 \\
(0.36)\end{array}$ & $\begin{array}{c}0.95 \\
(0.21)\end{array}$ & $\begin{array}{c}0.84 \\
(0.36)\end{array}$ & $\begin{array}{c}0.95 \\
(0.22)\end{array}$ & $\begin{array}{c}0.84 \\
(0.37)\end{array}$ \\
\hline Wage minimum & $\begin{array}{c}0.05 \\
(0.21)\end{array}$ & $\begin{array}{c}0.10 \\
(0.30)\end{array}$ & $\begin{array}{c}0.06 \\
(0.25)\end{array}$ & $\begin{array}{c}0.13 \\
(0.34)\end{array}$ & $\begin{array}{c}0.05 \\
(0.23)\end{array}$ & $\begin{array}{c}0.12 \\
(0.32)\end{array}$ & $\begin{array}{c}0.07 \\
(0.26)\end{array}$ & $\begin{array}{c}0.14 \\
(0.35)\end{array}$ \\
\hline Wage median & $\begin{array}{c}0.09 \\
(0.28)\end{array}$ & $\begin{array}{c}0.14 \\
(0.35)\end{array}$ & $\begin{array}{c}0.10 \\
(0.30)\end{array}$ & $\begin{array}{c}0.16 \\
(0.37)\end{array}$ & $\begin{array}{c}0.09 \\
(0.29)\end{array}$ & $\begin{array}{c}0.17 \\
(0.38)\end{array}$ & $\begin{array}{c}0.11 \\
(0.31)\end{array}$ & $\begin{array}{c}0.15 \\
(0.36)\end{array}$ \\
\hline Wage average & $\begin{array}{c}0.32 \\
(0.47)\end{array}$ & $\begin{array}{c}0.36 \\
(0.48)\end{array}$ & $\begin{array}{c}0.30 \\
(0.46)\end{array}$ & $\begin{array}{c}0.34 \\
(0.47)\end{array}$ & $\begin{array}{c}0.30 \\
(0.46)\end{array}$ & $\begin{array}{c}0.35 \\
(0.48)\end{array}$ & $\begin{array}{c}0.30 \\
(0.46)\end{array}$ & $\begin{array}{c}0.33 \\
(0.47)\end{array}$ \\
\hline Wage high & $\begin{array}{c}0.45 \\
(0.50)\end{array}$ & $\begin{array}{c}0.38 \\
(0.49)\end{array}$ & $\begin{array}{c}0.50 \\
(0.50)\end{array}$ & $\begin{array}{c}0.35 \\
(0.48)\end{array}$ & $\begin{array}{c}0.52 \\
(0.50)\end{array}$ & $\begin{array}{c}0.35 \\
(0.48)\end{array}$ & $\begin{array}{c}0.49 \\
(0.50)\end{array}$ & $\begin{array}{c}0.35 \\
(0.48)\end{array}$ \\
\hline Sample Size & 69891 & 68649 & 2640 & 2849 & 1435 & 1418 & 1161 & 1395 \\
\hline Weighted counts & 18433685 & 16083451 & 449684 & 460377 & 198875 & 231972 & 201522 & 223201 \\
\hline
\end{tabular}

Source: The Labour Force Survey, national monthly master file, September 2008 - May 2011.

Note: Restricted samples include only permanent paid employees, aged between 15 and 64 , who are single-job holders and who are not students. Survey sampling weights (abowt) applied in all analyses to account for complex survey design: stratification, clustering and non-response. The counts represent pooled populations for the recession time period. Standard deviations reported in parentheses. 
Appendix A 4: Mean Characteristics of Laid-Off Workers in the Main Samples, by Identity

\begin{tabular}{|c|c|c|c|c|c|c|c|c|}
\hline \multirow[t]{2}{*}{ Characteristics } & \multicolumn{2}{|c|}{$\begin{array}{c}\text { Non-Aboriginal } \\
\text { Identity }\end{array}$} & \multicolumn{2}{|c|}{$\begin{array}{c}\text { Aboriginal } \\
\text { Identity }\end{array}$} & \multicolumn{2}{|c|}{ Métis } & \multicolumn{2}{|c|}{ North American Indian } \\
\hline & Men & Women & Men & Women & Men & Women & Men & Women \\
\hline Less than h-school & $\begin{array}{c}0.22 \\
(0.41)\end{array}$ & $\begin{array}{c}0.14 \\
(0.35)\end{array}$ & $\begin{array}{c}0.28 \\
(0.45)\end{array}$ & $\begin{array}{c}0.23 \\
(0.42)\end{array}$ & $\begin{array}{c}0.25 \\
(0.44)\end{array}$ & $\begin{array}{c}0.19 \\
(0.39)\end{array}$ & $\begin{array}{c}0.31 \\
(0.46)\end{array}$ & $\begin{array}{c}0.25 \\
(0.44)\end{array}$ \\
\hline High school & $\begin{array}{c}0.25 \\
(0.43)\end{array}$ & $\begin{array}{c}0.23 \\
(0.42)\end{array}$ & $\begin{array}{l}0.27 \\
(0.44)\end{array}$ & $\begin{array}{c}0.22 \\
(0.41)\end{array}$ & $\begin{array}{c}0.28 \\
(0.45)\end{array}$ & $\begin{array}{c}0.21 \\
(0.41)\end{array}$ & $\begin{array}{c}0.27 \\
(0.44)\end{array}$ & $\begin{array}{c}0.24 \\
(0.43)\end{array}$ \\
\hline Incomplete pse & $\begin{array}{c}0.09 \\
(0.28)\end{array}$ & $\begin{array}{c}0.08 \\
(0.27)\end{array}$ & $\begin{array}{c}0.09 \\
(0.29)\end{array}$ & $\begin{array}{c}0.10 \\
(0.30)\end{array}$ & $\begin{array}{c}0.09 \\
(0.29)\end{array}$ & $\begin{array}{c}0.12 \\
(0.32)\end{array}$ & $\begin{array}{c}0.09 \\
(0.29)\end{array}$ & $\begin{array}{c}0.08 \\
(0.28)\end{array}$ \\
\hline Completed trade & $\begin{array}{c}0.20 \\
(0.40)\end{array}$ & $\begin{array}{c}0.10 \\
(0.29)\end{array}$ & $\begin{array}{c}0.20 \\
(0.40)\end{array}$ & $\begin{array}{c}0.12 \\
(0.32)\end{array}$ & $\begin{array}{c}0.24 \\
(0.42)\end{array}$ & $\begin{array}{c}0.15 \\
(0.36)\end{array}$ & $\begin{array}{c}0.16 \\
(0.37)\end{array}$ & $\begin{array}{c}0.09 \\
(0.28)\end{array}$ \\
\hline Completed college & $\begin{array}{c}0.15 \\
(0.36)\end{array}$ & $\begin{array}{c}0.25 \\
(0.43)\end{array}$ & $\begin{array}{c}0.13 \\
(0.34)\end{array}$ & $\begin{array}{c}0.25 \\
(0.44)\end{array}$ & $\begin{array}{c}0.10 \\
(0.30)\end{array}$ & $\begin{array}{c}0.25 \\
(0.43)\end{array}$ & $\begin{array}{c}0.15 \\
(0.36)\end{array}$ & $\begin{array}{c}0.26 \\
(0.44)\end{array}$ \\
\hline University degree & $\begin{array}{c}0.09 \\
(0.29)\end{array}$ & $\begin{array}{c}0.19 \\
(0.39)\end{array}$ & $\begin{array}{c}0.02 \\
(0.14)\end{array}$ & $\begin{array}{c}0.08 \\
(0.44)\end{array}$ & $\begin{array}{c}0.03 \\
(0.17)\end{array}$ & $\begin{array}{c}0.08 \\
(0.27)\end{array}$ & $\begin{array}{c}0.01 \\
(0.12)\end{array}$ & $\begin{array}{l}0.07 \\
(026)\end{array}$ \\
\hline Youth 15-24 & $\begin{array}{c}0.23 \\
(0.42)\end{array}$ & $\begin{array}{c}0.16 \\
(0.37)\end{array}$ & $\begin{array}{c}0.26 \\
(0.44)\end{array}$ & $\begin{array}{c}0.18 \\
(0.39)\end{array}$ & $\begin{array}{c}0.28 \\
(0.45)\end{array}$ & $\begin{array}{c}0.20 \\
(0.40)\end{array}$ & $\begin{array}{c}0.24 \\
(0.43)\end{array}$ & $\begin{array}{c}0.16 \\
(0.37)\end{array}$ \\
\hline Young 25-34 & $\begin{array}{c}0.23 \\
(0.42)\end{array}$ & $\begin{array}{c}0.19 \\
(0.39)\end{array}$ & $\begin{array}{c}0.26 \\
(0.44)\end{array}$ & $\begin{array}{c}0.24 \\
(0.42)\end{array}$ & $\begin{array}{c}0.22 \\
(0.42)\end{array}$ & $\begin{array}{c}0.25 \\
(0.44)\end{array}$ & $\begin{array}{c}0.29 \\
(0.45)\end{array}$ & $\begin{array}{c}0.22 \\
(0.41)\end{array}$ \\
\hline Young prime $35-44$ & $\begin{array}{c}0.18 \\
(0.38)\end{array}$ & $\begin{array}{c}0.21 \\
(0.40)\end{array}$ & $\begin{array}{c}0.19 \\
(0.39)\end{array}$ & $\begin{array}{c}0.24 \\
(043)\end{array}$ & $\begin{array}{c}0.18 \\
(0.39)\end{array}$ & $\begin{array}{c}0.21 \\
(0.41)\end{array}$ & $\begin{array}{c}0.19 \\
(0.40)\end{array}$ & $\begin{array}{c}0.27 \\
(0.44)\end{array}$ \\
\hline Mature prime $45-54$ & $\begin{array}{c}0.21 \\
(0.40)\end{array}$ & $\begin{array}{c}0.26 \\
(0.44)\end{array}$ & $\begin{array}{c}0.17 \\
(0.38)\end{array}$ & $\begin{array}{c}0.19 \\
(039)\end{array}$ & $\begin{array}{c}0.18 \\
(0.39)\end{array}$ & $\begin{array}{c}0.21 \\
(0.41)\end{array}$ & $\begin{array}{c}0.17 \\
(0.37)\end{array}$ & $\begin{array}{c}0.19 \\
(0.39)\end{array}$ \\
\hline Older worker 55-64 & $\begin{array}{c}0.16 \\
(0.36)\end{array}$ & $\begin{array}{c}0.18 \\
(0.38)\end{array}$ & $\begin{array}{c}0.11 \\
(0.32)\end{array}$ & $\begin{array}{c}0.14 \\
(035)\end{array}$ & $\begin{array}{c}0.12 \\
(0.33)\end{array}$ & $\begin{array}{c}0.12 \\
(0.33)\end{array}$ & $\begin{array}{c}0.10 \\
(0.30)\end{array}$ & $\begin{array}{c}0.16 \\
(0.36)\end{array}$ \\
\hline Married & $\begin{array}{c}0.51 \\
(0.50)\end{array}$ & $\begin{array}{c}0.61 \\
(0.49)\end{array}$ & $\begin{array}{c}0.48 \\
(0.50)\end{array}$ & $\begin{array}{c}0.60 \\
(0.49)\end{array}$ & $\begin{array}{c}0.49 \\
(0.50)\end{array}$ & $\begin{array}{c}0.59 \\
(0.49)\end{array}$ & $\begin{array}{c}0.48 \\
(0.50)\end{array}$ & $\begin{array}{c}0.61 \\
(0.49)\end{array}$ \\
\hline Widowed div/sep & $\begin{array}{c}0.07 \\
(0.25)\end{array}$ & $\begin{array}{c}0.11 \\
(0.32)\end{array}$ & $\begin{array}{c}0.08 \\
(0.26)\end{array}$ & $\begin{array}{c}0.09 \\
(0.29)\end{array}$ & $\begin{array}{c}0.07 \\
(0.26)\end{array}$ & $\begin{array}{c}0.10 \\
(0.31)\end{array}$ & $\begin{array}{c}0.08 \\
(0.27)\end{array}$ & $\begin{array}{c}0.08 \\
(0.27)\end{array}$ \\
\hline Single & $\begin{array}{c}0.42 \\
(0.49)\end{array}$ & $\begin{array}{c}0.27 \\
(0.44)\end{array}$ & $\begin{array}{c}0.44 \\
(0.50)\end{array}$ & $\begin{array}{c}0.31 \\
(0.46)\end{array}$ & $\begin{array}{c}0.44 \\
(0.50)\end{array}$ & $\begin{array}{c}0.30 \\
(0.46)\end{array}$ & $\begin{array}{c}0.44 \\
(0.50)\end{array}$ & $\begin{array}{c}0.31 \\
(0.46)\end{array}$ \\
\hline No children present & $\begin{array}{c}0.72 \\
(0.45)\end{array}$ & $\begin{array}{c}0.61 \\
(0.49)\end{array}$ & $\begin{array}{c}0.69 \\
(0.46)\end{array}$ & $\begin{array}{c}0.53 \\
(0.50)\end{array}$ & $\begin{array}{c}0.68 \\
(0.47)\end{array}$ & $\begin{array}{c}0.58 \\
(0.49)\end{array}$ & $\begin{array}{c}0.70 \\
(0.46)\end{array}$ & $\begin{array}{c}0.50 \\
(0.50)\end{array}$ \\
\hline Children $<6$ & $\begin{array}{c}0.10 \\
(0.31)\end{array}$ & $\begin{array}{c}0.11 \\
(0.31)\end{array}$ & $\begin{array}{c}0.14 \\
(0.35)\end{array}$ & $\begin{array}{c}0.15 \\
(0.35)\end{array}$ & $\begin{array}{c}0.14 \\
(0.34)\end{array}$ & $\begin{array}{c}0.15 \\
(0.36)\end{array}$ & $\begin{array}{c}0.14 \\
(0.35)\end{array}$ & $\begin{array}{c}0.14 \\
(0.35)\end{array}$ \\
\hline Children 6-14 & $\begin{array}{c}0.09 \\
(0.28)\end{array}$ & $\begin{array}{c}0.16 \\
(0.36)\end{array}$ & $\begin{array}{c}0.10 \\
(0.30)\end{array}$ & $\begin{array}{c}0.19 \\
(0.39)\end{array}$ & $\begin{array}{c}0.10 \\
(0.30)\end{array}$ & $\begin{array}{c}0.13 \\
(0.34)\end{array}$ & $\begin{array}{c}0.10 \\
(0.30)\end{array}$ & $\begin{array}{c}0.23 \\
(0.42)\end{array}$ \\
\hline Children 14-24 & $\begin{array}{c}0.08 \\
(0.27)\end{array}$ & $\begin{array}{c}0.12 \\
(0.33)\end{array}$ & $\begin{array}{c}0.06 \\
(0.25)\end{array}$ & $\begin{array}{c}0.13 \\
(0.34)\end{array}$ & $\begin{array}{c}0.08 \\
(0.27)\end{array}$ & $\begin{array}{c}0.13 \\
(0.34)\end{array}$ & $\begin{array}{c}0.05 \\
(0.23)\end{array}$ & $\begin{array}{c}0.12 \\
(0.33)\end{array}$ \\
\hline
\end{tabular}




\begin{tabular}{|c|c|c|c|c|c|c|c|c|}
\hline \multirow{2}{*}{$\begin{array}{l}\text { Appendix A4 } \\
\text { (continued) }\end{array}$} & \multicolumn{2}{|c|}{$\begin{array}{c}\text { Non-Aboriginal } \\
\text { Identity }\end{array}$} & \multicolumn{2}{|c|}{$\begin{array}{c}\text { Aboriginal } \\
\text { Identity }\end{array}$} & \multicolumn{2}{|c|}{ Métis } & \multicolumn{2}{|c|}{ North American Indian } \\
\hline & Men & Women & Men & Women & Men & Women & Men & Women \\
\hline Atlantic provinces & $\begin{array}{c}0.15 \\
(0.36)\end{array}$ & $\begin{array}{c}0.14 \\
(0.35)\end{array}$ & $\begin{array}{c}0.13 \\
(0.34)\end{array}$ & $\begin{array}{c}0.11 \\
(0.31)\end{array}$ & $\begin{array}{c}0.09 \\
(0.29)\end{array}$ & $\begin{array}{c}0.08 \\
(0.27)\end{array}$ & $\begin{array}{c}0.13 \\
(0.34)\end{array}$ & $\begin{array}{c}0.11 \\
(0.32)\end{array}$ \\
\hline Quebec & $\begin{array}{c}0.30 \\
(0.36)\end{array}$ & $\begin{array}{c}0.28 \\
(0.45)\end{array}$ & $\begin{array}{c}0.10 \\
(0.30)\end{array}$ & $\begin{array}{c}0.09 \\
(0.29)\end{array}$ & $\begin{array}{c}0.12 \\
(0.33)\end{array}$ & $\begin{array}{c}0.15 \\
(0.35)\end{array}$ & $\begin{array}{c}0.08 \\
(0.28)\end{array}$ & $\begin{array}{c}0.06 \\
(0.24)\end{array}$ \\
\hline Ontario & $\begin{array}{c}0.31 \\
(0.46)\end{array}$ & $\begin{array}{c}0.32 \\
(0.46)\end{array}$ & $\begin{array}{c}0.23 \\
(0.42)\end{array}$ & $\begin{array}{c}0.25 \\
(0.43)\end{array}$ & $\begin{array}{c}0.18 \\
(0.38)\end{array}$ & $\begin{array}{c}0.16 \\
(0.37)\end{array}$ & $\begin{array}{c}0.29 \\
(0.45)\end{array}$ & $\begin{array}{c}0.34 \\
(0.47)\end{array}$ \\
\hline Manitoba & $\begin{array}{c}0.02 \\
(0.14)\end{array}$ & $\begin{array}{c}0.03 \\
(0.16)\end{array}$ & $\begin{array}{c}0.11 \\
(0.31)\end{array}$ & $\begin{array}{c}0.12 \\
(0.32)\end{array}$ & $\begin{array}{c}0.14 \\
(0.35)\end{array}$ & $\begin{array}{c}0.15 \\
(0.36)\end{array}$ & $\begin{array}{c}0.08 \\
(0.27)\end{array}$ & $\begin{array}{c}0.08 \\
(0.28)\end{array}$ \\
\hline Saskatchewan & $\begin{array}{c}0.02 \\
(0.14)\end{array}$ & $\begin{array}{c}0.03 \\
(0.16)\end{array}$ & $\begin{array}{c}0.08 \\
(0.28)\end{array}$ & $\begin{array}{c}0.08 \\
(0.27)\end{array}$ & $\begin{array}{c}0.11 \\
(0.31)\end{array}$ & $\begin{array}{c}0.13 \\
(0.42)\end{array}$ & $\begin{array}{c}0.06 \\
(0.25)\end{array}$ & $\begin{array}{c}0.04 \\
(0.19)\end{array}$ \\
\hline Alberta & $\begin{array}{c}0.08 \\
(0.28)\end{array}$ & $\begin{array}{c}0.09 \\
(0.29)\end{array}$ & $\begin{array}{c}0.21 \\
(0.41)\end{array}$ & $\begin{array}{c}0.21 \\
(0.41)\end{array}$ & $\begin{array}{c}0.27 \\
(0.35)\end{array}$ & $\begin{array}{c}0.24 \\
(0.42)\end{array}$ & $\begin{array}{c}0.15 \\
(0.36)\end{array}$ & $\begin{array}{c}0.18 \\
(0.39)\end{array}$ \\
\hline British Columbia & $\begin{array}{c}0.10 \\
(0.30)\end{array}$ & $\begin{array}{c}0.10 \\
(0.30)\end{array}$ & $\begin{array}{c}0.14 \\
(0.34)\end{array}$ & $\begin{array}{c}0.13 \\
(0.34)\end{array}$ & $\begin{array}{c}0.08 \\
(0.27)\end{array}$ & $\begin{array}{c}0.09 \\
(0.29)\end{array}$ & $\begin{array}{c}0.19 \\
(0.40)\end{array}$ & $\begin{array}{c}0.17 \\
(0.38)\end{array}$ \\
\hline $\begin{array}{l}\text { Primary } \\
\text { industries }\end{array}$ & $\begin{array}{c}0.34 \\
(0.47)\end{array}$ & $\begin{array}{c}0.06 \\
(0.25)\end{array}$ & $\begin{array}{c}0.45 \\
(0.50)\end{array}$ & $\begin{array}{c}0.11 \\
(0.31)\end{array}$ & $\begin{array}{c}0.47 \\
(0.50)\end{array}$ & $\begin{array}{c}0.14 \\
(0.35)\end{array}$ & $\begin{array}{c}0.43 \\
(0.50)\end{array}$ & $\begin{array}{c}0.07 \\
(0.25)\end{array}$ \\
\hline Manufacturing & $\begin{array}{c}0.17 \\
(0.38)\end{array}$ & $\begin{array}{c}0.10 \\
(0.30)\end{array}$ & $\begin{array}{c}0.12 \\
(0.33)\end{array}$ & $\begin{array}{c}0.08 \\
(0.27)\end{array}$ & $\begin{array}{c}0.11 \\
(0.31)\end{array}$ & $\begin{array}{c}0.05 \\
(0.23)\end{array}$ & $\begin{array}{c}0.14 \\
(0.34)\end{array}$ & $\begin{array}{c}0.11 \\
(0.31)\end{array}$ \\
\hline Retail & $\begin{array}{c}0.15 \\
(0.36)\end{array}$ & $\begin{array}{c}0.24 \\
(0.43)\end{array}$ & $\begin{array}{c}0.13 \\
(0.33)\end{array}$ & $\begin{array}{c}0.33 \\
(0.47)\end{array}$ & $\begin{array}{c}0.12 \\
(0.33)\end{array}$ & $\begin{array}{c}0.34 \\
(0.47)\end{array}$ & $\begin{array}{c}0.14 \\
(0.34)\end{array}$ & $\begin{array}{c}0.31 \\
(0.46)\end{array}$ \\
\hline High skill services & $\begin{array}{c}0.11 \\
(0.31)\end{array}$ & $\begin{array}{c}0.13 \\
(0.33)\end{array}$ & $\begin{array}{c}0.10 \\
(0.31)\end{array}$ & $\begin{array}{c}0.10 \\
(0.30)\end{array}$ & $\begin{array}{c}0.10 \\
(0.30)\end{array}$ & $\begin{array}{c}0.10 \\
(0.30)\end{array}$ & $\begin{array}{c}0.12 \\
(0.32)\end{array}$ & $\begin{array}{c}0.10 \\
(0.30)\end{array}$ \\
\hline Public services & $\begin{array}{c}0.08 \\
(0.27)\end{array}$ & $\begin{array}{c}0.35 \\
(0.48)\end{array}$ & $\begin{array}{c}0.06 \\
(0.25)\end{array}$ & $\begin{array}{c}0.30 \\
(0.46)\end{array}$ & $\begin{array}{c}0.07 \\
(0.26)\end{array}$ & $\begin{array}{c}0.29 \\
(0.45)\end{array}$ & $\begin{array}{c}0.06 \\
(0.24)\end{array}$ & $\begin{array}{c}0.32 \\
(0.47)\end{array}$ \\
\hline Other services & $\begin{array}{c}0.14 \\
(0.35)\end{array}$ & $\begin{array}{c}0.11 \\
(0.32)\end{array}$ & $\begin{array}{c}0.12 \\
(0.33)\end{array}$ & $\begin{array}{c}0.08 \\
(0.28)\end{array}$ & $\begin{array}{c}0.12 \\
(0.33)\end{array}$ & $\begin{array}{c}0.08 \\
(0.27)\end{array}$ & $\begin{array}{c}0.11 \\
(0.32)\end{array}$ & $\begin{array}{c}0.08 \\
(0.28)\end{array}$ \\
\hline Management & $\begin{array}{c}0.03 \\
(0.17)\end{array}$ & $\begin{array}{c}0.03 \\
(0.18)\end{array}$ & $\begin{array}{c}0.02 \\
(0.14)\end{array}$ & $\begin{array}{c}0.03 \\
(0.16)\end{array}$ & $\begin{array}{c}0.02 \\
(0.13)\end{array}$ & $\begin{array}{c}0.03 \\
(0.17)\end{array}$ & $\begin{array}{c}0.02 \\
(0.14)\end{array}$ & $\begin{array}{c}0.02 \\
(0.15)\end{array}$ \\
\hline Professionals & $\begin{array}{c}0.05 \\
(0.23)\end{array}$ & $\begin{array}{c}0.14 \\
(0.35)\end{array}$ & $\begin{array}{c}0.02 \\
(0.15)\end{array}$ & $\begin{array}{c}0.08 \\
(0.28)\end{array}$ & $\begin{array}{c}0.02 \\
(0.15)\end{array}$ & $\begin{array}{c}0.09 \\
(0.29)\end{array}$ & $\begin{array}{c}0.02 \\
(0.15)\end{array}$ & $\begin{array}{c}0.08 \\
(0.27)\end{array}$ \\
\hline $\begin{array}{l}\text { Semi- } \\
\text { professionals }\end{array}$ & $\begin{array}{c}0.06 \\
(0.24)\end{array}$ & $\begin{array}{c}0.08 \\
(0.27)\end{array}$ & $\begin{array}{c}0.06 \\
(0.23)\end{array}$ & $\begin{array}{c}0.08 \\
(0.27)\end{array}$ & $\begin{array}{c}0.04 \\
(0.20)\end{array}$ & $\begin{array}{c}0.07 \\
(0.25)\end{array}$ & $\begin{array}{c}0.07 \\
(0.26)\end{array}$ & $\begin{array}{c}0.08 \\
(0.28)\end{array}$ \\
\hline Sales personnel & $\begin{array}{c}0.21 \\
(0.40)\end{array}$ & $\begin{array}{c}0.57 \\
(0.49)\end{array}$ & $\begin{array}{c}0.19 \\
(0.39)\end{array}$ & $\begin{array}{c}0.62 \\
(0.48)\end{array}$ & $\begin{array}{c}0.20 \\
(0.40)\end{array}$ & $\begin{array}{c}0.65 \\
(0.48)\end{array}$ & $\begin{array}{c}0.18 \\
(0.38)\end{array}$ & $\begin{array}{c}0.60 \\
(0.49)\end{array}$ \\
\hline Manual workers & $\begin{array}{c}0.63 \\
(0.48)\end{array}$ & $\begin{array}{c}0.14 \\
(0.35)\end{array}$ & $\begin{array}{c}0.69 \\
(0.46)\end{array}$ & $\begin{array}{c}0.17 \\
(0.37)\end{array}$ & $\begin{array}{c}0.70 \\
(0.46)\end{array}$ & $\begin{array}{c}0.15 \\
(0.36)\end{array}$ & $\begin{array}{c}0.69 \\
(0.46)\end{array}$ & $\begin{array}{c}0.18 \\
(0.38)\end{array}$ \\
\hline Union member & $\begin{array}{c}0.33 \\
(0.47)\end{array}$ & $\begin{array}{c}0.35 \\
(0.48)\end{array}$ & $\begin{array}{c}0.23 \\
(0.42)\end{array}$ & $\begin{array}{c}0.23 \\
(0.42)\end{array}$ & $\begin{array}{c}0.23 \\
(0.42)\end{array}$ & $\begin{array}{c}0.24 \\
(0.43)\end{array}$ & $\begin{array}{c}0.21 \\
(0.41)\end{array}$ & $\begin{array}{c}0.24 \\
(0.43)\end{array}$ \\
\hline
\end{tabular}




\begin{tabular}{|c|c|c|c|c|c|c|c|c|}
\hline \multirow{2}{*}{$\begin{array}{l}\text { Appendix A4 } \\
\text { (continued) }\end{array}$} & \multicolumn{2}{|c|}{$\begin{array}{c}\text { Non-Aboriginal } \\
\text { Identity }\end{array}$} & \multicolumn{2}{|c|}{$\begin{array}{c}\text { Aboriginal } \\
\text { Identity }\end{array}$} & \multicolumn{2}{|c|}{ Métis } & \multicolumn{2}{|c|}{ North American Indian } \\
\hline & Men & Women & Men & Women & Men & Women & Men & Women \\
\hline Small workplace & $\begin{array}{c}0.77 \\
(0.42)\end{array}$ & $\begin{array}{c}0.76 \\
(0.42)\end{array}$ & $\begin{array}{c}0.84 \\
(0.37)\end{array}$ & $\begin{array}{c}0.83 \\
(0.38)\end{array}$ & $\begin{array}{c}0.85 \\
(0.36)\end{array}$ & $\begin{array}{c}0.89 \\
(0.32)\end{array}$ & $\begin{array}{c}0.83 \\
(0.38)\end{array}$ & $\begin{array}{c}0.77 \\
(0.42)\end{array}$ \\
\hline $\begin{array}{l}\text { Medium } \\
\text { workplace }\end{array}$ & $\begin{array}{c}0.16 \\
(0.36)\end{array}$ & $\begin{array}{c}0.15 \\
(0.36)\end{array}$ & $\begin{array}{c}0.11 \\
(0.32)\end{array}$ & $\begin{array}{c}0.13 \\
(0.34)\end{array}$ & $\begin{array}{c}0.10 \\
(0.30)\end{array}$ & $\begin{array}{c}0.07 \\
(0.26)\end{array}$ & $\begin{array}{c}0.13 \\
(0.33)\end{array}$ & $\begin{array}{c}0.19 \\
(0.39)\end{array}$ \\
\hline Large workplace & $\begin{array}{c}0.07 \\
(0.25)\end{array}$ & $\begin{array}{c}0.08 \\
(0.27)\end{array}$ & $\begin{array}{c}0.05 \\
(0.21)\end{array}$ & $\begin{array}{c}0.04 \\
(0.20)\end{array}$ & $\begin{array}{c}0.05 \\
(0.22)\end{array}$ & $\begin{array}{c}0.04 \\
(0.20)\end{array}$ & $\begin{array}{c}0.04 \\
(0.20)\end{array}$ & $\begin{array}{c}0.04 \\
(0.21)\end{array}$ \\
\hline Private sector & $\begin{array}{c}0.91 \\
(0.29)\end{array}$ & $\begin{array}{c}0.71 \\
(0.45)\end{array}$ & $\begin{array}{c}0.89 \\
(0.31)\end{array}$ & $\begin{array}{c}0.74 \\
(0.44)\end{array}$ & $\begin{array}{c}0.88 \\
(0.32)\end{array}$ & $\begin{array}{c}0.74 \\
(0.44)\end{array}$ & $\begin{array}{c}0.90 \\
(0.30)\end{array}$ & $\begin{array}{c}0.71 \\
(0.45)\end{array}$ \\
\hline Full-time job & $\begin{array}{c}0.86 \\
(0.34)\end{array}$ & $\begin{array}{c}0.67 \\
(0.47)\end{array}$ & $\begin{array}{c}0.86 \\
(0.34)\end{array}$ & $\begin{array}{c}0.73 \\
(0.44)\end{array}$ & $\begin{array}{c}0.91 \\
(0.28)\end{array}$ & $\begin{array}{c}0.74 \\
(0.44)\end{array}$ & $\begin{array}{c}0.82 \\
(0.38)\end{array}$ & $\begin{array}{c}0.72 \\
(0.45)\end{array}$ \\
\hline Wage minimum & $\begin{array}{r}0.06 \\
(024)\end{array}$ & $\begin{array}{c}0.12 \\
(0.32)\end{array}$ & $\begin{array}{c}0.06 \\
(0.23)\end{array}$ & $\begin{array}{c}0.14 \\
(0.35)\end{array}$ & $\begin{array}{c}0.06 \\
(0.24)\end{array}$ & $\begin{array}{c}0.11 \\
(0.32)\end{array}$ & $\begin{array}{c}0.06 \\
(0.23)\end{array}$ & $\begin{array}{c}0.17 \\
(0.37)\end{array}$ \\
\hline Wage median & $\begin{array}{c}0.09 \\
(0.29)\end{array}$ & $\begin{array}{c}0.11 \\
(0.32)\end{array}$ & $\begin{array}{c}0.07 \\
(0.26)\end{array}$ & $\begin{array}{c}0.11 \\
(0.31)\end{array}$ & $\begin{array}{c}0.08 \\
(0.27)\end{array}$ & $\begin{array}{c}0.11 \\
(0.31)\end{array}$ & $\begin{array}{c}0.06 \\
(0.24)\end{array}$ & $\begin{array}{c}0.11 \\
(0.31)\end{array}$ \\
\hline Wage average & $\begin{array}{c}0.20 \\
(0.40)\end{array}$ & $\begin{array}{c}0.20 \\
(0.40)\end{array}$ & $\begin{array}{c}0.20 \\
(0.40)\end{array}$ & $\begin{array}{c}0.14 \\
(0.35)\end{array}$ & $\begin{array}{c}0.23 \\
(0.42)\end{array}$ & $\begin{array}{c}0.16 \\
(0.36)\end{array}$ & $\begin{array}{c}0.19 \\
(0.39)\end{array}$ & $\begin{array}{c}0.13 \\
(0.33)\end{array}$ \\
\hline Wage high & $\begin{array}{c}0.63 \\
(0.48)\end{array}$ & $\begin{array}{c}0.56 \\
(0.50)\end{array}$ & $\begin{array}{c}0.64 \\
(0.48)\end{array}$ & $\begin{array}{c}0.60 \\
(0.49)\end{array}$ & $\begin{array}{c}0.61 \\
(0.49)\end{array}$ & $\begin{array}{c}0.61 \\
(0.49)\end{array}$ & $\begin{array}{c}0.67 \\
(0.47)\end{array}$ & $\begin{array}{c}0.59 \\
(0.49)\end{array}$ \\
\hline Sample Size & 15007 & 10334 & 789 & 569 & 378 & 255 & 395 & 301 \\
\hline Weighted counts & 3504808 & 2421173 & 129422 & 86822 & 60372 & 40890 & 65980 & 44631 \\
\hline
\end{tabular}

Source: The Labour Force Survey, national monthly master file, September 2008 - May 2011.

Note: Main samples include all paid employees, aged between 15 and 64 , who are not full-time students. Survey sampling weights (abowt) applied to account for complex survey design: stratification, clustering and non-response. The counts represent pooled populations for the recession time period. Standard deviations reported in parentheses. 
Appendix A 5: Mean Characteristics of Laid-Off Workers in the Restricted Samples, by Identity

\begin{tabular}{|c|c|c|c|c|c|c|c|c|}
\hline \multirow[t]{2}{*}{ Characteristics } & \multicolumn{2}{|c|}{$\begin{array}{c}\text { Non-Aboriginal } \\
\text { Identity }\end{array}$} & \multicolumn{2}{|c|}{$\begin{array}{c}\text { Aboriginal } \\
\text { Identity }\end{array}$} & \multicolumn{2}{|c|}{ Métis } & \multicolumn{2}{|c|}{ North American Indian } \\
\hline & Men & Women & Men & Women & Men & Women & Men & Women \\
\hline Less than h-school & $\begin{array}{c}0.22 \\
(0.41)\end{array}$ & $\begin{array}{c}0.14 \\
(0.35)\end{array}$ & $\begin{array}{c}0.27 \\
(0.49)\end{array}$ & $\begin{array}{c}0.24 \\
(0.45)\end{array}$ & $\begin{array}{c}0.25 \\
(0.43)\end{array}$ & $\begin{array}{c}0.22 \\
(0.41)\end{array}$ & $\begin{array}{c}0.30 \\
(0.46)\end{array}$ & $\begin{array}{c}0.26 \\
(0.45)\end{array}$ \\
\hline High school & $\begin{array}{c}0.25 \\
(0.43)\end{array}$ & $\begin{array}{c}0.25 \\
(0.43)\end{array}$ & $\begin{array}{c}0.28 \\
(0.45)\end{array}$ & $\begin{array}{c}0.22 \\
(0.41)\end{array}$ & $\begin{array}{c}0.28 \\
(0.45)\end{array}$ & $\begin{array}{c}0.22 \\
(0.42)\end{array}$ & $\begin{array}{c}0.29 \\
(0.45)\end{array}$ & $\begin{array}{c}0.23 \\
(0.42)\end{array}$ \\
\hline Incomplete pse & $\begin{array}{c}0.86 \\
(0.28)\end{array}$ & $\begin{array}{c}0.07 \\
(0.26)\end{array}$ & $\begin{array}{c}0.08 \\
(0.28)\end{array}$ & $\begin{array}{c}0.09 \\
(0.28)\end{array}$ & $\begin{array}{c}0.10 \\
(0.30)\end{array}$ & $\begin{array}{c}0.09 \\
(0.29)\end{array}$ & $\begin{array}{c}0.08 \\
(0.27)\end{array}$ & $\begin{array}{c}0.08 \\
(0.28)\end{array}$ \\
\hline Completed trade & $\begin{array}{c}0.20 \\
(0.40)\end{array}$ & $\begin{array}{c}0.10 \\
(0.30)\end{array}$ & $\begin{array}{c}0.20 \\
(0.40)\end{array}$ & $\begin{array}{c}0.11 \\
(0.31)\end{array}$ & $\begin{array}{c}0.26 \\
(0.44)\end{array}$ & $\begin{array}{c}0.14 \\
(0.34)\end{array}$ & $\begin{array}{c}0.13 \\
(0.34)\end{array}$ & $\begin{array}{c}0.09 \\
(0.28)\end{array}$ \\
\hline Completed college & $\begin{array}{c}0.15 \\
(0.36)\end{array}$ & $\begin{array}{c}0.25 \\
(0.43)\end{array}$ & $\begin{array}{c}0.14 \\
(0.34)\end{array}$ & $\begin{array}{c}0.26 \\
(0.44)\end{array}$ & $\begin{array}{c}0.09 \\
(0.28)\end{array}$ & $\begin{array}{c}0.24 \\
(0.43)\end{array}$ & $\begin{array}{c}0.18 \\
(0.38)\end{array}$ & $\begin{array}{c}0.27 \\
(0.45)\end{array}$ \\
\hline University degree & $\begin{array}{c}0.09 \\
(0.29)\end{array}$ & $\begin{array}{c}0.18 \\
(0.38)\end{array}$ & $\begin{array}{c}0.02 \\
(0.15)\end{array}$ & $\begin{array}{c}0.08 \\
(0.27)\end{array}$ & $\begin{array}{c}0.03 \\
(0.18)\end{array}$ & $\begin{array}{c}0.08 \\
(0.28)\end{array}$ & $\begin{array}{c}0.02 \\
(0.13)\end{array}$ & $\begin{array}{c}0.06 \\
(0.24)\end{array}$ \\
\hline Youth 15-24 & $\begin{array}{c}0.21 \\
(0.41)\end{array}$ & $\begin{array}{c}0.15 \\
(0.35)\end{array}$ & $\begin{array}{c}0.27 \\
(0.44)\end{array}$ & $\begin{array}{c}0.16 \\
(0.37)\end{array}$ & $\begin{array}{c}0.28 \\
(0.45)\end{array}$ & $\begin{array}{c}0.19 \\
(0.39)\end{array}$ & $\begin{array}{c}0.26 \\
(0.44)\end{array}$ & $\begin{array}{c}0.13 \\
(0.34)\end{array}$ \\
\hline Young 25-34 & $\begin{array}{c}0.23 \\
(0.42)\end{array}$ & $\begin{array}{c}0.19 \\
(0.39)\end{array}$ & $\begin{array}{c}0.26 \\
(0.44)\end{array}$ & $\begin{array}{c}0.23 \\
(0.42)\end{array}$ & $\begin{array}{c}0.21 \\
(0.40)\end{array}$ & $\begin{array}{c}0.26 \\
(0.44)\end{array}$ & $\begin{array}{c}0.31 \\
(0.46)\end{array}$ & $\begin{array}{c}0.20 \\
(0.40)\end{array}$ \\
\hline Young prime $35-44$ & $\begin{array}{c}0.19 \\
(0.39)\end{array}$ & $\begin{array}{c}0.21 \\
(0.41)\end{array}$ & $\begin{array}{c}0.20 \\
(0.40)\end{array}$ & $\begin{array}{c}0.26 \\
(0.44)\end{array}$ & $\begin{array}{c}0.21 \\
(0.41)\end{array}$ & $\begin{array}{c}0.22 \\
(0.42)\end{array}$ & $\begin{array}{c}0.18 \\
(0.39)\end{array}$ & $\begin{array}{c}0.29 \\
(0.45)\end{array}$ \\
\hline Mature prime 45-54 & $\begin{array}{c}0.21 \\
(0.41)\end{array}$ & $\begin{array}{c}0.26 \\
(0.44)\end{array}$ & $\begin{array}{c}0.17 \\
(0.37)\end{array}$ & $\begin{array}{c}0.20 \\
(0.40)\end{array}$ & $\begin{array}{c}0.18 \\
(0.38)\end{array}$ & $\begin{array}{c}0.21 \\
(0.41)\end{array}$ & $\begin{array}{c}0.16 \\
(0.36)\end{array}$ & $\begin{array}{l}0.20 \\
(0.40)\end{array}$ \\
\hline Older worker 55-64 & $\begin{array}{c}0.16 \\
(0.36)\end{array}$ & $\begin{array}{c}0.19 \\
(0.39)\end{array}$ & $\begin{array}{c}0.11 \\
(0.31)\end{array}$ & $\begin{array}{c}0.15 \\
(036)\end{array}$ & $\begin{array}{c}0.12 \\
(0.33)\end{array}$ & $\begin{array}{l}0.12 \\
0.32)\end{array}$ & $\begin{array}{c}0.09 \\
(0.28)\end{array}$ & $\begin{array}{c}0.18 \\
(0.39)\end{array}$ \\
\hline Married & $\begin{array}{c}0.52 \\
(0.50)\end{array}$ & $\begin{array}{c}0.62 \\
(0.48)\end{array}$ & $\begin{array}{c}0.47 \\
(0.50)\end{array}$ & $\begin{array}{c}0.61 \\
(0.49)\end{array}$ & $\begin{array}{c}0.47 \\
(0.50)\end{array}$ & $\begin{array}{c}0.59 \\
(0.49)\end{array}$ & $\begin{array}{c}0.47 \\
(0.50)\end{array}$ & $\begin{array}{c}0.63 \\
(0.48)\end{array}$ \\
\hline Widowed divisep & $\begin{array}{c}0.07 \\
(0.26)\end{array}$ & $\begin{array}{c}0.12 \\
(0.32)\end{array}$ & $\begin{array}{c}0.08 \\
(0.27)\end{array}$ & $\begin{array}{c}0.09 \\
(0.29)\end{array}$ & $\begin{array}{c}0.08 \\
(0.27)\end{array}$ & $\begin{array}{c}0.11 \\
(0.31)\end{array}$ & $\begin{array}{c}0.08 \\
(0.27)\end{array}$ & $\begin{array}{c}0.08 \\
(0.27)\end{array}$ \\
\hline Single & $\begin{array}{c}0.41 \\
(0.49)\end{array}$ & $\begin{array}{c}0.25 \\
(0.44)\end{array}$ & $\begin{array}{c}0.45 \\
(0.50)\end{array}$ & $\begin{array}{c}0.30 \\
(0.46)\end{array}$ & $\begin{array}{c}0.45 \\
(0.50)\end{array}$ & $\begin{array}{c}0.30 \\
(0.46)\end{array}$ & $\begin{array}{c}0.45 \\
(0.50)\end{array}$ & $\begin{array}{c}0.30 \\
(0.46)\end{array}$ \\
\hline No children present & $\begin{array}{c}0.71 \\
(0.45)\end{array}$ & $\begin{array}{c}0.60 \\
(0.49)\end{array}$ & $\begin{array}{c}0.69 \\
(0.46)\end{array}$ & $\begin{array}{c}0.50 \\
(0.50)\end{array}$ & $\begin{array}{c}0.67 \\
(0.47)\end{array}$ & $\begin{array}{c}0.55 \\
(0.50)\end{array}$ & $\begin{array}{c}0.71 \\
(0.45)\end{array}$ & $\begin{array}{c}0.46 \\
(0.50)\end{array}$ \\
\hline Children $<6$ & $\begin{array}{c}0.11 \\
(0.31)\end{array}$ & $\begin{array}{c}0.11 \\
(0.31)\end{array}$ & $\begin{array}{c}0.14 \\
(0.35)\end{array}$ & $\begin{array}{c}0.15 \\
(0.36)\end{array}$ & $\begin{array}{c}0.13 \\
(0.34)\end{array}$ & $\begin{array}{c}0.16 \\
(0.37)\end{array}$ & $\begin{array}{c}0.15 \\
(0.36)\end{array}$ & $\begin{array}{c}0.13 \\
(0.34)\end{array}$ \\
\hline Children 6-14 & $\begin{array}{c}0.09 \\
(0.29)\end{array}$ & $\begin{array}{c}0.16 \\
(0.37)\end{array}$ & $\begin{array}{c}0.10 \\
(0.29)\end{array}$ & $\begin{array}{c}0.22 \\
(0.41)\end{array}$ & $\begin{array}{c}0.11 \\
(0.31)\end{array}$ & $\begin{array}{c}0.15 \\
(0.36)\end{array}$ & $\begin{array}{c}0.08 \\
(0.27)\end{array}$ & $\begin{array}{c}0.27 \\
(0.44)\end{array}$ \\
\hline Children 14-24 & $\begin{array}{c}0.08 \\
(0.28)\end{array}$ & $\begin{array}{c}0.13 \\
(0.33)\end{array}$ & $\begin{array}{c}0.07 \\
(0.25)\end{array}$ & $\begin{array}{c}0.13 \\
(0.33)\end{array}$ & $\begin{array}{c}0.09 \\
(0.29)\end{array}$ & $\begin{array}{c}0.12 \\
(0.33)\end{array}$ & $\begin{array}{c}0.05 \\
(0.22)\end{array}$ & $\begin{array}{c}0.13 \\
(0.34)\end{array}$ \\
\hline
\end{tabular}




\begin{tabular}{|c|c|c|c|c|c|c|c|c|}
\hline \multirow{2}{*}{$\begin{array}{l}\text { Appendix A5 } \\
\text { (continued) }\end{array}$} & \multicolumn{2}{|c|}{$\begin{array}{c}\text { Non-Aboriginal } \\
\text { Identity }\end{array}$} & \multicolumn{2}{|c|}{$\begin{array}{c}\text { Aboriginal } \\
\text { Identity }\end{array}$} & \multicolumn{2}{|c|}{ Métis } & \multicolumn{2}{|c|}{ North American Indian } \\
\hline & Men & Women & Men & Women & Men & Women & Men & Women \\
\hline Atlantic provinces & $\begin{array}{c}0.14 \\
(0.34)\end{array}$ & $\begin{array}{c}0.14 \\
(0.34)\end{array}$ & $\begin{array}{c}0.13 \\
(0.33)\end{array}$ & $\begin{array}{c}0.09 \\
(0.29)\end{array}$ & $\begin{array}{c}0.10 \\
(0.30)\end{array}$ & $\begin{array}{c}0.07 \\
(0.26)\end{array}$ & $\begin{array}{c}0.13 \\
(0.34)\end{array}$ & $\begin{array}{c}0.10 \\
(0.30)\end{array}$ \\
\hline Quebec & $\begin{array}{c}0.30 \\
(0.46)\end{array}$ & $\begin{array}{c}0.28 \\
(0.45)\end{array}$ & $\begin{array}{c}0.08 \\
(0.29)\end{array}$ & $\begin{array}{c}0.10 \\
(0.30)\end{array}$ & $\begin{array}{c}0.13 \\
(0.33)\end{array}$ & $\begin{array}{c}0.15 \\
(0.35)\end{array}$ & $\begin{array}{c}0.05 \\
(0.22)\end{array}$ & $\begin{array}{c}0.07 \\
(0.25)\end{array}$ \\
\hline Ontario & $\begin{array}{c}0.32 \\
(0.47)\end{array}$ & $\begin{array}{c}0.32 \\
(0.47)\end{array}$ & $\begin{array}{c}0.22 \\
(0.42)\end{array}$ & $\begin{array}{c}0.24 \\
(0.43)\end{array}$ & $\begin{array}{c}0.17 \\
(0.37)\end{array}$ & $\begin{array}{c}0.15 \\
(0.36)\end{array}$ & $\begin{array}{c}0.29 \\
(0.45)\end{array}$ & $\begin{array}{c}0.33 \\
(0.47)\end{array}$ \\
\hline Manitoba & $\begin{array}{c}0.02 \\
(0.14)\end{array}$ & $\begin{array}{c}0.03 \\
(0.16)\end{array}$ & $\begin{array}{c}0.09 \\
(0.29)\end{array}$ & $\begin{array}{c}0.11 \\
(0.32)\end{array}$ & $\begin{array}{c}0.12 \\
(0.33)\end{array}$ & $\begin{array}{c}0.14 \\
(0.35)\end{array}$ & $\begin{array}{c}0.07 \\
(0.26)\end{array}$ & $\begin{array}{c}0.09 \\
(0.28)\end{array}$ \\
\hline Saskatchewan & $\begin{array}{c}0.02 \\
(0.14)\end{array}$ & $\begin{array}{c}0.03 \\
(0.16)\end{array}$ & $\begin{array}{c}0.08 \\
(0.27)\end{array}$ & $\begin{array}{c}0.08 \\
(0.27)\end{array}$ & $\begin{array}{c}0.10 \\
(0.30)\end{array}$ & $\begin{array}{c}0.14 \\
(0.35)\end{array}$ & $\begin{array}{c}0.07 \\
(0.26)\end{array}$ & $\begin{array}{c}0.02 \\
(0.16)\end{array}$ \\
\hline Alberta & $\begin{array}{c}0.09 \\
(0.29)\end{array}$ & $\begin{array}{c}0.10 \\
(0.29)\end{array}$ & $\begin{array}{c}0.24 \\
(0.43)\end{array}$ & $\begin{array}{c}0.23 \\
(0.42)\end{array}$ & $\begin{array}{c}0.30 \\
(0.46)\end{array}$ & $\begin{array}{c}0.24 \\
(0.43)\end{array}$ & $\begin{array}{c}0.18 \\
(0.39)\end{array}$ & $\begin{array}{c}0.21 \\
(0.41)\end{array}$ \\
\hline British Columbia & $\begin{array}{c}0.11 \\
(0.31)\end{array}$ & $\begin{array}{c}0.10 \\
(0.30)\end{array}$ & $\begin{array}{c}0.14 \\
(0.35)\end{array}$ & $\begin{array}{c}0.13 \\
(0.34)\end{array}$ & $\begin{array}{c}0.08 \\
(0.28)\end{array}$ & $\begin{array}{c}0.10 \\
(0.30)\end{array}$ & $\begin{array}{c}0.20 \\
(0.40)\end{array}$ & $\begin{array}{c}0.17 \\
(0.38)\end{array}$ \\
\hline $\begin{array}{l}\text { Primary } \\
\text { industries }\end{array}$ & $\begin{array}{c}0.33 \\
(0.47)\end{array}$ & $\begin{array}{c}0.06 \\
(0.24)\end{array}$ & $\begin{array}{c}0.46 \\
(0.50)\end{array}$ & $\begin{array}{c}0.11 \\
(0.31)\end{array}$ & $\begin{array}{c}0.46 \\
(0.50)\end{array}$ & $\begin{array}{c}0.15 \\
(0.36)\end{array}$ & $\begin{array}{c}0.45 \\
(0.50)\end{array}$ & $\begin{array}{c}0.06 \\
(0.24)\end{array}$ \\
\hline Manufacturing & $\begin{array}{c}0.19 \\
(0.40)\end{array}$ & $\begin{array}{c}0.11 \\
(0.32)\end{array}$ & $\begin{array}{c}0.12 \\
(0.32)\end{array}$ & $\begin{array}{c}0.09 \\
(0.29)\end{array}$ & $\begin{array}{c}0.13 \\
(0.34)\end{array}$ & $\begin{array}{c}0.06 \\
(0.23)\end{array}$ & $\begin{array}{c}0.11 \\
(0.31)\end{array}$ & $\begin{array}{c}0.12 \\
(0.33)\end{array}$ \\
\hline Retail & $\begin{array}{c}0.16 \\
(0.37)\end{array}$ & $\begin{array}{c}0.25 \\
(0.43)\end{array}$ & $\begin{array}{c}0.13 \\
(0.33)\end{array}$ & $\begin{array}{c}0.34 \\
(0.47)\end{array}$ & $\begin{array}{c}0.13 \\
(0.34)\end{array}$ & $\begin{array}{c}0.35 \\
(0.48)\end{array}$ & $\begin{array}{c}0.12 \\
(0.33)\end{array}$ & $\begin{array}{c}0.32 \\
(0.47)\end{array}$ \\
\hline High skill services & $\begin{array}{c}0.11 \\
(0.31)\end{array}$ & $\begin{array}{c}0.14 \\
(0.34)\end{array}$ & $\begin{array}{c}0.11 \\
(0.31)\end{array}$ & $\begin{array}{c}0.10 \\
(0.31)\end{array}$ & $\begin{array}{c}0.08 \\
(0.28)\end{array}$ & $\begin{array}{c}0.10 \\
(0.29)\end{array}$ & $\begin{array}{c}0.13 \\
(0.33)\end{array}$ & $\begin{array}{c}0.11 \\
(0.32)\end{array}$ \\
\hline Public services & $\begin{array}{c}0.06 \\
(0.25)\end{array}$ & $\begin{array}{c}0.31 \\
(0.46)\end{array}$ & $\begin{array}{c}0.06 \\
(0.23)\end{array}$ & $\begin{array}{c}0.29 \\
(0.45)\end{array}$ & $\begin{array}{c}0.06 \\
(0.23)\end{array}$ & $\begin{array}{c}0.27 \\
(0.44)\end{array}$ & $\begin{array}{c}0.06 \\
(0.24)\end{array}$ & $\begin{array}{c}0.32 \\
(0.47)\end{array}$ \\
\hline Other services & $\begin{array}{c}0.14 \\
(0.34)\end{array}$ & $\begin{array}{c}0.12 \\
(0.32)\end{array}$ & $\begin{array}{c}0.13 \\
(0.34)\end{array}$ & $\begin{array}{c}0.07 \\
(0.25)\end{array}$ & $\begin{array}{c}0.13 \\
(0.34)\end{array}$ & $\begin{array}{c}0.08 \\
(0.27)\end{array}$ & $\begin{array}{c}0.13 \\
(0.34)\end{array}$ & $\begin{array}{c}0.05 \\
(0.23)\end{array}$ \\
\hline $\begin{array}{l}\text { Management } \\
\text { occup }\end{array}$ & $\begin{array}{c}0.04 \\
(0.19)\end{array}$ & $\begin{array}{c}0.04 \\
(0.19)\end{array}$ & $\begin{array}{c}0.02 \\
(0.16)\end{array}$ & $\begin{array}{c}0.03 \\
(0.18)\end{array}$ & $\begin{array}{c}0.02 \\
(0.15)\end{array}$ & $\begin{array}{c}0.04 \\
(0.19)\end{array}$ & $\begin{array}{c}0.03 \\
(0.16)\end{array}$ & $\begin{array}{c}0.03 \\
(0.16)\end{array}$ \\
\hline Professionals & $\begin{array}{c}0.05 \\
(0.22)\end{array}$ & $\begin{array}{c}0.13 \\
(0.33)\end{array}$ & $\begin{array}{c}0.02 \\
(0.13)\end{array}$ & $\begin{array}{c}0.08 \\
(0.27)\end{array}$ & $\begin{array}{c}0.01 \\
(0.10)\end{array}$ & $\begin{array}{c}0.07 \\
(0.26)\end{array}$ & $\begin{array}{c}0.03 \\
(0.16)\end{array}$ & $\begin{array}{c}0.09 \\
(0.28)\end{array}$ \\
\hline $\begin{array}{l}\text { Semi- } \\
\text { professionals }\end{array}$ & $\begin{array}{c}0.05 \\
(0.23)\end{array}$ & $\begin{array}{c}0.08 \\
(0.27)\end{array}$ & $\begin{array}{c}0.05 \\
(0.23)\end{array}$ & $\begin{array}{c}0.07 \\
(0.26)\end{array}$ & $\begin{array}{c}0.03 \\
(0.17)\end{array}$ & $\begin{array}{c}0.05 \\
(0.22)\end{array}$ & $\begin{array}{c}0.08 \\
(0.27)\end{array}$ & $\begin{array}{c}0.09 \\
(0.28)\end{array}$ \\
\hline Sales personnel & $\begin{array}{c}0.21 \\
(0.41)\end{array}$ & $\begin{array}{c}0.58 \\
(0.49)\end{array}$ & $\begin{array}{c}0.20 \\
(0.40)\end{array}$ & $\begin{array}{c}0.63 \\
(0.48)\end{array}$ & $\begin{array}{c}0.22 \\
(0.41)\end{array}$ & $\begin{array}{c}0.67 \\
(0.47)\end{array}$ & $\begin{array}{c}0.18 \\
(0.38)\end{array}$ & $\begin{array}{c}0.60 \\
(0.49)\end{array}$ \\
\hline Manual workers & $\begin{array}{c}0.63 \\
(0.48)\end{array}$ & $\begin{array}{c}0.14 \\
(0.35)\end{array}$ & $\begin{array}{c}0.69 \\
(0.46)\end{array}$ & $\begin{array}{c}0.16 \\
(0.37)\end{array}$ & $\begin{array}{c}0.70 \\
(0.46)\end{array}$ & $\begin{array}{c}0.16 \\
(0.36)\end{array}$ & $\begin{array}{c}0.68 \\
(0.47)\end{array}$ & $\begin{array}{c}0.17 \\
(0.38)\end{array}$ \\
\hline Union & $\begin{array}{c}0.34 \\
(0.47)\end{array}$ & $\begin{array}{c}0.34 \\
(0.47)\end{array}$ & $\begin{array}{c}0.21 \\
(0.41)\end{array}$ & $\begin{array}{c}0.26 \\
(0.44)\end{array}$ & $\begin{array}{c}0.19 \\
(0.40)\end{array}$ & $\begin{array}{c}0.25 \\
(0.44)\end{array}$ & $\begin{array}{c}0.21 \\
(0.41)\end{array}$ & $\begin{array}{c}0.29 \\
(0.46)\end{array}$ \\
\hline
\end{tabular}




\begin{tabular}{|c|c|c|c|c|c|c|c|c|}
\hline \multirow{2}{*}{$\begin{array}{l}\text { Appendix A5 } \\
\text { (continued) }\end{array}$} & \multicolumn{2}{|c|}{$\begin{array}{l}\text { Non-Aboriginal } \\
\text { Identity }\end{array}$} & \multicolumn{2}{|c|}{$\begin{array}{c}\text { Aboriginal } \\
\text { Identity }\end{array}$} & \multicolumn{2}{|c|}{ Métis } & \multicolumn{2}{|c|}{ North American Indian } \\
\hline & Men & Women & Men & Women & Men & Women & Men & Women \\
\hline Small workplace & $\begin{array}{c}0.76 \\
(0.43)\end{array}$ & $\begin{array}{c}0.78 \\
(0.41)\end{array}$ & $\begin{array}{c}0.86 \\
(0.34)\end{array}$ & $\begin{array}{c}0.86 \\
(0.35)\end{array}$ & $\begin{array}{c}0.86 \\
(0.34)\end{array}$ & $\begin{array}{c}0.92 \\
(0.28)\end{array}$ & $\begin{array}{c}0.86 \\
(0.34)\end{array}$ & $\begin{array}{c}0.79 \\
(0.41)\end{array}$ \\
\hline $\begin{array}{l}\text { Medium } \\
\text { workplace }\end{array}$ & $\begin{array}{c}0.18 \\
(0.38)\end{array}$ & $\begin{array}{c}0.16 \\
(0.37)\end{array}$ & $\begin{array}{c}0.08 \\
(0.27)\end{array}$ & $\begin{array}{c}0.10 \\
(0.31)\end{array}$ & $\begin{array}{c}0.09 \\
(0.29)\end{array}$ & $\begin{array}{c}0.05 \\
(0.23)\end{array}$ & $\begin{array}{c}0.06 \\
(0.24)\end{array}$ & $\begin{array}{c}0.17 \\
(0.38)\end{array}$ \\
\hline Large workplace & $\begin{array}{c}0.07 \\
(0.25)\end{array}$ & $\begin{array}{c}0.06 \\
(0.24)\end{array}$ & $\begin{array}{c}0.06 \\
(0.23)\end{array}$ & $\begin{array}{c}0.03 \\
(0.19)\end{array}$ & $\begin{array}{c}0.04 \\
(0.20)\end{array}$ & $\begin{array}{c}0.03 \\
(0.17)\end{array}$ & $\begin{array}{c}0.07 \\
(0.26)\end{array}$ & $\begin{array}{c}0.05 \\
(0.21)\end{array}$ \\
\hline Private sector & $\begin{array}{c}0.92 \\
(0.26)\end{array}$ & $\begin{array}{c}0.74 \\
(0.44)\end{array}$ & $\begin{array}{c}0.89 \\
(0.31)\end{array}$ & $\begin{array}{c}0.75 \\
(0.43)\end{array}$ & $\begin{array}{c}0.89 \\
(0.31)\end{array}$ & $\begin{array}{c}0.76 \\
(0.43)\end{array}$ & $\begin{array}{c}0.89 \\
(0.31)\end{array}$ & $\begin{array}{l}0.72 \\
0.45)\end{array}$ \\
\hline Full-time job & $\begin{array}{c}0.87 \\
(0.34)\end{array}$ & $\begin{array}{c}0.68 \\
(0.46)\end{array}$ & $\begin{array}{c}0.87 \\
(0.33)\end{array}$ & $\begin{array}{c}0.73 \\
(0.45)\end{array}$ & $\begin{array}{c}0.92 \\
(0.27)\end{array}$ & $\begin{array}{c}0.73 \\
(0.44)\end{array}$ & $\begin{array}{c}0.83 \\
(0.37)\end{array}$ & $\begin{array}{c}0.72 \\
(0.45)\end{array}$ \\
\hline Wage minimum & $\begin{array}{c}0.04 \\
(0.19)\end{array}$ & $\begin{array}{c}0.08 \\
(0.27)\end{array}$ & $\begin{array}{c}0.04 \\
(0.19)\end{array}$ & $\begin{array}{c}0.10 \\
(0.31)\end{array}$ & $\begin{array}{c}0.04 \\
(0.20)\end{array}$ & $\begin{array}{c}0.09 \\
(0.28)\end{array}$ & $\begin{array}{c}0.04 \\
(0.19)\end{array}$ & $\begin{array}{c}0.12 \\
(0.33)\end{array}$ \\
\hline Wage median & $\begin{array}{c}0.06 \\
(0.24)\end{array}$ & $\begin{array}{c}0.08 \\
(0.27)\end{array}$ & $\begin{array}{c}0.04 \\
(0.20)\end{array}$ & $\begin{array}{c}0.08 \\
(0.27)\end{array}$ & $\begin{array}{c}0.05 \\
(0.23)\end{array}$ & $\begin{array}{c}0.09 \\
(0.28)\end{array}$ & $\begin{array}{c}0.03 \\
(0.17)\end{array}$ & $\begin{array}{c}0.06 \\
(0.25)\end{array}$ \\
\hline Wage average & $\begin{array}{c}0.15 \\
(0.36)\end{array}$ & $\begin{array}{c}0.15 \\
(0.36)\end{array}$ & $\begin{array}{c}0.14 \\
(0.35)\end{array}$ & $\begin{array}{c}0.11 \\
(0.31)\end{array}$ & $\begin{array}{c}0.16 \\
(0.37)\end{array}$ & $\begin{array}{c}0.13 \\
(0.34)\end{array}$ & $\begin{array}{c}0.13 \\
(0.34)\end{array}$ & $\begin{array}{c}0.09 \\
(0.29)\end{array}$ \\
\hline Wage high & $\begin{array}{c}0.74 \\
(0.44)\end{array}$ & $\begin{array}{c}0.68 \\
(0.47)\end{array}$ & $\begin{array}{c}0.75 \\
(0.43)\end{array}$ & $\begin{array}{c}0.70 \\
(0.45)\end{array}$ & $\begin{array}{c}0.72 \\
(0.45)\end{array}$ & $\begin{array}{c}0.69 \\
(0.46)\end{array}$ & $\begin{array}{c}0.77 \\
(0.42)\end{array}$ & $\begin{array}{c}0.72 \\
(0.45)\end{array}$ \\
\hline Sample Size & 11332 & 7531 & 626 & 440 & 295 & 208 & 317 & 226 \\
\hline Weighted counts & 2705953 & 1802314 & 102590 & 68354 & 47108 & 33702 & 52995 & 34343 \\
\hline
\end{tabular}

Source: The Labour Force Survey, national monthly master file, September 2008 - May 2011.

Note: Restricted samples include only permanent paid employees, aged between 15 and 64, who are single-job holders and who are not students. Survey sampling weights (abowt) applied in all analyses to account for complex survey design: stratification, clustering and non-response. The counts represent pooled populations for the recession time period. Standard deviations reported in parentheses. 


\section{Appendix B}

Appendix B 1: Probit estimates used in decompositions of difference in layoffs for men

\begin{tabular}{|c|c|c|c|c|c|c|}
\hline \multirow{2}{*}{$\begin{array}{l}\text { Variables in the } \\
\text { equations }\end{array}$} & \multicolumn{2}{|c|}{$\begin{array}{l}\text { Non-Aboriginal } \\
\text { Men }\end{array}$} & \multicolumn{2}{|c|}{$\begin{array}{l}\text { Métis } \\
\text { Men }\end{array}$} & \multicolumn{2}{|c|}{$\begin{array}{c}\text { North American Indian } \\
\text { Men }\end{array}$} \\
\hline & $\begin{array}{c}\text { main } \\
\text { sample }\end{array}$ & $\begin{array}{l}\text { restricted } \\
\text { sample }\end{array}$ & $\begin{array}{c}\text { main } \\
\text { sample }\end{array}$ & $\begin{array}{l}\text { restricted } \\
\text { sample }\end{array}$ & $\begin{array}{c}\text { main } \\
\text { sample } \\
\end{array}$ & $\begin{array}{c}\text { restricted } \\
\text { sample }\end{array}$ \\
\hline $\begin{array}{l}\text { Less than } \\
\text { high school diploma }\end{array}$ & $\begin{array}{c}0.123 \\
(0.03)^{* * *}\end{array}$ & $\begin{array}{c}0.131 \\
(0.03)^{\ldots+1}\end{array}$ & $\begin{array}{l}0.037 \\
(0.17)\end{array}$ & $\begin{array}{l}0.044 \\
(0.20)\end{array}$ & $\begin{array}{r}-0.190 \\
(0.19)\end{array}$ & $\begin{array}{l}-0.274 \\
(0.21)\end{array}$ \\
\hline $\begin{array}{l}\text { Incomplete } \\
\text { postsecondary }\end{array}$ & $\begin{array}{l}0.025 \\
(0.04)\end{array}$ & $\begin{array}{l}-0.029 \\
(0.04)\end{array}$ & $\begin{array}{l}-0.102 \\
(0.20)\end{array}$ & $\begin{array}{l}-0.021 \\
(0.23)\end{array}$ & $\begin{array}{l}-0.504 \\
(0.25)^{*}\end{array}$ & $\begin{array}{l}-0.682 \\
(0.32)^{\star}\end{array}$ \\
\hline $\begin{array}{l}\text { Completed } \\
\text { trade certificate }\end{array}$ & $\begin{array}{l}-0.029 \\
(0.03)\end{array}$ & $\begin{array}{l}-0.044 \\
(0.03)\end{array}$ & $\begin{array}{l}0.073 \\
(0.19)\end{array}$ & $\begin{array}{l}0.178 \\
(0.21)\end{array}$ & $\begin{array}{l}-0.320 \\
(0.20)\end{array}$ & $\begin{array}{c}-0.684 \\
(0.25)^{\star \star}\end{array}$ \\
\hline $\begin{array}{l}\text { Completed } \\
\text { college diploma }\end{array}$ & $\begin{array}{c}-0.147 \\
(0.03)^{\star \star \star}\end{array}$ & $\begin{array}{c}-0.138 \\
(0.03)^{\star \star *}\end{array}$ & $\begin{array}{l}-0.065 \\
(0.20)\end{array}$ & $\begin{array}{l}-0.283 \\
(0.25)\end{array}$ & $\begin{array}{r}-0.205 \\
(0.21)\end{array}$ & $\begin{array}{l}0.049 \\
(0.22)\end{array}$ \\
\hline $\begin{array}{l}\text { Completed } \\
\text { university degree }\end{array}$ & $\begin{array}{c}-0.169 \\
(0.04)^{* * \star}\end{array}$ & $\begin{array}{c}-0.125 \\
(0.05)^{\star \star}\end{array}$ & $\begin{array}{l}-0.406 \\
(0.28)\end{array}$ & $\begin{array}{l}-0.439 \\
(0.37)\end{array}$ & $\begin{array}{c}-1.160 \\
(0.44)^{\star \star}\end{array}$ & $\begin{array}{l}-0.560 \\
(0.41)\end{array}$ \\
\hline Tenure $<2$ years & $\begin{array}{c}0.608 \\
(0.03)^{\star \star \star}\end{array}$ & $\begin{array}{c}0.591 \\
(0.04)^{\star \star * *}\end{array}$ & $\begin{array}{c}0.588 \\
(0.25)^{*}\end{array}$ & $\begin{array}{c}0.670 \\
(0.33)^{*}\end{array}$ & $\begin{array}{c}0.958 \\
(0.28)^{\star \star}\end{array}$ & $\begin{array}{c}0.684 \\
(0.30)^{*}\end{array}$ \\
\hline Tenure $2-5$ years & $\begin{array}{c}0.363 \\
(0.04)^{\star \star \star}\end{array}$ & $\begin{array}{c}0.539 \\
(0.04)^{* \star \star}\end{array}$ & $\begin{array}{l}0.212 \\
(0.26)\end{array}$ & $\begin{array}{l}0.499 \\
(0.32)\end{array}$ & $\begin{array}{c}0.564 \\
(0.29)^{*}\end{array}$ & $\begin{array}{l}0.494 \\
(0.30)\end{array}$ \\
\hline Tenure $5-10$ years & $\begin{array}{c}0.247 \\
(0.04)^{* * *}\end{array}$ & $\begin{array}{c}0.228 \\
(0.04)^{* * *}\end{array}$ & $\begin{array}{l}0.187 \\
(0.27)\end{array}$ & $\begin{array}{l}0.100 \\
(0.35)\end{array}$ & $\begin{array}{l}0.478 \\
(0.31)\end{array}$ & $\begin{array}{l}0.543 \\
(0.31)\end{array}$ \\
\hline Tenure $10-20$ years & $\begin{array}{c}0.167 \\
(0.04)^{* * *}\end{array}$ & $\begin{array}{c}0.162 \\
(0.04)^{\star \star \star}\end{array}$ & $\begin{array}{l}0.218 \\
(0.27)\end{array}$ & $\begin{array}{l}0.299 \\
(0.33)\end{array}$ & $\begin{array}{l}0.431 \\
(0.30)\end{array}$ & $\begin{array}{l}0.190 \\
(0.34)\end{array}$ \\
\hline Absent 1 week & $\begin{array}{l}0.002 \\
(0.06)\end{array}$ & $\begin{array}{l}-0.039 \\
(0.06)\end{array}$ & $\begin{array}{r}-0.144 \\
(0.27)\end{array}$ & $\begin{array}{r}-0.246 \\
(0.40)\end{array}$ & $\begin{array}{r}-0.255 \\
(0.35)\end{array}$ & - \\
\hline Absent 2 weeks & $\begin{array}{l}-0.008 \\
(0.09)\end{array}$ & $\begin{array}{l}-0.093 \\
(0.11)\end{array}$ & $\begin{array}{l}0.108 \\
(0.42)\end{array}$ & $\begin{array}{l}0.567 \\
(0.42)\end{array}$ & $\begin{array}{l}-0.196 \\
(0.66)\end{array}$ & - \\
\hline Absent 3 weeks & $\begin{array}{r}-0.095 \\
(0.12)\end{array}$ & $\begin{array}{r}-0.083 \\
(0.13)\end{array}$ & $\begin{array}{l}0.075 \\
(0.62)\end{array}$ & $\begin{array}{l}0.894 \\
(0.56)\end{array}$ & $\begin{array}{r}-0.062 \\
(0.46)\end{array}$ & $\begin{array}{l}0.136 \\
(0.66)\end{array}$ \\
\hline Absent 4 to 8 weeks & $\begin{array}{c}0.209 \\
(0.10)^{\star}\end{array}$ & $\begin{array}{c}0.329 \\
(0.11)^{\star \star}\end{array}$ & $\begin{array}{l}-0.156 \\
(0.37)\end{array}$ & $\begin{array}{l}-0.336 \\
(0.55)\end{array}$ & $\begin{array}{l}-0.245 \\
(0.54)\end{array}$ & $\begin{array}{l}0.442 \\
(0.47)\end{array}$ \\
\hline Absent > 8 weeks & $\begin{array}{l}-0.022 \\
(0.10)\end{array}$ & $\begin{array}{l}0.052 \\
(0.10)\end{array}$ & $\begin{array}{l}-0.570 \\
(0.69)\end{array}$ & $\begin{array}{l}0.222 \\
(0.48)\end{array}$ & $\begin{array}{l}-0.145 \\
(0.24)\end{array}$ & $\begin{array}{l}-0.061 \\
(0.49)\end{array}$ \\
\hline Age 15-24 & $\begin{array}{l}-0.019 \\
(0.04)\end{array}$ & $\begin{array}{l}0.059 \\
(0.04)\end{array}$ & $\begin{array}{l}0.303 \\
(0.22)\end{array}$ & $\begin{array}{l}0.409 \\
(0.25)\end{array}$ & $\begin{array}{l}0.217 \\
(0.20)\end{array}$ & $\begin{array}{l}-0.100 \\
(0.29)\end{array}$ \\
\hline Age $25-34$ & $\begin{array}{c}0.021 \\
(0.03)\end{array}$ & $\begin{array}{l}0.011 \\
(0.03)\end{array}$ & $\begin{array}{l}0.070 \\
(0.19)\end{array}$ & $\begin{array}{l}-0.015 \\
(0.22)\end{array}$ & $\begin{array}{l}0.031 \\
(0.21)\end{array}$ & $\begin{array}{l}0.268 \\
(0.23)\end{array}$ \\
\hline \multirow[t]{2}{*}{ Age $45-54$} & $\begin{array}{l}0.037 \\
(0.03)\end{array}$ & $\begin{array}{l}-0.013 \\
(0.03)\end{array}$ & $\begin{array}{l}-0.262 \\
(0.21)\end{array}$ & $\begin{array}{l}-0.411 \\
(0.27)\end{array}$ & $\begin{array}{l}-0.029 \\
(0.23)\end{array}$ & $\begin{array}{l}-0.129 \\
(0.25)\end{array}$ \\
\hline & & & & & & ... continut \\
\hline
\end{tabular}




\begin{tabular}{|c|c|c|c|c|c|c|}
\hline \multirow{2}{*}{$\begin{array}{l}\text { Appendix B1 } \\
\text { (continued) }\end{array}$} & \multicolumn{2}{|c|}{$\begin{array}{l}\text { Non-Aboriginal } \\
\text { Men }\end{array}$} & \multicolumn{2}{|c|}{$\begin{array}{l}\text { Métis } \\
\text { Men }\end{array}$} & \multicolumn{2}{|c|}{$\begin{array}{c}\text { North American Indian } \\
\text { Men }\end{array}$} \\
\hline & main sample & $\begin{array}{c}\text { restricted } \\
\text { sample }\end{array}$ & $\begin{array}{c}\text { main } \\
\text { sample }\end{array}$ & $\begin{array}{l}\text { restricted } \\
\text { sample }\end{array}$ & $\begin{array}{c}\text { main } \\
\text { sample }\end{array}$ & $\begin{array}{c}\text { restricted } \\
\text { sample }\end{array}$ \\
\hline Age $55-64$ & $\begin{array}{c}0.146 \\
(0.03)^{* * * *}\end{array}$ & $\begin{array}{c}0.083 \\
(0.04)^{*}\end{array}$ & $\begin{array}{l}0.228 \\
(0.24)\end{array}$ & $\begin{array}{l}0.079 \\
(0.29)\end{array}$ & $\begin{array}{l}-0.724 \\
(0.53)\end{array}$ & $\begin{array}{l}0.093 \\
(0.28)\end{array}$ \\
\hline Married & $\begin{array}{l}-0.063 \\
(0.03)^{*}\end{array}$ & $\begin{array}{l}-0.049 \\
(0.03)\end{array}$ & $\begin{array}{l}-0.336 \\
(0.20)\end{array}$ & $\begin{array}{l}-0.632 \\
(0.26)^{*}\end{array}$ & $\begin{array}{l}-0.181 \\
(0.19)\end{array}$ & $\begin{array}{l}-0.166 \\
(0.22)\end{array}$ \\
\hline $\begin{array}{l}\text { Widowed, } \\
\text { Divorced, separated }\end{array}$ & $\begin{array}{l}0.015 \\
(0.04)\end{array}$ & $\begin{array}{l}0.055 \\
(0.05)\end{array}$ & $\begin{array}{l}-0.182 \\
(0.26)\end{array}$ & $\begin{array}{l}-0.072 \\
(0.28)\end{array}$ & $\begin{array}{l}0.224 \\
(0.31)\end{array}$ & $\begin{array}{l}0.230 \\
(0.35)\end{array}$ \\
\hline Children < age 6 & $\begin{array}{l}-0.050 \\
(0.03)\end{array}$ & $\begin{array}{l}-0.055 \\
(0.04)\end{array}$ & $\begin{array}{l}0.109 \\
(0.22)\end{array}$ & $\begin{array}{l}0.200 \\
(0.28)\end{array}$ & $\begin{array}{l}0.085 \\
(0.23)\end{array}$ & $\begin{array}{l}-0.060 \\
(0.27)\end{array}$ \\
\hline Children aged 6-14 & $\begin{array}{l}-0.024 \\
(0.03)\end{array}$ & $\begin{array}{l}-0.029 \\
(0.04)\end{array}$ & $\begin{array}{l}0.283 \\
(0.23)\end{array}$ & $\begin{array}{l}0.497 \\
(0.29)\end{array}$ & $\begin{array}{l}0.029 \\
(0.22)\end{array}$ & $\begin{array}{l}-0.317 \\
(0.26)\end{array}$ \\
\hline Children aged 14-24 & $\begin{array}{l}-0.029 \\
(0.03)\end{array}$ & $\begin{array}{l}-0.007 \\
(0.04)\end{array}$ & $\begin{array}{c}0.481 \\
(0.24)^{*}\end{array}$ & $\begin{array}{c}0.700 \\
(0.29)^{*}\end{array}$ & $\begin{array}{l}0.093 \\
(0.25)\end{array}$ & $\begin{array}{r}0.229 \\
(0.27)\end{array}$ \\
\hline Atlantic provinces & $\begin{array}{c}0.141 \\
(0.02)^{* * *}\end{array}$ & $\begin{array}{c}0.078 \\
(0.05)^{* *}\end{array}$ & $\begin{array}{l}0.402 \\
(0.23)\end{array}$ & $\begin{array}{l}0.431 \\
(0.26)\end{array}$ & $\begin{array}{l}0.332 \\
(0.19)\end{array}$ & $\begin{array}{l}0.307 \\
(0.23)\end{array}$ \\
\hline Quebec & $\begin{array}{l}0.018 \\
(0.02)\end{array}$ & $\begin{array}{l}0.005 \\
(0.03)\end{array}$ & $\begin{array}{c}0.554 \\
(0.27)^{\star}\end{array}$ & $\begin{array}{l}0.378 \\
(0.30)\end{array}$ & $\begin{array}{l}0.450 \\
(0.28)\end{array}$ & $\begin{array}{l}0.062 \\
(0.41)\end{array}$ \\
\hline Manitoba & $\begin{array}{l}-0.218 \\
\left(0.040^{* * *}\right.\end{array}$ & $\begin{array}{l}-0.226 \\
(0.04)^{* * *}\end{array}$ & $\begin{array}{l}0.134 \\
(0.18)\end{array}$ & $\begin{array}{l}-0.072 \\
(0.22)\end{array}$ & $\begin{array}{r}-0.355 \\
(0.19)\end{array}$ & $\begin{array}{l}-0.486 \\
(0.26)\end{array}$ \\
\hline Saskatchewan & $\begin{array}{l}-0.184 \\
(0.03)^{\star \star \star}\end{array}$ & $\begin{array}{l}-0.191 \\
(0.04)^{* * *}\end{array}$ & $\begin{array}{l}0.103 \\
(0.22)\end{array}$ & $\begin{array}{l}-0.135 \\
(0.27)\end{array}$ & $\begin{array}{l}-0.050 \\
(0.23)\end{array}$ & $\begin{array}{l}0.110 \\
(0.25)\end{array}$ \\
\hline Alberta & $\begin{array}{c}-0.163 \\
(0.03)^{* * *}\end{array}$ & $\begin{array}{c}-0.132 \\
(0.04)^{* *}\end{array}$ & $\begin{array}{l}0.361 \\
(0.19)\end{array}$ & $\begin{array}{l}0.162 \\
(0.21)\end{array}$ & $\begin{array}{l}-0.382 \\
(0.25)\end{array}$ & $\begin{array}{l}-0.282 \\
(0.25)\end{array}$ \\
\hline British Columbia & $\begin{array}{c}-0.069 \\
(0.03)^{*}\end{array}$ & $\begin{array}{l}-0.058 \\
(0.04)\end{array}$ & $\begin{array}{l}-0.065 \\
(0.26)\end{array}$ & $\begin{array}{r}-0.225 \\
(0.31)\end{array}$ & $\begin{array}{l}-0.239 \\
(0.21)\end{array}$ & $\begin{array}{l}-0.146 \\
(0.24)\end{array}$ \\
\hline Primary industries & $\begin{array}{c}0.290 \\
(0.04)^{* * *}\end{array}$ & $\begin{array}{c}0.278 \\
(0.045)^{\star \star \star}\end{array}$ & $\begin{array}{c}0.508 \\
(0.22)^{*}\end{array}$ & $\begin{array}{c}0.846 \\
(0.29)^{* *}\end{array}$ & $\begin{array}{l}0.106 \\
(0.29)\end{array}$ & $\begin{array}{l}0.185 \\
(0.35)\end{array}$ \\
\hline Manufacturing & $\begin{array}{c}0.105 \\
(0.04)^{* *}\end{array}$ & $\begin{array}{l}0.066 \\
(0.05)\end{array}$ & $\begin{array}{l}0.310 \\
(0.26)\end{array}$ & $\begin{array}{c}0.728 \\
(0.31)^{*}\end{array}$ & $\begin{array}{l}0.145 \\
(0.31)\end{array}$ & $\begin{array}{l}-0.154 \\
(0.35)\end{array}$ \\
\hline Retail & $\begin{array}{l}-0.043 \\
(0.04)\end{array}$ & $\begin{array}{c}-0.100 \\
(0.04)^{*}\end{array}$ & $\begin{array}{l}-0.208 \\
(0.24)\end{array}$ & $\begin{array}{l}0.080 \\
(0.30)\end{array}$ & $\begin{array}{r}-0.367 \\
(0.30)\end{array}$ & $\begin{array}{l}-0.585 \\
(0.34)\end{array}$ \\
\hline Public services & $\begin{array}{c}0.132 \\
(0.05)^{\star \star}\end{array}$ & $\begin{array}{c}0.187 \\
(0.06)^{* *}\end{array}$ & $\begin{array}{r}-0.037 \\
(0.26)\end{array}$ & $\begin{array}{l}0.432 \\
(0.31)\end{array}$ & $\begin{array}{r}-0.365 \\
(0.34)\end{array}$ & $\begin{array}{r}-0.380 \\
(0.41)\end{array}$ \\
\hline Other services & $\begin{array}{l}0.0187 \\
(0.04)\end{array}$ & $\begin{array}{l}-0.069 \\
(0.05)\end{array}$ & $\begin{array}{l}-0.017 \\
(0.24)\end{array}$ & $\begin{array}{l}0.198 \\
(0.32)\end{array}$ & $\begin{array}{c}-0.426 \\
(0.30)\end{array}$ & $\begin{array}{l}-0.470 \\
(0.34)\end{array}$ \\
\hline Management & $\begin{array}{l}-0.095 \\
(0.05)\end{array}$ & $\begin{array}{l}-0.030 \\
(0.06)\end{array}$ & $\begin{array}{l}0.417 \\
(0.40)\end{array}$ & $\begin{array}{c}0.74 \\
(0.46)\end{array}$ & $\begin{array}{l}-0.483 \\
(0.50)\end{array}$ & $\begin{array}{l}0.021 \\
(0.46)\end{array}$ \\
\hline Semi-professionals & $\begin{array}{l}0.075 \\
(0.05)\end{array}$ & $\begin{array}{l}0.069 \\
(0.06)\end{array}$ & $\begin{array}{l}0.035 \\
(0.38)\end{array}$ & $\begin{array}{l}0.106 \\
(0.51)\end{array}$ & $\begin{array}{l}0.0854 \\
(0.39)\end{array}$ & $\begin{array}{l}0.578 \\
(0.37)\end{array}$ \\
\hline
\end{tabular}




\begin{tabular}{|c|c|c|c|c|c|c|}
\hline \multirow{2}{*}{$\begin{array}{l}\text { Appendix B1 } \\
\text { (continued) }\end{array}$} & \multicolumn{2}{|c|}{$\begin{array}{l}\text { Non-Aboriginal } \\
\text { Men }\end{array}$} & \multicolumn{2}{|c|}{$\begin{array}{l}\text { Métis } \\
\text { Men }\end{array}$} & \multicolumn{2}{|c|}{$\begin{array}{c}\text { North American Indian } \\
\text { Men }\end{array}$} \\
\hline & main sample & $\begin{array}{c}\begin{array}{c}\text { restricted } \\
\text { sample }\end{array} \\
\end{array}$ & $\begin{array}{c}\text { main } \\
\text { sample }\end{array}$ & $\begin{array}{c}\begin{array}{c}\text { restricted } \\
\text { sample }\end{array} \\
\end{array}$ & $\begin{array}{c}\text { main } \\
\text { sample }\end{array}$ & $\begin{array}{c}\begin{array}{c}\text { restricted } \\
\text { sample }\end{array} \\
\end{array}$ \\
\hline Sales personnel & $\begin{array}{l}-0.071 \\
(0.04)\end{array}$ & $\begin{array}{l}-0.041 \\
(0.05)\end{array}$ & $\begin{array}{l}0.297 \\
(0.31)\end{array}$ & $\begin{array}{c}0.836 \\
(0.36)^{*}\end{array}$ & $\begin{array}{l}-0.577 \\
(0.34)\end{array}$ & $\begin{array}{l}-0.185 \\
(0.35)\end{array}$ \\
\hline Manual workers & $\begin{array}{c}0.222 \\
(0.04)^{\star * *}\end{array}$ & $\begin{array}{l}0.273 \\
(0.05)^{* * *}\end{array}$ & $\begin{array}{l}0.275 \\
(0.32)\end{array}$ & $\begin{array}{l}0.536 \\
(0.38)\end{array}$ & $\begin{array}{l}-0.128 \\
(0.37)\end{array}$ & $\begin{array}{l}0.351 \\
(0.36)\end{array}$ \\
\hline Union member & $\begin{array}{l}0.140 \\
(0.02)^{ \pm \star *}\end{array}$ & $\begin{array}{c}0.162 \\
(0.03)^{* \pm *}\end{array}$ & $\begin{array}{l}-0.037 \\
(0.16)\end{array}$ & $\begin{array}{r}-0.040 \\
(0.18)\end{array}$ & $\begin{array}{l}0.100 \\
(0.17)\end{array}$ & $\begin{array}{l}0.109 \\
(0.19)\end{array}$ \\
\hline Union coverage & $\begin{array}{l}-0.011 \\
(0.06)\end{array}$ & - & $\begin{array}{l}-0.469 \\
(0.39)\end{array}$ & - & $\begin{array}{l}0.153 \\
(0.38)\end{array}$ & - \\
\hline Small size workplace & $\begin{array}{l}0.176 \\
(0.03)^{* * *}\end{array}$ & $\begin{array}{l}0.192 \\
(0.04)^{* \star *}\end{array}$ & $\begin{array}{l}0.260 \\
(0.22)\end{array}$ & $\begin{array}{l}0.441 \\
(0.24)\end{array}$ & $\begin{array}{l}0.506 \\
(0.31)\end{array}$ & $\begin{array}{l}0.195 \\
(0.28)\end{array}$ \\
\hline Medium size workplace & $\begin{array}{l}0.055 \\
(0.04)\end{array}$ & $\begin{array}{l}0.076 \\
(0.04)\end{array}$ & $\begin{array}{l}-0.109 \\
(0.24)\end{array}$ & $\begin{array}{l}-0.286 \\
(0.27)\end{array}$ & $\begin{array}{l}-0.038 \\
(0.34)\end{array}$ & $\begin{array}{l}-0.597 \\
(0.35)\end{array}$ \\
\hline Public sector & $\begin{array}{c}-0.263 \\
(0.04)^{m * *}\end{array}$ & $\begin{array}{c}-0.398 \\
(0.04)^{m+*}\end{array}$ & $\begin{array}{l}-0.023 \\
(0.20)\end{array}$ & $\begin{array}{l}-0.055 \\
(0.25)\end{array}$ & $\begin{array}{l}-0.150 \\
(0.23)\end{array}$ & $\begin{array}{l}-0.043 \\
(0.27)\end{array}$ \\
\hline Seasonal job & $\begin{array}{l}1.105 \\
(0.03)^{* * * *}\end{array}$ & - & $\begin{array}{c}1.284 \\
(0.20)^{* \star *}\end{array}$ & - & $\begin{array}{c}0.639 \\
(0.22)^{* *}\end{array}$ & - \\
\hline $\begin{array}{l}\text { Temporary, } \\
\text { contract, casual job }\end{array}$ & $\begin{array}{c}0.736 \\
(0.03)^{* * * *}\end{array}$ & - & $\begin{array}{l}0.605 \\
(0.19)^{* *}\end{array}$ & - & $\begin{array}{c}0.459 \\
(0.20)^{*}\end{array}$ & - \\
\hline Multiple job holder & $\begin{array}{l}-0.137 \\
(0.05)^{* *}\end{array}$ & - & $\begin{array}{r}-0.355 \\
(0.25)\end{array}$ & - & $\begin{array}{l}0.168 \\
(0.26)\end{array}$ & - \\
\hline Part-time at main job & $\begin{array}{l}0.218 \\
(0.04)^{* * * *}\end{array}$ & $\begin{array}{l}-.339 \\
(0.05)^{m+m}\end{array}$ & $\begin{array}{l}0.547 \\
(0.23)^{*}\end{array}$ & $\begin{array}{l}0.423 \\
(0.32)\end{array}$ & $\begin{array}{c}0.583 \\
(0.22)^{* *}\end{array}$ & $\begin{array}{l}0.371 \\
(0.32)\end{array}$ \\
\hline Wage below minimum & $\begin{array}{l}-0.041 \\
(0.10)\end{array}$ & $\begin{array}{l}-0.019 \\
(0.13)\end{array}$ & $\begin{array}{l}0.916 \\
(0.66)\end{array}$ & $\begin{array}{l}1.109 \\
(0.63)\end{array}$ & $\begin{array}{r}0.350 \\
(0.62)\end{array}$ & $\begin{array}{l}0.083 \\
(0.70)\end{array}$ \\
\hline Minimum wage & $\begin{array}{l}0.117 \\
(0.04)^{\star \star}\end{array}$ & $\begin{array}{c}0.190 \\
(0.05)^{* * *}\end{array}$ & $\begin{array}{l}0.108 \\
(0.26)\end{array}$ & $\begin{array}{l}0.039 \\
(0.32)\end{array}$ & $\begin{array}{l}-0.057 \\
(0.28)\end{array}$ & $\begin{array}{l}0.259 \\
(0.35)\end{array}$ \\
\hline Median wage & $\begin{array}{c}0.153 \\
(0.03)^{* * * *}\end{array}$ & $\begin{array}{c}0.184 \\
(0.04)^{\text {tat }}\end{array}$ & $\begin{array}{l}-0.118 \\
(0.22)\end{array}$ & $\begin{array}{l}-0.203 \\
(0.24)\end{array}$ & $\begin{array}{l}-158 \\
(0.23)\end{array}$ & $\begin{array}{l}-0.138 \\
(0.27)\end{array}$ \\
\hline Average wage & $\begin{array}{c}0.096 \\
(0.02)^{*}\end{array}$ & $\begin{array}{c}0.108 \\
(0.03)^{*+*}\end{array}$ & $\begin{array}{l}0.187 \\
(0.15)\end{array}$ & $\begin{array}{l}0.063 \\
(0.17)\end{array}$ & $\begin{array}{l}0.102 \\
(0.18)\end{array}$ & $\begin{array}{l}0.064 \\
(0.19)\end{array}$ \\
\hline Number of observations & $N=74158$ & $N=61448$ & $N=1469$ & $N=1185$ & $N=1094$ & $N=876$ \\
\hline Pseudo R2 & 0.1802 & 0.0965 & 0.2557 & 0.2540 & 0.2218 & 0.1870 \\
\hline Log pseudolikelihood & -4890533.7 & -3454008.7 & -68257.4 & -44091.8 & -62394.2 & -41074.8 \\
\hline
\end{tabular}

Source: The Labour Force Survey, national monthly master file, September 2008 - May 2011.

Note: Main samples include all paid employees, aged between 15 and 64, who are not full-time students. Restricted samples include only permanent paid employees, aged between 15 and 64, who are single-job holders and who are not students. Survey sampling weights (abowt) applied in all analyses. Standard errors reported in parentheses. 
Appendix B 2: Probit estimates used in decompositions of difference in layoffs for women

\begin{tabular}{|c|c|c|c|c|c|c|}
\hline \multirow{2}{*}{ Variables in the equations } & \multicolumn{2}{|c|}{$\begin{array}{l}\text { Non-Aboriginal } \\
\text { Women }\end{array}$} & \multicolumn{2}{|c|}{$\begin{array}{l}\text { Métis } \\
\text { Women }\end{array}$} & \multicolumn{2}{|c|}{$\begin{array}{c}\text { North American Indian } \\
\text { Women }\end{array}$} \\
\hline & $\begin{array}{c}\text { main } \\
\text { sample }\end{array}$ & $\begin{array}{c}\text { restricted } \\
\text { sample }\end{array}$ & $\begin{array}{c}\text { main } \\
\text { sample }\end{array}$ & $\begin{array}{c}\text { restricted } \\
\text { sample }\end{array}$ & main sample & $\begin{array}{c}\text { restricted } \\
\text { sample }\end{array}$ \\
\hline $\begin{array}{l}\text { Less than } \\
\text { high school diploma }\end{array}$ & $\begin{array}{c}0.084 \\
(0.04)^{*}\end{array}$ & $\begin{array}{l}0.062 \\
(0.04)\end{array}$ & $\begin{array}{l}-0.098 \\
(0.21)\end{array}$ & $\begin{array}{l}-0.028 \\
(0.23)\end{array}$ & $\begin{array}{l}0.110 \\
(0.17)\end{array}$ & $\begin{array}{l}0.127 \\
(0.20)\end{array}$ \\
\hline $\begin{array}{l}\text { Incomplete } \\
\text { postsecondary }\end{array}$ & $\begin{array}{l}-0.118 \\
(004)^{\star \star}\end{array}$ & $\begin{array}{r}-0.092 \\
(0.05)\end{array}$ & $\begin{array}{l}0.194 \\
(0.20)\end{array}$ & $\begin{array}{r}-0.220 \\
(0.24)\end{array}$ & $\begin{array}{l}-0.169 \\
(0.24)\end{array}$ & $\begin{array}{l}-0.027 \\
(0.28)\end{array}$ \\
\hline $\begin{array}{l}\text { Completed } \\
\text { trade certificate }\end{array}$ & $\begin{array}{l}-0.016 \\
(0.04)\end{array}$ & $\begin{array}{l}-0.038 \\
(0.04)\end{array}$ & $\begin{array}{l}0.312 \\
(0.21)\end{array}$ & $\begin{array}{l}0.188 \\
(0.24)\end{array}$ & $\begin{array}{l}-0.034 \\
(0.22)\end{array}$ & $\begin{array}{l}0.223 \\
(0.27)\end{array}$ \\
\hline $\begin{array}{l}\text { Completed } \\
\text { college diploma }\end{array}$ & $\begin{array}{l}-0.078 \\
(0.03)^{\star \star}\end{array}$ & $\begin{array}{r}-0.058 \\
(0.03)\end{array}$ & $\begin{array}{c}0.335 \\
(0.16)^{\star}\end{array}$ & $\begin{array}{l}0.338 \\
(0.19)\end{array}$ & $\begin{array}{l}-0.267 \\
(0.18)\end{array}$ & $\begin{array}{l}-0.206 \\
(0.21)\end{array}$ \\
\hline $\begin{array}{l}\text { Completed } \\
\text { university degree }\end{array}$ & $\begin{array}{c}-0.0618 \\
(0.04)\end{array}$ & $\begin{array}{l}-0.008 \\
(0.04)\end{array}$ & $\begin{array}{l}0.267 \\
(0.21)\end{array}$ & $\begin{array}{l}0.277 \\
(0.23)\end{array}$ & $\begin{array}{l}0.028 \\
(0.21)\end{array}$ & $\begin{array}{c}0.197 \\
(0.25)\end{array}$ \\
\hline Tenure $<2$ years & $\begin{array}{c}0.444 \\
(0.04)^{\star \star \star}\end{array}$ & $\begin{array}{c}0.463 \\
(0.05)^{\star \star \star \star}\end{array}$ & $\begin{array}{l}0.055 \\
(0.25)\end{array}$ & $\begin{array}{l}0.301 \\
(0.28)\end{array}$ & $\begin{array}{l}0.473 \\
(0.33)\end{array}$ & $\begin{array}{l}0.370 \\
(0.35)\end{array}$ \\
\hline Tenure $2-5$ years & $\begin{array}{c}0.235 \\
(0.04)^{\star \star \star}\end{array}$ & $\begin{array}{c}0.256 \\
(0.05)^{\star * *}\end{array}$ & $\begin{array}{l}0.046 \\
(0.25)\end{array}$ & $\begin{array}{l}0.207 \\
(0.29)\end{array}$ & $\begin{array}{l}0.160 \\
(0.34)\end{array}$ & $\begin{array}{l}0.106 \\
(0.36)\end{array}$ \\
\hline Tenure $5-10$ years & $\begin{array}{c}0.179 \\
(0.04)^{\star \star \star}\end{array}$ & $\begin{array}{c}0.173 \\
(0.04)^{* * * k}\end{array}$ & $\begin{array}{l}-0.046 \\
(0.27)\end{array}$ & $\begin{array}{l}0.141 \\
(0.30)\end{array}$ & $\begin{array}{l}0.267 \\
(0.33)\end{array}$ & $\begin{array}{l}0.463 \\
(0.35)\end{array}$ \\
\hline Tenure $10-20$ years & $\begin{array}{c}0.161 \\
(0.04)^{\star * \star}\end{array}$ & $\begin{array}{c}0.180 \\
(0.04)^{\star \star *}\end{array}$ & $\begin{array}{l}0.091 \\
(0.24)\end{array}$ & $\begin{array}{c}0.381 \\
(0.27)\end{array}$ & $\begin{array}{l}-0.041 \\
(0.33)\end{array}$ & $\begin{array}{l}-0.295 \\
(0.36)\end{array}$ \\
\hline Absent 1 week & $\begin{array}{c}0.196 \\
(0.05)^{\star \star \star}\end{array}$ & $\begin{array}{c}0.172 \\
(0.06)^{\star \star}\end{array}$ & $\begin{array}{l}0.250 \\
(0.31)\end{array}$ & $\begin{array}{c}0.22 \\
(0.37)\end{array}$ & $\begin{array}{c}0.784 \\
(0.32)^{\star}\end{array}$ & $\begin{array}{l}0.171 \\
(0.34)\end{array}$ \\
\hline Absent 2 weeks & $\begin{array}{l}0.008 \\
(0.08)\end{array}$ & $\begin{array}{l}0.055 \\
(0.09)\end{array}$ & $\begin{array}{l}0.929 \\
(0.48)\end{array}$ & $\begin{array}{l}0.473 \\
(0.65)\end{array}$ & $\begin{array}{l}0.215 \\
(0.37)\end{array}$ & - \\
\hline Absent 4 to 8 weeks & - & - & - & - & $\begin{array}{l}0.052 \\
(0.38)\end{array}$ & $\begin{array}{l}-0.107 \\
(0.50)\end{array}$ \\
\hline Absent $>8$ weeks & $\begin{array}{c}-0.193 \\
(0.06)^{\star \star}\end{array}$ & $\begin{array}{l}-0.102 \\
(0.07)\end{array}$ & $\begin{array}{l}-0.183 \\
(0.27)\end{array}$ & $\begin{array}{l}-0.298 \\
(0.32)\end{array}$ & $\begin{array}{l}0.133 \\
(0.40)\end{array}$ & $\begin{array}{l}0.262 \\
(0.38)\end{array}$ \\
\hline Age 15-24 & $\begin{array}{c}-0.151 \\
(0.04)^{\star *}\end{array}$ & $\begin{array}{l}0.012 \\
(0.05)\end{array}$ & $\begin{array}{c}0.475 \\
(0.22)^{\star}\end{array}$ & $\begin{array}{c}0.608 \\
(0.26)^{\star}\end{array}$ & $\begin{array}{l}-0.167 \\
(0.21)\end{array}$ & $\begin{array}{c}-0.08 \\
(0.25)\end{array}$ \\
\hline Age $25-34$ & $\begin{array}{c}-0.080 \\
(0.03)^{*}\end{array}$ & $\begin{array}{l}-0.067 \\
(0.04)\end{array}$ & $\begin{array}{c}0.354 \\
(0.17)^{*}\end{array}$ & $\begin{array}{l}0.327 \\
(0.20)\end{array}$ & $\begin{array}{l}-0.065 \\
(0.18)\end{array}$ & $\begin{array}{l}-0.209 \\
(0.22)\end{array}$ \\
\hline Age 45-54 & $\begin{array}{l}0.031 \\
(0.03)\end{array}$ & $\begin{array}{l}0.005 \\
(0.04)\end{array}$ & $\begin{array}{l}0.303 \\
(0.16)\end{array}$ & $\begin{array}{l}0.146 \\
(0.18)\end{array}$ & $\begin{array}{l}0.028 \\
(0.17)\end{array}$ & $\begin{array}{l}0.120 \\
(0.20)\end{array}$ \\
\hline \multirow[t]{2}{*}{ Age 55-64 } & $\begin{array}{c}0.101 \\
(0.04)^{\star * *}\end{array}$ & $\begin{array}{l}0.062 \\
(0.04)\end{array}$ & $\begin{array}{l}0.200 \\
(0.21)\end{array}$ & $\begin{array}{r}-0.045 \\
(0.25)\end{array}$ & $\begin{array}{r}-0.098 \\
(0.24)\end{array}$ & $\begin{array}{l}-0.120 \\
(0.31)\end{array}$ \\
\hline & & & & & & ...continue \\
\hline
\end{tabular}




\begin{tabular}{|c|c|c|c|c|c|c|}
\hline \multirow{2}{*}{$\begin{array}{l}\text { Appendix B2 } \\
\text { (continued) }\end{array}$} & \multicolumn{2}{|c|}{$\begin{array}{l}\text { Non-Aboriginal } \\
\text { Women }\end{array}$} & \multicolumn{2}{|c|}{$\begin{array}{l}\text { Métis } \\
\text { Women }\end{array}$} & \multicolumn{2}{|c|}{$\begin{array}{l}\text { North American Indian } \\
\text { Women }\end{array}$} \\
\hline & $\begin{array}{c}\text { main } \\
\text { sample }\end{array}$ & $\begin{array}{l}\text { restricted } \\
\text { sample }\end{array}$ & $\begin{array}{c}\text { main } \\
\text { sample }\end{array}$ & $\begin{array}{l}\text { restricted } \\
\text { sample }\end{array}$ & main sample & $\begin{array}{c}\text { restricted } \\
\text { sample }\end{array}$ \\
\hline Married & $\begin{array}{l}-0.106 \\
(0.06)\end{array}$ & $\begin{array}{l}-0.071 \\
(0.04)^{*}\end{array}$ & $\begin{array}{l}0.173 \\
(0.16)\end{array}$ & $\begin{array}{l}0.198 \\
(0.18)\end{array}$ & $\begin{array}{l}0.106 \\
(0.14)\end{array}$ & $\begin{array}{l}-0.123 \\
(0.17)\end{array}$ \\
\hline $\begin{array}{l}\text { Widowed, } \\
\text { Divorced, separated }\end{array}$ & $\begin{array}{l}-0.021 \\
(0.03)\end{array}$ & $\begin{array}{l}-0.016 \\
(0.05)\end{array}$ & $\begin{array}{l}-0.036 \\
(0.22)\end{array}$ & $\begin{array}{l}0.182 \\
(0.24)\end{array}$ & $\begin{array}{r}-0.001 \\
\langle 0.21\rangle\end{array}$ & $\begin{array}{l}-0.233 \\
(0.22)\end{array}$ \\
\hline Children < age 6 & $\begin{array}{l}0.014 \\
(0.04)\end{array}$ & $\begin{array}{l}-0.039 \\
(0.04)\end{array}$ & $\begin{array}{r}-0.022 \\
(0.20)\end{array}$ & $\begin{array}{l}-0.011 \\
(0.22)\end{array}$ & $\begin{array}{r}-0.077 \\
(019)\end{array}$ & $\begin{array}{r}-0.284 \\
(0.24)\end{array}$ \\
\hline Children aged 6-14 & $\begin{array}{l}0.018 \\
(0.04)\end{array}$ & $\begin{array}{l}-0.005 \\
(0.04)\end{array}$ & $\begin{array}{l}0.112 \\
(0.16)\end{array}$ & $\begin{array}{l}0.145 \\
(0.18)\end{array}$ & $\begin{array}{l}0.103 \\
(0.17)\end{array}$ & $\begin{array}{l}0.219 \\
(0.19)\end{array}$ \\
\hline Children aged 14-24 & $\begin{array}{l}0.007 \\
(0.03)\end{array}$ & $\begin{array}{l}0.017 \\
(0.04)\end{array}$ & $\begin{array}{c}0.391 \\
(0.18)^{*}\end{array}$ & $\begin{array}{l}0.370 \\
(0.20)\end{array}$ & $\begin{array}{l}0.001 \\
(0.19)\end{array}$ & $\begin{array}{l}-0.169 \\
(0.23)\end{array}$ \\
\hline Atlantic provinces & $\begin{array}{l}0.007 \\
(0.03)\end{array}$ & $\begin{array}{l}0.001 \\
(0.03)\end{array}$ & $\begin{array}{l}0.437 \\
(0.25)\end{array}$ & $\begin{array}{l}0.253 \\
(0.30)\end{array}$ & $\begin{array}{c}0.094 \\
(0.18)\end{array}$ & $\begin{array}{l}-0.158 \\
(0.24)\end{array}$ \\
\hline Quebec & $\begin{array}{l}0.043 \\
(0.02)\end{array}$ & $\begin{array}{r}-0.027 \\
(0.03)\end{array}$ & $\begin{array}{l}0.435 \\
(0.28)\end{array}$ & $\begin{array}{l}0.545 \\
(0.31)\end{array}$ & $\begin{array}{r}-0.386 \\
(0.29)\end{array}$ & $\begin{array}{l}-0.443 \\
(0.32)\end{array}$ \\
\hline Manitoba & $\begin{array}{l}-0.031 \\
(0.03)\end{array}$ & $\begin{array}{r}-0.013 \\
(0.04)\end{array}$ & $\begin{array}{l}0.329 \\
(0.19)\end{array}$ & $\begin{array}{l}0.389 \\
(0.23)\end{array}$ & $\begin{array}{l}-0.164 \\
(0.18)\end{array}$ & $\begin{array}{r}-0.144 \\
(0.21)\end{array}$ \\
\hline Saskatchewan & $\begin{array}{l}-0.020 \\
(0.04)\end{array}$ & $\begin{array}{l}0.043 \\
(0.04)\end{array}$ & $\begin{array}{c}0.478 \\
(0.22)^{\star}\end{array}$ & $\begin{array}{c}0.671 \\
(0.26)^{*}\end{array}$ & $\begin{array}{l}-0.407 \\
(0.21)\end{array}$ & $\begin{array}{l}-0.685 \\
(0.27)^{*}\end{array}$ \\
\hline Alberta & $\begin{array}{l}0.007 \\
(0.03)\end{array}$ & $\begin{array}{l}0.045 \\
(0.04)\end{array}$ & $\begin{array}{l}-0.102 \\
(0.21)\end{array}$ & $\begin{array}{l}-0.205 \\
(0.27)\end{array}$ & $\begin{array}{l}0.024 \\
(0.20)\end{array}$ & $\begin{array}{l}0.022 \\
(0.23)\end{array}$ \\
\hline British Columbia & $\begin{array}{l}0.016 \\
(0.04)\end{array}$ & $\begin{array}{l}0.013 \\
(0.04)\end{array}$ & $\begin{array}{l}0.190 \\
(0.22)\end{array}$ & $\begin{array}{l}0.273 \\
(0.25)\end{array}$ & $\begin{array}{r}-0.345 \\
(0.18)\end{array}$ & $\begin{array}{c}-0.425 \\
(0.20)^{\star}\end{array}$ \\
\hline Primary industries & $\begin{array}{l}-0.001 \\
(0.04)\end{array}$ & $\begin{array}{l}0.097 \\
(0.07)\end{array}$ & $\begin{array}{c}0.724 \\
(0.32)^{\star}\end{array}$ & $\begin{array}{c}0.850 \\
(0.35)^{*}\end{array}$ & $\begin{array}{l}0.527 \\
(0.32)\end{array}$ & $\begin{array}{c}0.711 \\
(0.34)^{*}\end{array}$ \\
\hline Manufacturing & $\begin{array}{l}0.102 \\
(0.06)\end{array}$ & $\begin{array}{c}0.197 \\
(0.06)^{* *}\end{array}$ & $\begin{array}{l}0.433 \\
(0.40)\end{array}$ & $\begin{array}{l}-0.303 \\
(0.53)\end{array}$ & $\begin{array}{l}0.116 \\
(0.54)\end{array}$ & $\begin{array}{l}0.623 \\
(0.61)\end{array}$ \\
\hline Retail & $\begin{array}{c}0.173 \\
(0.05)^{\star \star}\end{array}$ & $\begin{array}{l}-0.053 \\
(0.04)\end{array}$ & $\begin{array}{l}0.152 \\
(0.24)\end{array}$ & $\begin{array}{l}0.126 \\
(0.25)\end{array}$ & $\begin{array}{l}0.066 \\
(0.22)\end{array}$ & $\begin{array}{r}-0.040 \\
(0.25)\end{array}$ \\
\hline Public services & $\begin{array}{l}-0.047 \\
(0.04)\end{array}$ & $\begin{array}{l}0.0314 \\
(0.04)\end{array}$ & $\begin{array}{l}-0.017 \\
(0.21)\end{array}$ & $\begin{array}{l}0.651 \\
(0.23)\end{array}$ & $\begin{array}{c}-0.06 \\
(0.24)\end{array}$ & $\begin{array}{l}-0.446 \\
(0.29)\end{array}$ \\
\hline Other services & $\begin{array}{l}0.056 \\
(0.04)\end{array}$ & $\begin{array}{l}0.090 \\
(0.05)\end{array}$ & $\begin{array}{l}0.179 \\
(0.29)\end{array}$ & $\begin{array}{l}0.270 \\
(0.31)\end{array}$ & $\begin{array}{l}-0.356 \\
(0.30)\end{array}$ & $\begin{array}{l}0.510 \\
(0.35)\end{array}$ \\
\hline Management & $\begin{array}{c}0.05 \\
(0.05)\end{array}$ & - & - & - & $\begin{array}{l}-0.270 \\
(0.34)\end{array}$ & $\begin{array}{l}-0.265 \\
(0.39)\end{array}$ \\
\hline Semi-professionals & $\begin{array}{c}-0.124 \\
(0.04)^{\star \star}\end{array}$ & $\begin{array}{l}-0.060 \\
(0.05)\end{array}$ & $\begin{array}{r}-0.001 \\
(0.20)\end{array}$ & $\begin{array}{l}-0.103 \\
(0.23)\end{array}$ & $\begin{array}{l}0.124 \\
(0.22)\end{array}$ & $\begin{array}{l}0.192 \\
(0.28)\end{array}$ \\
\hline \multirow[t]{2}{*}{ Sales personnel } & $\begin{array}{c}-0.097 \\
(0.03)^{* *}\end{array}$ & $\begin{array}{r}-0.039 \\
(0.04)\end{array}$ & $\begin{array}{l}0.022 \\
(0.16)\end{array}$ & $\begin{array}{l}0.162 \\
(0.18)\end{array}$ & $\begin{array}{l}-0.248 \\
(0.20)\end{array}$ & $\begin{array}{r}-0.326 \\
(0.26)\end{array}$ \\
\hline & & & & & & ...continue \\
\hline
\end{tabular}




\begin{tabular}{|c|c|c|c|c|c|c|}
\hline \multirow{2}{*}{$\begin{array}{l}\text { Appendix B2 } \\
\text { (continued) }\end{array}$} & \multicolumn{2}{|c|}{$\begin{array}{l}\text { Non-Aboriginal } \\
\text { Women }\end{array}$} & \multicolumn{2}{|c|}{$\begin{array}{l}\text { Métis } \\
\text { Women }\end{array}$} & \multicolumn{2}{|c|}{$\begin{array}{l}\text { North American Indian } \\
\text { Women }\end{array}$} \\
\hline & $\begin{array}{l}\text { main } \\
\text { sample }\end{array}$ & $\begin{array}{c}\text { restricted } \\
\text { sample }\end{array}$ & $\begin{array}{c}\text { main } \\
\text { sample }\end{array}$ & $\begin{array}{c}\text { restricted } \\
\text { sample }\end{array}$ & main sample & $\begin{array}{c}\text { restricted } \\
\text { sample }\end{array}$ \\
\hline Manual workers & $\begin{array}{l}0.325 \\
(0.05)^{* * *}\end{array}$ & $\begin{array}{c}0.398 \\
(0.06)^{* * *}\end{array}$ & $\begin{array}{l}0.294 \\
(0.30)\end{array}$ & $\begin{array}{c}0.843 \\
(0.31)^{* * *}\end{array}$ & $\begin{array}{l}0.209 \\
(0.39)\end{array}$ & $\begin{array}{l}-0.578 \\
(0.57)\end{array}$ \\
\hline Union member & $\begin{array}{c}0.064 \\
(0.03)^{\star}\end{array}$ & $\begin{array}{l}0.036 \\
(0.03)\end{array}$ & $\begin{array}{l}-0.381 \\
(0.15)^{*}\end{array}$ & $\begin{array}{l}-0.294 \\
(0.16)\end{array}$ & $\begin{array}{l}-0.058 \\
(0.16)\end{array}$ & $\begin{array}{l}0.156 \\
(0.19)\end{array}$ \\
\hline Union coverage & $\begin{array}{l}-0.052 \\
(0.07)\end{array}$ & - & $\begin{array}{l}-0.803 \\
(0.43)\end{array}$ & - & $\begin{array}{c}0.792 \\
(0.38)^{*}\end{array}$ & - \\
\hline Small size workplace & $\begin{array}{l}0.308 \\
(0.03)^{\text {thth }}\end{array}$ & $\begin{array}{c}0.403 \\
(0.04)^{* * *}\end{array}$ & $\begin{array}{l}0.335 \\
(0.23)\end{array}$ & $\begin{array}{l}0.631 \\
(0.34)\end{array}$ & $\begin{array}{l}0.145 \\
(0.25)\end{array}$ & $\begin{array}{l}0.255 \\
(0.25)\end{array}$ \\
\hline Medium size workplace & $\begin{array}{l}0.122 \\
(0.04)^{* \star}\end{array}$ & $\begin{array}{c}0.210 \\
(0.05)^{\star \star \star \star}\end{array}$ & $\begin{array}{l}-0.213 \\
(0.27)\end{array}$ & $\begin{array}{l}-0.044 \\
(0.38)\end{array}$ & $\begin{array}{l}0.219 \\
(0.26)\end{array}$ & $\begin{array}{l}0.176 \\
(0.28)\end{array}$ \\
\hline Public sector & $\begin{array}{c}0.148 \\
(0.03)^{m+\ldots}\end{array}$ & $\begin{array}{c}0.217 \\
(0.04)^{\text {t***}}\end{array}$ & $\begin{array}{c}0.434 \\
(0.16)^{* *}\end{array}$ & $\begin{array}{l}0.325 \\
(0.17)\end{array}$ & $\begin{array}{l}0.218 \\
(0.17)\end{array}$ & $\begin{array}{c}0.589 \\
(0.23)^{\star}\end{array}$ \\
\hline Seasonal job & $\begin{array}{c}1.198 \\
(0.04)^{* * * *}\end{array}$ & - & $\begin{array}{l}0.632 \\
(0.36)\end{array}$ & - & $\begin{array}{c}1.711 \\
(0.30)^{\star \star *}\end{array}$ & - \\
\hline $\begin{array}{l}\text { Temporary, } \\
\text { contract, casual job }\end{array}$ & $\begin{array}{c}0.744 \\
(0.03)^{\star \star *}\end{array}$ & - & $\begin{array}{c}0.362 \\
(0.17)^{*}\end{array}$ & - & $\begin{array}{l}0.515 \\
(0.15)\end{array}$ & - \\
\hline Multiple job holder & $\begin{array}{l}-0.233 \\
(0.04)^{* * *}\end{array}$ & - & $\begin{array}{l}-0.402 \\
(0.21)\end{array}$ & - & $\begin{array}{l}0.064 \\
(0.22)\end{array}$ & - \\
\hline Part-time at main job & $\begin{array}{l}0.235 \\
(0.02)^{* * *}\end{array}$ & $\begin{array}{c}0.269 \\
(0.03)^{* * * *}\end{array}$ & $\begin{array}{c}0.522 \\
(0.13)^{*+* t}\end{array}$ & $\begin{array}{c}0.512 \\
(0.15)^{\star *}\end{array}$ & $\begin{array}{l}0.141 \\
(0.13)\end{array}$ & $\begin{array}{l}0.049 \\
(0.17)\end{array}$ \\
\hline Wage below minimum & $\begin{array}{c}0.363 \\
(0.09)^{* * *}\end{array}$ & - & $\begin{array}{l}-0.324 \\
(0.50)\end{array}$ & - & $\begin{array}{l}0.161 \\
(0.44)\end{array}$ & - \\
\hline Minimum wage & $\begin{array}{l}0.349 \\
(0.05)^{\star \star \star}\end{array}$ & $\begin{array}{c}0.328 \\
(0.05)^{* * *}\end{array}$ & $\begin{array}{l}0.308 \\
(0.25)\end{array}$ & $\begin{array}{l}0.201 \\
(0.28)\end{array}$ & $\begin{array}{c}0.898 \\
(0.24)^{+\ldots *}\end{array}$ & $\begin{array}{c}1.132 \\
(0.27)^{\star * * * *}\end{array}$ \\
\hline Median wage & $\begin{array}{l}0.298 \\
(0.04)^{*}\end{array}$ & $\begin{array}{c}0.255 \\
(0.05)^{\star *}\end{array}$ & $\begin{array}{l}0.100 \\
(0.21)\end{array}$ & $\begin{array}{r}-0.010 \\
(0.23)\end{array}$ & $\begin{array}{c}0.537 \\
(0.21)^{*}\end{array}$ & $\begin{array}{c}0.692 \\
(0.25)^{\star *}\end{array}$ \\
\hline Average wage & $\begin{array}{c}0.180 \\
(0.03)^{* * * *}\end{array}$ & $\begin{array}{c}0.153 \\
(0.04)^{* * *}\end{array}$ & $\begin{array}{l}0.079 \\
(0.16)\end{array}$ & $\begin{array}{l}0.330 \\
(0.48)\end{array}$ & $\begin{array}{c}0.382 \\
(0.17)^{\star}\end{array}$ & $\begin{array}{c}0.435 \\
(0.19)^{*}\end{array}$ \\
\hline Number of observations & $N=77487$ & $N=62317$ & $N=1526$ & $N=1194$ & $N=1460$ & $N=1136$ \\
\hline Pseudo R2 & 0.1345 & 0.0564 & 0.1679 & 0.1873 & 0.2001 & 0.1414 \\
\hline Log pseudolikelihood & -4213846.6 & -2812287.2 & -54119.3 & -36610.9 & -54539.5 & -35554.3 \\
\hline
\end{tabular}

Source: The Labour Force Survey, national monthly master file, September 2008 - May 2011.

Note: Main samples include all paid employees, aged between 15 and 64, who are not full-time students. Restricted samples include only permanent paid employees, aged between 15 and 64 , who are single-job holders and who are not students. Survey sampling weights (abowt) applied in all analyses. Standard errors reported in parentheses. 
Appendix B 3: Probit estimates used in reemployment decompositions, Aboriginal identity groups

\begin{tabular}{|c|c|c|c|c|c|c|c|c|}
\hline \multirow{3}{*}{$\begin{array}{l}\text { Variables in the } \\
\text { equations }\end{array}$} & \multicolumn{4}{|c|}{ Métis } & \multicolumn{4}{|c|}{ North American Indian } \\
\hline & \multicolumn{2}{|c|}{ Women } & \multicolumn{2}{|c|}{ Men } & \multicolumn{2}{|c|}{ Women } & \multicolumn{2}{|c|}{ Men } \\
\hline & $\begin{array}{l}\text { main } \\
\text { sample }\end{array}$ & $\begin{array}{c}\text { restricted } \\
\text { sample }\end{array}$ & $\begin{array}{c}\text { main } \\
\text { sample }\end{array}$ & $\begin{array}{c}\text { restricted } \\
\text { sample }\end{array}$ & $\begin{array}{c}\text { main } \\
\text { sample }\end{array}$ & $\begin{array}{l}\text { restricted } \\
\text { sample }\end{array}$ & $\begin{array}{c}\text { main } \\
\text { sample }\end{array}$ & $\begin{array}{c}\text { restricted } \\
\text { sample }\end{array}$ \\
\hline $\begin{array}{l}\text { Less than } \\
\text { high school } \\
\text { diploma }\end{array}$ & $\begin{array}{c}-2.301 \\
(0.49)^{\star * *}\end{array}$ & $\begin{array}{c}-2.222 \\
(0.49)^{* * *}\end{array}$ & $\begin{array}{l}-0.229 \\
(0.32)\end{array}$ & $\begin{array}{l}-0.217 \\
(0.32)\end{array}$ & $\begin{array}{l}-0.360 \\
(037)\end{array}$ & $\begin{array}{r}-0.254 \\
(0.39)\end{array}$ & $\begin{array}{l}-0.275 \\
(0.34)\end{array}$ & $\begin{array}{l}-0.295 \\
(0.30)\end{array}$ \\
\hline $\begin{array}{l}\text { Incomplete } \\
\text { postsecondary }\end{array}$ & $\begin{array}{l}0.730 \\
(0.55)\end{array}$ & $\begin{array}{l}0.832 \\
(0.56)\end{array}$ & $\begin{array}{l}-0.157 \\
(0.47)\end{array}$ & $\begin{array}{r}-0.209 \\
(0.46)\end{array}$ & $\begin{array}{c}2.306 \\
(0.69)^{\star \star}\end{array}$ & $\begin{array}{c}2.695 \\
(0.61)^{\star \star *}\end{array}$ & $\begin{array}{l}0.345 \\
(0.48)\end{array}$ & $\begin{array}{l}0.015 \\
(0.47)\end{array}$ \\
\hline $\begin{array}{l}\text { Completed } \\
\text { trade certificate }\end{array}$ & $\begin{array}{l}0.566 \\
(0.49)\end{array}$ & $\begin{array}{l}0.594 \\
(0.50)\end{array}$ & $\begin{array}{l}-0.260 \\
(0.36)\end{array}$ & $\begin{array}{l}-0.291 \\
(0.36)\end{array}$ & $\begin{array}{r}-0.463 \\
(0.63)\end{array}$ & $\begin{array}{l}0.179 \\
(0.60)\end{array}$ & $\begin{array}{l}0.380 \\
(0.38)\end{array}$ & $\begin{array}{l}0.347 \\
(0.38)\end{array}$ \\
\hline $\begin{array}{l}\text { Completed } \\
\text { college diploma }\end{array}$ & $\begin{array}{l}0.127 \\
(0.51)\end{array}$ & $\begin{array}{l}0.221 \\
(0.51)\end{array}$ & $\begin{array}{l}-0.618 \\
(0.48)\end{array}$ & $\begin{array}{l}-0.581 \\
(0.51)\end{array}$ & $\begin{array}{c}1.363 \\
(0.43)^{\star \star}\end{array}$ & $\begin{array}{c}1.570 \\
(0.45)^{\star \star \star}\end{array}$ & $\begin{array}{l}-0.183 \\
(0.39)\end{array}$ & $\begin{array}{r}-0.274 \\
(0.40)\end{array}$ \\
\hline $\begin{array}{l}\text { Completed } \\
\text { university degree }\end{array}$ & $\begin{array}{l}0.392 \\
(0.59)\end{array}$ & $\begin{array}{l}0.396 \\
(0.59)\end{array}$ & $\begin{array}{l}-0.170 \\
(0.81)\end{array}$ & $\begin{array}{l}-0.077 \\
(0.81)\end{array}$ & - & - & - & - \\
\hline Age 15-24 & $\begin{array}{l}0.515 \\
(0.53)\end{array}$ & $\begin{array}{l}0.419 \\
(0.54)\end{array}$ & $\begin{array}{l}0.089 \\
(0.45)\end{array}$ & $\begin{array}{l}0.131 \\
(0.45)\end{array}$ & $\begin{array}{l}-0.723 \\
(0.53)\end{array}$ & $\begin{array}{r}-1.057 \\
(0.59)\end{array}$ & $\begin{array}{c}0.872 \\
(0.37)^{\star}\end{array}$ & $\begin{array}{c}0.829 \\
(0.37)^{*}\end{array}$ \\
\hline Age 25-34 & $\begin{array}{l}0.195 \\
(048)\end{array}$ & $\begin{array}{l}0.195 \\
(0.48)\end{array}$ & $\begin{array}{l}0.051 \\
(0.42)\end{array}$ & $\begin{array}{l}0.075 \\
(0.42)\end{array}$ & $\begin{array}{l}-0.110 \\
(0.49)\end{array}$ & $\begin{array}{l}0.039 \\
(0.46)\end{array}$ & $\begin{array}{l}0.117 \\
(0.35)\end{array}$ & $\begin{array}{l}0.147 \\
(0.35)\end{array}$ \\
\hline Age $45-54$ & $\begin{array}{l}-0.274 \\
(0.53)\end{array}$ & $\begin{array}{l}-0.238 \\
(0.54)\end{array}$ & $\begin{array}{l}-0.133 \\
(0.41)\end{array}$ & $\begin{array}{l}-0.093 \\
(0.41)\end{array}$ & $\begin{array}{l}-0.300 \\
(0.40)\end{array}$ & $\begin{array}{r}-0.213 \\
(0.43)\end{array}$ & $\begin{array}{l}0.597 \\
(0.36)\end{array}$ & $\begin{array}{l}0.680 \\
(0.36)\end{array}$ \\
\hline Age $55-64$ & $\begin{array}{l}-0.816 \\
(0.55)\end{array}$ & $\begin{array}{l}-0.706 \\
(0.56)\end{array}$ & $\begin{array}{l}-0.276 \\
(0.47)\end{array}$ & $\begin{array}{l}-0.270 \\
(0.47)\end{array}$ & $\begin{array}{l}0.561 \\
(0.51)\end{array}$ & $\begin{array}{l}0.988 \\
(0.52)\end{array}$ & $\begin{array}{c}-1.414 \\
(0.50)^{\star \star}\end{array}$ & $\begin{array}{c}-1.405 \\
(0.51)^{\star \star}\end{array}$ \\
\hline Married & $\begin{array}{l}0.026 \\
(0.40)\end{array}$ & $\begin{array}{l}0.030 \\
(0.40)\end{array}$ & $\begin{array}{l}0.475 \\
(0.37)\end{array}$ & $\begin{array}{l}0.440 \\
(0.38)\end{array}$ & $\begin{array}{l}-0.535 \\
(0.35)\end{array}$ & $\begin{array}{l}-0.557 \\
(0.36)\end{array}$ & $\begin{array}{l}0.135 \\
(0.33)\end{array}$ & $\begin{array}{l}0.065 \\
(0.34)\end{array}$ \\
\hline $\begin{array}{l}\text { Widowed, } \\
\text { divorced. } \\
\text { separated }\end{array}$ & $\begin{array}{l}0.866 \\
(0.57)\end{array}$ & $\begin{array}{l}0.727 \\
(0.62)\end{array}$ & $\begin{array}{l}-0.214 \\
(0.50)\end{array}$ & $\begin{array}{l}-0.247 \\
(0.50)\end{array}$ & $\begin{array}{c}-2.746 \\
(0.78)^{\star \star \star *}\end{array}$ & $\begin{array}{c}-3.054 \\
(0.69)^{\star \star \star}\end{array}$ & $\begin{array}{l}0.182 \\
(0.52)\end{array}$ & $\begin{array}{l}0.077 \\
(0.53)\end{array}$ \\
\hline Children < age 6 & $\begin{array}{c}-1.646 \\
(0.46)^{\star \star \star}\end{array}$ & $\begin{array}{c}-1.622 \\
(0.47)^{\star \star}\end{array}$ & $\begin{array}{c}-0.959 \\
(0.42)^{\star}\end{array}$ & $\begin{array}{c}-0.974 \\
(0.42)^{\star}\end{array}$ & $\begin{array}{l}0.093 \\
(0.44)\end{array}$ & $\begin{array}{l}0.481 \\
(0.48)\end{array}$ & $\begin{array}{l}-0.471 \\
(0.39)\end{array}$ & $\begin{array}{r}-0.440 \\
(0.39)\end{array}$ \\
\hline $\begin{array}{l}\text { Children } \\
\text { aged 6-14 }\end{array}$ & $\begin{array}{l}0.474 \\
(0.55)\end{array}$ & $\begin{array}{l}0.540 \\
(0.56)\end{array}$ & $\begin{array}{l}0.912 \\
(0.55)\end{array}$ & $\begin{array}{l}0.876 \\
(0.55)\end{array}$ & $\begin{array}{l}0.147 \\
(0.42)\end{array}$ & $\begin{array}{l}0.394 \\
(0.43)\end{array}$ & $\begin{array}{l}-0.452 \\
(0.38)\end{array}$ & $\begin{array}{r}-0.435 \\
(0.38)\end{array}$ \\
\hline $\begin{array}{l}\text { Children } \\
\text { aged 14-24 }\end{array}$ & $\begin{array}{l}-0.557 \\
(0.57)\end{array}$ & $\begin{array}{l}-0.538 \\
(0.57)\end{array}$ & $\begin{array}{l}0.207 \\
(0.48)\end{array}$ & $\begin{array}{l}0.172 \\
(0.48)\end{array}$ & $\begin{array}{c}1.160 \\
(0.47)^{\star}\end{array}$ & $\begin{array}{c}1.067 \\
(0.49)^{*}\end{array}$ & $\begin{array}{l}-0.630 \\
(0.52)\end{array}$ & $\begin{array}{l}-0.615 \\
(0.52)\end{array}$ \\
\hline Atlantic provinces & $\begin{array}{l}0.481 \\
(0.54)\end{array}$ & $\begin{array}{l}0.294 \\
(0.56)\end{array}$ & $\begin{array}{l}-0.705 \\
(0.42)\end{array}$ & $\begin{array}{l}-0.762 \\
(0.42)\end{array}$ & $\begin{array}{c}0.958 \\
(0.39)^{\star}\end{array}$ & $\begin{array}{c}1.153 \\
(0.43)^{\star \star}\end{array}$ & $\begin{array}{l}0.344 \\
(0.30)\end{array}$ & $\begin{array}{l}0.332 \\
(0.30)\end{array}$ \\
\hline Quebec & $\begin{array}{l}0.103 \\
(0.63)\end{array}$ & $\begin{array}{l}0.070 \\
(0.63)\end{array}$ & $\begin{array}{l}-0.470 \\
(0.49)\end{array}$ & $\begin{array}{l}-0.478 \\
(0.52)\end{array}$ & $\begin{array}{c}1.518 \\
(0.73)^{*}\end{array}$ & $\begin{array}{c}1.939 \\
(0.73)^{\star *}\end{array}$ & $\begin{array}{l}-0.128 \\
(0.53)\end{array}$ & $\begin{array}{l}-0.100 \\
(0.53)\end{array}$ \\
\hline Manitoba & $\begin{array}{l}0.846 \\
(0.54)\end{array}$ & $\begin{array}{l}0.780 \\
(0.57)\end{array}$ & $\begin{array}{r}-0.498 \\
(0.35)\end{array}$ & $\begin{array}{l}-0.577 \\
(0.36)\end{array}$ & $\begin{array}{c}1.030 \\
(0.50)^{\star}\end{array}$ & $\begin{array}{c}1.626 \\
(0.55)^{\star \star}\end{array}$ & $\begin{array}{l}0.566 \\
(0.35)\end{array}$ & $\begin{array}{l}0.621 \\
(0.36)\end{array}$ \\
\hline
\end{tabular}




\begin{tabular}{|c|c|c|c|c|c|c|c|c|}
\hline \multirow{3}{*}{$\begin{array}{l}\text { Appendix B3 } \\
\text { (continued) }\end{array}$} & \multicolumn{4}{|c|}{ Métis } & \multicolumn{4}{|c|}{ North American Indian } \\
\hline & \multicolumn{2}{|c|}{ Women } & \multicolumn{2}{|c|}{ Men } & \multicolumn{2}{|c|}{ Women } & \multicolumn{2}{|c|}{ Men } \\
\hline & $\begin{array}{c}\text { main } \\
\text { sample }\end{array}$ & $\begin{array}{c}\text { restricted } \\
\text { sample }\end{array}$ & $\begin{array}{c}\text { main } \\
\text { sample }\end{array}$ & $\begin{array}{c}\text { restricted } \\
\text { sample }\end{array}$ & $\begin{array}{c}\text { main } \\
\text { sample }\end{array}$ & $\begin{array}{c}\text { restricted } \\
\text { sample }\end{array}$ & $\begin{array}{c}\text { main } \\
\text { sample }\end{array}$ & $\begin{array}{c}\text { restricted } \\
\text { sample }\end{array}$ \\
\hline Saskatchewan & $\begin{array}{l}0.443 \\
(0.57)\end{array}$ & $\begin{array}{l}0.377 \\
(0.58)\end{array}$ & $\begin{array}{l}-0.586 \\
(0.41)\end{array}$ & $\begin{array}{l}-0.664 \\
(0.41)\end{array}$ & - & - & - & - \\
\hline Alberta & $\begin{array}{r}-0.017 \\
(0.45)\end{array}$ & $\begin{array}{r}-0.093 \\
(0.46)\end{array}$ & $\begin{array}{l}0.100 \\
(0.39)\end{array}$ & $\begin{array}{l}0.087 \\
(0.41)\end{array}$ & $\begin{array}{r}-0.349 \\
(0.46)\end{array}$ & $\begin{array}{l}-0.096 \\
(0.47)\end{array}$ & $\begin{array}{r}-0.343 \\
(0.39)\end{array}$ & $\begin{array}{r}-0.396 \\
(0.39)\end{array}$ \\
\hline British Columbia & $\begin{array}{l}-0.314 \\
(0.58)\end{array}$ & $\begin{array}{l}-0.336 \\
(0.57)\end{array}$ & $\begin{array}{l}-0.871 \\
(0.49)\end{array}$ & $\begin{array}{l}-0.818 \\
(0.50)\end{array}$ & $\begin{array}{c}1.071 \\
(0.42)^{*}\end{array}$ & $\begin{array}{c}1.556 \\
(0.48)^{\star \star}\end{array}$ & $\begin{array}{r}-0.304 \\
(0.34)\end{array}$ & $\begin{array}{l}-0.430 \\
(0.34)\end{array}$ \\
\hline Full-time job & $\begin{array}{l}0.129 \\
(0.36)\end{array}$ & $\begin{array}{l}0.186 \\
(0.36)\end{array}$ & $\begin{array}{l}0.324 \\
(0.42)\end{array}$ & $\begin{array}{l}0.314 \\
(0.44)\end{array}$ & $\begin{array}{l}0.547 \\
(0.35)\end{array}$ & $\begin{array}{l}0.110 \\
(0.36)\end{array}$ & $\begin{array}{l}0.586 \\
(0.33)\end{array}$ & $\begin{array}{c}0.672 \\
(0.31)^{*}\end{array}$ \\
\hline Primary industries & $\begin{array}{r}-0.140 \\
(0.61)\end{array}$ & $\begin{array}{l}0.002 \\
(0.66)\end{array}$ & $\begin{array}{l}-1.014 \\
(0.46)^{\star}\end{array}$ & $\begin{array}{l}-0.930 \\
(0.46)^{\star}\end{array}$ & $\begin{array}{r}-0.346 \\
(0.72)\end{array}$ & $\begin{array}{r}-0.435 \\
(0.80)\end{array}$ & $\begin{array}{r}-0.267 \\
(0.43)\end{array}$ & $\begin{array}{l}-0.309 \\
(0.44)\end{array}$ \\
\hline Manufacturing & $\begin{array}{r}-0.836 \\
(0.64)\end{array}$ & $\begin{array}{r}-0.662 \\
(0.68)\end{array}$ & $\begin{array}{l}-1.070 \\
(0.50)^{\star}\end{array}$ & $\begin{array}{l}-1.048 \\
(0.50)^{\star}\end{array}$ & $\begin{array}{l}0.209 \\
(0.75)\end{array}$ & $\begin{array}{l}0.127 \\
(0.75)\end{array}$ & $\begin{array}{l}-1.112 \\
(0.48)^{*}\end{array}$ & $\begin{array}{l}-1.147 \\
(0.48)^{\star}\end{array}$ \\
\hline Retail & $\begin{array}{r}-0.735 \\
(0.55)\end{array}$ & $\begin{array}{r}-0.610 \\
(0.61)\end{array}$ & $\begin{array}{c}-1.219 \\
(0.53)^{\star}\end{array}$ & $\begin{array}{l}-1.152 \\
(0.54)^{\star}\end{array}$ & $\begin{array}{r}-0.765 \\
(0.55)\end{array}$ & $\begin{array}{r}-1.115 \\
(0.59)\end{array}$ & $\begin{array}{l}-0.443 \\
(0.46)\end{array}$ & $\begin{array}{l}-0.475 \\
(0.49)\end{array}$ \\
\hline Public services & $\begin{array}{l}0.890 \\
(0.68)\end{array}$ & $\begin{array}{l}0.960 \\
(0.68)\end{array}$ & $\begin{array}{l}-1.872 \\
(0.99)\end{array}$ & $\begin{array}{l}-1.860 \\
(1.01)\end{array}$ & $\begin{array}{r}-0.734 \\
(0.67)\end{array}$ & $\begin{array}{r}-0.700 \\
(0.71)\end{array}$ & $\begin{array}{r}-0.265 \\
(0.68)\end{array}$ & $\begin{array}{l}-0.290 \\
(0.70)\end{array}$ \\
\hline Other services & $\begin{array}{l}1.293 \\
(0.81)\end{array}$ & $\begin{array}{l}1.320 \\
(0.80)\end{array}$ & $\begin{array}{l}-1.128 \\
(0.53)^{\star}\end{array}$ & $\begin{array}{l}-1.092 \\
(0.53)^{k}\end{array}$ & $\begin{array}{l}1.284 \\
(072)\end{array}$ & $\begin{array}{l}0.558 \\
(0.79)\end{array}$ & $\begin{array}{l}0.465 \\
(0.49)\end{array}$ & $\begin{array}{l}0.477 \\
(0.49)\end{array}$ \\
\hline Management & - & - & - & - & $\begin{array}{c}-3.013 \\
(1.00)^{\star \star}\end{array}$ & - & $\begin{array}{l}-0.863 \\
(0.83)\end{array}$ & - \\
\hline $\begin{array}{l}\text { Semi- } \\
\text { professionals }\end{array}$ & $\begin{array}{c}1.581 \\
(078)^{\star}\end{array}$ & $\begin{array}{r}1.513 \\
(0.78)\end{array}$ & $\begin{array}{l}-0.230 \\
(0.78)\end{array}$ & $\begin{array}{l}-0.281 \\
(0.81)\end{array}$ & $\begin{array}{l}-1.864 \\
(0.84)^{\star}\end{array}$ & $\begin{array}{l}-0.937 \\
(0.79)\end{array}$ & $\begin{array}{l}0.018 \\
(0.71)\end{array}$ & $\begin{array}{r}-0.340 \\
(0.76)\end{array}$ \\
\hline Sales personnel & $\begin{array}{c}1.764 \\
(0.58)^{\star *}\end{array}$ & $\begin{array}{c}1.691 \\
\left(0.57^{\star \star}\right)\end{array}$ & $\begin{array}{l}-0.287 \\
(0.72)\end{array}$ & $\begin{array}{l}-0.239 \\
(0.73)\end{array}$ & $\begin{array}{c}-2.432 \\
(0.63)^{\star \star \star \hbar}\end{array}$ & $\begin{array}{l}-1.097 \\
(0.50)^{\star}\end{array}$ & $\begin{array}{l}-1.414 \\
(0.64)^{*}\end{array}$ & $\begin{array}{l}-1.049 \\
(0.60)\end{array}$ \\
\hline Manual workers & $\begin{array}{c}2.749 \\
(0.72)^{\star * *}\end{array}$ & $\begin{array}{c}2.727 \\
(0.71)^{\star * *}\end{array}$ & $\begin{array}{l}0.765 \\
(0.70)\end{array}$ & $\begin{array}{l}0.783 \\
(0.71)\end{array}$ & $\begin{array}{c}-3.344 \\
(0.83)^{\star * \star}\end{array}$ & $\begin{array}{c}-2.055 \\
(0.75)^{\star \star}\end{array}$ & $\begin{array}{r}-0.954 \\
(0.63)\end{array}$ & $\begin{array}{l}-0.528 \\
(0.59)\end{array}$ \\
\hline Public sector & $\begin{array}{l}-1.175 \\
(0.50)^{*}\end{array}$ & $\begin{array}{l}-1.133 \\
(0.50)^{*}\end{array}$ & $\begin{array}{l}0.063 \\
(0.48)\end{array}$ & $\begin{array}{c}0.0349 \\
(0.48)\end{array}$ & $\begin{array}{r}-0.244 \\
(0.50)\end{array}$ & $\begin{array}{l}-0.494 \\
(0.50)\end{array}$ & $\begin{array}{l}-0.108 \\
(0.52)\end{array}$ & $\begin{array}{l}-0.521 \\
(0.53)\end{array}$ \\
\hline $\begin{array}{l}\text { Number of } \\
\text { observations }\end{array}$ & $N=129$ & $N=126$ & $N=198$ & $N=193$ & $N=155$ & $N=148$ & $N=239$ & $N=236$ \\
\hline Pseudo R2 & 0.4639 & 0.4379 & 0.2592 & 0.2572 & 0.4105 & 0.4020 & 0.2134 & 0.2237 \\
\hline $\begin{array}{l}\text { Log } \\
\text { pseudolikelihood }\end{array}$ & -8333.22 & -8231.9 & -14444.9 & -14025.7 & -9944.42 & -9561.1 & -20692.3 & -20096.4 \\
\hline
\end{tabular}

Source: The Labour Force Survey, national monthly master file, September 2008 - May 2011.

Note: Main samples include all paid employees, aged between 15 and 64, who are not full-time students. Restricted samples include only permanent paid employees, aged between 15 and 64, who are single-job holders and who are not students. Survey sampling weights (abowt) applied in all analyses. Standard errors reported in parentheses. 


\section{References}

Ashenfelter, O. \& Oaxaca, R. (1987). The economics of discrimination: Economists enter the courtroom. American Economic Review, 77(2), 321-325.

Bauer, T. K. \& Sinning, M. (2008). An extension of the Blinder-Oaxaca decomposition to nonlinear models. Advances in Statistical Analysis, 92(1), 197-206.

Ben, J. (2008). The Blinder-Oaxaca decomposition for linear regression models. The Stata Journal, 8(4), 453-479.

Bernard, A. \& Galarneau, D. (2010). Layoffs in Canada. Perspectives on Labour and Income, 11(5), 5-17, Statistics Canada, Catalogue No. 75-001-X.

Bilodeau, D. (1997). Seasonal adjustment: Why, when, how? Institut de la Statistique du Québec.

Blau, F. D. \& Kahn, L. M. (1981). Causes and consequences of layoffs. Economic Inquiry, 19(1), 270-296.

Blinder, A. S. (1973). Wage discrimination: Reduced form and structural estimates. Journal of Human Resources, 8(4), 436-455.

Chan, P. C. W., Morissette, R. \& Frenette, M. (2011). Workers laid-off during the last three recessions: Who were they, and how did they fare? Statistics Canada, Social analysis Division, Analytical Studies Branch Research Paper Series.

Cornfield, D. B. (1982). Seniority, human capital, and layoffs: A case study. Industrial Relations, 21(3), 352-364.

Delic, S. \& Abele, F. (2010). The recession and Aboriginal workers. In G. B. Doern \& C. Stoney (Eds.), How Ottawa Spends 2010-2011: Recession, Realignment, and the 
New Deficit Era (pp. 187-216). Montreal \& Kingston: McGill-Queen's University Press.

Delic, S. (2009). Statistical information pertaining to socio-economic conditions of northern Aboriginal people in Canada: Sources and limitations. The Northern Review, 30(1), 119-150.

De Silva, A. (1999). Wage discrimination against natives. Canadian Public Policy, 25(1), 65-85.

Drost, H. (1994). Schooling, vocational training and unemployment: The case of Canadian Aboriginals. Canadian Public Policy, 20(1), 52-65.

Eaton, C. B. (1970). Studying mass layoff through Markov Chains. Industrial Relations, 9(4), 394-403.

Elsby, M., Hobijn, B. \& Sahin, A. (2010). The labour market in the great recession. Paper prepared for Brookings Panel on Economic Activity, March 18-19, 2010.

Elvira, M. M. \& Zatzick, C. D. (2002). Who's displaced first? The role of race in layoff decisions. Industrial relations, 41(2), 329-361.

Even, W. E. \& Macpherson, D. A. (1993). The decline of private-sector unionism and the gender wage gap. The Journal of Human Resources, 28(2), 279-296.

Fairlie, R. W. (2005). An extension of the Blinder-Oaxaca to logit and probit models. Journal of Economic and Social Measurement, 30(4), 305-316.

Fairlie, R. W. (1999). The absence of the African-American owned business: An analysis of the dynamics of self-employment. Journal of Labor Economics, 17(1), 80-108.

Fairlie, W. \& Kletzer, L. G. (1998). Jobs lost, jobs gained: An analysis of Black/White differences in job displacement in the 1980s. Industrial Relations, 37(4), 460-477. 
Galarneau, D. \& Stratychuk, L. M. (2001). After the layoff. Perspectives on Labour and Income, 2(10), 19-29.

Gang, I. N., Sen, K. \& Yun, M-S. (2002). Caste, ethnicity and poverty in rural India. The Institute for the Study of Labor (IZA). Discussion Paper No. 629, November 2002.

George, P. \& Kuhn, P. (1994). The size and structure of native-white wage differentials in Canada. The Canadian Journal of Economics, 27(1), 20-42.

Gibbons, R. \& Katz, L. F. (1991). Layoffs and lemons. Journal of Labour Economics, $9(4), 351-380$.

Gilmore, J. \& La Rochbelle-Cote, S. (2011). Inside the labour market downturn. Perspectives on Labour and Income, 23(1), 1-14.

Gomulka, J. \& Stern, N. (1990). The employment of married women in the United Kingdom 1970-83. Economica, 57(1), 171-199.

Government of Canada (2012). The Employment Equity Act. Current to March 6, 2012. Last amended on December 31, 2006. Published by the Minister of Justice. Ottawa: Government of Canada.

Gunderson, M., Hyatt, D. \& Riddell, C. (2000). Pay differences between the government and private sectors: Labour Force Survey and Census estimates. Human Resources in Government Series, CPRN Discussion Paper No. W|10.

Hamermesh, D. S. (1989). What do we know about worker displacement in the U. S.? Industrial Relations, 28(1), 51-60.

Hu, L. \& Taber, C. (2005). Layoffs, lemons, race and gender. The Institute for the Study of Labor (IZA). Discussion Paper No. 172., August 2005. 
Hull, J. (2009). Aboriginal post-secondary education and labour market outcomes in Canada based on 2006 Census data. Prologica Research Inc. Winnipeg, Manitoba, October 20, 2009.

Hull, J. (2000). Aboriginal post-secondary education and labour market outcomes Canada, 1996. Prologica Research Inc. Winnipeg, Manitoba, October 17, 2000.

Jones, S. R. G. \& Riddell, C. W. (2006). Unemployment and nonemployment: Heterogeneities in labour market states. The Review of Economics and Statistics, $88(2), 314-323$.

Jung, H. \& Pirog, A. M. (2011). Nonexperimental impact evaluations. In D. J. Besharov \& P. H. Cottingham (Eds.), The workforce investment act: Implementation experiences and evaluation findings (pp. 407-429). Kalamzoo: W.E. Upjohn Institute for Employment Research.

Kleinfeld, J. \& Kruse, J.A. (1982). Native Americans in the labor force: Hunting for an accurate measure. Monthly Labor Review, 105(7), 47-51.

Kletzer, L. (1991). Job displacement, 1979-86: How Blacks fared relative to whites. Monthly Labor Review, 114(7), 17-25.

Kuhn, P. \& Sweetman, A. (2002). Aboriginals as unwilling immigrants: Contact, assimilation and labour market outcomes. Journal of Population Economics, 15(2), 331-355.

Kuo, C. Y. (1976). The effect of education on the earnings of Indian, Eskimo, Métis, and White workers in the Mackenzie District of Northern Canada. Economic Development and Cultural Change, 24(2), 387-398. 
Mar, D. \& Ong, P. (1994). Race and rehiring in the high-tech industry. Review of Black Political Economy, 22(3), 43-54.

Merrill, S., Bruce, D. \& Marlin, A. (2010). Considerations for successful transitions between postsecondary education and the labour market for Aboriginal youth in Canada. Sackville, N. B.: Rural and Small Town Program, Mount Allison University.

Miller, P. W. (1987). Gender differences in observed and offered wages in Canada, 1980. Canadian Journal of Economics, 20(2), 225-244.

Moore, T. (1992). Racial differences in post displacement joblessness. Social Science Quarterly, 73(3), 674-689.

Nielsen, H. S. (1998). Discrimination and detailed decomposition in a logit model. Economic Letters, 61(1), 115-120.

Oaxaca, R. (1999). Identification in detailed wage decompositions. Review of Economics and Statistics, 81(1), 154-157.

Oaxaca, R. (1994). On discrimination and the decompositions of wage differentials. Journal of Econometrics, 61(1), 5-21.

Oaxaca, R. (1973). Male-female wage differentials in urban labour markets. International Economic Review, 14(3), 693-709.

Oaxaca, R. L. \& Ransom, M. R. (1988). Searching for the effect of unionism on the wages of union and nonunion workers. Journal of Labor Research, 9(2), 139-148.

Ornati, O. A. \& Giblin, E. J. (1975). Recession layoffs can be discriminatory. Management Review, 64(5), 25-33. 
Patrinos, H. A. \& Sakellariou, C. N. (1992). North American Indians in the Canadian labour market: A decomposition of wage differentials. Economics of Education Review, 11(3), 257-266.

Pendakur, K. \& Pendakur, R. (2011). Aboriginal income disparity in Canada. Canadian Public Policy, 37(1), 61-83.

Pendakur, K. \& Pendakur, R. (2007). Minority earnings across the distribution. Canadian Public Policy, 33(1), 41-62.

Pendakur, K. \& Pendakur, R. (2002). Colour my world: Have earnings gaps for Canadian-born ethnic minorities changed over time? Canadian Public Policy, 28(4), 489-512.

Pendakur, K. \& Pendakur, R. (1998). The colour of money: Earnings differentials among ethnic groups in Canada. Canadian Journal of Economics, 31(3), 518-548.

Picot, G., Lin, Z., \& Pyper, W. (1998). Permanent layoffs in Canada: overview and longitudinal analysis. Canadian Journal of Economics, 31(5), 1154-1178.

Picot, G. Lin, Z., \& Pyper, W. (1997). An overview of permanent layoffs. Perspectives on Labour and Income, 9(13), 46-52.

Picot, G. (1992). Workers on the move: Permanent layoffs. Perspectives on Labour and Income, 4(3), 1-12.

Powers, D. A., Yoshioka, H. \& Yun, M. (2011). mvdemp: Multivariate decomposition for nonlinear response models. The Stata Journal, 11(4), 556-576.

Rowe, G. \& Nguyen, H. (2004). Longitudinal analysis of labour force survey data. Proceedings of Statistics Canada Symposium 2002: Modeling Survey Data for 
Social and Economic Research. Statistics Canada, Socio-Economic Analysis and Modeling Division, Analysis and Development Branch.

Singh, G. \& Reid, F. (1998). Are seniority-based layoffs discriminatory? The adverse impact of layoffs on designated groups. Industrial Relations, 53(4), 730-749.

Sinning, M., Hahn, M. \& Bauer, T. K. (2008). The Blinder-Oaxaca decomposition for nonlinear regression models. The Stata Journal, 8(4), 480-492.

Statistics Canada (2011). Guide to Labour Force Survey 2009. Statistics Canada, Labour Statistics Division, Ottawa. Catalogue Number 71-543-G.

Statistics Canada (2011a). Study: Inside the labour market downturn, October 2008 to October 2010. The Daily, Wednesday, February 23, 2011.

Statistics Canada (2009). Statistics Canada quality guidelines: Seasonal adjustment and trend-cycle estimation. Fifth Edition - October 2009. Catalogue No. 12-539-X.

Statistics Canada (2007). How Statistics Canada identifies Aboriginal Peoples. Statistics Canada, Social and Aboriginal Statistics Division, Ottawa. Catalogue Number 12592-XIE.

Statistics Canada (2007a). TABS - Report with universe and large codesets (September 2007 implementation). Statistics Canada, September 2007.

Statistics Canada (2007b). National Occupational Classification for Statistics (NOC-S).

Statistics Canada, Standards Division, Ottawa. Catalogue Number 12-583-XIE.

Statistics Canada (2007c). North American Industry Classification System (NAICS) Canada 2007. Statistics Canada, Standards Division, Ottawa. Catalogue Number 12-501-XIE. 
Stolzenberg, R. M. \& Relles, D. A. (1997). Tools for intuition about sample selection bias and its correction. American Sociological Review, 62(3), 494-507.

Usalcas, J. (2011). Aboriginal people and the labour market: Estimates from the labour force survey, 2008-2010. Research paper, Aboriginal Labour Force Analysis Series, Statistics Canada, Labour Statistics Division, Ottawa. Catalogue Number 71-588-X, no. 3.

Walters, D., White, J., \& Maxim, P. (2004). Does postsecondary education benefit Aboriginal Canadians? An examination of earnings and employment outcomes for recent Aboriginal graduates. Canadian Public Policy, 15(3), 283-301.

White, J., Maxim, P., and Gyimah, S. O. (2003). Labour force activity of women in Canada: A comparative analysis of Aboriginal and non-Aboriginal women. Canadian Review of Sociology and Anthropology, 40(4), 391-415.

Wilson, G. (2005). Race and job dismissal: African American/White differences in their sources during the early work career. American Behavioural Scientist, 48(9), 1182-1199.

Wilkins, R. \& Wooden, M. (2011). Gender differences in rates of job dismissal: Why are men more likely to lose their jobs? Institute of the Study of Labor (IZA), Discussion Paper 6225.

Winship, C. \& Mare, R. D. (1992). Models for sample selection bias. Annual Review of Sociology, 18(1), 327-350.

Wooldridge, J. M. (2010). Econometric analysis of cross section and panel data $\left(2^{\text {nd }}\right.$ ed.). Cambridge, Massachusetts: The Massachusetts Institute of Technology Press. 
Yun, M-S. (2008). Identification problem and detailed Oaxaca decomposition: A general solution and inference. Journal of Economic and Social Measurement, 33(1), 2738.

Yun, M-S. (2005). Normalized equation and decomposition analysis: Computation and inference. The Institute for the Study of Labor (IZA). Discussion Paper No. 1822, October 2005.

Yun, M-S. (2004). Decomposing differences in the first moment. Economics Lectures, $82(1), 275-280$.

Yun, M-S. (2000). Decomposition analysis for a binary choice model. The Institute for the Study of Labor (IZA). Discussion Paper No. 145, April 2000.

Zietsma, D. (2010). Aboriginal people living off-reserve and the labour market: Estimates from the Labour Force Survey, 2008-2009. Statistics Canada, Labour Statistics Division, research paper, the Aboriginal labour force analysis series, Catalogue Number 71-588-X, no. 2. 


\title{
Essay II: The Determinants of the Employment Status of Aboriginal Peoples in Canada
}

\begin{abstract}
Using confidential microdata from the 2006 Census and the 2006 Aboriginal Peoples Survey, this essay examines the association between human and social capital and various cultural factors and the probability of employment of Aboriginal people, conditional on their labour market entry. Separate estimates are presented for men and women in the three identity populations, namely Inuit, Métis and North American Indians living on-reserve and off-reserve in urban, rural and northern communities across Canada. Two key conclusions arise from this analysis. First, the results reveal surprisingly large variation in the effects of different characteristics on the probability of employment for individuals belonging to different Aboriginal identity groups. Even within the same identity group, the effects are markedly different for men and women and for urban and rural residents. This suggests that disaggregated analysis is essential when examining the employment outcomes of Aboriginal Canadians. Second, the results reveal that the standard employment models that account only for the key human capital variables and some structural factors could be misleading for some subgroups of Aboriginal people because the effects of some of these variables change when one adds in social capital and cultural factors into the employment equations. This suggests that for some subgroups of Aboriginal people social capital and cultural factors are potentially important omitted variables in these employment equations.
\end{abstract}

Key words: Aboriginal workers, determinants of employment status, human capital, social capital, culture 


\section{Table of Contents}

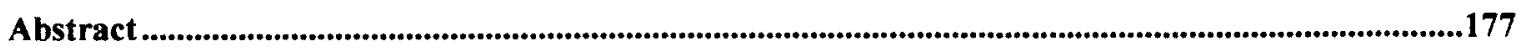

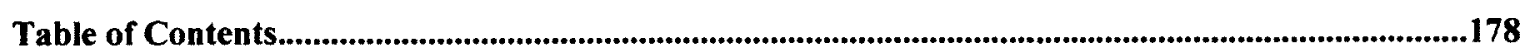

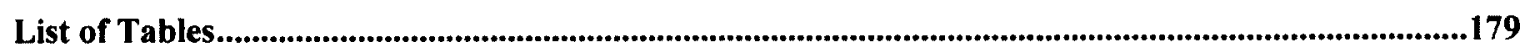

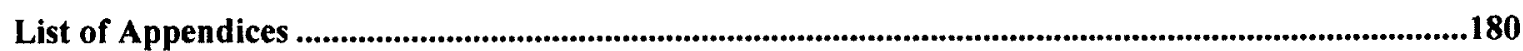

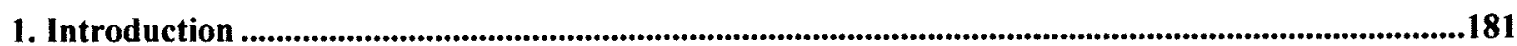

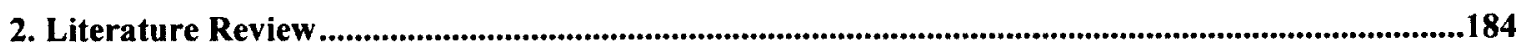

3. Data and Methodology ....................................................................................................................190

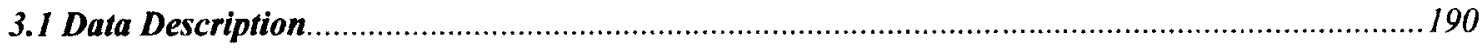

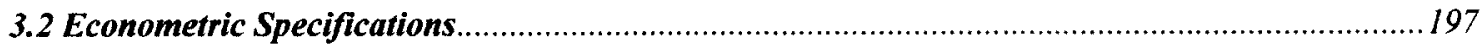

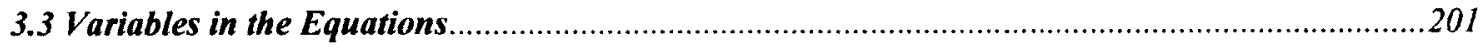

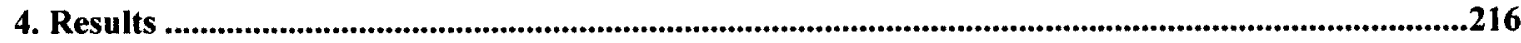

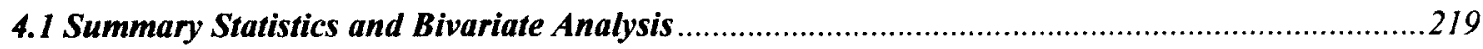

4.2 Multivariate Probit Regression Results for NAI Population ......................................................227

4.3 Multivariate Probit Regression Results for Métis Population .....................................................242

4.4 Multivariate Probit Regression Results for Inuit Population ................................................247

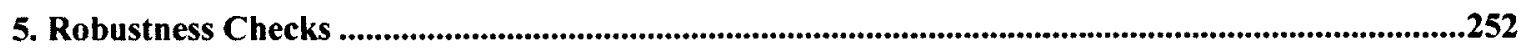

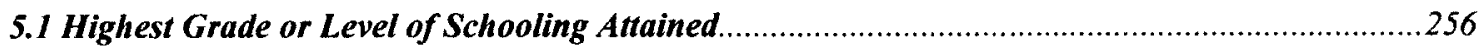

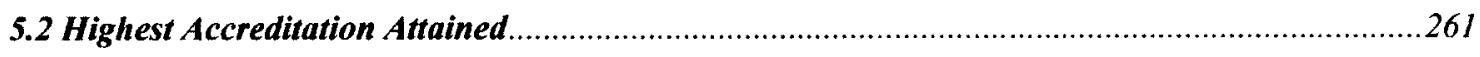

5.3 High School Certification and Highest Accreditation Attained ...............................................264

6. Discussion and Conclusions .............................................................................................................................268

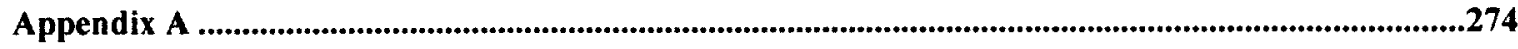

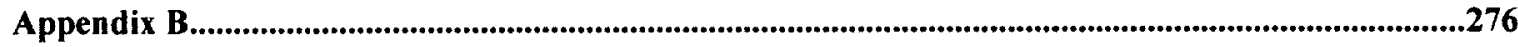

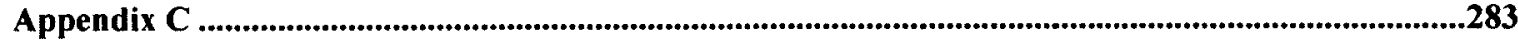

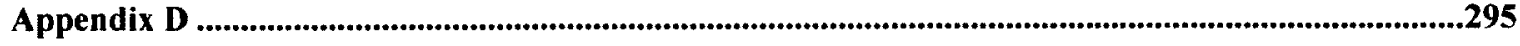

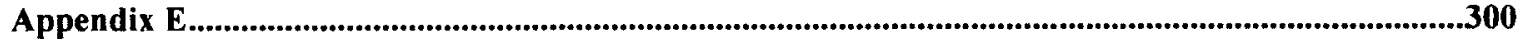

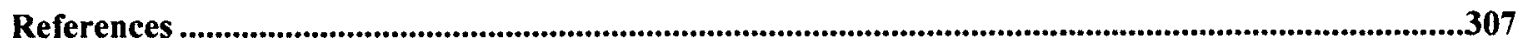




\section{List of Tables}

Table 1: The proportions of employed in the North American Indian, Métis and Inuit samples ......196

Table 2: Selected estimates from probit employment function for urban NAI, APS 2006..............228

Table 3: Selected estimates from probit employment function for rural NAI, APS 2006 ..............232

Table 4: Selected estimates from probit employment function for registered NAI, APS 2006.........235

Table 5: Selected estimates from probit employment function for non-registered NAI, APS 2006 ...239

Table 6: Selected estimates from probit employment function for Métis, APS $2006 \ldots \ldots \ldots \ldots \ldots \ldots \ldots . \ldots \ldots$

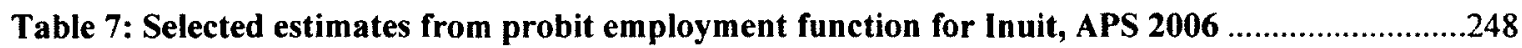

Table 8: Robustness checks for highest grade and highest level of schooling attained, APS 2006 _...258

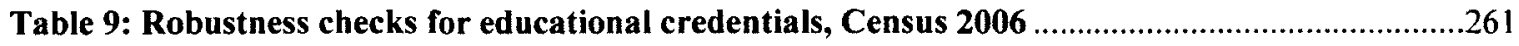

Table 10: Robustness checks for educational certification and credentials, Census $2006 \ldots \ldots \ldots \ldots \ldots . .265$ 


\section{List of Appendices}

\section{Appendix A}

Appendix A 1: Definitions of explanatory variables in the probit employment models, APS $2006 \ldots 274$

Appendix A 2: Definitions of explanatory variables in the robustness checks, Census 2006 .275

Appendix B

Appendix B 1: Sample means of all explanatory variables in the probit employment models, APS 2006 .

\section{Appendix C}

Appendix C 1: Detailed results from probit models predicting employment for urban NAI, APS 2006

Appendix C 2: Detailed results from probit models predicting employment for rural NAI, APS 2006

Appendix C 3: Detailed results from probit models predicting employment for registered NAI, APS 2006.

Appendix C 4: Detailed results from probit models predicting employment for non-registered NAI, APS 2006

Appendix C 5: Detailed results from probit models predicting employment for Métis, APS 2006...291

Appendix C 6: Detailed results from probit models predicting employment for Inuit, APS 2006 ....293

\section{Appendix D}

Appendix D 1: Detailed results from robustness checks for highest grade and highest level of schooling attained, APS 2006

\section{Appendix E}

Appendix E 1: Sample means of all explanatory variables in the probit employment models, Census

2006.

Appendix E 2: Detailed results from robustness checks for educational credentials, Census 2006 ...303

Appendix E 3: Detailed results from robustness checks for certification and credentials, Census 2006 


\section{Introduction}

Empirical studies in labour economics report overwhelming evidence of a positive correlation between human capital and labour market outcomes for the Canadian population as a whole (Ferrer \& Riddell, 2002). Studies probing into the outcomes of specific ethnic groups, however, find little or mixed evidence in support of such an association (Drost, 1994). This essay seeks to expand the analytical scope of the existing literature by adding variables from social capital theory as well as variables measuring some aspects of culture to employment models. The analysis is focused on the employment outcomes of an empirically under-researched (Pendakur \& Pendakur, 2011) segment of Canadian population, Aboriginal peoples.

The primary objective of the analysis is to establish if there is an association between various forms of capital - human, social, and cultural - and the probability of employment of individuals belonging to one of the three Aboriginal identity groups: Inuit, Métis, or North American Indian. ${ }^{44}$ The secondary objective is to determine if there is any variation in the association across groups, disaggregated by gender as well as by place of residence, urban/rural and on-reserve/off-reserve. The analysis is based on the confidential microdata from the 2006 Census and the 2006 Aboriginal Peoples Survey.

\footnotetext{
${ }^{44}$ Statistics Canada applies two methods to enumerate Aboriginal population: (1) using the general "ethnic origin" question, which allows the most inclusive definition of "Aboriginal ancestry" population, and (2) using a more specific "Aboriginal identity" question, which is the most precise indicator of an individual's affiliation with at least one of the three Aboriginal identity groups. On the 2006 Census form, this question was phrased in such a way as to accommodate multiple responses - that is, a respondent could have identified with more than one Aboriginal identity group. Data in this essay are restricted to respondents who reported membership in only one Aboriginal identity group - that is, only those respondents who identified with only one of the three groups of Aboriginal identity population (North American Indian, Inuit or Métis) are included and, throughout this essay, these respondents are referred to as the "single-reporting" Aboriginal identity population. The rationale for this restriction is explained in Section 2.
} 
As discussed in the Introduction of this thesis, the reported labour market statistics are markedly different for different Aboriginal identity groups. Previous research has uncovered some factors contributing to these outcomes but the conclusions reached are rather perplexing, stirring interest in further investigations. The central concern of this essay is to explore differences in the employment outcomes of individuals in each of the three Aboriginal identity groups and to assess the role different factors play in those outcomes, focusing in particular on factors laid out in human capital theory.

Conventional tests of human capital theory generally focuses on workers' human capital and its effect on the economic success in the form of earnings (Becker, 1993). The existing empirical tests of human capital model in the context of Aboriginal workers also involve examinations of the learning-earnings connection (Pendakur \& Pendakur, 1998, 2002, 2011). The learning-employment connection is generally overlooked. Arguably, for practical policy purposes, the learning-employment connection is equally important, if not more important, because growing awareness of the chronic employment gap between Aboriginal and non-Aboriginal people in Canada has motivated a variety of strategic gapreducing solution, mainly focused on job skills training and development programs. By focusing on the role different schooling plays in individuals' employment prospects, this essay will provide some useful information that can inform policy discussion in this area.

The essay is organized as follows: the following section briefly summarizes factors that have been identified in the existing literature on the topic and specifies more clearly the objectives and the contribution of the present analysis. Methodological considerations, including the description of the data sources as well as the econometric specifications and the theoretical underpinnings of the models are presented in Section 3. 
The main findings from multivariate regression analyses are presented in Section 4, separately for urban, rural and for the off-reserve North American Indian group, for Métis and for Inuit. Findings for the on-reserve North American Indian group and the robustness checks are discussed in Section 5 while Section 6 concludes the essay. 


\section{Literature Review}

What contributes to the gloomy picture of the labour market outcomes of the members of different Aboriginal identity groups in Canada? Relatively few survey-based analyses have examined the labour market outcomes of Aboriginal people in Canada. The analyses that exist focus almost exclusively on the income and earnings differentials (Kuo, 1976; Patrinos \& Sakellariou, 1992; George \& Kuhn, 1994; Bernier, 1997; De Silva, 1999; Maxim, White \& Beavon, 2003; Pendakur \& Pendakur, 1998, 2002, 2011). Only a few studies have looked specifically into the labour market activity — labour force participation, employment and unemployment - of Aboriginal people (Drost, 1994; Kuhn \& Sweetman, 2002; White, Maxim \& Gyimah, 2003; Walters, White, \& Maxim, 2004) and these studies made no inquiry into the specific circumstances of each identity group.

The findings from these studies, consistent with the descriptive evidence presented in the Introduction to this thesis, indicate that Aboriginal Canadians face substantially worse labour market prospects than non-Aboriginal Canadians - they are less likely to be employed, more likely to be unemployed or out of the labour force. Aboriginal men are reported to be at a greater disadvantage than Aboriginal women and the factors influencing the variations range from personal factors such as age and education to structural factors such as labour market conditions in different geographic locations. The rest of this section summarizes the key contributing factors reported in these studies and specifies the goals and potential contributions of the present analysis. 
Educational attainment is the most commonly mentioned factor contributing to the relative lack of employment success among Aboriginal people in Canada. All of the previous studies have reported that higher educational attainment significantly improves employment prospects of Aboriginal people (Drost, 1994; Kuhn \& Sweetman, 2002; White et al., 2003; Walters et al., 2004). However, there seems to be no consensus on the impact of different types and levels of educational attainment (Drost, 1994; Maxim \& White, 2006).

While Kuhn and Sweetman (2002) and White et al. (2003) find that higher educational attainment has a significant and monotonically positive effect on the employment status of Aboriginal people, Drost (1994) finds that "schooling that extends beyond the secondary school level does not steadily reduce the risk of unemployment among Aboriginals" (p. 59). ${ }^{45}$ Drost (1994) suggests that raising elementary and secondary school completion rates has the greatest potential for lowering the probability of unemployment among Aboriginal Canadians. In particular, Drost (1994) finds no evidence that vocational schooling and skill training at the post-secondary level produce any positive effect on the likelihood of employment among Aboriginal men. On the contrary, he finds that Aboriginal men with a trade school or community college certificate are more likely to be unemployed than Aboriginal men with a high school diploma as their highest educational qualification.

\footnotetext{
${ }^{45}$ Although not identical, the "single-origin" classification of Aboriginal ethnicity/ancestry in Kuhn and Sweetman (2002) is somewhat comparable with the term "single-reporting" classification of Aboriginal identity used in this study. The classification in Drost (1994), however, is not comparable at all as Drost's sample contains the total Aboriginal ethnic origin population that combines the single-origin and multiple origin ancestry. The classification in White et al. (2003) concerns only women and their sample is divided into non-Aboriginal, Registered/Status Indian, and Other Aboriginal women, excluding the Inuit.
} 
Drost (1994) also finds that positive influence of the university educational attainment on employment prospects is statistically significant only in the case of Aboriginal women - not in the case of Aboriginal men. Walters et al. (2004), on the other hand, report that "Aboriginals, both male and female, have better employment prospects if they have graduated from a trades or college program than from a university program ... [in fact, both] male and female Aboriginals with university degrees report the lowest levels of full-time employment and highest levels of unemployment, when compared with other university graduates" (p. 296).

In comparing employment rates between Aboriginal and non-Aboriginal youth, Maxim and White (2006) find that Aboriginal high school dropouts are significantly more likely to be employed than their non-Aboriginal counterparts. In regards to higher education, they find significant positive effects, particularly for First Nations people. ${ }^{46}$ The other commonly reported factor affecting the employment success of Aboriginal Canadians relate to their legal status and their place of residence. All of the previous studies have found the legal Indian Status, reserve and rural residence to be strongly associated with lower employment rates among Aboriginal Canadians. In fact, Kuhn and Sweetman (2002) and White et al. (2003) find these factors more important for the employment success of Aboriginal people than conventional human capital variables such as educational attainment.

\footnotetext{
${ }^{46}$ The term "First Nation(s)" pertains to the members of North American Indian identity group. The term "came into common usage in the 1970s to replace the word "Indian," which some people found offensive. Although the term First Nation is widely used, no legal definition of it exists. Among its uses, the term "First Nations peoples" refers to the Indian peoples in Canada, both Status [registered under the Indian Act] and Non-Status [not registered under the Indian Act]" (INAC, 2002, p.10). Statistics Canada collects this information using a separate question on the Census long-form questionnaire that asks respondents to selfidentify if they are a member of an Indian Band/First Nation (Statistics Canada, 2007, p.10).
} 
Controlling for the human capital variables, Kuhn and Sweetman (2002) and White et al. (2003) find that employment success is highest among non-Status Indians and Métis living in urban areas and lowest among Status Indians living on reserve and Inuit living in rural northern communities. The employment disadvantage was also found to be more pronounced for those with single Aboriginal ancestry, relative to those with multiple Aboriginal ancestries that includes some non-Aboriginal origin. In decomposing the overall Aboriginal to non-Aboriginal employment gap of 11.7 percentage points, Kuhn and Sweetman (2002), for instance, find that this gap "actually consists of only a 4.0 percentage point gap for those with multiple origins and a 25.2 percentage point gap for those with single origins" (p. 336).

With the exception of two studies, (Walters et al., 2004; Maxim \& White, 2006), which were based on data from the National Graduates Survey and the Youth in Transition Survey, respectively, all of the studies discussed above employed data from the publicly-available versions of the Canadian Census files, ranging from 1986 to 1996, and they focused primarily on the segment of Aboriginal ancestry population living in large urban centers. In fact, Kuhn and Sweetman (2002) exclude northern Aboriginal Canadians in their examination of the effect of social mobility on employment outcomes and White et al. (2003) do not include Inuit women in their analyses at all. Although Walters et al. (2004) include all three Aboriginal identity groups, they do not differentiate between registered and non-registered North American Indians.

In this essay, I employ confidential microdata from the 2006 Census and from the 2006 Aboriginal Peoples Survey and present multivariate analyses of the probability of employment for all three single-reporting Aboriginal identity groups, namely Inuit, Métis 
and North American Indian (NAI), living on-reserve and off-reserve in urban, rural and northern areas across Canada. This comprehensive geographic coverage as well as the fact that the study focuses on the single-reporting Aboriginal identity population will serve to fill an important gap in knowledge that is essential both for future empirical inquiries and for public policy considerations. Previous studies (Drost, 1994; Kuhn \& Sweetman, 2002; White et al., 2003) have relied on the census's ethnicity/ancestry/origin classifiers to identify their population samples and they have established that employment outcomes of the single-origin Aboriginal population are significantly poorer than the employment outcomes of the multiple-origin Aboriginal population. By relying on the Aboriginal identity classifier, which the census regards as the most accurate identifier of the group affiliation, this study will be able to provide a more accurate account of the differences among members of the three identity groups. The study also considers the registered and non-registered off-reserve North American Indian population and those living on-reserve, separately.

A further contribution to the existing literature on the topic is the wider theoretical scope of this study. As indicated earlier, the empirical examinations in the previous studies have focused almost exclusively on the role of human capital variables in affecting employment probabilities of Aboriginal people. This study simultaneously incorporates the variables from human and social capital theories as well as some cultural characteristics that have been identified as important in the employment outcomes of the general population (Sobel, 1982, 2002; Gerber, 1990; Becker \& Murphy, 2000; Gomez \& Santor, 2001; Annen, 2007). Assessing the relative impact of these variables on the probability of employment for different Aboriginal groups is potentially important as it 
could provide either evidence of omitted variable bias or a validation for their exclusion from the employment models.

A series of separate binomial probit models are constructed to estimate the relative impact of various sources of capital - human, social and cultural - on the probability of an Aboriginal identity individual being employed or unemployed, conditional on being in the labour force. The following section describes the data sources and specifies the variables in the equations. 


\section{Data and Methodology}

\subsection{Data Description}

The main results reported in this study are derived from the confidential master files of the 2006 Aboriginal Peoples Survey (APS). The results of robustness checks are based on data from the 2006 Census master file. The APS is a special post-censal survey whose target population, about 60,000 , was selected based on Aboriginal identity information provided on the 2006 Census long-form questionnaire (Statistics Canada, 2009). ${ }^{47}$ Because it combines a range of additional Aboriginal-specific information with the key census information, this survey offers the most comprehensive and current overview of the living conditions of the Aboriginal population, aged 6 and older, living off-reserve across Canada (Delic, 2009).

Previous research examining economic integration of Aboriginal Canadians was restricted to data from Canadian censuses, and most researchers relied exclusively on the census Public Use Microdata Files (PUMFs). ${ }^{48}$ Canadian censuses, however, contain only a small number of the variables that are needed for a detailed empirical examination of

\footnotetext{
${ }^{47}$ The APS was administered about five months after the 2006 Census enumeration day, which was May 16, 2006. The four questions used to identify Aboriginal population on the 2006 Census long-form questionnaire included the ethnic origin question (question 17), the Aboriginal self-reporting identity question (question 18), the Indian band or First Nation membership question (question 20), and the Treaty or Registered Indian question (question 21). The first question was used to derive the counts of the total Aboriginal ancestry population while the Aboriginal identity population, both single and multiple identity, was derived using the last three questions. Those who reported an Aboriginal origin without reporting an Aboriginal identity were defined as the Aboriginal ancestry-only population (Statistics Canada, 2009a).

${ }^{48}$ This data choice among earlier researchers is understandable, given the small size of the Aboriginal population, the historical difficulties in obtaining accurate and representative records on Aboriginal population in Canada as well as the difficulties of obtaining access to the Census master files. For those reasons, the census PUMFs have long been the only comprehensive and reliable data source for empirical analysis of issues that involve small population groups such as Aboriginal peoples in Canada. The author is grateful to the Canadian Research Data Centre Network (CRCDN) for granting her access to the confidential master files at the Carleton, Ottawa, Outaouais Local Research Data Centre (COOL RDC).
} 
complex determinants of the socioeconomic conditions of Aboriginal population. In fact, use of the PUMFs of Canadian censuses precludes much of the detailed analysis because of Statistics Canada's obligation to protect confidentiality of the information provided by individual respondents (Statistics Canada, 2006). Other data sources such as the monthly Labour Force Survey contain more detailed information on formal labour market activities but the sample sizes of Aboriginal identity populations in such surveys are often too small to allow any generalizable results, especially for Inuit (Patrinos \& Sakellariou, 1992; Pendakur \& Pendakur, 2002; Rowe \& Nguyen, 2004; Berg, 2005, Delic, 2009).

The APS was designed to address these data deficiencies by collecting comprehensive, nation-wide information through separate questionnaires that focus on a range of issues that are specifically relevant to each of the three main Aboriginal identity groups in Canada. Comprehensive information on issues such as language, housing, schooling and the use of communication technologies and traditional pursuits is not currently available in any other data set (Delic, 2009). These variables are important complements to the census-derived variables on geography, family structure, education and labour force activity, which have been appended to the APS analytical files. Inclusion of these previously unavailable explanatory variables in econometric models can potentially improve robustness of the findings and give a more complete picture of the complex factors affecting socioeconomic status of the heterogeneous Aboriginal population.

The analytical files of the 2006 APS survey contain individual records both for "Aboriginal ethnic ancestry/origin" population and for "Aboriginal identity" population. This study makes use of the latter definition, which refers to those individuals who 
reported identifying with at least one Aboriginal identity group, namely North American Indian, Métis or Inuit, or reported being a Treaty Indian or a Registered Indian, as defined by the Indian Act of Canada, or reported being members of an Indian band or First Nation (Statistics Canada, 2009). ${ }^{49}$ The APS analysis is focused on the population reporting single Aboriginal identity, which includes single Inuit identity, single Métis identity, and single North American Indian identity living off-reserve. ${ }^{50}$ The rest of this section presents more details on the contents of the individual data files used for model construction.

Three group-specific data files from the APS are used to build econometric models for each of the three identity groups. To model the probability of employment for the off-reserve North American Indian group, the "Adult Core" analytical file is used. This is the main data file that contains all core information on health, language, housing and communication technology from the APS and selected variables on education, labour market activity and income from the 2006 Census for all Aboriginal populations, aged 15 and over (Statistics Canada, 2008). Although this data file could be used to model the probability of employment for Métis and Inuit population as well, a choice was made to use their groups' specific files because they contain additional group-specific variables.

The model for Métis population is built using the "Métis Supplement" data file. This data file contains a number of Métis-specific variables such as family background,

\footnotetext{
${ }^{49}$ The former definition refers to those individuals who reported at least one ancestor who was North American Indian, Inuit or Métis. Based on the 2006 Census counts, most members of the Aboriginal identity population, 99 percent, also have Aboriginal ancestries while a substantial portion, 27 percent, of the Aboriginal ancestry population are not part of the identity population (Statistics Canada, 2009a).

${ }^{50}$ In the ten provinces, the 2006 APS targeted only Aboriginal population 6 years of age and older, living in private dwellings, excluding people living in Indian Settlements or on reserve. The survey target population in the three Territories, however, covered all of the Aboriginal residents, including the on-reserve residents (Statistics Canada, 2009a).
} 
household information and cultural background, in addition to all of the variables found in the Adult Core file (Statistics Canada, 2008a). The sets of variables in the model for Inuit population are derived from the "Arctic File," which contains all of the variables found in the Adult Core file and the data from the Arctic supplement that was administered to respondents living in the four Inuit regions, collectively called Inuit Nunangat (Statistics Canada, 2009b). ${ }^{51}$

The APS analytical samples of the three Aboriginal identity groups, as defined by single identities, in this study are restricted to labour force participants aged 15 to 64 who are not enrolled as full-time students and who are not recipients of basic income assistance. ${ }^{52}$ Excluded from these samples are the records of individuals with missing values on important variables such as Aboriginal identity, age or education. ${ }^{53}$ The Census

\footnotetext{
${ }^{51}$ The four Inuit regions include: (1) communities situated in the northern coastal Labrador region called Nunatsiavut, (2) communities in the northern Quebec region called Nunavik, (3) communities dispersed across the territory of Nunavut, and (4) communities in the Inuvialuit region of the Northwest Territories (Statistics Canada, 2009b).

${ }^{52}$ The APS question [D01E], which was posed to all respondents, asked: "During the year ending December 31,2005 , did you yourself receive any income from - social assistance or welfare benefits" (Statistics Canada, 2008, p. 105). Although Drost (1994) has found this variable to be highly significant in the unemployment equation of Aboriginal people, a decision was made in this study to filter out from the analytical samples all respondents who gave an affirmative answer to this question. The rationale for this decision is based on the a priori assumption that the determinants of employment outcomes of welfare recipients are likely to be different from those of non-recipients because the recipients are generally constrained by the rules and regulations of the assistance programs in their respective jurisdictions. Indeed, Christofides (2003) provides some evidence on the interrelation between the labour force and social assistance participation decisions for the general population in Canada. It would be interesting to explore this interrelation in an Aboriginal context but that would require a separate study. The exclusion of this variable reduced the total sample by 12.4 percent (about 3670 individuals in the Adult Core data file reported being welfare recipients). The sample was further reduced by 13.7 percent due to exclusion of 4040 full-time students. These numbers pertain to all groups in the Adult Core file, totaling to about 29520 .

${ }^{53}$ The missing values problem was present in each analytical file and was particularly pronounced in the Inuit samples. In the case of NAI women, for instance, about 27 percent of total cases selected for analysis contained missing values while in the case of Métis women and Inuit women, the proportion of cases with missing values were 30 percent and 45 percent, respectively. The proportion of cases with missing values in the NAI men sample was 21 percent. The same figures for Métis men and Inuit men were 25 percent and 46 percent, respectively. Imputing the missing values was deemed inappropriate (Greene, 2003) and the cases were excluded from the analyses.
} 
analytical samples are restricted to labour force participants aged 15 to 64 who are not

attending school. ${ }^{54}$

After the sample selection just described, the total APS samples that entered the regression model for North American Indian (NAI) identity were 2,610 for women and 2,420 for men. About 58 percent of NAI women and about 60 percent of NAI men were Registered or Treaty Indians. About 23 percent of NAI women and about 27 percent of NAI men lived in rural areas. The Métis estimation sample was composed of 2,250 women, about 28 percent of whom lived in rural areas and of 2,150 men, 30 percent of whom lived in rural areas. The Inuit samples contained 710 observations for women and 720 observations for men. The Census samples for NAI contained 68,750 observations for the on-reserve group and 102,310 for the urban off-reserve group, while for Métis and Inuit the samples were 29,410 and 12,060 labour force participants, respectively. Separate analyses were done for male and female members of each Aboriginal identity group, applying survey-proper weights. ${ }^{55}$ Further disaggregation, urban/rural and registered/non-registered, was done in the APS NAI samples, as suggested by the results

\footnotetext{
${ }^{54} \mathrm{~A}$ new version of detailed school attendance question was included in the 2006 Census but the variable was not released in the analytical files due to some data quality concerns (Statistics Canada, 2008b). Instead, a restricted dummy variable was provided and was used in this study to differentiate those attending from those not attending school, without accounting for part-time and full-time status. The welfare income information was not used because it was not possible to discern recipients from nonrecipients (Statistics Canada, 2008b).

${ }^{55}$ The APS has a complex survey design that involves stratifications, multiple stages of selection, unequal probabilities of selection, non-response and post-stratification adjustments (Statistics Canada, 2008). Because applying standard weights that do not account for these design features can lead to incorrect conclusions (Rao, 2006) the bootstrap resampling technique was applied in all analysis to obtain more accurate inferences (Fox, 2008). In each of the analytical samples, the procedure involved drawing 1000 bootstrap resamples by sampling from the individual observations with replacement from the original sample and then producing estimates for each subsample (Cameron \& Trivedi, 2010, p. 424). The procedure is intended to mimic sampling from the original population by treating the sample as if it were the population; in other words, "[t] he population is to the sample as the sample is to the bootstrap samples" (Fox, 2008, p.591, emphasis in the original). Individual composite weights (compw2) were applied in the Census estimation samples, along with clustering by census subdivision of current residence $(p c s d)$.
} 
of a Chow-like Test. ${ }^{56}$ In each model, the dependent variable was employment status, conditional on being in the labour force. For the purpose of this study, individuals are defined as employed if, during the week prior to the survey, they did any work for pay or for family business. The unemployed are those individuals who reported not having a job during the reference week but were actively searching for one. ${ }^{57}$

Table 1 presents the proportions of the employed in the APS and Census samples for each identity group separately. What is apparent in Table 1 is that the proportions of the employed for each identity group are notably smaller in the Census samples, relative to the APS samples. For men, the difference ranges from 5 to 13 percentage points and for women, the difference ranges from 5 to 11 percentage points. Another point that stands out in Table 1 is that, in each sample, women were more likely to be employed

\footnotetext{
${ }^{56}$ A generalized Hausman-type test (Wooldridge, 2010) was run using Stata's seemingly unrelated estimation command (suest) to verify if coefficients vary between NAI (urban/rural and registered/nonregistered) groups and between Métis (urban/rural) groups. The procedure entailed estimating separate regression models for each group, storing the results and then combining them into one matrix to test simultaneously whether coefficients differ significantly across groups. Except for a very small difference in the coefficient on presence of young children, no significant difference was detected in the Métis urban/rural model, as reflected in the overall test statistic for men $\left(\chi^{2}=54.30, p=0.1159\right)$ and for women $\left(\chi^{2}=54.81, p=0.1069\right)$. Many of the coefficients in the NAI urban/rural and registered/non-registered models were significantly different, and in particular those pertaining to the educational attainment. The overall test statistic for the NAl urban/rural model was $\left(\chi^{2}=72.94, p=0.0016\right)$ for men and $\left(\chi^{2}=71.22, p\right.$ $=0.0024)$ for women. Similarly, in the registered/non-registered model, the overall test statistic was $\left(\chi^{2}=\right.$ $63.76, p=0.0129)$ for men and $\left(\chi^{2}=66.52, p=0.0071\right)$ for women. Hence, separate analyses were done for these NAI groups.

${ }^{57}$ Note that this definition of employment includes both those employed full-time (more than 30 hours a week) and those employed part-time (less than 30 hours a week) and it follows the exact guidelines of the Statistics Canada's definition of employment. Under these guidelines, individuals are counted as employed if during the reference week (Sunday to Saturday) prior to Census Day (a) they did any work at all for pay as employees or as self-employed or as unpaid workers contributing directly to family business and if (b) they had a job but were absent in the reference week due to personal reasons an responsibilities, vacation, labour dispute or other reasons, excluding the reasons of layoff, between casual jobs, and waiting to take up a job at some future date. The definition of unemployment, however, does not strictly correspond to Statistics Canada's guidelines, which require the active job-seekers to also express their availability to take a job in order to be classified as unemployed. The employed and unemployed counts in this study come from the Census DEMPSTAT variable (Statistics Canada, 2008) which summarizes three categories: (1) respondents who reported working for pay or in self-employment, or being temporary absent from job; (2) respondents who reported not having a job but were looking for work; and (3) respondents who reported not having a job and not looking for one. These three categories in DEMPSTAT were derived from the questions $\mathrm{C} 01, \mathrm{CO} 3$, and $\mathrm{C} 04$, none of which inquired about respondent's availability to take a job.
} 
than men. The magnitude of the differences between men and women is smaller in the APS sample (three percentage points is the largest gap) than in Census samples (nine percentage points). Across identities, NAI living on reserve and Inuit had the smallest proportions employed while Métis had the highest proportions employed. ${ }^{58}$

Table 1: The proportions of employed in the North American Indian, Métis and Inuit samples

\begin{tabular}{|c|c|c|c|c|}
\hline \multirow{3}{*}{ Aboriginal identity groups } & \multicolumn{4}{|c|}{ Proportions Employed } \\
\hline & \multicolumn{2}{|c|}{$\begin{array}{c}\text { APS } 2006 \text { sample } \\
\text { Individuals, aged } 15 \text { to } 64 \text {, } \\
\text { who are not full-time students, } \\
\text { and who are not recipients of social assistance }\end{array}$} & \multicolumn{2}{|c|}{$\begin{array}{c}\text { Census } 2006 \text { sample } \\
\text { Individuals, aged } 15 \text { to } 64 \text {, } \\
\text { who are not enrolled in school }\end{array}$} \\
\hline & men & women & men & women \\
\hline On-reserve NAI & - & - & $\begin{array}{c}0.704 \\
(0.46)\end{array}$ & $\begin{array}{l}0.792 \\
(0.41)\end{array}$ \\
\hline Off-reserve, urban NAI & $\begin{array}{c}0.923 \\
(0.27)\end{array}$ & $\begin{array}{l}0.940 \\
(0.24)\end{array}$ & $\begin{array}{c}0.800 \\
(0.40)\end{array}$ & $\begin{array}{r}0.835 \\
(0.37)\end{array}$ \\
\hline Off-reserve, rural NAI & $\begin{array}{l}0.900 \\
(0.29)\end{array}$ & $\begin{array}{c}0.930 \\
(0.26)\end{array}$ & $\begin{array}{c}0.833 \\
(0.37)\end{array}$ & $\begin{array}{l}0.880 \\
(0.32)\end{array}$ \\
\hline Off-reserve, registered NAI & $\begin{array}{r}0.903 \\
(0.30)\end{array}$ & $\begin{array}{c}0.930 \\
(0.26)\end{array}$ & $\begin{array}{r}0.850 \\
(0.36)\end{array}$ & $\begin{array}{l}0.861 \\
(0.35)\end{array}$ \\
\hline Off-reserve, non-registered NAI & $\begin{array}{c}0.940 \\
(0.24)\end{array}$ & $\begin{array}{c}0.951 \\
(0.22)\end{array}$ & $\begin{array}{c}0.895 \\
(0.31)\end{array}$ & $\begin{array}{c}0.902 \\
(0.30)\end{array}$ \\
\hline Métis & $\begin{array}{c}0.948 \\
(0.22)\end{array}$ & $\begin{array}{r}0.956 \\
(0.20)\end{array}$ & $\begin{array}{c}0.901 \\
(0.30)\end{array}$ & $\begin{array}{c}0.914 \\
(0.28)\end{array}$ \\
\hline Inuit & $\begin{array}{l}0.880 \\
(0.33)\end{array}$ & $\begin{array}{l}0.906 \\
(0.29)\end{array}$ & $\begin{array}{l}0.755 \\
(0.43)\end{array}$ & $\begin{array}{l}0.831 \\
(0.37)\end{array}$ \\
\hline
\end{tabular}

Sources: APS, 2006 analytical files; bootstrap weights in effect. Census 2006, analytical file; individual composite weights in effect. Standard deviations in parentheses.

Note: The means reported in this table represent the proportions of observations of cases coded 1 in each sample. As such, the mean can also be interpreted as a probability that a randomly selected person from the sample will possess the characteristic measured by the mean. For instance, in the case of ten observations, 1111110000 , the mean is $6 / 10=.6$, which is the probability that any one case out of ten has a score of 1 .

While some these differences are likely due to the differences in the sample restrictions imposed in the two data sets, a separate regression analysis in each data set is done to gain an insight into other factors contributing to these outcomes. Econometric

\footnotetext{
${ }^{58}$ It should be noted here that the 'traditional sources of employment' such as hunting or fishing or work in other aspects of subsistence economy are unique to Aboriginal people and would not necessarily be reflected in these statistics, regardless of how inclusive the definition of employment is in these surveys.
} 
specifications of the regression model and theoretical considerations in the selection of explanatory variables are described in the following two sections, respectively.

\subsection{Econometric Specifications}

The key empirical question in this study is, "what determines the employment probability for an Aboriginal identity individual"? Do Aboriginal Canadians belonging to different Aboriginal identity groups have lower probabilities of employment because they are endowed with human and social capital characteristics generally associated with lower levels of attachment to the labour force? Are the employment probabilities of Aboriginal Canadians affected by unique features associated with their Aboriginality?

To gain some insight into these questions, four separate multivariate probits are run for each group in which blocks of explanatory variables are introduced sequentially to examine whether adding a block of social capital variables and cultural variables to the equation containing only human capital variables makes any significant contribution to the explanatory power of the models. ${ }^{59}$ Basic econometric specifications of this binomial probit model and the estimation procedure are explained below.

The conventional economic labour-leisure models suggest that individual $i$ would be observed in employment, rather than being unemployed, if his or her reservation wage ${ }^{60}\left(W_{r i}\right)$ is less than the offered wage $\left(W_{o i}\right)$. An individual's offered wage is

\footnotetext{
${ }^{59}$ The variables relating to the human capital theory are introduced in two separate blocks: the first block containing only the basic demographic characteristics such as education, age, official languages and the use of technology variables and the second block containing those four and all other human capital variables.

${ }^{60}$ Given the topic of investigation (the paid labour market) and the population group (Aboriginal Canadians) an uninformed reader may be tempted to make a connotation between the term "reservation wage" discussed in this section and the "Indian reservations," as they are sometimes called, where many Aboriginal people reside. This note is to clarify that the term "reservation wage," as discussed in this section, has no connection to the Indian reserves. Also, since the objective in this study is to predict an individual's employment probability, conditional on the individual's participation in the labour force, this
} 
determined in the labour market and is said to be influenced primarily by human capital factors such as education, labour market skills and work experience. An individual's reservation wage threshold is affected by various direct and indirect costs of working such as affordable childcare, transportation and other related costs. ${ }^{61}$ An increase in any of these costs means that the wage rate necessary to attract this individual into employment also must increase. Having exogenous income from other sources also tends to increase an individual's reservation wage, necessitating further increases in the offered wage in order to encourage the individual to take the employment offer.

Both the reservation and the offered wages here are taken as a function of particular characteristics $\left(\mathrm{X}_{r i}\right.$ and $\left.\mathrm{X}_{i i}\right)$ where the $o$ subscript refers to offered wages and the $r$ subscript refers to reservation wages. For those in employment then $W_{o i}>W_{r i}$ and for those not in employment $W_{o i}<W_{r i}$. Hence the probability of an $i$ th individual being in employment is $\pi(i)=\pi\left(W_{o i}>W_{r i}\right)$.

Theoretically, this dichotomous employment variable can be viewed as a proxy for an underlying variable that is really continuous; whether an individual succeeds or fails is really a matter of degree because some might be more successful than others. To predict this underlying latent variable, a binomial probit regression model (Wooldridge, 2006) is derived from an underlying latent variable model.$^{62}$ In the latent variable

reservation wage $\left(\mathrm{W}_{r i}\right)$ refers to the "minimum acceptable wage" and is not identical to the concept of reservation wage discussed in the context of the labour force participation decision. The minimum acceptable wage equates the marginal benefits and marginal costs of job search and determines when an individual will stop searching for a job and accept the job that offers this or better wage (Benjamin, Gunderson \& Riddell, 2002, p.536).

${ }^{61}$ Other related costs, in the case of Aboriginal people, could, for instance, include the availability of work in subsistence sector or any other cultural factor. The following section offers some discussion on that.

${ }^{62}$ An alternative was to use a logit model. The core difference between these two binary choice models lies in the assumption about the distribution of errors in a data set. In the probit model, errors are assumed to follow the standard normal distribution whereas in the logit model errors are assumed to follow the 
approach, the model is expressed with $\gamma^{*}$ as an unobserved variable, assumed to be linearly related to a set of factors $\chi$ and the error term $\mu$,

$$
\gamma_{i}^{*}=\beta_{0}+\beta_{1} \chi_{i 1}+\beta_{2} \chi_{i 2}+\ldots+\beta_{k} \chi_{i k}+\mu_{i}
$$

In this equation $\gamma^{*}$ is an unobservable magnitude which can be regarded the net benefit to individual $i$ of taking a particular course of action, in this case obtaining employment. While it is not possible to observe that net benefit, it is possible to observe the behavioural outcome whereby,

$$
\gamma_{i}= \begin{cases}0 & \text { if } \gamma_{i}^{*} \leq 0 \\ 1 & \text { if } \gamma_{i}^{*}>0\end{cases}
$$

That is, it is possible to observe whether the individual was employed or unemployed at the time of the survey. If the individual was employed, $\gamma_{i}=1$ would be observed, and that would be taken as evidence that a rational economic actor made a decision that improved his or her welfare based on rational calculations involving the reservation and offered wages.

Assuming that the individual observations $\left(x_{i}, y_{i}\right)$ are independently and identically distributed, that the explanatory variables are exogenous and that the error term is normally distributed and homoscedastic $\mu_{i} \mid \chi_{i} \sim N\left(0, \sigma^{2}\right)$, the employment response probability for $\gamma_{i}$, using the equations (1) and (2), is

$$
\begin{aligned}
\operatorname{Pr}\left(\gamma_{i}=1 \mid \chi_{i}\right) & =\operatorname{Pr}\left(\gamma_{i}^{*}>0 \mid \chi_{i}\right)=\operatorname{Pr}\left(\mu_{i}>-\beta_{0}-\beta_{1} \chi_{1_{i}}-\beta_{2} \chi_{2_{i}}-\ldots-\beta_{k} \chi_{k_{i}} \mid \chi_{i}\right) \\
& =1-\Phi\left(-\beta_{0}-\beta_{1} \chi_{1_{i}}-\beta_{2} \chi_{2_{i}}-\ldots-\beta_{k} \chi_{k_{i}}\right)
\end{aligned}
$$

standard logistic distribution. There are no strong theoretical grounds for choosing one over the other and empirically, the choice is arbitrary as there is generally very small difference in predicted probabilities from logit and probit models, though exceptions are possible (Greene, 2003, p.667; Wooldridge, 2006, p585). 


$$
=\Phi\left(\beta_{0}+\beta_{1} \chi_{1_{i}}+\beta_{2} \chi_{2_{i}}+\ldots \beta_{k} \chi_{k_{i}}\right)
$$

where $\Phi$ is the c.d.f. of the standard normal distribution.

Although the signs of each parameter $\beta$ here are informative, their magnitude is not particularly useful since they pertain to the latent model. The primary goal of this analysis is to estimate the magnitude of the effect of an independent variable in a block of factors $\chi$ on the probability of employment $\operatorname{Pr}(\gamma=1 \mid \chi)$ for different individuals, which requires estimating marginal effects for each observation.

Since all of the explanatory variables in the employment model are binary variables, to estimate the effect of, say, $\chi_{j}$ on the response probability requires finding the partial effect from changing $\chi_{j}$ from zero to one, holding all else constant. Using all of the data in the sample, the computation is specified as,

$$
\begin{aligned}
& \Phi\left(\beta_{0}+\beta_{1} \chi_{1}+\beta_{2} \chi_{2}+\ldots+\beta_{j-1} \chi_{j-1}+\beta_{j}+\beta_{j+1} \chi_{j+1}+\ldots+\beta_{k} \chi_{k}\right) \\
& -\Phi\left(\beta_{0}+\beta_{1} \chi_{1}+\beta_{2} \chi_{2}+\ldots+\beta_{j-1} \chi_{j-1}+\beta_{j+1} \chi_{j+1}+\ldots+\beta_{k} \chi_{k}\right)
\end{aligned}
$$

Because the marginal effect in equation (4) is dependent on the values of independent variables, to make the interpretation more meaningful, the preferred practice is to take the average of the marginal effects estimated for each observation and report the mean of the effects (Cameron \& Trivedi, 2010; Williams, 2012). ${ }^{63}$ The formula used for averaging the marginal effect of dummy variables over the entire sample (AMEs) is,

\footnotetext{
${ }^{63}$ The popular alternative, Marginal Effects at the Mean (MEMs) whereby the marginal effects are computed holding all independent variables at their means, was deemed inappropriate for the present analysis because of the high degree of heterogeneity within the Aboriginal population groups. The other alternative, Marginal Effects at Representative Values (MERs) whereby marginal effects are calculated using a range of values of one or more variables and then observing how the marginal effects differ across that range, could be informative but was not chosen because of potential cumbersomeness in specifying
} 
$n^{-1} \sum_{i=1}^{n}\left(\Phi\left(\chi_{-j} \hat{\beta}_{-j}+\chi_{j} \hat{\beta}_{j}\right)-\Phi\left(\chi_{-j} \widehat{\beta}_{\jmath}\right)\right)$

where the subscript $-j$ implies all variables excluding that indexed by $j .{ }^{64}$ The parameters are estimated by iterative maximum likelihood estimation procedure and the associated bootstrapped standard errors for the average marginal effects are obtained using the delta method (Cameron \& Trivedi, 2010). The following section describes the explanatory variables included in the models.

\subsection{Variables in the Equations}

As described in the previous section, the dependent variable in this study is the probability of employment, conditional on labour force participation, and is measured by a binary indicator, restricted to take on values of $\{0,1\}$ and coded as one if an individual reported being employed and zero if unemployed. For each Aboriginal identity group this dependent variable is regressed on a number of independent variables derived from the

representative values; even specifying only the variables of greatest interest such as educational attainment would be cumbersome as there are a number of classifications of that variable included in this study.

${ }^{64}$ An example using one of the variables from the model (Band Membership) can provide an intuitive insight into this procedure. The initial two steps of this procedure stipulate (1) to go to the first case in the sample and treat that individual as though the individual is a Band Member, regardless if that is true or not, leave all other independent variable values intact and compute the probability this person would be employed; (2) repeat the same procedure with the same individual, only this time treat the individual as though the individual is not a Band Member, regardless if that is true or not. The difference in the two computed probabilities of employment is the value of the marginal effect for that case. The same two steps are then repeated for every other case in the sample and the average is taken of all of the computed marginal effects and reported in the tables as the average marginal effect for the Band Membership variable. In essence, the comparison is done between two hypothetical populations (one with all Band Members in and one with all non-Band Members in) that have the exact same values on the other independent variables in the model. Since Band Membership is the only difference between these two populations, then that variable is assumed to be the reason behind the differences in their likelihood of employment. 
group-specific data files. The selection and organization of these independent variables is explained below.

As the existing literature suggests, the probability of being employed for an Aboriginal identity individual may be affected by a wide range of factors. The factors considered in this study are organized into three blocks of independent variables, two of which are based on the theoretical postulations of human and social capital theories while the third one relates to culture. Since the educational attainment is a primary variable of interest, various classification of this variable are included in the analysis. The definitions of this and of all other explanatory variables are presented in Appendix A, Table Al and Table A2 for the main and for the robustness checks analyses, respectively.

The first two blocks of independent variables take into account the key factors suggested by human capital theory. Educational attainment is the primary variable of interest in each block. The 2006 APS does not contain a continuous variable measuring years of full-time schooling, which was commonly included in most of the previous empirical work, but it does contain a comprehensive index measuring the completed educational qualifications (highest certificate, diploma or degree) and specifying different categories for the type of educational institution attended (high school, trades/apprenticeship, college, university). This index is of special importance to the analyses of labour market outcomes of Aboriginal people because it captures not only part-time education but also allows for an examination of the effect different programs may have on the employment probability of different individuals. The six dummy 
variables included in the main analysis describe the highest level of schooling attained and they range from some high school (or less) to a completed university education. ${ }^{65}$

Two additional classifications of the educational attainment variable are derived from the 2006 Census. One of these variables is qualitatively similar to the more descriptive variable in the APS, which contained insufficient counts to perform separate analysis for some groups, and in particular Inuit. As in the APS, this variable in Census data provides information on the individual's most advanced certificate, diploma or degree; the difference here is that, at the postsecondary non-university level of education, the variable gives more details on the length of the education program completed. ${ }^{66}$ The other variable is a new variable introduced for the first time in the 2006 Census with intent to identify more precisely the population with and without high school graduation certificate or its equivalent. This variable also collected information from these two populations on their completion of postsecondary education (Statistics Canada, 2008b).

\footnotetext{
${ }^{65}$ The APS analytical files contain two derived variables summarizing the highest level of schooling attained. One variable, DHLOSP, contains 14 categories that range from no schooling to earned doctorate while the other variable, DHLOSGP, contains seven categories that range from elementary or less to completed university. Since many of the categories from the first, more descriptive variable showed either zero or a small number of counts, especially in the gender-split samples of the three identity groups, the second variable was used to perform main analysis. Still, some amalgamation was deemed necessary because even in the reduced classification of categories a very small number of respondents reported some of the categories as their highest level of schooling attained. Thus the indicator for "completed university level of education' in the Métis and NAI samples is a composite that includes all 'completed' university educational attainment categories: That is, University certificate or diploma below Bachelor's level; Bachelor's degree; University certificate or diploma above Bachelor's degree; Master's degree; degree in medicine/dentistry/veterinary medicine/optometry; and earned doctorate. The 'university education' in the Inuit samples includes both 'completed' and 'some' university education because the sample sizes here were insufficient to allow separate analysis. Also, both Métis and NAI samples contain a very small proportion of respondents with only 'elementary or less' level of education so this category was combined with 'some high school' category. In the robustness checks analysis, the first variable, DHLOSP, is used for Métis and NAI while for Inuit, completed years of schooling is used (see Appendix A for details).

${ }^{66}$ The length here refers to months or years the college or other non-university certificate or diploma was designed to be completed not how long it took an individual to complete that program. The nine dummies constructed from this educational attainment variable are defined in Appendix A2.
} 
This new variable is of great value to the present analysis in that it permits individuals who have obtained registered apprenticeship or other trades certificates without completing high school diploma to be identified. Because these two Census variables are uniquely descriptive and important for the present study, separate analyses were done for each identity group - as well as for the on-reserve NAI group - to carry out robustness checks for the groups examined in the APS data files and to assess the effect of these variables on the probability of employment for the groups not examined in the APS data file, the on-reserve NAI group in particular. ${ }^{67}$

There are good theoretical and empirical grounds to expect a strong positive relationship between the level of schooling and the probability of being employed. An individual's educational attainment, according to human capital theory, might serve two major functions in determining his or her employment prospects. First, all else constant, completed educational credentials may indicate a person's high productivity, thereby making him or her more employable. Second, educational credentials may serve as a screening device, enabling employers to identify which individuals possess greater abilities in the given pool of job applicants. Under each scenario, a positive relationship between the likelihood of employment and educational attainment is expected (Becker, 1993). To a large extent, this positive relationship has been confirmed in empirical

\footnotetext{
${ }^{67}$ Eight categories were coded using the information from the newly introduced variable on education: (1) no high school diploma and no further schooling, (2) completed registered apprenticeship or other trades certificate or diploma, without high school graduation, (3) completed college certificate or diploma, without high school diploma, (4) the reference category - completed high school diploma, without further schooling, (5) completed high school diploma and registered apprenticeship or other trades certificate or diploma, (6) completed high school diploma and college certificate or diploma, (7) completed high school diploma and university certificate or diploma below Bachelor's degree, and (8) completed high school diploma and university degree or higher.
} 
studies involving general population (Riddell \& Song, 2009, 2011) although it remains unresolved in studies on ethnic minorities, as pointed out in the introduction to this essay. Human capital theory also suggests that there might be additional positive effects from higher educational credentials that accrue over the working life of an individual. Empirical research has shown that increases in the level of education significantly raise the rate of return to the post-educational acquisition of job-specific skills, which leads to further accumulation of human capital for that individual and makes him or her less likely to quit or be laid off (Becker, 1993). In practice, this variable is difficult to measure and in studies such as this one is generally approximated by the labour market experience gained from potential time spent working, which is calculated using the Mincer proxy, current age minus years of schooling minus six (Mincer \& Polachek, 1974).

The Mincer potential work experience variable, however, has been found to be a good proxy only in the case of men who have a strong labour force attachment. This proxy is inaccurate when continuous labour market participation is not evident and it biases the effects of schooling (Miller, 1993; Drolet, 2001). In the context of this study then, the Mincer potential work experience would most likely be a poor proxy for both Aboriginal men and Aboriginal women as it would overstate their actual work experience by not accounting for both voluntary and involuntary interruptions in their formal labour market participation. ${ }^{68}$

\footnotetext{
${ }^{68}$ Also, as mentioned earlier, the APS does not contain the continuous variable for years of schooling that is necessary for the calculation of the Mincer proxy. It does, however, contain the information on the age of respondents in years [DAGEYRS] and the age in years they last took elementary or high school courses [A15]. An attempt was made to use these two variables as an alternative for calculating the Mincer potential work experience. The computed variable, however, was highly correlated with education, with Variance Inflation Factor (VIF) values in excess of 20, and involved drastic reductions in the sample sizes due to missing values in the [A15] variable. Since the observations in [A15] are likely to be qualitatively different from those missing observations, imputing the missing values was deemed inappropriate (Greene,
} 
Included in the block of human capital variables is a special variable derived from a question that inquired about access to, and the use of, information technology. Although the 2006 APS contains a number of different variables that can be used to measure technological skills, the variable selected for this study relates to the use of the Internet at home and is expected to have a positive impact on the probability of employment of an individual (Kuhn, 2000; Kuhn \& Skuterud, 2000). ${ }^{69}$ Although this variable could be viewed as an indicator of an individual's socioeconomic status, it could also serve as a proxy for computer-specific human capital which is highly valued and rewarded in the contemporary labour market. ${ }^{70}$ In any case, having access to this information technology is likely to positively affect individuals' job search intensity, especially that of the unemployed, as it can enable them to integrate online job search into their regular job search methods, thus effectively reducing the cost of both acquiring a wider range of relevant employment information and the cost of responding to suitable job opportunities.

Aside from educational credentials and technological skills, the block of human capital variables also includes other individual characteristics such as age, marital status and the presence of dependent children in a household. The first two of these variables might be expected to represent a variety of influences, including discrimination and the relative degree of knowledge of how labour markets function. This knowledge is regarded as general human capital and is positively related to the individual's age. For

2003). Hence, the age categories included in the models are considered as reliable a proxy for work experience as any other conventional approximation of actual work experience would be.

${ }^{69}$ This information was solicited from individuals in all three identity groups and the questions asked whether or not they used computer and the Internet in the past 12 months, and if yes, where. The indicator included in this study measures the use of the Internet at home only.

${ }^{70}$ This variable could also be regarded as a proxy for social capital, and in particular for what literature labels as 'weak-ties' social capital, as it can facilitate an expansion of social networks and can afford a relatively-cheap access to membership clubs. Social capital, addressed in the Introduction Chapter of this thesis, is measured by specific variables, which are discussed later in this section of the essay. 
this reason, a positive relationship between the age and the likelihood of employment is expected. However, an individual's age may lead to discriminatory practices of certain employers or poor health conditions, which would dictate an inverse relationship. The age structure is entered into regression in the first block of human capital characteristics.

The individuals' marital status is likely to affect their range of employment opportunities, the types of work they would be offered or would be willing to accept as well as their level of motivation to maintain the employment status. This influence is further reinforced by the presence of young children and the incidence of single parenthood. Given the traditional pattern of the division of labour within households, the number of dependent children is expected to have an adverse effect on women's employment probability (Becker, 1993).

The relationship between being married and the probability of employment for men is difficult to determine a priori. The greater family responsibilities of married men, especially the additional financial responsibilities associated with having a greater number of children, may induce stability of their employment status (Becker, 1993) but additional children may also raise a man's welfare or other entitlements and thus reduce the incentive to work harder on maintaining his employment status (Garfinkel, Heintze \& Huang, 2000). To capture all of these possibilities, both the male and female employment models in this study control for the presence of children as well as for single-parent family structure. ${ }^{71}$ The Métis employment models also control for availability of safe and

\footnotetext{
${ }^{71}$ Since welfare recipients are excluded from the APS analytical samples, the welfare disincentive will not be captured in this analysis and any disincentive effect then would be assigned to other entitlements. The information on the presence of dependent children was taken from a derived APS variable, LFPRESCH, that contained 8 categories. Four indicators were created to capture the impact of having young, schoolaged or grownup children relative to not having children at all.
} 
affordable childcare. ${ }^{72}$ These characteristics are entered into regression in the second block of human capital variables.

Finally, a set of variables relating to individuals' self-reported health status are included in the second block of human capital variables, as suggested by human capital theory. Poor health, according to human capital theory, is likely to have an adverse effect on individuals' employment prospects for it directly affects their productivity level. Low productivity associated with poor health can lower individuals' earnings potential and their opportunity costs of leisure, the concept discussed in the previous section, and therefore their willingness to accept a job offer (Becker, 1993).

No attempt has been made in the previous empirical literature to relate any measure of health conditions of Aboriginal people to their labour market outcomes. Studies on general population have found a strong positive correlation, suggesting that better health significantly increases the probability of better labour market outcomes (Breslaw \& Stelcner, 1987; Harkness, 1993). Understanding the relationship between health and employment prospects of Aboriginal labour force participants has important policy implications, especially in the current context of Canada's demographic shift.

The APS contains a number of variables related to health conditions of Aboriginal people, including the self-assessed health variable. Instead of using the respondents' selfassessed measures of their health status, this study employs two special health indicators derived from a combination of two survey questions on long-term health conditions and

\footnotetext{
${ }^{72}$ This information was available only in the Métis Supplement of the APS and the question was posed to all respondents who reported that children under 15 years of age normally reside in their household. The question [J13] asked respondents to state "How difficult is it to find safe and affordable childcare for children in this community?". An indicator was created, coding the "Very difficult and Somewhat difficult" responses as one and "Not too difficult and Not difficult at all" responses as zero. To preserve the sample size, 'valid skip' responses were coded as zero.
} 
activity restrictions related to health. ${ }^{73}$ These two variables have the advantage of being more objective than self-assessed health status and hence less likely to be subject to the endogeneity problems associated with the respondents' self-assessed measures of health status (Breslaw \& Stelcner, 1987; Harkness, 1993). ${ }^{74}$

The third block of independent variables in this study accounts for some of the factors suggested by social capital theory, as discussed in the Introduction to this thesis. The APS contains several variables relating to access to social support networks. However, not all of these variables are present in all three group-specific data files and not all of them are worded clearly enough to classify them as weak or strong ties, as per the existing literature (Foley \& Edwards, 1999; Hunter, 2000; Gomez \& Santor, 2001; Glaeser, Laibson, \& Sacerdote, 2002; Durlauf, 2002; Nelson, Kaboolin \& Carver, 2003; Annen, 2004; Brough et al., 2006; Van Staveren \& Knorringa, 2007; Knorringa \& Van Staveren, 2007; Mignone, 2009). Nevertheless, three specific variables are selected to examine the association between social capital and the probability of employment of Aboriginal people.

The first variable, which was available in all three analytical files, is used to assess what literature defines as strong ties. This measure captures whether or not an

\footnotetext{
${ }^{73}$ The two indicators [DHLTHCON] and [DPAL] were derived from a range of health-related questions that inquired about the number of chronic health conditions and the degree of activity limitation. The latter variable was derived from the Census 2006 questionnaire that was introduced to identify a sample for the 2006 post-censal Participation and Activity Limitations Survey. In the APS, this variable classifies respondents based on the frequency with which they experience activity limitations imposed on them by condition(s) or health problem(s) in the areas of their lives such as work, school, home and leisure (Statistics Canada, 2009).

${ }^{74}$ A possible drawback here is that this derived variable may provide a narrower measure of health status than would a more subjective measure such as self-reported health status. This definition might exclude some individuals with poor health because poor health may not necessarily manifest itself in the form of activity restrictions or in chronic health indicator. Also, arguably, even the objective measures of health can be endogenous to an individual's labour market behavior (Cleary \& Angel, 1984; Angrist, 2001).
} 
individual reported having support from friends or family in a time of crises. ${ }^{75}$ It is hypothesized that this variable will have a positive impact on the individuals' employment probability, particularly in the case of Aboriginal women because this support could potentially be in the form of childcare; the lack of childcare, as White et al. (2003) have pointed out, is a significant impediment to their labour force activity.

The second variable measures the respondents' voluntary participation in the community. This weak-ties variable is included only in the Inuit and Métis employment equations because the information was supplied only in the Arctic and Métis supplements of the APS. ${ }^{76}$ The third variable is another proxy for what literature defines as weak ties and it measures civic participation. This variable, however, is included only in the Inuit employment models because the question was asked only in the Arctic supplement of the APS. ${ }^{77}$ Regardless of their classifications as being strong or weak ties, it is expected that all of the variables relating to social capital would have a positive impact on the probability of employment for Aboriginal identity individuals considered in this study.

Notwithstanding its importance, as discussed in the Introduction to this thesis, the empirical analysis of social capital is not a straightforward task. One obvious issue is that the potential endogeneity of social capital in relation to individual unobserved

\footnotetext{
${ }^{75}$ The question [E52F], which was posed to all respondents, asked: "People sometimes look to others for companionship, assistance, guidance or other types of support. Could you tell me how often this kind of support is available to you when you need it - someone to confide in or talk about yourself or your problems". An indicator was created by recoding the "all of the time, most of the time and some of the time" responses as one and "almost none of the time" as zero.

${ }^{76}$ In the Arctic Supplement, the question [K27A] asked: "Thinking of the last 12 months, did you volunteer for a community organization or group for example, for a radio station, a search and rescue team, a church group, a youth group, or other?" and in the Métis Supplement, the question [K07] asked: "Are you a member of any voluntary organizations or associations such as school groups, church groups, community centers, ethnic associations or social, civic or fraternal clubs?"

${ }^{77}$ The two indicators were created from [K28], which asked: "Did you vote in the most recent municipal election?" and from [K29], which asked: "Did you vote in the most recent provincial or territorial election?".
} 
heterogeneity in innate ability can make the identification of its impact on the probability of employment difficult. The efficacy of the social capital variables included in this analysis, especially of those concerning the weak-ties, might be compromised by the possible endogeneity associated with them (Poulsen \& Svendsen, 2005; Xue, 2008). For example, one can view individuals' preferences about civic participation and voluntary engagement in community organizations as codetermined with the amount of effort they put in finding the information about such opportunities, in which case social capital would emerge endogenously from the process of their preference creation. Also, since unemployed individuals have more time available than those who are working, they may invest some of that time at community centers or by interacting with their communities. This would create a problem in trying to estimate the impact of networks on individuals' probability of employment.

The final block of independent variables entered into the employment models relate to cultural factors and they are included primarily for exploratory purposes. In the context of the labour market, some of these factors such as knowledge of a traditional language, generally taken as a phenotypical or cultural characteristic that is a function of the group with which one identifies, can in fact be considered capital in the purest sense of the word because there exists a choice for acquiring it, especially for urban Aboriginal residents, and since fluency in traditional languages is potentially valued and rewarded more in some segments or geographical localities of the labour market than in others.

In the mainstream literature, cultural (or ethnic) capital is generally discussed by institutional economists, mostly in the context of segmented labour markets. As discussed in the Introduction to this thesis, segmented labour market theory holds that the labour 
market is divided into a more rewarding and a less rewarding segment and that access to the more rewarding segment is restricted not only by the individuals' human capital endowments but also by their personal characteristics such as their race, ethnicity and gender (Piore, 1975). From this theoretical point of view, formal educational credentials are seen as merely "screening devices" serving to "open the door" to upward mobility to the more advantaged groups of population in a society (Sobel, 1982). Under such screening conditions, ethnic or racial minorities have relatively small chances of successfully securing employment and are thus either pushed into less-rewarding segments of the labour market where employment contracts are brief and unstable or are discouraged from participating in the labour force at all.

The potential existence of racial discrimination in the labour market, as discussed in Introduction to this thesis, forms a significant aspect of the institutionalists' inquiry. The insitituionalist's approach to labour market analysis insists on understanding the dynamics by examining the whole social context in which labour market outcomes occur in order to understand how subjective perceptions of marginal benefits and marginal costs associated with a particular decision are formed by employers and by employees and how institutions affect those perceptions under uncertainty (North, 1991). As such, the approach opens up a way for making broader conjectures not only about the behaviour of employers but also about the behaviour of individual employees, especially if their cultural institutional arrangements dictate norms that are different from the norms generally observed in the larger society (Kleinfeld \& Kruse, 1982).

The approach allows for hypothesizing that, for instance, the relative lack of formal educational credentials among northern Aboriginal Canadians might be an 
indication of their own 'choice' not to aim at succeeding in the paid labour market but rather to aim at succeeding in the traditional labour market by prioritizing subsistence activities and kinship obligations as these might be more important to their daily existence, relative to the formal educational credentials which can be put into use in only limited ways. Considering the importance of the subsistence activity, in conjunction with acknowledging the limited opportunities to pursue higher education in the northern Aboriginal communities, one can further speculate that this could be a deliberate, community-supported act in which northern Aboriginal workers are encouraged to 'choose to succeed in the mixed economy' and use available (unstable or seasonal) wage employment as a strategy to attain that goal. In other words, it could be that traditional Aboriginal communities serve as critical institutions for the survival of what we generally consider 'disadvantaged' Aboriginal workers because their contribution to the Aboriginal society through traditional pursuits is valued by the community relatively more than would be their contribution through the formal labour market.

Cultural capital in this study is measured by a number of variables that capture perceived signs of cultural attachments among Aboriginal individuals. The primary variable of interest here is the individuals' engagement in traditional pursuits such as hunting. This variable is used to examine the relation between participation in traditional activities and the probability of employment in the paid labour market. ${ }^{78}$

\footnotetext{
${ }^{78}$ The information about the engagement in traditional pursuits such as hunting, fishing, trapping and gathering wild plants provided in the APS analytical files pertains to the recent engagement (in the 12 months prior to the survey) and the activities are classified based on whether the engagement was for commercial purposes, for food or medicinal/ceremonial uses, or for pleasure. Potentially, the first classification (for commercial purposes) could be endogenous as this form of engagement in traditional pursuits could be regarded as a form of labour force participation.
} 
The other variables of interest in the cultural capital block of variables pertain to traditional languages and they measure individuals' perceptions about the importance of and their ability to speak an Aboriginal language. ${ }^{79}$ The Aboriginal language variable, along with the formal and legal identity status such as being a registered member of an Indian Band or First Nation or being a member/beneficiary of a land claim agreement, could serve as a proxy to gain some insight into potential labour market discrimination because, presumably, potential employers could use these 'identity indicators' to identify an individual and make associations related to stereotypic behaviour of his/her particular group. This presumption is particularly relevant to the female employment models because a number of studies discussed earlier in this essay and some studies on ethnicity have found that Aboriginal women with legal Indian Status are at double disadvantage due to their gender and their Registered Status (Gerber, 1990; White et al., 2003).

In short, the expectations here are that cultural capital may affect both the supply of and the demand for the labour services of Aboriginal identity individuals. If there is a general perception on the demand side that cultural traits of an Aboriginal individual belonging to a specific Aboriginal identity group make him or her less achievement oriented or less committed to sustaining a long term employment, then this factor would normally be expected to lower the probability of employment success for that individual, regardless of whether the perception was based on correct or erroneous information.

On the supply side, the perceptions and the past experience of racial discrimination, or other structural factors, may discourage Aboriginal individuals from effectively seeking employment in the formal labour market. In some instances, it could

\footnotetext{
${ }^{79}$ The information on knowledge of and perceptions about importance of Aboriginal languages was supplied in all three analytical files.
} 
be that Aboriginal individuals may have chosen to specialize in skills and abilities that are simply not valued by the modern industrialized economies but are essential for the preservation of the traditional way of life. Non-employment in the formal labour market in this case then could be regarded as an individual's own utility maximization choice. All of the models also include standard variables to control for regional labour market characteristics, proficiency in official languages and income from other sources. ${ }^{80}$

\footnotetext{
${ }^{80}$ The geographic regions in the Inuit employment equations relate to the four Inuit land claims regions that comprise Inuit Nunangat listed earlier in this section. These four regions contain about 52 , generally small communities, which can be accessed only by air or by sea during the summer months (Statistics Canada, 2007). The geographic regions in the NAI and Métis employment equations represent six provinces (Quebec, Ontario, Manitoba, Saskatchewan, Alberta, and British Columbia) and two regions, Atlantic region (combining the four Atlantic Provinces) and Northern Region (combining the three Northern territories). The amalgamation was necessary in the two regions due to the small sample sizes of individuals with NAI identity and Métis identity. Other income indicates if, during the year ending December 31,2005 , the respondent received any (other than social assistance) income from sources such as other government income, child support, alimony, education allowances, scholarships, Northern Allowance, interest, or other. Affirmative responses given to separate questions that inquired about income from paid or self-employment, social assistance or welfare income, the EI, OAS/GIS, SA and CPP/QPP were not included in this study. Two additional indicators were included in each sample, indicating if the respondent is the first household maintainer and if the respondent lived in a household that had more than one maintainer.
} 


\section{Results}

Given the complex survey design of the data sets used in this study and the choices made in constructing the analytical samples, several important limitations threatening both the internal validity (accuracy) and the external validity (generalizability) of the findings need to be emphasized here. As pointed out in the preceding section, missing data due to both the undercoverage of the on-reserve population groups and the self-selection of participants in responding to survey questions pose significant concerns for the present analysis. ${ }^{81}$ Also, a potential bias was entered in the study design process whereby the analysis was restricted to the sample of labour force participants (individuals who were either employed or unemployed) who were not students (full-time/full-time or par-time) and who were not recipients of social assistance.

Labour force participation is a voluntary act whereby some people choose not to work while others might be unable to find work they consider suitable for them. If labour force participants and non-participants are systematically different, then the results may be biased in unknown ways thus compromising both the internal and the external validity of the study. Similarly, if collectively the individuals living on the participating reserves are systematically different from those individuals living on the reserves that did not

\footnotetext{
${ }^{81}$ The Census data on North American Indians residing on-reserve involve important limitations that must be emphasized here. Historically, population counts from a number of Indian reserves and settlements have been excluded from the Canadian Census data because enumeration was incomplete. In other, more serious cases, the population counts were missing because some reserves, including the most populous reserve, have never participated in the Census. Although the number of reserves affected by this issue has decreased substantially, from 77 reserves in 1996 to 30 reserves in 2001, undercoverage is still an issue. The 2006 Census data employed in this study do not contain information on 22 reserves (Statistics Canada, 2008b).
} 
participate in the Census data collection, the sample of the on-reserve NAI groups may be biased. ${ }^{82}$ The same concern applies for the excluded welfare recipients and students.

Selection bias presents a threat to internal validity if the included independent variables are correlated with the disturbance term, the error term $\mu$ in equation (1) presented in the previous section, and an analysis based on a biased sample can lead to inaccurate estimates of the regression coefficients, the $\beta$ parameters in the same equation. Thus the effects of the examined relationship between variables maybe attributed to exogenous variables that actually are due to selection factors. External validity is threatened because of the possibility that the inferences derived from biased samples might not be generalizable to the population of interest. Because of the restrictions applied, the samples included in the present analysis might be underrepresenting highly disadvantaged individuals and overrepresenting more advantaged individuals in terms of their socioeconomic characteristics.

General practice in the empirical literature addressing the detection and correction of selection bias of this form has been to apply the Heckman two-step procedure for linear models or some variation of it for non-linear models (Heckman, 1979; Dubin \& Rivers, 1989). These selection bias correction methods, however, are based on sets of assumptions and work well only if those assumptions hold in the applied data sets. If the source of selection is unknown and if assumptions do not hold, correction makes little or no difference in the results (Jung \& Pirog, 2011). In fact, evidence exist showing that

\footnotetext{
${ }^{82}$ Possibly, this particular issue may not present a major bias because, in their earlier work, White and Maxim (2003) "have determined that the communities that did not participate in the census are not unique in their characteristics" (p. 29).
} 
correction sometimes worsens rather than improves the biased estimates, even under ordinary conditions (Winship \& Mare, 1992; Stolzenberg \& Relles, 1997).

Potential selection problem in relation to the welfare recipients is partly addressed in the robustness checks section of this study where the welfare recipients are included in the analytical samples. No attempt has been made to address other aspects of the discussed selectivity in the present analysis. Hence, the findings presented here should be interpreted with caution as they could be confounded by the potential selection issues. 


\subsection{Summary Statistics and Bivariate Analysis}

The mean characteristics of all individuals in the APS samples are presented in Appendix B1 while the Census samples' characteristics are presented in Appendix E1. This section will summarize some of the main characteristics from the APS samples related to human and social capital and culture that form the core of the models in the main part of the analysis. The section will also present some of the findings from a bivariate analysis, performed as a preliminary check to detect potential correlations and to determine the variables to be included in the probit regression models. ${ }^{83}$ The mean characteristics for the Census samples will be presented in the robustness checks section.

A brief look at the educational measure rows in the sample means table in Appendix B1 reveals that the Inuit population has substantially higher proportions of individuals with lower levels of education. As presented in the first row of the table, about 52 percent of Inuit men and 40 percent of Inuit women have less than high school education. The corresponding figures for Métis are 22 percent for men and 16 percent for women. The figures for the off-reserve NAI population with less than high school education vary depending on their place of residence (higher proportions are among those living in rural areas) and their legal Indian status (higher proportions are among

\footnotetext{
${ }^{83} \mathrm{~A}$ simple chi-square test of independence was performed to examine the relations between independent variables and the probability of employment. The analysis involved testing the joint distribution of two variables to see whether or not they have a statistically significant relationship. More precisely, the analysis involved comparing the empirically observed frequencies against the frequencies that are expected by chance alone to see whether this difference is statistically significant. Chi-square test formula is

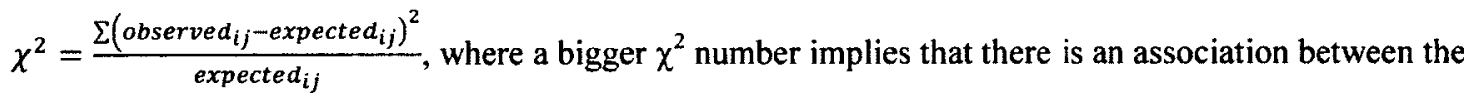
two variables and $p \leq .05$ means that the difference cannot be assigned to chance (Wooldridge, 2006). Only selected variables are discussed in this section; other results can be made available, upon request.
} 
registered Indians) but in each case these proportions are substantially higher for NAI men than they are for NAI women.

Likewise, a completed high school diploma as the highest level of schooling attained was reported by 8 percent of Inuit men and 13 percent of Inuit women. ${ }^{84}$ The corresponding figures for Métis are 21 percent for each gender. Among the NAI population, the proportions of individuals with completed high school diploma or equivalency are highest for non-registered NAI women (21\%) and lowest for urban NAI $\operatorname{men}(15 \%)$.

At the other end of the educational spectrum, a very small proportion of Inuit ( $2 \%$ of men and $8 \%$ of women) have completed a university degree. The corresponding figures are 10 percent for the urban and non-registered off-reserve NAI men and for Métis men, while for the registered and for rural NAI men, these figures are 5 percent and 8 percent, respectively. The proportions of women with completed university degree are larger, relative to men, in NAI and Métis population groups and they range from 11 percent for NAI women living in rural areas to 16 percent for Métis and registered NAI women.

In regards to completion of other postsecondary schooling, men in each Aboriginal identity group were more likely than women to have completed a diploma or certificate from trades school or registered apprenticeship program; the proportions range from 14 percent for Inuit, non-registered NAI and NAI men living in urban areas to 17 percent for NAI men living in rural areas. Women, on the other hand, were more likely than men to have a completed diploma or certificate from other non-university

\footnotetext{
${ }^{84}$ The last classification of the educational attainment in Appendix B 1 indicates that relatively large proportions of Inuit men (18\%) and women (32\%) have incomplete grade 12 or 13 . Given that full-time students are excluded from the analysis, these cannot be assigned to the young population in the sample.
} 
institutions; the proportions here range from 23 percent for Inuit women to 30 percent for NAI women living in rural areas. The proportions among men, however, are not negligible as they range from 11 percent for Inuit men to 17 percent for Métis men. ${ }^{85}$

The proportions of populations with incomplete vocational schooling or other non-university type of education range from 9 to 10 percent for NAI and Métis women to 11 percent for Inuit women. The same figures for men range from 10 percent for Métis men to 14 percent for non-registered NAI men. The proportions of individuals with incomplete postsecondary schooling at the university level are smaller. About equal percentages $(5 \%)$ of NAI and Métis women had incomplete university education; this proportion ranged from 2 percent for NAI men living in rural areas to 5 percent for NAI men living in urban areas.

In relation to other characteristics considered in the human capital blocks of variables, the table in Appendix B1 reveals that, relative to other groups, Inuit had considerably smaller proportions of individuals with access to information technology at home, 44 percent of men and 49 percent of women. These proportions for Métis and nonregistered NAI are 77 percent for each gender. The proportions varied for the other NAI groups, ranging from about 60 percent for the rural population to 76 percent for the urban population.

A significantly smaller proportion of Inuit men (32\%) reported having a chronic health condition, compared to Métis and urban NAI men (48\%) or non-registered NAI men (50\%). A similar pattern appears for women; while 46 percent of Inuit women

\footnotetext{
${ }^{85}$ Relatively large proportions of Aboriginal populations in the categories of non-university education could be due to government incentives "for lesser educated persons to take training courses in order to maintain eligibility for government programs" (White et al., 2003, p. 398). This might have an impact on the observed effect these credentials have on the probability of employment for these individuals.
} 
reported having one or more chronic health conditions, the corresponding figures were 53 percent for Métis, urban and non-registered NAI women and 51 percent for registered and rural NAI women. Similarly, about 29 percent of Inuit men and 22 percent of Inuit women reported having a health condition that limits the range of their physical activity. The corresponding figures for other Aboriginal identity men range from 29 percent for registered NAI men to 33 percent for Métis men. The proportions for women range from 31 percent for Métis to 33 percent for urban NAI women.

The incidence of lone parenthood appears quite a bit higher among women than among men; for instance, the proportion of lone parents amounts to 18 percent of registered NAI women and 17 percent of Inuit women while the corresponding proportions for registered NAI men and for Inuit men are 5 and 6 percent, respectively. Among Métis and non-registered NAI men, only 3 percent are single parents.

The proportions of individuals with other sources of income are also distributed quite differently across identity and in some cases across gender as well. While about equal percentage of Métis men (16\%) and Métis women (15\%) had other sources of income, the corresponding figures among Inuit were 34 percent for men and 47 percent for women. Likewise, while about 27 percent of NAI women had other sources of income, the corresponding figures for NAI men ranged from 12 percent for nonregistered men to 17 percent for registered men.

In relation to social capital, it is surprising that only 56 percent of Inuit men and 58 percent of Inuit women reported having someone to turn to in times of crisis. Among the other Aboriginal identity groups, these proportions ranged from 94 percent for urban and registered NAI men to 99 percent for Métis women. Greater proportions of Inuit 
( $45 \%$ of men and $46 \%$ of women) reported volunteering in their community, compared with 20 percent of Métis men and 26 percent of Métis women. Civic participation was slightly more prevalent among Inuit men (76\%) then among Inuit women (72\%).

Finally, in relation to cultural factors, formal membership was more prevalent among registered NAI ( 93 percent for each gender) and Inuit ( $89 \%$ for men and $92 \%$ for women) than among Métis (23\% for men and $20 \%$ for women). A slightly larger proportions of NAI women were registered under the Indian Act (61\% in rural areas and $59 \%$ in urban areas), compared to NAI men (59\% in rural areas and $56 \%$ in urban areas).

Not surprisingly, a greater proportion of Inuit population speak an Aboriginal language (85\%), relative to Métis ( $9 \%$ of men and $10 \%$ of women) and to non-registered NAI ( $7 \%$ of men and $4 \%$ of women). The proportions among other NAI groups range from 15 percent for urban NAI women to 29 percent for registered NAI men. While, as expected, the great majority of Inuit ( $95 \%$ of men and $97 \%$ of women) stated that it is important for them to keep, learn or re-learn their Aboriginal language, the proportions among other Aboriginal identity groups are large as well. About 45 percent of Métis men and 49 percent of Métis women gave a positive response to that question. Among NAI groups, the proportions ranged from 43 percent of non-registered NAI women to 76 percent of registered women.

The pursuit of traditional activities for commercial purposes was somewhat more prevalent among Inuit (24\% of men and $8 \%$ of women) and among NAI men living in rural areas (9\%). Registered NAI men (5\%) and Métis men (4\%) were also engaged in traditional pursuits for commercial purposes while for the other groups the proportions were small, about 2 percent of the population. Although substantially larger proportions 
of Inuit ( $94 \%$ of men and $86 \%$ of women) were engaged in traditional pursuits for food and medicinal purposes, the proportions in the other groups are also fairly large, ranging from 35 percent for non-registered NAI women to 67 percent for NAI men living in rural areas. Also, the engagement in traditional pursuits for pleasure was reported in each sample, with proportions ranging from the smallest 34 percent in the sample of NAI women living in urban areas to the largest 76 percent in the sample of Inuit women.

These apparent differences in the distribution of characteristics among individuals in different Aboriginal identity groups could have important implications for their employment outcomes. The following few paragraphs discuss some results from bivariate analysis examining possible relations between some of these characteristics and the probability of employment. ${ }^{86}$ The primary focus is the level of educational attainment.

The results from the chi-square test of independence suggest an important relationship seems to exist between some levels of education and the probability of employment for some group members, but not for all. At the level of completed university education, for instance, the chi-square for NAI men $\chi^{2}(1,2570)=14.880$, is highly significant, at $\mathrm{p}<.000$, as is the chi-square for NAI women $\chi^{2}(1,2710)=12.708$, at $\mathrm{p}<.000$, suggesting possible positive association at this level of education. Likewise, the chi-square for NAI men with less than high school education is highly significant, $\chi^{2}(1,2570)=23.165, \mathrm{p}<.000$, as is the chi-square for NAI women $\chi^{2}(1,2710)=64.549$, $p<.000$. The strength of this latter negative relationship appears slightly higher for women, $\varphi=-.157, \mathrm{p}<.000$, than for men, $\varphi=-.097, \mathrm{p}<.000$.

\footnotetext{
${ }^{86}$ It should be emphasized here that this chi-square statistic is highly sensitive to sample size. Given that the problem of missing values was present in each group to a different degree, the chi-square results presented in this section should be taken with special caution as this will have a bearing on the outcome.
} 
This association for NAI group, however, is not as consistent for other levels of education as human capital theory would suggest. While the completion of high school and non-university diploma is significantly and positively associated with the probability of employment for NAI women, the same relation is not statistically significant in the case of NAI men. ${ }^{87}$ Also, there seems to be no significant relation between having incomplete university or non-university education and the probability of employment for NAI group.

Similarly, the completed university education is significantly associated with higher probability of employment, as is the incomplete high school education associated with lower probability of employment, for Métis people. ${ }^{88}$ The completion of the nonuniversity education, however, is significantly associated with higher probability of employment but only for Métis men, $\chi^{2}(1,2540)=9.912, p<.002$, not for Métis women, $\chi^{2}(1,2480)=.128, p=.721$. Incomplete postsecondary education does not appear to be significantly associated with the probability of employment for Métis.

Perhaps the most surprising finding from this bivariate analysis is that, in the Inuit samples, none of the educational variables have significant association with the probability of employment. ${ }^{89}$ This implies that employment prospects for Inuit people

\footnotetext{
${ }^{87}$ For NAI women, the chi-square and the significance level for completed high school education level are $\chi^{2}(1,2710)=11.623, p<.001$ and $\chi^{2}(1,2710)=16.259, p<.000$ for completed non-university education level. The same statistics for NAI men are $\chi^{2}(1,2570)=1.343$, with $p=.247$ and $\chi^{2}(1,2570)=.008$, with $p$ $=.927$ for completed high school education and for completed non-university education, respectively.

${ }^{88}$ For the completed university level of education, the chi-square for Métis men $\chi^{2}(1,2540)=7.577$ is significant at $p<.006$ and the chi-square for Métis women $\chi^{2}(1,2480)=10.728$ is significant at $p<.001$. For the less than high school level of education, the chi-square is $\chi^{2}(1,2540)=15.638, p<.000$ for Métis men and $\chi^{2}(1,2480)=31.388, p<.000$ for Métis women.

${ }^{89}$ The chi-square for Inuit men with incomplete high school education is $\chi^{2}(1,910)=3.276, p=.070$ and for Inuit women it is $\chi^{2}(1,910)=3.511, p=.061$. Likewise, the chi-square for Inuit men with some or completed university education is $\chi^{2}(1,910)=2.526, p=.112$ while for women it is $\chi^{2}(1,910)=.481, p=$
} 
depend largely on some other, possibly unobserved factors. Although there is not much empirical literature examining the labour market activity of Inuit, studies done on income and earnings have found that Inuit, in fact, do face quite different patterns from those of other Aboriginal identity groups (Bernier, 1997; Pendakur \& Pendakur, 2011).

Although informative, these bivariate associations do not reveal much as no controls are introduced at this stage. The regression models presented in the following sections provide estimated net effects of all independent variables on the probability of employment for members of different identity groups. In each presented table, the Model 1 includes only the key human capital variables; Model 2 adds in the health, family and other structural factors; Model 3 adds in the social capital variables while the Full Model accounts for the cultural factors as well.

This multivariate analysis examines whether the group differentials can be explained by some measurable factors included in the blocks of human and social capital and the cultural factors. ${ }^{90}$ For space reasons, only the key factors of interest from each block of variables are presented in these tables; full models are presented in the relevant appendices. $^{91}$

.488. The chi-square values for the completed high school and non-university education are extremely small, for both Inuit men and Inuit women.

${ }^{90} \mathrm{~A}$ test for each identity groups was performed using a linear regression model with all independent variables included to assess the extent of multicollinearity and the results can be made available, upon request. In this test the Variance Inflation Factor (VIF) values describe how inflated the variance of coefficient is in the presence of multicollinearity, compared to what it would be if there were no multicollinearity. These values are always $\geq 1$ and there is no formal cutoff value for determining presence of multicollinearity (Wooldridge, 2006). VIF values exceeding 10, however, are generally regarded as indicating multicollinearity (Baum, 2008), although Allison (1999) posits that in generalized linear models the VIF values greater than 2.5 may be a cause for concern. The VIF values of age variable for all identity groups in this study exceed this 2.5 threshold but not by much.

${ }^{91}$ The interaction between age and educational attainment was tested in each model; no significant interaction was detected. 


\subsection{Multivariate Probit Regression Results for NAI Population}

The main results from the multivariate probit regression analysis for the four groups of off-reserve NAI population (urban, rural, registered and no-registered) are presented in this section of the essay, separately for men and for women, in Tables 2-5. The full results for these four groups are presented in Appendix C (Appendix C1, C2, C3 and $\mathrm{C} 4$, respectively). The estimated Average Marginal Effects (AMEs) and the associated bootstrapped standard errors appear in the tables, and, as in the preceding section, the discussion centers on assessing the difference in the effects of the key human capital, social capital and cultural factors across groups. ${ }^{92}$ The effects of some of the important factors from the full models, not discussed in this section, will be addressed in the concluding remarks section.

Considering first the urban NAI group, the results from multivariate regression analysis presented in Table 2 seem to be in line with the results from the bivariate analysis - and for the most part, in line with the results reported in the previous literature examining the relation between the level of education and the probability of employment for NAI group. As the second column of Table 2 shows, the estimates for urban NAI women almost monotonically reflect what human capital theory would suggest: the probability of employment increases with an increase in educational level.

\footnotetext{
${ }^{92}$ This comparison is made under the assumption that the unobserved errors have common variance. This standard assumption, inherent in all generalized linear models, might be an unreasonable assumption here, given the extent of heterogeneity within Aboriginal population. If this assumption is incorrect, comparing the magnitude of the observed difference in coefficients would be misleading since it is not possible to discern how much of the apparent difference is due to the underlying effect of the covariate and how much of it is due to the variation in the unobserved heterogeneity in the probit models across groups (Allison, 1999a; Hoetker, 2003; Williams, 2009). To account for this in some way, the differences in coefficients are discussed only in relation to key variables; in most of the discussions that follow, the directional association and the statistical significance is used as the primary method for comparison. This approach is more correct since the difference in signs of coefficients cannot be explained by the difference in the residual variation.
} 
Table 2: Selected estimates from probit employment function for urban NAI, APS 2006

\begin{tabular}{|c|c|c|c|c|c|c|c|c|}
\hline \multirow{3}{*}{$\begin{array}{l}\text { Selected variables in the } \\
\text { probit equations }\end{array}$} & \multicolumn{8}{|c|}{ Average Marginal Effects } \\
\hline & \multicolumn{2}{|c|}{ Model 1} & \multicolumn{2}{|c|}{ Model 2} & \multicolumn{2}{|c|}{ Model 3} & \multicolumn{2}{|c|}{ Full Model } \\
\hline & Men & Women & Men & Women & Men & Women & Men & Women \\
\hline $\begin{array}{l}\text { Less than } \\
\text { high school education }\end{array}$ & $\begin{array}{c}-0.054 \\
(0.008)^{\star \star \hbar \hbar}\end{array}$ & $\begin{array}{c}-0.038 \\
(0.007)^{\star * *}\end{array}$ & $\begin{array}{c}-0.056 \\
(0.008)^{\star \star \star}\end{array}$ & $\begin{array}{c}-0.036 \\
(0.007)^{\star * *}\end{array}$ & $\begin{array}{c}-0.058 \\
(0.008)^{\star \star \star}\end{array}$ & $\begin{array}{c}-0.030 \\
(0.006)^{\star \star \star *}\end{array}$ & $\begin{array}{c}-0.050 \\
(0.007)^{* \star \star}\end{array}$ & $\begin{array}{c}-0.034 \\
(0.006)^{* * *}\end{array}$ \\
\hline $\begin{array}{l}\text { Some } \\
\text { non-university education }\end{array}$ & $\begin{array}{c}-0.030 \\
10.011^{* \star}\end{array}$ & $\begin{array}{c}0.045 \\
(0.004)^{\cdots *}\end{array}$ & $\begin{array}{c}-0.030 \\
(0.010)^{\star \star}\end{array}$ & $\begin{array}{c}0.044 \\
(0.004)^{* * *}\end{array}$ & $\begin{array}{c}-0.031 \\
(0.010)^{* *}\end{array}$ & $\begin{array}{c}0.044 \\
(0.004)^{\star \star \star}\end{array}$ & $\begin{array}{c}-0.029 \\
(0.010)^{* *}\end{array}$ & $\begin{array}{c}0.042 \\
(0.004)^{\star * *}\end{array}$ \\
\hline $\begin{array}{l}\text { Completed, } \\
\text { non-university education }\end{array}$ & $\begin{array}{c}-0.048 \\
(0.009)^{* * *}\end{array}$ & $\begin{array}{c}0.059 \\
(0.005)^{\star \star \star}\end{array}$ & $\begin{array}{c}-0.048 \\
(0.008)^{* * *}\end{array}$ & $\begin{array}{c}0.061 \\
(0.004)^{\star * *}\end{array}$ & $\begin{array}{c}-0.050 \\
(0.008)^{* * *}\end{array}$ & $\begin{array}{c}0.062 \\
(0.004)^{\star * *}\end{array}$ & $\begin{array}{c}-0.046 \\
(0.008)^{\star * \star}\end{array}$ & $\begin{array}{c}0.060 \\
(0.004)^{\star \star \star}\end{array}$ \\
\hline $\begin{array}{l}\text { Some } \\
\text { university education }\end{array}$ & $\begin{array}{c}-0.0527 \\
(0.011)^{* *}\end{array}$ & $\begin{array}{c}0.050 \\
(0.003)^{\star *}\end{array}$ & $\begin{array}{c}-0.043 \\
(0.011)^{* * *}\end{array}$ & $\begin{array}{c}0.051 \\
(0.003)^{* * *}\end{array}$ & $\begin{array}{c}-0.045 \\
(0.011)^{* * *}\end{array}$ & $\begin{array}{c}0.052 \\
(0.003)^{* *}\end{array}$ & $\begin{array}{c}-0.043 \\
(0.011)^{* * *}\end{array}$ & $\begin{array}{c}0.051 \\
(0.003)^{* * *}\end{array}$ \\
\hline $\begin{array}{l}\text { Completed } \\
\text { university education }\end{array}$ & $\begin{array}{c}0.037 \\
(0.006)^{\star \star \star}\end{array}$ & $\begin{array}{c}0.055 \\
(0.004)^{* * *}\end{array}$ & $\begin{array}{c}0.034 \\
(0.007)^{\star \star \star}\end{array}$ & $\begin{array}{c}0.053 \\
(0.004)^{\star \star *}\end{array}$ & $\begin{array}{c}0.034 \\
(0.007)^{\star * \star}\end{array}$ & $\begin{array}{c}0.055 \\
(0.003)^{* * *}\end{array}$ & $\begin{array}{c}0.037 \\
(0.006)^{\star \star \star *}\end{array}$ & $\begin{array}{c}0.054 \\
(0.003)^{* * *}\end{array}$ \\
\hline Age 15 to 19 & $\begin{array}{c}-0.103 \\
(0.015)^{\star \star \star}\end{array}$ & $\begin{array}{c}-0.108 \\
(0.018)^{\star * \star}\end{array}$ & $\begin{array}{c}-0.075 \\
(0.013)^{\star \star \star}\end{array}$ & $\begin{array}{c}-0.085 \\
(0.016)^{\star \star \star}\end{array}$ & $\begin{array}{c}-0.080 \\
(0.013)^{\star \star \star}\end{array}$ & $\begin{array}{c}-0.086 \\
(0.016)^{\star * \star}\end{array}$ & $\begin{array}{c}-0.088 \\
(0.013)^{* * * *}\end{array}$ & $\begin{array}{c}-0.084 \\
(0.016)^{\star \star * *}\end{array}$ \\
\hline Age 25 to 34 & $\begin{array}{c}0.034 \\
(0.006)^{* * *}\end{array}$ & $\begin{array}{c}-0.110 \\
(0.014)^{\star \star \star}\end{array}$ & $\begin{array}{c}0.035 \\
(0.006)^{\star \star \star}\end{array}$ & $\begin{array}{c}-0.094 \\
(0.012)^{\star * \star}\end{array}$ & $\begin{array}{c}0.033 \\
(0.006)^{\star \star *}\end{array}$ & $\begin{array}{c}-0.085 \\
(0.011)^{\star \star \star}\end{array}$ & $\begin{array}{c}0.033 \\
(0.006)^{\star \star *}\end{array}$ & $\begin{array}{c}-0.079 \\
(0.011)^{\star \star \star}\end{array}$ \\
\hline Age 35 to 44 & $\begin{array}{c}0.045 \\
(0.006)^{\star \star \star}\end{array}$ & $\begin{array}{c}-0.062 \\
(0.011)^{\star * k \star}\end{array}$ & $\begin{array}{c}0.043 \\
(0.006)^{\star \star \star}\end{array}$ & $\begin{array}{c}-0.027 \\
(0.009)^{\star *}\end{array}$ & $\begin{array}{c}0.041 \\
(0.006)^{\star \star \star}\end{array}$ & $\begin{array}{c}-0.014 \\
(0.008)\end{array}$ & $\begin{array}{c}0.044 \\
(0.006)^{\star * * *}\end{array}$ & $\begin{array}{l}-0.008 \\
(0.008)\end{array}$ \\
\hline Age 45 to 54 & $\begin{array}{c}0.044 \\
(0.006)^{* * *}\end{array}$ & $\begin{array}{c}-0.049 \\
(0.010)^{\star \star \star}\end{array}$ & $\begin{array}{c}0.020 \\
(0.008)^{* *}\end{array}$ & $\begin{array}{c}-0.019 \\
(0.009)^{*}\end{array}$ & $\begin{array}{c}0.019 \\
(0.008)^{*}\end{array}$ & $\begin{array}{l}-0.013 \\
(0.009)\end{array}$ & $\begin{array}{c}0.022 \\
(0.008)^{\star \star}\end{array}$ & $\begin{array}{c}-0.009 \\
(0.008)\end{array}$ \\
\hline Age 55 to 64 & $\begin{array}{c}0.073 \\
(0.003)^{\star * \star}\end{array}$ & $\begin{array}{l}0.0001 \\
(0.011)\end{array}$ & $\begin{array}{c}0.058 \\
(0.005)^{\star \star \star}\end{array}$ & $\begin{array}{c}0.014 \\
(0.009)\end{array}$ & $\begin{array}{c}0.057 \\
(0.005)^{* * *}\end{array}$ & $\begin{array}{c}0.020 \\
(0.008)^{*}\end{array}$ & $\begin{array}{c}0.060 \\
(0.005)^{\star \star *}\end{array}$ & $\begin{array}{c}0.023 \\
(0.008)^{* *}\end{array}$ \\
\hline Access/use technology & $\begin{array}{c}0.029 \\
(0.006)^{* * *}\end{array}$ & $\begin{array}{c}-0.0046 \\
(0.004)\end{array}$ & $\begin{array}{c}0.013 \\
(0.005)^{*}\end{array}$ & $\begin{array}{c}-0.008 \\
(0.004)^{\star}\end{array}$ & $\begin{array}{c}0.014 \\
(0.005)^{*}\end{array}$ & $\begin{array}{c}-0.006 \\
(0.004)\end{array}$ & $\begin{array}{c}0.009 \\
(0.005)\end{array}$ & $\begin{array}{c}-0.010 \\
(0.004)^{\star}\end{array}$ \\
\hline Chronic health condition & & & $\begin{array}{c}-0.007 \\
(0.004)\end{array}$ & $\begin{array}{c}0.014 \\
(0.003)^{* * *}\end{array}$ & $\begin{array}{c}-0.005 \\
(0.005)\end{array}$ & $\begin{array}{c}0.013 \\
(0.003)^{* * *}\end{array}$ & $\begin{array}{c}-0.003 \\
(0.004)\end{array}$ & $\begin{array}{c}0.012 \\
(0.003)^{*}\end{array}$ \\
\hline Health activity limitation & & & $\begin{array}{c}-0.0154 \\
(0.004)^{\star \star}\end{array}$ & $\begin{array}{c}-0.014 \\
(0.004)^{\star \star *}\end{array}$ & $\begin{array}{c}-0.018 \\
(0.005)^{\star * * *}\end{array}$ & $\begin{array}{c}-0.016 \\
(0.004)^{\star * *}\end{array}$ & $\begin{array}{c}-0.020 \\
(0.004)^{\star \star \star}\end{array}$ & $\begin{array}{c}-0.017 \\
(0.004)^{\text {**** }}\end{array}$ \\
\hline Has someone to confide in & & & & & $\begin{array}{c}-0.029 \\
(0.005)^{\star \star \star}\end{array}$ & $\begin{array}{c}0.035 \\
(0.011)^{* * *}\end{array}$ & $\begin{array}{c}-0.033 \\
(0.005)^{* * *}\end{array}$ & $\begin{array}{c}0.038 \\
(0.011)^{\star \cdots \star}\end{array}$ \\
\hline Band member & & & & & & & $\begin{array}{c}0.015 \\
(0.009)\end{array}$ & $\begin{array}{c}-0.022 \\
(0.004)^{\ldots}\end{array}$ \\
\hline Registered Indian & & & & & & & $\begin{array}{r}-0.005 \\
(0.009)\end{array}$ & $\begin{array}{c}-0.022 \\
(0.004)^{\star \star}\end{array}$ \\
\hline $\begin{array}{l}\text { Speaks Aboriginal } \\
\text { languages }\end{array}$ & & & & & & & $\begin{array}{c}-0.057 \\
(0.007)^{* * *}\end{array}$ & $\begin{array}{l}-0.002 \\
(0.004)\end{array}$ \\
\hline $\begin{array}{l}\text { Values Aboriginal } \\
\text { languages }\end{array}$ & & & & & & & $\begin{array}{c}0.021 \\
(0.005)^{*+* *}\end{array}$ & $\begin{array}{c}0.011 \\
(0.004)^{*}\end{array}$ \\
\hline $\begin{array}{l}\text { Traditional pursuits } \\
\text { for commerce }\end{array}$ & & & & & & & $\begin{array}{c}-0.044 \\
(0.014)^{\star \star}\end{array}$ & $\begin{array}{c}0.013 \\
(0.008)\end{array}$ \\
\hline $\begin{array}{l}\text { Traditional pursuits } \\
\text { for food }\end{array}$ & & & & & & & $\begin{array}{c}-0.011 \\
(0.005)^{\star}\end{array}$ & $\begin{array}{c}-0.013 \\
(0.004)^{\star \star * *}\end{array}$ \\
\hline $\begin{array}{l}\text { Traditional pursuits } \\
\text { for pleasure }\end{array}$ & & & & & & & $\begin{array}{c}0.027 \\
(0.004)^{* * *}\end{array}$ & $\begin{array}{c}0.005 \\
(0.003)\end{array}$ \\
\hline Number of observations & 1590 & 1730 & 1560 & 1710 & 1550 & 1700 & 1530 & 1690 \\
\hline
\end{tabular}

Source: Statistics Canada, 2006 Aboriginal Peoples Survey, Adult Core analytical file. Bootstrapped (1000 replications) standard errors in parentheses. Statistical significance ${ }^{* * *} p<.001 ;{ }^{* *} p<.01 ;{ }^{*} p<.05$.

Note: Estimates for all other variables are presented in Appendix $\mathbf{C l}$. 
The trend for urban NAI men, however, is not only not monotonic but the associations between both completed and incomplete postsecondary schooling below university level and the probability of employment are in direct opposition to what human capital theory would suggest - ceteris paribus, urban NAI men who possess some or completed postsecondary schooling below completed university level are less likely to be employed than are their counterparts who have only a high school diploma. This peculiar finding for urban NAI remains after social capital (Model 3) and cultural characteristics (Full Model) are considered. Another noteworthy point here is that, even at the university level of education, where the effect is positive for both genders, the magnitude of the effect, albeit not very large for either genders, is consistently larger for NAI women than it is for NAI men. ${ }^{93}$ As estimated in the Full Model, ceteris paribus, university-educated urban NAI women are on average about five percentage points more likely to be employed than are their counterparts who have only a high school diploma completed. All else held constant, the mean expected probability of urban NAI men being employed increases by less than four percentage points as the level of education changes from a completed high school to a completed university education.

The effects of the other characteristics from human capital blocks of variables are also intriguing, both for NAI men and for NAI women. When only basic human capital characteristics are considered (Model 1) the use of information technology seems to be positively associated with the probability of employment for urban NAI men. This effect

\footnotetext{
${ }^{93}$ A note here is warranted to highlight that the average marginal effects (AMEs) presented in these tables entail the same type of limitation in conveying the accurate outcome for different individuals within each gender group as do marginal effects estimated at the mean (MEMs). As Williams (2012) points out, however defined, the averages can distort difference in effects across individuals. Given the heterogeneity within Aboriginal population, individual marginal effects likely involve large variation so that true magnitude of the effect for some individuals is not being reflected in the AMEs presented here.
} 
remains positive and statistically significant in the ensuing two models when health (Model 2) and social capital (Model 3) characteristics are considered but when cultural factors are accounted for (Full Model) this effect becomes not statistically different from zero for urban NAI men. For urban NAI women, the information technology variable is negatively associated with the probability of employment and its effect becomes statistically significant only when health characteristics are included (Model 2) and when all cultural factors are included (Full Model) in the model.

The effects of the two health-related variables in the urban NAI samples vary. The results for the first measure, capturing the presence of chronic health conditions, indicate that this factor is only statistically significant for NAI women and, unexpectedly, the association is positive. The results for the other measure, capturing activity limitation, is, as expected, negative and statistically significant in all models, suggesting that, all else constant, conditions that impose activity limitations on individuals decrease the probability of their being employed, relative to those individuals who report having no activity limiting condition.

The association between the social capital variable (support networks) and the probability of employment is statistically significant in all models but positive only in the case of urban NAI women, suggesting that ceteris paribus, average expected probability of urban NAI women being employed increases by about four percentage points (Full Model) as the indicator for social capital changes from not having someone to confide in to having someone to turn to in times of crisis. Interestingly, for urban NAI men, this association is negative, as displayed in Table 2, possibly suggesting that this might not be a good measure of social capital in this case. 
Finally, estimated results for the block of cultural characteristics, displayed in the last two columns of Table 2, suggest notable variations in the effect on probability of employment for urban NAI. As shown in Table 2, the engagement in traditional pursuits for food and medicinal purposes appears to have an adverse impact on the probability of employment for both genders. Urban NAI men are also less likely to be employed if they engaged in traditional pursuits for commercial purposes but more likely to be employed if they engaged in traditional pursuits for pleasure. The proficiency in Aboriginal languages is negatively associated with the probability of employment for urban NAI men; having favourable perceptions about importance of Aboriginal languages, however, is positively associated with the probability of employment for both genders. Band membership appears to have a significantly negative effect on the probability of employment, but only for urban NAI women.

In short, the predictions of human capital theory do not hold up when the associated empirical models are applied to urban NAI men. This finding, in agreement with previous literature, leaves the main research question unresolved. Adding social and cultural factors, as measured in this study, to the human capital model does not seem to add much to our understanding of employment probability of urban NAI. With some exceptions, the perplexity remains as the effects are not what the theory would suggest. This, however, might be a measurement issue, implying that further investigations using different variables could prove informative. 
Table 3: Selected estimates from probit employment function for rural NAI, APS 2006

\begin{tabular}{|c|c|c|c|c|c|c|c|c|}
\hline \multirow{3}{*}{$\begin{array}{l}\text { Selected variables in the } \\
\text { probit equations }\end{array}$} & \multicolumn{8}{|c|}{ Average Marginal Effects } \\
\hline & \multicolumn{2}{|c|}{ Model 1} & \multicolumn{2}{|c|}{ Model 2} & \multicolumn{2}{|c|}{ Model 3} & \multicolumn{2}{|c|}{ Full Model } \\
\hline & Men & Women & Men & Women & Men & Women & Men & Women \\
\hline $\begin{array}{l}\text { Less than } \\
\text { high school education }\end{array}$ & $\begin{array}{c}-0.023 \\
(0.008)^{* *}\end{array}$ & $\begin{array}{c}0.048 \\
(0.009)^{* \cdots *}\end{array}$ & $\begin{array}{c}-0.028 \\
(0.008)^{* * *}\end{array}$ & $\begin{array}{c}-0.054 \\
(0.009)^{* * *}\end{array}$ & $\begin{array}{c}-0.024 \\
(0.008)^{* *}\end{array}$ & $\begin{array}{c}-0.053 \\
(0.009)^{\star \star *}\end{array}$ & $\begin{array}{c}-0.032 \\
(0.008)^{* * *}\end{array}$ & $\begin{array}{c}-0.054 \\
(0.009)^{\star \star \star}\end{array}$ \\
\hline $\begin{array}{l}\text { Some } \\
\text { non-university education }\end{array}$ & $\begin{array}{c}0.009 \\
(0.009)\end{array}$ & $\begin{array}{c}-0.040 \\
(0.013)^{\star *}\end{array}$ & $\begin{array}{l}-0.009 \\
(0.010)\end{array}$ & $\begin{array}{c}-0.049 \\
(0.12)^{\star \star \star}\end{array}$ & $\begin{array}{r}-0.009 \\
(0.010)\end{array}$ & $\begin{array}{c}-0.050 \\
(0.012)^{\star \star \star \star}\end{array}$ & $\begin{array}{l}-0.001 \\
(0.009)\end{array}$ & $\begin{array}{c}-0.50 \\
(0.012)^{* * *}\end{array}$ \\
\hline $\begin{array}{l}\text { Completed, } \\
\text { non-university education }\end{array}$ & $\begin{array}{c}-0.001 \\
(0.008)\end{array}$ & $\begin{array}{c}-0.028 \\
(0.007)^{* * *}\end{array}$ & $\begin{array}{l}-0.007 \\
(0.007)\end{array}$ & $\begin{array}{c}-0.021 \\
(0.007)^{\star \star}\end{array}$ & $\begin{array}{r}-0.003 \\
(0.007)\end{array}$ & $\begin{array}{c}-0.017 \\
(0.007)^{\star}\end{array}$ & $\begin{array}{r}-0.009 \\
(0.007)\end{array}$ & $\begin{array}{c}-0.017 \\
(0.007)^{*}\end{array}$ \\
\hline $\begin{array}{l}\text { Some } \\
\text { university education }\end{array}$ & $\begin{array}{l}-0.015 \\
(0.019)\end{array}$ & $\begin{array}{c}-0.016 \\
(0.015)\end{array}$ & $\begin{array}{c}-0.011 \\
(0.019)\end{array}$ & $\begin{array}{l}-0.006 \\
(0.014)\end{array}$ & $\begin{array}{c}0.006 \\
(0.017)\end{array}$ & $\begin{array}{c}-0.007 \\
(0.014)\end{array}$ & $\begin{array}{c}-0.003 \\
(0.018)\end{array}$ & $\begin{array}{c}0.003 \\
(0.012)\end{array}$ \\
\hline $\begin{array}{l}\text { Completed } \\
\text { university education }\end{array}$ & $\begin{array}{c}0.004 \\
(0.012)\end{array}$ & $\begin{array}{c}0.035 \\
(0.006)^{\star \star \star}\end{array}$ & $\begin{array}{r}-0.009 \\
(0.012)\end{array}$ & $\begin{array}{c}0.032 \\
(0.006)^{\star \star \star}\end{array}$ & $\begin{array}{c}-0.007 \\
(0.012)\end{array}$ & $\begin{array}{c}0.032 \\
(0.005)^{\star \star \star *}\end{array}$ & $\begin{array}{l}-0.0018 \\
(0.011)\end{array}$ & $\begin{array}{c}0.037 \\
(0.005)^{\star \star \star}\end{array}$ \\
\hline Age 15 to 19 & $\begin{array}{c}-0.005 \\
(0.012)\end{array}$ & $\begin{array}{c}-0.078 \\
(0.018)^{\star \star \star}\end{array}$ & $\begin{array}{c}0.002 \\
(0.011)\end{array}$ & $\begin{array}{c}-0.059 \\
(0.016)^{* \star \star *}\end{array}$ & $\begin{array}{l}0.0002 \\
(0.010)\end{array}$ & $\begin{array}{c}-0.058 \\
(0.016)^{\star \star \star \star}\end{array}$ & $\begin{array}{r}-0.0002 \\
(0.010)\end{array}$ & $\begin{array}{c}-0.050 \\
(0.014)^{\star \star}\end{array}$ \\
\hline Age 25 to 34 & $\begin{array}{c}0.054 \\
(0.007)^{\star \star \star}\end{array}$ & $\begin{array}{c}0.031 \\
(0.007)^{\star \star \star}\end{array}$ & $\begin{array}{c}0.048 \\
(0.007)^{\star \star \star}\end{array}$ & $\begin{array}{c}-0.001 \\
(0.008)\end{array}$ & $\begin{array}{c}0.054 \\
(0.007)^{\star * *}\end{array}$ & $\begin{array}{c}-0.001 \\
(0.008)\end{array}$ & $\begin{array}{c}0.050 \\
(0.007)^{\star * *}\end{array}$ & $\begin{array}{c}-0.010 \\
(0.008)\end{array}$ \\
\hline Age 35 to 44 & $\begin{array}{c}0.061 \\
(0.007)^{\star \star \star}\end{array}$ & $\begin{array}{c}0.078 \\
(0.007)^{\star \star \star \star}\end{array}$ & $\begin{array}{c}0.028 \\
(0.009)^{\star \star}\end{array}$ & $\begin{array}{c}0.020 \\
(0.008)^{\star \star}\end{array}$ & $\begin{array}{c}0.031 \\
(0.009)^{\star *}\end{array}$ & $\begin{array}{c}0.030 \\
(0.007)^{\star \star \star \star}\end{array}$ & $\begin{array}{c}0.032 \\
(0.009)^{\star \star \star \star}\end{array}$ & $\begin{array}{c}0.024 \\
(0.007)^{\star \star *}\end{array}$ \\
\hline Age 45 to 54 & $\begin{array}{c}0.080 \\
(0.007)^{* * *}\end{array}$ & $\begin{array}{c}0.060 \\
(0.007)^{\star \star \star *}\end{array}$ & $\begin{array}{c}0.047 \\
(0.009)^{\star * *}\end{array}$ & $\begin{array}{c}0.020 \\
(0.008)^{\star}\end{array}$ & $\begin{array}{c}0.047 \\
(0.009)^{\star * *}\end{array}$ & $\begin{array}{c}0.025 \\
(0.008)^{* *}\end{array}$ & $\begin{array}{c}0.048 \\
(0.009)^{* \star \star}\end{array}$ & $\begin{array}{c}0.021 \\
(0.008)^{* *}\end{array}$ \\
\hline Age 55 to 64 & $\begin{array}{c}0.097 \\
(0.005)^{\star \star *}\end{array}$ & $\begin{array}{c}0.075 \\
(0.004)^{\star \star \star \star}\end{array}$ & $\begin{array}{c}0.088 \\
(0.006)^{\star \star \star}\end{array}$ & $\begin{array}{c}0.063 \\
(0.005)^{\star \star k \star}\end{array}$ & $\begin{array}{c}0.090 \\
(0.006)^{* * \star}\end{array}$ & $\begin{array}{c}0.064 \\
(0.005)^{\star * \star}\end{array}$ & $\begin{array}{c}0.094 \\
(0.006)^{* * *}\end{array}$ & $\begin{array}{c}0.062 \\
(0.005)^{\star \star *}\end{array}$ \\
\hline Access/use technology & $\begin{array}{c}0.046 \\
(0.006)^{\star \star *}\end{array}$ & $\begin{array}{c}0.018 \\
(0.005)^{* \pi *}\end{array}$ & $\begin{array}{c}0.033 \\
(0.006)^{* * *}\end{array}$ & $\begin{array}{c}0.014 \\
(0.004)^{\star *}\end{array}$ & $\begin{array}{c}0.023 \\
(0.005)^{\star \star *}\end{array}$ & $\begin{array}{c}0.015 \\
(0.004)^{\star \star}\end{array}$ & $\begin{array}{c}0.017 \\
(0.005)^{\star \star}\end{array}$ & $\begin{array}{c}0.016 \\
(0.004)^{\star \star \star}\end{array}$ \\
\hline Chronic health condition & & & $\begin{array}{c}-0.002 \\
(0.005)\end{array}$ & $\begin{array}{c}-0.053 \\
(0.004)^{\star \star \star}\end{array}$ & $\begin{array}{c}-0.006 \\
(0.005)\end{array}$ & $\begin{array}{c}-0.052 \\
(0.004)^{\star \star \star \star}\end{array}$ & $\begin{array}{c}-0.007 \\
(0.005)\end{array}$ & $\begin{array}{c}-0.056 \\
(0.004)^{\star * *}\end{array}$ \\
\hline Health activity limitation & & & $\begin{array}{c}0.009 \\
(0.005)\end{array}$ & $\begin{array}{c}0.018 \\
(0.004)^{\star \star \star}\end{array}$ & $\begin{array}{c}0.011 \\
(0.005)^{*}\end{array}$ & $\begin{array}{c}0.014 \\
(0.004)^{\star \star *}\end{array}$ & $\begin{array}{c}0.018 \\
(0.005)^{\star \star \star}\end{array}$ & $\begin{array}{c}0.013 \\
(0.004)^{\star *}\end{array}$ \\
\hline Has someone to confide in & & & & & $\begin{array}{c}0.011 \\
(0.014)\end{array}$ & $\begin{array}{c}-0.022 \\
(0.007)^{\star \star}\end{array}$ & $\begin{array}{c}-0.022 \\
(0.009)^{\star}\end{array}$ & $\begin{array}{c}-0.022 \\
(0.007)^{\star \star}\end{array}$ \\
\hline Band member & & & & & & & $\begin{array}{c}-0.0247 \\
(0.007)^{* \star \star}\end{array}$ & $\begin{array}{c}-0.032 \\
(0.006)^{\star *}\end{array}$ \\
\hline Registered Indian & & & & & & & $\begin{array}{c}-0.050 \\
(0.006)^{* * *}\end{array}$ & $\begin{array}{c}0.017 \\
(0.007)^{\star}\end{array}$ \\
\hline $\begin{array}{l}\text { Speaks Aboriginal } \\
\text { languages }\end{array}$ & & & & & & & $\begin{array}{c}-0.062 \\
(0.007)^{\star \star \star *}\end{array}$ & $\begin{array}{c}0.014 \\
(0.005)^{\text {k* }}\end{array}$ \\
\hline $\begin{array}{l}\text { Values Aboriginal } \\
\text { languages }\end{array}$ & & & & & & & $\begin{array}{c}-0.048 \\
(0.005)^{\star \star \star}\end{array}$ & $\begin{array}{c}0.036 \\
(0.005)^{\star * \star}\end{array}$ \\
\hline $\begin{array}{l}\text { Traditional pursuits } \\
\text { for commerce }\end{array}$ & & & & & & & $\begin{array}{c}0.031 \\
(0.006)^{\star * *}\end{array}$ & $\begin{array}{c}0.008 \\
(0.010)\end{array}$ \\
\hline $\begin{array}{l}\text { Traditional pursuits } \\
\text { for food }\end{array}$ & & & & & & & $\begin{array}{c}-0.032 \\
(0.006)^{* \star \star \star}\end{array}$ & $\begin{array}{c}-0.015 \\
(0.005)^{* *}\end{array}$ \\
\hline $\begin{array}{l}\text { Traditional pursuits } \\
\text { for pleasure }\end{array}$ & & & & & & & $\begin{array}{c}-0.029 \\
(0.006)^{\star * *}\end{array}$ & $\begin{array}{c}0.017 \\
(0.005)^{* \star}\end{array}$ \\
\hline Number of observations & 940 & 960 & 920 & 940 & 910 & 940 & 900 & 940 \\
\hline
\end{tabular}

Source: Statistics Canada, 2006 Aboriginal Peoples Survey, Adult Core analytical file. Bootstrapped (1000 replications) standard errors in parentheses. Statistical significance ${ }^{* *} p<.001 ;{ }^{* *} p<.01 ;{ }^{*} p<.05$.

Note: Estimates for all other variables are presented in Appendix C2. 
The average marginal effects of the probit estimation for the rural NAI group are presented next in Table 3. Most noteworthy in the rural NAI sample are the striking differences in the effects of educational attainment between rural men and rural women as well as between rural and urban NAI. As displayed in Table 3, none of the estimates on postsecondary education are statistically significant in the case of rural NAI men. In the case of rural NAI women, the effects on two categories (some or completed nonuniversity education) are statistically significant but, as it was the case for urban NAI men, the association here is also in contradiction to what human capital theory would suggest - the association suggests that, holding all else constant, the probability of rural NAI women being employed declines as the indicator for educational attainment changes from completed high school diploma to some non-university postsecondary education or to completed non-university postsecondary education. The association between completed university education and the probability of employment of rural NAI women, however, is positive and statistically significant.

The effect of the variable measuring the access to and the use of information technology in the rural samples is positive and statistically significant in each model and for each gender. In the rural male sample, however, the magnitude of the effect of this variable diminishes steadily from $4.7 \%$ in Model 1 to $1.7 \%$ in the Full Model.

The first of the health-related measures in the model (chronic health condition) is statistically significant only in the case of rural NAI women and the association is strong and negative, as hypothesized a priori. The association between the other health-related measure (activity limitation) and the probability of employment is surprisingly positive both for men and for women, suggesting that the probability of employment increases as 
the indicator on this health-related measure changes from no activity limitation to activity limitation. This effect for rural NAI men is only observed in the last two models when the social capital and cultural characteristics are included.

As shown in Table 3, the measure for social capital (social networks) is negatively correlated with the probability of employment, both for rural NAI women and for rural NAI men (in the Full Model). It is interesting to note though that the association between social networks and the probability of employment for rural NAI men changes from nonnegative and statistically not different from zero in the initial model (Model 3) to negative and statistically significant in the final model (Full Model) where all cultural characteristics are included.

Finally, some large differences in the effect are notable in the block of variables relating to cultural aspects. As it was the case in the urban samples, if the engagement in traditional pursuits is done for food and medicinal purposes, it has a negative effect on the probability of employment for both genders in the rural samples. However, if the engagement in traditional pursuits is done for pleasure, the association between that activity and the probability of employment appears positive for rural NAI women and negative for rural NAI men. The association between the engagements in traditional pursuits for commercial purposes is positive and statistically significant, but only in the model of rural NAI men, possibly indicating that this form of traditional pursuit was reported as formal employment. Both variables relating to Aboriginal languages are statistically significant for both genders but the association is negative in the model of rural NAI men and positive in the model of rural NAI women. Band membership exerts a significantly negative effect on the probability of employment of rural NAI people. 
Table 4: Selected estimates from probit employment function for registered NAI, APS 2006

\begin{tabular}{|c|c|c|c|c|c|c|c|c|}
\hline \multirow{3}{*}{$\begin{array}{l}\text { Selected variables in the } \\
\text { probit equations }\end{array}$} & \multicolumn{8}{|c|}{ Average Marginal Effects } \\
\hline & \multicolumn{2}{|c|}{ Model 1} & \multicolumn{2}{|c|}{ Model 2} & \multicolumn{2}{|c|}{ Model 3} & \multicolumn{2}{|c|}{ Full Model } \\
\hline & Men & Women & Men & Women & Men & Women & Men & Women \\
\hline $\begin{array}{l}\text { Less than } \\
\text { high school education }\end{array}$ & $\begin{array}{c}-0.048 \\
(0.008)^{* \star *}\end{array}$ & $\begin{array}{c}-0.024 \\
(0.007)^{* *}\end{array}$ & $\begin{array}{c}-0.051 \\
(0.008)^{\star \star \star \star}\end{array}$ & $\begin{array}{c}-0.017 \\
(0.006)^{\star \star}\end{array}$ & $\begin{array}{c}-0.053 \\
(0.008)^{\star * *}\end{array}$ & $\begin{array}{c}-0.017 \\
(0.006)^{\star *}\end{array}$ & $\begin{array}{c}-0.051 \\
(0.008)^{* \cdots *}\end{array}$ & $\begin{array}{c}-0.022 \\
(0.006)^{\star *}\end{array}$ \\
\hline $\begin{array}{l}\text { Some } \\
\text { non-university education }\end{array}$ & $\begin{array}{r}-0.012 \\
(0.009)\end{array}$ & $\begin{array}{c}0.056 \\
(0.004)^{\star * *}\end{array}$ & $\begin{array}{c}-0.024 \\
(0.010)^{\star}\end{array}$ & $\begin{array}{c}0.058 \\
(0.004)^{\text {k*k* }}\end{array}$ & $\begin{array}{c}-0.024 \\
(0.010)^{\star}\end{array}$ & $\begin{array}{c}0.057 \\
(0.004)^{\star \star * \star}\end{array}$ & $\begin{array}{r}-0.019 \\
(0.010)\end{array}$ & $\begin{array}{c}0.055 \\
(0.004)^{\star \star \star \star}\end{array}$ \\
\hline $\begin{array}{l}\text { Completed, } \\
\text { non-university education }\end{array}$ & $\begin{array}{c}-0.037 \\
(0.009)^{* * *}\end{array}$ & $\begin{array}{c}0.070 \\
(0.006)^{* * * *}\end{array}$ & $\begin{array}{c}-0.049 \\
(0.009)^{\star \star \star}\end{array}$ & $\begin{array}{c}0.078 \\
(0.005)^{\star \star \star}\end{array}$ & $\begin{array}{c}-0.051 \\
(0.009)^{* \star \star}\end{array}$ & $\begin{array}{c}0.078 \\
(0.005)^{* * *}\end{array}$ & $\begin{array}{c}-0.048 \\
(0.008)^{\star * * *}\end{array}$ & $\begin{array}{c}0.072 \\
(0.005)^{\star * \star}\end{array}$ \\
\hline $\begin{array}{l}\text { Some } \\
\text { university education }\end{array}$ & $\begin{array}{c}-0.067 \\
(0.013)^{\star * *}\end{array}$ & $\begin{array}{c}0.061 \\
(0.004)^{\star \star \star}\end{array}$ & $\begin{array}{c}-0.083 \\
(0.014)^{\star * * \star}\end{array}$ & $\begin{array}{c}0.057 \\
(0.004)^{\star \star \star}\end{array}$ & $\begin{array}{c}-0.085 \\
(0.014)^{\star \star \star}\end{array}$ & $\begin{array}{c}0.057 \\
(0.005)^{* * *}\end{array}$ & $\begin{array}{c}-0.085 \\
(0.014)^{\star * * *}\end{array}$ & $\begin{array}{c}0.056 \\
(0.004)^{\star \star \star}\end{array}$ \\
\hline $\begin{array}{l}\text { Completed } \\
\text { university education }\end{array}$ & $\begin{array}{c}0.047 \\
(0.007)^{\star \star *}\end{array}$ & $\begin{array}{c}0.064 \\
(0.005)^{* * *}\end{array}$ & $\begin{array}{c}0.040 \\
(0.007)^{\star \star \star *}\end{array}$ & $\begin{array}{c}0.065 \\
(0.004)^{* * *}\end{array}$ & $\begin{array}{c}0.040 \\
(0.007)^{\star * * \star}\end{array}$ & $\begin{array}{c}0.064 \\
(0.004)^{\star \star \star}\end{array}$ & $\begin{array}{c}0.042 \\
(0.007)^{\star \star \star}\end{array}$ & $\begin{array}{c}0.063 \\
(0.004)^{\star * *}\end{array}$ \\
\hline Age 15 to 19 & $\begin{array}{c}0.100 \\
(0.015)^{\star \star \star}\end{array}$ & $\begin{array}{c}-0.144 \\
(0.021)^{\star \star \star}\end{array}$ & $\begin{array}{c}-0.075 \\
(0.014)^{\star \star *}\end{array}$ & $\begin{array}{c}-0.145 \\
(0.019)^{*+* *}\end{array}$ & $\begin{array}{c}-0.083 \\
(0.014)^{\star * *}\end{array}$ & $\begin{array}{c}-0.145 \\
(0.020)^{\star \star \star}\end{array}$ & $\begin{array}{c}-0.085 \\
(0.014)^{\star * * *}\end{array}$ & $\begin{array}{c}-0.142 \\
(0.019)^{\star * *}\end{array}$ \\
\hline Age 25 to 34 & $\begin{array}{c}0.0245 \\
(0.007)^{* *}\end{array}$ & $\begin{array}{c}-0.076 \\
(0.011)^{\star \star *}\end{array}$ & $\begin{array}{c}0.027 \\
(0.008)^{\star \star \star \star}\end{array}$ & $\begin{array}{c}-0.077 \\
(0.010)^{\text {kn*k}}\end{array}$ & $\begin{array}{c}0.026 \\
(0.008)^{\star *}\end{array}$ & $\begin{array}{c}-0.074 \\
(0.010)^{\star * *}\end{array}$ & $\begin{array}{c}0.026 \\
(0.008)^{\star \star}\end{array}$ & $\begin{array}{c}-0.072 \\
(0.010)^{\star \star \star}\end{array}$ \\
\hline Age 35 to 44 & $\begin{array}{c}0.063 \\
(0.006)^{\star * \star}\end{array}$ & $\begin{array}{c}-0.064 \\
(0.009)^{\ldots * *}\end{array}$ & $\begin{array}{c}0.058 \\
(0.007)^{\star * * *}\end{array}$ & $\begin{array}{c}-0.062 \\
(0.009)^{\star * * *}\end{array}$ & $\begin{array}{c}0.057 \\
(0.007)^{* * *}\end{array}$ & $\begin{array}{c}-0.055 \\
(0.009)^{\star \star \star}\end{array}$ & $\begin{array}{c}0.055 \\
(0.007)^{\star \star \star}\end{array}$ & $\begin{array}{c}-0.052 \\
(0.009)^{\star \star \star}\end{array}$ \\
\hline Age 45 to 54 & $\begin{array}{c}0.061 \\
(0.006)^{\star \star *}\end{array}$ & $\begin{array}{c}-0.038 \\
(0.008)^{\star * \star *}\end{array}$ & $\begin{array}{c}0.052 \\
(0.008)^{\star \star \star \star}\end{array}$ & $\begin{array}{c}-0.046 \\
(0.010)^{\star \star \star \star}\end{array}$ & $\begin{array}{c}0.050 \\
(0.008)^{\star * *}\end{array}$ & $\begin{array}{c}-0.038 \\
(0.009)^{* * *}\end{array}$ & $\begin{array}{c}0.052 \\
(0.008)^{\star \star \star *}\end{array}$ & $\begin{array}{c}-0.035 \\
(0.009)^{\star \star \star \star}\end{array}$ \\
\hline Age 55 to 64 & $\begin{array}{c}0.089 \\
(0.004)^{* * *}\end{array}$ & $\begin{array}{c}0.025 \\
(0.008)^{\star \star}\end{array}$ & $\begin{array}{c}0.087 \\
(0.005)^{* * *}\end{array}$ & $\begin{array}{c}0.003 \\
(0.011)\end{array}$ & $\begin{array}{c}0.087 \\
(0.005)^{* * *}\end{array}$ & $\begin{array}{c}0.008 \\
(0.010)\end{array}$ & $\begin{array}{c}0.089 \\
(0.005)^{\star \star *}\end{array}$ & $\begin{array}{c}0.009 \\
(0.009)\end{array}$ \\
\hline Access/use technology & $\begin{array}{c}0.069 \\
(0.006)^{* * *}\end{array}$ & $\begin{array}{c}0.009 \\
(0.005)\end{array}$ & $\begin{array}{c}0.053 \\
(0.005)^{\star * *}\end{array}$ & $\begin{array}{c}0.002 \\
(0.004)\end{array}$ & $\begin{array}{c}0.052 \\
(0.006)^{\star \star \star *}\end{array}$ & $\begin{array}{c}0.003 \\
(0.004)\end{array}$ & $\begin{array}{c}0.049 \\
(0.005)^{* * *}\end{array}$ & $\begin{array}{c}-0.001 \\
(0.004)\end{array}$ \\
\hline Chronic health condition & & & $\begin{array}{c}-0.004 \\
(0.005)\end{array}$ & $\begin{array}{c}0.015 \\
(0.004)^{m * *}\end{array}$ & $\begin{array}{c}-0.001 \\
(0.005)\end{array}$ & $\begin{array}{c}0.015 \\
(0.004)^{\star * *}\end{array}$ & $\begin{array}{c}0.004 \\
(0.005)\end{array}$ & $\begin{array}{c}0.015 \\
(0.004)^{k \star \star}\end{array}$ \\
\hline Health activity limitation & & & $\begin{array}{c}-0.032 \\
(0.005)^{* * *}\end{array}$ & $\begin{array}{c}-0.010 \\
(0.004)^{*}\end{array}$ & $\begin{array}{c}-0.035 \\
(0.005)^{\star \star * *}\end{array}$ & $\begin{array}{c}-0.010 \\
(0.004)^{\star}\end{array}$ & $\begin{array}{c}-0.040 \\
(0.005)^{\star \star *}\end{array}$ & $\begin{array}{c}-0.012 \\
(0.004)^{\star \star}\end{array}$ \\
\hline Rural place of residence & & & $\begin{array}{c}-0.056 \\
(0.005)^{* \star *}\end{array}$ & $\begin{array}{c}0.008 \\
(0.003)^{*}\end{array}$ & $\begin{array}{c}-0.055 \\
(0.005)^{\star * \star}\end{array}$ & $\begin{array}{c}0.011 \\
(0.004)^{* *}\end{array}$ & $\begin{array}{c}-0.049 \\
(0.005)^{* * *}\end{array}$ & $\begin{array}{c}0.012 \\
(0.003)^{\star \star}\end{array}$ \\
\hline Has someone to confide in & & & & & $\begin{array}{c}-0.013 \\
(0.007)\end{array}$ & $\begin{array}{c}0.046 \\
(0.011)^{\star * *}\end{array}$ & $\begin{array}{c}-0.028 \\
(0.006)^{* * *}\end{array}$ & $\begin{array}{c}0.050 \\
(0.012)^{\star \star \star}\end{array}$ \\
\hline Band member & & & & & & & $\begin{array}{c}0.022 \\
(0.010)^{\star}\end{array}$ & $\begin{array}{c}-0.032 \\
(0.005)^{* * *}\end{array}$ \\
\hline $\begin{array}{l}\text { Speaks Aboriginal } \\
\text { languages }\end{array}$ & & & & & & & $\begin{array}{c}-0.041 \\
(0.005)^{* * *}\end{array}$ & $\begin{array}{l}0.0001 \\
(0.004)\end{array}$ \\
\hline $\begin{array}{l}\text { Values Aboriginal } \\
\text { languages }\end{array}$ & & & & & & & $\begin{array}{c}0.019 \\
(0.005)^{\star \star *}\end{array}$ & $\begin{array}{c}0.025 \\
(0.005)^{\star * *}\end{array}$ \\
\hline $\begin{array}{l}\text { Traditional pursuits } \\
\text { for commerce }\end{array}$ & & & & & & & $\begin{array}{c}-0.022 \\
(0.009)^{*}\end{array}$ & $\begin{array}{c}0.036 \\
(0.007)^{* * *}\end{array}$ \\
\hline $\begin{array}{l}\text { Traditional pursuits } \\
\text { for food }\end{array}$ & & & & & & & $\begin{array}{c}0.003 \\
(0.005)\end{array}$ & $\begin{array}{c}-0.014 \\
(0.004)^{\star * *}\end{array}$ \\
\hline $\begin{array}{l}\text { Traditional pursuits } \\
\text { for pleasure }\end{array}$ & & & & & & & $\begin{array}{c}0.009 \\
(0.005)\end{array}$ & $\begin{array}{c}0.009 \\
(0.004)^{*}\end{array}$ \\
\hline Number of observations & 1680 & 1840 & 1650 & 1820 & 1630 & 1810 & 1610 & 1800 \\
\hline
\end{tabular}

Source: Statistics Canada, 2006 Aboriginal Peoples Survey, Adult Core analytical file. Bootstrapped (1000 replications) standard errors in parentheses. Statistical significance ${ }^{* * *} p<.001 ;{ }^{* *} p<.01 ;{ }^{*} p<.05$.

Note: Estimates for all other variables are presented in Appendix C3. 
The estimated results for registered NAI, presented in Table 4, are very similar to those of the urban NAI, presented earlier in this section, particularly in regard to the effects of educational attainment on the probability of employment for men and for women. As shown in Table 4, the association between postsecondary schooling and the probability of employment of registered NAI men is only positive at the completed university education level - at all other levels of education, the association is negative. One peculiar exception is that the effect on incomplete non-university education for registered NAI men is not consistently statistically significant, as it was in the previously presented model for urban NAI men. As shown in Table 4, the effect is only significant in the expanded human capital model (Model 2) and in the subsequent model (Model 3) where social capital variables are added in. In the initial human capital block of variables and in the final full model, the effect on this category of education is not statistically different from zero for registered NAI men.

In the case of registered NAI women, the effects of postsecondary educational attainment are consistently positive and statistically significant, as it was the case in the previously discussed model for urban NAI women. In addition, the magnitude of the effects is consistently slightly larger in the case of registered NAI women, when compared to that of urban NAI women, suggesting that, assuming no difference in residual variation, the returns to postsecondary schooling are highest for registered NAI women, particularly at the level of completed non-university education. These effects are also consistent across all four models.

An interesting insight in regards to other variables in this block is that the information technology seems to have a significantly positive effect on the probability of 
employment for registered NAI men, but not for registered NAI women. As shown in Table 4, when only the key human capital variables are assessed (Model 1), holding all else constant, the probability of registered NAI men being employed increases by about seven percentage points as the indicator for information technology changes from not having access to the Internet at home to having access. The magnitude of this effect, however, decreases by about two percentage points in the Full Model where all other observable characteristics are taken into account.

Having a chronic health condition appears to have a positive impact on the probability of employment for registered NAI women only. Having a health condition that limits the range of physical activity, however, is negatively associated with the probability of employment both for registered men and for registered women. Although generally small, the magnitudes of this effect in each model are larger for registered NAI men than they are for registered NAI women.

In relation to social capital, a surprising finding is that social support networks seem to have opposite impacts on the probability of employment for registered NAI men and for registered NAI women. As shown in Table 4, the variable measuring social support in the form of strong ties has a positive impact on the probability of employment for registered NAI women and a negative impact on the probability of employment for registered men. This finding is consistent with that reported earlier for urban NAI group.

The estimates for the final block of variables relating to culture indicate that traditional pursuits only have a significant impact on the probability of employment of registered NAI men if they are done for commercial purposes. As shown in Table 4, the mean expected probability of registered NAI men being employed decreases by about 
two percentage points when the indicator on traditional pursuits for commercial purposes changes from zero to one, holding all else constant. An interesting finding is that the mean expected probability of registered $\mathrm{NAI}$ women being employed increases by about four percentage points when the same indicator changes from zero to one, holding all else constant. The effect of the engagement in traditional pursuits for pleasure is also positively related with the probability of employment for registered NAI women while the engagement in traditional pursuits for food and medicinal purposes is negative.

Finally, the findings from Table 4 suggest that fluency in an Aboriginal language has an overall negative impact on the probability of employment for registered NAI men. Favourable valuation of the Aboriginal languages, on the other hand, has a positive effect on the probability of employment, both for registered NAI men and for registered NAI women. The effect of Band membership appears positive for registered NAI men and negative for registered NAI women, suggesting that registered NAI men who are Band members are more likely to be employed than registered NAI men who are not Band members. The reverse seems to be the case for registered NAI women. 
Table 5: Selected estimates from probit employment function for non-registered NAI, APS 2006

\begin{tabular}{|c|c|c|c|c|c|c|c|c|}
\hline \multirow{3}{*}{$\begin{array}{l}\text { Selected variables in the } \\
\text { probit equations }\end{array}$} & \multicolumn{8}{|c|}{ Average Marginal Effects } \\
\hline & \multicolumn{2}{|c|}{ Model 1} & \multicolumn{2}{|c|}{ Model 2} & \multicolumn{2}{|c|}{ Model 3} & \multicolumn{2}{|c|}{ Full Model } \\
\hline & Men & Women & Men & Women & Men & Women & Men & Women \\
\hline $\begin{array}{l}\text { Less than } \\
\text { high school education }\end{array}$ & $\begin{array}{c}-0.042 \\
(0.009)^{\star * \star *}\end{array}$ & $\begin{array}{c}-0.033 \\
(0.009)^{* * *}\end{array}$ & $\begin{array}{c}-0.052 \\
(0.009)^{* * *}\end{array}$ & $\begin{array}{c}-0.039 \\
(0.008)^{* * *}\end{array}$ & $\begin{array}{c}-0.062 \\
(0.010)^{\star \star * *}\end{array}$ & $\begin{array}{c}-0.036 \\
(0.007)^{\star \star \star}\end{array}$ & $\begin{array}{c}-0.044 \\
(0.008)^{* * *}\end{array}$ & $\begin{array}{c}-0.038 \\
(0.007)^{\text {n+k }}\end{array}$ \\
\hline $\begin{array}{l}\text { Some } \\
\text { non-university education }\end{array}$ & $\begin{array}{c}-0.010 \\
(0.012)\end{array}$ & $\begin{array}{c}0.003 \\
(0.008)\end{array}$ & $\begin{array}{l}-0.010 \\
(0.009)\end{array}$ & $\begin{array}{c}0.004 \\
(0.008)\end{array}$ & $\begin{array}{c}-0.014 \\
(0.010)\end{array}$ & $\begin{array}{c}0.002 \\
(0.007)\end{array}$ & $\begin{array}{l}-0.019 \\
(0.010)\end{array}$ & $\begin{array}{c}0.002 \\
(0.007)\end{array}$ \\
\hline $\begin{array}{l}\text { Completed, } \\
\text { non-university education }\end{array}$ & $\begin{array}{c}-0.020 \\
(0.008)^{*}\end{array}$ & $\begin{array}{c}0.015 \\
(0.006)^{*}\end{array}$ & $\begin{array}{l}-0.016 \\
(0.007)^{*}\end{array}$ & $\begin{array}{c}0.017 \\
(0.005)^{\star *}\end{array}$ & $\begin{array}{c}-0.020 \\
(0.007)^{* \star}\end{array}$ & $\begin{array}{c}0.021 \\
(0.005)^{* \star *}\end{array}$ & $\begin{array}{c}-0.024 \\
(0.007)^{\star \star}\end{array}$ & $\begin{array}{c}0.021 \\
(0.005)^{*}\end{array}$ \\
\hline $\begin{array}{l}\text { Some } \\
\text { university education }\end{array}$ & $\begin{array}{c}0.027 \\
(0.007)^{\star \star \star}\end{array}$ & $\begin{array}{c}0.022 \\
(0.006)^{* k \star}\end{array}$ & $\begin{array}{c}0.034 \\
(0.008)^{\star \star \star}\end{array}$ & $\begin{array}{c}0.032 \\
(0.005)^{ \pm \star *}\end{array}$ & $\begin{array}{c}0.032 \\
(0.008)^{\star \star *}\end{array}$ & $\begin{array}{c}0.032 \\
(0.004)^{\star * *}\end{array}$ & $\begin{array}{c}0.032 \\
(0.008)^{\star * \star}\end{array}$ & $\begin{array}{c}0.030 \\
(0.005)^{\star \star \star \star}\end{array}$ \\
\hline $\begin{array}{l}\text { Completed } \\
\text { university education }\end{array}$ & $\begin{array}{c}0.015 \\
(0.007)^{\star}\end{array}$ & $\begin{array}{c}0.046 \\
(0.004)^{\star \star \star}\end{array}$ & $\begin{array}{c}0.022 \\
(0.007)^{\star \star}\end{array}$ & $\begin{array}{c}0.042 \\
(0.004)^{\star \star \star \star}\end{array}$ & $\begin{array}{c}0.021 \\
(0.007)^{\star *}\end{array}$ & $\begin{array}{c}0.041 \\
(0.003)^{\star \star \star}\end{array}$ & $\begin{array}{c}0.029 \\
(0.006)^{* * *}\end{array}$ & $\begin{array}{c}0.041 \\
(0.003)^{\star \star *}\end{array}$ \\
\hline Age 15 to 19 & $\begin{array}{c}-0.047 \\
(0.014)^{* *}\end{array}$ & $\begin{array}{c}-0.037 \\
(0.012)^{* *}\end{array}$ & $\begin{array}{c}-0.024 \\
(0.011)^{*}\end{array}$ & $\begin{array}{c}-0.026 \\
(0.010)^{* *}\end{array}$ & $\begin{array}{c}-0.030 \\
(0.011)^{\star \star}\end{array}$ & $\begin{array}{c}-0.023 \\
(0.009)^{*}\end{array}$ & $\begin{array}{c}-0.027 \\
(0.011)^{\star}\end{array}$ & $\begin{array}{c}-0.020 \\
(0.009)^{\star}\end{array}$ \\
\hline Age 25 to 34 & $\begin{array}{c}0.064 \\
(0.005)^{\star \star \star}\end{array}$ & $\begin{array}{c}-0.044 \\
(0.010)^{\star \star \star}\end{array}$ & $\begin{array}{c}0.065 \\
(0.005)^{\star \star *}\end{array}$ & $\begin{array}{c}-0.039 \\
(0.009)^{\star \star *}\end{array}$ & $\begin{array}{c}0.062 \\
(0.005)^{\star \star \star \star}\end{array}$ & $\begin{array}{c}-0.031 \\
(0.008)^{\star \star *}\end{array}$ & $\begin{array}{c}0.059 \\
(0.005)^{\star \star \star}\end{array}$ & $\begin{array}{c}-0.030 \\
(0.008)^{\star \star *}\end{array}$ \\
\hline Age 35 to 44 & $\begin{array}{c}0.030 \\
(0.006)^{\star \star \star}\end{array}$ & $\begin{array}{c}0.042 \\
(0.005)^{\star * *}\end{array}$ & $\begin{array}{c}0.027 \\
(0.006)^{\star \star \star}\end{array}$ & $\begin{array}{c}0.055 \\
(0.006)^{* * *}\end{array}$ & $\begin{array}{c}0.023 \\
(0.007)^{* *}\end{array}$ & $\begin{array}{c}0.065 \\
(0.005)^{\star \star \star}\end{array}$ & $\begin{array}{c}0.031 \\
(0.006)^{\star \star}\end{array}$ & $\begin{array}{c}0.068 \\
(0.006)^{\star \star \star}\end{array}$ \\
\hline Age 45 to 54 & $\begin{array}{c}0.039 \\
(0.006)^{\star * *}\end{array}$ & $\begin{array}{c}0.004 \\
(0.008)\end{array}$ & $\begin{array}{c}0.009 \\
(0.009)\end{array}$ & $\begin{array}{c}0.039 \\
(0.007)^{\star \star \star}\end{array}$ & $\begin{array}{c}0.005 \\
(0.009)\end{array}$ & $\begin{array}{c}0.045 \\
(0.007)^{\star * *}\end{array}$ & $\begin{array}{l}0.0006 \\
(0.009)\end{array}$ & $\begin{array}{c}0.049 \\
(0.007)^{*}\end{array}$ \\
\hline Age 55 to 64 & $\begin{array}{c}0.062 \\
(0.003)^{\star \star \star}\end{array}$ & $\begin{array}{c}0.020 \\
(0.008)^{\star}\end{array}$ & $\begin{array}{c}0.052 \\
(0.004)^{\star \star \star}\end{array}$ & $\begin{array}{c}0.045 \\
(0.005)^{\star \star \star}\end{array}$ & $\begin{array}{c}0.052 \\
(0.005)^{\star \star \star}\end{array}$ & $\begin{array}{c}0.050 \\
(0.004)^{* * \star}\end{array}$ & $\begin{array}{c}0.054 \\
(0.004)^{\star \star \star}\end{array}$ & $\begin{array}{c}0.053 \\
(0.004)^{\star * *}\end{array}$ \\
\hline Access/use technology & $\begin{array}{c}-0.018 \\
(0.005)^{\star *}\end{array}$ & $\begin{array}{c}-0.019 \\
(0.004)^{\star \star \star *}\end{array}$ & $\begin{array}{c}-0.030 \\
(0.005)^{* *}\end{array}$ & $\begin{array}{c}-0.020 \\
(0.003)^{\star * * *}\end{array}$ & $\begin{array}{c}-0.030 \\
(0.005)^{\star \star \star}\end{array}$ & $\begin{array}{c}-0.015 \\
(0.003)^{\star \star \star}\end{array}$ & $\begin{array}{c}-0.030 \\
(0.005)^{\star \star \star}\end{array}$ & $\begin{array}{c}-0.015 \\
(0.003)^{\star \star \star}\end{array}$ \\
\hline Chronic health condition & & & $\begin{array}{c}-0.020 \\
(0.005)^{\star * *}\end{array}$ & $\begin{array}{c}-0.032 \\
(0.004)^{\star * *}\end{array}$ & $\begin{array}{c}-0.023 \\
(0.005)^{\star \star \star}\end{array}$ & $\begin{array}{c}-0.027 \\
(0.004)^{*}\end{array}$ & $\begin{array}{c}-0.033 \\
(0.004)^{* * *}\end{array}$ & $\begin{array}{c}-0.028 \\
(0.004)^{\star \star \star}\end{array}$ \\
\hline Health activity limitation & & & $\begin{array}{c}0.016 \\
(0.005)^{\star *}\end{array}$ & $\begin{array}{c}-0.009 \\
(0.004)^{\star}\end{array}$ & $\begin{array}{c}0.017 \\
(0.005)^{\star * \star}\end{array}$ & $\begin{array}{c}-0.01 \\
(0.004)^{\star \star \star}\end{array}$ & 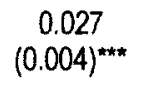 & $\begin{array}{c}-0.016 \\
(0.004)^{\star * * *}\end{array}$ \\
\hline Rural place of residence & & & $\begin{array}{c}0.020 \\
(0.004)^{\star \star \star \star}\end{array}$ & $\begin{array}{c}-0.029 \\
(0.005)^{\star \star \star \star}\end{array}$ & $\begin{array}{c}0.023 \\
(0.004)^{\star \star \star}\end{array}$ & $\begin{array}{c}-0.028 \\
(0.005)^{\star * *}\end{array}$ & $\begin{array}{c}0.018 \\
(0.004)^{\star \star \star \star}\end{array}$ & $\begin{array}{c}-0.032 \\
(0.005)^{\star \star \star}\end{array}$ \\
\hline Has someone to confide in & & & & & $\begin{array}{c}-0.045 \\
(0.004)^{\star * *}\end{array}$ & $\begin{array}{c}0.011 \\
(0.013)\end{array}$ & $\begin{array}{c}-0.047 \\
(0.004)^{\star * \star *}\end{array}$ & $\begin{array}{c}0.012 \\
(0.013)\end{array}$ \\
\hline Band member & & & & & & & $\begin{array}{c}0.006 \\
(0.006)\end{array}$ & $\begin{array}{c}-0.012 \\
(0.004)^{\star \star}\end{array}$ \\
\hline $\begin{array}{l}\text { Speaks Aboriginal } \\
\text { languages }\end{array}$ & & & & & & & $\begin{array}{c}-0.116 \\
(0.012)^{\star \star \star}\end{array}$ & $\begin{array}{c}0.020 \\
(0.005)^{\star \star *}\end{array}$ \\
\hline $\begin{array}{l}\text { Values Aboriginal } \\
\text { languages }\end{array}$ & & & & & & & $\begin{array}{l}-0.007 \\
(0.005)\end{array}$ & $\begin{array}{c}0.012 \\
(0.004)^{\star \star}\end{array}$ \\
\hline $\begin{array}{l}\text { Traditional pursuits } \\
\text { for commerce }\end{array}$ & & & & & & & $\begin{array}{c}-0.002 \\
(0.010)\end{array}$ & $\begin{array}{c}-0.048 \\
(0.019)^{\star}\end{array}$ \\
\hline $\begin{array}{l}\text { Traditional pursuits } \\
\text { for food }\end{array}$ & & & & & & & $\begin{array}{c}-0.053 \\
(0.007)^{\star \star \star}\end{array}$ & $\begin{array}{c}-0.030 \\
(0.006)^{* * *}\end{array}$ \\
\hline $\begin{array}{l}\text { Traditional pursuits } \\
\text { for pleasure }\end{array}$ & & & & & & & $\begin{array}{c}0.043 \\
(0.005)^{\star \star \star \star}\end{array}$ & $\begin{array}{c}0.020 \\
(0.004)^{* k *}\end{array}$ \\
\hline Number of observations & 840 & 850 & 830 & 840 & 830 & 840 & 820 & 830 \\
\hline
\end{tabular}

Source: Statistics Canada, 2006 Aboriginal Peoples Survey, Adult Core analytical file. Bootstrapped (1000 replications) standard errors in parentheses. Statistical significance ${ }^{* * *} p<.001 ;{ }^{* *} p<.01 ;{ }^{*} p<.05$.

Note: Estimates for all other variables are presented in Appendix C4. 
The results presented in Table 5 for the non-registered NAI group differ markedly from those just presented for the registered NAI group. As displayed in the first column of Table 5, postsecondary educational attainment at the university level (both incomplete and completed) has a positive effect on the probability of employment for non-registered NAI men. At the level of completed non-university education, however, non-registered NAI men are less likely to be employed, relative to their counterparts with only a high school diploma as their highest level of education attained. The associations between these three levels of postsecondary education and the probability of employment for nonregistered NAI women, as displayed in the second column of Table 5, are all positive and statistically significant. The effect of having some non-university education, however, is positive but not significantly different from zero in the employment model of nonregistered NAI women.

Access to and the use of information technology appears negatively associated with the probability of employment, both for non-registered NAI men and for nonregistered NAI women. Likewise, the association between having one or more chronic health conditions and the probability of employment is negative, both for non-registered NAI men and for non-registered NAI women. With regards to second measure of health condition (activity limitation) the association remains negative for non-registered NAI women but for non-registered NAI men the association is positive. The measure relating to social capital (social networks) appears to be negatively associated with the probability of employment for non-registered NAI men. The same measure appears insignificant in the employment model of non-registered NAI women. 
As in the previous models for the other NAI groups, the traditional pursuit, if done for food and medicinal purposes, are negatively associated with the probability of employment, both for non-registered NAI men and for non-registered NAI women. This association becomes positive for both genders if the traditional pursuits are done for pleasure. No relationship seems to exist between the engagement in traditional pursuits for commercial purposes and the probability of employment for non-registered NAI men; for non-registered NAI women, the relationship is negative and statistically significant.

Finally, the effects of both measures relating to Aboriginal languages are positive for non-registered NAI women. For non-registered NAI men, on the other hand, the effect on the first measure is large and negative - as shown in Table 5, the probability of non-registered NAI men being employed drops by about 12 percentage points when the indicator relating to fluency in an Aboriginal language changes from zero to one, holding all else constant. There seems to be no relationship between Band membership and the probability of employment for non-registered NAI men. For non-registered NAI women, however, Band membership exerts significantly negative effect on the probability of employment.

As pointed out earlier in this essay, there are reasons to suspect that the estimates presented above may not be accurately representing the outcomes of different group members, particularly in regard to the effect of educational attainment as classified using the standard six categories. The following two sections present the results for the other two Aboriginal identity groups, Métis and Inuit, using the standard classification of the educational attainment; robustness checks will be performed using alternative measures of educational attainment for each group. 


\subsection{Multivariate Probit Regression Results for Métis Population}

Selected average marginal effects from probit estimates for Métis population are presented in Table 6, while Appendix C5 contains the results from the full model. As above, the discussion here centers on the key characteristics from each block of variables, with the level of educational attainment, social networks and traditional pursuits being the primary variables of interest. Two important points related to the regression results for postsecondary educational attainment of Métis population are worth highlighting here. In short, the two points suggest that, for Métis people, only completed university education makes a difference.

First, as Table 6 makes apparent, the association between the level of postsecondary schooling and the probability of employment for Métis men seems to follow the same direction as that of the urban NAI men presented in the preceding section. However, with the exception of the level of completed university education, this association in the regression models of Métis men does not appear to be important. The negative statistically significant association between the level of incomplete nonuniversity education and the probability of employment for Métis men (Model 1) does not exist in the columns of the subsequent models when the other observed human capital characteristics (Model 2), social capital characteristics (Model 3) and cultural factors (Full Model) are taken into account. 
Table 6: Selected estimates from probit employment function for Métis, APS 2006

\begin{tabular}{|c|c|c|c|c|c|c|c|c|}
\hline \multirow{3}{*}{$\begin{array}{l}\text { Selected variables in the } \\
\text { probit equations }\end{array}$} & \multicolumn{8}{|c|}{ Average Marginal Effects } \\
\hline & \multicolumn{2}{|c|}{ Model 1} & \multicolumn{2}{|c|}{ Model 2} & \multicolumn{2}{|c|}{ Model 3} & \multicolumn{2}{|c|}{ Full Model } \\
\hline & Men & Women & Men & Women & Men & Women & Men & Women \\
\hline $\begin{array}{l}\text { Less than } \\
\text { high school education }\end{array}$ & $\begin{array}{c}-0.027 \\
(0.005)^{\star * *}\end{array}$ & $\begin{array}{c}-0.045 \\
(0.006)^{* k \star}\end{array}$ & $\begin{array}{c}-0.010 \\
(0.004)^{\star}\end{array}$ & $\begin{array}{c}-0.036 \\
(0.005)^{* \star * *}\end{array}$ & $\begin{array}{l}-0.007 \\
(0.004)\end{array}$ & $\begin{array}{c}-0.036 \\
(0.005)^{\star \star \star}\end{array}$ & $\begin{array}{l}-0.004 \\
(0.004)\end{array}$ & $\begin{array}{c}-0.027 \\
(0.004)^{* * *}\end{array}$ \\
\hline $\begin{array}{l}\text { Some } \\
\text { non-university education }\end{array}$ & $\begin{array}{c}-0.025 \\
(0.007)^{* \star}\end{array}$ & $\begin{array}{r}-0.005 \\
(0.004)\end{array}$ & $\begin{array}{l}-0.007 \\
(0.006)\end{array}$ & $\begin{array}{l}-0005 \\
(0.004)\end{array}$ & $\begin{array}{l}-0.007 \\
(0.006)\end{array}$ & $\begin{array}{l}0.0003 \\
(0.004)\end{array}$ & $\begin{array}{l}-0.006 \\
(0.006)\end{array}$ & $\begin{array}{c}0.002 \\
(0.004)\end{array}$ \\
\hline $\begin{array}{l}\text { Completed, } \\
\text { non-university education }\end{array}$ & $\begin{array}{l}-0.0009 \\
(0.004)\end{array}$ & $\begin{array}{c}-0.011 \\
(0.004)^{\star *}\end{array}$ & $\begin{array}{c}0.008 \\
(0.004)^{*}\end{array}$ & $\begin{array}{c}-0.002 \\
(0.003)\end{array}$ & $\begin{array}{c}0.007 \\
(0.004)\end{array}$ & $\begin{array}{l}-0.0008 \\
(0.003)\end{array}$ & $\begin{array}{c}0.013 \\
(0.003)^{\star \star *}\end{array}$ & $\begin{array}{l}-0.001 \\
(0.003)\end{array}$ \\
\hline $\begin{array}{l}\text { Some } \\
\text { university education }\end{array}$ & $\begin{array}{l}-0.004 \\
(0.009)\end{array}$ & $\begin{array}{c}-0.031 \\
(0.010)^{\star *}\end{array}$ & $\begin{array}{c}0.019 \\
(0.006)^{\star \star}\end{array}$ & $\begin{array}{c}0.006 \\
(0.004)\end{array}$ & $\begin{array}{c}0.017 \\
(0.006)^{\star \star}\end{array}$ & $\begin{array}{c}0.007 \\
(0.004)\end{array}$ & $\begin{array}{c}0.017 \\
(0.006)^{\star \star}\end{array}$ & $\begin{array}{c}0.006 \\
(0.004)\end{array}$ \\
\hline $\begin{array}{l}\text { Completed } \\
\text { university education }\end{array}$ & $\begin{array}{c}0.023 \\
(0.006)^{* * *}\end{array}$ & $\begin{array}{c}0.032 \\
(0.003)^{\star * *}\end{array}$ & $\begin{array}{c}0.017 \\
(0.006)^{\star *}\end{array}$ & $\begin{array}{c}0.027 \\
(0.003)^{\star * *}\end{array}$ & $\begin{array}{c}0.015 \\
(0.006)^{*}\end{array}$ & $\begin{array}{c}0.028 \\
(0.003)^{\star \star \star}\end{array}$ & $\begin{array}{c}0.013 \\
(0.006)^{*}\end{array}$ & $\begin{array}{c}0.027 \\
(0.003)^{\star \star \star}\end{array}$ \\
\hline Age 15 to 19 & $\begin{array}{c}-0.006 \\
(0.006)\end{array}$ & $\begin{array}{c}-0.099 \\
(0.011)^{\star \star \star \star}\end{array}$ & $\begin{array}{c}0.007 \\
(0.005)\end{array}$ & $\begin{array}{c}-0.065 \\
(0.009)^{* * *}\end{array}$ & $\begin{array}{l}0.0051 \\
(0.005)\end{array}$ & $\begin{array}{c}-0.063 \\
(0.009)^{\star \star \star}\end{array}$ & $\begin{array}{c}0.005 \\
(0.005)\end{array}$ & $\begin{array}{c}-0.056 \\
(0.008)^{\star \star *}\end{array}$ \\
\hline Age 25 to 34 & $\begin{array}{c}0.063 \\
(0.004)^{\star \star \star}\end{array}$ & $\begin{array}{c}-0.002 \\
(0.004)\end{array}$ & $\begin{array}{c}0.034 \\
(0.003)^{\star \star * \star}\end{array}$ & $\begin{array}{c}0.007 \\
(0.004)\end{array}$ & $\begin{array}{c}0.035 \\
(0.003)^{\star * * *}\end{array}$ & $\begin{array}{c}0.008 \\
(0.004)\end{array}$ & $\begin{array}{c}0.035 \\
(0.003)^{\star \star \star}\end{array}$ & $\begin{array}{c}0.006 \\
(0.004)\end{array}$ \\
\hline Age 35 to 44 & $\begin{array}{c}0.047 \\
(0.004)^{* * *}\end{array}$ & $\begin{array}{c}-0.003 \\
(0.004)\end{array}$ & $\begin{array}{c}0.011 \\
(0.005)\end{array}$ & $\begin{array}{c}0.006 \\
(0.004)\end{array}$ & $\begin{array}{c}0.012 \\
(0.005)^{*}\end{array}$ & $\begin{array}{c}0.007 \\
(0.005)\end{array}$ & $\begin{array}{c}0.014 \\
(0.005)^{\star *}\end{array}$ & $\begin{array}{c}0.002 \\
(0.004)^{\star \star \star}\end{array}$ \\
\hline Age 45 to 54 & $\begin{array}{c}0.051 \\
(0.004)^{\star \star \star}\end{array}$ & $\begin{array}{c}0.011 \\
(0.003)^{\star *}\end{array}$ & $\begin{array}{c}0.016 \\
(0.005)^{\star \star}\end{array}$ & $\begin{array}{c}0.007 \\
(0.004)\end{array}$ & $\begin{array}{c}0.014 \\
(0.005)^{* *}\end{array}$ & $\begin{array}{c}0.009 \\
(0.004)^{\star}\end{array}$ & $\begin{array}{c}0.014 \\
(0.005)^{\star \star}\end{array}$ & $\begin{array}{c}0.016 \\
(0.004)^{\star * *}\end{array}$ \\
\hline Age 55 to 64 & $\begin{array}{c}0.065 \\
(0.003)^{\star * *}\end{array}$ & $\begin{array}{l}-0.0006 \\
(0.005)\end{array}$ & $\begin{array}{c}0.036 \\
(0.004)^{* * *}\end{array}$ & $\begin{array}{c}-0.021 \\
(0.007)^{\star \star}\end{array}$ & $\begin{array}{c}0.035 \\
(0.004)^{\star \star \star}\end{array}$ & $\begin{array}{c}-0.019 \\
(0.007)^{\star}\end{array}$ & $\begin{array}{c}0.034 \\
(0.004)^{\star \star \star}\end{array}$ & $\begin{array}{r}-0.012 \\
(0.007)\end{array}$ \\
\hline Access/use technology & $\begin{array}{c}0.046 \\
(0.005)^{\star \star \star}\end{array}$ & $\begin{array}{c}0.010 \\
(0.003)^{* *}\end{array}$ & $\begin{array}{c}0.049 \\
(0.004)^{\star \star \star}\end{array}$ & $\begin{array}{c}0.009 \\
(0.003)^{\star \star}\end{array}$ & $\begin{array}{c}0.044 \\
(0.004)^{\star \star \star \star}\end{array}$ & $\begin{array}{c}0.010 \\
(0.003)^{\star \star}\end{array}$ & $\begin{array}{c}0.046 \\
(0.004)^{k \star \star}\end{array}$ & $\begin{array}{c}0.011 \\
(0.003)^{\star \star \star}\end{array}$ \\
\hline Chronic health condition & & & $\begin{array}{c}0.002 \\
(0.003)\end{array}$ & $\begin{array}{c}-0.016 \\
(0.002)^{* * \star}\end{array}$ & $\begin{array}{l}0.0006 \\
(0.003)\end{array}$ & $\begin{array}{c}-0.016 \\
(0.002)^{* \star}\end{array}$ & $\begin{array}{c}0.003 \\
(0.003)\end{array}$ & $\begin{array}{c}-0.020 \\
(0.002)^{* * *}\end{array}$ \\
\hline Health activity limitation & & & $\begin{array}{c}-0.005 \\
(0.003)\end{array}$ & $\begin{array}{c}0.005 \\
(0.002)\end{array}$ & $\begin{array}{c}-0.003 \\
(0.003)\end{array}$ & $\begin{array}{c}0.004 \\
(0.002)\end{array}$ & $\begin{array}{c}0.002 \\
(0.003)\end{array}$ & $\begin{array}{c}0.005 \\
(0.002)^{*}\end{array}$ \\
\hline Rural place of residence & & & $\begin{array}{c}-0.021 \\
(0.003)^{\star \star \star}\end{array}$ & $\begin{array}{c}-0.004 \\
(0.002)\end{array}$ & $\begin{array}{c}-0.021 \\
(0.003)^{\star \star *}\end{array}$ & $\begin{array}{c}-0.003 \\
(0.002)\end{array}$ & $\begin{array}{c}-0.014 \\
(0.003)^{\star k k}\end{array}$ & $\begin{array}{c}-0.004 \\
(0.002)\end{array}$ \\
\hline Has someone to confide in & & & & & $\begin{array}{c}0.031 \\
(0.009)^{* *}\end{array}$ & $\begin{array}{c}-0.004 \\
(0.005)\end{array}$ & $\begin{array}{c}0.042 \\
(0.01)^{* * * \star}\end{array}$ & $\begin{array}{c}-0.004 \\
(0.005)\end{array}$ \\
\hline $\begin{array}{l}\text { Volunteering club } \\
\text { membership }\end{array}$ & & & & & $\begin{array}{c}0.022 \\
(0.003)^{\star \star \star}\end{array}$ & $\begin{array}{c}-0.009 \\
(0.003)^{\star \star}\end{array}$ & $\begin{array}{c}0.025 \\
(0.003)^{\star \star \star}\end{array}$ & $\begin{array}{c}-0.009 \\
(0.003)^{\star \star}\end{array}$ \\
\hline $\begin{array}{l}\text { Member in Métis } \\
\text { organizations }\end{array}$ & & & & & & & $\begin{array}{r}-0.002 \\
(0.004)\end{array}$ & $\begin{array}{l}-0.003 \\
(0.003)\end{array}$ \\
\hline Spouse is Aboriginal & & & & & & & $\begin{array}{c}-0.034 \\
(0.005)^{\star \star \star}\end{array}$ & $\begin{array}{c}-0.044 \\
(0.004)^{\prime}\end{array}$ \\
\hline $\begin{array}{l}\text { Speaks Aboriginal } \\
\text { languages }\end{array}$ & & & & & & & $\begin{array}{c}0.016 \\
(0.004)^{\star * \star}\end{array}$ & $\begin{array}{c}0.003 \\
(0.004)\end{array}$ \\
\hline $\begin{array}{l}\text { Values Aboriginal } \\
\text { languages }\end{array}$ & & & & & & & $\begin{array}{c}-0.019 \\
(0.003)^{\star \star \star}\end{array}$ & $\begin{array}{c}-0.011 \\
(0.002)^{\star \star \star}\end{array}$ \\
\hline $\begin{array}{l}\text { Traditional pursuits } \\
\text { for commerce }\end{array}$ & & & & & & & $\begin{array}{c}-0.033 \\
(0.009)^{\star \star \star}\end{array}$ & $\begin{array}{c}-0.082 \\
(0.02)^{\star \star \star}\end{array}$ \\
\hline $\begin{array}{l}\text { Traditional pursuits } \\
\text { for food }\end{array}$ & & & & & & & $\begin{array}{c}0.014 \\
(0.004)^{* * *}\end{array}$ & $\begin{array}{c}-0.008 \\
(0.003)^{\star \star}\end{array}$ \\
\hline $\begin{array}{l}\text { Traditional pursuits } \\
\text { for pleasure }\end{array}$ & & & & & & & $\begin{array}{c}-0.028 \\
(0.003)^{*}\end{array}$ & $\begin{array}{c}0.024 \\
(0.003)^{* * *} \\
\end{array}$ \\
\hline Number of observations & 2450 & 2500 & 2220 & 2310 & 2200 & 2300 & 2150 & 2250 \\
\hline
\end{tabular}

Source: Statistics Canada, 2006 Aboriginal Peoples Survey, Métis Supplement analytical file. Bootstrapped (1000 replications) standard errors in parentheses. Statistical significance ${ }^{* * *} p<.001 ;{ }^{* *} p<.01 ;{ }^{*} p<.05$.

Note: Estimates for all other variables are presented in Appendix C5. 
Furthermore, in the expanded human capital model for Métis men (Model 2) estimates for two levels of educational attainment (completed non-university education and incomplete university education) which in the basic human capital model (Model 1) were not statistically different from zero become not only statistically significant but the sign of their association shifts from negative to positive, as shown in the third column of Table 6. Also, while the positive association and the statistical significance is maintained in the following two models for the level of incomplete university education, for the level of completed non-university education the association disappears when social capital variables are added in (Model 3) and reappears again when all, including the cultural factors (Full Model) are considered. Finally, and perhaps most puzzlingly, as shown in Table 6, the estimated effect for the less than high school diploma is not significantly different from zero in the last two models (Model 3 and Full Model).

The second important point relating to the regression results for postsecondary education of Métis people that needs to be highlighted here is that, the association between postsecondary schooling and the probability of employment for Métis women appears in sharp contrast to that of urban NAI women - postsecondary education below completed university level appears either unassociated or negatively associated with the probability of employment for Métis women. The associations, however, exist only for two levels of postsecondary education (completed non-university and incomplete university education levels) and are present only in the first block of human capital characteristics (Model 1). As shown in Table 6, the sign on one of these associations (incomplete university education) changes from negative to positive while the statistical significance disappears for both variables in the succeeding models. 
The access to and the use of information technology is positively associated with the probability of employment, both for Métis men and for Métis women, but appears more important for men. No relation exists between either of the two variables measuring the effects of health condition on the probability of employment for Métis men. For Métis women, however, having one or more chronic health conditions exerts a negative effect on the probability of employment. Oddly, health condition in the form of activity limitation appears to increase the probability of employment for Métis women; this effect, however, is very small and it appears only in the Full Model when all observed characteristics are taken into account.

As pointed out in Section 3.3, Métis employment models include an additional variable measuring social capital (weak ties) in addition to the one (strong ties) included in the employment models of NAI. The estimated results from Métis employment models suggest that the first measure of social capital (social networks) has a positive effect on the probability of employment, but only in the employment models of Métis men. The second measure (volunteering in the community) has a positive effect on the probability of employment for Métis men but a negative effect on the probability of employment for Métis women. The magnitude of this negative effect in the employment models of Métis women, however, is rather small.

As shown in Table 6, an association exists between traditional pursuits and the probability of employment for Métis people but the effect differs across gender. In the employment models of Métis men, the association is positive if the traditional pursuits are done for food and medicinal purposes while in the employment models of Métis women, this effect is negative. Conversely, in the employment models of Métis men, the 
association if negative if traditional pursuits are done for pleasure while in the employment models of Métis women this effect is positive. If the traditional pursuits are done for commercial purposes, however, the effect is negative for both genders.

Finally, while being fluent in an Aboriginal language has a significant positive effect on the probability of employment for Métis men, holding a view that it is important to learn Aboriginal languages has a negative effect on the employment probability, both for Métis men and for Métis women. Also, having a non-Aboriginal spouse seems to yield an advantage for Métis people, a finding that corresponds to that in Kuhn and Sweetman (2002). As shown in the last two columns of Table 6, the probability of employment both for Métis men and for Métis women declines when the indicator for spouse changes from non-Aboriginal to Aboriginal spouse. 


\subsection{Multivariate Probit Regression Results for Inuit Population}

The estimation results for selected variables from probit model of employment for the Inuit population are presentation in Table 7. As in the previous sections for other Aboriginal identity groups, the following discussion focuses primarily on the assessment of changes in the probability of employment associated with a change in the level of educational attainment. Incorporated into this discussion are also the effects of some other factors from human capital theory as well as some social capital and cultural characteristics. The estimation results for all variables are presented in Appendix C6.

Contrary to the insights derived from the bivariate analysis presented earlier in this section, as displayed in the first column of Table 7, the multivariate estimation results for Inuit men suggest an overall positive association between postsecondary schooling and the probability of employment. This finding, in sharp contrast with the findings in previous literature (Drost, 1994; Kuhn and Sweetman, 2002), remains stable in the subsequent models (Model 2, Model 3, and Full Model) which take other observed characteristics into account. The magnitude of the effect is also noticeably larger from the magnitudes of the related variable discussed in the previous two section pertaining to NAI and Métis groups - as estimated in Model 1, the probability of employment for Inuit men increases by 11.4 percentage points when the indicator for educational attainment changes from completed high school diploma to completed postsecondary education, as measured at the combined university and non-university level. 
Table 7: Selected estimates from probit employment function for Inuit, APS 2006

\begin{tabular}{|c|c|c|c|c|c|c|c|c|}
\hline \multirow{3}{*}{$\begin{array}{l}\text { Selected variables in the } \\
\text { probit equations }\end{array}$} & \multicolumn{8}{|c|}{ Average Marginal Effects } \\
\hline & \multicolumn{2}{|c|}{ Model 1} & \multicolumn{2}{|c|}{ Model 2} & \multicolumn{2}{|c|}{ Model 3} & \multicolumn{2}{|c|}{ Full Model } \\
\hline & Men & Women & Men & Women & Men & Women & Men & Women \\
\hline $\begin{array}{l}\text { Less than } \\
\text { high school education }\end{array}$ & $\begin{array}{c}0.074 \\
(0.013)^{\star \star \star *}\end{array}$ & $\begin{array}{l}-0.013 \\
(0.009)\end{array}$ & $\begin{array}{c}0.100 \\
(0.013)^{m+m}\end{array}$ & $\begin{array}{r}-0.005 \\
(0.009)\end{array}$ & $\begin{array}{c}0.068 \\
(0.012)^{* * *}\end{array}$ & $\begin{array}{c}-0.019 \\
(0.009)^{*}\end{array}$ & $\begin{array}{c}0.049 \\
(0.012)^{\star \star \star *}\end{array}$ & $\begin{array}{l}-0.005 \\
(0.009)\end{array}$ \\
\hline $\begin{array}{l}\text { Some } \\
\text { non-university education }\end{array}$ & $\begin{array}{c}0.088 \\
(0.009)^{\star \star * \star}\end{array}$ & $\begin{array}{c}-0.044 \\
(0.016)^{\star *}\end{array}$ & $\begin{array}{c}0.090 \\
(0.009)^{* * * *}\end{array}$ & $\begin{array}{c}-0.028 \\
(0.014)^{*}\end{array}$ & $\begin{array}{c}0.061 \\
(0.010)^{* * *}\end{array}$ & $\begin{array}{c}-0.053 \\
(0.015)^{\star *}\end{array}$ & $\begin{array}{c}0.043 \\
(0.010)^{\star \star \star *}\end{array}$ & $\begin{array}{c}-0.036 \\
(0.014)^{*}\end{array}$ \\
\hline $\begin{array}{l}\text { Completed } \\
\text { non-university or university }\end{array}$ & $\begin{array}{c}0.114 \\
(0.011)^{\text {*+*k}}\end{array}$ & $\begin{array}{c}-0.046 \\
(0.011)^{\star \star \star}\end{array}$ & $\begin{array}{c}0.119 \\
(0.010)^{\star \star \star \star}\end{array}$ & $\begin{array}{r}-0.013 \\
(0.009)\end{array}$ & $\begin{array}{c}0.092 \\
(0.010)^{+\ldots+k}\end{array}$ & $\begin{array}{l}-0.017 \\
(0.009)\end{array}$ & $\begin{array}{c}0.081 \\
(0.010)^{\star \star \star}\end{array}$ & $\begin{array}{c}-0.019 \\
(0.008)^{\star}\end{array}$ \\
\hline Age 15 to 19 & $\begin{array}{c}-0.044 \\
(0.016)^{\star \star}\end{array}$ & $\begin{array}{c}-0.002 \\
(0.011)\end{array}$ & $\begin{array}{c}-0.037 \\
(0.014)^{* *}\end{array}$ & $\begin{array}{c}-0.038 \\
(0.015)^{*}\end{array}$ & $\begin{array}{c}-0.032 \\
(0.014)^{*}\end{array}$ & $\begin{array}{c}-0.049 \\
(0.016)^{* * *}\end{array}$ & $\begin{array}{c}-0.072 \\
(0.017)^{\ldots * *}\end{array}$ & $\begin{array}{c}-0.071 \\
(0.016)^{\star \star \star}\end{array}$ \\
\hline Age 25 to 34 & $\begin{array}{c}0.045 \\
(0.010)^{\star * *}\end{array}$ & $\begin{array}{c}0.114 \\
(0.008)^{\star \star \star}\end{array}$ & $\begin{array}{c}0.060 \\
(0.009)^{\star * \star}\end{array}$ & $\begin{array}{c}0.104 \\
(0.007)^{\star \star \star}\end{array}$ & $\begin{array}{c}0.0253 \\
(0.010)^{\star}\end{array}$ & $\begin{array}{c}0.097 \\
(0.007)^{\star \star \star}\end{array}$ & $\begin{array}{c}0.008 \\
(0.011)\end{array}$ & $\begin{array}{c}0.093 \\
(0.007)^{\star \star *}\end{array}$ \\
\hline Age 35 to 44 & $\begin{array}{r}-0.002 \\
(0.012)\end{array}$ & $\begin{array}{c}0.081 \\
(0.008)^{\star \star \star}\end{array}$ & $\begin{array}{c}0.008 \\
(0.012)\end{array}$ & $\begin{array}{c}0.047 \\
(0.010)^{\star \star \star}\end{array}$ & $\begin{array}{c}-0.052 \\
(0.014)^{\star \cdots \star \star}\end{array}$ & $\begin{array}{c}0.034 \\
(0.011)^{* *}\end{array}$ & $\begin{array}{c}-0.078 \\
(0.015)^{*+*}\end{array}$ & $\begin{array}{c}0.023 \\
(0.010)^{\star}\end{array}$ \\
\hline Age 45 to 54 & $\begin{array}{c}0.017 \\
(0.011)\end{array}$ & $\begin{array}{c}0.105 \\
(0.007)^{\star \star \star}\end{array}$ & $\begin{array}{c}0.017 \\
(0.013)\end{array}$ & $\begin{array}{c}0.079 \\
(0.008)^{\star \star \star}\end{array}$ & $\begin{array}{c}-0.056 \\
(0.017)^{\star *}\end{array}$ & $\begin{array}{c}0.068 \\
(0.009)^{\star * \star}\end{array}$ & $\begin{array}{c}-0.080 \\
(0.017)^{\star * \star *}\end{array}$ & $\begin{array}{c}0.079 \\
(0.008)^{* * *}\end{array}$ \\
\hline Age 55 to 64 & $\begin{array}{c}0.087 \\
(0.009)^{\star \star \star}\end{array}$ & $\begin{array}{c}0.065 \\
(0.008)^{\star \star *}\end{array}$ & $\begin{array}{c}0.082 \\
(0.009)^{\star * * *}\end{array}$ & $\begin{array}{c}0.043 \\
(0.011)^{\star \star \star *}\end{array}$ & $\begin{array}{c}0.031 \\
(0.014)^{*}\end{array}$ & $\begin{array}{c}0.027 \\
(0.013)^{*}\end{array}$ & $\begin{array}{c}0.007 \\
(0.016)\end{array}$ & $\begin{array}{c}0.040 \\
(0.010)^{* * *}\end{array}$ \\
\hline Access/use technology & $\begin{array}{c}0.015 \\
(0.007)^{\star}\end{array}$ & $\begin{array}{c}0.0690 \\
(0.006)^{* * *}\end{array}$ & $\begin{array}{c}0.028 \\
(0.007)^{\star \star * *}\end{array}$ & $\begin{array}{c}0.062 \\
(0.006)^{\star \star \star}\end{array}$ & $\begin{array}{c}0.040 \\
(0.006)^{\star \star * \hbar}\end{array}$ & $\begin{array}{c}0.060 \\
(0.006)^{* * *}\end{array}$ & $\begin{array}{c}0.044 \\
(0.006)^{\star * \star}\end{array}$ & $\begin{array}{c}0.057 \\
(0.005)^{\star \star \star}\end{array}$ \\
\hline Chronic health condition & & & $\begin{array}{c}-0.010 \\
(0.008)\end{array}$ & $\begin{array}{c}-0.015 \\
(0.005)^{\star \star}\end{array}$ & $\begin{array}{r}-0.0005 \\
(0.007)\end{array}$ & $\begin{array}{c}-0.011 \\
(0.005)^{\star}\end{array}$ & $\begin{array}{c}0.004 \\
(0.007)\end{array}$ & $\begin{array}{c}-0.020 \\
(0.005)^{\star \star}\end{array}$ \\
\hline Health activity limitation & & & $\begin{array}{c}0.024 \\
(0.008)\end{array}$ & $\begin{array}{c}-0.053 \\
(0.007)^{* * *}\end{array}$ & $\begin{array}{l}-0.0002 \\
(0.007)\end{array}$ & $\begin{array}{c}-0.042 \\
(0.007)^{\star \star \star *}\end{array}$ & $\begin{array}{r}-0.002 \\
(0.007)\end{array}$ & $\begin{array}{c}-0.052 \\
(0.007)^{\star * *}\end{array}$ \\
\hline Has someone to confide in & & & & & $\begin{array}{c}-0.046 \\
(0.006)^{\star * \star}\end{array}$ & $\begin{array}{c}0.048 \\
(0.006)^{k * * *}\end{array}$ & $\begin{array}{c}-0.048 \\
(0.006)^{\star * *}\end{array}$ & $\begin{array}{c}0.047 \\
(0.006)^{\star \star \star}\end{array}$ \\
\hline $\begin{array}{l}\text { Volunteering club } \\
\text { membership }\end{array}$ & & & & & $\begin{array}{c}-0.036 \\
(0.006)^{\star \star \star *}\end{array}$ & $\begin{array}{c}0.004 \\
(0.006)\end{array}$ & $\begin{array}{c}-0.037 \\
(0.006)^{\star * *}\end{array}$ & $\begin{array}{c}0.006 \\
(0.006)\end{array}$ \\
\hline Voting, municipal & & & & & $\begin{array}{c}0.032 \\
(0.010)^{\star * *}\end{array}$ & $\begin{array}{c}0.127 \\
(0.011)^{\star \star \star}\end{array}$ & $\begin{array}{c}0.025 \\
(0.009)^{\star \star}\end{array}$ & $\begin{array}{c}0.103 \\
(0.010)^{\star * *}\end{array}$ \\
\hline Voting, provincial & & & & & $\begin{array}{c}0.066 \\
(0.011)^{\star \star \star}\end{array}$ & $\begin{array}{c}-0.084 \\
(0.006)^{\star \star \star \star}\end{array}$ & $\begin{array}{c}0.083 \\
(0.011)^{\star k+k}\end{array}$ & $\begin{array}{c}-0.076 \\
(0.007)^{\star \star \star *}\end{array}$ \\
\hline $\begin{array}{l}\text { Member in Inuit } \\
\text { organizations }\end{array}$ & & & & & & & $\begin{array}{c}-0.045 \\
(0.007)^{* * *}\end{array}$ & $\begin{array}{c}0.041 \\
(0.011)^{\star \star \star}\end{array}$ \\
\hline $\begin{array}{l}\text { Speaks Aboriginal } \\
\text { languages }\end{array}$ & & & & & & & $\begin{array}{c}0.070 \\
(0.015)^{\star \star \star}\end{array}$ & $\begin{array}{c}0.027 \\
(0.010)^{\star \star}\end{array}$ \\
\hline $\begin{array}{l}\text { Values Aboriginal } \\
\text { languages }\end{array}$ & & & & & & & $\begin{array}{c}-0.011 \\
(0.015)\end{array}$ & $\begin{array}{c}0.065 \\
(0.023)^{\star \star}\end{array}$ \\
\hline $\begin{array}{l}\text { Traditional pursuits } \\
\text { for commerce }\end{array}$ & & & & & & & $\begin{array}{l}-0.008 \\
(0.008)\end{array}$ & $\begin{array}{c}-0.182 \\
(0.017)^{\star \star \star}\end{array}$ \\
\hline $\begin{array}{l}\text { Traditional pursuits } \\
\text { for food }\end{array}$ & & & & & & & $\begin{array}{c}0.013 \\
(0.015)\end{array}$ & $\begin{array}{c}0.037 \\
(0.012)^{\star \star}\end{array}$ \\
\hline $\begin{array}{l}\text { Traditional pursuits } \\
\text { for pleasure }\end{array}$ & & & & & & & $\begin{array}{r}-0.003 \\
(0.007)\end{array}$ & $\begin{array}{c}-0.031 \\
(0.007)^{\star * *}\end{array}$ \\
\hline Number of observations & 840 & 840 & 800 & 790 & 750 & 720 & 720 & 710 \\
\hline
\end{tabular}

Source: Statistics Canada, 2006 Aboriginal Peoples Survey, analytical files, Arctic File. Bootstrapped (1000 replications) standard errors in parentheses. Statistical significance ${ }^{* * *} p<.001 ;{ }^{* *} p<.01 ;{ }^{*} p<.05$.

Note: Estimates for all other variables are presented in Appendix C6. 
However, the interpretation of the effects of educational attainment variable on the probability of employment for Inuit men warrants caution. As shown in the first row of the same column in Table 7, the estimate for the level of less than high school education is also positive - suggesting that those with less than a high school diploma are more likely to be employed than those with a completed high school diploma. Clearly, this is not what human capital theory would suggest.

The estimated results for Inuit women are also peculiar in two ways. First, as illustrated in the first row of Table 7, although the association between having less than high school diploma and the probability of employment for Inuit women is as expected negative, this association is statistically significant only in Model 3. Second, as illustrated in the following two rows of Table 7, the association between postsecondary schooling (both incomplete and completed) and the probability of employment for Inuit women is negative. Furthermore, while the finding on some postsecondary schooling remains stable in all of the subsequent models, the finding on completed postsecondary schooling does not - the statistical significance observed with the reduced human capital block of variables (Model 1) disappears in the following two models when other human capital characteristics are included (Model 2) and when social capital characteristics are included (Model 3) but reappears again in the final model (Full Model) when all observed factors are accounted for. The educational attainment findings for Inuit women should thus be interpreted with caution as well. It could be that Inuit face different patterns, as indicated in the existing literature on income and earning disparity (Penadakur \& Pendakur, 2011).

The variable measuring the access to and the use of information technology appears positively related to the probability of employment, both for Inuit men and for 
Inuit women. There seems to be no association between the two measures of health and the probability of employment for Inuit men. For Inuit women, however, the association is negative and statistically significant, both for the measure of chronic health conditions and for the measure of activity limitation, which is in accord with the associated theory.

The reported estimates on the effect of the three measures of social capital indicate different associations for Inuit men and for Inuit women. As shown in Table 7, the measure for strong ties (social networks) is positively associated with the probability of employment for Inuit women and negatively associated with the probability of employment for Inuit men. The first measure of weak ties (volunteering in the community) seems to have no impact on the probability of employment for Inuit women. For Inuit men, however, the association exists but its direction is in direct contrast to what social capital theory would suggest — the estimated average marginal effect for this measure of social capital suggests that the probability of Inuit men being employed decreases as this indicator changes from not volunteering to volunteering in the community.

Civic participation (at the municipal level, as another measure of weak ties, appears to have a positive effect on the probability of employment, both for Inuit men and for Inuit women, with the magnitude of the effect being quite large for Inuit women. As shown in Table 7, the probability of employment of Inuit women increases by 12.7 percentage points (Model 3) or 10.3 percentage points (Full Model) as this indicator changes from not voting in municipal elections to voting in municipal elections. At the provincial level, however, the association of civic participation variable is positive for Inuit men and negative for Inuit women. 
A particularly interesting finding from the final block of variables that relate to Aboriginal culture is that the engagement in traditional pursuits has no association with the probability of employment for Inuit men. For Inuit women, however, the association between traditional pursuits for commercial purposes and the probability of employment is negative and the magnitude of the effect is relatively large - as presented in Table 7, the probability of employment for Inuit women decreases by over 18 percentage points as this indicator changes from not participating in traditional pursuits to participating for commercial purposes. Inuit women are also less likely to be employed, all else constant, if they engaged in traditional pursuits for pleasure but more likely to be employed if they engaged in traditional pursuits for food and medicinal purposes, relative to Inuit women who did not engage in any traditional pursuits in the 12 months preceding to the survey.

As expected, fluency in an Aboriginal language is positively associated with the probability of employment, both for Inuit men and for Inuit women. Being a member or beneficiary of a land claim agreement, however, seems to be positively associated with the probability of employment for Inuit women but negatively associated with the probability of employment for Inuit men. As displayed in Table 7, all else held constant, the mean expected probability of Inuit men being employed decreases by about four percentage points as this indicator changes from not being a member to being a member or beneficiary of a land claim agreement. 


\section{Robustness Checks}

In the following three sections, selected estimates are reported from a sensitivity test in which the association between each explanatory variable and the probability of employment for each identity group was examined more closely by varying the measure for educational attainment. In each model, different and generally more detailed classifications of the educational attainment variable are used in place of the standard sixcategory measure just discussed in order to probe further into the peculiarity of the findings presented in the preceding results sections. As discussed above, the results for postsecondary education obtained using the standard six-category classification are not uniformly positive and increasing for any of the examined groups of Aboriginal people.

The reported estimates in the first of these three sections are based on data from the 2006 APS and they pertain to the same groups of Aboriginal identity population whose sample means and regression findings are presented in Section 4 . The estimates presented in the other two sections are based on data from the 2006 Census and they pertain to four Aboriginal identity groups: the on-reserve NAI group, the urban offreserve NAI group, Métis and Inuit. The mean proportions of the employed in each of the analytical samples have been summarized earlier in this essay, in Table 1. The mean proportions pertaining to educational attainment in each sample are summarized next. The sample means of all other explanatory variables are presented in Appendix E1.

The analytical samples drawn from the 2006 Census reveal that Inuit and the onreserve NAI group differ in important ways from the other two Aboriginal identity 
groups considered in this part of the analysis. ${ }^{94}$ In terms of their observed characteristics related to highest accreditation attained, the on-reserve NAI group has the largest proportion of people with no educational credentials (59\% of men and $54 \%$ of women), followed by Inuit group (57\% for each gender). These figures for Métis amount to 32 percent for men and 27 percent for women. In the urban off-reserve NAI group, about 54 percent of men and about 44 percent of women are without any educational credentials.

More women than men in each sample have completed university degree; about 4 percent of women in the Inuit and in the on-reserve NAI samples have completed university degree, compared to 2 percent of men. In the Métis sample, these figures correspond to 8 percent of women and 6 percent of men while in the urban off-reserve NAI sample the figures are 7 percent of women and 4 percent of men. A slightly larger proportion of urban NAI women (4\%) completed university certificate below Bachelor's level, compared to the proportions in other samples (ranging from one percent of Inuit men to 3 percent of Métis and the on-reserve NAI women).

Completed high school diploma is the highest accreditation attained for 14 percent of the on-reserve NAI men and 17 percent of the on-reserve NAI women. In the Inuit sample, these figures correspond to 13 percent of men and 15 percent of women. In Métis sample, 26 percent of men and 28 percent of women report high school diploma as their highest accreditation attained while in the urban off-reserve NAI sample, these figures are 19 percent of men and 22 percent of women.

\footnotetext{
${ }^{94}$ As indicated earlier, the results discussed in this section pertain to Inuit and Métis and to two NAI groups, those living on-reserve and those living off-reserve in urban areas. The sample for the latter NAI group was further disaggregated and separate analyses were done for registered and non-registered NAI as well as for rural off-reserve NAI. For space considerations, the results from these analyses are not presented here but can be made available, upon request.
} 
In relation to vocational schooling, a relatively small proportion of NAI men have completed registered apprenticeship certificates (3\% on-reserve and $4 \%$ off-reserve), compared to the proportion that completed other trades certificates $(11 \%$ on reserve and $9 \%$ off-reserve). Similarly, 8 percent of Métis men have completed registered apprenticeship certificate and 11 percent completed other trades certificate. These figures for Inuit men amount to 4 percent for registered apprenticeship and 10 percent for other trades diploma.

A revealing insight here is that the on-reserve NAI male sample $(8 \%)$ and the Inuit male sample (9\%) contain larger proportions of these vocational schooling certificate holders who obtained their certificates without first obtaining a high school diploma or equivalency. The proportions of those who obtained these certificates upon completing high school education are 6 percent for on-reserve NAI men and 5 percent for Inuit men.

The reverse seems to be the case among Métis men and among urban NAI men. In the Métis male sample, 12 percent of the vocational schooling certificate holders have obtained their certificate after completing high school, compared to 6 percent who obtained their certificate without completing high school. Similarly, in the urban NAI male sample, 7 percent of the vocational schooling certificate holders have obtained their certification upon completion of high school diploma, compared 6 percent who obtained their certificate without first completing high school diploma.

In relation to other postsecondary certifications, the proportions of individuals who obtained their college diplomas without first completing a high school diploma are comparatively very small, ranging from one percent in the urban NAI male sample to 
about 5 percent in the Inuit female sample. The proportion of individuals who completed their college diplomas upon completing high school education are substantially larger, ranging from 7 percent in the on-reserve NAI male sample to 20 percent in the Métis female sample.

In relation to the length of the college program, the proportions are similar (ranging from 3 to 5 percent) across identity groups for college program that lasted over two years. For a college program that lasted between one and two years, the proportions are largest among Métis ( $8 \%$ for men and $12 \%$ for women) and smallest among the onreserve NAI ( $4 \%$ for men and $7 \%$ for women). The same distribution appears for a college program that lasted between 3 to 12 months - the proportions are highest among Métis (6\% for women and $2 \%$ for men) and smallest among the on-reserve NAI (3\% for women and $2 \%$ for men). In each group, women were more likely than men to have college diploma completed. These differences could have some bearing on the regression results presented in the previous section and they will be examined here in more detail. 


\subsection{Highest Grade or Level of Schooling Attained}

The results from the multivariate analysis presented in Section 4 have revealed several unusual and somewhat unexpected effects of different levels of educational attainment on the probability of employment for different Aboriginal identity groups. The overall conclusion, consistent to a large extent with the previous empirical work, is that postsecondary schooling below university level has an adverse effect on the probability of employment for some Aboriginal identity groups, and in particular for registered and for urban NAI men. For urban and registered NAI women, on the other hand, the results suggest that any schooling beyond high school diploma increases the probability of employment for them. For the other two groups, Métis and Inuit, the estimated effects are ambiguous. In no case did more education consistently lead to higher probabilities of employment, which is what human capital theory predicts.

The estimated effects presented in Section 4 were derived using the standard sixcategory measure of educational attainment found in the analytical files of the 2006 APS. As indicated earlier in Section 4, there are reasons to suspect that this classification might be concealing some important differences that exist, particularly with regard to the vocational schooling category, between men and women. The descriptive evidence presented in Section 4.1 has revealed that the distribution in this regard differs clearly across gender.

This section presents the results from robustness checks in which two more detailed measures of educational attainment from the 2006 APS were used in place of the standard six-category measure of educational attainment. The contents and the coding of these measures have been described earlier in this essay, in Section 3.3. Further details on 
these variables are provided in Appendix A1 and Appendix A2. The discussion here is restricted to assessing the change in estimated average marginal effects of the educational attainment while the full regression results are presented in Appendix D1.

As shown in Table 8, when educational attainment classification is expanded to allow a distinction between two types of non-university education, and two types of completed non-university schooling, no change is observed in the estimated effects for urban NAI women - at every level and with every type of postsecondary schooling (completed and incomplete) the probability of employment for urban NAI women is higher, holding all else constant, compared to those with only high school diploma. The same holds true for the registered NAI women, as shown in Table 8.

For urban NAI men, a change emerges when the incomplete non-university educational classification is expanded to distinguish incomplete vocational schooling from other non-university education. As shown in Table 8, the association between other incomplete non-university education and the probability of employment for urban NAI men remains negative but the association between incomplete vocational schooling and the probability of employment of urban NAI men is positive, suggesting that, ceteris paribus, the probability of urban NAI men being employed increases by about five percentage points as the educational classification indicator changes from high school diploma to some vocational schooling. The association between completed trades certificate and the probability of employment for urban NAI men, however, is oddly negative, suggesting that urban NAI men with completed trades certificates are less likely to be employed than are urban NAI men who have only high school diplomas completed. 
Table 8: Robustness checks for highest grade and highest level of schooling attained, APS 2006

\begin{tabular}{|c|c|c|c|c|c|c|c|c|c|c|c|c|}
\hline \multirow{4}{*}{$\begin{array}{l}\text { Selected education } \\
\text { variables in the equations }\end{array}$} & \multicolumn{12}{|c|}{ Average Marginal Effects } \\
\hline & \multicolumn{8}{|c|}{ North American Indian } & \multicolumn{2}{|c|}{ Métis } & \multicolumn{2}{|c|}{ Inuit } \\
\hline & \multicolumn{2}{|c|}{ Urban } & \multicolumn{2}{|c|}{ Rural } & \multicolumn{2}{|c|}{ Registered } & \multicolumn{2}{|c|}{ Non-registered } & \multirow[b]{2}{*}{ Men } & \multirow[b]{2}{*}{ Women } & \multirow[b]{2}{*}{ Men } & \multirow[b]{2}{*}{ Women } \\
\hline & Men & Women & Men & Women & Men & Women & Men & Women & & & & \\
\hline $\begin{array}{l}\text { Less than } \\
\text { high school education }\end{array}$ & $\begin{array}{c}-0.048 \\
(0.007)^{\star \star *}\end{array}$ & $\begin{array}{c}-0.034 \\
(0.006)^{\star \star *}\end{array}$ & $\begin{array}{c}-0.032 \\
(0.008)^{\star \star \star \star}\end{array}$ & $\begin{array}{c}-0.054 \\
(0.009)^{* * *}\end{array}$ & $\begin{array}{c}-0.050 \\
(0.007)^{\star \star \star \star}\end{array}$ & $\begin{array}{c}-0.022 \\
(0.006)^{\star \star}\end{array}$ & $\begin{array}{c}-0.042 \\
(0.008)^{\star \star \star \star}\end{array}$ & $\begin{array}{l}-0.049 \\
(0.008)^{\star \star \star}\end{array}$ & $\begin{array}{l}-0.004 \\
(0.004)\end{array}$ & $\begin{array}{c}-0.027 \\
(0.004)^{\star \star \star *}\end{array}$ & - & - \\
\hline $\begin{array}{l}\text { Some } \\
\text { vocational schooling }\end{array}$ & $\begin{array}{c}0.055 \\
(0.005)^{\star \star \star *}\end{array}$ & $\begin{array}{c}0.043 \\
(0.005)^{\star \star \star}\end{array}$ & $\begin{array}{l}-0.018 \\
(0.022)\end{array}$ & $\begin{array}{l}-0.096 \\
(0.028)^{\star \star}\end{array}$ & $\begin{array}{c}0.066 \\
(0.007)^{\star \star \star \star}\end{array}$ & $\begin{array}{c}0.021 \\
(0.008)^{*}\end{array}$ & $\begin{array}{c}0.017 \\
(0.011)\end{array}$ & - & - & - & - & - \\
\hline $\begin{array}{l}\text { Some } \\
\text { non-university education }\end{array}$ & $\begin{array}{c}-0.045 \\
(0.012)^{* * *}\end{array}$ & $\begin{array}{c}0.041 \\
(0.004)^{* * * *}\end{array}$ & $\begin{array}{c}0.002 \\
(0.010)\end{array}$ & $\begin{array}{c}-0.046 \\
(0.013)^{* * *}\end{array}$ & $\begin{array}{c}-0.033 \\
(0.011)^{\star *}\end{array}$ & $\begin{array}{c}0.059 \\
(0.004)^{\star \star \star}\end{array}$ & $\begin{array}{l}-0.028 \\
(0.012)\end{array}$ & $\begin{array}{c}-0.022 \\
(0.009)^{*}\end{array}$ & $\begin{array}{l}-0.005 \\
(0.005)\end{array}$ & $\begin{array}{c}0.002 \\
(0.004)\end{array}$ & - & - \\
\hline $\begin{array}{l}\text { Some } \\
\text { university education }\end{array}$ & $\begin{array}{c}-0.043 \\
(0.011)^{\star \star \star \star}\end{array}$ & $\begin{array}{c}0.051 \\
(0.003)^{\star \star \star *}\end{array}$ & $\begin{array}{r}-0.002 \\
(0.018)\end{array}$ & $\begin{array}{c}0.003 \\
(0.012)\end{array}$ & $\begin{array}{c}-0.085 \\
(0.014)^{* \star \star \star}\end{array}$ & $\begin{array}{c}0.056 \\
(0.004)^{\star \star \star}\end{array}$ & $\begin{array}{c}0.030 \\
(0.008)^{\star \star \star *}\end{array}$ & $\begin{array}{c}0.025 \\
(0.005)^{\star \star \star *}\end{array}$ & $\begin{array}{c}0.017 \\
(0.006)^{* *}\end{array}$ & $\begin{array}{l}0.006 \\
(0.004)\end{array}$ & - & - \\
\hline $\begin{array}{l}\text { Completed } \\
\text { trades certificate or diploma }\end{array}$ & $\begin{array}{c}-0.031 \\
(0.009)^{\star \star}\end{array}$ & $\begin{array}{c}0.038 \\
(0.004)^{* * * *}\end{array}$ & $\begin{array}{c}-0.021 \\
(0.008)^{*}\end{array}$ & $\begin{array}{c}0.016 \\
(0.008)^{*}\end{array}$ & $\begin{array}{c}-0.048 \\
(0.010)^{* * *}\end{array}$ & $\begin{array}{c}0.064 \\
(0.003)^{\star \star \star}\end{array}$ & $\begin{array}{r}-0.012 \\
(0.007)\end{array}$ & $\begin{array}{c}-0.012 \\
(0.008)\end{array}$ & $\begin{array}{l}-0.0003 \\
(0.004)\end{array}$ & $\begin{array}{l}-0.001 \\
(0.005)\end{array}$ & - & - \\
\hline $\begin{array}{l}\text { Completed } \\
\text { non-university diploma }\end{array}$ & $\begin{array}{c}-0.068 \\
(0.011)^{\star * *}\end{array}$ & $\begin{array}{c}0.060 \\
(0.004)^{\star \star \star}\end{array}$ & $\begin{array}{c}0.005 \\
(0.008)\end{array}$ & $\begin{array}{c}-0.024 \\
(0.007)^{* *}\end{array}$ & $\begin{array}{c}-0.055 \\
(0.010)^{* \ldots \hbar}\end{array}$ & $\begin{array}{c}0.065 \\
(0.005)^{\star k+k}\end{array}$ & $\begin{array}{c}-0.034 \\
(0.010)^{\star \star}\end{array}$ & $\begin{array}{c}0.022 \\
(0.004)^{\star \star \star}\end{array}$ & $\begin{array}{c}0.026 \\
(0.003)^{n+*}\end{array}$ & $\begin{array}{r}-0.001 \\
(0.003)\end{array}$ & - & - \\
\hline $\begin{array}{l}\text { Completed } \\
\text { university degree }\end{array}$ & $\begin{array}{c}0.038 \\
(0.006)^{* * *}\end{array}$ & $\begin{array}{c}0.055 \\
(0.003)^{\star \star * \star}\end{array}$ & $\begin{array}{r}-0.003 \\
(0.012)\end{array}$ & $\begin{array}{c}0.040 \\
(0.005)^{* * * *}\end{array}$ & $\begin{array}{c}0.049 \\
(0.007)^{\star \star \star * *}\end{array}$ & $\begin{array}{c}0.065 \\
(0.004)^{\star * *}\end{array}$ & $\begin{array}{c}0.029 \\
(0.006)^{\star \star \star \star}\end{array}$ & $\begin{array}{c}0.033 \\
(0.004)^{* \star *}\end{array}$ & $\begin{array}{c}0.014 \\
(0.006)^{*}\end{array}$ & $\begin{array}{c}0.027 \\
(0.008)^{\star * * *}\end{array}$ & - & - \\
\hline Grade 6 or less & - & - & - & - & - & - & - & - & - & - & $\begin{array}{c}0.037 \\
(0.009)^{* * * *}\end{array}$ & $\begin{array}{c}0.017 \\
(0.008)^{*}\end{array}$ \\
\hline Grade 9 & - & - & - & - & - & - & - & - & - & - & $\begin{array}{c}0.020 \\
(0.009)^{*}\end{array}$ & $\begin{array}{c}0.031 \\
(0.007)^{\star \star \star *}\end{array}$ \\
\hline Grade 10 & - & - & - & - & - & - & - & - & - & - & $\begin{array}{c}-0.001 \\
(0.009)\end{array}$ & $\begin{array}{c}-0.035 \\
(0.011)^{\star *}\end{array}$ \\
\hline Grade 11 & - & - & - & - & - & - & - & - & - & - & $\begin{array}{c}0.018 \\
(0.010)\end{array}$ & $\begin{array}{c}0.063 \\
(0.006)^{* * *}\end{array}$ \\
\hline Grade 12 or 13 , incomplete & - & - & - & - & - & - & - & - & - & - & $\begin{array}{c}0.036 \\
(0.008)^{\star \star *}\end{array}$ & $\begin{array}{c}-0.037 \\
(0.010)^{* * *}\end{array}$ \\
\hline Number of observations & 1530 & 1690 & 900 & 940 & 1610 & 1800 & 820 & 830 & 2150 & 2250 & 710 & 700 \\
\hline
\end{tabular}

Source: Statistics Canada, 2006 APS, analytical files. Bootstrapped (1000 replications) standard errors in parentheses. Statistical significance ${ }^{* * *} p<.001 ;{ }^{* *} p<.01 ;{ }^{*} p<.05$

Note: Estimates for all other variables are presented in Appendix D1. 
Another new insight that can be derived from Table 8 for NAI men is that completion of a university diploma below Bachelor's degree also exerts a positive impact on the probability of employment for urban NAI men. The overall results for registered NAI men are very similar, with one important exception. As shown in the fifth column of Table 8, the association between completed university diploma below Bachelor's degree and the probability of employment for registered NAI men, although positive, is not significantly different from zero.

No significant change appears in the estimated effects for the non-registered NAI men from those presented in the preceding section. For the non-registered NAI women, however, the association between incomplete non-university education and the probability of employment is negative and statistically significant. This association was unstable in the previous analysis in Section 4 but in no model was it statistically different from zero. In the model for rural NAI men, no significant association was observed in the previous analysis for any of the postsecondary education categories. In Table 8, however, there appears to be a significant negative association between completed trades certificate and the probability of employment. This effect, as shown in the fourth column of Table 8, for rural NAI women is positive as is the effect of completed university diploma below Bachelor's level. The other effects remain as in earlier models.

The estimated effects of educational attainment for Métis remain highly variable as in the previous analysis. While the positive effects found in the Full Model for Métis men in previous analysis pertaining to two categories of education (completed nonuniversity education and incomplete university education) remain positive in the present analysis, the estimated effect of the first category, as displayed in the first row of Table 8, 
also remains not statistically different from zero, as it was in the previous analysis. No change from previous results appears in the estimated model for Métis women, suggesting that the findings are robust.

The sensitivity test for Inuit population is done using the highest completed grade of elementary and secondary schooling, with the combined completed grade 7 or 8 as the reference category. Although informative, and to an extent in accord with previous literature on the topic, the findings from this test should be interpreted with caution as well since the ambiguity remains even with this measure of educational attainment.

As presented in Table 8, the estimated average marginal effect on the incomplete grade 12 or 13 category suggests that the probability of employment for Inuit men increases as the completed grades indicator changes from completed grade 7 or 8 to incomplete grade 12 or 13 , holding all else constant. This finding coincides with that in Drost (1994) for Aboriginal population. However, the estimated average marginal effect on the completed grade 6 or less category suggests that the results should be treated with caution - it appears that the probability of employment for Inuit men also increases when the indicator on completed grades changes from completed grade 7 or 8 to completed grade 6 or less, which contradicts what human capital theory would suggest. The same counterintuitive conclusion is reached when assessing the estimated average marginal effects for Inuit women, implying that this measure of educational attainment is not much useful in helping us understand the effect of education on the probability of employment for Inuit population. In the following two sections, two different measures of educational attainment are applied to probe further into this issue. 


\subsection{Highest Accreditation Attained}

This section presents estimated average marginal effects from the robustness checks probit analysis of employment for two NAI identity groups, the on-reserve NAI and urban off-reserve NAl, as well as for Métis and for Inuit using the Census measure of educational attainment — highest accreditation attained - which qualitatively corresponds closely to the expanded measure from the APS just presented. As in the preceding section, discussion here centers on the change in estimated effects of highest accreditation attained on the probability of employment. The estimated effects for all other variables included in the models for each group are presented in Appendix E2.

Table 9: Robustness checks for educational credentials, Census 2006

\begin{tabular}{|c|c|c|c|c|c|c|c|c|}
\hline \multirow{3}{*}{$\begin{array}{l}\text { Highest accreditation } \\
\text { attained }\end{array}$} & \multicolumn{8}{|c|}{ Average Marginal Effects } \\
\hline & \multicolumn{2}{|c|}{$\begin{array}{c}\text { On-reserve } \\
\text { NAl }\end{array}$} & \multicolumn{2}{|c|}{$\begin{array}{c}\text { Urban off-reserve } \\
\text { NAI }\end{array}$} & \multicolumn{2}{|c|}{ Métis } & \multicolumn{2}{|c|}{ Inuit } \\
\hline & Men & Women & Men & Women & Men & Women & Men & Women \\
\hline No education credentials & $\begin{array}{c}-0.100 \\
(0.009)^{\star \star *}\end{array}$ & $\begin{array}{c}-0.091 \\
(0.007)^{\star \star \star}\end{array}$ & $\begin{array}{c}-0.099 \\
(0.009)^{\star \star \star}\end{array}$ & $\begin{array}{c}-0.082 \\
(0.008)^{\star \star \star}\end{array}$ & $\begin{array}{c}-0.056 \\
(0.008)^{\star \star \star}\end{array}$ & $\begin{array}{c}-0.050 \\
(0.008)^{\star \star *}\end{array}$ & $\begin{array}{c}-0.135 \\
(0.021)^{\star \star \star}\end{array}$ & $\begin{array}{c}-0.104 \\
(0.017)^{\star * * *}\end{array}$ \\
\hline $\begin{array}{l}\text { Completed } \\
\text { other trades diploma }\end{array}$ & $\begin{array}{c}-0.053 \\
(0.012)^{\star \star \star *}\end{array}$ & $\begin{array}{r}-0.006 \\
(0.011)\end{array}$ & $\begin{array}{c}-0.066 \\
(0.010)^{\star \star \star}\end{array}$ & $\begin{array}{c}-0.011 \\
(0.010)\end{array}$ & $\begin{array}{c}-0.022 \\
(0.011)^{\star}\end{array}$ & $\begin{array}{c}0.010 \\
(0.010)\end{array}$ & $\begin{array}{c}-0.096 \\
(0.028)^{\star \star \star}\end{array}$ & $\begin{array}{r}-0.065 \\
(0.039)\end{array}$ \\
\hline $\begin{array}{l}\text { Completed } \\
\text { apprenticeship certificate }\end{array}$ & $\begin{array}{c}-0.041 \\
(0.015)^{\star \star}\end{array}$ & $\begin{array}{l}-0.007 \\
(0.020)\end{array}$ & $\begin{array}{r}-0.004 \\
(0.013)\end{array}$ & $\begin{array}{r}-0.008 \\
(0.024)\end{array}$ & $\begin{array}{c}0.002 \\
(0.012)\end{array}$ & $\begin{array}{c}0.011 \\
(0.020)\end{array}$ & $\begin{array}{l}-0.048 \\
(0.038)\end{array}$ & $\begin{array}{r}-0.050 \\
(0.059)\end{array}$ \\
\hline $\begin{array}{l}\text { Completed } \\
\text { college program, } 3-12 \text { months }\end{array}$ & $\begin{array}{l}-0.0001 \\
(0.020)\end{array}$ & $\begin{array}{c}0.034 \\
(0.013)^{\star *}\end{array}$ & $\begin{array}{r}-0.030 \\
(0.017)\end{array}$ & $\begin{array}{c}0.011 \\
(0.011)\end{array}$ & $\begin{array}{c}0.009 \\
(0.018)\end{array}$ & $\begin{array}{c}0.002 \\
(0.010)\end{array}$ & $\begin{array}{r}-0.059 \\
(0.055)\end{array}$ & $\begin{array}{r}-0.025 \\
(0.029)\end{array}$ \\
\hline $\begin{array}{l}\text { Completed } \\
\text { college program, } 1-2 \text { years }\end{array}$ & $\begin{array}{c}0.051 \\
(0.012)^{* \star \star}\end{array}$ & $\begin{array}{c}0.059 \\
(0.009)^{\star \star \star}\end{array}$ & $\begin{array}{c}0.014 \\
(0.012)\end{array}$ & $\begin{array}{c}0.025 \\
(0.009)^{* *}\end{array}$ & $\begin{array}{l}0.0015 \\
(0.010)\end{array}$ & $\begin{array}{c}0.020 \\
(0.011)\end{array}$ & $\begin{array}{c}0.048 \\
(0.048)\end{array}$ & $\begin{array}{c}0.026 \\
(0.029)\end{array}$ \\
\hline $\begin{array}{l}\text { Completed } \\
\text { college program, over } 2 \text { years }\end{array}$ & $\begin{array}{c}0.078 \\
(0.015)^{\star * *}\end{array}$ & $\begin{array}{c}0.055 \\
(0.010)^{\star \star *}\end{array}$ & $\begin{array}{c}0.029 \\
(0.013)^{*}\end{array}$ & $\begin{array}{c}0.037 \\
(0.010)^{\star * *}\end{array}$ & $\begin{array}{c}0.034 \\
(0.010)^{* *}\end{array}$ & $\begin{array}{c}0.041 \\
(0.010)^{\star * *}\end{array}$ & $\begin{array}{r}-0.034 \\
(0.053)\end{array}$ & $\begin{array}{c}0.004 \\
(0.034)\end{array}$ \\
\hline $\begin{array}{l}\text { Completed } \\
\text { university certificate }\end{array}$ & $\begin{array}{c}0.065 \\
(0.019)^{\star \star}\end{array}$ & $\begin{array}{c}0.058 \\
(0.011)^{* * *}\end{array}$ & $\begin{array}{c}0.024 \\
(0.016)\end{array}$ & $\begin{array}{c}0.015 \\
(0.012)\end{array}$ & $\begin{array}{c}0.023 \\
(0.018)\end{array}$ & $\begin{array}{c}0.013 \\
(0.014)\end{array}$ & $\begin{array}{l}-0.006 \\
(0.063)\end{array}$ & $\begin{array}{c}0.008 \\
(0.045)\end{array}$ \\
\hline $\begin{array}{l}\text { Completed } \\
\text { university degree }\end{array}$ & $\begin{array}{c}0.134 \\
(0.017)^{* * *}\end{array}$ & $\begin{array}{c}0.105 \\
(0.010)^{* * * *}\end{array}$ & $\begin{array}{c}0.078 \\
(0.012)^{k \star \star}\end{array}$ & $\begin{array}{c}0.062 \\
(0.009)^{\star \star \star}\end{array}$ & $\begin{array}{c}0.058 \\
(0.009)^{\star \star \star *}\end{array}$ & $\begin{array}{c}0.049 \\
(0.007)^{* * *}\end{array}$ & $\begin{array}{c}0.102 \\
(0.053)\end{array}$ & $\begin{array}{c}0.081 \\
(0.024)^{* *}\end{array}$ \\
\hline Number of observations & 36920 & 31830 & 51750 & 50560 & 15320 & 14090 & 6090 & 5970 \\
\hline
\end{tabular}

Source: Statistics Canada, 2006 Census analytical file. Individual composite weights in effect. Standard errors (adjusted for clustering) in parentheses. Statistical significance ${ }^{* * *} p<.001 ;{ }^{* *} p<.01 ;{ }^{*} p<.05$.

Note: Estimates for all other variables are presented in Appendix E2. 
The first point to highlight from Table 9 is that the estimated average marginal effects on the first classification category (no educational credentials) for each examined group are statistically significant and in accord with what human capital theory would suggest - ceteris paribus, the probability of employment decreases as the indicator for highest accreditation attained changes from completed high school diploma to no educational credentials. The magnitude of this effect is particularly large for Inuit men $(13 \%)$ and for Inuit women $(10 \%)$ as well as for the on-reserve NAI men $(10 \%)$.

The estimated average marginal effects on the other end of the educational spectrum (completed university degree) are also in accord with human capital theory for all groups, although in the case of Inuit men, the association is not significantly different from zero. For the on-reserve NAI, the magnitude of this effect is particularly large. As displayed in Table 10, all else constant, the probability of employment for the on-reserve NAI men increases by about 13 percentage points as the indicator for highest credentials attained changes from completed high school diploma to completed university degree.

The estimated effects of other postsecondary credentials differ across groups and across gender. As shown in Table 10, the association between completed apprenticeship certificate and the probability of employment is negative for all groups except for Métis but is statistically significant only in the case of the on-reserve NAI men. On the other hand, the association between completed other trades certificate and the probability of employment is negative and statistically significant for all Aboriginal identity men. The magnitude of this effect is particularly large $(10 \%)$ in the case of Inuit men.

The estimated average marginal effects of completed university diploma below Bachelor's degree are positive and statistically significant for the on-reserve NAI group; 
with the exception of Inuit men, the relationship between this educational credential and the probability of employment is also positive but not statistically significant from zero.

Completed college diploma from program that lasted over two years is positively associated with the probability of employment for the two NAI groups and for Métis. The positive association between completed college diploma from program that lasted between one and two years exists only for the on-reserve NAI group and for the offreserve NAI women. For the other groups, this association is not statistically different from zero. Finally, completed college diploma from program that lasted between 3-12 months is positively associated with the probability of employment only for the onreserve NAI women. Notably, for NAI men, as well as for Inuit men and women, the effect of this credential is negative, albeit not significantly different from zero.

The following section looks at this classification of accreditation from a different angle using the newly designed variable from the 2006 Census. As pointed out in Section 3.3 , this classification of education is particularly useful for the present inquiry as it permits differentiating between high school graduates who hold vocational schooling certificates or diplomas from high school dropouts who also hold these vocational schooling credentials. Setting aside the limitations in the Census data discussed earlier in Section 4 , the estimated results pertaining to the effects of education on the probability of employment from this final robustness checks can be regarded as the most informative for all groups examined here. 


\subsection{High School Certification and Highest Accreditation Attained}

This final section presents the estimated average marginal effects from the robustness checks on the probit analysis of employment for the same two NAI identity groups, the on-reserve NAI and urban off-reserve NAI, as well as for Métis and for Inuit. The estimated effects pertain to the Census's newly introduced classification of educational attainment that takes high school graduation into account when defining different groups holding different postsecondary educational credentials. More precisely, the classification lists separately individuals who have obtained postsecondary schooling credentials upon obtaining their high school diplomas from those who have obtained postsecondary schooling credentials without first graduating from high school. As such, this newest measure of educational attainment has a potential to reveal more useful insights into the effects of postsecondary education on the probability of employment for all groups examined in this essay. As in the previous sections of the robustness checks, the discussion here is restricted to assessing the change in estimated effects for this measure. The estimated effects for all other variables included in the models are presented in Appendix E3.

As displayed in the first and in the last rows of Table 10, the patterns of the estimated average marginal effects of the first and of the last category of educational attainment remain as presented in the previous section in Table 9, with almost identical coefficients, thus allowing for two clear conclusions to be drawn from this analysis: (1) ceteris paribus, the probability of employment of an Aboriginal identity person decreases as the indicator for educational attainment changes from completed high school diploma without further schooling to no high school diploma and no further schooling, and (2) all 
else constant, the probability of employment of an Aboriginal identity person, excluding Inuit men, increases as the indicator for educational attainment changes from completed high school diploma with no further schooling to completed university degree with completed high school diploma.

Table 10: Robustness checks for educational certification and credentials, Census 2006

\begin{tabular}{|c|c|c|c|c|c|c|c|c|}
\hline \multirow{3}{*}{$\begin{array}{l}\text { High school certification and } \\
\text { accreditation attained }\end{array}$} & \multicolumn{8}{|c|}{ Average Marginal Effects } \\
\hline & \multicolumn{2}{|c|}{$\begin{array}{l}\text { On-reserve } \\
\text { NAl }\end{array}$} & \multicolumn{2}{|c|}{$\begin{array}{c}\text { Urban off-reserve } \\
\text { NAI }\end{array}$} & \multicolumn{2}{|c|}{ Métis } & \multicolumn{2}{|c|}{ Inuit } \\
\hline & Men & Women & Men & Women & Men & Women & Men & Women \\
\hline $\begin{array}{l}\text { No high school diploma and no } \\
\text { further schooling }\end{array}$ & $\begin{array}{c}-0.100 \\
(0.009)^{\star * *}\end{array}$ & $\begin{array}{c}-0.091 \\
(0.007)^{\star \star *}\end{array}$ & $\begin{array}{c}-0.099 \\
(0.009)^{* \star \star}\end{array}$ & $\begin{array}{c}-0.082 \\
(0.008)^{\star \star *}\end{array}$ & $\begin{array}{c}-0.056 \\
(0.008)^{\star * * *}\end{array}$ & $\begin{array}{c}-0.050 \\
(0.008)^{* \cdots *}\end{array}$ & $\begin{array}{c}-0.138 \\
(0.021)^{\star \star \star}\end{array}$ & $\begin{array}{c}-0.106 \\
(0.017)^{* * *}\end{array}$ \\
\hline $\begin{array}{l}\text { Registered apprenticeship or } \\
\text { other trade certificate, without } \\
\text { high school diploma }\end{array}$ & $\begin{array}{c}-0.083 \\
(0.013)^{\text {***}}\end{array}$ & $\begin{array}{c}-0.032 \\
(0.014)^{*}\end{array}$ & $\begin{array}{c}-0.091 \\
(0.012)^{\star \star \star \star}\end{array}$ & $\begin{array}{c}-0.062 \\
(0.015)^{\star \star \star}\end{array}$ & $\begin{array}{c}-0.034 \\
(0.014)^{*}\end{array}$ & $\begin{array}{c}0.004 \\
(0.016)\end{array}$ & $\begin{array}{c}-0.132 \\
(0.029)^{\star \star \star}\end{array}$ & $\begin{array}{c}-0.086 \\
(0.035)^{\star}\end{array}$ \\
\hline $\begin{array}{l}\text { College certificate, without high } \\
\text { school diploma }\end{array}$ & $\begin{array}{c}-0.020 \\
(0.0019)\end{array}$ & $\begin{array}{c}0.012 \\
(0.018)\end{array}$ & $\begin{array}{c}-0.071 \\
(0.022)^{\star *}\end{array}$ & $\begin{array}{l}-0.019 \\
(0.016)\end{array}$ & $\begin{array}{l}-0.016 \\
(0.023)\end{array}$ & $\begin{array}{c}-0.019 \\
(0.017)\end{array}$ & $\begin{array}{c}-0.077 \\
(0.035)^{\star}\end{array}$ & $\begin{array}{l}-0.037 \\
(0.027)\end{array}$ \\
\hline $\begin{array}{l}\text { High school diploma and } \\
\text { registered apprenticeship or } \\
\text { other trade certificate }\end{array}$ & $\begin{array}{c}-0.007 \\
(0.012)\end{array}$ & $\begin{array}{c}0.012 \\
(0.012)\end{array}$ & $\begin{array}{c}-0.013 \\
(0.010)\end{array}$ & $\begin{array}{c}0.012 \\
(0.012)\end{array}$ & $\begin{array}{c}-0.003 \\
(0.010)\end{array}$ & $\begin{array}{c}0.012 \\
(0.011)\end{array}$ & $\begin{array}{c}0.004 \\
(0.028)\end{array}$ & $\begin{array}{r}-0.030 \\
(0.055)\end{array}$ \\
\hline $\begin{array}{l}\text { High school diploma and } \\
\text { college certificate }\end{array}$ & $\begin{array}{c}0.065 \\
(0.011)^{\star \star * *}\end{array}$ & $\begin{array}{c}0.062 \\
(0.007)^{\star \star \star}\end{array}$ & $\begin{array}{c}0.023 \\
(0.009)^{*}\end{array}$ & $\begin{array}{c}0.033 \\
(0.007)^{\star \star \star}\end{array}$ & $\begin{array}{c}0.015 \\
(0.008)\end{array}$ & $\begin{array}{c}0.024 \\
(0.007)^{\star \star \star}\end{array}$ & $\begin{array}{l}-0.037 \\
(0.046)\end{array}$ & $\begin{array}{c}0.029 \\
(0.026)\end{array}$ \\
\hline $\begin{array}{l}\text { High school diploma and } \\
\text { university certificate }\end{array}$ & $\begin{array}{c}0.065 \\
(0.019)^{* \star}\end{array}$ & $\begin{array}{c}0.058 \\
(0.011)^{* * *}\end{array}$ & $\begin{array}{l}0.023 \\
(0.016)\end{array}$ & $\begin{array}{c}0.015 \\
(0.012)\end{array}$ & $\begin{array}{c}0.023 \\
(0.018)\end{array}$ & $\begin{array}{c}0.013 \\
(0.014)\end{array}$ & $\begin{array}{c}-0.006 \\
(0.063)\end{array}$ & $\begin{array}{c}0.008 \\
(0.045)\end{array}$ \\
\hline $\begin{array}{l}\text { High school diploma and } \\
\text { university degree }\end{array}$ & $\begin{array}{c}0.133 \\
(0.017)^{\star \star \star \star}\end{array}$ & $\begin{array}{c}0.104 \\
(0.010)^{\star \star \star}\end{array}$ & $\begin{array}{c}0.078 \\
(0.012)^{\star \star \star \star}\end{array}$ & $\begin{array}{c}0.062 \\
(0.009)^{\star \star \star}\end{array}$ & $\begin{array}{c}0.058 \\
(0.009)^{\star \star *}\end{array}$ & $\begin{array}{c}0.049 \\
(0.007)^{\star \star \star}\end{array}$ & $\begin{array}{c}0.102 \\
(0.053)\end{array}$ & $\begin{array}{c}0.082 \\
(0.024)^{\star \star}\end{array}$ \\
\hline Number of observations & 36920 & 31830 & 51750 & 50560 & 15320 & 14090 & 6090 & 5970 \\
\hline
\end{tabular}

Source: Statistics Canada, 2006 Census analytical file. Individual composite weights in effect. Standard errors (adjusted for clustering) in parentheses. Statistical significance ${ }^{* * *} p<.001 ;{ }^{* *} p<.01 ;{ }^{*} p<.05$.

Note: Estimates for all other variables are presented in Appendix E3.

The insights on the effects of the other levels of educational attainment, however, are not as uniform across groups. As shown in Table 10, the completion of a university certificate below Bachelor's degree is significantly associated with higher probability of employment only for NAI living on reserve - for all other groups, this association is not significantly different from zero, although it remains positive, except for Inuit men.

Also, for the NAI group, a positive and statistically significant association exists between completed college diploma and the probability of employment - this, however, 
is true only if the college diploma was obtained upon completion of high school diploma. As shown in Table 10, the association between the college diploma that was obtained without first obtaining a high school diploma is in fact negative for urban NAI men.

The association between having a registered apprenticeship or other trades certificate and the probability of employment for NAI men is negative, regardless if these credentials were obtained with or without completed high school diploma; this negative association, however, is not statistically different from zero for the credentials obtained with completed high school diploma.

For NAI women, an important new insight is that the positive association of the registered apprenticeship or other trades certificate obtained upon completion of high school diploma is not statistically different from zero but the negative association of the same credentials obtained without high school diploma is statistically significant, both for the on-reserve NAI women and for the urban off-reserve NAI women.

In the Métis employment equations, the positive association between college diplomas obtained upon completion of high school is statistically significant only for Métis women. Furthermore, the probability of employment of Métis men who have obtained registered apprenticeship or other trades certificate without first completing high school diploma is lower than the probability of employment of Métis men who have no high school diploma and no further schooling, all else constant. The same conclusion applies both to Inuit men and to Inuit women who have completed registered apprenticeship or other trades certificate without first completing high school diploma.

Finally, the association between completed college diploma, without high school diploma, and the probability of employment for Inuit men is significantly negative, 
implying that the probability of employment for Inuit men decreases as the indicator on educational credentials changes from completed high school diploma without further schooling to completed college certificate without high school diploma, all else held constant. This association for Inuit women is also negative, albeit not significant.

In short, the results from this robustness check suggest that the possession of postsecondary credentials such as registered apprenticeship or other trade certificates and college diplomas is of no use to Aboriginal people unless those credentials are obtained upon completion of a high school diploma. This conclusion speaks directly to a part of the conclusion reached by Drost (1994) where he says that "the largest gains in lowering the unemployment probability of Aboriginals can be achieved by raising elementary and secondary school completion rates" (p. 59). The counterintuitive results on the effects of years of completed elementary school on the probability of employment of Inuit people, however, do not support the other part of Drost's conclusion.

The results from this robustness check also shed some light on the conclusion Drost reached in regard to the effect of completed university education on the probability of employment of Aboriginal people. Drost's conclusion was that "the attainment of a university degree/certificate reduces the risk of unemployment for male Aboriginals, [but unlike for female Aboriginals] the effect [for male Aboriginals] is statistically not significant" (Drost, 1994, p. 59). This puzzlement could have resulted from the choice of educational classification used in Drost (1994). As examined in this study, the effect of having completed university degree on the probability of employment is statistically significant for all groups of Aboriginal people, except for Inuit men. The effect of completed university certificate, however, is different for different groups. 


\section{Discussion and Conclusions}

Employment is widely recognized as a source of economic empowerment and a crucial pathway out of poverty, especially for historically disadvantaged groups such as the Aboriginal groups in Canada. Empirical literature on the employment outcomes of Aboriginal groups, however, is relatively scarce. What exists tends to emphasize the importance of the key human capital factors such as education and other basic sociodemographic characteristics of individuals, as postulated in human capital theory.

This essay has taken on a wider theoretical scope by simultaneously incorporating factors from human capital theory and from social capital theory as well as some cultural characteristics that have not been accounted for in any of the empirical works to date. The study has also expanded the analytical and geographic scope by assessing the impact the addition of a block of variables from the social and cultural theoretical stances has on the estimated effects of variables from human capital theory and by conducting separate analysis of the probability of employment for Inuit, Métis and North American Indian men and women living on-and-off-reserve, in urban and rural areas across Canada.

The single most important conclusion arising from this analysis is that policy makers should bear in mind that the effects of the examined factors on the probability of employment are markedly different across identity groups and in some instances they are counterintuitive. The estimated results presented in this essay affirm the importance of distinguishing between (1) employment equations of Aboriginal men and Aboriginal women, (2) employment equations of NAI living in urban areas and NAI living in rural 
areas, (3) employment equations of registered NAI and non-registered NAI, and, perhaps most importantly, (4) the levels and types of postsecondary educational attainment.

As presented in Section 4, the estimated effects of educational attainment (measured by the standard six-category classification) on the probability of employment of urban and registered NAI men are strikingly different from those of urban and registered NAI women - it appears that the returns to postsecondary schooling are consistently positive for urban and registered NAI women while for urban and registered NAI men the returns to postsecondary schooling are positive only at the level of completed university education - at every other level of postsecondary education, the estimated average marginal effects are negative for urban and for registered NAI men.

As puzzling as it is, this finding is not a complete surprise since, to an extent, it is consistent with the previous empirical work on the topic. However, the robustness checks suggest that the effects of postsecondary education on the probability of employment for NAI women are possibly biased upwards and for NAI men possibly biased downwards.

As presented in Section 5.2, while the results from robustness checks on educational attainment (measured by an expanded nine-category classification) yield essentially the same conclusion for the effects of postsecondary education on the probability of employment for urban and registered NAI women as those presented in Section 4, a new insight surfaces for urban and registered NAI men - it appears that the effects of completed university diploma below Bachelor's degree (for urban NAI men) and of some vocational schooling (for urban and for registered NAI men) are positively associated with the probability of employment. 
The results from the other two robustness checks (based on more detailed measures of educational attainment) yield very different conclusions about these effects, both for men and for women. For instance, in the assessment of highest accreditation attained, the effect of a completed diploma from a college program that lasted over two years appears positively associated with the probability of employment of all NAI, men and women, living on-reserve and off-reserve. Similarly, a completed diploma from a college program that lasted between one and two years is positively associated with the probability of employment both for NAI men (on-reserve) and for NAI women (onreserve and off-reserve). Also, while the negative association between vocational schooling and the probability of employment of NAI men is maintained here, the positive association in relation to this level of schooling that was observed in Section 4 for NAI women is not present anymore - on the contrary, it appears that the association is negative, although the finding is not statistically different from zero.

In the assessment of high school certification and postsecondary accreditation, it becomes clear that the association between vocational schooling - when obtained without completion of a high school diploma - and the probability of employment is significantly negative both for men and for women. Interestingly, when the same credential is obtained upon completion of a high school diploma, the association becomes positive in the case of women and negative in the case of men - but in neither case is the effect statistically significant. When obtained after completion of high school, college diplomas (for all NAI groups) and university certificates (for on-reserve NAI) have positive effects on the probability of employment, both for men and for women. In the end, the returns to postsecondary education are not as drastically different for NAI men 
and for NAI women as one would conclude when assessing collapsed categories of educational attainment.

The general conclusion, however, remains that the predictions of human capital theory hold only at the two extreme ends of the educational attainment spectrum - no high school certification and completed university degree. Findings for all other levels of postsecondary schooling seem to contradict human capital theory.

Beyond this, important exceptions were observed - estimates in the Inuit employment equations, for instance, simply do not look at all like what human capital theory postulates. Even the robustness tests have produced some unexpected associations, as noted in the assessment of the effects of completed grades and completed university education on the probability of employment for Inuit men. Métis employment equations also seem to produce counterintuitive and highly variable results in relation to educational attainment, as discussed in Section 4.3. Although the last robustness tests estimated expected associations for the first and for the last category of educational attainment in Métis models, aside from a completed college diploma in the female employment equation, none of the in-between categories of educational attainment was significantly related to employment success of Métis.

In conjunction with the finding from previous literature on the topic, the findings from this study give rise to serious concerns in light of the fact that much of the current public policy centers on 'improving' employability of Aboriginal people through administration of various job training and skills development programs in partnership with postsecondary institutions such as community colleges. These concerns are only accentuated when one considers the possibility of Aboriginal high school dropouts being 
encouraged (or tempted) to undertake those skills development and training programs. As examined in this study, college certificates are useless for improving the employment prospects of Aboriginal people, unless they are obtained upon completion of a high school diploma.

Aside from the insight on the effects of different educational attainments, the main analysis has also established quite a few new factors such as access to information technology, social capital, non-Aboriginal spouse (in Métis models only), fluency in Aboriginal languages and engagement in some forms of traditional pursuits to have an overall positive impact on the employment success of some groups of Aboriginal people. For other groups, however, the effects of these new factors appear only occasionally and inconsistently important and in some instances the effects appear negative.

Surprisingly, for Inuit men, no association seems to exist between engagement in any of the three considered forms of traditional pursuits and the probability of employment. For Inuit women, however, the association between engagement in traditional pursuits for commercial purposes and the probability of employment is, as expected, negative, possibly suggesting that the engagement in this form of traditional activity is deemed but not reported as employment. The estimated effects on the two health-related measures are also mixed across groups, although for some (Métis and Inuit women) the effects are negative, as expected.

Overall, the addition of social and cultural factors (as measured in this study) to the human capital model does not add much to our understanding of employment probabilities for all Aboriginal groups. For some groups, the addition provides some insights but the main research question remains unresolved for most, and particularly for 
men. A number of factors could be contributing to the observed differences in the impacts of the newly assessed social capital and cultural factors and to the observed puzzlement in the effects of different levels of educational attainment on the probability of employment of individuals belonging to different Aboriginal identity groups. Indeed, some of the contributing factors could be immeasurable or unobservable in the used data sets. As discussed in the Introduction to this thesis, institutional factors, not observable in the statistical information, could play an important role given the history of Aboriginal people in Canada and a qualitative inquiry might prove useful. 


\section{Appendix A}

Appendix A 1: Definitions of explanatory variables in the probit employment models, APS 2006

\begin{tabular}{|c|c|}
\hline Variable & Definition \\
\hline $\begin{array}{l}\text { Education } \\
\text { Some high school or less } \\
\text { Some non-university } \\
\text { Completed non-university } \\
\text { Some university } \\
\text { Completed university }\end{array}$ & $\begin{array}{l}\text { Reference group is respondents with completed high school diploma or equivalency } \\
\text { Dummy }=1 \text { if highest level of schooling attained is some high school or less } \\
\text { Dummy }=1 \text { if highest level of schooling attained is some postsecondary non-university } \\
\text { Dummy }=1 \text { if highest level of schooling attained is completed postsecondary non-university } \\
\text { Dummy }=1 \text { if highest level of schooling attained is some university education } \\
\text { Dummy }=1 \text { if highest level of schooling attained is completed university education }\end{array}$ \\
\hline $\begin{array}{l}\text { Age } \\
\text { Age } 15-19 \\
\text { Age } 25-34 \\
\text { Age } 35-44 \\
\text { Age } 45-54 \\
\text { Age } 55-64\end{array}$ & $\begin{array}{l}\text { Reference group is respondents in the age category } 20-24 \\
\text { Dummy }=1 \text { if respondent is in the age category } 15-19 \\
\text { Dummy }=1 \text { if respondent is in the age category } 25-34 \\
\text { Dummy }=1 \text { if respondent is in the age category } 35-44 \\
\text { Dummy }=1 \text { if respondent is in the age category } 45-54 \\
\text { Dummy }=1 \text { if respondent is in the age category } 55-64\end{array}$ \\
\hline Official Languages & Three dummies for official languages (reference is English only) \\
\hline Technology (access/use) & Dummy $=1$ if respondent used the Internet at home in the past 12 months \\
\hline $\begin{array}{l}\text { Marital Status } \\
\text { Common-law } \\
\text { Legally married } \\
\text { Other marital status }\end{array}$ & $\begin{array}{l}\text { Reference group is respondents who are single, never married } \\
\text { Dummy }=1 \text { if respondent is in a common-law relationship } \\
\text { Dummy }=1 \text { if respondent is married and not separated } \\
\text { Dummy }=1 \text { if respondent is separated, divorced or widowed }\end{array}$ \\
\hline $\begin{array}{l}\text { Presence of Children } \\
\text { Young children } \\
\text { School-aged children } \\
\text { Adult children }\end{array}$ & $\begin{array}{l}\text { Reference group is respondents without dependent children in the household } \\
\text { Dummy }=1 \text { if one or more children are younger than } 6 \\
\text { Dummy }=1 \text { if one or more children are aged } 6 \text { to } 14 \\
\text { Dummy }=1 \text { if one or more children are } 15 \text { or older }\end{array}$ \\
\hline Family structure & Dummy $=1$ if respondent is a single parent \\
\hline Childcare & Dummy $=1$ if respondent had access to safe, affordable childcare \\
\hline $\begin{array}{l}\text { Chronic condition(s) } \\
\text { Activity limitation(s) }\end{array}$ & $\begin{array}{l}\text { Dummy }=1 \text { if respondent has one or more chronic health condition(s) } \\
\text { Dummy }=1 \text { if health condition(s) limit(s) activity of the respondent }\end{array}$ \\
\hline $\begin{array}{l}\text { Social Capital } \\
\text { Decision-making } \\
\text { Club membership } \\
\text { Civic participation }\end{array}$ & $\begin{array}{l}\text { Dummy }=1 \text { if respondent has a network of friends and family to confide in } \\
\text { Dummy }=1 \text { if respondent volunteered in the community in the past } 12 \text { months } \\
\text { Two dummies for voting in recent municipal and provincial elections }\end{array}$ \\
\hline $\begin{array}{l}\text { Cultural Factors } \\
\text { Membership } \\
\text { Aboriginal language/s } \\
\text { Aboriginal language/k } \\
\text { Traditional Activities }\end{array}$ & $\begin{array}{l}\text { Dummy }=1 \text { if member/beneficiary of land claim agreement/band member } \\
\text { Dummy }=1 \text { if respondent speaks an Aboriginal language } \\
\text { Dummy }=1 \text { if respondent holds important to know an Aboriginal language } \\
\text { Three dummies for engaged in any traditional activities in the past } 12 \text { months }\end{array}$ \\
\hline Other Income & Dummy = 1 if respondent has income from other sources \\
\hline Other support & Dummy $=1$ if there are more than one maintainers in the household \\
\hline Area of Residence & $\begin{array}{l}\text { Three dummies for Inuit area of residence (reference is Inuvialuit region) } \\
\text { Seven dummies for other regions (reference is province of Ontario) }\end{array}$ \\
\hline Rural/Urban residence & Dummy $=1$ if respondent lives in rural area \\
\hline
\end{tabular}


Appendix A 2: Definitions of explanatory variables in the robustness checks, Census 2006

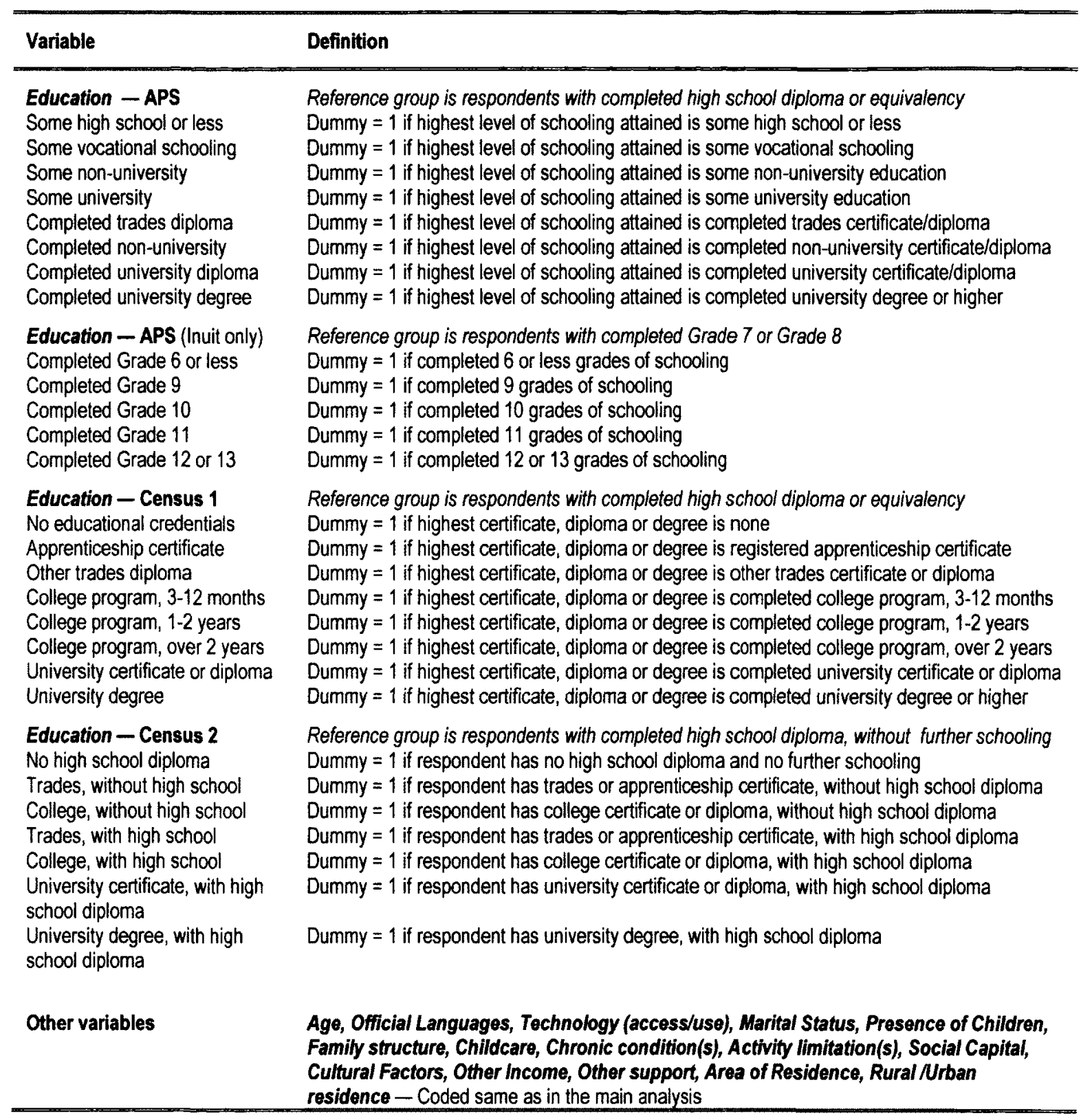




\section{Appendix B}

Appendix B 1: Sample means of all explanatory variables in the probit employment models, APS 2006

\begin{tabular}{|c|c|c|c|c|c|c|c|c|c|c|c|c|}
\hline \multirow{3}{*}{ Characteristics } & \multicolumn{8}{|c|}{ North American Indian } & \multicolumn{2}{|c|}{ Métis } & \multicolumn{2}{|c|}{ Inuit } \\
\hline & \multicolumn{2}{|c|}{ Urban } & \multicolumn{2}{|c|}{ Rural } & \multicolumn{2}{|c|}{ Registered } & \multicolumn{2}{|c|}{ Non-registered } & \multirow[b]{2}{*}{ Men } & \multirow[b]{2}{*}{ Women } & \multirow[b]{2}{*}{ Men } & \multirow[b]{2}{*}{ Women } \\
\hline & Men & Women & Men & Women & Men & Women & Men & Women & & & & \\
\hline \multicolumn{13}{|l|}{ Main education measure } \\
\hline $\begin{array}{l}\text { Less than } \\
\text { high school education }\end{array}$ & $\begin{array}{l}0.258 \\
(0.44)\end{array}$ & $\begin{array}{l}0.131 \\
(0.34)\end{array}$ & $\begin{array}{l}0.290 \\
(0.44)\end{array}$ & $\begin{array}{l}0.172 \\
(0.38)\end{array}$ & $\begin{array}{l}0.281 \\
(0.45)\end{array}$ & $\begin{array}{l}0.159 \\
(0.36)\end{array}$ & $\begin{array}{l}0.246 \\
(0.43)\end{array}$ & $\begin{array}{l}0.112 \\
(0.31)\end{array}$ & $\begin{array}{l}0.221 \\
(0.41)\end{array}$ & $\begin{array}{l}0.159 \\
(0.37)\end{array}$ & $\begin{array}{l}0.516 \\
(0.50)\end{array}$ & $\begin{array}{l}0.403 \\
(0.48)\end{array}$ \\
\hline $\begin{array}{l}\text { Completed } \\
\text { high school education }\end{array}$ & $\begin{array}{l}0.155 \\
(0.36)\end{array}$ & $\begin{array}{l}0.174 \\
(0.38)\end{array}$ & $\begin{array}{l}0.179 \\
(0.37)\end{array}$ & $\begin{array}{l}0.169 \\
(0.38)\end{array}$ & $\begin{array}{l}0.158 \\
(0.37)\end{array}$ & $\begin{array}{l}0.147 \\
(0.35)\end{array}$ & $\begin{array}{l}0.164 \\
(0.37)\end{array}$ & $\begin{array}{l}0.211 \\
(0.41)\end{array}$ & $\begin{array}{l}0.214 \\
(0.41)\end{array}$ & $\begin{array}{l}0.212 \\
(0.41)\end{array}$ & $\begin{array}{l}0.081 \\
(0.28)\end{array}$ & $\begin{array}{l}0.130 \\
(0.33)\end{array}$ \\
\hline $\begin{array}{l}\text { Some } \\
\text { non-university education }\end{array}$ & $\begin{array}{l}0.135 \\
(0.34)\end{array}$ & $\begin{array}{l}0.089 \\
(0.28)\end{array}$ & $\begin{array}{l}0.097 \\
(0.29)\end{array}$ & $\begin{array}{l}0.105 \\
(0.31)\end{array}$ & $\begin{array}{l}0.116 \\
(0.32)\end{array}$ & $\begin{array}{l}0.091 \\
(0.29)\end{array}$ & $\begin{array}{l}0.138 \\
(0.34)\end{array}$ & $\begin{array}{l}0.094 \\
(0.29)\end{array}$ & $\begin{array}{l}0.099 \\
(0.29)\end{array}$ & $\begin{array}{l}0.104 \\
(0.31)\end{array}$ & $\begin{array}{l}0.130 \\
(0.34)\end{array}$ & $\begin{array}{r}0.112 \\
(0.31)\end{array}$ \\
\hline $\begin{array}{l}\text { Completed, } \\
\text { non-university education }\end{array}$ & $\begin{array}{l}0.279 \\
(0.45)\end{array}$ & $\begin{array}{l}0.377 \\
(0.48)\end{array}$ & $\begin{array}{l}0.334 \\
(0.46)\end{array}$ & $\begin{array}{l}0.368 \\
(0.49)\end{array}$ & $\begin{array}{l}0.296 \\
(0.45)\end{array}$ & $\begin{array}{l}0.366 \\
(0.48)\end{array}$ & $\begin{array}{l}0.288 \\
(0.45)\end{array}$ & $\begin{array}{l}0.388 \\
(0.49)\end{array}$ & $\begin{array}{l}0.328 \\
(0.46)\end{array}$ & $\begin{array}{l}0.322 \\
(0.47)\end{array}$ & - & - \\
\hline $\begin{array}{l}\text { Some } \\
\text { university education }\end{array}$ & $\begin{array}{l}0.049 \\
(0.22)\end{array}$ & $\begin{array}{r}0.052 \\
(0.22)\end{array}$ & $\begin{array}{l}0.023 \\
(0.15)\end{array}$ & $\begin{array}{l}0.038 \\
(0.19)\end{array}$ & $\begin{array}{l}0.049 \\
(0.22)\end{array}$ & $\begin{array}{l}0.050 \\
(0.22)\end{array}$ & $\begin{array}{l}0.034 \\
(0.18)\end{array}$ & $\begin{array}{l}0.047 \\
(0.21)\end{array}$ & $\begin{array}{l}0.035 \\
(0.18)\end{array}$ & $\begin{array}{l}0.047 \\
(0.21)\end{array}$ & - & - \\
\hline $\begin{array}{l}\text { Completed } \\
\text { university education }\end{array}$ & $\begin{array}{l}0.124 \\
(0.33)\end{array}$ & $\begin{array}{l}0.177 \\
(0.38\end{array}$ & $\begin{array}{l}0.077 \\
(0.26)\end{array}$ & $\begin{array}{l}0.148 \\
(0.36)\end{array}$ & $\begin{array}{l}0.099 \\
(0.30)\end{array}$ & $\begin{array}{l}0.186 \\
(0.39)\end{array}$ & $\begin{array}{l}0.129 \\
(0.33)\end{array}$ & $\begin{array}{l}0.147 \\
(0.35)\end{array}$ & $\begin{array}{l}0.103 \\
(0.30)\end{array}$ & $\begin{array}{l}0.156 \\
(0.36)\end{array}$ & - & - \\
\hline $\begin{array}{l}\text { Completed } \\
\text { non-university or university }\end{array}$ & - & - & - & - & - & - & - & - & - & - & $\begin{array}{l}0.273 \\
(0.45)\end{array}$ & $\begin{array}{l}0.354 \\
(0.47)\end{array}$ \\
\hline \multicolumn{13}{|c|}{ Education robustness checks I } \\
\hline $\begin{array}{l}\text { Less than } \\
\text { high school education }\end{array}$ & $\begin{array}{l}0.258 \\
(0.44)\end{array}$ & $\begin{array}{l}0.131 \\
(0.34)\end{array}$ & $\begin{array}{r}0.290 \\
(0.44)\end{array}$ & $\begin{array}{l}0.172 \\
(0.38)\end{array}$ & $\begin{array}{l}0.281 \\
(0.45)\end{array}$ & $\begin{array}{l}0.159 \\
(0.36)\end{array}$ & $\begin{array}{l}0.246 \\
(0.43)\end{array}$ & $\begin{array}{l}0.112 \\
(0.31)\end{array}$ & $\begin{array}{l}0.221 \\
(0.41)\end{array}$ & $\begin{array}{l}0.159 \\
(0.37)\end{array}$ & $\begin{array}{l}0.516 \\
(0.50)\end{array}$ & $\begin{array}{l}0.403 \\
(0.48)\end{array}$ \\
\hline $\begin{array}{l}\text { Completed } \\
\text { high school diploma }\end{array}$ & $\begin{array}{l}0.155 \\
(0.36)\end{array}$ & $\begin{array}{l}0.174 \\
(0.38)\end{array}$ & $\begin{array}{l}0.179 \\
(0.37)\end{array}$ & $\begin{array}{l}0.169 \\
(0.38)\end{array}$ & $\begin{array}{l}0.158 \\
\langle 0.37\rangle\end{array}$ & $\begin{array}{l}0.147 \\
(0.35)\end{array}$ & $\begin{array}{l}0.164 \\
(0.37)\end{array}$ & $\begin{array}{l}0.211 \\
(0.41)\end{array}$ & $\begin{array}{l}0.214 \\
(0.41)\end{array}$ & $\begin{array}{l}0.212 \\
(0.41)\end{array}$ & $\begin{array}{l}0.081 \\
(0.27)\end{array}$ & $\begin{array}{l}0.130 \\
(0.33)\end{array}$ \\
\hline $\begin{array}{l}\text { Some } \\
\text { vocational schooling }\end{array}$ & $\begin{array}{l}0.025 \\
(0.16)\end{array}$ & $\begin{array}{l}0.016 \\
(0.12)\end{array}$ & $\begin{array}{l}0.019 \\
(0.13)\end{array}$ & $\begin{array}{l}0.006 \\
(0.08)\end{array}$ & $\begin{array}{l}0.018 \\
(0.13)\end{array}$ & $\begin{array}{l}0.014 \\
(0.12)\end{array}$ & $\begin{array}{l}0.031 \\
(0.17)\end{array}$ & $\begin{array}{l}0.013 \\
(0.11)\end{array}$ & $\begin{array}{l}0.099 \\
(0.29)\end{array}$ & $\begin{array}{l}0.104 \\
(0.31)\end{array}$ & $\begin{array}{l}0.130 \\
(0.34)\end{array}$ & $\begin{array}{l}0.112 \\
(0.31)\end{array}$ \\
\hline
\end{tabular}




\begin{tabular}{|c|c|c|c|c|c|c|c|c|c|c|c|c|}
\hline \multirow{3}{*}{$\begin{array}{l}\text { Appendix B1 } \\
\text { (continued) }\end{array}$} & \multicolumn{8}{|c|}{ North American Indian } & \multicolumn{2}{|c|}{ Métis } & \multicolumn{2}{|c|}{ Inuit } \\
\hline & \multicolumn{2}{|c|}{ Urban } & \multicolumn{2}{|c|}{ Rural } & \multicolumn{2}{|c|}{ Registered } & \multicolumn{2}{|c|}{ Non-registered } & \multirow[b]{2}{*}{ Men } & \multirow[b]{2}{*}{ Women } & \multirow[b]{2}{*}{ Men } & \multirow[b]{2}{*}{ Women } \\
\hline & Men & Women & Men & Women & Men & Women & Men & Women & & & & \\
\hline \multicolumn{13}{|c|}{ Education robustness checks I } \\
\hline $\begin{array}{l}\text { Some } \\
\text { non-university education }\end{array}$ & $\begin{array}{l}0.110 \\
(0.31)\end{array}$ & $\begin{array}{l}0.072 \\
(0.26)\end{array}$ & $\begin{array}{l}0.078 \\
(0.26)\end{array}$ & $\begin{array}{l}0.99 \\
(0.30)\end{array}$ & $\begin{array}{l}0.098 \\
(0.30)\end{array}$ & $\begin{array}{l}0.077 \\
(0.26)\end{array}$ & $\begin{array}{l}0.107 \\
(0.31)\end{array}$ & $\begin{array}{l}0.081 \\
(0.27)\end{array}$ & - & - & - & - \\
\hline $\begin{array}{l}\text { Some } \\
\text { university education }\end{array}$ & $\begin{array}{l}0.049 \\
(0.22)\end{array}$ & $\begin{array}{l}0.052 \\
(0.22)\end{array}$ & $\begin{array}{l}0.023 \\
(0.15)\end{array}$ & $\begin{array}{l}0.038 \\
(0.19)\end{array}$ & $\begin{array}{l}0.049 \\
(0.22)\end{array}$ & $\begin{array}{l}0.050 \\
(0.22)\end{array}$ & $\begin{array}{l}0.034 \\
(0.18)\end{array}$ & $\begin{array}{l}0.047 \\
(0.21)\end{array}$ & $\begin{array}{l}0.035 \\
(0.18)\end{array}$ & $\begin{array}{l}0.047 \\
(0.18)\end{array}$ & - & - \\
\hline $\begin{array}{l}\text { Completed } \\
\text { trades certificate or diploma }\end{array}$ & $\begin{array}{l}0.137 \\
(0.34)\end{array}$ & $\begin{array}{l}0.092 \\
(0.29)\end{array}$ & $\begin{array}{l}0.172 \\
(0.37)\end{array}$ & $\begin{array}{l}0.070 \\
(0.26)\end{array}$ & $\begin{array}{l}0.147 \\
(0.36)\end{array}$ & $\begin{array}{l}0.078 \\
(0.27)\end{array}$ & $\begin{array}{l}0.143 \\
(0.35)\end{array}$ & $\begin{array}{l}0.100 \\
(0.30)\end{array}$ & $\begin{array}{l}0.155 \\
(0.36)\end{array}$ & $\begin{array}{l}0.070 \\
(0.25)\end{array}$ & $\begin{array}{l}0.144 \\
(0.35)\end{array}$ & $\begin{array}{l}0.048 \\
(0.21)\end{array}$ \\
\hline $\begin{array}{l}\text { Completed, } \\
\text { non-university diploma }\end{array}$ & $\begin{array}{l}0.142 \\
(0.35)\end{array}$ & $\begin{array}{l}0.285 \\
(0.45)\end{array}$ & $\begin{array}{l}0.162 \\
(0.36)\end{array}$ & $\begin{array}{l}0.298 \\
(0.47)\end{array}$ & $\begin{array}{l}0.149 \\
(0.36)\end{array}$ & $\begin{array}{l}0.288 \\
(0.45)\end{array}$ & $\begin{array}{r}0.145 \\
(0.35)\end{array}$ & $\begin{array}{l}0.288 \\
(0.45)\end{array}$ & $\begin{array}{l}0.173 \\
(0.37)\end{array}$ & $\begin{array}{l}0.254 \\
(0.44)\end{array}$ & $\begin{array}{l}0.109 \\
(0.31)\end{array}$ & $\begin{array}{l}0.226 \\
(0.41)\end{array}$ \\
\hline $\begin{array}{l}\text { Completed } \\
\text { university diploma }\end{array}$ & $\begin{array}{l}0.018 \\
(0.13)\end{array}$ & $\begin{array}{l}0.023 \\
(0.15)\end{array}$ & $\begin{array}{l}0.022 \\
(0.14)\end{array}$ & $\begin{array}{l}0.037 \\
(0.19)\end{array}$ & $\begin{array}{l}0.015 \\
(0.12)\end{array}$ & $\begin{array}{l}0.030 \\
(0.17)\end{array}$ & $\begin{array}{l}0.024 \\
(0.15)\end{array}$ & $\begin{array}{l}0.020 \\
(0.14)\end{array}$ & - & - & - & - \\
\hline $\begin{array}{l}\text { Completed } \\
\text { university degree }\end{array}$ & $\begin{array}{l}0.105 \\
(0.31)\end{array}$ & $\begin{array}{l}0.154 \\
(0.36)\end{array}$ & $\begin{array}{l}0.055 \\
(0.22)\end{array}$ & $\begin{array}{l}0.111 \\
(0.32)\end{array}$ & $\begin{array}{l}0.083 \\
(0.28)\end{array}$ & $\begin{array}{l}0.157 \\
(0.36)\end{array}$ & $\begin{array}{l}0.105 \\
(0.31)\end{array}$ & $\begin{array}{l}0.127 \\
(0.33)\end{array}$ & $\begin{array}{l}0.103 \\
(0.30)\end{array}$ & $\begin{array}{l}0.156 \\
(0.36)\end{array}$ & $\begin{array}{l}0.020 \\
(0.14)\end{array}$ & $\begin{array}{l}0.080 \\
(0.27)\end{array}$ \\
\hline \multicolumn{13}{|c|}{ Education robustness checks II } \\
\hline Grade 6 or less & - & - & - & - & - & - & - & - & - & - & $\begin{array}{l}0.165 \\
(0.37)\end{array}$ & $\begin{array}{l}0.090 \\
(0.28)\end{array}$ \\
\hline Grade 7 to Grade 8 & - & - & - & - & - & - & - & - & - & - & $\begin{array}{l}0.170 \\
(0.38)\end{array}$ & $\begin{array}{l}0.168 \\
(0.37)\end{array}$ \\
\hline Grade 9 & - & - & - & - & - & - & - & - & - & - & $\begin{array}{l}0.144 \\
(0.35)\end{array}$ & $\begin{array}{l}0.168 \\
(0.37)\end{array}$ \\
\hline Grade 10 & - & - & - & - & - & - & - & - & - & - & $\begin{array}{l}0.188 \\
(0.39)\end{array}$ & $\begin{array}{l}0.167 \\
(0.37)\end{array}$ \\
\hline Grade 11 & - & - & - & - & - & - & - & - & - & - & $\begin{array}{l}0.156 \\
(0.37)\end{array}$ & $\begin{array}{l}0.087 \\
(0.28)\end{array}$ \\
\hline
\end{tabular}




\begin{tabular}{|c|c|c|c|c|c|c|c|c|c|c|c|c|}
\hline \multirow{3}{*}{$\begin{array}{l}\text { Appendix B1 } \\
\text { (continued) }\end{array}$} & \multicolumn{8}{|c|}{ North American Indian } & \multicolumn{2}{|c|}{ Métis } & \multicolumn{2}{|c|}{ Inuit } \\
\hline & \multicolumn{2}{|c|}{ Urban } & \multicolumn{2}{|c|}{ Rural } & \multicolumn{2}{|c|}{ Registered } & \multicolumn{2}{|c|}{ Non-registered } & \multirow[b]{2}{*}{ Men } & \multirow[b]{2}{*}{ Women } & \multirow[b]{2}{*}{ Men } & \multirow[b]{2}{*}{ Women } \\
\hline & Men & Women & Men & Women & Men & Women & Men & Women & & & & \\
\hline \multicolumn{13}{|c|}{ Education robustness checks II } \\
\hline Grade 12 or 13 , incomplete & - & - & - & - & - & - & - & - & - & - & $\begin{array}{l}0.176 \\
(0.38)\end{array}$ & $\begin{array}{l}0.320 \\
(0.46)\end{array}$ \\
\hline Age 15-19 & $\begin{array}{l}0.059 \\
(0.24)\end{array}$ & $\begin{array}{l}0.056 \\
(0.23)\end{array}$ & $\begin{array}{l}0.052 \\
(0.22)\end{array}$ & $\begin{array}{l}0.039 \\
(0.20\rangle\end{array}$ & $\begin{array}{l}0.063 \\
(0.24)\end{array}$ & $\begin{array}{l}0.035 \\
(0.18)\end{array}$ & $\begin{array}{l}0.049 \\
(0.21)\end{array}$ & $\begin{array}{l}0.079 \\
(0.27)\end{array}$ & $\begin{array}{l}0.053 \\
(0.22)\end{array}$ & $\begin{array}{l}0.049 \\
(0.22)\end{array}$ & $\begin{array}{l}0.058 \\
(0.24)\end{array}$ & $\begin{array}{l}0.075 \\
(0.26)\end{array}$ \\
\hline Age 20-24 & $\begin{array}{l}0.111 \\
(0.31)\end{array}$ & $\begin{array}{l}0.083 \\
(0.27)\end{array}$ & $\begin{array}{l}0.088 \\
(0.28)\end{array}$ & $\begin{array}{l}0.071 \\
(0.26)\end{array}$ & $\begin{array}{l}0.105 \\
(0.31)\end{array}$ & $\begin{array}{l}0.080 \\
(0.27)\end{array}$ & $\begin{array}{l}0.106 \\
(0.31)\end{array}$ & $\begin{array}{l}0.081 \\
(0.27)\end{array}$ & $\begin{array}{l}0.085 \\
(0.28)\end{array}$ & $\begin{array}{l}0.085 \\
(0.28)\end{array}$ & $\begin{array}{l}0.120 \\
(0.33)\end{array}$ & $\begin{array}{l}0.100 \\
(0.29)\end{array}$ \\
\hline Age 25-34 & $\begin{array}{l}0.150 \\
(0.43)\end{array}$ & $\begin{array}{l}0.226 \\
(0.42)\end{array}$ & $\begin{array}{l}0.194 \\
(0.39)\end{array}$ & $\begin{array}{l}0.199 \\
(0.41)\end{array}$ & $\begin{array}{l}0.244 \\
(0.43)\end{array}$ & $\begin{array}{l}0.242 \\
(0.43)\end{array}$ & $\begin{array}{l}0.225 \\
(0.42)\end{array}$ & $\begin{array}{l}0.188 \\
(0.39)\end{array}$ & $\begin{array}{l}0.241 \\
(0.42)\end{array}$ & $\begin{array}{l}0.223 \\
(0.42)\end{array}$ & $\begin{array}{l}0.218 \\
(0.42)\end{array}$ & $\begin{array}{l}0.298 \\
(0.45)\end{array}$ \\
\hline Age $35-44$ & $\begin{array}{l}0.278 \\
(0.45)\end{array}$ & $\begin{array}{l}0.302 \\
(0.46)\end{array}$ & $\begin{array}{l}0.272 \\
(0.44)\end{array}$ & $\begin{array}{l}0.308 \\
(0.47)\end{array}$ & $\begin{array}{l}0.275 \\
(0.45)\end{array}$ & $\begin{array}{l}0.299 \\
(0.45)\end{array}$ & $\begin{array}{l}0.278 \\
(0.45)\end{array}$ & $\begin{array}{l}0.312 \\
(0.46)\end{array}$ & $\begin{array}{l}0.240 \\
(0.42)\end{array}$ & $\begin{array}{l}0.269 \\
(0.45)\end{array}$ & $\begin{array}{l}0.285 \\
(0.46)\end{array}$ & $\begin{array}{l}0.296 \\
(0.45)\end{array}$ \\
\hline Age $45-54$ & $\begin{array}{l}0.221 \\
(0.42)\end{array}$ & $\begin{array}{l}0.226 \\
(0.42)\end{array}$ & $\begin{array}{l}0.271 \\
(0.44)\end{array}$ & $\begin{array}{l}0.276 \\
(0.46)\end{array}$ & $\begin{array}{l}0.208 \\
(0.41)\end{array}$ & $\begin{array}{l}0.230 \\
(0.42)\end{array}$ & $\begin{array}{l}0.268 \\
(0.44)\end{array}$ & $\begin{array}{l}0.249 \\
(0.43)\end{array}$ & $\begin{array}{l}0.244 \\
(0.42)\end{array}$ & $\begin{array}{l}0.282 \\
(0.45)\end{array}$ & $\begin{array}{l}0.207 \\
(0.41)\end{array}$ & $\begin{array}{l}0.175 \\
(0.37)\end{array}$ \\
\hline Age $55-64$ & $\begin{array}{l}0.080 \\
(0.27)\end{array}$ & $\begin{array}{l}0.106 \\
(0.31)\end{array}$ & $\begin{array}{l}0.122 \\
(0.32)\end{array}$ & $\begin{array}{l}0.106 \\
(0.31)\end{array}$ & $\begin{array}{l}0.103 \\
(0.31)\end{array}$ & $\begin{array}{l}0.114 \\
(0.32)\end{array}$ & $\begin{array}{l}0.073 \\
(0.26)\end{array}$ & $\begin{array}{l}0.093 \\
(0.29)\end{array}$ & $\begin{array}{l}0.137 \\
(0.34)\end{array}$ & $\begin{array}{l}0.093 \\
(0.29)\end{array}$ & $\begin{array}{l}0.110 \\
(0.32)\end{array}$ & $\begin{array}{l}0.057 \\
(0.23)\end{array}$ \\
\hline English only & $\begin{array}{l}0.851 \\
(0.36)\end{array}$ & $\begin{array}{l}0.820 \\
(0.39)\end{array}$ & $\begin{array}{l}0.836 \\
(0.36)\end{array}$ & $\begin{array}{l}0.856 \\
(0.36)\end{array}$ & $\begin{array}{l}0.898 \\
(0.30)\end{array}$ & $\begin{array}{l}0.881 \\
(0.32)\end{array}$ & $\begin{array}{l}0.781 \\
(0.41)\end{array}$ & $\begin{array}{l}0.740 \\
(0.44)\end{array}$ & $\begin{array}{l}0.723 \\
(0.44)\end{array}$ & $\begin{array}{l}0.722 \\
(0.45)\end{array}$ & $\begin{array}{l}0.927 \\
(0.26)\end{array}$ & $\begin{array}{l}0.923 \\
(0.26)\end{array}$ \\
\hline French only & $\begin{array}{l}0.036 \\
(0.19)\end{array}$ & $\begin{array}{l}0.037 \\
(0.19)\end{array}$ & $\begin{array}{l}0.068 \\
(0.25)\end{array}$ & $\begin{array}{l}0.028 \\
(0.17)\end{array}$ & $\begin{array}{l}0.037 \\
(0.19)\end{array}$ & $\begin{array}{l}0.025 \\
(0.15)\end{array}$ & $\begin{array}{l}0.053 \\
(0.22)\end{array}$ & $\begin{array}{l}0.051 \\
(0.22)\end{array}$ & $\begin{array}{l}0.054 \\
(0.22)\end{array}$ & $\begin{array}{l}0.052 \\
(0.22)\end{array}$ & $\begin{array}{l}0.015 \\
(0.12)\end{array}$ & $\begin{array}{l}0.017 \\
(0.13)\end{array}$ \\
\hline Both official languages & $\begin{array}{l}0.113 \\
(0.32)\end{array}$ & $\begin{array}{l}0.147 \\
(0.35)\end{array}$ & $\begin{array}{l}0.095 \\
(0.29)\end{array}$ & $\begin{array}{l}0.116 \\
(0.33)\end{array}$ & $\begin{array}{l}0.065 \\
(0.25)\end{array}$ & $\begin{array}{l}0.094 \\
(0.29)\end{array}$ & $\begin{array}{l}0.165 \\
(0.37)\end{array}$ & $\begin{array}{l}0.209 \\
(0.41)\end{array}$ & $\begin{array}{l}0.223 \\
(0.41)\end{array}$ & $\begin{array}{l}0.226 \\
(0.42)\end{array}$ & $\begin{array}{l}0.058 \\
(0.23)\end{array}$ & $\begin{array}{l}0.060 \\
(0.23)\end{array}$ \\
\hline Access/use of technology & $\begin{array}{l}0.761 \\
(0.43)\end{array}$ & $\begin{array}{l}0.759 \\
(0.43)\end{array}$ & $\begin{array}{l}0.605 \\
(0.48)\end{array}$ & $\begin{array}{l}0.705 \\
(0.46)\end{array}$ & $\begin{array}{l}0.681 \\
(0.47)\end{array}$ & $\begin{array}{c}0.732 \\
(0.44)\end{array}$ & $\begin{array}{l}0.776 \\
(0.42)\end{array}$ & $\begin{array}{l}0.769 \\
(0.42)\end{array}$ & $\begin{array}{l}0.771 \\
(0.42)\end{array}$ & $\begin{array}{l}0.772 \\
(0.42)\end{array}$ & $\begin{array}{l}0.438 \\
(0.50)\end{array}$ & $\begin{array}{l}0.491 \\
(0.49)\end{array}$ \\
\hline
\end{tabular}




\begin{tabular}{|c|c|c|c|c|c|c|c|c|c|c|c|c|}
\hline \multirow{3}{*}{$\begin{array}{l}\text { Appendix B1 } \\
\text { (continued) }\end{array}$} & \multicolumn{8}{|c|}{ North American Indian } & \multicolumn{2}{|c|}{ Métis } & \multicolumn{2}{|c|}{ Inuit } \\
\hline & \multicolumn{2}{|c|}{ Urban } & \multicolumn{2}{|c|}{ Rural } & \multicolumn{2}{|c|}{ Registered } & \multicolumn{2}{|c|}{ Non-registered } & \multirow[b]{2}{*}{ Men } & \multirow[b]{2}{*}{ Women } & \multirow[b]{2}{*}{ Men } & \multirow[b]{2}{*}{ Women } \\
\hline & Men & Women & Men & Women & Men & Women & Men & Women & & & & \\
\hline Legally married & $\begin{array}{l}0.370 \\
(0.48)\end{array}$ & $\begin{array}{l}0.331 \\
(0.47)\end{array}$ & $\begin{array}{c}0.464 \\
(0.49)\end{array}$ & $\begin{array}{l}0.487 \\
(0.51)\end{array}$ & $\begin{array}{l}0.354 \\
(0.48)\end{array}$ & $\begin{array}{l}0.336 \\
(0.47)\end{array}$ & $\begin{array}{l}0.446 \\
(0.50)\end{array}$ & $\begin{array}{l}0.411 \\
(0.49)\end{array}$ & $\begin{array}{l}0.469 \\
(0.49)\end{array}$ & $\begin{array}{l}0.463 \\
(0.50)\end{array}$ & $\begin{array}{l}0.409 \\
(0.50)\end{array}$ & $\begin{array}{l}0.400 \\
(0.48)\end{array}$ \\
\hline Common-law & $\begin{array}{l}0.207 \\
(0.41)\end{array}$ & $\begin{array}{l}0.200 \\
(0.40)\end{array}$ & $\begin{array}{l}0.207 \\
(0.40)\end{array}$ & $\begin{array}{l}0.190 \\
(0.40)\end{array}$ & $\begin{array}{l}0.217 \\
(0.41)\end{array}$ & $\begin{array}{l}0.205 \\
(0.40)\end{array}$ & $\begin{array}{l}0.192 \\
(0.39)\end{array}$ & $\begin{array}{l}0.184 \\
(0.39)\end{array}$ & $\begin{array}{l}0.166 \\
(0.37)\end{array}$ & $\begin{array}{l}0.188 \\
(0.39)\end{array}$ & $\begin{array}{l}0.255 \\
(0.44)\end{array}$ & $\begin{array}{c}0.219 \\
(0.41)\end{array}$ \\
\hline Divorced-separated & $\begin{array}{l}0.123 \\
(0.33)\end{array}$ & $\begin{array}{l}0.196 \\
(0.39)\end{array}$ & $\begin{array}{l}0.104 \\
(0.30)\end{array}$ & $\begin{array}{l}0.176 \\
(0.39)\end{array}$ & $\begin{array}{l}0.110 \\
(0.31)\end{array}$ & $\begin{array}{l}0.195 \\
(0.39)\end{array}$ & $\begin{array}{l}0.130 \\
(0.33)\end{array}$ & $\begin{array}{l}0.186 \\
(0.39)\end{array}$ & $\begin{array}{l}0.128 \\
(0.33)\end{array}$ & $\begin{array}{l}0.175 \\
(0.38)\end{array}$ & $\begin{array}{l}0.057 \\
(0.23)\end{array}$ & $\begin{array}{l}0.068 \\
(0.25)\end{array}$ \\
\hline Single & $\begin{array}{l}0.506 \\
(0.50)\end{array}$ & $\begin{array}{l}0.473 \\
(0.50)\end{array}$ & $\begin{array}{l}0.432 \\
(0.48)\end{array}$ & $\begin{array}{l}0.337 \\
(0.48)\end{array}$ & $\begin{array}{l}0.535 \\
(0.50)\end{array}$ & $\begin{array}{l}0.469 \\
(0.50)\end{array}$ & $\begin{array}{l}0.424 \\
(0.49)\end{array}$ & $\begin{array}{l}0.403 \\
(0.49)\end{array}$ & $\begin{array}{l}0.402 \\
(0.48)\end{array}$ & $\begin{array}{l}0.361 \\
(0.48)\end{array}$ & $\begin{array}{c}0.534 \\
(0.50)\end{array}$ & $\begin{array}{l}0.532 \\
(0.49)\end{array}$ \\
\hline No children present & $\begin{array}{l}0.585 \\
(0.49)\end{array}$ & $\begin{array}{l}0.538 \\
(0.50)\end{array}$ & $\begin{array}{l}0.525 \\
(0.49)\end{array}$ & $\begin{array}{l}0.429 \\
(0.50)\end{array}$ & $\begin{array}{l}0.565 \\
(0.50)\end{array}$ & $\begin{array}{l}0.491 \\
(0.50)\end{array}$ & $\begin{array}{l}0.577 \\
(0.49)\end{array}$ & $\begin{array}{l}0.548 \\
(0.50)\end{array}$ & $\begin{array}{l}0.571 \\
(0.49)\end{array}$ & $\begin{array}{l}0.509 \\
(0.50)\end{array}$ & $\begin{array}{l}0.327 \\
(0.47)\end{array}$ & $\begin{array}{l}0.265 \\
(0.44)\end{array}$ \\
\hline Children $<6$ & $\begin{array}{l}0.165 \\
(0.37)\end{array}$ & $\begin{array}{l}0.135 \\
(0.34)\end{array}$ & $\begin{array}{l}0.165 \\
(0.36)\end{array}$ & $\begin{array}{l}0.174 \\
(0.39)\end{array}$ & $\begin{array}{l}0.161 \\
(0.37)\end{array}$ & $\begin{array}{l}0.144 \\
(0.35)\end{array}$ & $\begin{array}{l}0.171 \\
(0.38)\end{array}$ & $\begin{array}{l}0.143 \\
(0.35)\end{array}$ & $\begin{array}{l}0.131 \\
(0.33)\end{array}$ & $\begin{array}{l}0.147 \\
(0.36)\end{array}$ & $\begin{array}{c}0.312 \\
(0.47)\end{array}$ & $\begin{array}{l}0.358 \\
(0.47)\end{array}$ \\
\hline Children 6-14 & $\begin{array}{l}0.149 \\
(0.36)\end{array}$ & $\begin{array}{l}0.179 \\
(0.38)\end{array}$ & $\begin{array}{l}0.190 \\
(0.38)\end{array}$ & $\begin{array}{l}0.233 \\
(0.43)\end{array}$ & $\begin{array}{c}0.164 \\
(0.37)\end{array}$ & $\begin{array}{l}0.207 \\
(0.40)\end{array}$ & $\begin{array}{l}0.153 \\
(0.36)\end{array}$ & $\begin{array}{l}0.168 \\
(0.37)\end{array}$ & $\begin{array}{c}0.164 \\
(0.37)\end{array}$ & $\begin{array}{l}0.188 \\
(0.39)\end{array}$ & $\begin{array}{l}0.236 \\
(0.43)\end{array}$ & $\begin{array}{l}0.295 \\
(0.45)\end{array}$ \\
\hline Children 14-24 & $\begin{array}{l}0.100 \\
(0.30)\end{array}$ & $\begin{array}{l}0.147 \\
(0.35)\end{array}$ & $\begin{array}{l}0.120 \\
(0.32)\end{array}$ & $\begin{array}{l}0.163 \\
(0.38)\end{array}$ & $\begin{array}{l}0.110 \\
(0.31)\end{array}$ & $\begin{array}{l}0.157 \\
(0.36)\end{array}$ & $\begin{array}{l}0.099 \\
(0.30)\end{array}$ & $\begin{array}{l}0.141 \\
(0.35)\end{array}$ & $\begin{array}{l}0.133 \\
(0.34)\end{array}$ & $\begin{array}{l}0.156 \\
(0.37)\end{array}$ & $\begin{array}{l}0.125 \\
(0.33)\end{array}$ & $\begin{array}{l}0.082 \\
(0.27)\end{array}$ \\
\hline Lone parent & $\begin{array}{l}0.042 \\
(0.20)\end{array}$ & $\begin{array}{l}0.159 \\
(0.36)\end{array}$ & $\begin{array}{l}0.033 \\
(0.17)\end{array}$ & $\begin{array}{l}0.127 \\
(0.34)\end{array}$ & $\begin{array}{l}0.047 \\
(0.21)\end{array}$ & $\begin{array}{l}0.180 \\
(0.38)\end{array}$ & $\begin{array}{l}0.030 \\
(0.17)\end{array}$ & $\begin{array}{l}0.111 \\
(0.31)\end{array}$ & $\begin{array}{c}0.034 \\
(0.18)\end{array}$ & $\begin{array}{l}0.113 \\
(0.32)\end{array}$ & $\begin{array}{l}0.062 \\
(0.24)\end{array}$ & $\begin{array}{l}0.172 \\
(0.37)\end{array}$ \\
\hline Access to childcare & - & - & - & - & - & - & - & - & $\begin{array}{l}0.160 \\
(0.36)\end{array}$ & $\begin{array}{c}0.211 \\
(0.41)\end{array}$ & - & - \\
\hline Chronic health condition & $\begin{array}{l}0.479 \\
(0.50)\end{array}$ & $\begin{array}{l}0.526 \\
(0.50)\end{array}$ & $\begin{array}{l}0.458 \\
(0.49)\end{array}$ & $\begin{array}{l}0.508 \\
(0.51)\end{array}$ & $\begin{array}{c}0.457 \\
(0.50)\end{array}$ & $\begin{array}{l}0.514 \\
(0.50)\end{array}$ & $\begin{array}{c}0.497 \\
(0.50)\end{array}$ & $\begin{array}{l}0.534 \\
(0.50)\end{array}$ & $\begin{array}{l}0.476 \\
(0.49)\end{array}$ & $\begin{array}{l}0.528 \\
(0.50)\end{array}$ & $\begin{array}{l}0.319 \\
(0.47)\end{array}$ & $\begin{array}{l}0.460 \\
(0.49)\end{array}$ \\
\hline
\end{tabular}




\begin{tabular}{|c|c|c|c|c|c|c|c|c|c|c|c|c|}
\hline \multirow{3}{*}{$\begin{array}{l}\text { Appendix B1 } \\
\text { (continued) }\end{array}$} & \multicolumn{8}{|c|}{ North American Indian } & \multicolumn{2}{|c|}{ Métis } & \multicolumn{2}{|c|}{ Inuit } \\
\hline & \multicolumn{2}{|c|}{ Urban } & \multicolumn{2}{|c|}{ Rural } & \multicolumn{2}{|c|}{ Registered } & \multicolumn{2}{|c|}{ Non-registered } & \multirow[b]{2}{*}{ Men } & \multirow[b]{2}{*}{ Women } & \multirow[b]{2}{*}{ Men } & \multirow[b]{2}{*}{ Women } \\
\hline & Men & Women & Men & Women & Men & Women & Men & Women & & & & \\
\hline Health activity limitation & $\begin{array}{l}0.296 \\
(0.46)\end{array}$ & $\begin{array}{l}0.326 \\
(0.47)\end{array}$ & $\begin{array}{l}0.324 \\
(0.46)\end{array}$ & $\begin{array}{l}0.321 \\
(0.48)\end{array}$ & $\begin{array}{l}0.291 \\
(0.46)\end{array}$ & $\begin{array}{l}0.326 \\
(0.47)\end{array}$ & $\begin{array}{l}0.319 \\
(0.46)\end{array}$ & $\begin{array}{l}0.323 \\
(0.47)\end{array}$ & $\begin{array}{l}0.326 \\
(0.46)\end{array}$ & $\begin{array}{l}0.310 \\
(0.47)\end{array}$ & $\begin{array}{l}0.294 \\
(0.46)\end{array}$ & $\begin{array}{l}0.224 \\
(0.41)\end{array}$ \\
\hline Other sources of income & $\begin{array}{l}0.143 \\
(0.35)\end{array}$ & $\begin{array}{l}0.268 \\
(0.44)\end{array}$ & $\begin{array}{l}0.162 \\
(0.36)\end{array}$ & $\begin{array}{l}0.267 \\
(0.45)\end{array}$ & $\begin{array}{l}0.168 \\
(0.37)\end{array}$ & $\begin{array}{l}0.264 \\
(0.44)\end{array}$ & $\begin{array}{l}0.120 \\
(0.32)\end{array}$ & $\begin{array}{l}0.275 \\
(0.45)\end{array}$ & $\begin{array}{l}0.160 \\
(0.36)\end{array}$ & $\begin{array}{l}0.155 \\
(0.44)\end{array}$ & $\begin{array}{l}0.340 \\
(0.48)\end{array}$ & $\begin{array}{l}0.473 \\
(0.49)\end{array}$ \\
\hline Household maintainers & $\begin{array}{l}0.503 \\
(0.50)\end{array}$ & $\begin{array}{l}0.472 \\
(0.50)\end{array}$ & $\begin{array}{l}0.511 \\
(0.49)\end{array}$ & $\begin{array}{l}0.488 \\
(0.51)\end{array}$ & $\begin{array}{l}0.454 \\
(0.50)\end{array}$ & $\begin{array}{l}0.445 \\
(0.49)\end{array}$ & $\begin{array}{l}0.572 \\
(0.49)\end{array}$ & $\begin{array}{l}0.521 \\
(0.50)\end{array}$ & $\begin{array}{l}0.524 \\
(0.49)\end{array}$ & $\begin{array}{l}0.538 \\
(0.50)\end{array}$ & $\begin{array}{l}0.382 \\
(0.49)\end{array}$ & $\begin{array}{l}0.377 \\
(0.48)\end{array}$ \\
\hline Respondent maintainer & $\begin{array}{l}0.572 \\
(0.50)\end{array}$ & $\begin{array}{l}0.456 \\
(0.50)\end{array}$ & $\begin{array}{l}0.563 \\
(0.49)\end{array}$ & $\begin{array}{l}0.408 \\
(0.50)\end{array}$ & $\begin{array}{l}0.540 \\
(0.50)\end{array}$ & $\begin{array}{c}0.484 \\
(0.50)\end{array}$ & $\begin{array}{l}0.610 \\
(0.49)\end{array}$ & $\begin{array}{l}0.387 \\
(0.49)\end{array}$ & $\begin{array}{l}0.626 \\
(0.48)\end{array}$ & $\begin{array}{l}0.384 \\
(0.49)\end{array}$ & $\begin{array}{l}0.526 \\
(0.50)\end{array}$ & $\begin{array}{l}0.490 \\
(0.49)\end{array}$ \\
\hline Atlantic provinces & $\begin{array}{l}0.044 \\
(0.20)\end{array}$ & $\begin{array}{l}0.047 \\
(0.21)\end{array}$ & $\begin{array}{l}0.151 \\
(0.35)\end{array}$ & $\begin{array}{l}0.152 \\
(0.37)\end{array}$ & $\begin{array}{l}0.047 \\
(0.21)\end{array}$ & $\begin{array}{l}0.041 \\
(0.20)\end{array}$ & $\begin{array}{l}0.101 \\
(0.30)\end{array}$ & $\begin{array}{l}0.115 \\
(0.32)\end{array}$ & $\begin{array}{l}0.028 \\
(0.16)\end{array}$ & $\begin{array}{l}0.025 \\
(0.16)\end{array}$ & - & - \\
\hline Northern Territories & $\begin{array}{l}0.013 \\
(0.11)\end{array}$ & $\begin{array}{l}0.013 \\
(0.11)\end{array}$ & $\begin{array}{l}0.100 \\
(0.29)\end{array}$ & $\begin{array}{l}0.103 \\
(0.31)\end{array}$ & $\begin{array}{l}0.056 \\
(0.23)\end{array}$ & $\begin{array}{l}0.052 \\
(0.22)\end{array}$ & $\begin{array}{l}0.007 \\
(0.08)\end{array}$ & $\begin{array}{l}0.007 \\
(0.08)\end{array}$ & $\begin{array}{l}0.006 \\
(0.07)\end{array}$ & $\begin{array}{l}0.006 \\
(0.08)\end{array}$ & - & - \\
\hline Quebec & $\begin{array}{l}0.090 \\
(0.29)\end{array}$ & $\begin{array}{l}0.102 \\
(0.30)\end{array}$ & $\begin{array}{l}0.108 \\
(0.30)\end{array}$ & $\begin{array}{c}0.70 \\
(0.26)\end{array}$ & $\begin{array}{l}0.068 \\
(0.25)\end{array}$ & $\begin{array}{l}0.071 \\
(0.25)\end{array}$ & $\begin{array}{l}0.129 \\
(0.33)\end{array}$ & $\begin{array}{l}0.130 \\
(0.34)\end{array}$ & $\begin{array}{l}0.135 \\
(0.34)\end{array}$ & $\begin{array}{l}0.127 \\
(0.34)\end{array}$ & - & - \\
\hline Ontario & $\begin{array}{l}0.333 \\
(0.47)\end{array}$ & $\begin{array}{l}0.342 \\
(0.47)\end{array}$ & $\begin{array}{l}0.269 \\
(0.43)\end{array}$ & $\begin{array}{l}0.286 \\
(0.46)\end{array}$ & $\begin{array}{l}0.250 \\
(0.43)\end{array}$ & $\begin{array}{l}0.260 \\
(0.44)\end{array}$ & $\begin{array}{l}0.406 \\
(0.49)\end{array}$ & $\begin{array}{l}0.432 \\
(0.50)\end{array}$ & $\begin{array}{l}0.191 \\
(0.39)\end{array}$ & $\begin{array}{l}0.177 \\
(0.38)\end{array}$ & - & - \\
\hline Manitoba & $\begin{array}{l}0.087 \\
(0.28)\end{array}$ & $\begin{array}{l}0.082 \\
(0.27)\end{array}$ & $\begin{array}{l}0.084 \\
(0.27)\end{array}$ & $\begin{array}{l}0.079 \\
(0.27)\end{array}$ & $\begin{array}{l}0.124 \\
(0.33)\end{array}$ & $\begin{array}{l}0.114 \\
(0.32)\end{array}$ & $\begin{array}{l}0.036 \\
(0.19)\end{array}$ & $\begin{array}{l}0.032 \\
(0.18)\end{array}$ & $\begin{array}{l}0.239 \\
(0.42)\end{array}$ & $\begin{array}{l}0.213 \\
(0.41)\end{array}$ & - & - \\
\hline Saskatchewan & $\begin{array}{l}0.072 \\
(0.26)\end{array}$ & $\begin{array}{l}0.059 \\
(0.23)\end{array}$ & $\begin{array}{l}0.048 \\
(0.21)\end{array}$ & $\begin{array}{l}0.067 \\
(0.25)\end{array}$ & $\begin{array}{l}0.093 \\
(0.29)\end{array}$ & $\begin{array}{l}0.086 \\
(0.28)\end{array}$ & $\begin{array}{l}0.029 \\
(0.17)\end{array}$ & $\begin{array}{l}0.023 \\
(0.15)\end{array}$ & $\begin{array}{l}0.115 \\
(0.32)\end{array}$ & $\begin{array}{l}0.129 \\
(0.34)\end{array}$ & - & - \\
\hline Alberta & $\begin{array}{l}0.171 \\
(0.38)\end{array}$ & $\begin{array}{l}0.133 \\
(0.34)\end{array}$ & $\begin{array}{l}0.116 \\
(0.31)\end{array}$ & $\begin{array}{l}0.102 \\
(0.31)\end{array}$ & $\begin{array}{l}0.171 \\
(0.38)\end{array}$ & $\begin{array}{l}0.149 \\
(0.35)\end{array}$ & $\begin{array}{l}0.138 \\
(0.34)\end{array}$ & $\begin{array}{l}0.092 \\
(0.29)\end{array}$ & $\begin{array}{l}0.252 \\
(0.43)\end{array}$ & $\begin{array}{l}0.275 \\
(0.45)\end{array}$ & - & - \\
\hline British Columbia & $\begin{array}{l}0.190 \\
(0.39)\end{array}$ & $\begin{array}{l}0.221 \\
(0.41)\end{array}$ & $\begin{array}{l}0.122 \\
(0.32)\end{array}$ & $\begin{array}{l}0.139 \\
(0.35)\end{array}$ & $\begin{array}{l}0.189 \\
(0.39)\end{array}$ & $\begin{array}{l}0.226 \\
(0.42)\end{array}$ & $\begin{array}{l}0.153 \\
(0.36)\end{array}$ & $\begin{array}{l}0.168 \\
(0.37)\end{array}$ & $\begin{array}{l}0.034 \\
(0.18)\end{array}$ & $\begin{array}{l}0.048 \\
(0.22)\end{array}$ & - & - \\
\hline
\end{tabular}




\begin{tabular}{|c|c|c|c|c|c|c|c|c|c|c|c|c|}
\hline \multirow{3}{*}{$\begin{array}{l}\text { Appendix B1 } \\
\text { (continued) }\end{array}$} & \multicolumn{8}{|c|}{ North American Indian } & \multicolumn{2}{|c|}{ Métis } & \multicolumn{2}{|c|}{ Inuit } \\
\hline & \multicolumn{2}{|c|}{ Untan } & \multicolumn{2}{|c|}{ Rural } & \multicolumn{2}{|c|}{ Registered } & \multicolumn{2}{|c|}{ Non-registered } & \multirow[b]{2}{*}{ Men } & \multirow[b]{2}{*}{ Women } & \multirow[b]{2}{*}{ Men } & \multirow[b]{2}{*}{ Women } \\
\hline & Men & Women & Men & Women & Men & Women & Men & Women & & & & \\
\hline Rural place of residence & - & - & - & - & $\begin{array}{l}0.259 \\
(0.44)\end{array}$ & $\begin{array}{l}0.230 \\
(0.42)\end{array}$ & $\begin{array}{l}0.241 \\
(0.43)\end{array}$ & $\begin{array}{l}0.216 \\
(0.41)\end{array}$ & $\begin{array}{l}0.356 \\
(0.47)\end{array}$ & $\begin{array}{l}0.347 \\
(0.48)\end{array}$ & - & - \\
\hline Nunatsiavut & - & - & - & - & - & - & - & - & - & - & $\begin{array}{l}0.047 \\
(0.21)\end{array}$ & $\begin{array}{l}0.046 \\
(0.21)\end{array}$ \\
\hline Nunavik & - & - & - & - & - & - & - & - & - & - & $\begin{array}{l}0.228 \\
(0.42)\end{array}$ & $\begin{array}{l}0.217 \\
(0.41)\end{array}$ \\
\hline Nunavut & - & - & - & - & - & - & - & - & - & - & $\begin{array}{l}0.543 \\
(0.50)\end{array}$ & $\begin{array}{l}0.527 \\
(0.49)\end{array}$ \\
\hline Inuvialuit & - & - & - & - & - & - & - & - & - & - & $\begin{array}{l}0.182 \\
(0.39)\end{array}$ & $\begin{array}{l}0.210 \\
(0.40)\end{array}$ \\
\hline Has someone to confide in & $\begin{array}{l}0.944 \\
(0.23)\end{array}$ & $\begin{array}{l}0.966 \\
(0.18)\end{array}$ & $\begin{array}{l}0.970 \\
(0.17)\end{array}$ & $\begin{array}{l}0.966 \\
(0.18)\end{array}$ & $\begin{array}{l}0.944 \\
(0.23)\end{array}$ & $\begin{array}{l}0.968 \\
(0.18)\end{array}$ & $\begin{array}{l}0.959 \\
(0.20)\end{array}$ & $\begin{array}{l}0.964 \\
(0.18)\end{array}$ & $\begin{array}{l}0.967 \\
(0.18)\end{array}$ & $\begin{array}{l}0.986 \\
(0.12)\end{array}$ & $\begin{array}{l}0.565 \\
(0.50)\end{array}$ & $\begin{array}{l}0.580 \\
(0.49)\end{array}$ \\
\hline Volunteering club membership & - & - & - & - & - & - & - & - & $\begin{array}{l}0.204 \\
(0.40)\end{array}$ & $\begin{array}{l}0.265 \\
(0.44)\end{array}$ & $\begin{array}{l}0.453 \\
(0.50)\end{array}$ & $\begin{array}{l}0.459 \\
(0.49)\end{array}$ \\
\hline $\begin{array}{l}\text { Métis / Inuit organization } \\
\text { membership }\end{array}$ & - & - & - & - & - & - & - & - & $\begin{array}{l}0.228 \\
(0.41)\end{array}$ & $\begin{array}{l}0.203 \\
(0.41)\end{array}$ & $\begin{array}{l}0.895 \\
(0.31)\end{array}$ & $\begin{array}{l}0.918 \\
(0.27)\end{array}$ \\
\hline Has an Aboriginal identity spouse & - & - & - & - & - & - & - & - & $\begin{array}{l}0.177 \\
(0.38)\end{array}$ & $\begin{array}{l}0.165 \\
(0.37)\end{array}$ & - & - \\
\hline Voting, municipal & - & - & - & - & - & - & - & - & - & - & $\begin{array}{l}0.762 \\
(0.43)\end{array}$ & $\begin{array}{l}0.725 \\
(0.44)\end{array}$ \\
\hline Voting, provincial & - & - & - & - & - & - & - & - & - & - & $\begin{array}{l}0.757 \\
(0.43)\end{array}$ & $\begin{array}{l}0.715 \\
(0.45)\end{array}$ \\
\hline Band membership & $\begin{array}{l}0.571 \\
(0.50)\end{array}$ & $\begin{array}{l}0.597 \\
(0.49)\end{array}$ & $\begin{array}{l}0.579 \\
(0.48)\end{array}$ & $\begin{array}{l}0.641 \\
(0.49)\end{array}$ & $\begin{array}{l}0.926 \\
(0.26)\end{array}$ & $\begin{array}{l}0.927 \\
(0.26)\end{array}$ & $\begin{array}{l}0.107 \\
(0.31)\end{array}$ & $\begin{array}{l}0.129 \\
(0.33)\end{array}$ & - & - & - & - \\
\hline
\end{tabular}




\begin{tabular}{|c|c|c|c|c|c|c|c|c|c|c|c|c|}
\hline \multirow{3}{*}{$\begin{array}{l}\text { Appendix B1 } \\
\text { (continued) }\end{array}$} & \multicolumn{8}{|c|}{ North American Indian } & \multicolumn{2}{|c|}{ Métis } & \multicolumn{2}{|c|}{ Inuit } \\
\hline & \multicolumn{2}{|c|}{ Urban } & \multicolumn{2}{|c|}{ Rural } & \multicolumn{2}{|c|}{ Registered } & \multicolumn{2}{|c|}{ Non-registered } & \multirow[b]{2}{*}{ Men } & \multirow[b]{2}{*}{ Women } & \multirow[b]{2}{*}{ Men } & \multirow[b]{2}{*}{ Women } \\
\hline & Men & Women & Men & Women & Men & Women & Men & Women & & & & \\
\hline Registered Indian Status & $\begin{array}{l}0.563 \\
(0.50)\end{array}$ & $\begin{array}{l}0.594 \\
(0.49)\end{array}$ & $\begin{array}{l}0.586 \\
(0.48)\end{array}$ & $\begin{array}{l}0.613 \\
(0.50)\end{array}$ & - & - & - & - & - & - & - & - \\
\hline Speaks Aboriginal languages & $\begin{array}{l}0.189 \\
(0.39)\end{array}$ & $\begin{array}{l}0.150 \\
(0.35)\end{array}$ & $\begin{array}{l}0.222 \\
(0.41)\end{array}$ & $\begin{array}{l}0.247 \\
(0.44)\end{array}$ & $\begin{array}{l}0.294 \\
(0.46)\end{array}$ & $\begin{array}{l}0.261 \\
(0.44)\end{array}$ & $\begin{array}{l}0.070 \\
(0.25)\end{array}$ & $\begin{array}{l}0.038 \\
(0.19)\end{array}$ & $\begin{array}{l}0.088 \\
(0.28)\end{array}$ & $\begin{array}{l}0.097 \\
(0.30)\end{array}$ & $\begin{array}{r}0.854 \\
(0.36)\end{array}$ & $\begin{array}{l}0.848 \\
(0.35)\end{array}$ \\
\hline Values Aboriginal languages & $\begin{array}{l}0.608 \\
(0.49)\end{array}$ & $\begin{array}{l}0.614 \\
(0.48)\end{array}$ & $\begin{array}{r}0.626 \\
(0.47)\end{array}$ & $\begin{array}{l}0.685 \\
(0.47)\end{array}$ & $\begin{array}{l}0.717 \\
(0.45)\end{array}$ & $\begin{array}{l}0.764 \\
(0.42)\end{array}$ & $\begin{array}{r}0.473 \\
(0.50)\end{array}$ & $\begin{array}{l}0.430 \\
(0.49)\end{array}$ & $\begin{array}{r}0.453 \\
(0.49)\end{array}$ & $\begin{array}{l}0.490 \\
(0.50)\end{array}$ & $\begin{array}{l}0.952 \\
(0.22)\end{array}$ & $\begin{array}{l}0.970 \\
(0.17)\end{array}$ \\
\hline $\begin{array}{l}\text { Traditional pursuits } \\
\text { for commerce }\end{array}$ & $\begin{array}{l}0.024 \\
(0.15)\end{array}$ & $\begin{array}{l}0.017 \\
(0.13)\end{array}$ & $\begin{array}{l}0.093 \\
(0.28)\end{array}$ & $\begin{array}{l}0.020 \\
(0.14)\end{array}$ & $\begin{array}{l}0.049 \\
(0.22)\end{array}$ & $\begin{array}{l}0.021 \\
(0.14)\end{array}$ & $\begin{array}{l}0.031 \\
(0.17)\end{array}$ & $\begin{array}{l}0.012 \\
(0.11)\end{array}$ & $\begin{array}{l}0.040 \\
(0.19)\end{array}$ & $\begin{array}{l}0.018 \\
(0.13)\end{array}$ & $\begin{array}{l}0.237 \\
(0.43)\end{array}$ & $\begin{array}{l}0.080 \\
(0.27)\end{array}$ \\
\hline $\begin{array}{l}\text { Traditional pursuits } \\
\text { for food and medicinal purposes }\end{array}$ & $\begin{array}{l}0.425 \\
(0.50)\end{array}$ & $\begin{array}{l}0.370 \\
(0.48)\end{array}$ & $\begin{array}{l}0.670 \\
(0.46)\end{array}$ & $\begin{array}{l}0.555 \\
(0.51)\end{array}$ & $\begin{array}{l}0.527 \\
(0.50)\end{array}$ & $\begin{array}{l}0.451 \\
(0.49)\end{array}$ & $\begin{array}{l}0.433 \\
(0.49)\end{array}$ & $\begin{array}{l}0.353 \\
(0.48)\end{array}$ & $\begin{array}{l}0.556 \\
(0.49)\end{array}$ & $\begin{array}{l}0.437 \\
(0.50)\end{array}$ & $\begin{array}{l}0.937 \\
(0.24)\end{array}$ & $\begin{array}{l}0.861 \\
(0.34)\end{array}$ \\
\hline $\begin{array}{l}\text { Traditional pursuits } \\
\text { for pleasure }\end{array}$ & $\begin{array}{l}0.428 \\
(0.50)\end{array}$ & $\begin{array}{l}0.342 \\
(0.47)\end{array}$ & $\begin{array}{l}0.601 \\
(0.48)\end{array}$ & $\begin{array}{l}0.506 \\
(0.51)\end{array}$ & $\begin{array}{l}0.452 \\
(0.50)\end{array}$ & $\begin{array}{l}0.376 \\
(0.48)\end{array}$ & $\begin{array}{l}0.496 \\
(0.50)\end{array}$ & $\begin{array}{l}0.384 \\
(0.49)\end{array}$ & $\begin{array}{l}0.589 \\
(0.49)\end{array}$ & $\begin{array}{l}0.450 \\
(0.50)\end{array}$ & $\begin{array}{l}0.659 \\
(0.48)\end{array}$ & $\begin{array}{l}0.759 \\
(0.42)\end{array}$ \\
\hline Weighted Counts & 63440 & 71000 & 21330 & 20520 & 48210 & 54780 & 36560 & 36730 & 78290 & 79130 & 23740 & 24460 \\
\hline
\end{tabular}

Source: Statistics Canada, 2006 Aboriginal Peoples Survey, analytical files. Bootstrap weights in effect. Standard deviation in parentheses.

Note: Since all of the independent variables in this study are dummy variables, coded 1 and 0 , the means reported in this table represent the proportions of observations of cases coded 1 in each sample. This mean can also be interpreted as a probability that a randomly selected person from the sample will possess the characteristic measured by the mean. For instance, in the case of ten observations, 1111110000 , the mean is $6 / 10=.6$, which is the probability that any one case out of ten has a score of 1 . 


\section{Appendix C}

Appendix C 1: Detailed results from probit models predicting employment for urban NAI, APS 2006

\begin{tabular}{|c|c|c|c|c|c|c|c|c|}
\hline \multirow{3}{*}{ Variables in equations } & \multicolumn{8}{|c|}{ Average Marginal Effects } \\
\hline & \multicolumn{2}{|c|}{ Model 1} & \multicolumn{2}{|c|}{ Model 2} & \multicolumn{2}{|c|}{ Model 3} & \multicolumn{2}{|c|}{ Full Model } \\
\hline & Men & Women & Men & Women & Men & Women & Men & Women \\
\hline $\begin{array}{l}\text { Less than } \\
\text { high school education }\end{array}$ & $\begin{array}{c}-0.054 \\
(0.008)^{\star \star * *}\end{array}$ & $\begin{array}{c}-0.038 \\
(0.007)^{\star * *}\end{array}$ & $\begin{array}{c}-0.056 \\
(0.008)^{*+4=}\end{array}$ & $\begin{array}{c}-0.036 \\
(0.007)^{* * * *}\end{array}$ & $\begin{array}{c}-0.058 \\
(0.008)^{* * *}\end{array}$ & $\begin{array}{c}-0.030 \\
(0.006)^{* * *}\end{array}$ & $\begin{array}{c}-0.050 \\
(0.007)^{* \cdots}\end{array}$ & $\begin{array}{c}-0.034 \\
(0.006)^{* * *}\end{array}$ \\
\hline $\begin{array}{l}\text { Some } \\
\text { non-university education }\end{array}$ & $\begin{array}{c}-0.030 \\
10.011^{\star \star}\end{array}$ & $\begin{array}{c}0.045 \\
(0.004)^{ \pm * *}\end{array}$ & $\begin{array}{c}-0.030 \\
(0.010)^{* *}\end{array}$ & $\begin{array}{c}0.044 \\
(0.004)^{\star k \star *}\end{array}$ & $\begin{array}{c}-0.031 \\
(0.010)^{\star \star}\end{array}$ & $\begin{array}{c}0.044 \\
(0.004)^{\star \star \star *}\end{array}$ & $\begin{array}{c}-0.029 \\
(0.010)^{\star \star *}\end{array}$ & $\begin{array}{c}0.042 \\
(0.004)^{\star * *}\end{array}$ \\
\hline $\begin{array}{l}\text { Completed, } \\
\text { non-university education }\end{array}$ & $\begin{array}{c}-0.048 \\
(0.009)^{\star \star \star \star}\end{array}$ & $\begin{array}{c}0.059 \\
(0.005)^{* * *}\end{array}$ & $\begin{array}{c}-0.048 \\
(0.008)^{\star * * *}\end{array}$ & $\begin{array}{c}0.061 \\
(0.004)^{\star \star \star *}\end{array}$ & $\begin{array}{c}-0.050 \\
(0.008)^{\star \star \star}\end{array}$ & $\begin{array}{c}0.062 \\
(0.004)^{* \star *}\end{array}$ & $\begin{array}{c}-0.046 \\
(0.008)^{\star * *}\end{array}$ & $\begin{array}{c}0.060 \\
(0.004)^{\star \star \star}\end{array}$ \\
\hline $\begin{array}{l}\text { Some } \\
\text { university education }\end{array}$ & $\begin{array}{c}-0.0527 \\
(0.011)^{\star * *}\end{array}$ & $\begin{array}{c}0.050 \\
(0.003)^{\star \star \star}\end{array}$ & $\begin{array}{c}-0.043 \\
(0.011)^{\star \star \star}\end{array}$ & $\begin{array}{c}0.051 \\
(0.003)^{\star \star \star}\end{array}$ & $\begin{array}{c}-0.045 \\
(0.011)^{\star \star \star}\end{array}$ & $\begin{array}{c}0.052 \\
(0.003)^{\star \star *}\end{array}$ & $\begin{array}{c}-0.043 \\
(0.011)^{\star \star \star}\end{array}$ & $\begin{array}{c}0.051 \\
(0.003)^{* \star \star}\end{array}$ \\
\hline $\begin{array}{l}\text { Completed } \\
\text { university education }\end{array}$ & $\begin{array}{c}0.037 \\
(0.006)^{\star * *}\end{array}$ & $\begin{array}{c}0.055 \\
(0.004)^{* * *}\end{array}$ & $\begin{array}{c}0.034 \\
(0.007)^{\star \star * \star}\end{array}$ & $\begin{array}{c}0.053 \\
(0.004)^{\star \star \star}\end{array}$ & $\begin{array}{c}0.034 \\
(0.007)^{* * *}\end{array}$ & $\begin{array}{c}0.055 \\
(0.003)^{\star * \star}\end{array}$ & $\begin{array}{c}0.037 \\
(0.006)^{* k \star}\end{array}$ & $\begin{array}{c}0.054 \\
(0.003)^{\star \star *}\end{array}$ \\
\hline Age 15 to 19 & $\begin{array}{c}-0.103 \\
(0.015)^{* * *}\end{array}$ & $\begin{array}{c}-0.108 \\
(0.018)^{\star \star \star *}\end{array}$ & $\begin{array}{c}-0.075 \\
(0.013)^{* * *}\end{array}$ & $\begin{array}{c}-0.085 \\
(0.016)^{* * *}\end{array}$ & $\begin{array}{c}-0.080 \\
(0.013)^{\star \star \star \star}\end{array}$ & $\begin{array}{c}-0.086 \\
(0.016)^{\star * \star}\end{array}$ & $\begin{array}{c}-0.088 \\
(0.013)^{* * *}\end{array}$ & $\begin{array}{c}-0.084 \\
(0.016)^{\star * *}\end{array}$ \\
\hline Age 25 to 34 & $\begin{array}{c}0.034 \\
(0.006)^{\star \pm \star}\end{array}$ & $\begin{array}{c}-0.110 \\
(0.014)^{\star \star \star}\end{array}$ & $\begin{array}{c}0.035 \\
(0.006)^{\text {*k** }}\end{array}$ & $\begin{array}{c}-0.094 \\
(0.012)^{\star * *}\end{array}$ & $\begin{array}{c}0.033 \\
(0.006)^{\star \star \star}\end{array}$ & $\begin{array}{c}-0.085 \\
(0.011)^{\star * \star}\end{array}$ & $\begin{array}{c}0.033 \\
(0.006)^{\star \star \star}\end{array}$ & $\begin{array}{c}-0.079 \\
(0.011)^{\star * *}\end{array}$ \\
\hline Age 35 to 44 & $\begin{array}{c}0.045 \\
(0.006)^{* \star *}\end{array}$ & $\begin{array}{c}-0.062 \\
(0.011)^{\star * *}\end{array}$ & $\begin{array}{c}0.043 \\
(0.006)^{\star * *}\end{array}$ & $\begin{array}{c}-0.027 \\
(0.009)^{* *}\end{array}$ & $\begin{array}{c}0.041 \\
(0.006)^{* * *}\end{array}$ & $\begin{array}{c}-0.014 \\
(0.008)\end{array}$ & $\begin{array}{c}0.044 \\
(0.006)^{\star \star \star}\end{array}$ & $\begin{array}{c}-0.008 \\
(0.008)\end{array}$ \\
\hline Age 45 to 54 & $\begin{array}{c}0.044 \\
(0.006)^{\star * *}\end{array}$ & $\begin{array}{c}-0.049 \\
(0.010)^{* * *}\end{array}$ & $\begin{array}{c}0.020 \\
(0.008)^{* \star}\end{array}$ & $\begin{array}{c}-0.019 \\
(0.009)^{*}\end{array}$ & $\begin{array}{c}0.019 \\
(0.008)^{*}\end{array}$ & $\begin{array}{c}-0.013 \\
(0.009)\end{array}$ & $\begin{array}{c}0.022 \\
(0.008)^{\star \star}\end{array}$ & $\begin{array}{c}-0.009 \\
(0.008)\end{array}$ \\
\hline Age 55 to 64 & $\begin{array}{c}0.073 \\
(0.003)^{\star * \hbar}\end{array}$ & $\begin{array}{l}0.0001 \\
(0.011)\end{array}$ & $\begin{array}{c}0.058 \\
(0.005)^{\star \star \star}\end{array}$ & $\begin{array}{c}0.014 \\
(0.009)\end{array}$ & $\begin{array}{c}0.057 \\
(0.005)^{\star \star \star}\end{array}$ & $\begin{array}{c}0.020 \\
(0.008)^{\star}\end{array}$ & $\begin{array}{c}0.060 \\
(0.005)^{\star \star \star *}\end{array}$ & $\begin{array}{c}0.023 \\
(0.008)^{\star \star}\end{array}$ \\
\hline Languages - French & $\begin{array}{c}0.017 \\
(0.008)^{*}\end{array}$ & $\begin{array}{c}0.050 \\
(0.003)^{\star \star \star t}\end{array}$ & $\begin{array}{c}0.018 \\
(0.018)\end{array}$ & $\begin{array}{c}0.060 \\
(0.002)^{* * *}\end{array}$ & $\begin{array}{c}0.018 \\
(0.018)\end{array}$ & $\begin{array}{c}0.060 \\
(0.002)^{\star \star \star}\end{array}$ & $\begin{array}{c}0.025 \\
(0.015)\end{array}$ & $\begin{array}{c}0.060 \\
(0.002)^{\star \star \star}\end{array}$ \\
\hline Languages - Both & $\begin{array}{c}0.011 \\
(0.010)\end{array}$ & $\begin{array}{c}0.026 \\
(0.003)^{\star \star \star}\end{array}$ & $\begin{array}{c}0.012 \\
(0.007)\end{array}$ & $\begin{array}{c}0.043 \\
(0.004)^{\star * *}\end{array}$ & $\begin{array}{c}0.012 \\
(0.007)\end{array}$ & $\begin{array}{c}0.039 \\
(0.004)^{* * *}\end{array}$ & $\begin{array}{c}0.012 \\
(0.007)\end{array}$ & $\begin{array}{c}0.041 \\
(0.004)^{* * *}\end{array}$ \\
\hline Access/use technology & $\begin{array}{c}0.029 \\
(0.006)^{\star \star \star}\end{array}$ & $\begin{array}{r}-0.0046 \\
(0.004)\end{array}$ & $\begin{array}{c}0.013 \\
(0.005)^{*}\end{array}$ & $\begin{array}{c}-0.008 \\
(0.004)^{*}\end{array}$ & $\begin{array}{c}0.014 \\
(0.005)^{\star \star}\end{array}$ & $\begin{array}{c}-0.006 \\
(0.004)\end{array}$ & $\begin{array}{c}0.009 \\
(0.005)\end{array}$ & $\begin{array}{c}-0.010 \\
(0.004)^{\star}\end{array}$ \\
\hline Legally married & & & $\begin{array}{c}0.064 \\
(0.008)^{\star * *}\end{array}$ & $\begin{array}{c}-0.019 \\
(0.007)^{\star \star}\end{array}$ & $\begin{array}{c}0.066 \\
(0.008)^{\star \star \star \star}\end{array}$ & $\begin{array}{c}0.020 \\
(0.007)^{\star \star *}\end{array}$ & $\begin{array}{c}0.070 \\
(0.008)^{\star * \star}\end{array}$ & $\begin{array}{c}-0.021 \\
(0.007)^{\star \star}\end{array}$ \\
\hline Common-law & & & $\begin{array}{c}0.044 \\
(0.05)^{\star \star \star}\end{array}$ & $\begin{array}{c}-0.038 \\
(0.007)^{\star \star \star \star}\end{array}$ & $\begin{array}{c}0.046 \\
(0.005)^{\star * \star}\end{array}$ & $\begin{array}{c}-0.034 \\
(0.006)^{\star \star *}\end{array}$ & $\begin{array}{c}0.048 \\
(0.005)^{\star * *}\end{array}$ & $\begin{array}{c}-0.038 \\
(0.007)^{\star \star \star \star}\end{array}$ \\
\hline Divorced-separated & & & $\begin{array}{r}-0.0001 \\
(0.008)\end{array}$ & $\begin{array}{c}-0.035 \\
(0.007)^{* * *}\end{array}$ & $\begin{array}{c}0.002 \\
(0.007)\end{array}$ & $\begin{array}{c}-0.043 \\
(0.007)^{* * *}\end{array}$ & $\begin{array}{c}0.003 \\
(0.007)\end{array}$ & $\begin{array}{c}-0.043 \\
(0.007)^{\star * *}\end{array}$ \\
\hline Children $<6$ & & & $\begin{array}{c}-0.080 \\
(0.010)^{\star \star * *}\end{array}$ & $\begin{array}{c}0.010 \\
(0.005)\end{array}$ & $\begin{array}{c}-0.078 \\
(0.010)^{\text {t*k}}\end{array}$ & $\begin{array}{c}0.007 \\
(0.005)\end{array}$ & $\begin{array}{c}-0.073 \\
(0.010)^{* * *}\end{array}$ & $\begin{array}{c}0.009 \\
(0.005)\end{array}$ \\
\hline Children 6-14 & & & $\begin{array}{c}-0.073 \\
(0.013)^{\star * *}\end{array}$ & $\begin{array}{c}0.003 \\
(0.005)\end{array}$ & $\begin{array}{c}-0.074 \\
(0.013)^{\star *}\end{array}$ & $\begin{array}{c}-0.0002 \\
(0.007)\end{array}$ & $\begin{array}{c}-0.076 \\
(0.013)^{* * *}\end{array}$ & $\begin{array}{l}0.0007 \\
(0.007)\end{array}$ \\
\hline Children $14-24$ & & & $\begin{array}{c}0.005 \\
(0.009)\end{array}$ & $\begin{array}{c}-0.045 \\
(0.008)^{\star * *}\end{array}$ & $\begin{array}{c}0.002 \\
(0.009)\end{array}$ & $\begin{array}{c}-0.041 \\
(0.008)^{\star \star \star \star}\end{array}$ & $\begin{array}{c}0.001 \\
(0.009)\end{array}$ & $\begin{array}{c}-0.039 \\
(0.008)^{\star \star \star \star}\end{array}$ \\
\hline Lone parent & & & $\begin{array}{c}0.043 \\
(0.007)^{\star * \star}\end{array}$ & $\begin{array}{c}-0.042 \\
(0.011)^{\star * * *}\end{array}$ & $\begin{array}{c}0.041 \\
(0.007)^{\star \star \star}\end{array}$ & $\begin{array}{c}-0.029 \\
(0.010)^{\star *}\end{array}$ & $\begin{array}{c}0.042 \\
(0.007)^{\star * *}\end{array}$ & $\begin{array}{c}-0.037 \\
(0.010)^{\star * * *}\end{array}$ \\
\hline
\end{tabular}




\begin{tabular}{|c|c|c|c|c|c|c|c|c|}
\hline \multirow{3}{*}{$\begin{array}{l}\text { Appendix C1 } \\
\text { (continued) }\end{array}$} & \multicolumn{8}{|c|}{ Average Marginal Effects } \\
\hline & \multicolumn{2}{|c|}{ Model 1} & \multicolumn{2}{|c|}{ Model 2} & \multicolumn{2}{|c|}{ Model 3} & \multicolumn{2}{|c|}{ Full Model } \\
\hline & Men & Women & Men & Women & Men & Women & Men & Women \\
\hline Chronic health condition & & & $\begin{array}{l}-0.007 \\
(0.004)\end{array}$ & $\begin{array}{c}0.014 \\
(0.003)^{\star \star \star *}\end{array}$ & $\begin{array}{l}-0.005 \\
(0.005)\end{array}$ & $\begin{array}{c}0.013 \\
(0.003)^{* * *}\end{array}$ & $\begin{array}{l}-0.003 \\
(0.004)\end{array}$ & $\begin{array}{c}0.012 \\
(0.003)^{\star \star \star}\end{array}$ \\
\hline Health activity limitation & & & $\begin{array}{c}-0.0154 \\
(0.004)^{\star \star}\end{array}$ & $\begin{array}{c}-0.014 \\
(0.004)^{\star \star \star *}\end{array}$ & $\begin{array}{c}-0.018 \\
(0.005)^{* * *}\end{array}$ & $\begin{array}{c}-0.016 \\
(0.004)^{\star \star \star}\end{array}$ & $\begin{array}{c}-0.020 \\
(0.004)^{\star \star \star}\end{array}$ & $\begin{array}{c}-0.017 \\
(0.004)^{\star * *}\end{array}$ \\
\hline Other sources of income & & & $\begin{array}{c}0.025 \\
(0.004)^{\star *}\end{array}$ & $\begin{array}{c}-0.030 \\
(0.005)^{\star * *}\end{array}$ & $\begin{array}{c}0.026 \\
(0.004)^{* * *}\end{array}$ & $\begin{array}{c}-0.035 \\
(0.005)^{\star \star \star}\end{array}$ & $\begin{array}{c}0.028 \\
(0.004)^{*}\end{array}$ & $\begin{array}{c}-0.034 \\
(0.005)^{\star \star *}\end{array}$ \\
\hline Two or more maintainers & & & $\begin{array}{l}-0.004 \\
(0.005)\end{array}$ & $\begin{array}{c}0.026 \\
(0.004)^{\star * *}\end{array}$ & $\begin{array}{l}-0.001 \\
(0.005)\end{array}$ & $\begin{array}{c}0.025 \\
(0.004)^{\star \star \star}\end{array}$ & $\begin{array}{l}-0.006 \\
(0.005)\end{array}$ & $\begin{array}{c}0.023 \\
(0.004)^{\star \star \star}\end{array}$ \\
\hline First maintainer & & & $\begin{array}{c}-0.001 \\
(0.005)\end{array}$ & $\begin{array}{c}0.032 \\
(0.004)^{* * *}\end{array}$ & $\begin{array}{c}-0.002 \\
(0.005)\end{array}$ & $\begin{array}{c}0.034 \\
(0.004)^{\star * *}\end{array}$ & $\begin{array}{c}-0.004 \\
(0.005)\end{array}$ & $\begin{array}{c}0.034 \\
(0.004)^{\star * *}\end{array}$ \\
\hline Atlantic provinces & & & $\begin{array}{c}-0.087 \\
(0.012)^{\star \star *}\end{array}$ & $\begin{array}{c}0.021 \\
(0.004)^{* * * *}\end{array}$ & $\begin{array}{c}-0.090 \\
(0.012)^{\star * \star}\end{array}$ & $\begin{array}{c}0.021 \\
(0.004)^{\star \star \star}\end{array}$ & $\begin{array}{c}-0.090 \\
(0.012)^{\star * \star}\end{array}$ & $\begin{array}{c}0.019 \\
(0.004)^{\star \star \star}\end{array}$ \\
\hline Northem Territories & & & $\begin{array}{c}-0.082 \\
(0.015)^{\star * \star}\end{array}$ & $\begin{array}{c}0.026 \\
(0.008)^{* *}\end{array}$ & $\begin{array}{c}-0.082 \\
(0.015)^{\star *}\end{array}$ & $\begin{array}{c}0.034 \\
(0.006)^{\star \star \star}\end{array}$ & $\begin{array}{c}-0.061 \\
(0.013)^{* * \star}\end{array}$ & $\begin{array}{c}0.038 \\
(0.005)^{\star \star \star \star}\end{array}$ \\
\hline Quebec & & & $\begin{array}{c}-0.048 \\
(0.014)^{\star}\end{array}$ & $\begin{array}{c}-0.039 \\
(0.009)^{* * *}\end{array}$ & $\begin{array}{c}-0.048 \\
(0.024)^{\star}\end{array}$ & $\begin{array}{c}-0.036 \\
(0.009)^{\star \star \star \star}\end{array}$ & $\begin{array}{c}-0.057 \\
(0.023)^{\star}\end{array}$ & $\begin{array}{c}-0.034 \\
(0.009)^{* * *}\end{array}$ \\
\hline Manitoba & & & $\begin{array}{c}-0.115 \\
(0.013)^{\star * *}\end{array}$ & $\begin{array}{c}0.037 \\
(0.004)^{\star \star \star}\end{array}$ & $\begin{array}{c}-0.124 \\
(0.014)^{\text {N1***}}\end{array}$ & $\begin{array}{c}0.047 \\
(0.003)^{* * *}\end{array}$ & $\begin{array}{c}-0.199 \\
(0.013)^{* *}\end{array}$ & $\begin{array}{c}0.048 \\
(0.003)^{*+* *}\end{array}$ \\
\hline Saskatchewan & & & $\begin{array}{c}0.071 \\
(0.011)^{\star \star \star}\end{array}$ & $\begin{array}{c}-0.012 \\
(0.006)^{\star}\end{array}$ & $\begin{array}{c}-0.071 \\
(0.011)^{* * *}\end{array}$ & $\begin{array}{c}0.002 \\
(0.005)\end{array}$ & $\begin{array}{c}-0.060 \\
(0.010)^{\star \star *}\end{array}$ & $\begin{array}{c}0.004 \\
(0.005)\end{array}$ \\
\hline Alberta & & & $\begin{array}{c}-0.026 \\
(0.008)^{\star \star}\end{array}$ & $\begin{array}{c}0.016 \\
(0.004)^{* * *}\end{array}$ & $\begin{array}{c}-0.027 \\
(0.008)^{\star \star}\end{array}$ & $\begin{array}{c}0.020 \\
(0.004)^{\star * *}\end{array}$ & $\begin{array}{c}-0.015 \\
(0.007)^{*}\end{array}$ & $\begin{array}{c}0.026 \\
(0.004)^{* * *}\end{array}$ \\
\hline British Columbia & & & $\begin{array}{l}-0.028 \\
(0.008)\end{array}$ & $\begin{array}{c}0.015 \\
(0.005)^{* *}\end{array}$ & $\begin{array}{c}-0.027 \\
(0.008)^{\star \star * \pi *}\end{array}$ & $\begin{array}{c}0.027 \\
(0.004)^{\star \star \star}\end{array}$ & $\begin{array}{c}-0.025 \\
(0.008)^{\star \star}\end{array}$ & $\begin{array}{c}0.030 \\
(0.004)^{\star \star *}\end{array}$ \\
\hline Has someone to confide in & & & & & $\begin{array}{c}-0.029 \\
(0.005)^{\star \star * k *}\end{array}$ & $\begin{array}{c}0.035 \\
(0.011)^{\star *}\end{array}$ & $\begin{array}{c}-0.033 \\
(0.005)^{* k \star}\end{array}$ & $\begin{array}{c}0.038 \\
(0.011)^{\star \star \star}\end{array}$ \\
\hline Band member & & & & & & & $\begin{array}{c}0.015 \\
(0.009)\end{array}$ & $\begin{array}{c}-0.022 \\
(0.004)^{\star \star \star}\end{array}$ \\
\hline Registered Indian & & & & & & & $\begin{array}{l}-0.005 \\
(0.009)\end{array}$ & $\begin{array}{c}-0.022 \\
(0.004)^{\star \star \star \star}\end{array}$ \\
\hline $\begin{array}{l}\text { Speaks Aboriginal } \\
\text { languages }\end{array}$ & & & & & & & $\begin{array}{c}-0.057 \\
(0.007)^{* * *}\end{array}$ & $\begin{array}{l}-0.002 \\
(0.004)\end{array}$ \\
\hline $\begin{array}{l}\text { Values Aboriginal } \\
\text { languages }\end{array}$ & & & & & & & $\begin{array}{c}0.021 \\
(0.005)^{\text {k** }}\end{array}$ & $\begin{array}{c}0.011 \\
(0.004)^{*}\end{array}$ \\
\hline $\begin{array}{l}\text { Traditional pursuits } \\
\text { for commerce }\end{array}$ & & & & & & & $\begin{array}{c}-0.044 \\
(0.014)^{\star \star}\end{array}$ & $\begin{array}{c}0.013 \\
(0.008)\end{array}$ \\
\hline $\begin{array}{l}\text { Traditional pursuits } \\
\text { for food }\end{array}$ & & & & & & & $\begin{array}{c}-0.011 \\
(0.005)^{\star}\end{array}$ & $\begin{array}{c}-0.013 \\
(0.004)^{\star \star \star \star}\end{array}$ \\
\hline $\begin{array}{l}\text { Traditional pursuits } \\
\text { for pleasure }\end{array}$ & & & & & & & $\begin{array}{c}0.027 \\
(0.004)^{\star \star \star}\end{array}$ & $\begin{array}{c}0.005 \\
(0.003) \\
\end{array}$ \\
\hline Number of observations & 1590 & 1730 & 1560 & 1710 & 1550 & 1700 & 1530 & 1690 \\
\hline
\end{tabular}

Source: Statistics Canada, 2006 Aboriginal Peoples Survey, Adult Core analytical file. Bootstrapped (1000 replications) standard errors in parentheses. Statistical significance ${ }^{* * *} p<.001 ;{ }^{* *} p<.01 ;{ }^{*} p<.05$. 
Appendix C 2: Detailed results from probit models predicting employment for rural NAI, APS 2006

\begin{tabular}{|c|c|c|c|c|c|c|c|c|}
\hline \multirow{3}{*}{ Variables in equations } & \multicolumn{8}{|c|}{ Average Marginal Effects } \\
\hline & \multicolumn{2}{|c|}{ Model 1} & \multicolumn{2}{|c|}{ Model 2} & \multicolumn{2}{|c|}{ Model 3} & \multicolumn{2}{|c|}{ Full Model } \\
\hline & Men & Women & Men & Women & Men & Women & Men & Women \\
\hline $\begin{array}{l}\text { Less than } \\
\text { high school education }\end{array}$ & $\begin{array}{c}-0.023 \\
(0.008)^{\star *}\end{array}$ & $\begin{array}{c}-0.048 \\
(0.009)^{\star \star \star}\end{array}$ & $\begin{array}{c}-0.028 \\
(0.008)^{\star * *}\end{array}$ & $\begin{array}{c}-0.054 \\
(0.009)^{* * *}\end{array}$ & $\begin{array}{c}-0.024 \\
(0.008)^{\star \star *}\end{array}$ & $\begin{array}{c}-0.053 \\
(0.009)^{* * *}\end{array}$ & $\begin{array}{c}-0.032 \\
(0.008)^{\star * k *}\end{array}$ & $\begin{array}{c}-0.054 \\
(0.009)^{\star \star *}\end{array}$ \\
\hline $\begin{array}{l}\text { Some } \\
\text { non-university education }\end{array}$ & $\begin{array}{c}0.009 \\
(0.009)\end{array}$ & $\begin{array}{c}-0.040 \\
(0.013)^{* *}\end{array}$ & $\begin{array}{c}-0.009 \\
(0.010)\end{array}$ & $\begin{array}{c}-0.049 \\
(0.12)^{* * *}\end{array}$ & $\begin{array}{l}-0.009 \\
(0.010)\end{array}$ & $\begin{array}{c}-0.050 \\
(0.012)^{* * *}\end{array}$ & $\begin{array}{l}-0.001 \\
(0.009)\end{array}$ & $\begin{array}{c}-0.50 \\
(0.012)^{* \cdots *}\end{array}$ \\
\hline $\begin{array}{l}\text { Completed, } \\
\text { non-university education }\end{array}$ & $\begin{array}{l}-0.001 \\
(0.008)\end{array}$ & $\begin{array}{c}-0.028 \\
(0.007)^{\star \star \star *}\end{array}$ & $\begin{array}{l}-0.007 \\
(0.007)\end{array}$ & $\begin{array}{c}-0.021 \\
(0.007)^{\star *}\end{array}$ & $\begin{array}{r}-0.003 \\
(0.007)\end{array}$ & $\begin{array}{c}-0.017 \\
(0.007)^{\star}\end{array}$ & $\begin{array}{r}-0.009 \\
(0.007)\end{array}$ & $\begin{array}{c}-0.017 \\
(0.007\rangle^{\star}\end{array}$ \\
\hline $\begin{array}{l}\text { Some } \\
\text { university education }\end{array}$ & $\begin{array}{l}-0.015 \\
(0.019)\end{array}$ & $\begin{array}{l}-0.016 \\
(0.015)\end{array}$ & $\begin{array}{l}-0.011 \\
(0.019)\end{array}$ & $\begin{array}{l}-0.006 \\
(0.014)\end{array}$ & $\begin{array}{c}0.006 \\
(0.017)\end{array}$ & $\begin{array}{l}-0.007 \\
(0.014)\end{array}$ & $\begin{array}{l}-0.003 \\
(0.018)\end{array}$ & $\begin{array}{c}0.003 \\
(0.012)\end{array}$ \\
\hline $\begin{array}{l}\text { Completed } \\
\text { university education }\end{array}$ & $\begin{array}{c}0.004 \\
(0.012)\end{array}$ & $\begin{array}{c}0.035 \\
(0.006)^{* * * *}\end{array}$ & $\begin{array}{c}-0.009 \\
(0.012)\end{array}$ & $\begin{array}{c}0.032 \\
(0.006)^{* * *}\end{array}$ & $\begin{array}{c}-0.007 \\
(0.012)\end{array}$ & $\begin{array}{c}0.032 \\
(0.005)^{* * *}\end{array}$ & $\begin{array}{r}-0.0018 \\
(0.011)\end{array}$ & $\begin{array}{c}0.037 \\
(0.005)^{\star * *}\end{array}$ \\
\hline Age 15 to 19 & $\begin{array}{c}-0.005 \\
(0.012)\end{array}$ & $\begin{array}{c}-0.078 \\
(0.018)^{\star * * *}\end{array}$ & $\begin{array}{c}0.002 \\
(0.011)\end{array}$ & $\begin{array}{c}-0.059 \\
(0.016)^{\star \star \star}\end{array}$ & $\begin{array}{l}0.0002 \\
(0.010)\end{array}$ & $\begin{array}{c}-0.058 \\
(0.016)^{\star \star \star}\end{array}$ & $\begin{array}{l}-0.0002 \\
(0.010)\end{array}$ & $\begin{array}{c}-0.050 \\
(0.014)^{\star *}\end{array}$ \\
\hline Age 25 to 34 & $\begin{array}{c}0.054 \\
(0.007)^{\star * *}\end{array}$ & $\begin{array}{c}0.031 \\
(0.007)^{\star \star *}\end{array}$ & $\begin{array}{c}0.048 \\
(0.007)^{\star \star \star}\end{array}$ & $\begin{array}{c}-0.001 \\
(0.008)\end{array}$ & $\begin{array}{c}0.054 \\
(0.007)^{\star * *}\end{array}$ & $\begin{array}{c}-0.001 \\
(0.008)\end{array}$ & $\begin{array}{c}0.050 \\
(0.007)^{\star * *}\end{array}$ & $\begin{array}{c}-0.010 \\
(0.008)\end{array}$ \\
\hline Age 35 to 44 & $\begin{array}{c}0.061 \\
(0.007)^{\star \star \star}\end{array}$ & $\begin{array}{c}0.078 \\
(0.007)^{\star \star *}\end{array}$ & $\begin{array}{c}0.028 \\
(0.009)^{\star \star}\end{array}$ & $\begin{array}{c}0.020 \\
(0.008)^{\star *}\end{array}$ & $\begin{array}{c}0.031 \\
(0.009)^{\star \star}\end{array}$ & $\begin{array}{c}0.030 \\
(0.007)^{* * * *}\end{array}$ & $\begin{array}{c}0.032 \\
(0.009)^{* * *}\end{array}$ & $\begin{array}{c}0.024 \\
(0.007)^{\star \star}\end{array}$ \\
\hline Age 45 to 54 & $\begin{array}{c}0.080 \\
(0.007)^{\star * *}\end{array}$ & $\begin{array}{c}0.060 \\
(0.007)^{\star \star \star}\end{array}$ & $\begin{array}{c}0.047 \\
(0.009)^{\star \star \star}\end{array}$ & $\begin{array}{c}0.020 \\
(0.008)^{*}\end{array}$ & $\begin{array}{c}0.047 \\
(0.009)^{* * *}\end{array}$ & $\begin{array}{c}0.025 \\
(0.008)^{* *}\end{array}$ & $\begin{array}{c}0.048 \\
(0.009)^{\star * * *}\end{array}$ & $\begin{array}{c}0.021 \\
(0.008)^{\star \star}\end{array}$ \\
\hline Age 55 to 64 & $\begin{array}{c}0.097 \\
(0.005)^{\star \star *}\end{array}$ & $\begin{array}{c}0.075 \\
(0.004)^{\star \star \star *}\end{array}$ & $\begin{array}{c}0.088 \\
(0.006)^{\star \star \star}\end{array}$ & $\begin{array}{c}0.063 \\
(0.005)^{\star \star \star}\end{array}$ & $\begin{array}{c}0.090 \\
(0.006)^{\star \star \star}\end{array}$ & $\begin{array}{c}0.064 \\
(0.005)^{* * *}\end{array}$ & $\begin{array}{c}0.094 \\
(0.006)^{\star \star \star \star}\end{array}$ & $\begin{array}{c}0.062 \\
(0.005)^{\star \star \star \star}\end{array}$ \\
\hline Languages - French & $\begin{array}{c}-0.046 \\
(0.014)^{\star \star}\end{array}$ & $\begin{array}{c}-0.034 \\
(0.013)^{\star \star}\end{array}$ & $\begin{array}{c}-0.139 \\
(0.025)^{\star \star \star}\end{array}$ & $\begin{array}{c}-0.027 \\
(0.008)\end{array}$ & $\begin{array}{c}-0.185 \\
(0.028)^{\star \star \star}\end{array}$ & $\begin{array}{c}-0.032 \\
(0.017)\end{array}$ & $\begin{array}{c}-0.210 \\
(0.026)^{\star \star * \star}\end{array}$ & $\begin{array}{c}-0.023 \\
(0.016)\end{array}$ \\
\hline Languages - Both & $\begin{array}{c}-0.007 \\
(0.009)\end{array}$ & $\begin{array}{c}0.019 \\
(0.006)^{\star \star}\end{array}$ & $\begin{array}{c}-0.040 \\
(0.012)^{\star \star}\end{array}$ & $\begin{array}{c}0.019 \\
(0.008)^{\star}\end{array}$ & $\begin{array}{c}-0.085 \\
(0.014)^{\star \star \star}\end{array}$ & $\begin{array}{c}0.014 \\
(0.008)\end{array}$ & $\begin{array}{c}-0.079 \\
(0.013)^{* \cdots *}\end{array}$ & $\begin{array}{c}0.013 \\
(0.008)\end{array}$ \\
\hline Access/use technology & $\begin{array}{c}0.046 \\
(0.006)^{\star \star \star}\end{array}$ & $\begin{array}{c}0.018 \\
(0.005)^{\star \star \star}\end{array}$ & $\begin{array}{c}0.033 \\
(0.006)^{\star \star \star}\end{array}$ & $\begin{array}{c}0.014 \\
(0.004)^{\star \star}\end{array}$ & $\begin{array}{c}0.023 \\
(0.005)^{\star \star \star}\end{array}$ & $\begin{array}{c}0.015 \\
(0.004)^{\star \star}\end{array}$ & $\begin{array}{c}0.017 \\
(0.005)^{\star \star}\end{array}$ & $\begin{array}{c}0.016 \\
(0.004)^{\star \star \star}\end{array}$ \\
\hline Legally married & & & $\begin{array}{c}0.013 \\
(0.010)\end{array}$ & $\begin{array}{c}0.061 \\
(0.007)^{* * *}\end{array}$ & $\begin{array}{c}0.012 \\
(0.010)\end{array}$ & $\begin{array}{c}0.057 \\
(0.007)^{\star * *}\end{array}$ & $\begin{array}{c}-0.004 \\
(0.010)\end{array}$ & $\begin{array}{c}0.063 \\
(0.007)^{\star \star \star}\end{array}$ \\
\hline Common-law & & & $\begin{array}{c}-0.023 \\
(0.010)^{*}\end{array}$ & $\begin{array}{c}0.018 \\
(0.007)^{\star}\end{array}$ & $\begin{array}{c}-0.016 \\
(0.009)\end{array}$ & $\begin{array}{c}0.016 \\
(0.007)^{*}\end{array}$ & $\begin{array}{c}-0.026 \\
(0.009)^{\star *}\end{array}$ & $\begin{array}{c}0.018 \\
(0.007)^{\star \star}\end{array}$ \\
\hline Divorced-separated & & & $\begin{array}{c}0.056 \\
(0.006)^{\star \star \star}\end{array}$ & $\begin{array}{c}0.033 \\
(0.005)^{\star \star \star}\end{array}$ & $\begin{array}{c}0.051 \\
(0.006)^{\star * \star}\end{array}$ & $\begin{array}{c}0.027 \\
(0.005)^{\star * * *}\end{array}$ & $\begin{array}{c}0.042 \\
(0.006)^{\prime}\end{array}$ & $\begin{array}{c}0.026 \\
(0.005)^{\star \star \star}\end{array}$ \\
\hline Children $<6$ & & & $\begin{array}{c}0.012 \\
(0.008)\end{array}$ & $\begin{array}{c}0.005 \\
(0.007)\end{array}$ & $\begin{array}{c}0.010 \\
(0.008)\end{array}$ & $\begin{array}{c}0.006 \\
(0.007)\end{array}$ & $\begin{array}{c}0.027 \\
(0.007)^{\star * *}\end{array}$ & $\begin{array}{c}0.006 \\
(0.007)\end{array}$ \\
\hline Children 6-14 & & & $\begin{array}{c}0.067 \\
(0.006)^{\star \star \star}\end{array}$ & $\begin{array}{c}0.044 \\
(0.005)^{\star * *}\end{array}$ & $\begin{array}{c}0.070 \\
(0.006)^{\star \star \star}\end{array}$ & $\begin{array}{c}0.042 \\
(0.005)^{\star * \star *}\end{array}$ & $\begin{array}{c}0.088 \\
(0.005)^{\star \star \star}\end{array}$ & $\begin{array}{c}0.039 \\
(0.005)^{* * *}\end{array}$ \\
\hline Children 14-24 & & & $\begin{array}{c}-0.022 \\
(0.010)^{*}\end{array}$ & $\begin{array}{c}0.026 \\
(0.005)^{\star \star *}\end{array}$ & $\begin{array}{c}-0.018 \\
(0.010)\end{array}$ & $\begin{array}{c}0.021 \\
(0.006)^{* \star *}\end{array}$ & $\begin{array}{c}-0.004 \\
(0.009)\end{array}$ & $\begin{array}{c}0.020 \\
(0.005)^{\star \star *}\end{array}$ \\
\hline Lone parent & & & $\begin{array}{c}-0.108 \\
(0.025)^{\star \star \star}\end{array}$ & $\begin{array}{c}-0.030 \\
(0.011)^{\star \star}\end{array}$ & $\begin{array}{c}-0.126 \\
(0.027)^{\star \star \star \star}\end{array}$ & $\begin{array}{r}-0.019 \\
(0.010)\end{array}$ & $\begin{array}{c}-0.101 \\
(0.024)^{\star \star \star \star}\end{array}$ & $\begin{array}{c}-0.016 \\
(0.010)\end{array}$ \\
\hline Chronic health condition & & & $\begin{array}{c}-0.002 \\
(0.005)\end{array}$ & $\begin{array}{c}-0.053 \\
(0.004)^{* * *}\end{array}$ & $\begin{array}{c}-0.006 \\
(0.005)\end{array}$ & $\begin{array}{c}-0.052 \\
(0.004)^{* * *}\end{array}$ & $\begin{array}{c}-0.007 \\
(0.005)\end{array}$ & $\begin{array}{c}-0.056 \\
(0.004)^{\star \star \star}\end{array}$ \\
\hline Health activity limitation & & & $\begin{array}{c}0.009 \\
(0.005)\end{array}$ & $\begin{array}{c}0.018 \\
(0.004)^{\star \star \star}\end{array}$ & $\begin{array}{c}0.011 \\
(0.005)^{*}\end{array}$ & $\begin{array}{c}0.014 \\
(0.004)^{*}\end{array}$ & $\begin{array}{c}0.018 \\
(0.005)^{\star * \star *}\end{array}$ & $\begin{array}{c}0.013 \\
(0.004)^{\star \star}\end{array}$ \\
\hline
\end{tabular}




\begin{tabular}{|c|c|c|c|c|c|c|c|c|}
\hline \multirow{3}{*}{$\begin{array}{l}\text { Appendix C2 } \\
\text { (continued) }\end{array}$} & \multicolumn{8}{|c|}{ Average Marginal Effects } \\
\hline & \multicolumn{2}{|c|}{ Model 1} & \multicolumn{2}{|c|}{ Model 2} & \multicolumn{2}{|c|}{ Model 3} & \multicolumn{2}{|c|}{ Full Model } \\
\hline & Men & Women & Men & Women & Men & Women & Men & Women \\
\hline Other sources of income & & & $\begin{array}{c}-0.048 \\
(0.008)^{* * *}\end{array}$ & $\begin{array}{c}0.019 \\
(0.004)^{* * *}\end{array}$ & $\begin{array}{c}-0.038 \\
(0.007)^{*+* *}\end{array}$ & $\begin{array}{c}0.023 \\
(0.004)^{* * *}\end{array}$ & $\begin{array}{c}-0.031 \\
(0.007)^{\star \star * \star}\end{array}$ & $\begin{array}{c}0.024 \\
(0.004)^{* * * *}\end{array}$ \\
\hline Two or more maintainers & & & $\begin{array}{c}0.026 \\
(0.005)^{\star * *}\end{array}$ & $\begin{array}{c}0.016 \\
(0.004)^{* * \star}\end{array}$ & $\begin{array}{c}0.019 \\
(0.005)^{* * *}\end{array}$ & $\begin{array}{c}0.016 \\
(0.004)^{\star * *}\end{array}$ & $\begin{array}{c}0.019 \\
(0.005)^{\star * *}\end{array}$ & $\begin{array}{c}0.018 \\
(0.004)^{m * *}\end{array}$ \\
\hline First maintainer & & & $\begin{array}{c}0.031 \\
(0.006)^{\star \star *}\end{array}$ & $\begin{array}{c}0.024 \\
(0.004)^{* * *}\end{array}$ & $\begin{array}{c}0.027 \\
(0.005)^{* * * *}\end{array}$ & $\begin{array}{c}0.025 \\
(0.004)^{\star \star \star \star}\end{array}$ & $\begin{array}{c}0.028 \\
(0.005)^{\star \star \star *}\end{array}$ & $\begin{array}{c}0.028 \\
(0.004)^{* * * *}\end{array}$ \\
\hline Atlantic provinces & & & $\begin{array}{c}-0.102 \\
(0.011)^{* * *}\end{array}$ & $\begin{array}{c}-0.091 \\
(0.009)^{* * *}\end{array}$ & $\begin{array}{c}-0.116 \\
(0.011)^{\text {twkt }}\end{array}$ & $\begin{array}{c}-0.089 \\
(0.009)^{\star \star \star \star}\end{array}$ & $\begin{array}{c}-0.117 \\
(0.010)^{\star \star \star \star}\end{array}$ & $\begin{array}{c}-0.088 \\
(0.009)^{\star \star \star *}\end{array}$ \\
\hline Northern Territories & & & $\begin{array}{c}0.007 \\
(0.009)\end{array}$ & $\begin{array}{c}-0.070 \\
(0.10)^{\star * *}\end{array}$ & $\begin{array}{c}0.022 \\
(0.008)^{* * *}\end{array}$ & $\begin{array}{c}-0.052 \\
(0.009)^{* * *}\end{array}$ & $\begin{array}{c}0.050 \\
(0.007)^{* * *}\end{array}$ & $\begin{array}{c}-0.069 \\
(0.010)^{* * * *}\end{array}$ \\
\hline Quebec & & & $\begin{array}{c}0.046 \\
(0.010)^{\star * *}\end{array}$ & $\begin{array}{c}-0.014 \\
(0.014)\end{array}$ & $\begin{array}{c}0.042 \\
(0.011)^{* * \star}\end{array}$ & $\begin{array}{r}-0.010 \\
(0.013)\end{array}$ & $\begin{array}{c}0.062 \\
(0.008)^{* \star * *}\end{array}$ & $\begin{array}{l}-0.008 \\
(0.012)\end{array}$ \\
\hline Manitoba & & & $\begin{array}{c}-0.097 \\
(0.015)^{* * *}\end{array}$ & - & $\begin{array}{c}-0.077 \\
(0.014)^{n+* *}\end{array}$ & - & $\begin{array}{c}-0.062 \\
(0.012)^{* * *}\end{array}$ & - \\
\hline Saskatchewan & & & $\begin{array}{c}-0.147 \\
(0.017)^{\star \star *}\end{array}$ & $\begin{array}{c}-0.110 \\
(0.013)^{\star \star \star \star}\end{array}$ & $\begin{array}{c}-0.132 \\
(0.016)^{* * *}\end{array}$ & $\begin{array}{c}-0.107 \\
(0.013)^{\star \pm * *}\end{array}$ & $\begin{array}{c}-0.075 \\
(0.013)^{\star * * *}\end{array}$ & $\begin{array}{c}-0.0118 \\
(0.014)^{\star * * *}\end{array}$ \\
\hline Alberta & & & $\begin{array}{c}0.029 \\
(0.009)^{\star *}\end{array}$ & $\begin{array}{c}-0.038 \\
(0.011)^{* *}\end{array}$ & $\begin{array}{c}0.032 \\
(0.009)^{* * * *}\end{array}$ & $\begin{array}{c}-0.036 \\
(0.011)^{\star *}\end{array}$ & $\begin{array}{c}0.059 \\
(0.007)^{\star * *}\end{array}$ & $\begin{array}{l}-0.035 \\
(0.011)^{* *}\end{array}$ \\
\hline British Columbia & & & $\begin{array}{c}-0.005 \\
(0.010)\end{array}$ & $\begin{array}{c}-0.035 \\
(0.009)^{* * *}\end{array}$ & $\begin{array}{c}-0.001 \\
(0.010)\end{array}$ & $\begin{array}{c}-0.032 \\
(0.009)^{\star * * *}\end{array}$ & $\begin{array}{c}0.008 \\
(0.009)\end{array}$ & $\begin{array}{c}-0.029 \\
(0.009)^{* *}\end{array}$ \\
\hline Has someone to confide in & & & & & $\begin{array}{c}0.011 \\
(0.014)\end{array}$ & $\begin{array}{c}-0.022 \\
(0.007)^{* * *}\end{array}$ & $\begin{array}{c}-0.022 \\
(0.009)^{*}\end{array}$ & $\begin{array}{c}-0.022 \\
(0.007)^{*+}\end{array}$ \\
\hline Band member & & & & & & & $\begin{array}{c}-0.0247 \\
(0.007)^{* * * *}\end{array}$ & $\begin{array}{c}-0.032 \\
(0.006)^{*+* *+}\end{array}$ \\
\hline Registered Indian & & & & & & & $\begin{array}{c}-0.050 \\
(0.006)^{* * *}\end{array}$ & $\begin{array}{c}0.017 \\
(0.007)^{*}\end{array}$ \\
\hline $\begin{array}{l}\text { Speaks Aboriginal } \\
\text { languages }\end{array}$ & & & & & & & $\begin{array}{c}-0.062 \\
(0.007)^{\star \star * \star}\end{array}$ & $\begin{array}{c}0.014 \\
(0.005)^{* *}\end{array}$ \\
\hline $\begin{array}{l}\text { Values Aboriginal } \\
\text { languages }\end{array}$ & & & & & & & $\begin{array}{c}-0.048 \\
(0.005)^{*+*}\end{array}$ & $\begin{array}{c}0.036 \\
(0.005)^{\text {tw*t }}\end{array}$ \\
\hline $\begin{array}{l}\text { Traditional pursuits } \\
\text { for commerce }\end{array}$ & & & & & & & $\begin{array}{c}0.031 \\
(0.006)^{* k *}\end{array}$ & $\begin{array}{c}0.008 \\
(0.010)\end{array}$ \\
\hline $\begin{array}{l}\text { Traditional pursuits } \\
\text { for food }\end{array}$ & & & & & & & $\begin{array}{c}-0.032 \\
(0.006)^{* * * *}\end{array}$ & $\begin{array}{c}-0.015 \\
(0.005)^{* *}\end{array}$ \\
\hline $\begin{array}{l}\text { Traditional pursuits } \\
\text { for pleasure }\end{array}$ & & & & & & & $\begin{array}{c}-0.029 \\
(0.006)^{* * * *}\end{array}$ & $\begin{array}{c}0.017 \\
(0.005)^{* *}\end{array}$ \\
\hline Number of observations & 940 & 960 & 920 & 940 & 910 & 940 & 900 & 940 \\
\hline
\end{tabular}

Source: Statistics Canada, 2006 Aboriginal Peoples Survey, Adult Core analytical file. Bootstrapped ( 1000 replications) standard errors in parentheses. Statistical significance ${ }^{* * *} p<.001 ;{ }^{* *} p<.01 ;{ }^{*} p<.05$. 
Appendix C 3: Detailed results from probit models predicting employment for registered NAl, APS 2006

\begin{tabular}{|c|c|c|c|c|c|c|c|c|}
\hline \multirow{3}{*}{ Variables in equations } & \multicolumn{8}{|c|}{ Average Marginal Effects } \\
\hline & \multicolumn{2}{|c|}{ Model 1} & \multicolumn{2}{|c|}{ Model 2} & \multicolumn{2}{|c|}{ Model 3} & \multicolumn{2}{|c|}{ Full Model } \\
\hline & Men & Women & Men & Women & Men & Women & Men & Women \\
\hline $\begin{array}{l}\text { Less than } \\
\text { high school education }\end{array}$ & $\begin{array}{c}-0.048 \\
(0.008)^{\star * *}\end{array}$ & $\begin{array}{c}-0.024 \\
(0.007)^{\star \star}\end{array}$ & $\begin{array}{c}-0.051 \\
(0.008)^{\star * *}\end{array}$ & $\begin{array}{c}-0.017 \\
(0.006)^{\star \star}\end{array}$ & $\begin{array}{c}-0.053 \\
(0.008)^{* * *}\end{array}$ & $\begin{array}{c}-0.017 \\
(0.006)^{\star \star}\end{array}$ & $\begin{array}{c}-0.051 \\
(0.008)^{\star \star *}\end{array}$ & $\begin{array}{c}-0.022 \\
(0.006)^{\star \star}\end{array}$ \\
\hline $\begin{array}{l}\text { Some } \\
\text { non-university education }\end{array}$ & $\begin{array}{r}-0.012 \\
(0.009)\end{array}$ & $\begin{array}{c}0.056 \\
(0.004)^{* * *}\end{array}$ & $\begin{array}{c}-0.024 \\
(0.010)^{*}\end{array}$ & $\begin{array}{c}0.058 \\
(0.004)^{\star \star \star \star}\end{array}$ & $\begin{array}{c}-0.024 \\
(0.010)^{\star}\end{array}$ & $\begin{array}{c}0.057 \\
(0.004)^{\star \star \star \star}\end{array}$ & $\begin{array}{r}-0.019 \\
(0.010)\end{array}$ & $\begin{array}{c}0.055 \\
(0.004)^{* * *}\end{array}$ \\
\hline $\begin{array}{l}\text { Completed, } \\
\text { non-university education }\end{array}$ & $\begin{array}{c}-0.037 \\
(0.009)^{\star \star \star}\end{array}$ & $\begin{array}{c}0.070 \\
(0.006)^{* * *}\end{array}$ & $\begin{array}{c}-0.049 \\
(0.009)^{\star * \star}\end{array}$ & $\begin{array}{c}0.078 \\
(0.005)^{\star \star \star}\end{array}$ & $\begin{array}{c}-0.051 \\
(0.009)^{\star \star \star}\end{array}$ & $\begin{array}{c}0.078 \\
(0.005)^{\star * *}\end{array}$ & $\begin{array}{c}-0.048 \\
(0.008)^{k \star \star k}\end{array}$ & $\begin{array}{c}0.072 \\
(0.005)^{\star \star \star *}\end{array}$ \\
\hline $\begin{array}{l}\text { Some } \\
\text { university education }\end{array}$ & $\begin{array}{c}-0.067 \\
(0.013)^{\star \star \star \star}\end{array}$ & $\begin{array}{c}0.061 \\
(0.004)^{\star \star \star}\end{array}$ & $\begin{array}{c}-0.083 \\
(0.014)^{\star \star \star}\end{array}$ & $\begin{array}{c}0.057 \\
(0.004)^{\star \star \star \star}\end{array}$ & $\begin{array}{c}-0.085 \\
(0.014)^{\star \star \star}\end{array}$ & $\begin{array}{c}0.057 \\
(0.005)^{\star * *}\end{array}$ & $\begin{array}{c}-0.085 \\
(0.014)^{\star \star \star}\end{array}$ & $\begin{array}{c}0.056 \\
(0.004)^{\star * *}\end{array}$ \\
\hline $\begin{array}{l}\text { Completed } \\
\text { university education }\end{array}$ & $\begin{array}{c}0.047 \\
(0.007)^{\star \star \star}\end{array}$ & $\begin{array}{c}0.064 \\
(0.005)^{\star \star \star}\end{array}$ & $\begin{array}{c}0.040 \\
(0.007)^{\star \star \star}\end{array}$ & $\begin{array}{c}0.065 \\
(0.004)^{\star \star \star}\end{array}$ & $\begin{array}{c}0.040 \\
(0.007)^{\star \star \star \star}\end{array}$ & $\begin{array}{c}0.064 \\
(0.004)^{\star * \star}\end{array}$ & $\begin{array}{c}0.042 \\
(0.007)^{\star \star *}\end{array}$ & $\begin{array}{c}0.063 \\
(0.004)^{\star * \star}\end{array}$ \\
\hline Age 15 to 19 & $\begin{array}{c}0.100 \\
(0.015)^{\star \star \star}\end{array}$ & $\begin{array}{c}-0.144 \\
(0.021)^{\star \star \star}\end{array}$ & $\begin{array}{c}-0.075 \\
(0.014)^{\star * *}\end{array}$ & $\begin{array}{c}-0.145 \\
(0.019)^{\star \star \star}\end{array}$ & $\begin{array}{c}-0.083 \\
(0.014)^{\star \star \star}\end{array}$ & $\begin{array}{c}-0.145 \\
(0.020)^{* * *}\end{array}$ & $\begin{array}{c}-0.085 \\
(0.014)^{\star \star \star \star}\end{array}$ & $\begin{array}{c}-0.142 \\
(0.019)^{\star * \star}\end{array}$ \\
\hline Age 25 to 34 & $\begin{array}{c}0.0245 \\
(0.007)^{\star \star}\end{array}$ & $\begin{array}{c}-0.076 \\
(0.041)^{\star \star \star *}\end{array}$ & $\begin{array}{c}0.027 \\
(0.008)^{\star \star *}\end{array}$ & $\begin{array}{c}-0.077 \\
(0.010)^{\star \star * *}\end{array}$ & $\begin{array}{c}0.026 \\
(0.008)^{\star \star}\end{array}$ & $\begin{array}{c}-0.074 \\
(0.010)^{* *}\end{array}$ & $\begin{array}{c}0.026 \\
(0.008)^{* *}\end{array}$ & $\begin{array}{c}-0.072 \\
(0.010)^{* * *}\end{array}$ \\
\hline Age 35 to 44 & $\begin{array}{c}0.063 \\
(0.006)^{\star \star \star}\end{array}$ & $\begin{array}{c}-0.064 \\
(0.009)^{\star \star \star \star}\end{array}$ & $\begin{array}{c}0.058 \\
(0.007)^{* *}\end{array}$ & $\begin{array}{c}-0.062 \\
(0.009)^{\star \star \star}\end{array}$ & $\begin{array}{c}0.057 \\
(0.007)^{\star \star \star}\end{array}$ & $\begin{array}{c}-0.055 \\
(0.009)^{\star * k}\end{array}$ & $\begin{array}{c}0.055 \\
(0.007)^{\star * \star}\end{array}$ & $\begin{array}{c}-0.052 \\
(0.009)^{\star \star \star}\end{array}$ \\
\hline Age 45 to 54 & $\begin{array}{c}0.061 \\
(0.006)^{\star \star \star}\end{array}$ & $\begin{array}{c}-0.038 \\
(0.008)^{\star \star \star}\end{array}$ & $\begin{array}{c}0.052 \\
(0.008)^{\star \star \star}\end{array}$ & $\begin{array}{c}-0.046 \\
(0.010)^{\star * * *}\end{array}$ & $\begin{array}{c}0.050 \\
(0.008)^{\star \star \star \star}\end{array}$ & $\begin{array}{c}-0.038 \\
(0.009)^{* * *}\end{array}$ & $\begin{array}{c}0.052 \\
(0.008)^{\star \star \star}\end{array}$ & $\begin{array}{c}-0.035 \\
(0.009)^{\star \star \star}\end{array}$ \\
\hline Age 55 to 64 & $\begin{array}{c}0.089 \\
(0.004)^{\star * * *}\end{array}$ & $\begin{array}{c}0.025 \\
(0.008)^{\star *}\end{array}$ & $\begin{array}{c}0.087 \\
(0.005)^{* * \star}\end{array}$ & $\begin{array}{c}0.003 \\
(0.011)\end{array}$ & $\begin{array}{c}0.087 \\
(0.005)^{\star \star \star}\end{array}$ & $\begin{array}{c}0.008 \\
(0.010)\end{array}$ & $\begin{array}{c}0.089 \\
(0.005)^{\star * * *}\end{array}$ & $\begin{array}{c}0.009 \\
(0.009)\end{array}$ \\
\hline Languages - French & $\begin{array}{c}0.040 \\
(0.007)^{\star \star \star}\end{array}$ & $\begin{array}{c}0.036 \\
(0.005)^{\star \star \star}\end{array}$ & $\begin{array}{c}-0.017 \\
(0.018)\end{array}$ & $\begin{array}{c}0.070 \\
(0.003)^{\star * *}\end{array}$ & $\begin{array}{c}-0.009 \\
(0.018)\end{array}$ & $\begin{array}{c}0.069 \\
(0.003)^{\star \star \star}\end{array}$ & $\begin{array}{c}-0.004 \\
(0.017)\end{array}$ & $\begin{array}{c}0.069 \\
(0.003)^{\star * \star}\end{array}$ \\
\hline Languages - Both & $\begin{array}{c}0.027 \\
(0.006)^{\star \star \star *}\end{array}$ & $\begin{array}{c}0.038 \\
(0.004)^{\star \star \star}\end{array}$ & $\begin{array}{c}0.003 \\
(0.008)\end{array}$ & $\begin{array}{c}0.060 \\
(0.004)^{\star * *}\end{array}$ & $\begin{array}{c}0.006 \\
(0.008)\end{array}$ & $\begin{array}{c}0.057 \\
(0.004)^{* * *}\end{array}$ & $\begin{array}{c}0.002 \\
(0.008)\end{array}$ & $\begin{array}{c}0.057 \\
(0.004)^{\star \star *}\end{array}$ \\
\hline Access/use technology & $\begin{array}{c}0.069 \\
(0.006)^{\star \star *}\end{array}$ & $\begin{array}{c}0.009 \\
(0.005)\end{array}$ & $\begin{array}{c}0.053 \\
(0.005)^{\star * *}\end{array}$ & $\begin{array}{c}0.002 \\
(0.004)\end{array}$ & $\begin{array}{c}0.052 \\
(0.006)^{\star \star \star \star}\end{array}$ & $\begin{array}{c}0.003 \\
(0.004)\end{array}$ & $\begin{array}{c}0.049 \\
(0.005)^{\star * *}\end{array}$ & $\begin{array}{c}-0.001 \\
(0.004)\end{array}$ \\
\hline Legally married & & & $\begin{array}{c}0.031 \\
(0.007)^{\star * *}\end{array}$ & $\begin{array}{c}0.031 \\
(0.006)^{* * *}\end{array}$ & $\begin{array}{c}0.031 \\
(0.007)^{* * *}\end{array}$ & $\begin{array}{c}0.028 \\
(0.006)^{* * *}\end{array}$ & $\begin{array}{c}0.031 \\
(0.007)^{\star \star \star}\end{array}$ & $\begin{array}{c}0.029 \\
(0.006)^{* * *}\end{array}$ \\
\hline Common-law & & & $\begin{array}{c}0.038 \\
(0.006)^{\star \star *}\end{array}$ & $\begin{array}{r}-0.009 \\
(0.006)\end{array}$ & $\begin{array}{c}0.038 \\
(0.006)^{\star \star \star}\end{array}$ & $\begin{array}{c}-0.008 \\
(0.006)\end{array}$ & $\begin{array}{c}0.040 \\
(0.005)^{\star \star \star}\end{array}$ & $\begin{array}{l}-0.009 \\
(0.006)\end{array}$ \\
\hline Divorced-separated & & & $\begin{array}{c}0.041 \\
(0.005)^{\star \star \star}\end{array}$ & $\begin{array}{c}-0.027 \\
(0.006)^{k \star \star}\end{array}$ & $\begin{array}{c}0.042 \\
(0.005)^{* \star * *}\end{array}$ & $\begin{array}{c}-0.031 \\
(0.006)^{* \star \star}\end{array}$ & $\begin{array}{c}0.044 \\
(0.005)^{* * *}\end{array}$ & $\begin{array}{c}-0.030 \\
(0.006)^{* * *}\end{array}$ \\
\hline Children $<6$ & & & $\begin{array}{c}-0.034 \\
(0.009)^{* *}\end{array}$ & $\begin{array}{c}-0.046 \\
(0.008)^{* \star \star \star}\end{array}$ & $\begin{array}{c}-0.033 \\
(0.008)^{\star \star \star}\end{array}$ & $\begin{array}{c}-0.044 \\
(0.008)^{\star \star \star \star}\end{array}$ & $\begin{array}{c}-0.024 \\
(0.008)^{\star \star}\end{array}$ & $\begin{array}{c}-0.046 \\
(0.008)^{\star * *}\end{array}$ \\
\hline Children 6-14 & & & $\begin{array}{c}0.020 \\
(0.007)^{\star \star}\end{array}$ & $\begin{array}{r}-0.014 \\
(0.008)\end{array}$ & $\begin{array}{c}0.020 \\
(0.007)^{\star *}\end{array}$ & $\begin{array}{c}-0.015 \\
(0.008)\end{array}$ & $\begin{array}{c}0.027 \\
(0.007)^{\star \star * *}\end{array}$ & $\begin{array}{c}-0.016 \\
(0.008)^{*}\end{array}$ \\
\hline Children 14-24 & & & $\begin{array}{c}-0.020 \\
(0.010)^{*}\end{array}$ & $\begin{array}{c}-0.066 \\
(0.009)^{* * *}\end{array}$ & $\begin{array}{c}-0.021 \\
(0.010)^{*}\end{array}$ & $\begin{array}{c}-0.072 \\
(0.010)^{\star * *}\end{array}$ & $\begin{array}{r}-0.015 \\
(0.009)\end{array}$ & $\begin{array}{c}-0.072 \\
(0.010)^{\star * *}\end{array}$ \\
\hline Lone parent & & & $\begin{array}{c}-0.001 \\
(0.012)\end{array}$ & $\begin{array}{r}-0.0009 \\
(0.008)\end{array}$ & $\begin{array}{r}-0.003 \\
(0.013)\end{array}$ & $\begin{array}{c}0.005 \\
(0.008)\end{array}$ & $\begin{array}{c}-0.005 \\
(0.013)\end{array}$ & $\begin{array}{c}0.002 \\
(0.008)\end{array}$ \\
\hline Chronic health condition & & & $\begin{array}{c}-0.004 \\
(0.005)\end{array}$ & $\begin{array}{c}0.015 \\
(0.004)^{\star \star \star}\end{array}$ & $\begin{array}{c}-0.001 \\
(0.005)\end{array}$ & $\begin{array}{c}0.015 \\
(0.004)^{*}\end{array}$ & $\begin{array}{c}0.004 \\
(0.005)\end{array}$ & $\begin{array}{c}0.015 \\
(0.004)^{\star \star \star \star}\end{array}$ \\
\hline Health activity limitation & & & $\begin{array}{c}-0.032 \\
(0.005)^{\star \star \star}\end{array}$ & $\begin{array}{c}-0.010 \\
(0.004)^{\star}\end{array}$ & $\begin{array}{c}-0.035 \\
(0.005)^{\star \star \star *}\end{array}$ & $\begin{array}{c}-0.010 \\
(0.004)^{*}\end{array}$ & $\begin{array}{c}-0.040 \\
(0.005)^{\star \star \star}\end{array}$ & $\begin{array}{c}-0.012 \\
(0.004)^{\star \star}\end{array}$ \\
\hline
\end{tabular}




\begin{tabular}{|c|c|c|c|c|c|c|c|c|}
\hline \multirow{3}{*}{$\begin{array}{l}\text { Appendix C3 } \\
\text { (continued) }\end{array}$} & \multicolumn{8}{|c|}{ Average Marginal Effects } \\
\hline & \multicolumn{2}{|c|}{ Model 1} & \multicolumn{2}{|c|}{ Model 2} & \multicolumn{2}{|c|}{ Model 3} & \multicolumn{2}{|c|}{ Full Model } \\
\hline & Men & Women & Men & Women & Men & Women & Men & Women \\
\hline Other sources of income & & & $\begin{array}{c}0.005 \\
(0.005)\end{array}$ & $\begin{array}{c}-0.007 \\
(0.004)\end{array}$ & $\begin{array}{c}0.06 \\
(0.005)\end{array}$ & $\begin{array}{c}-0.005 \\
(0.004)\end{array}$ & $\begin{array}{c}0.006 \\
(0.005)\end{array}$ & $\begin{array}{c}-0.001 \\
(0.004)\end{array}$ \\
\hline Two or more maintainers & & & $\begin{array}{c}0.024 \\
(0.005)^{\star * *}\end{array}$ & $\begin{array}{c}0.016 \\
(0.004)^{\star \star \star}\end{array}$ & $\begin{array}{c}0.027 \\
(0.005)^{\star \star *}\end{array}$ & $\begin{array}{c}0.016 \\
(0.004)^{* * *}\end{array}$ & $\begin{array}{c}0.024 \\
(0.004)^{\star \star \star}\end{array}$ & $\begin{array}{c}0.018 \\
(0.004)^{* \cdots *}\end{array}$ \\
\hline First maintainer & & & $\begin{array}{c}-0.007 \\
(0.005)\end{array}$ & $\begin{array}{c}0.030 \\
(0.004)^{* * *}\end{array}$ & $\begin{array}{c}-0.008 \\
(0.005)\end{array}$ & $\begin{array}{c}0.028 \\
(0.004)^{m}\end{array}$ & $\begin{array}{c}-0.012 \\
(0.005)^{*}\end{array}$ & $\begin{array}{c}0.028 \\
(0.004)^{\star \star \star *}\end{array}$ \\
\hline Atlantic provinces & & & $\begin{array}{c}0.088 \\
(0.010)^{\star \star \star *}\end{array}$ & $\begin{array}{c}-0.024 \\
(0.008)^{\star *}\end{array}$ & $\begin{array}{c}-0.088 \\
(0.010)^{\star \star \star}\end{array}$ & $\begin{array}{c}-0.027 \\
(0.008)^{\star *}\end{array}$ & $\begin{array}{c}-0.092 \\
(0.010)^{* k *}\end{array}$ & $\begin{array}{c}-0.025 \\
(0.008)^{\star \star}\end{array}$ \\
\hline Northern Territories & & & $\begin{array}{l}-0.0008 \\
(0.008)\end{array}$ & $\begin{array}{c}-0.011 \\
(0.007)\end{array}$ & $\begin{array}{r}-0.0002 \\
(0.008)\end{array}$ & $\begin{array}{l}-0.0008 \\
(0.007)\end{array}$ & $\begin{array}{c}0.005 \\
(0.008)\end{array}$ & $\begin{array}{r}-0.0001 \\
(0.007)\end{array}$ \\
\hline Quebec & & & $\begin{array}{c}0.025 \\
(0.011)^{*}\end{array}$ & $\begin{array}{c}-0.063 \\
(0.015)^{\star * *}\end{array}$ & $\begin{array}{c}0.024 \\
(0.011)^{\star}\end{array}$ & $\begin{array}{c}-0.064 \\
(0.015)^{* * *}\end{array}$ & $\begin{array}{c}0.020 \\
(0.012)\end{array}$ & $\begin{array}{c}-0.060 \\
(0.014)^{\star \star \star}\end{array}$ \\
\hline Manitoba & & & $\begin{array}{c}-0.104 \\
(0.012)^{\star \star \star}\end{array}$ & $\begin{array}{c}-0.052 \\
(0.004)^{\star \star \star}\end{array}$ & $\begin{array}{c}-0.108 \\
(0.012)^{\star \star \star}\end{array}$ & $\begin{array}{c}0.055 \\
(0.004)^{\star * *}\end{array}$ & $\begin{array}{c}-0.101 \\
(0.012)^{* * * *}\end{array}$ & $\begin{array}{c}0.056 \\
(0.004)^{* * *}\end{array}$ \\
\hline Saskatchewan & & & $\begin{array}{c}-0.088 \\
(0.011)^{\star \star \star}\end{array}$ & $\begin{array}{r}-0.0004 \\
(0.006)\end{array}$ & $\begin{array}{c}-0.085 \\
(0.011)^{\star \star \star}\end{array}$ & $\begin{array}{r}-0.0001 \\
(0.006)\end{array}$ & $\begin{array}{c}-0.076 \\
(0.010)^{* * * k}\end{array}$ & $\begin{array}{l}0.0008 \\
(0.006)\end{array}$ \\
\hline Alberta & & & $\begin{array}{c}-0.031 \\
(0.009)^{* *}\end{array}$ & $\begin{array}{c}0.017 \\
(0.005)^{\star \star}\end{array}$ & $\begin{array}{c}-0.030 \\
(0.009)^{\star *}\end{array}$ & $\begin{array}{c}0.014 \\
(0.005)^{\star *}\end{array}$ & $\begin{array}{c}0.019 \\
(0.009)^{*}\end{array}$ & $\begin{array}{c}0.020 \\
(0.005)^{* *}\end{array}$ \\
\hline British Columbia & & & $\begin{array}{c}-0.017 \\
(0.008)^{*}\end{array}$ & $\begin{array}{c}0.014 \\
(0.006)^{*}\end{array}$ & $\begin{array}{c}-0.014 \\
(0.008)\end{array}$ & $\begin{array}{c}0.014 \\
(0.006)\end{array}$ & $\begin{array}{c}-0.013 \\
(0.008)\end{array}$ & $\begin{array}{c}0.016 \\
(0.005)^{\star \star}\end{array}$ \\
\hline Rural place of residence & & & $\begin{array}{c}-0.056 \\
(0.005)^{* \star \star *}\end{array}$ & $\begin{array}{c}0.008 \\
(0.003)^{\star}\end{array}$ & $\begin{array}{c}-0.055 \\
(0.005)^{\star \star \star \star}\end{array}$ & $\begin{array}{c}0.011 \\
(0.004)^{\star *}\end{array}$ & $\begin{array}{c}-0.049 \\
(0.005)^{* * *}\end{array}$ & $\begin{array}{c}0.012 \\
(0.003)^{\star \star}\end{array}$ \\
\hline Has someone to confide in & & & & & $\begin{array}{c}-0.013 \\
(0.007)\end{array}$ & $\begin{array}{c}0.046 \\
(0.011)^{\star \star \star}\end{array}$ & $\begin{array}{c}-0.028 \\
(0.006)^{* * *}\end{array}$ & $\begin{array}{c}0.050 \\
(0.012)^{* * * *}\end{array}$ \\
\hline Band member & & & & & & & $\begin{array}{c}0.022 \\
(0.010)^{\star}\end{array}$ & $\begin{array}{c}-0.032 \\
(0.005)^{* \cdots \hbar}\end{array}$ \\
\hline $\begin{array}{l}\text { Speaks Aboriginal } \\
\text { languages }\end{array}$ & & & & & & & $\begin{array}{c}-0.041 \\
(0.005)^{\star * * *}\end{array}$ & $\begin{array}{l}0.0001 \\
(0.004)\end{array}$ \\
\hline $\begin{array}{l}\text { Values Aboriginal } \\
\text { languages }\end{array}$ & & & & & & & $\begin{array}{c}0.019 \\
(0.005)^{\star *}\end{array}$ & $\begin{array}{c}0.025 \\
(0.005)^{\star \star \star}\end{array}$ \\
\hline $\begin{array}{l}\text { Traditional pursuits } \\
\text { for commerce }\end{array}$ & & & & & & & $\begin{array}{c}-0.022 \\
(0.009)^{\star}\end{array}$ & $\begin{array}{c}0.036 \\
(0.007)^{* *}\end{array}$ \\
\hline $\begin{array}{l}\text { Traditional pursuits } \\
\text { for food }\end{array}$ & & & & & & & $\begin{array}{c}0.003 \\
(0.005)\end{array}$ & $\begin{array}{c}-0.014 \\
(0.004)^{\star \star \hbar}\end{array}$ \\
\hline $\begin{array}{l}\text { Traditional pursuits } \\
\text { for pleasure }\end{array}$ & & & & & & & $\begin{array}{c}0.009 \\
(0.005)\end{array}$ & $\begin{array}{c}0.009 \\
(0.004)^{\star}\end{array}$ \\
\hline Number of observations & 1680 & 1840 & 1650 & 1820 & 1630 & 1810 & 1610 & 1800 \\
\hline
\end{tabular}

Source: Statistics Canada, 2006 Aboriginal Peoples Survey, Adult Core analytical file. Bootstrapped (1000 replications) standard errors in parentheses. Statistical significance ${ }^{* *} p<.001 ;{ }^{* *} p<.01 ;{ }^{*} p<.05$. 
Appendix C 4: Detailed results from probit models predicting employment for non-registered NAI, APS 2006

\begin{tabular}{|c|c|c|c|c|c|c|c|c|}
\hline \multirow{3}{*}{ Variables in equations } & \multicolumn{8}{|c|}{ Average Marginal Effects } \\
\hline & \multicolumn{2}{|c|}{ Model 1} & \multicolumn{2}{|c|}{ Model 2} & \multicolumn{2}{|c|}{ Model 3} & \multicolumn{2}{|c|}{ Full Model } \\
\hline & Men & Women & Men & Women & Men & Women & Men & Women \\
\hline $\begin{array}{l}\text { Less than } \\
\text { high school education }\end{array}$ & $\begin{array}{c}-0.042 \\
(0.009)^{* * * *}\end{array}$ & $\begin{array}{c}-0.033 \\
(0.009)^{* * *}\end{array}$ & $\begin{array}{c}-0.052 \\
(0.009)^{* * * *}\end{array}$ & $\begin{array}{c}-0.039 \\
(0.008)^{+* * *}\end{array}$ & $\begin{array}{c}-0.062 \\
(0.010)^{* * \star}\end{array}$ & $\begin{array}{c}-0.036 \\
(0.007)^{* \star * *}\end{array}$ & $\begin{array}{c}-0.044 \\
(0.008)^{* \star \star}\end{array}$ & $\begin{array}{c}-0.038 \\
(0.007)^{*+*}\end{array}$ \\
\hline $\begin{array}{l}\text { Some } \\
\text { non-university education }\end{array}$ & $\begin{array}{l}-0.010 \\
(0.012)\end{array}$ & $\begin{array}{c}0.003 \\
(0.008)\end{array}$ & $\begin{array}{r}-0.010 \\
(0.009)\end{array}$ & $\begin{array}{c}0.004 \\
(0.008)\end{array}$ & $\begin{array}{l}-0.014 \\
(0.010)\end{array}$ & $\begin{array}{c}0.002 \\
(0.007)\end{array}$ & $\begin{array}{l}-0.019 \\
(0.010)\end{array}$ & $\begin{array}{c}0.002 \\
(0.007)\end{array}$ \\
\hline $\begin{array}{l}\text { Completed, } \\
\text { non-university education }\end{array}$ & $\begin{array}{c}-0.020 \\
(0.008)^{*}\end{array}$ & $\begin{array}{c}0.015 \\
(0.006)^{*}\end{array}$ & $\begin{array}{l}-0.016 \\
(0.007)^{\star}\end{array}$ & $\begin{array}{c}0.017 \\
(0.005)^{\star \star}\end{array}$ & $\begin{array}{c}-0.020 \\
(0.007)^{* \star}\end{array}$ & $\begin{array}{c}0.021 \\
(0.005)^{\star \star \star \star}\end{array}$ & $\begin{array}{c}-0.024 \\
(0.007)^{* *}\end{array}$ & $\begin{array}{c}0.021 \\
(0.005)^{* * *}\end{array}$ \\
\hline $\begin{array}{l}\text { Some } \\
\text { university education }\end{array}$ & $\begin{array}{c}0.027 \\
(0.007)^{* * *}\end{array}$ & $\begin{array}{c}0.022 \\
(0.006)^{* * * *}\end{array}$ & $\begin{array}{c}0.034 \\
(0.008)^{n+* *}\end{array}$ & $\begin{array}{c}0.032 \\
(0.005)^{\star * * *}\end{array}$ & $\begin{array}{c}0.032 \\
(0.008)^{* * *}\end{array}$ & $\begin{array}{c}0.032 \\
(0.004)^{* * * *}\end{array}$ & $\begin{array}{c}0.032 \\
(0.008)^{* * *}\end{array}$ & $\begin{array}{c}0.030 \\
(0.005)^{* * *}\end{array}$ \\
\hline $\begin{array}{l}\text { Completed } \\
\text { university education }\end{array}$ & $\begin{array}{c}0.015 \\
(0.007)^{\star}\end{array}$ & $\begin{array}{c}0.046 \\
(0.004)^{\star * *}\end{array}$ & $\begin{array}{c}0.022 \\
(0.007)^{\star \star}\end{array}$ & $\begin{array}{c}0.042 \\
(0.004)^{\star * *}\end{array}$ & $\begin{array}{c}0.021 \\
(0.007)^{\star *}\end{array}$ & $\begin{array}{c}0.041 \\
(0.003)^{* * * *}\end{array}$ & $\begin{array}{c}0.029 \\
(0.006)^{\star \star *}\end{array}$ & $\begin{array}{c}0.041 \\
(0.003)^{*+* *}\end{array}$ \\
\hline Age 15 to 19 & $\begin{array}{c}-0.047 \\
(0.014)^{* *}\end{array}$ & $\begin{array}{c}-0.037 \\
(0.012)^{\star *}\end{array}$ & $\begin{array}{c}-0.024 \\
(0.011)^{*}\end{array}$ & $\begin{array}{c}-0.026 \\
(0.010)^{* *}\end{array}$ & $\begin{array}{c}-0.030 \\
(0.011)^{* *}\end{array}$ & $\begin{array}{c}-0.023 \\
(0.009)^{\star}\end{array}$ & $\begin{array}{l}-0.027 \\
(0.011)^{*}\end{array}$ & $\begin{array}{c}-0.020 \\
(0.009)^{*}\end{array}$ \\
\hline Age 25 to 34 & $\begin{array}{c}0.064 \\
(0.005)^{* * \star}\end{array}$ & $\begin{array}{c}-0.044 \\
(0.010)^{* * * *}\end{array}$ & $\begin{array}{c}0.065 \\
(0.005)^{n+* *}\end{array}$ & $\begin{array}{l}-0.039 \\
(0.009)^{* * *}\end{array}$ & $\begin{array}{c}0.062 \\
(0.005)^{* * *}\end{array}$ & $\begin{array}{c}-0.031 \\
(0.008)^{* * *}\end{array}$ & $\begin{array}{c}0.059 \\
(0.005)^{* * *}\end{array}$ & $\begin{array}{c}-0.030 \\
(0.008)^{* * * *}\end{array}$ \\
\hline Age 35 to 44 & $\begin{array}{c}0.030 \\
(0.006)^{* * * *}\end{array}$ & $\begin{array}{c}0.042 \\
(0.005)^{* * *}\end{array}$ & $\begin{array}{c}0.027 \\
(0.006)^{* * * *}\end{array}$ & $\begin{array}{c}0.055 \\
(0.006)^{* * * *}\end{array}$ & $\begin{array}{c}0.023 \\
(0.007)^{* *}\end{array}$ & $\begin{array}{c}0.065 \\
(0.005)^{+* * *}\end{array}$ & $\begin{array}{c}0.031 \\
(0.006)^{* * *}\end{array}$ & $\begin{array}{c}0.068 \\
(0.006)^{* * * *}\end{array}$ \\
\hline Age 45 to 54 & $\begin{array}{c}0.039 \\
(0.006)^{\star \star \star}\end{array}$ & $\begin{array}{c}0.004 \\
(0.008)\end{array}$ & $\begin{array}{c}0.009 \\
(0.009)\end{array}$ & $\begin{array}{c}0.039 \\
(0.007)^{\star \star \star \star}\end{array}$ & $\begin{array}{l}0.005 \\
(0.009)\end{array}$ & $\begin{array}{c}0.045 \\
(0.007)^{\star \star * *}\end{array}$ & $\begin{array}{l}0.0006 \\
(0.009)\end{array}$ & $\begin{array}{c}0.049 \\
(0.007)^{+* t *}\end{array}$ \\
\hline Age 55 to 64 & $\begin{array}{c}0.062 \\
(0.003)^{* * *}\end{array}$ & $\begin{array}{c}0.020 \\
(0.008)^{*}\end{array}$ & $\begin{array}{c}0.052 \\
(0.004)^{\star \star * *}\end{array}$ & $\begin{array}{c}0.045 \\
(0.005)^{* * *}\end{array}$ & $\begin{array}{c}0.052 \\
(0.005)^{* * *}\end{array}$ & $\begin{array}{c}0.050 \\
(0.004)^{* * *}\end{array}$ & $\begin{array}{c}0.054 \\
(0.004)^{* * * *}\end{array}$ & $\begin{array}{c}0.053 \\
(0.004)^{* * *}\end{array}$ \\
\hline Languages - French & $\begin{array}{c}-0.069 \\
(0.013)^{* * *}\end{array}$ & $\begin{array}{c}0.020 \\
(0.006)^{* *}\end{array}$ & $\begin{array}{c}-0.092 \\
(0.023)^{* * *}\end{array}$ & $\begin{array}{l}-0.015 \\
(0.012)\end{array}$ & $\begin{array}{c}-0.105 \\
(0.025)^{* * *}\end{array}$ & $\begin{array}{c}-0.017 \\
(0.012)\end{array}$ & $\begin{array}{c}-0.084 \\
(0.019)^{* * *}\end{array}$ & $\begin{array}{c}-0.015 \\
(0.012)\end{array}$ \\
\hline Languages - Both & $\begin{array}{c}-0.029 \\
(0.012)^{*}\end{array}$ & $\begin{array}{l}0.0006 \\
(0.004)\end{array}$ & $\begin{array}{c}-0.032 \\
(0.009)^{*+\ldots}\end{array}$ & $\begin{array}{l}-0.001 \\
(0.005)\end{array}$ & $\begin{array}{c}-0.042 \\
(0.009)^{*+*}\end{array}$ & $\begin{array}{l}-0.004 \\
(0.006)\end{array}$ & $\begin{array}{c}-0.031 \\
(0.008)^{*+*}\end{array}$ & $\begin{array}{l}-0.003 \\
(0.006)\end{array}$ \\
\hline Access/use technology & $\begin{array}{c}-0.018 \\
(0.005)^{\star \star}\end{array}$ & $\begin{array}{c}-0.019 \\
(0.004)^{\star \star \star *}\end{array}$ & $\begin{array}{c}-0.030 \\
(0.005)^{\star \star *}\end{array}$ & $\begin{array}{c}-0.020 \\
(0.003)^{\star \star \star}\end{array}$ & $\begin{array}{c}-0.030 \\
(0.005)^{\star \star * *}\end{array}$ & $\begin{array}{c}-0.015 \\
(0.003)^{* * *}\end{array}$ & $\begin{array}{c}-0.030 \\
(0.005)^{\star * *}\end{array}$ & $\begin{array}{c}-0.015 \\
(0.003)^{+*+*}\end{array}$ \\
\hline Legaily married & & & $\begin{array}{c}0.051 \\
(0.010)^{* \star * *}\end{array}$ & $\begin{array}{c}-0.055 \\
(0.011)^{*+* *}\end{array}$ & $\begin{array}{c}0.051 \\
(0.010)^{\star \star *}\end{array}$ & $\begin{array}{c}-0.064 \\
(0.011)^{\star \star \star *}\end{array}$ & $\begin{array}{c}0.047 \\
(0.010)^{* * *}\end{array}$ & $\begin{array}{c}-0.064 \\
(0.012)^{\star \Delta * t+}\end{array}$ \\
\hline Common-law & & & $\begin{array}{c}0.019 \\
(0.007)^{* *}\end{array}$ & $\begin{array}{c}-0.025 \\
(0.008)^{* *}\end{array}$ & $\begin{array}{c}0.022 \\
(0.007)^{* *}\end{array}$ & $\begin{array}{c}-0.022 \\
(0.008)^{* *}\end{array}$ & $\begin{array}{c}0.025 \\
(0.006)^{* * *}\end{array}$ & $\begin{array}{c}-0.020 \\
(0.008)^{* *}\end{array}$ \\
\hline Divorced-separated & & & $\begin{array}{c}-0.043 \\
(0.011)^{\star \star * *}\end{array}$ & $\begin{array}{c}0.023 \\
(0.009)^{*}\end{array}$ & $\begin{array}{c}-0.045 \\
(0.012)^{* * * *}\end{array}$ & $\begin{array}{c}-0.038 \\
(0.010)^{* * * *}\end{array}$ & $\begin{array}{c}-0.052 \\
(0.011)^{* * *}\end{array}$ & $\begin{array}{c}-0.039 \\
(0.010)^{* * * *}\end{array}$ \\
\hline Children $<6$ & & & $\begin{array}{c}-0.059 \\
(0.011)^{* \star \star}\end{array}$ & $\begin{array}{c}0.053 \\
(0.004)^{* * *}\end{array}$ & $\begin{array}{c}-0.058 \\
(0.011)^{* * *}\end{array}$ & $\begin{array}{c}0.050 \\
(0.004)^{*+* *}\end{array}$ & $\begin{array}{c}-0.064 \\
(0.011)^{* \pm *}\end{array}$ & $\begin{array}{c}0.051 \\
(0.004)^{* * *}\end{array}$ \\
\hline Children 6-14 & & & $\begin{array}{c}-0.068 \\
(0.014)^{\star \star \star}\end{array}$ & $\begin{array}{c}0.046 \\
(0.004)^{* * *}\end{array}$ & $\begin{array}{c}-0.062 \\
(0.013)^{\star * *}\end{array}$ & $\begin{array}{c}0.040 \\
(0.04)^{\star \star \star \star}\end{array}$ & $\begin{array}{c}-0.063 \\
(0.013)^{* * * *}\end{array}$ & $\begin{array}{c}0.037 \\
(0.004)^{* * *}\end{array}$ \\
\hline Children 14-24 & & & $\begin{array}{c}0.028 \\
(0.006)^{* * *}\end{array}$ & $\begin{array}{c}0.004 \\
(0.006)\end{array}$ & $\begin{array}{c}0.030 \\
(0.006)^{\star * * *}\end{array}$ & $\begin{array}{c}0.011 \\
(0.005)\end{array}$ & $\begin{array}{c}0.021 \\
(0.006)^{* *}\end{array}$ & $\begin{array}{c}0.008 \\
(0.006)\end{array}$ \\
\hline Lone parent & & & $\begin{array}{c}0.044 \\
(0.007)^{* * * *}\end{array}$ & $\begin{array}{c}-0.161 \\
(0.022)^{*+* *}\end{array}$ & $\begin{array}{c}0.043 \\
(0.007)^{* * * *}\end{array}$ & $\begin{array}{c}-0.126 \\
(0.019)^{* * *}\end{array}$ & $\begin{array}{c}0.053 \\
(0.005)^{* * *}\end{array}$ & $\begin{array}{c}-0.118 \\
(0.019)^{* * * *}\end{array}$ \\
\hline Chronic health condition & & & $\begin{array}{c}-0.020 \\
(0.005)^{\star \star \star *}\end{array}$ & $\begin{array}{c}-0.032 \\
(0.004)^{* * *}\end{array}$ & $\begin{array}{c}-0.023 \\
(0.005)^{* * * *}\end{array}$ & $\begin{array}{c}-0.027 \\
(0.004)^{*+\cdots *}\end{array}$ & $\begin{array}{c}-0.033 \\
(0.004)^{* \star * *}\end{array}$ & $\begin{array}{c}-0.028 \\
(0.004)^{*+4 t}\end{array}$ \\
\hline Health activity limitation & & & $\begin{array}{c}0.016 \\
(0.005)^{* *}\end{array}$ & $\begin{array}{c}-0.009 \\
(0.004)^{*}\end{array}$ & $\begin{array}{c}0.017 \\
(0.005)^{\star \star \star}\end{array}$ & $\begin{array}{c}-0.01 \\
(0.004)^{t+* t}\end{array}$ & $\begin{array}{c}0.027 \\
(0.004)^{* * *}\end{array}$ & $\begin{array}{c}-0.016 \\
(0.004)^{* * * *}\end{array}$ \\
\hline
\end{tabular}




\begin{tabular}{|c|c|c|c|c|c|c|c|c|}
\hline \multirow{3}{*}{$\begin{array}{l}\text { Appendix C4 } \\
\text { (continued) }\end{array}$} & \multicolumn{8}{|c|}{ Average Marginal Effects } \\
\hline & \multicolumn{2}{|c|}{ Model 1} & \multicolumn{2}{|c|}{ Model 2} & \multicolumn{2}{|c|}{ Model 3} & \multicolumn{2}{|c|}{ Full Model } \\
\hline & Men & Women & Men & Women & Men & Women & Men & Women \\
\hline Other sources of income & & & $\begin{array}{c}0.003 \\
(0.006)\end{array}$ & $\begin{array}{c}-0.045 \\
(0.006)^{* \star *}\end{array}$ & $\begin{array}{c}0.008 \\
(0.006)\end{array}$ & $\begin{array}{c}-0.051 \\
(0.006)^{* * *}\end{array}$ & $\begin{array}{c}0.012 \\
(0.006)\end{array}$ & $\begin{array}{c}-0.052 \\
(0.006)^{* * *}\end{array}$ \\
\hline Two or more maintainers & & & $\begin{array}{c}-0.025 \\
(0.006)^{* * *}\end{array}$ & $\begin{array}{c}0.021 \\
(0.004)^{* * *}\end{array}$ & $\begin{array}{c}-0.022 \\
(0.006)^{* * * *}\end{array}$ & $\begin{array}{c}0.016 \\
(0.004)^{* * *}\end{array}$ & $\begin{array}{c}-0.022 \\
(0.005)^{\star * *}\end{array}$ & $\begin{array}{c}0.014 \\
(0.004)^{\star \star *}\end{array}$ \\
\hline First maintainer & & & $\begin{array}{c}0.024 \\
(0.005)^{\star \star \star}\end{array}$ & $\begin{array}{c}0.036 \\
(0.004)^{* k \star}\end{array}$ & $\begin{array}{c}0.024 \\
(0.005)^{\star \star \star}\end{array}$ & $\begin{array}{c}0.037 \\
(0.004)^{\star \star \star *}\end{array}$ & $\begin{array}{c}0.030 \\
(0.005)^{\star \star \star *}\end{array}$ & $\begin{array}{c}0.035 \\
(0.004)^{\star \star \star}\end{array}$ \\
\hline Atlantic provinces & & & $\begin{array}{c}-0.137 \\
(0.011)^{* k *}\end{array}$ & $\begin{array}{c}-0.011 \\
(0.005)^{*}\end{array}$ & $\begin{array}{c}-0.139 \\
(0.011)^{\star * *}\end{array}$ & $\begin{array}{c}-0.011 \\
(0.004)^{\star}\end{array}$ & $\begin{array}{c}-0.123 \\
(0.011)^{* * * *}\end{array}$ & $\begin{array}{l}-0.007 \\
(0.004)\end{array}$ \\
\hline Northern Territories & & & $\begin{array}{c}-0.011 \\
(0.025)^{\star \star \star \star}\end{array}$ & - & $\begin{array}{c}-0.094 \\
(0.026)^{\star \star \star}\end{array}$ & - & $\begin{array}{l}-0.027 \\
(0.015)\end{array}$ & - \\
\hline Quebec & & & $\begin{array}{l}-0.013 \\
(0.013)\end{array}$ & $\begin{array}{c}0.001 \\
(0.008)\end{array}$ & $\begin{array}{l}-0.089 \\
(0.012)\end{array}$ & $\begin{array}{l}-0.0009 \\
(0.007)\end{array}$ & $\begin{array}{r}-0.023 \\
(0.013)\end{array}$ & $\begin{array}{c}0.003 \\
(0.007)\end{array}$ \\
\hline Manitoba & & & $\begin{array}{c}-0.140 \\
(0.021)^{\star \star \star}\end{array}$ & - & $\begin{array}{c}-0.126 \\
(0.022)^{\star \star \star}\end{array}$ & - & $\begin{array}{c}-0.138 \\
(0.023)^{\star \star \star \star}\end{array}$ & - \\
\hline Saskatchewan & & & $\begin{array}{c}-0.083 \\
(0.024)^{\star \star}\end{array}$ & $\begin{array}{c}-0.060 \\
(0.012)^{\star \star \star \star}\end{array}$ & $\begin{array}{c}-0.078 \\
(0.023)^{\star \star}\end{array}$ & $\begin{array}{c}-0.057 \\
(0.012)^{* * *}\end{array}$ & $\begin{array}{c}-0.040 \\
(0.016)^{\star}\end{array}$ & $\begin{array}{c}-0.053 \\
(0.010)^{\star \star \star \star}\end{array}$ \\
\hline Alberta & & & $\begin{array}{c}0.011 \\
(0.007)\end{array}$ & $\begin{array}{c}0.018 \\
(0.005)^{\star \star \star}\end{array}$ & $\begin{array}{c}0.011 \\
(0.007)\end{array}$ & $\begin{array}{c}0.017 \\
(0.005)^{\star \star \star \star}\end{array}$ & $\begin{array}{c}0.020 \\
(0.007)^{\star \star}\end{array}$ & $\begin{array}{c}0.017 \\
(0.005)^{\star \star \star}\end{array}$ \\
\hline British Columbia & & & $\begin{array}{c}-0.026 \\
(0.011)^{\star}\end{array}$ & $\begin{array}{c}0.028 \\
(0.004)^{\star \star \star}\end{array}$ & $\begin{array}{c}-0.024 \\
(0.010)^{\star}\end{array}$ & $\begin{array}{c}0.039 \\
(0.004)^{\star \star \star \star}\end{array}$ & $\begin{array}{c}-0.019 \\
(0.010)^{*}\end{array}$ & $\begin{array}{c}0.041 \\
(0.004)^{* * *}\end{array}$ \\
\hline Rural place of residence & & & $\begin{array}{c}0.020 \\
(0.004)^{* * *}\end{array}$ & $\begin{array}{c}-0.029 \\
(0.005)^{\star \star \star}\end{array}$ & $\begin{array}{c}0.023 \\
(0.004)^{* * * *}\end{array}$ & $\begin{array}{c}-0.028 \\
(0.005)^{\star * * *}\end{array}$ & $\begin{array}{c}0.018 \\
(0.004)^{\star \star \star}\end{array}$ & $\begin{array}{c}-0.032 \\
(0.005)^{* * * *}\end{array}$ \\
\hline Has someone to confide in & & & & & $\begin{array}{c}-0.045 \\
(0.004)^{\star \star \star}\end{array}$ & $\begin{array}{c}0.011 \\
(0.013)\end{array}$ & $\begin{array}{c}-0.047 \\
(0.004)^{\star * \star}\end{array}$ & $\begin{array}{c}0.012 \\
(0.013)\end{array}$ \\
\hline Band member & & & & & & & $\begin{array}{c}0.006 \\
(0.006)\end{array}$ & $\begin{array}{c}-0.012 \\
(0.004)^{\star \star}\end{array}$ \\
\hline $\begin{array}{l}\text { Speaks Aboriginal } \\
\text { languages }\end{array}$ & & & & & & & $\begin{array}{c}-0.116 \\
(0.012)^{* * *}\end{array}$ & $\begin{array}{c}0.020 \\
(0.005)^{* * * *}\end{array}$ \\
\hline $\begin{array}{l}\text { Values Aboriginal } \\
\text { languages }\end{array}$ & & & & & & & $\begin{array}{l}-0.007 \\
(0.005)\end{array}$ & $\begin{array}{c}0.012 \\
(0.004)^{\star *}\end{array}$ \\
\hline $\begin{array}{l}\text { Traditional pursuits } \\
\text { for commerce }\end{array}$ & & & & & & & $\begin{array}{r}-0.002 \\
(0.010)\end{array}$ & $\begin{array}{c}-0.048 \\
(0.019)^{\star}\end{array}$ \\
\hline $\begin{array}{l}\text { Traditional pursuits } \\
\text { for food }\end{array}$ & & & & & & & $\begin{array}{c}-0.053 \\
(0.007)^{* * *}\end{array}$ & $\begin{array}{c}-0.030 \\
(0.006)^{* * *}\end{array}$ \\
\hline $\begin{array}{l}\text { Traditional pursuits } \\
\text { for pleasure }\end{array}$ & & & & & & & $\begin{array}{c}0.043 \\
(0.005)^{\star \star \star \star}\end{array}$ & $\begin{array}{c}0.020 \\
(0.004)^{\text {\#** }}\end{array}$ \\
\hline Number of observations & 840 & 850 & 830 & 840 & 830 & 840 & 820 & 830 \\
\hline
\end{tabular}

Source: Statistics Canada, 2006 Aboriginal Peoples Survey, Adult Core analytical file. Bootstrapped ( 1000 replications) standard errors in parentheses. Statistical significance ${ }^{* * *} p<.001 ;{ }^{* *} p<.01 ;{ }^{*} p<.05$. 
Appendix C 5: Detailed results from probit models predicting employment for Métis, APS 2006

\begin{tabular}{|c|c|c|c|c|c|c|c|c|}
\hline \multirow{3}{*}{ Variables in equations } & \multicolumn{8}{|c|}{ Average Marginal Effects } \\
\hline & \multicolumn{2}{|c|}{ Model 1} & \multicolumn{2}{|c|}{ Model 2} & \multicolumn{2}{|c|}{ Model 3} & \multicolumn{2}{|c|}{ Full Model } \\
\hline & Men & Women & Men & Women & Men & Women & Men & Women \\
\hline $\begin{array}{l}\text { Less than } \\
\text { high school education }\end{array}$ & $\begin{array}{c}-0.027 \\
(0.005)^{\star \star \star \star}\end{array}$ & $\begin{array}{c}-0.045 \\
(0.006)^{\star \star \star}\end{array}$ & $\begin{array}{c}-0.010 \\
(0.004)^{\star}\end{array}$ & $\begin{array}{c}-0.036 \\
(0.005)^{\star \star *}\end{array}$ & $\begin{array}{c}-0.007 \\
(0.004)\end{array}$ & $\begin{array}{c}-0.036 \\
(0.005)^{\star \cdots}\end{array}$ & $\begin{array}{l}-0.004 \\
(0.004)\end{array}$ & $\begin{array}{c}-0.027 \\
(0.004)^{*}\end{array}$ \\
\hline $\begin{array}{l}\text { Some } \\
\text { non-university education }\end{array}$ & $\begin{array}{c}-0.025 \\
(0.007)^{* *}\end{array}$ & $\begin{array}{l}-0.005 \\
(0.004)\end{array}$ & $\begin{array}{l}-0.007 \\
(0.006)\end{array}$ & $\begin{array}{c}-0005 \\
(0.004)\end{array}$ & $\begin{array}{l}-0.007 \\
(0.006)\end{array}$ & $\begin{array}{l}0.0003 \\
(0.004)\end{array}$ & $\begin{array}{l}-0.006 \\
(0.006)\end{array}$ & $\begin{array}{c}0.002 \\
(0.004)\end{array}$ \\
\hline $\begin{array}{l}\text { Completed, } \\
\text { non-university education }\end{array}$ & $\begin{array}{l}-0.0009 \\
(0.004)\end{array}$ & $\begin{array}{c}-0.011 \\
(0.004)^{\star *}\end{array}$ & $\begin{array}{c}0.008 \\
(0.004)^{*}\end{array}$ & $\begin{array}{l}-0.002 \\
(0.003)\end{array}$ & $\begin{array}{c}0.007 \\
(0.004)\end{array}$ & $\begin{array}{r}-0.0008 \\
(0.003)\end{array}$ & $\begin{array}{c}0.013 \\
(0.003)^{\star \star \star \star}\end{array}$ & $\begin{array}{r}-0.001 \\
(0.003)\end{array}$ \\
\hline $\begin{array}{l}\text { Some } \\
\text { university education }\end{array}$ & $\begin{array}{c}-0.004 \\
(0.009)\end{array}$ & $\begin{array}{c}-0.031 \\
(0.010)^{* *}\end{array}$ & $\begin{array}{c}0.019 \\
(0.006)^{* *}\end{array}$ & $\begin{array}{c}0.006 \\
(0.004)\end{array}$ & $\begin{array}{c}0.017 \\
(0.006)^{* *}\end{array}$ & $\begin{array}{c}0.007 \\
(0.004)\end{array}$ & $\begin{array}{c}0.017 \\
(0.006)^{\star *}\end{array}$ & $\begin{array}{c}0.006 \\
(0.004)\end{array}$ \\
\hline $\begin{array}{l}\text { Completed } \\
\text { university education }\end{array}$ & $\begin{array}{c}0.023 \\
(0.006)^{* * *}\end{array}$ & $\begin{array}{c}0.032 \\
(0.003)^{\star \star \star}\end{array}$ & $\begin{array}{c}0.017 \\
(0.006)^{\star \star}\end{array}$ & $\begin{array}{c}0.027 \\
(0.003)^{\star \star \star \star}\end{array}$ & $\begin{array}{c}0.015 \\
(0.006)^{*}\end{array}$ & $\begin{array}{c}0.028 \\
(0.003)^{\star \star \star}\end{array}$ & $\begin{array}{c}0.013 \\
(0.006)^{\star}\end{array}$ & $\begin{array}{c}0.027 \\
(0.003)^{\star \star \star}\end{array}$ \\
\hline Age 15 to 19 & $\begin{array}{c}-0.006 \\
(0.006)\end{array}$ & $\begin{array}{c}-0.099 \\
(0.011)^{\star * *}\end{array}$ & $\begin{array}{c}0.007 \\
(0.005)\end{array}$ & $\begin{array}{c}-0.065 \\
(0.009)^{\star \star \star}\end{array}$ & $\begin{array}{c}0.0051 \\
(0.005)\end{array}$ & $\begin{array}{c}-0.063 \\
(0.009)^{\star * *}\end{array}$ & $\begin{array}{c}0.005 \\
(0.005)\end{array}$ & $\begin{array}{c}-0.056 \\
(0.008)^{\star * \star}\end{array}$ \\
\hline Age 25 to 34 & $\begin{array}{c}0.063 \\
(0.004)^{\star \star \star}\end{array}$ & $\begin{array}{c}-0.002 \\
(0.004)\end{array}$ & $\begin{array}{c}0.034 \\
(0.003)^{\star * *}\end{array}$ & $\begin{array}{c}0.007 \\
(0.004)\end{array}$ & $\begin{array}{c}0.035 \\
(0.003)^{\star * \star *}\end{array}$ & $\begin{array}{c}0.008 \\
(0.004)\end{array}$ & $\begin{array}{c}0.035 \\
(0.003)^{* * *}\end{array}$ & $\begin{array}{c}0.006 \\
(0.004)\end{array}$ \\
\hline Age 35 to 44 & $\begin{array}{c}0.047 \\
(0.004)^{* * *}\end{array}$ & $\begin{array}{c}-0.003 \\
(0.004)\end{array}$ & $\begin{array}{c}0.011 \\
(0.005)\end{array}$ & $\begin{array}{c}0.006 \\
(0.004)\end{array}$ & $\begin{array}{c}0.012 \\
(0.005)^{*}\end{array}$ & $\begin{array}{c}0.007 \\
(0.005)\end{array}$ & $\begin{array}{c}0.014 \\
(0.005)^{* *}\end{array}$ & $\begin{array}{c}0.002 \\
(0.004)^{* * *}\end{array}$ \\
\hline Age 45 to 54 & $\begin{array}{c}0.051 \\
(0.004)^{* * * *}\end{array}$ & $\begin{array}{c}0.011 \\
(0.003)^{\star \star}\end{array}$ & $\begin{array}{c}0.016 \\
(0.005)^{\star \star}\end{array}$ & $\begin{array}{c}0.007 \\
(0.004)\end{array}$ & $\begin{array}{c}0.014 \\
(0.005)^{\star *}\end{array}$ & $\begin{array}{c}0.009 \\
(0.004)^{*}\end{array}$ & $\begin{array}{c}0.014 \\
(0.005)^{* *}\end{array}$ & $\begin{array}{c}0.016 \\
(0.004)^{\star \star \star}\end{array}$ \\
\hline Age 55 to 64 & $\begin{array}{c}0.065 \\
(0.003)^{* \cdots *}\end{array}$ & $\begin{array}{c}-0.0006 \\
(0.005)\end{array}$ & $\begin{array}{c}0.036 \\
(0.004)^{\star \star \star *}\end{array}$ & $\begin{array}{c}-0.021 \\
(0.007)^{* *}\end{array}$ & $\begin{array}{c}0.035 \\
(0.004)^{\star \star \star}\end{array}$ & $\begin{array}{c}-0.019 \\
(0.007)^{\star}\end{array}$ & $\begin{array}{c}0.034 \\
(0.004)^{\star \star *}\end{array}$ & $\begin{array}{c}-0.012 \\
(0.007)\end{array}$ \\
\hline Languages - French & $\begin{array}{c}-0.006 \\
(0.005)\end{array}$ & $\begin{array}{c}-0.060 \\
(0.008)^{\star \star *}\end{array}$ & $\begin{array}{c}0.032 \\
(0.004)^{* * *}\end{array}$ & $\begin{array}{c}0.013 \\
(0.005)^{\star}\end{array}$ & $\begin{array}{c}0.032 \\
(0.004)^{n * * *}\end{array}$ & $\begin{array}{c}0.013 \\
(0.005)^{*}\end{array}$ & $\begin{array}{c}0.030 \\
(0.004)^{\star \star \star *}\end{array}$ & $\begin{array}{c}0.010 \\
(0.005)^{*}\end{array}$ \\
\hline Languages - Both & $\begin{array}{r}-0.0009 \\
(0.004)\end{array}$ & $\begin{array}{c}-0.004 \\
(0.004)\end{array}$ & $\begin{array}{c}0.025 \\
(0.004)^{* * *}\end{array}$ & $\begin{array}{c}0.026 \\
(0.003)^{\star \star \star \star}\end{array}$ & $\begin{array}{c}0.024 \\
(0.004)^{* \star *}\end{array}$ & $\begin{array}{c}0.026 \\
(0.03)^{* * *}\end{array}$ & $\begin{array}{c}0.026 \\
(0.003)^{\star \star \star \star}\end{array}$ & $\begin{array}{c}0.028 \\
(0.003)^{\star * *}\end{array}$ \\
\hline Access/use technology & $\begin{array}{c}0.046 \\
(0.005)^{\star * *}\end{array}$ & $\begin{array}{c}0.010 \\
(0.003)^{\star \star}\end{array}$ & $\begin{array}{c}0.049 \\
(0.004)^{\star \star}\end{array}$ & $\begin{array}{c}0.009 \\
(0.003)^{\star \star}\end{array}$ & $\begin{array}{c}0.044 \\
(0.004)^{* * * *}\end{array}$ & $\begin{array}{c}0.010 \\
(0.003)^{* * *}\end{array}$ & $\begin{array}{c}0.046 \\
(0.004)^{\star \star \star}\end{array}$ & $\begin{array}{c}0.011 \\
(0.003)^{* * *}\end{array}$ \\
\hline Legally married & & & $\begin{array}{c}0.022 \\
(0.004)\end{array}$ & $\begin{array}{c}0.037 \\
(0.005)^{\star \star \star}\end{array}$ & $\begin{array}{c}0.021 \\
(0.004)^{* * *}\end{array}$ & $\begin{array}{c}0.038 \\
(0.005)^{\star \star \star *}\end{array}$ & $\begin{array}{c}0.021 \\
(0.004)^{\star \star \star \star}\end{array}$ & $\begin{array}{c}0.039 \\
(0.005)^{\star \star *}\end{array}$ \\
\hline Common-law & & & $\begin{array}{c}0.005 \\
(0.005)\end{array}$ & $\begin{array}{c}0.038 \\
(0.003)^{\star \star \star}\end{array}$ & $\begin{array}{c}0.005 \\
(0.005)\end{array}$ & $\begin{array}{c}0.037 \\
(0.003)^{\star \star \star}\end{array}$ & $\begin{array}{c}0.011 \\
(0.004)^{\star *}\end{array}$ & $\begin{array}{c}0.041 \\
(0.003)^{\star * *}\end{array}$ \\
\hline Divorced-separated & & & $\begin{array}{c}0.024 \\
(0.004)^{\star \star \star}\end{array}$ & $\begin{array}{c}0.015 \\
(0.003)^{\star \star \star}\end{array}$ & $\begin{array}{c}0.026 \\
(0.004)\end{array}$ & $\begin{array}{c}0.015 \\
(0.003)^{* * *}\end{array}$ & $\begin{array}{c}0.025 \\
(0.004)^{\star \star \star *}\end{array}$ & $\begin{array}{c}0.011 \\
(0.003)^{\star \star}\end{array}$ \\
\hline Children $<6$ & & & $\begin{array}{c}0.005 \\
(0.005)\end{array}$ & $\begin{array}{c}-0.031 \\
(0.008)^{\star \star \star}\end{array}$ & $\begin{array}{l}0.0009 \\
(0.005)\end{array}$ & $\begin{array}{c}-0.030 \\
(0.008)^{\star * *}\end{array}$ & $\begin{array}{c}0.005 \\
(0.005)\end{array}$ & $\begin{array}{c}0.020 \\
(0.007)^{\star *}\end{array}$ \\
\hline Children 6-14 & & & $\begin{array}{c}-0.012 \\
(0.007)\end{array}$ & $\begin{array}{c}-0.046 \\
(0.008)^{* \star \star}\end{array}$ & $\begin{array}{c}-0.017 \\
(0.007)^{\star}\end{array}$ & $\begin{array}{c}-0.047 \\
(0.008)^{\star \star *}\end{array}$ & $\begin{array}{l}-0.008 \\
(0.006)\end{array}$ & $\begin{array}{c}-0.034 \\
(0.006)^{\star \star *}\end{array}$ \\
\hline Children 14-24 & & & $\begin{array}{c}0.002 \\
(0.005)\end{array}$ & $\begin{array}{c}-0.027 \\
(0.005)^{\star \star \star}\end{array}$ & $\begin{array}{c}-0.0003 \\
(0.005)\end{array}$ & $\begin{array}{c}-0.027 \\
(0.005)^{\star * *}\end{array}$ & $\begin{array}{c}0.006 \\
(0.005)\end{array}$ & $\begin{array}{c}-0.028 \\
(0.005)^{* * *}\end{array}$ \\
\hline Lone parent & & & $\begin{array}{c}0.052 \\
(0.002)^{\star * *}\end{array}$ & $\begin{array}{l}-0.003 \\
(0.005)\end{array}$ & $\begin{array}{c}0.052 \\
(0.002)^{* *}\end{array}$ & $\begin{array}{c}-0.004 \\
(0.005)\end{array}$ & $\begin{array}{c}0.050 \\
(0.002)^{\star * *}\end{array}$ & $\begin{array}{c}-0.008 \\
(0.005)\end{array}$ \\
\hline Access to childcare & & & $\begin{array}{c}-0.005 \\
(0.005)\end{array}$ & $\begin{array}{c}-0.005 \\
(0.004)\end{array}$ & $\begin{array}{c}-0.002 \\
(0.005)\end{array}$ & $\begin{array}{r}-0.004 \\
(0.004)\end{array}$ & $\begin{array}{c}0.005 \\
(0.004)\end{array}$ & $\begin{array}{c}-0.004 \\
(0.004)\end{array}$ \\
\hline Chronic health condition & & & $\begin{array}{c}0.002 \\
(0.003)\end{array}$ & $\begin{array}{c}0.016 \\
(0.002)^{* * *}\end{array}$ & $\begin{array}{l}0.0006 \\
(0.003)\end{array}$ & $\begin{array}{c}-0.016 \\
(0.002)^{* * *}\end{array}$ & $\begin{array}{c}0.003 \\
(0.003)\end{array}$ & $\begin{array}{c}-0.020 \\
(0.002)^{* * *}\end{array}$ \\
\hline
\end{tabular}




\begin{tabular}{|c|c|c|c|c|c|c|c|c|}
\hline \multirow{3}{*}{$\begin{array}{l}\text { Appendix C5 } \\
\text { (continued) }\end{array}$} & \multicolumn{8}{|c|}{ Average Marginal Effects } \\
\hline & \multicolumn{2}{|c|}{ Model 1} & \multicolumn{2}{|c|}{ Model 2} & \multicolumn{2}{|c|}{ Model 3} & \multicolumn{2}{|c|}{ Full Model } \\
\hline & Men & Women & Men & Women & Men & Women & Men & Women \\
\hline Health activity limitation & & & $\begin{array}{l}-0.005 \\
(0.003)\end{array}$ & $\begin{array}{c}0.005 \\
(0.002)\end{array}$ & $\begin{array}{l}-0.003 \\
(0.003)\end{array}$ & $\begin{array}{c}0.004 \\
(0.002)\end{array}$ & $\begin{array}{c}0.002 \\
(0.003)\end{array}$ & $\begin{array}{c}0.005 \\
(0.002)^{*}\end{array}$ \\
\hline Other sources of income & & & $\begin{array}{c}-0.018 \\
(0.005)^{\star * *}\end{array}$ & $\begin{array}{c}0.003 \\
(0.003)\end{array}$ & $\begin{array}{c}-0.018 \\
(0.005)^{\star * * *}\end{array}$ & $\begin{array}{c}0.004 \\
(0.003)\end{array}$ & $\begin{array}{c}-0.016 \\
(0.005)^{\star * *}\end{array}$ & $\begin{array}{l}-0.0003 \\
(0.003)\end{array}$ \\
\hline Two or more maintainers & & & $\begin{array}{c}0.027 \\
(0.003)^{\star * *}\end{array}$ & $\begin{array}{c}-0.011 \\
(0.003)^{* * *}\end{array}$ & $\begin{array}{c}0.025 \\
(0.003)^{\star * * *}\end{array}$ & $\begin{array}{c}-0.011 \\
(0.003)^{\star * *}\end{array}$ & $\begin{array}{c}0.023 \\
(0.003)^{* * * *}\end{array}$ & $\begin{array}{l}-0.005 \\
(0.002)\end{array}$ \\
\hline First maintainer & & & $\begin{array}{c}0.032 \\
(0.003)^{\star * *}\end{array}$ & $\begin{array}{c}0.015 \\
(0.002)^{* * *}\end{array}$ & $\begin{array}{c}0.032 \\
(0.003)^{* * * *}\end{array}$ & $\begin{array}{c}0.015 \\
(0.002)^{* * * *}\end{array}$ & $\begin{array}{c}0.031 \\
(0.003)^{\star * *}\end{array}$ & $\begin{array}{c}0.017 \\
(0.002)^{n+m+*}\end{array}$ \\
\hline Atlantic provinces & & & $\begin{array}{c}-0.026 \\
(0.007)^{* * *}\end{array}$ & $\begin{array}{c}-0.051 \\
(0.01)^{* * *}\end{array}$ & $\begin{array}{c}-0.027 \\
(0.007)^{* * *}\end{array}$ & $\begin{array}{c}-0.051 \\
(0.010)^{* * *}\end{array}$ & $\begin{array}{c}-0.024 \\
(0.006)^{* * *}\end{array}$ & $\begin{array}{c}-0.031 \\
(0.008)^{* * * *}\end{array}$ \\
\hline Northern Territories & & & $\begin{array}{c}0.001 \\
(0.006)\end{array}$ & $\begin{array}{c}0.026 \\
(0.003)^{\star \star \star}\end{array}$ & $\begin{array}{l}0.0003 \\
(0.006)\end{array}$ & $\begin{array}{c}0.026 \\
(0.003)^{\text {\#**}}\end{array}$ & $\begin{array}{c}0.002 \\
(0.005)\end{array}$ & $\begin{array}{c}0.030 \\
(0.003)^{*+* * *}\end{array}$ \\
\hline Quebec & & & $\begin{array}{c}-0.029 \\
(0.008)^{* \star *}\end{array}$ & $\begin{array}{c}-0.081 \\
(0.012)^{\star \star \star}\end{array}$ & $\begin{array}{c}-0.029 \\
(0.008)^{* * *}\end{array}$ & $\begin{array}{c}-0.083 \\
(0.012)^{* * * *}\end{array}$ & $\begin{array}{l}-0.018 \\
(0.007)\end{array}$ & $\begin{array}{l}-0.067 \\
(0.009)^{* * * *}\end{array}$ \\
\hline Manitoba & & & $\begin{array}{c}0.033 \\
(0.004)^{\star * *}\end{array}$ & $\begin{array}{c}0.006 \\
(0.004)\end{array}$ & $\begin{array}{c}0.032 \\
(0.004)^{\star \star \star *}\end{array}$ & $\begin{array}{c}0.006 \\
(0.004)\end{array}$ & $\begin{array}{c}0.034 \\
(0.004)^{\star * *}\end{array}$ & $\begin{array}{c}0.009 \\
(0.004)^{* *}\end{array}$ \\
\hline Saskatchewan & & & $\begin{array}{c}0.016 \\
(0.004)^{* * *}\end{array}$ & $\begin{array}{c}0.011 \\
(0.003)^{* *}\end{array}$ & $\begin{array}{c}0.019 \\
(0.004)^{* * * *}\end{array}$ & $\begin{array}{c}0.011 \\
(0.003)^{* * t}\end{array}$ & $\begin{array}{c}0.016 \\
(0.004)^{n \star \star \star}\end{array}$ & $\begin{array}{c}0.017 \\
(0.003)^{\text {tat* }}\end{array}$ \\
\hline Alberta & & & $\begin{array}{c}0.036 \\
(0.003)^{\star \star * *}\end{array}$ & $\begin{array}{c}0.016 \\
(0.003)^{n+* *}\end{array}$ & $\begin{array}{c}0.036 \\
(0.003)^{\star * \star *}\end{array}$ & $\begin{array}{c}0.017 \\
(0.003)^{\star \star * *}\end{array}$ & $\begin{array}{c}0.037 \\
(0.003)^{\star \star \star *}\end{array}$ & $\begin{array}{c}0.020 \\
(0.003)^{*+*+*}\end{array}$ \\
\hline British Columbia & & & $\begin{array}{c}0.037 \\
(0.002)^{\star * \star}\end{array}$ & $\begin{array}{c}0.015 \\
(0.003)^{* * * *}\end{array}$ & $\begin{array}{c}0.036 \\
(0.003)^{* * * *}\end{array}$ & $\begin{array}{c}0.015 \\
(0.003)^{* * *}\end{array}$ & $\begin{array}{c}0.031 \\
(0.003)^{* * *}\end{array}$ & $\begin{array}{c}0.016 \\
(0.003)^{* * * *}\end{array}$ \\
\hline Rural place of residence & & & $\begin{array}{c}-0.021 \\
(0.003)^{\star \star * *}\end{array}$ & $\begin{array}{c}-0.004 \\
(0.002)\end{array}$ & $\begin{array}{c}-0.021 \\
(0.003)^{* * *}\end{array}$ & $\begin{array}{l}-0.003 \\
(0.002)\end{array}$ & $\begin{array}{c}-0.014 \\
(0.003)^{\star \star *}\end{array}$ & $\begin{array}{c}-0.004 \\
(0.002)\end{array}$ \\
\hline Has someone to confide in & & & & & $\begin{array}{c}0.031 \\
(0.009)^{* * *}\end{array}$ & $\begin{array}{l}-0.004 \\
(0.005)\end{array}$ & $\begin{array}{c}0.042 \\
(0.01)^{* * *}\end{array}$ & $\begin{array}{c}-0.004 \\
(0.005)\end{array}$ \\
\hline $\begin{array}{l}\text { Volunteering club } \\
\text { membership }\end{array}$ & & & & & $\begin{array}{c}0.022 \\
(0.003)^{* * * *}\end{array}$ & $\begin{array}{c}-0.009 \\
(0.003)^{* *}\end{array}$ & $\begin{array}{c}0.025 \\
(0.003)^{* * *}\end{array}$ & $\begin{array}{c}-0.009 \\
(0.003)^{* *}\end{array}$ \\
\hline $\begin{array}{l}\text { Member in Métis } \\
\text { organizations }\end{array}$ & & & & & & & $\begin{array}{c}-0.002 \\
(0.004)\end{array}$ & $\begin{array}{l}-0.003 \\
(0.003)\end{array}$ \\
\hline Spouse is Aboriginal & & & & & & & $\begin{array}{c}-0.034 \\
(0.005)^{* * * *}\end{array}$ & $\begin{array}{c}-0.044 \\
(0.004)^{*+* *}\end{array}$ \\
\hline $\begin{array}{l}\text { Speaks Aboriginal } \\
\text { languages }\end{array}$ & & & & & & & $\begin{array}{c}0.016 \\
(0.004)^{\star * * *}\end{array}$ & $\begin{array}{c}0.003 \\
(0.004)\end{array}$ \\
\hline $\begin{array}{l}\text { Values Aboriginal } \\
\text { languages }\end{array}$ & & & & & & & $\begin{array}{c}-0.019 \\
(0.003)^{* * * *}\end{array}$ & $\begin{array}{c}-0.011 \\
(0.002)^{* * * *}\end{array}$ \\
\hline $\begin{array}{l}\text { Traditional pursuits } \\
\text { for commerce }\end{array}$ & & & & & & & $\begin{array}{c}-0.033 \\
(0.009)^{\star * * *}\end{array}$ & $\begin{array}{c}-0.082 \\
(0.02)^{* * *}\end{array}$ \\
\hline $\begin{array}{l}\text { Traditional pursuits } \\
\text { for food }\end{array}$ & & & & & & & $\begin{array}{c}0.014 \\
(0.004)^{* * *}\end{array}$ & $\begin{array}{c}-0.008 \\
(0.003)^{* *}\end{array}$ \\
\hline $\begin{array}{l}\text { Traditional pursuits } \\
\text { for pleasure }\end{array}$ & & & & & & & $\begin{array}{c}-0.028 \\
(0.003)^{+* * *}\end{array}$ & $\begin{array}{c}0.024 \\
(0.003)^{*+*}\end{array}$ \\
\hline Number of observations & 2450 & 2500 & 2220 & 2310 & 2200 & 2300 & 2150 & 2250 \\
\hline
\end{tabular}

Source: Statistics Canada, 2006 Aboriginal Peoples Survey, Métis Supplement analytical file. Bootstrapped (1000 replications) standard errors in parentheses. Statistical significance ${ }^{* * *} p<.001 ;{ }^{* *} p<.01 ;{ }^{*} p<.05$. 
Appendix C 6: Detailed results from probit models predicting employment for Inuit, APS 2006

\begin{tabular}{|c|c|c|c|c|c|c|c|c|}
\hline \multirow{3}{*}{ Variables in equations } & \multicolumn{8}{|c|}{ Average Marginal Effects } \\
\hline & \multicolumn{2}{|c|}{ Model 1} & \multicolumn{2}{|c|}{ Model 2} & \multicolumn{2}{|c|}{ Model 3} & \multicolumn{2}{|c|}{ Full Model } \\
\hline & Men & Women & Men & Women & Men & Women & Men & Women \\
\hline $\begin{array}{l}\text { Less than } \\
\text { high school education }\end{array}$ & $\begin{array}{c}0.074 \\
(0.013)^{* * *}\end{array}$ & $\begin{array}{r}-0.013 \\
(0.009)\end{array}$ & $\begin{array}{c}0.100 \\
(0.013)^{\star \star \star}\end{array}$ & $\begin{array}{l}-0.005 \\
(0.009)\end{array}$ & $\begin{array}{c}0.068 \\
(0.012)^{\star \star \star}\end{array}$ & $\begin{array}{c}-0.019 \\
(0.009)^{\star}\end{array}$ & $\begin{array}{c}0.049 \\
(0.012)^{* * *}\end{array}$ & $\begin{array}{r}-0.005 \\
(0.009)\end{array}$ \\
\hline $\begin{array}{l}\text { Some } \\
\text { non-university education }\end{array}$ & $\begin{array}{c}0.088 \\
(0.009)^{* * \star}\end{array}$ & $\begin{array}{c}-0.044 \\
(0.016)^{* *}\end{array}$ & $\begin{array}{c}0.090 \\
(0.009)^{* * * *}\end{array}$ & $\begin{array}{c}-0.028 \\
(0.014)^{\star}\end{array}$ & $\begin{array}{c}0.061 \\
(0.010)^{\star * *}\end{array}$ & $\begin{array}{c}-0.053 \\
(0.015)^{\star \star}\end{array}$ & $\begin{array}{c}0.043 \\
(0.010)^{\star \star \star \star}\end{array}$ & $\begin{array}{c}-0.036 \\
(0.014)^{\star}\end{array}$ \\
\hline $\begin{array}{l}\text { Completed } \\
\text { non-university or } \\
\text { university }\end{array}$ & $\begin{array}{c}0.114 \\
(0.011)^{\star \star \star}\end{array}$ & $\begin{array}{c}-0.046 \\
(0.011)^{\star * *}\end{array}$ & $\begin{array}{c}0.119 \\
(0.010)^{\star \star \star *}\end{array}$ & $\begin{array}{r}-0.013 \\
(0.009)\end{array}$ & $\begin{array}{c}0.092 \\
(0.010)^{\star \star \star}\end{array}$ & $\begin{array}{l}-0.017 \\
(0.009)\end{array}$ & $\begin{array}{c}0.081 \\
(0.010)^{\star \star \star}\end{array}$ & $\begin{array}{c}-0.019 \\
(0.008)^{*}\end{array}$ \\
\hline Age 15 to 19 & $\begin{array}{c}-0.044 \\
(0.016)^{* *}\end{array}$ & $\begin{array}{c}-0.002 \\
(0.011)\end{array}$ & $\begin{array}{c}-0.037 \\
(0.014)^{\star \star}\end{array}$ & $\begin{array}{c}-0.038 \\
(0.015)^{\star}\end{array}$ & $\begin{array}{c}0.032 \\
(0.014)^{\star}\end{array}$ & $\begin{array}{c}-0.049 \\
(0.016)^{* * *}\end{array}$ & $\begin{array}{c}-0.072 \\
(0.017)^{\star \star \star}\end{array}$ & $\begin{array}{c}-0.071 \\
(0.016)^{* * *}\end{array}$ \\
\hline Age 25 to 34 & $\begin{array}{c}0.045 \\
(0.010)^{\star \star \star}\end{array}$ & $\begin{array}{c}0.114 \\
(0.008)^{\star \star \star \star}\end{array}$ & $\begin{array}{c}0.060 \\
(0.009)^{* * *}\end{array}$ & $\begin{array}{c}0.104 \\
(0.007)^{\star \star * *}\end{array}$ & $\begin{array}{c}0.0253 \\
(0.010)^{*}\end{array}$ & $\begin{array}{c}0.097 \\
(0.007)^{\star \star \star}\end{array}$ & $\begin{array}{c}0.008 \\
(0.011)\end{array}$ & $\begin{array}{c}0.093 \\
(0.007)^{\star \star * \star}\end{array}$ \\
\hline Age 35 to 44 & $\begin{array}{c}-0.002 \\
(0.012)\end{array}$ & $\begin{array}{c}0.081 \\
(0.008)^{\star \star \star}\end{array}$ & $\begin{array}{c}0.008 \\
(0.012)\end{array}$ & $\begin{array}{c}0.047 \\
(0.010)^{\star \star \star}\end{array}$ & $\begin{array}{c}-0.052 \\
(0.014)^{\star \star \star *}\end{array}$ & $\begin{array}{c}0.034 \\
(0.011)^{* *}\end{array}$ & $\begin{array}{c}-0.078 \\
(0.015)^{\star \star \star}\end{array}$ & $\begin{array}{c}0.023 \\
(0.010)^{\star}\end{array}$ \\
\hline Age 45 to 54 & $\begin{array}{c}0.017 \\
(0.011)\end{array}$ & $\begin{array}{c}0.105 \\
(0.007)^{\star * *}\end{array}$ & $\begin{array}{c}0.017 \\
(0.013)\end{array}$ & $\begin{array}{c}0.079 \\
(0.008)^{\star \star *}\end{array}$ & $\begin{array}{c}-0.056 \\
(0.017)^{\star k}\end{array}$ & $\begin{array}{c}0.068 \\
(0.009)^{\star \star \star}\end{array}$ & $\begin{array}{c}-0.080 \\
(0.017)^{\star \star \star}\end{array}$ & $\begin{array}{c}0.079 \\
(0.008)^{* * * *}\end{array}$ \\
\hline Age 55 to 64 & $\begin{array}{c}0.087 \\
(0.009)^{\star * *}\end{array}$ & $\begin{array}{c}0.065 \\
(0.008)^{* * *}\end{array}$ & $\begin{array}{c}0.082 \\
(0.009)^{* * * *}\end{array}$ & $\begin{array}{c}0.043 \\
(0.011)^{\star * *}\end{array}$ & $\begin{array}{c}0.031 \\
(0.014)^{*}\end{array}$ & $\begin{array}{c}0.027 \\
(0.013)^{*}\end{array}$ & $\begin{array}{c}0.007 \\
(0.016)\end{array}$ & $\begin{array}{c}0.040 \\
(0.010)^{* * *}\end{array}$ \\
\hline Languages - French & $\begin{array}{c}-0.443 \\
(0.05)^{\star \star \star \star}\end{array}$ & $\begin{array}{c}0.069 \\
(0.008)^{\star \star \star}\end{array}$ & $\begin{array}{c}-0.051 \\
(0.048)^{\star \star \star}\end{array}$ & $\begin{array}{c}0.034 \\
(0.016)^{*}\end{array}$ & $\begin{array}{c}-0.568 \\
(0.043)^{\star \star \star}\end{array}$ & $\begin{array}{c}0.022 \\
(0.012)\end{array}$ & $\begin{array}{c}-0.551 \\
(0.045)^{\star \star \star}\end{array}$ & $\begin{array}{c}0.011 \\
(0.021)\end{array}$ \\
\hline Languages - Both & $\begin{array}{c}-0.038 \\
(0.017)^{\star}\end{array}$ & $\begin{array}{c}0.043 \\
(0.008)^{\star \star \star}\end{array}$ & $\begin{array}{c}-0.118 \\
(0.022)^{* * *}\end{array}$ & $\begin{array}{c}-0.023 \\
(0.017)\end{array}$ & $\begin{array}{c}-0.153 \\
(0.023)^{* * *}\end{array}$ & $\begin{array}{c}0.022 \\
(0.011)\end{array}$ & $\begin{array}{c}-0.173 \\
(0.024)^{\star * \star}\end{array}$ & $\begin{array}{c}0.020 \\
(0.010)\end{array}$ \\
\hline Access/use technology & $\begin{array}{c}0.015 \\
(0.007)^{\star}\end{array}$ & $\begin{array}{c}0.0690 \\
(0.006)^{\star * *}\end{array}$ & $\begin{array}{c}0.028 \\
(0.007)^{* * *}\end{array}$ & $\begin{array}{c}0.062 \\
(0.006)^{m}\end{array}$ & $\begin{array}{c}0.040 \\
(0.006)^{* * *}\end{array}$ & $\begin{array}{c}0.060 \\
(0.006)^{\star \star \star}\end{array}$ & $\begin{array}{c}0.044 \\
(0.006)^{\star \star \star}\end{array}$ & $\begin{array}{c}0.057 \\
(0.005)^{* * *}\end{array}$ \\
\hline Legally married & & & $\begin{array}{c}-0.020 \\
(0.015)\end{array}$ & $\begin{array}{c}0.125 \\
(0.015)^{\star \star \star}\end{array}$ & $\begin{array}{c}-0.005 \\
(0.015)\end{array}$ & $\begin{array}{c}0.171 \\
(0.014)^{\star \star \star}\end{array}$ & $\begin{array}{c}-0.019 \\
(0.015)\end{array}$ & $\begin{array}{c}0.172 \\
(0.013)^{\star * *}\end{array}$ \\
\hline Common-law & & & $\begin{array}{c}-0.017 \\
(0.014)\end{array}$ & $\begin{array}{c}0.074 \\
(0.009)^{* * *}\end{array}$ & $\begin{array}{c}-0.021 \\
(0.015)\end{array}$ & $\begin{array}{c}0.093 \\
(0.008)^{* * *}\end{array}$ & $\begin{array}{c}-0.042 \\
(0.016)^{* *}\end{array}$ & $\begin{array}{c}0.095 \\
(0.008)^{* * *}\end{array}$ \\
\hline Divorced-separated & & & $\begin{array}{c}-0.019 \\
(0.015)\end{array}$ & $\begin{array}{l}0.0169 \\
(0.013)\end{array}$ & $\begin{array}{c}-0.035 \\
(0.015)^{\star}\end{array}$ & $\begin{array}{c}0.019 \\
(0.012)\end{array}$ & $\begin{array}{c}-0.028 \\
(0.016)\end{array}$ & $\begin{array}{c}0.018 \\
(0.011)\end{array}$ \\
\hline Children $<6$ & & & $\begin{array}{c}-0.018 \\
(0.012)\end{array}$ & $\begin{array}{c}-0.083 \\
(0.015)^{\star \star *}\end{array}$ & $\begin{array}{l}0.0009 \\
(0.012)\end{array}$ & $\begin{array}{c}-0.120 \\
(0.016)^{\star \star \star}\end{array}$ & $\begin{array}{c}0.018 \\
(0.012)\end{array}$ & $\begin{array}{c}-0.115 \\
(0.015)^{\star * *}\end{array}$ \\
\hline Children 6-14 & & & $\begin{array}{c}-0.049 \\
(0.015)^{\star \star}\end{array}$ & $\begin{array}{c}-0.072 \\
(0.016)^{\star * *}\end{array}$ & $\begin{array}{c}-0.029 \\
(0.014)^{*}\end{array}$ & $\begin{array}{c}-0.111 \\
(0.016)^{\star \star \star}\end{array}$ & $\begin{array}{c}-0.008 \\
(0.013)\end{array}$ & $\begin{array}{c}-0.089 \\
(0.014)^{* * *}\end{array}$ \\
\hline Children 14-24 & & & $\begin{array}{c}0.022 \\
(0.013)\end{array}$ & $\begin{array}{c}-0.063 \\
(0.021)^{\star \star}\end{array}$ & $\begin{array}{c}0.031 \\
(0.013)^{*}\end{array}$ & $\begin{array}{c}-0.094 \\
(0.022)^{\star \star \star}\end{array}$ & $\begin{array}{c}0.033 \\
(0.013)^{*}\end{array}$ & $\begin{array}{c}-0.111 \\
(0.022)^{\star \star \star \star}\end{array}$ \\
\hline Lone parent & & & $\begin{array}{c}0.035 \\
(0.013)^{\star *}\end{array}$ & $\begin{array}{c}0.076 \\
(0.010)^{\star \star * \star}\end{array}$ & $\begin{array}{c}0.062 \\
(0.010)^{\star \star}\end{array}$ & $\begin{array}{c}0.091 \\
(0.010)^{* * *}\end{array}$ & $\begin{array}{c}0.052 \\
(0.011)^{* * *}\end{array}$ & $\begin{array}{c}0.094 \\
(0.009)^{\star * *}\end{array}$ \\
\hline Chronic health condition & & & $\begin{array}{c}-0.010 \\
(0.008)\end{array}$ & $\begin{array}{c}-0.015 \\
(0.005)^{\star \star}\end{array}$ & $\begin{array}{r}-0.0005 \\
(0.007)\end{array}$ & $\begin{array}{c}-0.011 \\
(0.005)^{\star}\end{array}$ & $\begin{array}{c}0.004 \\
(0.007)\end{array}$ & $\begin{array}{c}-0.020 \\
(0.005)^{* * *}\end{array}$ \\
\hline Health activity limitation & & & $\begin{array}{c}0.024 \\
(0.008)\end{array}$ & $\begin{array}{c}-0.053 \\
(0.007)^{\star * *}\end{array}$ & $\begin{array}{c}-0.0002 \\
(0.007)\end{array}$ & $\begin{array}{c}-0.042 \\
(0.007)^{\star \star}\end{array}$ & $\begin{array}{l}-0.002 \\
(0.007)\end{array}$ & $\begin{array}{c}-0.052 \\
(0.007)^{\star * *}\end{array}$ \\
\hline \multirow[t]{2}{*}{ Other sources of income } & & & $\begin{array}{c}-0.014 \\
(0.006)^{\star}\end{array}$ & $\begin{array}{c}-0.050 \\
(0.007)^{\star \star \star}\end{array}$ & $\begin{array}{c}-0.029 \\
(0.007)^{\star \star *}\end{array}$ & $\begin{array}{c}-0.059 \\
(0.007)^{\star * \star}\end{array}$ & $\begin{array}{c}-0.020 \\
(0.007)^{\star \star}\end{array}$ & $\begin{array}{c}-0.060 \\
(0.006)^{\star \star \star}\end{array}$ \\
\hline & & & & & & & & ...continued \\
\hline
\end{tabular}




\begin{tabular}{|c|c|c|c|c|c|c|c|c|}
\hline \multirow{3}{*}{$\begin{array}{l}\text { Appendix C6 } \\
\text { (continued) }\end{array}$} & \multicolumn{8}{|c|}{ Average Marginal Effects } \\
\hline & \multicolumn{2}{|c|}{ Model 1} & \multicolumn{2}{|c|}{ Model 2} & \multicolumn{2}{|c|}{ Model 3} & \multicolumn{2}{|c|}{ Full Model } \\
\hline & Men & Women & Men & Women & Men & Women & Men & Women \\
\hline Two or more maintainers & & & $\begin{array}{c}0.028 \\
(0.006)^{* * *}\end{array}$ & $\begin{array}{c}0.011 \\
(0.006)\end{array}$ & $\begin{array}{c}0.031 \\
(0.006)^{m * *}\end{array}$ & $\begin{array}{c}0.016 \\
(0.006)^{*}\end{array}$ & $\begin{array}{c}0.021 \\
(0.006)^{* *}\end{array}$ & $\begin{array}{c}0.015 \\
(0.006)^{* *}\end{array}$ \\
\hline First maintainer & & & $\begin{array}{c}0.090 \\
(0.007)^{\star * *}\end{array}$ & $\begin{array}{l}0.0003) \\
(0.006)\end{array}$ & $\begin{array}{c}0.098 \\
(0.007)^{\text {ntw }}\end{array}$ & $\begin{array}{l}-0.008 \\
(0.006)\end{array}$ & $\begin{array}{c}0.091 \\
(0.007)^{* * * *}\end{array}$ & $\begin{array}{l}-0.012 \\
(0.006)^{*}\end{array}$ \\
\hline Nunatsiavut & & & $\begin{array}{c}-0.314 \\
(0.025)^{\star \star *}\end{array}$ & $\begin{array}{c}-0.105 \\
(0.018)^{\star \star * \star}\end{array}$ & $\begin{array}{c}-0.418 \\
(0.026)^{* * *}\end{array}$ & $\begin{array}{c}-0.132 \\
(0.018)^{\star \star \star \star}\end{array}$ & $\begin{array}{c}-0.448 \\
(0.025)^{* * *}\end{array}$ & $\begin{array}{r}-0.124 \\
(0.017)^{* *+}\end{array}$ \\
\hline Nunavik & & & $\begin{array}{c}0.021 \\
(0.10)^{*}\end{array}$ & $\begin{array}{c}0.084 \\
(0.009)^{* * \star}\end{array}$ & $\begin{array}{c}-0.039 \\
(0.014)^{* *}\end{array}$ & $\begin{array}{c}0.082 \\
(0.008)^{* * * *}\end{array}$ & $\begin{array}{c}-0.110 \\
(0.020)^{* * * *}\end{array}$ & $\begin{array}{c}0.078 \\
(0.009)^{* * *}\end{array}$ \\
\hline Nunavut & & & $\begin{array}{c}-0.040 \\
(0.009)^{\star \star \star *}\end{array}$ & $\begin{array}{c}0.046 \\
(0.009)^{\star \star * \hbar}\end{array}$ & $\begin{array}{c}-0.072 \\
(0.010)^{*+* k}\end{array}$ & $\begin{array}{c}0.077 \\
(0.009)^{* \star * \star}\end{array}$ & $\begin{array}{c}-0.130 \\
(0.012)^{*+* k}\end{array}$ & $\begin{array}{c}0.058 \\
(0.010)^{* *+1}\end{array}$ \\
\hline Has someone to confide in & & & & & $\begin{array}{c}-0.046 \\
(0.006)^{*+*}\end{array}$ & $\begin{array}{c}0.048 \\
(0.006)^{* \ldots *}\end{array}$ & $\begin{array}{c}-0.048 \\
(0.006)^{*+\ldots}\end{array}$ & $\begin{array}{c}0.047 \\
(0.006)^{* * *}\end{array}$ \\
\hline $\begin{array}{l}\text { Volunteering club } \\
\text { membership }\end{array}$ & & & & & $\begin{array}{c}-0.036 \\
(0.006)^{\star \star \star \star}\end{array}$ & $\begin{array}{c}0.004 \\
(0.006)\end{array}$ & $\begin{array}{c}-0.037 \\
(0.006)^{\star \star \star \star}\end{array}$ & $\begin{array}{c}0.006 \\
(0.006)\end{array}$ \\
\hline Voting, municipal & & & & & $\begin{array}{c}0.032 \\
(0.010)^{* \star}\end{array}$ & $\begin{array}{c}0.127 \\
(0.011)^{* * *}\end{array}$ & $\begin{array}{c}0.025 \\
(0.009)^{* *}\end{array}$ & $\begin{array}{c}0.103 \\
(0.010)^{* *+1}\end{array}$ \\
\hline Voting, provincial & & & & & $\begin{array}{c}0.066 \\
(0.011)^{* * *}\end{array}$ & $\begin{array}{c}-0.084 \\
(0.006)^{\star \star * *}\end{array}$ & $\begin{array}{c}0.083 \\
(0.011)^{* * *}\end{array}$ & $\begin{array}{c}-0.076 \\
(0.007)^{\star \star \star *}\end{array}$ \\
\hline $\begin{array}{l}\text { Member in Inuit } \\
\text { organizations }\end{array}$ & & & & & & & $\begin{array}{c}-0.045 \\
(0.007)^{\star \star *}\end{array}$ & $\begin{array}{c}0.041 \\
(0.011)^{* * *}\end{array}$ \\
\hline $\begin{array}{l}\text { Speaks Aboriginal } \\
\text { languages }\end{array}$ & & & & & & & $\begin{array}{c}0.070 \\
(0.015)^{\star \star \star}\end{array}$ & $\begin{array}{l}0.027 \\
(0.010)^{* *}\end{array}$ \\
\hline $\begin{array}{l}\text { Values Aboriginal } \\
\text { languages }\end{array}$ & & & & & & & $\begin{array}{l}-0.011 \\
(0.015)\end{array}$ & $\begin{array}{c}0.065 \\
(0.023)^{* *}\end{array}$ \\
\hline $\begin{array}{l}\text { Traditional pursuits } \\
\text { for commerce }\end{array}$ & & & & & & & $\begin{array}{l}-0.008 \\
(0.008)\end{array}$ & $\begin{array}{r}-0.182 \\
(0.017)^{* *}\end{array}$ \\
\hline $\begin{array}{l}\text { Traditional pursuits } \\
\text { for food }\end{array}$ & & & & & & & $\begin{array}{c}0.013 \\
(0.015)\end{array}$ & $\begin{array}{c}0.037 \\
(0.012)^{* *}\end{array}$ \\
\hline $\begin{array}{l}\text { Traditional pursuits } \\
\text { for pleasure }\end{array}$ & & & & & & & $\begin{array}{l}-0.003 \\
(0.007)\end{array}$ & $\begin{array}{c}-0.031 \\
(0.007)^{* \pm+1}\end{array}$ \\
\hline Number of observations & 840 & 840 & 800 & 790 & 750 & 720 & 720 & 710 \\
\hline
\end{tabular}

Source: Statistics Canada, 2006 Aboriginal Peoples Survey, analytical files, Arctic File. Bootstrapped (1000 replications) standard errors in parentheses. Statistical significance ${ }^{* * *} p<.001 ;{ }^{* *} p<.01 ;{ }^{*} p<.05$ 


\section{Appendix D}

Appendix D 1: Detailed results from robustness checks for highest grade and highest level of schooling attained, APS 2006

\begin{tabular}{|c|c|c|c|c|c|c|c|c|c|c|c|c|}
\hline \multirow{4}{*}{ Variables in equations } & \multicolumn{12}{|c|}{ Average Marginal Effects } \\
\hline & \multicolumn{8}{|c|}{ North American Indian } & \multicolumn{2}{|c|}{ Métis } & \multicolumn{2}{|c|}{ Inuit } \\
\hline & \multicolumn{2}{|c|}{ Urban } & \multicolumn{2}{|c|}{ Rural } & \multicolumn{2}{|c|}{ Registered } & \multicolumn{2}{|c|}{ Non-registered } & \multirow[b]{2}{*}{ Men } & \multirow[b]{2}{*}{ Women } & \multirow[b]{2}{*}{ Men } & \multirow[b]{2}{*}{ Women } \\
\hline & Men & Women & Men & Women & Men & Women & Men & Women & & & & \\
\hline $\begin{array}{l}\text { Less than } \\
\text { high school education }\end{array}$ & $\begin{array}{c}-0.048 \\
(0.007)^{\star * *}\end{array}$ & $\begin{array}{c}-0.034 \\
(0.006)^{* * * *}\end{array}$ & $\begin{array}{c}-0.032 \\
(0.008)^{* * * *}\end{array}$ & $\begin{array}{c}-0.054 \\
(0.009)^{* * *}\end{array}$ & $\begin{array}{c}-0.050 \\
(0.007)^{* * *}\end{array}$ & $\begin{array}{c}-0.022 \\
(0.006)^{\star *}\end{array}$ & $\begin{array}{c}-0.042 \\
(0.008)^{\star \star \star}\end{array}$ & $\begin{array}{c}-0.049 \\
(0.008)^{\star * *}\end{array}$ & $\begin{array}{r}-0.004 \\
(0.004)\end{array}$ & $\begin{array}{c}-0.027 \\
(0.004)^{\star \star \star}\end{array}$ & - & - \\
\hline $\begin{array}{l}\text { Some } \\
\text { vocational schooling }\end{array}$ & $\begin{array}{c}0.055 \\
(0.005)^{\star \star \star}\end{array}$ & $\begin{array}{c}0.043 \\
(0.005)^{\star \star \star}\end{array}$ & $\begin{array}{l}-0.018 \\
(0.022)\end{array}$ & $\begin{array}{c}-0.096 \\
(0.028)^{\star *}\end{array}$ & $\begin{array}{c}0.066 \\
(0.007)^{\star * *}\end{array}$ & $\begin{array}{c}0.021 \\
(0.008)^{*}\end{array}$ & $\begin{array}{c}0.017 \\
(0.011)\end{array}$ & - & - & - & - & - \\
\hline $\begin{array}{l}\text { Some } \\
\text { non-university education }\end{array}$ & $\begin{array}{c}-0.045 \\
(0.012)^{\star \star *}\end{array}$ & $\begin{array}{c}0.041 \\
(0.004)^{* * *}\end{array}$ & $\begin{array}{c}0.002 \\
(0.010)\end{array}$ & $\begin{array}{c}-0.046 \\
(0.013)^{\star * *}\end{array}$ & $\begin{array}{c}-0.033 \\
(0.011)^{* *}\end{array}$ & $\begin{array}{c}0.059 \\
(0.004)^{\star \star \star \star}\end{array}$ & $\begin{array}{l}-0.028 \\
(0.012)\end{array}$ & $\begin{array}{c}-0.022 \\
(0.009)^{*}\end{array}$ & $\begin{array}{l}-0.005 \\
(0.005)\end{array}$ & $\begin{array}{c}0.002 \\
(0.004)\end{array}$ & - & - \\
\hline $\begin{array}{l}\text { Some } \\
\text { university education }\end{array}$ & $\begin{array}{c}-0.043 \\
(0.011)^{\star k \star}\end{array}$ & $\begin{array}{c}0.051 \\
(0.003)^{\star * *}\end{array}$ & $\begin{array}{c}-0.002 \\
(0.018)\end{array}$ & $\begin{array}{c}0.003 \\
(0.012)\end{array}$ & $\begin{array}{c}-0.085 \\
(0.014)^{\star * \star}\end{array}$ & $\begin{array}{c}0.056 \\
(0.004)^{\star \star \star}\end{array}$ & $\begin{array}{c}0.030 \\
(0.008)^{\star \star *}\end{array}$ & $\begin{array}{c}0.025 \\
(0.005)^{\star \star \star}\end{array}$ & $\begin{array}{c}0.017 \\
(0.006)^{* *}\end{array}$ & $\begin{array}{c}0.006 \\
(0.004)\end{array}$ & - & - \\
\hline $\begin{array}{l}\text { Completed } \\
\text { trades certificate or diploma }\end{array}$ & $\begin{array}{c}-0.031 \\
(0.009)^{\star \star *}\end{array}$ & $\begin{array}{c}0.038 \\
(0.004)^{* * *}\end{array}$ & $\begin{array}{c}-0.021 \\
(0.008)^{*}\end{array}$ & $\begin{array}{c}0.016 \\
(0.008)^{\star}\end{array}$ & $\begin{array}{c}-0.048 \\
(0.010)^{* * *}\end{array}$ & $\begin{array}{c}0.064 \\
(0.003)^{\star \star *}\end{array}$ & $\begin{array}{r}-0.012 \\
(0.007)\end{array}$ & $\begin{array}{l}-0.012 \\
(0.008)\end{array}$ & $\begin{array}{r}-0.0003 \\
(0.004)\end{array}$ & $\begin{array}{l}-0.001 \\
(0.005)\end{array}$ & - & - \\
\hline $\begin{array}{l}\text { Completed } \\
\text { non-university diploma }\end{array}$ & $\begin{array}{c}-0.068 \\
(0.011)^{* * *}\end{array}$ & $\begin{array}{c}0.060 \\
(0.004)^{* * *}\end{array}$ & $\begin{array}{c}0.005 \\
(0.008)\end{array}$ & $\begin{array}{c}-0.024 \\
(0.007)^{\star \star}\end{array}$ & $\begin{array}{c}-0.055 \\
(0.010)^{* k * k}\end{array}$ & $\begin{array}{c}0.065 \\
(0.005)^{\star \star \star}\end{array}$ & $\begin{array}{c}-0.034 \\
(0.010)^{\text {n* }}\end{array}$ & $\begin{array}{c}0.022 \\
(0.004)^{\star \star \star \star}\end{array}$ & $\begin{array}{c}0.026 \\
(0.003)^{* * *}\end{array}$ & $\begin{array}{l}-0.001 \\
(0.003)\end{array}$ & - & - \\
\hline $\begin{array}{l}\text { Completed } \\
\text { university diploma }\end{array}$ & $\begin{array}{c}0.025 \\
(0.010)^{*}\end{array}$ & $\begin{array}{c}0.039 \\
(0.004)^{* * *}\end{array}$ & $\begin{array}{c}0.007 \\
(0.016)\end{array}$ & $\begin{array}{c}0.021 \\
(0.010)^{\star}\end{array}$ & $\begin{array}{c}0.007 \\
(0.016)\end{array}$ & $\begin{array}{c}0.039 \\
(0.006)^{\star \star \star}\end{array}$ & $\begin{array}{c}0.023 \\
(0.008)^{\star *}\end{array}$ & - & - & - & - & - \\
\hline $\begin{array}{l}\text { Completed } \\
\text { university degree }\end{array}$ & $\begin{array}{c}0.038 \\
(0.006)^{* * *}\end{array}$ & $\begin{array}{c}0.055 \\
(0.003)^{* * *}\end{array}$ & $\begin{array}{l}-0.003 \\
(0.012)\end{array}$ & $\begin{array}{c}0.040 \\
(0.005)^{* * * *}\end{array}$ & $\begin{array}{c}0.049 \\
(0.007)^{* * * *}\end{array}$ & $\begin{array}{c}0.065 \\
(0.004)^{* * *}\end{array}$ & $\begin{array}{c}0.029 \\
(0.006)^{* \star \star}\end{array}$ & $\begin{array}{c}0.033 \\
(0.004)^{* * *}\end{array}$ & $\begin{array}{c}0.014 \\
(0.006)^{\star}\end{array}$ & $\begin{array}{c}0.027 \\
(0.008)^{* * * *}\end{array}$ & - & - \\
\hline Grade 6 or less & - & - & - & - & - & - & - & - & - & - & $\begin{array}{c}0.037 \\
(0.009)^{* \star *}\end{array}$ & $\begin{array}{c}0.017 \\
(0.008)^{\star}\end{array}$ \\
\hline Grade 9 & - & - & - & - & - & - & - & - & - & - & $\begin{array}{c}0.020 \\
(0.009)^{*}\end{array}$ & $\begin{array}{c}0.031 \\
(0.007)^{\cdots}\end{array}$ \\
\hline Grade 10 & - & - & - & - & - & - & - & - & - & - & $\begin{array}{l}-0.001 \\
(0.009)\end{array}$ & $\begin{array}{c}-0.035 \\
(0.011)^{\star *}\end{array}$ \\
\hline
\end{tabular}




\begin{tabular}{|c|c|c|c|c|c|c|c|c|c|c|c|c|}
\hline \multirow{2}{*}{$\begin{array}{l}\text { Appendix D1 } \\
\text { (continued) }\end{array}$} & \multicolumn{8}{|c|}{ North American Indian } & \multicolumn{2}{|c|}{ Métis } & \multicolumn{2}{|c|}{ Inuit } \\
\hline & Men & Women & Men & Women & Men & Women & Men & Women & Men & Women & Men & Women \\
\hline Grade 11 & - & - & - & - & - & - & - & - & - & - & $\begin{array}{c}0.018 \\
(0.010)\end{array}$ & $\begin{array}{c}0.063 \\
(0.006)^{* * * \star}\end{array}$ \\
\hline Age 15 to 19 & $\begin{array}{c}-0.095 \\
(0.013)^{\star * *}\end{array}$ & $\begin{array}{c}-0.088 \\
(0.016)^{* \star * *}\end{array}$ & $\begin{array}{c}-0.01 \\
(0.010)\end{array}$ & $\begin{array}{c}-0.048 \\
(0.014)^{* *}\end{array}$ & $\begin{array}{c}-0.089 \\
(0.008)^{* * *}\end{array}$ & $\begin{array}{c}-0.139 \\
(0.019)^{* * * *}\end{array}$ & $\begin{array}{c}-0.032 \\
(0.011)^{* *}\end{array}$ & $\begin{array}{c}-0.026 \\
(0.010)^{* *}\end{array}$ & $\begin{array}{c}0.005 \\
(0.005)\end{array}$ & $\begin{array}{c}-0.056 \\
(0.008)^{\star \star *}\end{array}$ & $\begin{array}{c}-0.075 \\
(0.018)^{\star \star *}\end{array}$ & $\begin{array}{c}-0.081 \\
(0.017)^{\star * * \star}\end{array}$ \\
\hline Age 25 to 34 & $\begin{array}{c}0.031 \\
(0.006)^{\star * *}\end{array}$ & $\begin{array}{c}-0.081 \\
(0.011)^{\star \star * *}\end{array}$ & $\begin{array}{c}0.048 \\
(0.007)^{* * *}\end{array}$ & $\begin{array}{c}-0.007 \\
(0.008)\end{array}$ & $\begin{array}{c}0.026 \\
(0.007)^{* * *}\end{array}$ & $\begin{array}{c}-0.071 \\
(0.009)^{* * *}\end{array}$ & $\begin{array}{c}0.057 \\
(0.005)^{* * *}\end{array}$ & $\begin{array}{c}-0.030 \\
(0.008)^{\star * * *}\end{array}$ & $\begin{array}{c}0.035 \\
(0.003)^{* * *}\end{array}$ & $\begin{array}{c}0.006 \\
(0.004)\end{array}$ & $\begin{array}{c}0.014 \\
(0.011)\end{array}$ & $\begin{array}{c}0.079 \\
(0.007)^{* * * *}\end{array}$ \\
\hline Age 35 to 44 & $\begin{array}{c}0.042 \\
(0.006)^{\star \star \star}\end{array}$ & $\begin{array}{l}-0.010 \\
(0.008)\end{array}$ & $\begin{array}{c}0.029 \\
\left(0.009^{* \star}\right.\end{array}$ & $\begin{array}{c}0.025 \\
(0.007)^{* *}\end{array}$ & $\begin{array}{c}0.055 \\
(0.007)^{\star \star \star}\end{array}$ & $\begin{array}{c}-0.051 \\
(0.008)^{* * *}\end{array}$ & $\begin{array}{c}0.027 \\
(0.007)^{\star \star * *}\end{array}$ & $\begin{array}{c}0.070 \\
(0.006)^{\star \star * *}\end{array}$ & $\begin{array}{c}0.013 \\
(0.005)^{* *}\end{array}$ & $\begin{array}{c}0.016 \\
(0.004)^{* \star *}\end{array}$ & $\begin{array}{c}-0.058 \\
(0.015)^{* * *}\end{array}$ & $\begin{array}{c}-0.007 \\
(0.011)\end{array}$ \\
\hline Age 45 to 54 & $\begin{array}{c}0.021 \\
(0.008)^{* \star}\end{array}$ & $\begin{array}{c}-0.010 \\
(0.008)\end{array}$ & $\begin{array}{c}0.046 \\
(0.009)^{* * *}\end{array}$ & $\begin{array}{c}0.022 \\
(0.008)^{* *}\end{array}$ & $\begin{array}{c}0.051 \\
(0.008)^{\star \star \star}\end{array}$ & $\begin{array}{c}-0.033 \\
(0.009)^{\star \star \star \star}\end{array}$ & $\begin{array}{c}-0.001 \\
(0.009)\end{array}$ & $\begin{array}{c}0.050 \\
(0.007)^{\star \star \star *}\end{array}$ & $\begin{array}{c}0.014 \\
(0.005)^{* *}\end{array}$ & $\begin{array}{c}0.016 \\
(0.004)^{\star \star \star *}\end{array}$ & $\begin{array}{c}-0.059 \\
(0.018)^{\star \star}\end{array}$ & $\begin{array}{c}0.052 \\
(0.010)^{\star \star \star}\end{array}$ \\
\hline Languages - Both & $\begin{array}{c}0.016 \\
(0.007)^{*}\end{array}$ & $\begin{array}{c}0.039 \\
(0.004)^{\star \star * *}\end{array}$ & $\begin{array}{c}-0.081 \\
(0.013)^{* * *}\end{array}$ & $\begin{array}{c}0.014 \\
(0.007)\end{array}$ & $\begin{array}{c}0.003 \\
(0.008)\end{array}$ & $\begin{array}{c}0.057 \\
(0.004)^{\star \star \star \star}\end{array}$ & $\begin{array}{c}-0.027 \\
(0.008)^{\star \star}\end{array}$ & $\begin{array}{c}-0.004 \\
(0.006)\end{array}$ & $\begin{array}{c}0.025 \\
(0.003)^{* * * *}\end{array}$ & $\begin{array}{c}0.028 \\
(0.003)^{n+*}\end{array}$ & $\begin{array}{c}-0.191 \\
(0.026)^{\star * *}\end{array}$ & $\begin{array}{c}0.010 \\
(0.011)\end{array}$ \\
\hline Access/use technology & $\begin{array}{c}0.012 \\
(0.005)^{*}\end{array}$ & $\begin{array}{c}-0.010 \\
(0.004)^{\star}\end{array}$ & $\begin{array}{c}0.015 \\
(0.005)^{* * *}\end{array}$ & $\begin{array}{c}0.016 \\
(0.004)^{* * *}\end{array}$ & $\begin{array}{c}0.051 \\
(0.005)^{* * *}\end{array}$ & $\begin{array}{c}-0.002 \\
(0.004)\end{array}$ & $\begin{array}{c}-0.029 \\
(0.005)^{* * * *}\end{array}$ & $\begin{array}{c}-0.016 \\
(0.003)^{* * * *}\end{array}$ & $\begin{array}{c}0.046 \\
(0.004)^{* * *}\end{array}$ & $\begin{array}{c}0.011 \\
(0.003)^{* * *}\end{array}$ & $\begin{array}{c}0.044 \\
(0.006)^{* * *}\end{array}$ & $\begin{array}{c}0.060 \\
(0.005)^{* * * *}\end{array}$ \\
\hline Legally married & $\begin{array}{c}0.069 \\
(0.007)^{\star * * *}\end{array}$ & $\begin{array}{c}-0.020 \\
(0.007)^{\star \star}\end{array}$ & $\begin{array}{r}-0.004 \\
(0.009)\end{array}$ & $\begin{array}{c}0.061 \\
(0.007)^{*+*}\end{array}$ & $\begin{array}{c}0.030 \\
(0.007)^{\star \star * *}\end{array}$ & $\begin{array}{c}0.030 \\
(0.006)^{\star \star \star \star}\end{array}$ & $\begin{array}{c}0.046 \\
(0.009)^{* * *}\end{array}$ & $\begin{array}{c}-0.061 \\
(0.012)^{\star \star *}\end{array}$ & $\begin{array}{c}0.022 \\
(0.004)^{* \star *}\end{array}$ & $\begin{array}{c}0.039 \\
(0.005)^{\star * t *}\end{array}$ & $\begin{array}{l}-0.010 \\
(0.015)\end{array}$ & $\begin{array}{c}0.168 \\
(0.013)^{* * \star}\end{array}$ \\
\hline \multirow[t]{2}{*}{ Common-law } & $\begin{array}{c}0.045 \\
(0.005)^{* * *}\end{array}$ & $\begin{array}{c}-0.037 \\
(0.006)^{* * * *}\end{array}$ & $\begin{array}{c}-0.025 \\
(0.008)^{* *}\end{array}$ & $\begin{array}{c}0.016 \\
(0.007)^{*}\end{array}$ & $\begin{array}{c}0.038 \\
(0.006)^{* \star * *}\end{array}$ & $\begin{array}{c}-0.009 \\
(0.006)\end{array}$ & $\begin{array}{c}0.021 \\
(0.006)^{* * \star}\end{array}$ & $\begin{array}{c}-0.017 \\
(0.007)^{*}\end{array}$ & $\begin{array}{c}0.011 \\
(0.004)^{* *}\end{array}$ & $\begin{array}{c}0.041 \\
(0.003)^{\star \star \star \star}\end{array}$ & $\begin{array}{c}-0.039 \\
(0.015)^{*}\end{array}$ & $\begin{array}{c}0.093 \\
(0.008)^{* * *}\end{array}$ \\
\hline & & & & & & & & & & & & ...continued \\
\hline
\end{tabular}




\begin{tabular}{|c|c|c|c|c|c|c|c|c|c|c|c|c|}
\hline \multirow{4}{*}{$\begin{array}{l}\text { Appendix D1 } \\
\text { (continued) }\end{array}$} & \multicolumn{12}{|c|}{ Average Marginal Effects } \\
\hline & \multicolumn{8}{|c|}{ North American Indian } & \multicolumn{2}{|c|}{ Métis } & \multicolumn{2}{|c|}{ Inuit } \\
\hline & \multicolumn{2}{|c|}{ Urban } & \multicolumn{2}{|c|}{ Rural } & \multicolumn{2}{|c|}{ Registered } & \multicolumn{2}{|c|}{ Non-registered } & \multirow[b]{2}{*}{ Men } & \multirow[b]{2}{*}{ Women } & \multirow[b]{2}{*}{ Men } & \multirow[b]{2}{*}{ Women } \\
\hline & Men & Women & Men & Women & Men & Women & Men & Women & & & & \\
\hline Divorced-separated & $\begin{array}{c}0.005 \\
(0.007)\end{array}$ & $\begin{array}{c}-0.044 \\
(0.007)^{\star \star * *}\end{array}$ & $\begin{array}{c}0.042 \\
(0.006)^{*+*}\end{array}$ & $\begin{array}{c}0.025 \\
(0.005)^{* * *}\end{array}$ & $\begin{array}{c}0.045 \\
(0.005)^{* * *}\end{array}$ & $\begin{array}{c}-0.028 \\
(0.006)^{* * *}\end{array}$ & $\begin{array}{c}-0.051 \\
(0.010)^{* * *}\end{array}$ & $\begin{array}{c}-0.037 \\
\langle 0.010)^{* * *}\end{array}$ & $\begin{array}{c}0.024 \\
(0.004)^{* * *}\end{array}$ & $\begin{array}{c}0.011 \\
(0.003)^{* *}\end{array}$ & $\begin{array}{c}-0.010 \\
(0.014)\end{array}$ & $\begin{array}{c}0.020 \\
(0.011)\end{array}$ \\
\hline Children $<6$ & $\begin{array}{c}-0.073 \\
(0.010)^{\star \star \star}\end{array}$ & $\begin{array}{c}0.008 \\
(0.005)\end{array}$ & $\begin{array}{c}0.026 \\
(0.007)^{*+* t}\end{array}$ & $\begin{array}{c}0.008 \\
(0.007)\end{array}$ & $\begin{array}{c}-0.025 \\
(0.008)^{* *}\end{array}$ & $\begin{array}{c}-0.044 \\
(0.008)^{* * *}\end{array}$ & $\begin{array}{c}-0.060 \\
(0.010)^{* * *}\end{array}$ & $\begin{array}{c}0.051 \\
(0.004)^{* \star *}\end{array}$ & $\begin{array}{c}0.006 \\
(0.005)\end{array}$ & $\begin{array}{c}-0.020 \\
(0.007)^{* *}\end{array}$ & $\begin{array}{c}0.014 \\
(0.012)\end{array}$ & $\begin{array}{c}-0.114 \\
(0.015)^{* * *}\end{array}$ \\
\hline Children 6-14 & $\begin{array}{c}-0.076 \\
(0.013)^{* * *}\end{array}$ & $\begin{array}{l}0.0002 \\
(0.007)\end{array}$ & $\begin{array}{c}0.088 \\
(0.005)^{*+1 *}\end{array}$ & $\begin{array}{c}0.040 \\
(0.005)^{* \star * *}\end{array}$ & $\begin{array}{c}0.027 \\
(0.007)^{*+*}\end{array}$ & $\begin{array}{c}-0.016 \\
(0.008)^{*}\end{array}$ & $\begin{array}{c}-0.060 \\
(0.012)^{* * * *}\end{array}$ & $\begin{array}{c}0.034 \\
(0.004)^{* * *}\end{array}$ & $\begin{array}{c}-0.009 \\
(0.006)\end{array}$ & $\begin{array}{c}-0.034 \\
(0.006)^{* * * *}\end{array}$ & $\begin{array}{c}-0.024 \\
(0.015)\end{array}$ & $\begin{array}{c}-0.091 \\
(0.014)^{* * *}\end{array}$ \\
\hline Children 14-24 & $\begin{array}{c}0.001 \\
(0.009)\end{array}$ & $\begin{array}{c}-0.038 \\
(0.008)^{\star \star \star \star}\end{array}$ & $\begin{array}{c}-0.003 \\
(0.009)\end{array}$ & $\begin{array}{c}0.022 \\
(0.005)^{\star \star \star *}\end{array}$ & $\begin{array}{l}-0.015 \\
(0.009)\end{array}$ & $\begin{array}{c}-0.073 \\
(0.009)^{*+*}\end{array}$ & $\begin{array}{c}0.022 \\
(0.006)^{* *}\end{array}$ & $\begin{array}{c}0.008 \\
(0.006)\end{array}$ & $\begin{array}{c}0.005 \\
(0.005)\end{array}$ & $\begin{array}{c}-0.028 \\
(0.005)^{* * *}\end{array}$ & $\begin{array}{c}0.019 \\
(0.014)\end{array}$ & $\begin{array}{c}-0.098 \\
(0.021)^{* * *}\end{array}$ \\
\hline Lone parent & $\begin{array}{c}0.041 \\
(0.007)^{* * \star}\end{array}$ & $\begin{array}{c}-0.037 \\
(0.008)^{* * *}\end{array}$ & $\begin{array}{c}-0.102 \\
(0.024)^{* * *}\end{array}$ & $\begin{array}{c}-0.018 \\
(0.004)\end{array}$ & $\begin{array}{c}-0.005 \\
(0.013)\end{array}$ & $\begin{array}{c}0.002 \\
(0.008)\end{array}$ & $\begin{array}{c}0.051 \\
(0.005)^{*+*}\end{array}$ & $\begin{array}{c}-0.122 \\
(0.019)^{m+m}\end{array}$ & $\begin{array}{c}0.050 \\
(0.002)^{* * * *}\end{array}$ & $\begin{array}{c}-0.008 \\
(0.005)\end{array}$ & $\begin{array}{c}0.068 \\
(0.010)^{* * *}\end{array}$ & $\begin{array}{c}0.0938 \\
(0.009)^{* * *}\end{array}$ \\
\hline Access to childcare & - & - & - & - & - & - & - & - & $\begin{array}{c}0.005 \\
(0.004)\end{array}$ & $\begin{array}{c}-0.003 \\
(0.004)\end{array}$ & - & - \\
\hline Chronic health condition & $\begin{array}{l}-0.003 \\
(0.004)\end{array}$ & $\begin{array}{c}0.011 \\
(0.003)^{* *}\end{array}$ & $\begin{array}{c}-0.008 \\
(0.005)\end{array}$ & $\begin{array}{c}-0.056 \\
(0.004)^{* * *}\end{array}$ & $\begin{array}{c}0.004 \\
(0.005)\end{array}$ & $\begin{array}{c}0.016 \\
(0.003)^{* * *}\end{array}$ & $\begin{array}{c}-0.032 \\
(0.004)^{* * *}\end{array}$ & $\begin{array}{c}-0.027 \\
(0.004)^{* * *}\end{array}$ & $\begin{array}{c}0.002 \\
(0.003)\end{array}$ & $\begin{array}{c}-0.020 \\
(0.002)^{* * * *}\end{array}$ & $\begin{array}{c}0.004 \\
(0.007)\end{array}$ & $\begin{array}{c}-0.014 \\
(0.005)^{* *}\end{array}$ \\
\hline Health activity limitation & $\begin{array}{c}-0.021 \\
(0.004)^{\star \star \star *}\end{array}$ & $\begin{array}{c}-0.016 \\
(0.004)^{\star \star * *}\end{array}$ & $\begin{array}{c}0.018 \\
(0.005)^{\star \star * *}\end{array}$ & $\begin{array}{c}0.014 \\
(0.004)^{* *}\end{array}$ & $\begin{array}{c}-0.038 \\
(0.005)^{*+*}\end{array}$ & $\begin{array}{c}-0.014 \\
(0.004)^{* *}\end{array}$ & $\begin{array}{c}0.024 \\
(0.004)^{* * *}\end{array}$ & $\begin{array}{c}-0.013 \\
(0.004)^{\star \star *}\end{array}$ & $\begin{array}{c}0.004 \\
(0.003)\end{array}$ & $\begin{array}{c}0.005 \\
(0.002)^{\star}\end{array}$ & $\begin{array}{c}0.007 \\
(0.007)\end{array}$ & $\begin{array}{c}-0.061 \\
(0.007)^{*+*+}\end{array}$ \\
\hline Other sources of income & $\begin{array}{c}0.029 \\
(0.004)^{* * * *}\end{array}$ & $\begin{array}{c}-0.033 \\
(0.005)^{* * *}\end{array}$ & $\begin{array}{c}-0.030 \\
(0.007)^{* * * *}\end{array}$ & $\begin{array}{c}0.024 \\
(0.004)^{* * *}\end{array}$ & $\begin{array}{c}0.007 \\
(0.005)\end{array}$ & $\begin{array}{l}-0.0007 \\
(0.004)\end{array}$ & $\begin{array}{c}0.012 \\
(0.006)^{*}\end{array}$ & $\begin{array}{c}-0.050 \\
(0.006)^{*+*+*}\end{array}$ & $\begin{array}{c}-0.016 \\
(0.005)^{* *}\end{array}$ & $\begin{array}{l}-0.0003 \\
(0.003)\end{array}$ & $\begin{array}{c}-0.020 \\
(0.007)^{\star \star}\end{array}$ & $\begin{array}{c}-0.049 \\
(0.006)^{* *+*}\end{array}$ \\
\hline Two or more maintainers & $\begin{array}{c}-0.005 \\
(0.004)\end{array}$ & $\begin{array}{c}0.022 \\
(0.003)^{m * *}\end{array}$ & $\begin{array}{c}0.020 \\
(0.005)^{+* *}\end{array}$ & $\begin{array}{c}0.018 \\
(0.004)^{* * *}\end{array}$ & $\begin{array}{c}0.025 \\
(0.004)^{* * *}\end{array}$ & $\begin{array}{c}0.018 \\
(0.004)^{* * *}\end{array}$ & $\begin{array}{c}-0.022 \\
(0.005)^{* * *}\end{array}$ & $\begin{array}{c}0.011 \\
(0.004)^{* \star}\end{array}$ & $\begin{array}{c}0.022 \\
(0.003)^{* * *}\end{array}$ & $\begin{array}{c}-0.005 \\
(0.002)\end{array}$ & $\begin{array}{c}0.016 \\
(0.006)^{*}\end{array}$ & $\begin{array}{c}0.008 \\
(0.006)\end{array}$ \\
\hline First maintainer & $\begin{array}{c}-0.003 \\
(0.005)\end{array}$ & $\begin{array}{c}0.033 \\
(0.004)^{* \star * *}\end{array}$ & $\begin{array}{c}0.028 \\
(0.005)^{+*}\end{array}$ & $\begin{array}{c}0.026 \\
(0.004)^{* * \star}\end{array}$ & $\begin{array}{c}-0.011 \\
(0.005)^{*}\end{array}$ & $\begin{array}{c}0.029 \\
(0.004)^{* * *}\end{array}$ & $\begin{array}{c}0.031 \\
(0.005)^{* * *}\end{array}$ & $\begin{array}{c}0.036 \\
(0.004)^{* \star *}\end{array}$ & $\begin{array}{c}0.031 \\
(0.003)^{* * *}\end{array}$ & $\begin{array}{c}0.017 \\
(0.002)^{* * * *}\end{array}$ & $\begin{array}{c}0.088 \\
(0.007)^{* * * *}\end{array}$ & $\begin{array}{c}-0.011 \\
(0.006)\end{array}$ \\
\hline Allantic provinces & $\begin{array}{c}-0.093 \\
(0.012)^{\star \star *}\end{array}$ & $\begin{array}{c}0.018 \\
(0.004)^{\star \star * *}\end{array}$ & $\begin{array}{c}-0.113 \\
(0.010)^{* * *}\end{array}$ & $\begin{array}{c}-0.090 \\
(0.009)^{* \star *}\end{array}$ & $\begin{array}{c}-0.095 \\
(0.010)^{*+* t}\end{array}$ & $\begin{array}{c}-0.027 \\
(0.008)^{* *}\end{array}$ & $\begin{array}{c}-0.123 \\
(0.011)^{* * *}\end{array}$ & $\begin{array}{c}-0.008 \\
(0.004)\end{array}$ & $(0.007)^{-0.027}$ & $\begin{array}{c}-0.031 \\
(0.008)^{* \ldots *}\end{array}$ & - & - \\
\hline Northem Territories & $\begin{array}{c}-0.060 \\
(0.013)^{* \star *}\end{array}$ & $\begin{array}{c}0.038 \\
(0.005)^{* * *}\end{array}$ & $\begin{array}{c}0.051 \\
(0.007)^{* * *}\end{array}$ & $\begin{array}{c}-0.064 \\
(0.010)^{* * *}\end{array}$ & $\begin{array}{c}0.005 \\
(0.008)\end{array}$ & $\begin{array}{c}0.001 \\
(0.007)\end{array}$ & $\begin{array}{c}-0.032 \\
(0.016)^{*}\end{array}$ & - & $\begin{array}{c}0.003 \\
(0.005)\end{array}$ & $\begin{array}{c}0.030 \\
(0.003)^{+\hbar+*}\end{array}$ & - & - \\
\hline
\end{tabular}




\begin{tabular}{|c|c|c|c|c|c|c|c|c|c|c|c|c|}
\hline \multirow{4}{*}{$\begin{array}{l}\text { Appendix D1 } \\
\text { (continued) }\end{array}$} & \multicolumn{12}{|c|}{ Average Marginal Effects } \\
\hline & \multicolumn{8}{|c|}{ North American Indian } & \multicolumn{2}{|c|}{ Métis } & \multicolumn{2}{|c|}{ Inuit } \\
\hline & \multicolumn{2}{|c|}{ Urban } & \multicolumn{2}{|c|}{ Rural } & \multicolumn{2}{|c|}{ Registered } & \multicolumn{2}{|c|}{ Non-registered } & \multirow[b]{2}{*}{ Men } & \multirow[b]{2}{*}{ Women } & \multirow[b]{2}{*}{ Men } & \multirow[b]{2}{*}{ Women } \\
\hline & Men & Women & Men & Women & Men & Women & Men & Women & & & & \\
\hline Quebec & $\begin{array}{c}-0.600 \\
(0.024)^{*}\end{array}$ & $\begin{array}{c}-0.033 \\
(0.009)^{* * *}\end{array}$ & $\begin{array}{c}0.063 \\
(0.008)^{* * * *}\end{array}$ & $\begin{array}{c}-0.008 \\
(0.012)\end{array}$ & $\begin{array}{c}0.018 \\
(0.012)\end{array}$ & $\begin{array}{c}-0.064 \\
(0.015)^{\star \star * *}\end{array}$ & $\begin{array}{l}-0.022 \\
(0.012)\end{array}$ & $\begin{array}{l}0.0007 \\
(0.008)\end{array}$ & $\begin{array}{c}-0.016 \\
(0.007)^{*}\end{array}$ & $\begin{array}{c}-0.067 \\
(0.009)^{* * *}\end{array}$ & - & - \\
\hline Manitoba & $\begin{array}{c}-0.118 \\
(0.013)^{* * *}\end{array}$ & $\begin{array}{c}0.049 \\
(0.003)^{* * *}\end{array}$ & $\begin{array}{c}-0.060 \\
(0.012)^{* * * *}\end{array}$ & - & $\begin{array}{c}-0.102 \\
(0.012)^{* * *}\end{array}$ & $\begin{array}{c}0.056 \\
(0.006)^{* * *}\end{array}$ & $\begin{array}{c}-0.133 \\
(0.022)^{* * *}\end{array}$ & - & $\begin{array}{c}0.034 \\
(0.004)^{* * *}\end{array}$ & $\begin{array}{c}0.009 \\
(0.003)^{\star *}\end{array}$ & - & - \\
\hline Saskatchewan & $\begin{array}{c}-0.061 \\
(0.010)^{* * * *}\end{array}$ & $\begin{array}{c}0.005 \\
(0.005)\end{array}$ & $\begin{array}{c}-0.070 \\
(0.013)^{* * * *}\end{array}$ & $\begin{array}{c}-0.116 \\
(0.013)^{* * * *}\end{array}$ & $\begin{array}{c}-0.079 \\
(0.010)^{* * *}\end{array}$ & $\begin{array}{c}0.001 \\
(0.006)\end{array}$ & $\begin{array}{c}-0.041 \\
(0.016)^{*}\end{array}$ & $\begin{array}{c}-0.052 \\
(0.010)^{* * *}\end{array}$ & $\begin{array}{c}0.016 \\
(0.004)^{* * * *}\end{array}$ & $\begin{array}{c}0.017 \\
(0.003)^{* * *}\end{array}$ & - & - \\
\hline Alberta & $\begin{array}{c}-0.017 \\
(0.007)^{*}\end{array}$ & $\begin{array}{c}0.026 \\
(0.004)^{* * *}\end{array}$ & $\begin{array}{c}0.061 \\
(0.007)^{* * *}\end{array}$ & $\begin{array}{c}-0.033 \\
(0.011)^{\star *}\end{array}$ & $\begin{array}{c}-0.020 \\
(0.009)^{*}\end{array}$ & $\begin{array}{c}0.021 \\
(0.005)^{* * * *}\end{array}$ & $\begin{array}{c}0.020 \\
(0.007)^{* *}\end{array}$ & $\begin{array}{c}0.019 \\
(0.005)^{* * *}\end{array}$ & $\begin{array}{c}0.037 \\
(0.003)^{* * *}\end{array}$ & $\begin{array}{c}0.020 \\
(0.003)^{* * *}\end{array}$ & - & - \\
\hline British Columbia & $\begin{array}{c}-0.027 \\
(0.008)^{* *}\end{array}$ & $\begin{array}{c}0.029 \\
(0.004)^{* * * *}\end{array}$ & $\begin{array}{c}0.011 \\
(0.009)^{* * * *}\end{array}$ & $\begin{array}{c}-0.029 \\
(0.009)^{* *}\end{array}$ & $\begin{array}{c}-0.014 \\
(0.008)\end{array}$ & $\begin{array}{c}0.017 \\
(0.005)^{\star \star}\end{array}$ & $\begin{array}{c}-0.019 \\
(0.009)\end{array}$ & $\begin{array}{c}0.043 \\
(0.003)^{* * *}\end{array}$ & $\begin{array}{c}0.032 \\
(0.003)^{* * * *}\end{array}$ & $\begin{array}{c}0.016 \\
(0.003)^{* * *}\end{array}$ & - & - \\
\hline Rural place of residence & - & - & - & - & $\begin{array}{c}-0.048 \\
(0.005)^{* * *}\end{array}$ & $\begin{array}{c}0.012 \\
(0.003)^{\star \star}\end{array}$ & $\begin{array}{c}0.016 \\
(0.004)^{* * *}\end{array}$ & $\begin{array}{c}-0.036 \\
(0.005)^{* * *}\end{array}$ & $\begin{array}{c}-0.013 \\
(0.003)^{*+* k}\end{array}$ & $\begin{array}{c}-0.004 \\
(0.002)\end{array}$ & - & - \\
\hline Nunatsiavut & - & - & - & - & - & - & - & - & - & - & $\begin{array}{c}-0.444 \\
(0.025)^{* * *}\end{array}$ & $\begin{array}{c}-0.130 \\
(0.018)^{\star * * *}\end{array}$ \\
\hline Nunavik & - & - & - & - & - & - & - & - & - & - & $\begin{array}{c}-0.120 \\
(0.020)^{\star * * *}\end{array}$ & $\begin{array}{c}0.073 \\
(0.008)^{* *+*}\end{array}$ \\
\hline Nunavut & - & - & - & - & - & - & - & - & - & - & $\begin{array}{c}-0.133 \\
(0.012)^{* * *}\end{array}$ & $\begin{array}{c}0.055 \\
(0.009)^{* * * *}\end{array}$ \\
\hline Has someone to confide in & $\begin{array}{c}-0.033 \\
(0.005)^{* * *}\end{array}$ & $\begin{array}{c}0.037 \\
(0.011)^{* *}\end{array}$ & $\begin{array}{c}-0.021 \\
(0.010)^{*}\end{array}$ & $\begin{array}{c}-0.023 \\
(0.007)^{\star *}\end{array}$ & $\begin{array}{c}-0.027 \\
(0.006)^{\star * * *}\end{array}$ & $\begin{array}{c}0.052 \\
(0.012)^{* * *}\end{array}$ & $\begin{array}{c}-0.047 \\
(0.004)^{* * * *}\end{array}$ & $\begin{array}{c}0.016 \\
(0.015)\end{array}$ & $\begin{array}{c}0.040 \\
(0.009)^{* * * *}\end{array}$ & $\begin{array}{c}-0.004 \\
(0.005)\end{array}$ & $\begin{array}{c}-0.054 \\
(0.006)^{* * *}\end{array}$ & $\begin{array}{c}0.042 \\
(0.005)^{* * * *}\end{array}$ \\
\hline Band member & $\begin{array}{c}0.017 \\
(0.009)\end{array}$ & $\begin{array}{c}-0.023 \\
(0.004)^{* * *}\end{array}$ & $\begin{array}{c}-0.025 \\
(0.007)^{* * *}\end{array}$ & $\begin{array}{l}-0.030 \\
(0.06)^{* * *}\end{array}$ & $\begin{array}{c}0.021 \\
(0.010)^{*}\end{array}$ & $\begin{array}{c}-0.032 \\
(0.005)^{\star \star * *}\end{array}$ & $\begin{array}{c}0.006 \\
(0.006)\end{array}$ & $\begin{array}{c}-0.013 \\
(0.004)^{\star \star}\end{array}$ & - & - & - & - \\
\hline Registered Indian & $\begin{array}{l}-0.006 \\
(0.009)\end{array}$ & $\begin{array}{c}-0.022 \\
(0.004)^{* * * *}\end{array}$ & $\begin{array}{c}-0.050 \\
(0.006)^{* * * *}\end{array}$ & $\begin{array}{c}0.016 \\
(0.007)^{\star}\end{array}$ & - & - & - & - & - & - & - & - \\
\hline Volunteering club membership & - & - & - & - & - & - & - & - & $\begin{array}{c}0.023 \\
(0.003)^{*+* * *}\end{array}$ & $\begin{array}{c}-0.009 \\
(0.003)^{* *}\end{array}$ & $\begin{array}{c}-0.038 \\
(0.006)^{* \pm *}\end{array}$ & $\begin{array}{l}0.007 \\
(0.006)\end{array}$ \\
\hline
\end{tabular}




\begin{tabular}{|c|c|c|c|c|c|c|c|c|c|c|c|c|}
\hline \multirow{2}{*}{$\begin{array}{l}\text { Appendix D1 } \\
\text { (continued) }\end{array}$} & \multicolumn{8}{|c|}{ North American Indian } & \multicolumn{2}{|c|}{ Métis } & \multicolumn{2}{|c|}{ Inuit } \\
\hline & Men & Women & Men & Women & Men & Women & Men & Women & Men & Women & Men & Women \\
\hline $\begin{array}{l}\text { Member in Métis/nuit } \\
\text { organizations }\end{array}$ & - & - & - & - & - & - & - & - & $\begin{array}{l}-0.004 \\
(0.004)\end{array}$ & $\begin{array}{c}-0.003 \\
(0.003)^{*+*}\end{array}$ & $\begin{array}{l}-0.047 \\
(0.008)^{* * \star}\end{array}$ & $\begin{array}{c}0.061 \\
(0.012)^{*+*+}\end{array}$ \\
\hline Voting, provincial & - & - & - & - & - & - & - & - & - & - & $\begin{array}{c}0.079 \\
(0.010)^{* \text { twt }}\end{array}$ & $\begin{array}{l}-0.072 \\
(0.007)^{* * * *}\end{array}$ \\
\hline Speaks Aboriginal languages & $\begin{array}{c}-0.060 \\
(0.007)^{* \star * *}\end{array}$ & $\begin{array}{l}-0.002 \\
(0.004)\end{array}$ & $\begin{array}{c}-0.064 \\
(0.007)^{\star \star \star \star}\end{array}$ & $\begin{array}{c}0.012 \\
(0.005)^{\star}\end{array}$ & $\begin{array}{c}-0.040 \\
(0.005)^{* * *}\end{array}$ & $\begin{array}{l}0.0006 \\
(0.004)\end{array}$ & $\begin{array}{c}-0.118 \\
(0.013)^{\star \star *}\end{array}$ & $\begin{array}{c}0.020 \\
(0.005)^{\star * *}\end{array}$ & $\begin{array}{c}0.016 \\
(0.004)^{\star \star \star \star}\end{array}$ & $\begin{array}{c}0.003 \\
(0.004)\end{array}$ & $\begin{array}{c}0.076 \\
(0.014)^{\star \star \star k}\end{array}$ & $\begin{array}{c}0.018 \\
(0.010)\end{array}$ \\
\hline Values Aboriginal languages & $\begin{array}{c}0.023 \\
(0.005)^{* * *}\end{array}$ & $\begin{array}{c}0.010 \\
(0.004)^{*}\end{array}$ & $\begin{array}{c}-0.050 \\
(0.005)^{* * *}\end{array}$ & $\begin{array}{c}0.036 \\
(0.005)^{* * * *}\end{array}$ & $\begin{array}{c}0.020 \\
(0.005)^{* * * *}\end{array}$ & $\begin{array}{c}0.025 \\
(0.005)^{\star \star \star *}\end{array}$ & $\begin{array}{l}-0.007 \\
(0.004)\end{array}$ & $\begin{array}{c}0.009 \\
(0.004)^{*}\end{array}$ & $\begin{array}{c}-0.018 \\
(0.003)^{* * *}\end{array}$ & $\begin{array}{c}-0.011 \\
(0.002)^{n * * *}\end{array}$ & $\begin{array}{c}-0.012 \\
(0.016)\end{array}$ & $\begin{array}{c}0.074 \\
(0.023)^{* *}\end{array}$ \\
\hline $\begin{array}{l}\text { Traditional pursuits } \\
\text { for pleasure }\end{array}$ & $\begin{array}{c}0.027 \\
(0.004)^{* * *}\end{array}$ & $\begin{array}{r}0.005 \\
(0.003) \\
\end{array}$ & $\begin{array}{c}-0.029 \\
(0.006)^{* * *}\end{array}$ & $\begin{array}{c}0.015 \\
(0.005)^{* *}\end{array}$ & $\begin{array}{r}0.009 \\
(0.005) \\
\end{array}$ & $\begin{array}{l}0.008 \\
(0.004) \\
\end{array}$ & $\begin{array}{c}0.041 \\
(0.005)^{* * *} \\
\end{array}$ & $\begin{array}{c}0.021 \\
(0.004)^{* \pm *}\end{array}$ & $\begin{array}{c}-0.028 \\
(0.003)^{*+*}\end{array}$ & $\begin{array}{c}0.024 \\
(0.003)^{* * t}\end{array}$ & $\begin{array}{l}-0.0006 \\
(0.007)\end{array}$ & $\begin{array}{r}-0.031 \\
(0.007)^{* * * *}\end{array}$ \\
\hline Number of observations & 1530 & 1690 & 900 & 940 & 1610 & 1800 & 820 & 830 & 2150 & 2250 & 710 & 700 \\
\hline
\end{tabular}

Source: Statistics Canada, 2006 Aboriginal Peoples Survey, analytical files: Adult Core, Métis Supplement and Arctic File. Bootstrapped (1000 replications) standard errors in parentheses. Statistical significance ${ }^{* * *} p<.001 ;{ }^{* *} p<.01 ;{ }^{*} p<.05$. 
Appendix E

Appendix E 1: Sample means of all explanatory variables in the probit employment models, Census 2006

\begin{tabular}{|c|c|c|c|c|c|c|c|c|}
\hline \multirow[t]{2}{*}{ Characteristics } & \multicolumn{2}{|c|}{$\begin{array}{c}\text { On-reserve } \\
\text { NAI }\end{array}$} & \multicolumn{2}{|c|}{$\begin{array}{c}\text { Urban off-reserve } \\
\text { NAI }\end{array}$} & \multicolumn{2}{|c|}{ Métis } & \multicolumn{2}{|c|}{ Inuit } \\
\hline & Men & Women & Men & Women & Men & Women & Men & Women \\
\hline $\begin{array}{l}\text { Highest accreditation attained } \\
\text { No education credentials }\end{array}$ & $\begin{array}{l}0.587 \\
(0.49)\end{array}$ & $\begin{array}{l}0.541 \\
(0.50)\end{array}$ & $\begin{array}{l}0.507 \\
(0.50)\end{array}$ & $\begin{array}{l}0.437 \\
(0.50)\end{array}$ & $\begin{array}{l}0.323 \\
(0.47)\end{array}$ & $\begin{array}{l}0.272 \\
(0.44)\end{array}$ & $\begin{array}{l}0.574 \\
(0.43)\end{array}$ & $\begin{array}{l}0.566 \\
(0.49)\end{array}$ \\
\hline $\begin{array}{l}\text { Completed } \\
\text { high school diploma }\end{array}$ & $\begin{array}{l}0.141 \\
(0.35)\end{array}$ & $\begin{array}{l}0.173 \\
(0.38)\end{array}$ & $\begin{array}{l}0.195 \\
(0.39)\end{array}$ & $\begin{array}{l}0.217 \\
(0.41)\end{array}$ & $\begin{array}{l}0.258 \\
(0.44)\end{array}$ & $\begin{array}{l}0.279 \\
(0.45)\end{array}$ & $\begin{array}{l}0.134 \\
(0.34)\end{array}$ & $\begin{array}{r}0.149 \\
(0.36)\end{array}$ \\
\hline $\begin{array}{l}\text { Completed } \\
\text { other trades diploma }\end{array}$ & $\begin{array}{l}0.112 \\
(0.31)\end{array}$ & $\begin{array}{l}0.058 \\
(0.23)\end{array}$ & $\begin{array}{l}0.092 \\
(0.29)\end{array}$ & $\begin{array}{l}0.061 \\
(0.24)\end{array}$ & $\begin{array}{l}0.110 \\
(0.31)\end{array}$ & $\begin{array}{l}0.088 \\
(0.28)\end{array}$ & $\begin{array}{l}0.102 \\
(0.30)\end{array}$ & $\begin{array}{l}0.056 \\
(0.23)\end{array}$ \\
\hline $\begin{array}{l}\text { Completed } \\
\text { apprenticeship certificate }\end{array}$ & $\begin{array}{l}0.033 \\
(0.18)\end{array}$ & $\begin{array}{l}0.013 \\
(0.11)\end{array}$ & $\begin{array}{l}0.041 \\
(0.20)\end{array}$ & $\begin{array}{l}0.014 \\
(0.12)\end{array}$ & $\begin{array}{l}0.076 \\
(0.26)\end{array}$ & $\begin{array}{l}0.021 \\
(0.14)\end{array}$ & $\begin{array}{l}0.043 \\
(0.20)\end{array}$ & $\begin{array}{l}0.018 \\
(0.13)\end{array}$ \\
\hline $\begin{array}{l}\text { Completed } \\
\text { college program, } 3-12 \text { months }\end{array}$ & $\begin{array}{l}0.017 \\
(0.13)\end{array}$ & $\begin{array}{l}0.029 \\
(0.17)\end{array}$ & $\begin{array}{l}0.018 \\
(0.13)\end{array}$ & $\begin{array}{l}0.037 \\
(0.19)\end{array}$ & $\begin{array}{l}0.022 \\
(0.15)\end{array}$ & $\begin{array}{l}0.062 \\
(0.24)\end{array}$ & $\begin{array}{l}0.024 \\
(0.15)\end{array}$ & $\begin{array}{l}0.042 \\
(0.20)\end{array}$ \\
\hline $\begin{array}{l}\text { Completed } \\
\text { college program, } 1-2 \text { years }\end{array}$ & $\begin{array}{l}0.041 \\
(0.20)\end{array}$ & $\begin{array}{l}0.069 \\
(0.25)\end{array}$ & $\begin{array}{l}0.053 \\
(0.11)\end{array}$ & $\begin{array}{l}0.085 \\
(0.28)\end{array}$ & $\begin{array}{l}0.084 \\
(0.28)\end{array}$ & $\begin{array}{l}0.124 \\
(0.33)\end{array}$ & $\begin{array}{l}0.054 \\
(0.22)\end{array}$ & $\begin{array}{l}0.079 \\
(0.27)\end{array}$ \\
\hline $\begin{array}{l}\text { Completed } \\
\text { college program, over } 2 \text { years }\end{array}$ & $\begin{array}{l}0.029 \\
(0.17)\end{array}$ & $\begin{array}{l}0.037 \\
(0.19)\end{array}$ & $\begin{array}{l}0.033 \\
(0.18)\end{array}$ & $\begin{array}{l}0.040 \\
(0.20)\end{array}$ & $\begin{array}{l}0.046 \\
(0.21)\end{array}$ & $\begin{array}{l}0.040 \\
(0.19)\end{array}$ & $\begin{array}{l}0.037 \\
(0.19)\end{array}$ & $\begin{array}{l}0.033 \\
(0.18)\end{array}$ \\
\hline $\begin{array}{l}\text { Completed } \\
\text { university certificate }\end{array}$ & $\begin{array}{l}0.020 \\
(0.14)\end{array}$ & $\begin{array}{l}0.034 \\
(0.18)\end{array}$ & $\begin{array}{l}0.022 \\
(0.15)\end{array}$ & $\begin{array}{l}0.037 \\
(0.19)\end{array}$ & $\begin{array}{l}0.021 \\
(0.14)\end{array}$ & $\begin{array}{l}0.032 \\
(0.18)\end{array}$ & $\begin{array}{l}0.011 \\
(0.10)\end{array}$ & $\begin{array}{l}0.019 \\
(0.14)\end{array}$ \\
\hline $\begin{array}{l}\text { Completed } \\
\text { university degree }\end{array}$ & $\begin{array}{l}0.020 \\
(0.14)\end{array}$ & $\begin{array}{l}0.045 \\
(0.21)\end{array}$ & $\begin{array}{l}0.038 \\
(0.19)\end{array}$ & $\begin{array}{l}0.070 \\
(0.25)\end{array}$ & $\begin{array}{l}0.060 \\
(0.24)\end{array}$ & $\begin{array}{l}0.081 \\
(0.27)\end{array}$ & $\begin{array}{l}0.020 \\
(0.14)\end{array}$ & $\begin{array}{l}0.037 \\
(0.19)\end{array}$ \\
\hline \multicolumn{9}{|l|}{$\begin{array}{l}\text { Certification and } \\
\text { accreditation }\end{array}$} \\
\hline $\begin{array}{l}\text { No high school diploma and } \\
\text { no further schooling }\end{array}$ & $\begin{array}{l}0.587 \\
(0.49)\end{array}$ & $\begin{array}{l}0.541 \\
(0.50)\end{array}$ & $\begin{array}{l}0.507 \\
(0.50)\end{array}$ & $\begin{array}{l}0.437 \\
(0.50)\end{array}$ & $\begin{array}{l}0.323 \\
(0.47)\end{array}$ & $\begin{array}{l}0.272 \\
(0.44)\end{array}$ & $\begin{array}{l}0.574 \\
(0.49)\end{array}$ & $\begin{array}{l}0.566 \\
(0.49)\end{array}$ \\
\hline $\begin{array}{l}\text { Registered apprenticeship or } \\
\text { other trade certificate, without } \\
\text { high school diploma }\end{array}$ & $\begin{array}{l}0.084 \\
(0.28)\end{array}$ & $\begin{array}{l}0.031 \\
(0.17)\end{array}$ & $\begin{array}{l}0.058 \\
(0.23)\end{array}$ & $\begin{array}{l}0.024 \\
(0.15)\end{array}$ & $\begin{array}{l}0.064 \\
(0.24)\end{array}$ & $\begin{array}{l}0.029 \\
(0.17)\end{array}$ & $\begin{array}{l}0.093 \\
(0.29)\end{array}$ & $\begin{array}{l}0.042 \\
(0.20)\end{array}$ \\
\hline $\begin{array}{l}\text { College certificate, without high } \\
\text { school diploma }\end{array}$ & $\begin{array}{l}0.017 \\
(0.13)\end{array}$ & $\begin{array}{l}0.027 \\
(0.16)\end{array}$ & $\begin{array}{l}0.014 \\
(0.12)\end{array}$ & $\begin{array}{l}0.023 \\
(0.15)\end{array}$ & $\begin{array}{l}0.014 \\
(0.12)\end{array}$ & $\begin{array}{l}0.021 \\
(0.14)\end{array}$ & $\begin{array}{l}0.030 \\
(0.17)\end{array}$ & $\begin{array}{l}0.049 \\
(0.22)\end{array}$ \\
\hline $\begin{array}{l}\text { High school diploma and } \\
\text { no further schooling }\end{array}$ & $\begin{array}{l}0.141 \\
(0.35)\end{array}$ & $\begin{array}{l}0.173 \\
(0.38)\end{array}$ & $\begin{array}{l}0.195 \\
(0.39)\end{array}$ & $\begin{array}{l}0.217 \\
(0.41)\end{array}$ & $\begin{array}{l}0.258 \\
(0.44)\end{array}$ & $\begin{array}{l}0.279 \\
(0.45)\end{array}$ & $\begin{array}{l}0.134 \\
(0.34)\end{array}$ & $\begin{array}{l}0.149 \\
(0.36)\end{array}$ \\
\hline $\begin{array}{l}\text { High school diploma and } \\
\text { registered apprenticeship or } \\
\text { other trade certificate }\end{array}$ & $\begin{array}{l}0.061 \\
(0.24)\end{array}$ & $\begin{array}{l}0.039 \\
(0.19)\end{array}$ & $\begin{array}{l}0.075 \\
(0.26)\end{array}$ & $\begin{array}{l}0.051 \\
(0.22)\end{array}$ & $\begin{array}{l}0.122 \\
(0.33)\end{array}$ & $\begin{array}{l}0.080 \\
(0.27)\end{array}$ & $\begin{array}{l}0.051 \\
(0.22)\end{array}$ & $\begin{array}{l}0.032 \\
(0.18)\end{array}$ \\
\hline $\begin{array}{l}\text { High school diploma and } \\
\text { college certificate }\end{array}$ & $\begin{array}{l}0.070 \\
(0.25)\end{array}$ & $\begin{array}{l}0.109 \\
(0.31)\end{array}$ & $\begin{array}{l}0.090 \\
(0.29)\end{array}$ & $\begin{array}{l}0.140 \\
(0.35)\end{array}$ & $\begin{array}{l}0.138 \\
(0.34)\end{array}$ & $\begin{array}{l}0.204 \\
(0.40)\end{array}$ & $\begin{array}{l}0.085 \\
(0.28)\end{array}$ & $\begin{array}{l}0.105 \\
(0.31)\end{array}$ \\
\hline $\begin{array}{l}\text { High school diploma and } \\
\text { university certificate }\end{array}$ & $\begin{array}{l}0.020 \\
(0.14)\end{array}$ & $\begin{array}{l}0.034 \\
(0.18)\end{array}$ & $\begin{array}{l}0.022 \\
(0.15)\end{array}$ & $\begin{array}{l}0.037 \\
(0.19)\end{array}$ & $\begin{array}{l}0.021 \\
(0.14)\end{array}$ & $\begin{array}{l}0.032 \\
(0.18)\end{array}$ & $\begin{array}{l}0.011 \\
(0.10)\end{array}$ & $\begin{array}{l}0.193 \\
(0.14)\end{array}$ \\
\hline \multirow[t]{2}{*}{$\begin{array}{l}\text { High school diploma and } \\
\text { university degree }\end{array}$} & $\begin{array}{l}0.019 \\
(0.14)\end{array}$ & $\begin{array}{l}0.045 \\
(0.21)\end{array}$ & $\begin{array}{l}0.038 \\
(0.19)\end{array}$ & $\begin{array}{l}0.070 \\
(0.25)\end{array}$ & $\begin{array}{l}0.060 \\
(0.24)\end{array}$ & $\begin{array}{l}0.081 \\
(0.27)\end{array}$ & $\begin{array}{l}0.020 \\
(0.14)\end{array}$ & $\begin{array}{l}0.037 \\
(0.19)\end{array}$ \\
\hline & & & & & & & & ...continue \\
\hline
\end{tabular}




\begin{tabular}{|c|c|c|c|c|c|c|c|c|}
\hline \multirow{2}{*}{$\begin{array}{l}\text { Appendix E1 } \\
\text { (continued) }\end{array}$} & \multicolumn{2}{|c|}{$\begin{array}{c}\text { On-reserve } \\
\text { NAl }\end{array}$} & \multicolumn{2}{|c|}{$\begin{array}{c}\text { Urban off-reserve } \\
\text { NAl }\end{array}$} & \multicolumn{2}{|c|}{ Métis } & \multicolumn{2}{|c|}{ Inuit } \\
\hline & Men & Women & Men & Women & Men & Women & Men & Women \\
\hline Age $15-19$ & $\begin{array}{l}0.078 \\
(0.27)\end{array}$ & $\begin{array}{l}0.076 \\
(0.26)\end{array}$ & $\begin{array}{l}0.183 \\
(0.39)\end{array}$ & $\begin{array}{l}0.156 \\
(0.36)\end{array}$ & $\begin{array}{l}0.050 \\
(0.22)\end{array}$ & $\begin{array}{l}0.048 \\
(0.21)\end{array}$ & $\begin{array}{l}0.089 \\
(0.28)\end{array}$ & $\begin{array}{l}0.082 \\
(0.27)\end{array}$ \\
\hline Age $20-24$ & $\begin{array}{l}0.126 \\
(0.33)\end{array}$ & $\begin{array}{l}0.122 \\
(0.33)\end{array}$ & $\begin{array}{c}0.128 \\
(0.33)\end{array}$ & $\begin{array}{l}0.124 \\
(0.33)\end{array}$ & $\begin{array}{c}0.100 \\
(0.30)\end{array}$ & $\begin{array}{l}0.093 \\
(0.29)\end{array}$ & $\begin{array}{l}0.151 \\
(0.36)\end{array}$ & $\begin{array}{l}0.139 \\
(0.35)\end{array}$ \\
\hline Age $25-34$ & $\begin{array}{l}0.231 \\
(0.42)\end{array}$ & $\begin{array}{l}0.235 \\
(0.42)\end{array}$ & $\begin{array}{l}0.211 \\
(0.41)\end{array}$ & $\begin{array}{l}0.221 \\
(0.41)\end{array}$ & $\begin{array}{l}0.218 \\
(0.41)\end{array}$ & $\begin{array}{l}0.217 \\
(0.41)\end{array}$ & $\begin{array}{l}0.253 \\
(0.43)\end{array}$ & $\begin{array}{l}0.263 \\
(0.44)\end{array}$ \\
\hline Age $35-44$ & $\begin{array}{c}0.250 \\
(0.43)\end{array}$ & $\begin{array}{l}0.252 \\
(0.43)\end{array}$ & $\begin{array}{c}0.215 \\
(0.41)\end{array}$ & $\begin{array}{l}0.223 \\
(0.42)\end{array}$ & $\begin{array}{c}0.241 \\
(0.43)\end{array}$ & $\begin{array}{l}0.259 \\
(0.44)\end{array}$ & $\begin{array}{l}0.251 \\
(0.43)\end{array}$ & $\begin{array}{l}0.253 \\
(0.43)\end{array}$ \\
\hline Age 45-54 & $\begin{array}{l}0.195 \\
(0.40)\end{array}$ & $\begin{array}{c}0.194 \\
(0.39)\end{array}$ & $\begin{array}{l}0.167 \\
(0.37)\end{array}$ & $\begin{array}{l}0.177 \\
(0.38)\end{array}$ & $\begin{array}{l}0.241 \\
(0.43)\end{array}$ & $\begin{array}{l}0.247 \\
(0.43)\end{array}$ & $\begin{array}{l}0.159 \\
(0.36)\end{array}$ & $\begin{array}{l}0.173 \\
(0.38)\end{array}$ \\
\hline Age $55-64$ & $\begin{array}{l}0.120 \\
(0.32)\end{array}$ & $\begin{array}{l}0.120 \\
(0.32)\end{array}$ & $\begin{array}{l}0.094 \\
(0.29)\end{array}$ & $\begin{array}{l}0.098 \\
(0.30)\end{array}$ & $\begin{array}{l}0.150 \\
(0.36)\end{array}$ & $\begin{array}{l}0.135 \\
(0.34)\end{array}$ & $\begin{array}{l}0.097 \\
(0.30)\end{array}$ & $\begin{array}{l}0.091 \\
(0.29)\end{array}$ \\
\hline Atlantic provinces & $\begin{array}{c}0.052 \\
(0.22)\end{array}$ & $\begin{array}{l}0.057 \\
(0.23)\end{array}$ & $\begin{array}{l}0.053 \\
(0.22)\end{array}$ & $\begin{array}{l}0.055 \\
(0.23)\end{array}$ & $\begin{array}{l}0.056 \\
(0.23)\end{array}$ & $\begin{array}{l}0.049 \\
(0.22)\end{array}$ & $\begin{array}{l}0.124 \\
(0.33)\end{array}$ & $\begin{array}{l}0.121 \\
(0.33)\end{array}$ \\
\hline Other provinces & - & - & - & - & - & - & $\begin{array}{l}0.075 \\
(0.26)\end{array}$ & $\begin{array}{l}0.092 \\
(0.29)\end{array}$ \\
\hline Northwest Territories & - & - & - & - & - & - & $\begin{array}{l}0.097 \\
(0.30)\end{array}$ & $\begin{array}{l}0.111 \\
(0.31)\end{array}$ \\
\hline Nunavut & - & - & - & - & - & - & $\begin{array}{l}0.479 \\
(0.50)\end{array}$ & $\begin{array}{l}0.461 \\
(0.50)\end{array}$ \\
\hline Northern territories & $\begin{array}{l}0.037 \\
(0.19)\end{array}$ & $\begin{array}{l}0.036 \\
(0.18)\end{array}$ & $\begin{array}{l}0.275 \\
(0.16)\end{array}$ & $\begin{array}{l}0.026 \\
(0.16)\end{array}$ & $\begin{array}{l}0.012 \\
(0.11)\end{array}$ & $\begin{array}{l}0.011 \\
(0.10)\end{array}$ & - & - \\
\hline Quebec & $\begin{array}{l}0.113 \\
(0.32)\end{array}$ & $\begin{array}{l}0.119 \\
(0.32)\end{array}$ & $\begin{array}{l}0.101 \\
(0.30)\end{array}$ & $\begin{array}{l}0.097 \\
(0.30)\end{array}$ & $\begin{array}{c}0.08 \\
(0.27)\end{array}$ & $\begin{array}{l}0.070 \\
(0.25)\end{array}$ & $\begin{array}{l}0.224 \\
(0.42)\end{array}$ & $\begin{array}{l}0.214 \\
(0.41)\end{array}$ \\
\hline Ontario & $\begin{array}{l}0.163 \\
(0.37)\end{array}$ & $\begin{array}{r}0.163 \\
(0.37)\end{array}$ & $\begin{array}{l}0.231 \\
(0.42)\end{array}$ & $\begin{array}{l}0.234 \\
(0.42)\end{array}$ & $\begin{array}{l}0.202 \\
(0.40)\end{array}$ & $\begin{array}{l}0.196 \\
(0.40)\end{array}$ & - & - \\
\hline Manitoba & $\begin{array}{l}0.181 \\
(0.38)\end{array}$ & $\begin{array}{l}0.179 \\
(0.38)\end{array}$ & $\begin{array}{c}0.133 \\
(0.34)\end{array}$ & $\begin{array}{l}0.136 \\
(0.34)\end{array}$ & $\begin{array}{c}0.177 \\
(0.38)\end{array}$ & $\begin{array}{c}0.177 \\
(0.38)\end{array}$ & - & - \\
\hline Saskatchewan & $\begin{array}{l}0.145 \\
(0.35)\end{array}$ & $\begin{array}{c}0.147 \\
(0.35)\end{array}$ & $\begin{array}{l}0.119 \\
(0.32)\end{array}$ & $\begin{array}{l}0.120 \\
(0.32)\end{array}$ & $\begin{array}{c}0.117 \\
(0.32)\end{array}$ & $\begin{array}{c}0.115 \\
(0.32)\end{array}$ & - & - \\
\hline Alberta & $\begin{array}{c}0.125 \\
(0.33)\end{array}$ & $\begin{array}{l}0.127 \\
(0.33)\end{array}$ & $\begin{array}{l}0.136 \\
(0.34\end{array}$ & $\begin{array}{l}0.138 \\
(0.34)\end{array}$ & $\begin{array}{c}0.214 \\
(0.41)\end{array}$ & $\begin{array}{l}0.224 \\
(0.42)\end{array}$ & - & - \\
\hline British Columbia & $\begin{array}{r}0.183 \\
(0.39)\end{array}$ & $\begin{array}{r}0.170 \\
(0.37)\end{array}$ & $\begin{array}{c}0.198 \\
(0.40)\end{array}$ & $\begin{array}{l}0.192 \\
(0.39)\end{array}$ & $\begin{array}{l}0.143 \\
(0.35)\end{array}$ & $\begin{array}{c}0.156 \\
(0.36)\end{array}$ & - & - \\
\hline English only & $\begin{array}{c}0.913 \\
(0.28)\end{array}$ & $\begin{array}{c}0.908 \\
(0.29)\end{array}$ & $\begin{array}{l}0.882 \\
(0.32)\end{array}$ & $\begin{array}{l}0.877 \\
(0.33)\end{array}$ & $\begin{array}{l}0.771 \\
(0.42)\end{array}$ & $\begin{array}{l}0.778 \\
(0.41)\end{array}$ & $\begin{array}{l}0.890 \\
(0.31)\end{array}$ & $\begin{array}{c}0.877 \\
(0.33)\end{array}$ \\
\hline French only & $\begin{array}{l}0.047 \\
(0.21)\end{array}$ & $\begin{array}{l}0.052 \\
(0.22)\end{array}$ & $\begin{array}{l}0.042 \\
(0.20)\end{array}$ & $\begin{array}{c}0.043 \\
(0.20)\end{array}$ & $\begin{array}{l}0.042 \\
(0.20)\end{array}$ & $\begin{array}{l}0.0387 \\
(0.19)\end{array}$ & $\begin{array}{l}0.019 \\
(0.13)\end{array}$ & $\begin{array}{r}0.018 \\
(0.13)\end{array}$ \\
\hline Both official languages & $\begin{array}{l}0.033 \\
(0.18)\end{array}$ & $\begin{array}{l}0.032 \\
(0.17)\end{array}$ & $\begin{array}{c}0.073 \\
(0.26)\end{array}$ & $\begin{array}{l}0.077 \\
(0.27)\end{array}$ & $\begin{array}{l}0.186 \\
(0.39)\end{array}$ & $\begin{array}{l}0.183 \\
(0.39)\end{array}$ & $\begin{array}{l}0.059 \\
(0.23)\end{array}$ & $\begin{array}{c}0.064 \\
(0.24)\end{array}$ \\
\hline None official language & $\begin{array}{l}0.007 \\
(0.08)\end{array}$ & $\begin{array}{l}0.008 \\
(0.09)\end{array}$ & $\begin{array}{l}0.003 \\
(0.06)\end{array}$ & $\begin{array}{l}0.003 \\
(0.05)\end{array}$ & - & 一 & $\begin{array}{l}0.033 \\
(0.18)\end{array}$ & $\begin{array}{l}0.041 \\
(0.20)\end{array}$ \\
\hline
\end{tabular}




\begin{tabular}{|c|c|c|c|c|c|c|c|c|}
\hline \multirow{2}{*}{$\begin{array}{l}\text { Appendix E1 } \\
\text { (continued) }\end{array}$} & \multicolumn{2}{|c|}{$\begin{array}{c}\text { On-reserve } \\
\text { NAI }\end{array}$} & \multicolumn{2}{|c|}{$\begin{array}{c}\text { Urban off-reserve } \\
\text { NAl }\end{array}$} & \multicolumn{2}{|c|}{ Métis } & \multicolumn{2}{|c|}{ Inuit } \\
\hline & Men & Women & Men & Women & Men & Women & Men & Women \\
\hline Legally married & $\begin{array}{l}0.293 \\
(0.45)\end{array}$ & $\begin{array}{l}0.320 \\
(0.47)\end{array}$ & $\begin{array}{l}0.249 \\
(0.43)\end{array}$ & $\begin{array}{l}0.260 \\
(0.44)\end{array}$ & $\begin{array}{l}0.413 \\
(0.49)\end{array}$ & $\begin{array}{l}0.424 \\
(0.49)\end{array}$ & $\begin{array}{l}0.301 \\
(0.46)\end{array}$ & $\begin{array}{l}0.327 \\
(0.47)\end{array}$ \\
\hline Single & $\begin{array}{l}0.612 \\
(0.49)\end{array}$ & $\begin{array}{l}0.551 \\
(0.50)\end{array}$ & $\begin{array}{l}0.652 \\
(0.48)\end{array}$ & $\begin{array}{l}0.584 \\
(0.49)\end{array}$ & $\begin{array}{l}0.448 \\
(0.50)\end{array}$ & $\begin{array}{l}0.378 \\
(0.48)\end{array}$ & $\begin{array}{l}0.642 \\
(0.48)\end{array}$ & $\begin{array}{l}0.568 \\
(0.49)\end{array}$ \\
\hline Divorced-separated & $\begin{array}{l}0.094 \\
(0.29)\end{array}$ & $\begin{array}{l}0.129 \\
(0.33)\end{array}$ & $\begin{array}{c}0.99 \\
(0.30)\end{array}$ & $\begin{array}{l}0.155 \\
(0.36)\end{array}$ & $\begin{array}{l}0.139 \\
(0.35)\end{array}$ & $\begin{array}{l}0.198 \\
(0.40)\end{array}$ & $\begin{array}{l}0.057 \\
(0.23)\end{array}$ & $\begin{array}{l}0.105 \\
(0.31)\end{array}$ \\
\hline No children present & $\begin{array}{l}0.524 \\
(0.50)\end{array}$ & $\begin{array}{l}0.358 \\
(0.48)\end{array}$ & $\begin{array}{l}0.633 \\
(0.48)\end{array}$ & $\begin{array}{l}0.483 \\
(0.50)\end{array}$ & $\begin{array}{l}0.612 \\
(0.49)\end{array}$ & $\begin{array}{l}0.496 \\
(0.50)\end{array}$ & $\begin{array}{l}0.492 \\
(0.50)\end{array}$ & $\begin{array}{l}0.337 \\
(0.47)\end{array}$ \\
\hline Children $<6$ & $\begin{array}{l}0.203 \\
(0.40)\end{array}$ & $\begin{array}{l}0.273 \\
(0.44)\end{array}$ & $\begin{array}{l}0.156 \\
(0.36)\end{array}$ & $\begin{array}{l}0.217 \\
(0.41)\end{array}$ & $\begin{array}{l}0.130 \\
(0.34)\end{array}$ & $\begin{array}{l}0.169 \\
(0.37)\end{array}$ & $\begin{array}{l}0.258 \\
(0.44)\end{array}$ & $\begin{array}{l}0.321 \\
(0.47)\end{array}$ \\
\hline Children 6-14 & $\begin{array}{l}0.164 \\
(0.37)\end{array}$ & $\begin{array}{l}0.215 \\
(0.41)\end{array}$ & $\begin{array}{l}0.126 \\
(0.33)\end{array}$ & $\begin{array}{l}0.178 \\
(0.38)\end{array}$ & $\begin{array}{l}0.134 \\
(0.34)\end{array}$ & $\begin{array}{l}0.179 \\
(0.38)\end{array}$ & $\begin{array}{l}0.170 \\
(0.38)\end{array}$ & $\begin{array}{l}0.229 \\
(0.42)\end{array}$ \\
\hline Children $14-24$ & $\begin{array}{r}0.109 \\
(0.31)\end{array}$ & $\begin{array}{l}0.154 \\
(0.36)\end{array}$ & $\begin{array}{l}0.085 \\
(0.28)\end{array}$ & $\begin{array}{r}0.122 \\
(0.33)\end{array}$ & $\begin{array}{l}0.124 \\
(0.33)\end{array}$ & $\begin{array}{c}0.157 \\
(0.36)\end{array}$ & $\begin{array}{l}0.079 \\
(0.27)\end{array}$ & $\begin{array}{l}0.113 \\
(0.32)\end{array}$ \\
\hline Lone parent & $\begin{array}{l}0.227 \\
(0.42)\end{array}$ & $\begin{array}{l}0.312 \\
(0.46)\end{array}$ & $\begin{array}{l}0.237 \\
(0.42)\end{array}$ & $\begin{array}{l}0.338 \\
(0.47)\end{array}$ & $\begin{array}{l}0.122 \\
(0.33)\end{array}$ & $\begin{array}{l}0.223 \\
(0.42)\end{array}$ & $\begin{array}{l}0.176 \\
(0.38)\end{array}$ & $\begin{array}{l}0.251 \\
(0.43)\end{array}$ \\
\hline Health limitation & $\begin{array}{c}0.120 \\
(0.32)\end{array}$ & $\begin{array}{l}0.130 \\
(0.33)\end{array}$ & $\begin{array}{l}0.136 \\
(0.34)\end{array}$ & $\begin{array}{l}0.148 \\
(0.35)\end{array}$ & $\begin{array}{l}0.178 \\
(0.38)\end{array}$ & $\begin{array}{c}0.177 \\
(0.38)\end{array}$ & $\begin{array}{c}0.128 \\
(0.33)\end{array}$ & $\begin{array}{c}0.124 \\
(0.33)\end{array}$ \\
\hline Household maintainers, one & $\begin{array}{c}0.678 \\
(0.47)\end{array}$ & $\begin{array}{c}0.682 \\
(0.46)\end{array}$ & $\begin{array}{l}0.625 \\
(0.48)\end{array}$ & $\begin{array}{l}0.646 \\
(0.48)\end{array}$ & $\begin{array}{l}0.520 \\
(0.50)\end{array}$ & $\begin{array}{c}0.561 \\
(0.50)\end{array}$ & $\begin{array}{l}0.650 \\
(0.48)\end{array}$ & $\begin{array}{c}0.662 \\
(0.47)\end{array}$ \\
\hline Household maintainers, two + & $\begin{array}{l}0.322 \\
(0.47)\end{array}$ & $\begin{array}{l}0.318 \\
(0.46)\end{array}$ & $\begin{array}{l}0.375 \\
(0.48)\end{array}$ & $\begin{array}{l}0.353 \\
(0.48)\end{array}$ & $\begin{array}{l}0.479 \\
(0.50)\end{array}$ & $\begin{array}{c}0.438 \\
(0.50)\end{array}$ & $\begin{array}{l}0.350 \\
(0.48)\end{array}$ & $\begin{array}{l}0.338 \\
(0.47)\end{array}$ \\
\hline Respondent maintainer & $\begin{array}{l}0.880 \\
(0.32)\end{array}$ & $\begin{array}{l}0.884 \\
(0.32)\end{array}$ & $\begin{array}{l}0.892 \\
(0.31)\end{array}$ & $\begin{array}{c}0.904 \\
(0.29)\end{array}$ & $\begin{array}{l}0.915 \\
(0.28)\end{array}$ & $\begin{array}{l}0.920 \\
(0.27)\end{array}$ & $\begin{array}{l}0.818 \\
(0.39)\end{array}$ & $\begin{array}{c}0.836 \\
(0.37)\end{array}$ \\
\hline Weighted counts & 74290 & 68900 & 200090 & 222580 & 104630 & 103800 & 11940 & 12320 \\
\hline
\end{tabular}

Source: Statistics Canada, 2006 Census analytical file. Individual composite weights in effect. Standard deviations in parentheses. 
Appendix E 2: Detailed results from robustness checks for educational credentials, Census 2006

\begin{tabular}{|c|c|c|c|c|c|c|c|c|}
\hline \multirow{3}{*}{$\begin{array}{l}\text { Highest accreditation } \\
\text { attained }\end{array}$} & \multicolumn{8}{|c|}{ Average Marginal Effects } \\
\hline & \multicolumn{2}{|c|}{$\begin{array}{c}\text { On-reserve } \\
\text { NAI }\end{array}$} & \multicolumn{2}{|c|}{$\begin{array}{c}\text { Urban off-reserve } \\
\text { NAI }\end{array}$} & \multicolumn{2}{|c|}{ Mètis } & \multicolumn{2}{|c|}{ Inuit } \\
\hline & Men & Women & Men & Women & Men & Women & Men & Women \\
\hline No education credentials & $\begin{array}{c}-0.100 \\
(0.009)^{* * *}\end{array}$ & $\begin{array}{c}-0.091 \\
(0.007)^{\star \star \star \star}\end{array}$ & $\begin{array}{c}-0.0987 \\
(0.009)^{* * *}\end{array}$ & $\begin{array}{c}-0.082 \\
(0.008)^{\star \star * *}\end{array}$ & $\begin{array}{c}-0.056 \\
(0.008)^{\star \star *}\end{array}$ & $\begin{array}{c}-0.050 \\
(0.008)^{* * *}\end{array}$ & $\begin{array}{c}-0.135 \\
(0.021)^{\star * *}\end{array}$ & $\begin{array}{c}-0.104 \\
(0.017)^{\star \star \star \star}\end{array}$ \\
\hline $\begin{array}{l}\text { Completed } \\
\text { other trades diploma }\end{array}$ & $\begin{array}{c}-0.053 \\
(0.012)^{* * *}\end{array}$ & $\begin{array}{c}-0.006 \\
(0.011)\end{array}$ & $\begin{array}{c}-0.066 \\
(0.010)^{* * *}\end{array}$ & $\begin{array}{l}-0.011 \\
(0.010)\end{array}$ & $\begin{array}{c}-0.022 \\
(0.011)^{*}\end{array}$ & $\begin{array}{c}0.010 \\
(0.010)\end{array}$ & $\begin{array}{c}-0.096 \\
(0.028)^{\star \star}\end{array}$ & $\begin{array}{l}-0.065 \\
(0.039)\end{array}$ \\
\hline $\begin{array}{l}\text { Completed } \\
\text { apprenticeship certificate }\end{array}$ & $\begin{array}{c}-0.041 \\
(0.015)^{* *}\end{array}$ & $\begin{array}{c}-0.007 \\
(0.020)\end{array}$ & $\begin{array}{c}-0.004 \\
(0.013)\end{array}$ & $\begin{array}{l}-0.008 \\
(0.024)\end{array}$ & $\begin{array}{c}0.002 \\
(0.012)\end{array}$ & $\begin{array}{c}0.011 \\
(0.020)\end{array}$ & $\begin{array}{l}-0.048 \\
(0.038)\end{array}$ & $\begin{array}{r}-0.050 \\
(0.059)\end{array}$ \\
\hline $\begin{array}{l}\text { Completed } \\
\text { college program, 3-12 } \\
\text { months }\end{array}$ & $\begin{array}{r}-0.0001 \\
(0.020)\end{array}$ & $\begin{array}{c}0.034 \\
(0.013)^{* *}\end{array}$ & $\begin{array}{r}-0.030 \\
(0.017)\end{array}$ & $\begin{array}{c}0.011 \\
(0.011)\end{array}$ & $\begin{array}{c}0.009 \\
(0.018)\end{array}$ & $\begin{array}{c}0.002 \\
(0.010)\end{array}$ & $\begin{array}{l}-0.059 \\
(0.055)\end{array}$ & $\begin{array}{c}-0.025 \\
(0.029)\end{array}$ \\
\hline $\begin{array}{l}\text { Completed } \\
\text { college program, 1-2 } \\
\text { years }\end{array}$ & $\begin{array}{c}0.051 \\
(0.012)^{\star \star \star}\end{array}$ & $\begin{array}{c}0.059 \\
(0.009)^{* * *}\end{array}$ & $\begin{array}{c}0.014 \\
(0.012)\end{array}$ & $\begin{array}{c}0.025 \\
(0.009)^{\star \star}\end{array}$ & $\begin{array}{l}0.0015 \\
(0.010)\end{array}$ & $\begin{array}{c}0.020 \\
(0.011)\end{array}$ & $\begin{array}{c}0.048 \\
(0.048)\end{array}$ & $\begin{array}{c}0.026 \\
(0.029)\end{array}$ \\
\hline $\begin{array}{l}\text { Completed } \\
\text { college program, over } 2 \\
\text { years }\end{array}$ & $\begin{array}{c}0.078 \\
(0.015)^{\star \star \star}\end{array}$ & $\begin{array}{c}0.055 \\
(0.010)^{\star \star \star}\end{array}$ & $\begin{array}{c}0.029 \\
(0.013)^{\star}\end{array}$ & $\begin{array}{c}0.037 \\
(0.010)^{\star \star \star}\end{array}$ & $\begin{array}{c}0.034 \\
(0.010)^{\star *}\end{array}$ & $\begin{array}{c}0.041 \\
(0.010)^{\star * *}\end{array}$ & $\begin{array}{l}-0.034 \\
(0.053)\end{array}$ & $\begin{array}{c}0.004 \\
(0.034)\end{array}$ \\
\hline $\begin{array}{l}\text { Completed } \\
\text { university certificate }\end{array}$ & $\begin{array}{c}0.065 \\
(0.019)^{\star \star}\end{array}$ & $\begin{array}{c}0.058 \\
(0.011)^{\star \star \star}\end{array}$ & $\begin{array}{c}0.024 \\
(0.016)\end{array}$ & $\begin{array}{c}0.015 \\
(0.012)\end{array}$ & $\begin{array}{c}0.023 \\
(0.018)\end{array}$ & $\begin{array}{c}0.013 \\
(0.014)\end{array}$ & $\begin{array}{l}-0.006 \\
(0.063)\end{array}$ & $\begin{array}{c}0.008 \\
(0.045)\end{array}$ \\
\hline $\begin{array}{l}\text { Completed } \\
\text { university degree }\end{array}$ & $\begin{array}{c}0.134 \\
(0.017)^{\star \star *}\end{array}$ & $\begin{array}{c}0.105 \\
(0.010)^{\star \star \star \star}\end{array}$ & $\begin{array}{c}0.078 \\
(0.012)^{\star * \star}\end{array}$ & $\begin{array}{c}0.062 \\
(0.009)^{* * *}\end{array}$ & $\begin{array}{c}0.058 \\
(0.009)^{* * *}\end{array}$ & $\begin{array}{c}0.049 \\
(0.007)^{\star \star \star}\end{array}$ & $\begin{array}{c}0.102 \\
(0.053)\end{array}$ & $\begin{array}{c}0.081 \\
(0.024)^{\star *}\end{array}$ \\
\hline Age $15-19$ & $\begin{array}{c}-0.072 \\
(0.014)^{\star \star * *}\end{array}$ & $\begin{array}{c}-0.074 \\
(0.015)^{* \star * k}\end{array}$ & $\begin{array}{c}0.019 \\
(0.011)\end{array}$ & $\begin{array}{c}0.012 \\
(0.011)\end{array}$ & $\begin{array}{c}-0.031 \\
(0.018)\end{array}$ & $\begin{array}{l}-0.007 \\
(0.015)\end{array}$ & $\begin{array}{c}0.004 \\
(0.030)\end{array}$ & $\begin{array}{l}-0.026 \\
(0.029)\end{array}$ \\
\hline Age $25-34$ & $\begin{array}{c}0.048 \\
(0.008)^{\star * *}\end{array}$ & $\begin{array}{c}0.055 \\
(0.008)^{k \times *}\end{array}$ & $\begin{array}{c}0.031 \\
(0.009)^{* *}\end{array}$ & $\begin{array}{c}0.046 \\
(0.008)^{\star \star \star}\end{array}$ & $\begin{array}{c}0.017 \\
(0.009)\end{array}$ & $\begin{array}{c}0.012 \\
(0.009)\end{array}$ & $\begin{array}{c}0.045 \\
(0.016)^{* *}\end{array}$ & $\begin{array}{c}0.019 \\
(0.020)\end{array}$ \\
\hline Age $35-44$ & $\begin{array}{c}0.086 \\
(0.009)^{\star \star \star}\end{array}$ & $\begin{array}{c}0.098 \\
(0.008)^{\text {tk*}}\end{array}$ & $\begin{array}{c}0.049 \\
(0.009)^{\star \star \star}\end{array}$ & $\begin{array}{c}0.069 \\
(0.009)^{\star \star \star *}\end{array}$ & $\begin{array}{c}0.026 \\
(0.010)^{*}\end{array}$ & $\begin{array}{c}0.026 \\
(0.009)^{* *}\end{array}$ & $\begin{array}{c}0.058 \\
(0.018)^{\star \star}\end{array}$ & $\begin{array}{c}0.049 \\
(0.019)^{*}\end{array}$ \\
\hline Age $45-54$ & $\begin{array}{c}0.117 \\
(0.009)^{\star \star *}\end{array}$ & $\begin{array}{c}0.125 \\
(0.008)^{\star * * \star}\end{array}$ & $\begin{array}{c}0.067 \\
(0.010)^{\star \star \star}\end{array}$ & $\begin{array}{c}0.093 \\
(0.008)^{\star \star \star}\end{array}$ & $\begin{array}{c}0.027 \\
(0.011)\end{array}$ & $\begin{array}{c}0.022 \\
(0.010)^{\star}\end{array}$ & $\begin{array}{c}0.051 \\
(0.021)^{*}\end{array}$ & $\begin{array}{c}0.083 \\
(0.019)^{\star \star \star *}\end{array}$ \\
\hline Age $55-64$ & $\begin{array}{c}0.121 \\
(0.011)^{\star \star * k}\end{array}$ & $\begin{array}{c}0.146 \\
(0.009)^{\star \star \star}\end{array}$ & $\begin{array}{c}0.060 \\
(0.011)^{\star \star \star}\end{array}$ & $\begin{array}{c}0.100 \\
(0.010)^{\star \star \star}\end{array}$ & $\begin{array}{c}0.015 \\
(0.014)\end{array}$ & $\begin{array}{c}0.023 \\
(0.012)^{*}\end{array}$ & $\begin{array}{c}.088 \\
(0.023)^{\star \star \star}\end{array}$ & $\begin{array}{c}0.0126 \\
(0.016)^{* * * *}\end{array}$ \\
\hline Atlantic provinces & $\begin{array}{c}-0.132 \\
(0.037)^{\star \star \star}\end{array}$ & $\begin{array}{c}-0.103 \\
(0.024)^{* * *}\end{array}$ & $\begin{array}{c}-0.110 \\
(0.029)^{\star * *}\end{array}$ & $\begin{array}{c}-0.072 \\
(0.020)^{*}\end{array}$ & $\begin{array}{c}-0.094 \\
(0.030)^{* *}\end{array}$ & $\begin{array}{c}-0.095 \\
(0.023)^{\star \star *}\end{array}$ & $\begin{array}{c}-0.200 \\
(0.036)^{\star \star * \star}\end{array}$ & $\begin{array}{c}-0.107 \\
(0.053)^{*}\end{array}$ \\
\hline Other provinces & - & - & - & - & 一 & - & $\begin{array}{c}0.041 \\
(0.058)\end{array}$ & $\begin{array}{c}-0.125 \\
(0.057)^{\star}\end{array}$ \\
\hline Northwest Territories & - & - & - & - & - & - & $\begin{array}{c}-0.034 \\
(0.031)\end{array}$ & $\begin{array}{c}-0.088 \\
(0.042)^{*}\end{array}$ \\
\hline Nunavut & - & - & - & - & - & - & $\begin{array}{c}-0.036 \\
(0.020)\end{array}$ & $\begin{array}{c}-0.042 \\
(0.023)\end{array}$ \\
\hline Northern territories & $\begin{array}{c}-0.124 \\
(0.023)^{* * * *}\end{array}$ & $\begin{array}{l}-0.008 \\
(0.015)\end{array}$ & $\begin{array}{c}-0.126 \\
(0.018)^{* * * *}\end{array}$ & $\begin{array}{c}-0.037 \\
(0.013)^{*}\end{array}$ & $\begin{array}{l}-0.031 \\
(0.011)\end{array}$ & $\begin{array}{c}0.025 \\
(0.009)^{* *}\end{array}$ & - & - \\
\hline Quebec & $\begin{array}{c}-0.054 \\
(0.023)^{\star}\end{array}$ & $\begin{array}{c}0.049 \\
(0.021)^{\star}\end{array}$ & $\begin{array}{c}-0.087 \\
(0.019)^{\text {*** }}\end{array}$ & $\begin{array}{c}0.022 \\
(0.017)\end{array}$ & $\begin{array}{c}-0.032 \\
(0.023)\end{array}$ & $\begin{array}{c}-0.064 \\
(0.029)^{*}\end{array}$ & - & - \\
\hline Manitoba & $\begin{array}{c}-0.128 \\
(0.022)^{* * *}\end{array}$ & $\begin{array}{c}-0.060 \\
(0.016)^{\star * * *}\end{array}$ & $\begin{array}{c}-0.104 \\
(0.023)^{* * *}\end{array}$ & $\begin{array}{c}-0.033 \\
(0.013)^{\star}\end{array}$ & $\begin{array}{c}0.015 \\
(0.008)\end{array}$ & $\begin{array}{c}0.016 \\
(0.008)^{*}\end{array}$ & - & - \\
\hline Saskatchewan & $\begin{array}{c}-0.130 \\
(0.020)^{* * *}\end{array}$ & $\begin{array}{c}-0.071 \\
(0.016)^{\star * *}\end{array}$ & $\begin{array}{c}-0.099 \\
(0.019)^{* * *}\end{array}$ & $\begin{array}{c}0.070 \\
(0.013)^{\star \star *}\end{array}$ & $\begin{array}{l}-0.012 \\
(0.011)\end{array}$ & $\begin{array}{c}0.023 \\
(0.009)^{\star \star *}\end{array}$ & - & - \\
\hline
\end{tabular}




\begin{tabular}{|c|c|c|c|c|c|c|c|c|}
\hline \multirow{3}{*}{$\begin{array}{l}\text { Appendix E2 } \\
\text { (continued) }\end{array}$} & \multicolumn{8}{|c|}{ Average Marginal Effects } \\
\hline & \multicolumn{2}{|c|}{$\begin{array}{c}\text { On-reserve } \\
\text { NAI }\end{array}$} & \multicolumn{2}{|c|}{$\begin{array}{c}\text { Urban off-reserve } \\
\text { NAl }\end{array}$} & \multicolumn{2}{|c|}{ Métis } & \multicolumn{2}{|c|}{ Inuit } \\
\hline & Men & Women & Men & Women & Men & Women & Men & Women \\
\hline Alberta & $\begin{array}{c}-0.049 \\
(0.022)^{*}\end{array}$ & $\begin{array}{c}-0.067 \\
(0.019)^{* * * *}\end{array}$ & $\begin{array}{c}0.006 \\
(0.018)\end{array}$ & $\begin{array}{l}-0.002 \\
(0.012)\end{array}$ & $\begin{array}{c}0.042 \\
(0.009)^{+*+*}\end{array}$ & $\begin{array}{c}0.029 \\
(0.008)^{* * *}\end{array}$ & - & - \\
\hline British Columbia & $\begin{array}{c}-0.080 \\
(0.021)^{\star \star \star}\end{array}$ & $\begin{array}{c}-0.054 \\
(0.015)^{\star \star \star *}\end{array}$ & $\begin{array}{c}-0.030 \\
(0.014)^{*}\end{array}$ & $\begin{array}{c}-0.024 \\
(0.009)^{\text {** }}\end{array}$ & $\begin{array}{c}0.009 \\
(0.010)\end{array}$ & $\begin{array}{l}0.0087 \\
(0.010)\end{array}$ & - & - \\
\hline French only & $\begin{array}{l}-0.020 \\
(0.037)\end{array}$ & $\begin{array}{c}-0.119 \\
(0.041)^{* *}\end{array}$ & $\begin{array}{c}0.047 \\
(0.021)^{\star}\end{array}$ & $\begin{array}{l}-0.023 \\
(0.030)\end{array}$ & $\begin{array}{c}0.002 \\
(0.020)\end{array}$ & $\begin{array}{c}0.026 \\
(0.020)\end{array}$ & $\begin{array}{c}0.078 \\
(0.032)^{*}\end{array}$ & $\begin{array}{c}0.036 \\
(0.027)\end{array}$ \\
\hline Both official languages & $\begin{array}{c}0.012 \\
(0.014)\end{array}$ & $\begin{array}{c}-0.027 \\
(0.017)\end{array}$ & $\begin{array}{c}0.065 \\
(0.012)^{* * *}\end{array}$ & $\begin{array}{c}0.013 \\
(0.013)\end{array}$ & $\begin{array}{c}0.015 \\
(0.010)\end{array}$ & $\begin{array}{c}0.027 \\
(0.007)^{\star \star \star}\end{array}$ & $\begin{array}{c}0.054 \\
(0.024)^{*}\end{array}$ & $\begin{array}{c}0.019 \\
(0.029)\end{array}$ \\
\hline None official language & $\begin{array}{c}-0.806 \\
(0.045)\end{array}$ & $\begin{array}{l}-0.105 \\
(0.058)\end{array}$ & - & - & - & - & $\begin{array}{c}0.027 \\
(0.037)\end{array}$ & $\begin{array}{c}0.005 \\
(0.039)\end{array}$ \\
\hline Legally married & $\begin{array}{c}0.069 \\
(0.007)^{* * *}\end{array}$ & $\begin{array}{c}0.038 \\
(0.006)^{* * *}\end{array}$ & $\begin{array}{c}0.072 \\
(0.007)^{\star * \star *}\end{array}$ & $\begin{array}{c}0.044 \\
(0.007)^{\star * * *}\end{array}$ & $\begin{array}{c}0.054 \\
(0.008)^{* * *}\end{array}$ & $\begin{array}{c}0.036 \\
(0.008)^{* * * *}\end{array}$ & $\begin{array}{c}0.100 \\
(0.175)^{* * * *}\end{array}$ & $\begin{array}{c}0.059 \\
(0.015)^{* * *}\end{array}$ \\
\hline $\begin{array}{l}\text { Divorced-separated } \\
\text { Children }<6\end{array}$ & $\begin{array}{c}0.017 \\
(0.011) \\
0.033 \\
(0.007)^{\star \star \star}\end{array}$ & $\begin{array}{c}0.004 \\
(0.009) \\
-0.0064 \\
(0.007)\end{array}$ & $\begin{array}{c}0.024 \\
(0.009)^{*} \\
-0.001 \\
(0.007)\end{array}$ & $\begin{array}{c}0.022 \\
(0.009)^{\star} \\
-0.055 \\
(0.008)^{\star \star *}\end{array}$ & $\begin{array}{c}0.040 \\
(0.008)^{\star \star \star} \\
-0.003 \\
(0.008)\end{array}$ & $\begin{array}{c}0.017 \\
(0.008)^{\star} \\
-0.060 \\
(0.010)^{\star \star \star}\end{array}$ & $\begin{array}{c}0.012 \\
(0.35) \\
0.030 \\
(0.021)\end{array}$ & $\begin{array}{c}0.008 \\
(0.020) \\
-0.004 \\
(0.019)\end{array}$ \\
\hline Children 6-14 & $\begin{array}{c}0.040 \\
(0.007)^{\star * *}\end{array}$ & $\begin{array}{c}0.027 \\
(0.007)^{\star * \star}\end{array}$ & $\begin{array}{c}0.015 \\
(0.007)^{*}\end{array}$ & $\begin{array}{c}-0.016 \\
(0.009)\end{array}$ & $\begin{array}{c}0.021 \\
(0.008)^{*}\end{array}$ & $\begin{array}{c}-0.013 \\
(0.008)\end{array}$ & $\begin{array}{c}0.053 \\
(0.022)^{\star}\end{array}$ & $\begin{array}{c}0.036 \\
(0.020)\end{array}$ \\
\hline Children 14-24 & $\begin{array}{c}0.049 \\
(0.010)^{* \star *}\end{array}$ & $\begin{array}{c}0.018 \\
(0.009)^{*}\end{array}$ & $\begin{array}{c}0.016 \\
(0.009)\end{array}$ & $\begin{array}{l}0.0001 \\
(0.009)\end{array}$ & $\begin{array}{c}0.022 \\
(0.008)^{\star \star *}\end{array}$ & $\begin{array}{c}0.015 \\
(0.008)\end{array}$ & $\begin{array}{c}0.034 \\
(0.028)\end{array}$ & $\begin{array}{c}-0.021 \\
(0.029)\end{array}$ \\
\hline Lone parent & $\begin{array}{c}-0.040 \\
(0.008)^{* * *}\end{array}$ & $\begin{array}{c}-0.014 \\
(0.007)^{*}\end{array}$ & $\begin{array}{c}-0.037 \\
(0.007)^{* * * *}\end{array}$ & $\begin{array}{c}-0.010 \\
(0.007)\end{array}$ & $\begin{array}{c}-0.039 \\
(0.010)^{* * *}\end{array}$ & $\begin{array}{c}0.010 \\
(0.010)\end{array}$ & $\begin{array}{c}-0.059 \\
(0.022)^{* \star}\end{array}$ & $\begin{array}{c}0.016 \\
(0.015)\end{array}$ \\
\hline Health activity limitation & $\begin{array}{l}-0.018 \\
(0.009)\end{array}$ & $\begin{array}{c}-0.035 \\
(0.010)^{* * *}\end{array}$ & $\begin{array}{c}-0.046 \\
(0.010)^{* * *}\end{array}$ & $\begin{array}{c}-0.055 \\
(0.009)^{* * * *}\end{array}$ & $\begin{array}{c}-0.017 \\
(0.009)^{*}\end{array}$ & $\begin{array}{c}-0.036 \\
(0.011)^{* *}\end{array}$ & $\begin{array}{l}-0.016 \\
(0.021)\end{array}$ & $\begin{array}{l}-0.067 \\
(0.027)^{*}\end{array}$ \\
\hline Household maintainer one & $\begin{array}{c}-0.043 \\
(0.008)^{* * *}\end{array}$ & $\begin{array}{c}-0.034 \\
(0.007)^{* * *}\end{array}$ & $\begin{array}{c}-0.056 \\
(0.006)^{\star \star * *}\end{array}$ & $\begin{array}{c}-0.055 \\
(0.006)^{* * * *}\end{array}$ & $\begin{array}{c}0.018 \\
(0.006)^{\star *}\end{array}$ & $\begin{array}{c}-0.049 \\
(0.008)^{* * *}\end{array}$ & $\begin{array}{c}-0.002 \\
(0.015)\end{array}$ & $\begin{array}{c}-0.026 \\
(0.013)\end{array}$ \\
\hline Respondent maintainer & $\begin{array}{l}0.0048 \\
(0.009)\end{array}$ & $\begin{array}{l}0.0004 \\
(0.009)\end{array}$ & $\begin{array}{c}-0.010 \\
(0.007)\end{array}$ & $\begin{array}{c}0.008 \\
(0.008)\end{array}$ & $\begin{array}{c}0.020 \\
(0.010)\end{array}$ & $\begin{array}{c}0.014 \\
(0.011)\end{array}$ & $\begin{array}{c}-0.024 \\
(0.012)\end{array}$ & $\begin{array}{c}0.025 \\
(0.015)\end{array}$ \\
\hline Number of observations & 36920 & 31830 & 51750 & 50560 & 15320 & 14090 & 6090 & 5970 \\
\hline
\end{tabular}

Source: Statistics Canada, 2006 Census analytical file. Individual composite weights in effect. Standard errors (adjusted for clustering) in parentheses. Statistical significance ${ }^{* * *} p<.001 ;{ }^{* *} p<.01 ;{ }^{*} p<.05$. 
Appendix E 3: Detailed results from robustness checks for certification and credentials, Census 2006

\begin{tabular}{|c|c|c|c|c|c|c|c|c|}
\hline \multirow{3}{*}{$\begin{array}{l}\text { High school } \\
\text { certification and } \\
\text { accreditation attained }\end{array}$} & \multicolumn{8}{|c|}{ Average Marginal Effects } \\
\hline & \multicolumn{2}{|c|}{$\begin{array}{l}\text { On-reserve } \\
\text { NAI }\end{array}$} & \multicolumn{2}{|c|}{$\begin{array}{c}\text { Urban off-reserve } \\
\text { NAI }\end{array}$} & \multicolumn{2}{|c|}{ Métis } & \multicolumn{2}{|c|}{ Inuit } \\
\hline & Men & Women & Men & Women & Men & Women & Men & Women \\
\hline $\begin{array}{l}\text { No high school diploma } \\
\text { and no further schooling }\end{array}$ & $\begin{array}{c}-0.100 \\
(0.009)^{*+\cdots}\end{array}$ & $\begin{array}{c}-0.091 \\
(0.007)^{*+*}\end{array}$ & $\begin{array}{c}-0.099 \\
(0.009)^{* * *}\end{array}$ & $\begin{array}{c}-0.082 \\
(0.008)^{* * *}\end{array}$ & $\begin{array}{l}-0.056 \\
(0.008)^{*+*}\end{array}$ & $\begin{array}{c}-0.050 \\
(0.008)^{* * * *}\end{array}$ & $\begin{array}{c}-0.138 \\
(0.021)^{n+1+}\end{array}$ & $\begin{array}{l}-0.106 \\
(0.017)^{* * *}\end{array}$ \\
\hline $\begin{array}{l}\text { Registered } \\
\text { apprenticeship or other } \\
\text { trade certificate, without } \\
\text { high school diploma }\end{array}$ & $\begin{array}{c}-0.083 \\
(0.013)^{* * * t}\end{array}$ & $\begin{array}{c}-0.032 \\
(0.014)^{\star}\end{array}$ & $\begin{array}{c}-0.091 \\
(0.012)^{* * *}\end{array}$ & $\begin{array}{c}-0.062 \\
(0.015)^{* * * *}\end{array}$ & $\begin{array}{l}-0.034 \\
(0.014)^{*}\end{array}$ & $\begin{array}{c}0.004 \\
(0.016)\end{array}$ & $\begin{array}{c}-0.132 \\
(0.029)^{+t+*}\end{array}$ & $\begin{array}{c}-0.086 \\
(0.035)^{*}\end{array}$ \\
\hline $\begin{array}{l}\text { College certificate, } \\
\text { without high school } \\
\text { diploma }\end{array}$ & $\begin{array}{c}-0.020 \\
(0.0019)\end{array}$ & $\begin{array}{c}0.012 \\
(0.018)\end{array}$ & $\begin{array}{c}-0.071 \\
(0.022)^{* *}\end{array}$ & $\begin{array}{r}-0.019 \\
(0.016)\end{array}$ & $\begin{array}{l}-0.016 \\
(0.023)\end{array}$ & $\begin{array}{c}-0.019 \\
(0.017)\end{array}$ & $\begin{array}{c}-0.077 \\
(0.035)^{\star}\end{array}$ & $\begin{array}{l}-0.037 \\
(0.027)\end{array}$ \\
\hline $\begin{array}{l}\text { High school diploma and } \\
\text { registered } \\
\text { apprenticeship or other } \\
\text { trade certificate }\end{array}$ & $\begin{array}{l}-0.007 \\
(0.012)\end{array}$ & $\begin{array}{c}0.012 \\
(0.012)\end{array}$ & $\begin{array}{c}-0.013 \\
(0.010)\end{array}$ & $\begin{array}{c}0.012 \\
(0.012)\end{array}$ & $\begin{array}{l}-0.003 \\
(0.010)\end{array}$ & $\begin{array}{c}0.012 \\
(0.011)\end{array}$ & $\begin{array}{c}0.004 \\
(0.028)\end{array}$ & $\begin{array}{l}-0.030 \\
(0.055)\end{array}$ \\
\hline $\begin{array}{l}\text { High school diploma and } \\
\text { college certificate }\end{array}$ & $\begin{array}{c}0.065 \\
(0.011)^{\star * *}\end{array}$ & $\begin{array}{c}0.062 \\
(0.007)^{\star * *}\end{array}$ & $\begin{array}{c}0.023 \\
(0.009)^{\star}\end{array}$ & $\begin{array}{c}0.033 \\
(0.007)^{\star * \star}\end{array}$ & $\begin{array}{c}0.015 \\
(0.008)\end{array}$ & $\begin{array}{c}0.024 \\
(0.007)^{\star \star}\end{array}$ & $\begin{array}{l}-0.037 \\
(0.046)\end{array}$ & $\begin{array}{c}0.029 \\
(0.026)\end{array}$ \\
\hline $\begin{array}{l}\text { High school diploma and } \\
\text { university certificate }\end{array}$ & $\begin{array}{c}0.065 \\
(0.019)^{\star \star}\end{array}$ & $\begin{array}{c}0.058 \\
(0.011)^{\star \star \star}\end{array}$ & $\begin{array}{c}0.023 \\
(0.016)\end{array}$ & $\begin{array}{c}0.015 \\
(0.012)\end{array}$ & $\begin{array}{c}0.023 \\
(0.018)\end{array}$ & $\begin{array}{c}0.013 \\
(0.014)\end{array}$ & $\begin{array}{l}-0.006 \\
(0.063)\end{array}$ & $\begin{array}{c}0.008 \\
(0.045)\end{array}$ \\
\hline $\begin{array}{l}\text { High school diploma and } \\
\text { university degree }\end{array}$ & $\begin{array}{c}0.133 \\
(0.017)^{\star \star \star *}\end{array}$ & $\begin{array}{c}0.104 \\
(0.010)^{* * *}\end{array}$ & $\begin{array}{c}0.078 \\
(0.012)^{\star \star \star}\end{array}$ & $\begin{array}{c}0.062 \\
(0.009)^{* * *}\end{array}$ & $\begin{array}{c}0.058 \\
(0.009)^{* \star *}\end{array}$ & $\begin{array}{c}0.049 \\
(0.007)^{\text {tw* }}\end{array}$ & $\begin{array}{c}0.102 \\
(0.053)\end{array}$ & $\begin{array}{c}0.082 \\
(0.024)^{* *}\end{array}$ \\
\hline Age $15-19$ & $\begin{array}{c}-0.071 \\
(0.014)\end{array}$ & $\begin{array}{c}-0.074 \\
(0.014)^{\star \star * *}\end{array}$ & $\begin{array}{c}0.020 \\
(0.011)\end{array}$ & $\begin{array}{c}0.013 \\
(0.011)\end{array}$ & $\begin{array}{l}-0.030 \\
(0.017)\end{array}$ & $\begin{array}{l}-0.006 \\
(0.015)\end{array}$ & $\begin{array}{c}0.004 \\
(0.029)\end{array}$ & $\begin{array}{l}-0.024 \\
(0.029)\end{array}$ \\
\hline Age $25-34$ & $\begin{array}{c}0.048 \\
(0.008)^{\star \star * *}\end{array}$ & $\begin{array}{c}0.055 \\
(0.008)^{\star \star \star \star}\end{array}$ & $\begin{array}{c}0.031 \\
(0.009)^{* * *}\end{array}$ & $\begin{array}{c}0.046 \\
(0.008)^{* * *}\end{array}$ & $\begin{array}{c}0.017 \\
(0.009)\end{array}$ & $\begin{array}{c}0.012 \\
(0.010)\end{array}$ & $\begin{array}{c}0.043 \\
(0.016)^{\star *}\end{array}$ & $\begin{array}{c}0.020 \\
(0.020)\end{array}$ \\
\hline Age $35-44$ & $\begin{array}{c}0.087 \\
(0.009)^{* \star \star *}\end{array}$ & $\begin{array}{c}0.100 \\
(0.008)^{* * * *}\end{array}$ & $\begin{array}{c}0.049 \\
(0.009)^{* * *}\end{array}$ & $\begin{array}{c}0.070 \\
(0.009)^{\text {****}}\end{array}$ & $\begin{array}{c}0.026 \\
(0.010)\end{array}$ & $\begin{array}{c}0.027 \\
(0.009)^{* *}\end{array}$ & $\begin{array}{c}0.059 \\
(0.018)^{\star * t}\end{array}$ & $\begin{array}{c}0.50 \\
(0.019)^{*}\end{array}$ \\
\hline Age 45-54 & $\begin{array}{c}0.119 \\
(0.009)^{\star \star * \star}\end{array}$ & $\begin{array}{c}0.126 \\
(0.008)^{\star * * *}\end{array}$ & $\begin{array}{c}0.069 \\
(0.010)^{\star k * *}\end{array}$ & $\begin{array}{c}0.093 \\
(0.008)^{\star * *}\end{array}$ & $\begin{array}{c}0.028 \\
(0.011)\end{array}$ & $\begin{array}{c}0.023 \\
(0.010)^{*}\end{array}$ & $\begin{array}{c}0.050 \\
(0.022)^{*}\end{array}$ & $\begin{array}{c}0.084 \\
(0.019)^{* * * *}\end{array}$ \\
\hline Age $55-64$ & $\begin{array}{c}0.122 \\
(0.010)^{\star \star *}\end{array}$ & $\begin{array}{c}0.146 \\
(0.009)^{* \star *}\end{array}$ & $\begin{array}{c}0.062 \\
(0.011)^{* * *}\end{array}$ & $\begin{array}{c}0.101 \\
(0.009)^{\star \star \star}\end{array}$ & $\begin{array}{c}0.017 \\
(0.014)\end{array}$ & $\begin{array}{c}0.024 \\
(0.012)^{*}\end{array}$ & $\begin{array}{c}0.088 \\
(0.023)^{* * \star}\end{array}$ & $\begin{array}{c}0.126 \\
(0.016)^{* * *}\end{array}$ \\
\hline Atlantic provinces & $\begin{array}{l}-0.137 \\
(0.037)^{*+*}\end{array}$ & $\begin{array}{c}-0.104 \\
(0.024)^{* * *}\end{array}$ & $\begin{array}{c}-0.110 \\
(0.029)^{* * *}\end{array}$ & $\begin{array}{c}-0.073 \\
(0.020)^{*+* *}\end{array}$ & $\begin{array}{c}-0.094 \\
(0.030)^{* *}\end{array}$ & $\begin{array}{c}-0.097 \\
(0.023)^{\star \star * *}\end{array}$ & $\begin{array}{c}-0.214 \\
(0.037)^{* * *}\end{array}$ & $\begin{array}{r}-0.115 \\
(0.054)^{*}\end{array}$ \\
\hline Other provinces & - & - & - & - & - & - & $\begin{array}{c}0.032 \\
(0.059)\end{array}$ & $\begin{array}{c}-0.128 \\
(0.057)^{*}\end{array}$ \\
\hline Northwest Territories & - & - & - & - & - & - & $\begin{array}{r}-0.043 \\
(0.030)\end{array}$ & $\begin{array}{c}-0.092 \\
(0.042)^{*}\end{array}$ \\
\hline Nunavut & - & - & - & - & - & - & $\begin{array}{c}-0.037 \\
(0.020)\end{array}$ & $\begin{array}{c}-0.042 \\
(0.022)\end{array}$ \\
\hline Northern territories & $\begin{array}{c}-0.120 \\
(0.023)^{* * * *}\end{array}$ & $\begin{array}{l}-0.006 \\
(0.016)\end{array}$ & $\begin{array}{c}-0.121 \\
(0.017)^{* * *}\end{array}$ & $\begin{array}{c}-0.034 \\
(0.013)^{*}\end{array}$ & $\begin{array}{l}-0.002 \\
(0.012)\end{array}$ & $\begin{array}{c}0.026 \\
(0.008)^{* *}\end{array}$ & - & - \\
\hline Quebec & $\begin{array}{c}-0.054 \\
(0.023)^{*}\end{array}$ & $\begin{array}{c}0.050 \\
(0.021)^{\star}\end{array}$ & $\begin{array}{c}-0.086 \\
(0.019)^{* * *}\end{array}$ & $\begin{array}{c}0.022 \\
(0.017)\end{array}$ & $\begin{array}{c}-0.034 \\
(0.023)\end{array}$ & $\begin{array}{c}-0.063 \\
(0.029)^{\star}\end{array}$ & - & - \\
\hline Manitoba & $\begin{array}{c}-0.128 \\
(0.022)^{\star \star \star}\end{array}$ & $\begin{array}{c}-0.060 \\
(0.016)^{\star \star *}\end{array}$ & $\begin{array}{c}-0.104 \\
(0.023)^{* * *}\end{array}$ & $\begin{array}{c}-0.033 \\
(0.013)^{*}\end{array}$ & $\begin{array}{c}0.016 \\
(0.008)\end{array}$ & $\begin{array}{c}0.014 \\
(0.008)\end{array}$ & - & - \\
\hline Saskatchewan & $\begin{array}{c}0.131 \\
(0.020)^{* * *}\end{array}$ & $\begin{array}{c}-0.072 \\
(0.016)^{* * *}\end{array}$ & $\begin{array}{l}-0.010 \\
(0.019)^{* * * *}\end{array}$ & $\begin{array}{c}-0.70 \\
(0.013)^{*+*}\end{array}$ & $\begin{array}{l}-0.011 \\
(0.011)\end{array}$ & $\begin{array}{c}0.021 \\
(0.009)^{*}\end{array}$ & - & - \\
\hline Alberta & $\begin{array}{c}-0.049 \\
(0.022)^{*}\end{array}$ & $\begin{array}{c}-0.067 \\
(0.019)^{* * * *}\end{array}$ & $\begin{array}{c}0.008 \\
(0.018)\end{array}$ & $\begin{array}{c}-0.002 \\
(0.012)\end{array}$ & $\begin{array}{c}0.044 \\
(0.009)^{* \ldots *}\end{array}$ & $\begin{array}{c}0.029 \\
(0.008)^{\star \star}\end{array}$ & - & - \\
\hline
\end{tabular}




\begin{tabular}{|c|c|c|c|c|c|c|c|c|}
\hline \multirow{3}{*}{$\begin{array}{l}\text { Appendix E3 } \\
\text { (continued) }\end{array}$} & \multicolumn{8}{|c|}{ Average Marginal Effects } \\
\hline & \multicolumn{2}{|c|}{$\begin{array}{c}\text { On-reserve } \\
\text { NAI }\end{array}$} & \multicolumn{2}{|c|}{$\begin{array}{c}\text { Urban off-reserve } \\
\text { NAI }\end{array}$} & \multicolumn{2}{|c|}{ Métis } & \multicolumn{2}{|c|}{ Inuit } \\
\hline & Men & Women & Men & Women & Men & Women & Men & Women \\
\hline British Columbia & $\begin{array}{c}-0.082 \\
(0.021)^{* * * *}\end{array}$ & $\begin{array}{c}-0.055 \\
(0.015)^{* * * *}\end{array}$ & $\begin{array}{c}-0.030 \\
(0.014)^{*}\end{array}$ & $\begin{array}{c}-0.025 \\
(0.009)^{* *}\end{array}$ & $\begin{array}{c}0.010 \\
(0.010)\end{array}$ & $\begin{array}{l}0.007 \\
(0.010)\end{array}$ & - & - \\
\hline French only & $\begin{array}{c}-0.019 \\
(0.037)\end{array}$ & $\begin{array}{c}-0.119 \\
(0.041)^{* * *}\end{array}$ & $\begin{array}{c}0.045 \\
(0.021)^{*}\end{array}$ & $\begin{array}{c}-0.022 \\
(0.030)\end{array}$ & $\begin{array}{c}0.03 \\
(0.020)\end{array}$ & $\begin{array}{c}0.026 \\
(0.020)\end{array}$ & $\begin{array}{c}0.075 \\
(0.032)^{*}\end{array}$ & $\begin{array}{c}0.033 \\
(0.027)\end{array}$ \\
\hline Both official languages & $\begin{array}{c}0.012 \\
(0.014)\end{array}$ & $\begin{array}{l}-0.028 \\
(0.017)\end{array}$ & $\begin{array}{c}0.063 \\
(0.012)^{* * * *}\end{array}$ & $\begin{array}{c}0.011 \\
(0.013)\end{array}$ & $\begin{array}{c}0.014 \\
(0.010)\end{array}$ & $\begin{array}{c}0.027 \\
(0.007)^{m+* *}\end{array}$ & $\begin{array}{c}0.048 \\
(0.025)\end{array}$ & $\begin{array}{l}-0.023 \\
(0.028)\end{array}$ \\
\hline None official language & $\begin{array}{c}-0.081 \\
(0.044)\end{array}$ & $\begin{array}{c}-0.106 \\
(0.058)\end{array}$ & - & - & - & - & $\begin{array}{c}0.028 \\
(0.036)\end{array}$ & $\begin{array}{c}0.004 \\
(0.039)\end{array}$ \\
\hline Legally married & $\begin{array}{c}0.069 \\
(0.007)^{* * *}\end{array}$ & $\begin{array}{c}0.039 \\
(0.006)^{*+* * *}\end{array}$ & $\begin{array}{c}0.072 \\
(0.006)^{* * *}\end{array}$ & $\begin{array}{c}0.044 \\
(0.007)^{\star * \star *}\end{array}$ & $\begin{array}{c}0.054 \\
(0.008)^{* * *}\end{array}$ & $\begin{array}{c}0.036 \\
(0.008)^{* * *}\end{array}$ & $\begin{array}{c}0.096 \\
(0.017)^{* * * *}\end{array}$ & $\begin{array}{c}0.062 \\
(0.015)^{* * *}\end{array}$ \\
\hline Divorced-separated & $\begin{array}{c}0.017 \\
(0.011)\end{array}$ & $\begin{array}{c}0.004 \\
(0.009)\end{array}$ & $\begin{array}{c}0.023 \\
(0.009)^{*}\end{array}$ & $\begin{array}{c}0.021 \\
(0.009)^{\star}\end{array}$ & $\begin{array}{c}0.040 \\
(0.008)^{\star \star \star \star}\end{array}$ & $\begin{array}{c}0.017 \\
(0.008)^{*}\end{array}$ & $\begin{array}{c}0.014 \\
(0.036)\end{array}$ & $\begin{array}{c}0.009 \\
(0.020)\end{array}$ \\
\hline Children $<6$ & $\begin{array}{c}0.033 \\
(0.007)^{* * * *}\end{array}$ & $\begin{array}{c}-0.006 \\
(0.007)\end{array}$ & $\begin{array}{l}0.0003 \\
(0.007)\end{array}$ & $\begin{array}{c}-0.054 \\
(0.008)^{* * * *}\end{array}$ & $\begin{array}{l}0.0002 \\
(0.008)\end{array}$ & $\begin{array}{c}-0.059 \\
(0.010)^{* * * *}\end{array}$ & $\begin{array}{c}0.032 \\
(0.021)\end{array}$ & $\begin{array}{c}-0.004 \\
(0.018)\end{array}$ \\
\hline Children 6-14 & $\begin{array}{c}0.040 \\
(0.007)^{\star \star * *}\end{array}$ & $\begin{array}{c}0.027 \\
(0.007)^{\star \star \star}\end{array}$ & $\begin{array}{c}0.015 \\
(0.007)^{*}\end{array}$ & $\begin{array}{c}-0.015 \\
(0.009)\end{array}$ & $\begin{array}{c}0.021 \\
(0.008)^{*}\end{array}$ & $\begin{array}{c}-0.013 \\
(0.008)\end{array}$ & $\begin{array}{c}0.058 \\
(0.022)^{* *}\end{array}$ & $\begin{array}{c}0.037 \\
(0.020)\end{array}$ \\
\hline Children 14-24 & $\begin{array}{c}0.049 \\
(0.010)^{\star \star * \star}\end{array}$ & $\begin{array}{c}0.019 \\
(0.009)^{*}\end{array}$ & $\begin{array}{c}0.017 \\
(0.009)\end{array}$ & $\begin{array}{l}0.0009 \\
(0.009)\end{array}$ & $\begin{array}{c}0.022 \\
(0.008)^{* \star}\end{array}$ & $\begin{array}{c}0.015 \\
(0.008)\end{array}$ & $\begin{array}{c}0.036 \\
(0.029)\end{array}$ & $\begin{array}{c}-0.021 \\
(0.029)\end{array}$ \\
\hline Lone parent & $\begin{array}{c}-0.041 \\
(0.008)^{\star \star n \star}\end{array}$ & $\begin{array}{c}-0.015 \\
(0.007)^{*}\end{array}$ & $\begin{array}{c}-0.040 \\
(0.007)^{\star \star \star}\end{array}$ & $\begin{array}{l}-0.010 \\
(0.007)\end{array}$ & $\begin{array}{c}-0.039 \\
(0.010)^{\star \star \star \star}\end{array}$ & $\begin{array}{c}0.010 \\
(0.010)\end{array}$ & $\begin{array}{c}-0.061 \\
(0.022)^{* *}\end{array}$ & $\begin{array}{c}0.018 \\
(0.015)\end{array}$ \\
\hline Health activity limitation & $\begin{array}{l}-0.017 \\
(0.009)\end{array}$ & $\begin{array}{c}-0.035 \\
(0.010)^{\star t *}\end{array}$ & $\begin{array}{c}-0.046 \\
(0.010)^{* * *}\end{array}$ & $\begin{array}{c}-0.055 \\
(0.009)^{* * *}\end{array}$ & $\begin{array}{c}-0.02 \\
(0.009)\end{array}$ & $\begin{array}{c}-0.036 \\
(0.010)^{* *}\end{array}$ & $\begin{array}{l}-0.015 \\
(0.022)\end{array}$ & $\begin{array}{l}-0.067 \\
(0.027)^{*}\end{array}$ \\
\hline Household maintainer one & $\begin{array}{c}-0.043 \\
(0.008)^{* * *}\end{array}$ & $\begin{array}{c}-0.034 \\
(0.007)^{\star \star \star \star}\end{array}$ & $\begin{array}{c}-0.055 \\
(0.006)^{* * *}\end{array}$ & $\begin{array}{c}-0.055 \\
(0.006)^{* \star *}\end{array}$ & $\begin{array}{c}-0.02 \\
(0.006)^{* *}\end{array}$ & $\begin{array}{c}-0.048 \\
(0.008)^{*+* *}\end{array}$ & $\begin{array}{c}-0.002 \\
(0.015)\end{array}$ & $\begin{array}{c}-0.025 \\
(0.013)\end{array}$ \\
\hline Respondent maintainer & $\begin{array}{c}0.005 \\
(0.009)\end{array}$ & $\begin{array}{l}0.0005 \\
(0.009) \\
\end{array}$ & $\begin{array}{l}-0.010 \\
(0.007)\end{array}$ & $\begin{array}{c}0.008 \\
(0.008)\end{array}$ & $\begin{array}{c}0.02 \\
(0.010)\end{array}$ & $\begin{array}{c}-0.014 \\
(0.011)\end{array}$ & $\begin{array}{l}-0.023 \\
(0.012)\end{array}$ & $\begin{array}{c}0.023 \\
(0.015)\end{array}$ \\
\hline Number of observations & 36920 & 31830 & 51750 & 50560 & 15320 & 14090 & 6090 & 5970 \\
\hline
\end{tabular}

Source: Statistics Canada, 2006 Census analytical file. Individual composite weights in effect. Standard errors (adjusted for clustering) in parentheses. Statistical significance ${ }^{* * *} p<.001 ;{ }^{* *} p<.01 ;{ }^{*} p<.05$. 


\section{References}

Allison, P. D. (1999). Logistic regression using the SAS system: Theory and application. Cary, NC: SAS Institute Inc.

Allison, P. D. (1999a). Comparing logit and probit coefficients across groups. Sociological Methods \& Research, 28(2), 186-2008.

Angrist, D. J. (2001). Estimation of limited dependent variable models with dummy endogenous regressors. Journal of Business and Economic Statistics, 19(1), 2-28.

Annen, K. (2007). Economic returns to social capital in the urban informal sector in developing countries: Micro evidence from small textile producers in Bolivia. Unpublished Manuscript. University of Guelph, Department of Economics. Retrieved March 2009: http://www.economics.uoguelph.ca/kannen/ Informal\%20Firms.pdf

Baum. C. F. (2006). An introduction to modern econometrics using Stata. College Station, Texas: Stata Press.

Becker, G. S. (1993). The human capital: A theoretical and empirical analysis, with special reference to education ( $3^{\text {rd }}$ ed.). Chicago: University of Chicago Press.

Becker, G. S. \& Murphy, K. M. (2000). Social economics: Market behavior in a social environment. Cambridge: The Balknap Press of Harvard University Press.

Benjamin, D., Gunderson, M., \& Riddell, C. W. (2002). Labour market economics:

Theory, evidence and policy in Canada $\left(5^{\text {th }}\right.$ ed.). Toronto: McGraw-Hill Ryerson Limited.

Berg, N. (2005). Non-response Bias. In K. Kempf-Leonard (ed.), Encyclopedia of Social Measurement, vol. 2, (pp. 865-873). London: Academic Press. 
Bernier, R. (1997). The dimensions of wage inequality among Aboriginal Peoples. Research Paper No. 109, Statistics Canada, Analytical Studies Branch - Research Paper Series. ISSN: 1200-5223, No. 11F0019MPE No. 109.

Breslaw, J.A. \& Stelcner, M. (1987). The effect of health on the labor force behavior of elderly men in Canada. The Journal of Human Racecourses, 22(4), 490-517.

Brough, M., Bond, C., Hunt, J., Jenkins, D. Shannon, S., \& Schubert, L. (2006). Social capital meets identity: Aboriginality in an urban setting. Journal of Sociology, 42(4), 396-411.

Cameron, C. A. \& Trivedi, P. K. (2010). Microeconometrics using Stata, Revised edition. Texas: StataCorp LP.

Christofides, L. N. (2003). Social assistance and labour supply. Canadian Journal of Economics, 33(3), 715-741.

Cleary, P. D. \& Angel, R. (1984). The analysis of relationships involving dichotomous dependent variables. Journal of Health and Social Behavior, 25(3), 334-348.

Delic, S. (2009). Statistical information pertaining to socio-economic conditions of northern Aboriginal people in Canada: Sources and limitations. The Northern Review, 30(1), 119-150.

De Silva, A. (1999). Wage discrimination against natives. Canadian Public Policy, 25(1), 65-85.

Drolet, M. (2001). The male-female wage gap. Perspectives on Labour and Income, 2(12). The online edition, Statistics Canada catalogue No. 75-001-XIE.

Drost, H. (1994). Schooling, vocational training and unemployment: The case of Canadian Aboriginals. Canadian Public Policy, 20(1), 52-65. 
Dubin, J. A. \& Rivers, D. (1989). Selection bias in linear regression, logit and probit models. Sociological Methods and Research, 18(2 \& 3), 360-390.

Durlauf, N. S. (2002). On the empirics of social capital. The Economic Journal, $112(483), 459-479$.

Ferrer, A. M. \& Riddell, C. W. (2002). The role of credentials in the Canadian labour market. Canadian Journal of Economics, 35(4), 879-905.

Foley, W. M. \& Edwards, B. (1999). Is it time to disinvest in social capital? Journal of Public Policy, 19(2),141-73.

Fox, J. (2008). Applied regression analysis and generalized linear models, 2nd edition. Thousand Oaks: SAGE Publication Inc.

Garfinkel, I., Heintze, T. \& Huang, C. C. (2000). Child support enforcement: Incentives and well-being. Conference on incentive effects of tax and transfer policies, Washington, D.C., December 8, 2000.

George, P. \& Kuhn, P. (1994). The size and structure of native-white wage differentials in Canada. The Canadian Journal of Economics, 27(1), 20-42.

Gerber, L. (1990). Multiple jeopardy: A socio-economic comparison of men and women among Indian, Métis and Inuit Peoples of Canada. Canadian Ethnic Studies, 22(3), 69-84.

Glaeser, L. E, Laibson, \& Sacerdote, B. (2002). An economic approach to social capital. The Economic Journal, 112(483), 437-458.

Gomez, R. \& Santor, E. (2001). Membership has its privileges: The effect of social capital and neighbourhood characteristics on the earnings of microfinance borrowers. Canadian Journal of Economics, 34(4), 943-966. 
Greene, W. H. (2003). Econometric analysis (5 $5^{\text {th }}$ ed.). New Jersey: Prentice Hall.

Harkness, J. (1993). Labour force participation by disabled males in Canada. Canadian Journal of Economics, 26(4), 878-889.

Heckman, J. J. (1979). Sample selection bias as a specification error. Econometrica, $47(1), 153-161$.

Hoetker, G. (2003). Confounded coefficients: Accurately comparing logit and probit coefficients across groups. University of Illinois, College of Business, Working Papers, version October 22, 2004.

Hunter, B. H. (2000). Social exclusion, social capital and Indigenous Australians: Measuring the social costs of unemployment. Canberra: Centre for Aboriginal Economic Policy Research, Australian National University. Discussion Paper No. 204.

INAC (2002). Words first: An evolving terminology relating to Aboriginal Peoples in Canada. Indian and Northern Affairs Canada, Communication Branch, Ottawa, October 2002.

Jung, H. \& Pirog, A. M. (2011). Nonexperimental impact evaluations. In D. J. Besharov \& P. H. Cottingham (Eds.), The workforce investment act: Implementation experiences and evaluation findings (pp. 407-429). Kalamzoo: W.E. Upjohn Institute for Employment Research.

Kleinfeld, J. \& Kruse, A. (1982). Native Americans in the labor force: Hunting for an accurate measure. Monthly Labor Review, 105(7), 47-51.

Knorringa, P. \& Van Staveren, I. (2007). Beyond social capital: A critical approach. Review of Social Economy, 65(1), 1-9. 
Kuhn, P. (2000). Policies for an Internet labour market. Policy Options, 21(8), 42-47.

Kuhn, P. \& Skuterud, M. S. (2000). Job search methods: Internet versus traditional. Monthly Labor Review, 123(10), 3-11.

Kuhn, P. \& Sweetman, A. (2002). Aboriginals as unwilling immigrants: Contact, assimilation and labour market outcomes. Journal of Population Economics, $15(2), 331-355$.

Kuo, C. Y. (1976). The effect of education on the earnings of Indian, Eskimo, Métis, and White workers in the Mackenzie District of Northern Canada. Economic Development and Cultural Change, 24(2), 387-398.

Maxim, P. S., White, J. P. \& Beavon, D. (2003). Dispersion and polarization of income among Aboriginal and non-Aboriginal Canadians. In J. P. White, P. S. Maxim, \& D. Beavon (Eds.), Aboriginal conditions: Research as a foundation for public policy (pp. 222-247). Vancouver, British Columbia: UBC Press.

Maxim, P. \& White, J. (2006). School completion and workforce transitions among urban Aboriginal youth. In J. P. White, S. Wingert, D. Beavon, \& P. Maxim (Eds.), Aboriginal policy research: Moving forward, making a difference, Volume 3, (pp. 33-52). Toronto: Thompson Educational Publishing.

Mignone, J. (2009). Social capital and Aboriginal communities: A critical assessment. Journal of Aboriginal Health, 5(3), 100-147.

Miller, C. (1993). Actual experience, potential experience or age, and labor force participation by married women. Atlantic Economic Journal, 21(4), 60-66.

Mincer, J. \& Polachek, S. (1974). Family investment in human capital: Earnings of women. Journal of Political Economy, 82(2), 76-108. 
Nelson, B. J., Kaboolian, L. \& Carver, K. A. (2003). The concord handbook: How to build social capital across communities. The Concord Project, UCLA School of Public Policy and Social Research, University of California, Los Angeles.

North, D. (1991). Institutions. Journal of Economic Perspectives, 5(1), 97-112.

Patrinos, H. A. \& Sakellariou, C. N. (1992). North American Indians in the Canadian labour market: A Decomposition of wage differentials. Economics of Education Review, 11(3), 257-266.

Pendakur, K. \& Pendakur, R. (2011). Aboriginal income disparity in Canada. Canadian Public Policy, 37(1), 61-83.

Pendakur, K. \& Pendakur, R. (2002). Colour my world: Have earnings gaps for Canadian-born ethnic minorities changed over time? Canadian Public Policy, $28(4), 489-512$.

Pendakur, K. \& Pendakur, R. (1998). The colour of money: Earnings differentials among ethnic groups in Canada. Canadian Journal of Economics, 31(3), 518-548.

Piore, M. J. (1975). Notes for a theory of labor market stratification. In R. C. Edwards, M. Reich, \& D. M. Gordon (Eds.), Labor market segmentation (pp. 125-150). Lexington: D. C. Health and Company.

Poulsen, A. \& Svendsen, G. T. (2005). Social capital and endogenous preferences. Public Choice, 123(1), 171-196.

Rao, J. N. K. (2006). Bootstrap methods for analyzing complex survey data. Proceedings of Statistics Canada Symposium 2006: Methodological Issues in Measuring Population Health, Statistics Canada, Catalogue Number 11-522-XIE. 
Riddell, C. W. \& Song, X. (2011). The impact of education on unemployment incidence and re-employment success: Evidence from U.S. labour market. The Institute for the Study of Labor (IZA), Discussion Paper No. 5572, March 2011.

Riddell, C. W. \& Song, X. (2009). The causal effects of education on adaptability to employment shocks: Evidence from the Canadian labour market. Canadian Labour Market and Skills Researcher Network, Working Paper No. 8.

Rowe, G. \& Nguyen, H. (2004). Longitudinal analysis of Labour Force Survey data. Statistics Canada. Proceedings of Statistics Canada Symposium 2002: Modeling Survey Data for Social and Economic Research.

Sobel, I. (1982). Human capital and institutional theories of the labor market: Rivals or complements? Journal of Economic Issues, 16(1), 255-272.

Sobel, J. (2002). Can we trust social capital? Journal of Economic Literature, 40(1), 139154.

Statistics Canada (2009). Aboriginal Peoples Survey 2006: Concepts and methods guide. Statistics Canada, Social and Aboriginal Statistics Division, January 2009, Cat. No. $89-637-X 2008003$.

Statistics Canada (2009a). Aboriginal Peoples Survey 2006: User's guide to the analytical files. Statistics Canada, Social and Aboriginal Statistics Division.

Statistics Canada (2009b). Aboriginal People's Survey 2006: Arctic analytical file, no frequencies. January 2009.

Statistics Canada (2008). Aboriginal People's Survey 2006: Adult core analytical file, no frequencies. December 2008. 
Statistics Canada (2008a). Aboriginal People's Survey 2006: Métis analytical file, no frequencies. December 2008.

Statistics Canada (2008b). Research Data Centers (RDC): 2006 Census code book. Statistics Canada, Census Operations Division, October 2008.

Statistics Canada (2007). How Statistics Canada identifies Aboriginal Peoples. Ottawa, Minister of Industry, Catalogue No. 12-592-XIE.

Statistics Canada (2006). The 2001 Census Public Use Microdata File: Individuals File User Documentation, $2^{\text {nd }}$ revision. Catalogue no. 95M0016XCB.

Stolzenberg, R. M. \& Relles, D. A. (1997). Tools for intuition about sample selection bias and its correction. American Sociological Review, 62(3), 494-507.

Van Staveren, I. \& Knorringa, P. (2007). Unpacking social capital in economic development: How social relations matter. Review of Social Economy, 65(1), $107-$ 135.

Walters, D., White, J., \& Maxim, P. (2004). Does postsecondary education benefit Aboriginal Canadians? An examination of earnings and employment outcomes for recent Aboriginal graduates. Canadian Public Policy, 15(3), 283-301.

White, J., Maxim, P. \& Gyimah, S. O. (2003). Labour force activity of women in Canada: A comparative analysis of Aboriginal and non-Aboriginal women. The Canadian Review of Sociology and Anthropology, 40(4), 391- 415.

White, J. P. \& Maxim, P. S. (2003). Social capital, social cohesion, and population outcomes in Canada's First Nations communities. In J. P. White, P. S. Maxim, \& D. Beavon (Eds.), Aboriginal conditions: Research as a foundation for public policy (pp. 7-33). Vancouver, British Columbia: UBC Press. 
Williams, R. (2012). Using Stata's margins command to estimate and interpret adjusted and marginal effects. The Stata Journal, 12(2), 308-331.

Williams, R. (2009). Using heterogeneous choice models to compare logit and probit coefficients across groups. Sociological Methods \& Research, 37(4), 531-559.

Winship, C. \& Mare, R. D. (1992). Models for sample selection bias. Annual Review of Sociology, 18(1), 327-350.

Wooldridge, J. M. (2010). Econometric analysis of cross section and panel data $\left(2^{\text {nd }}\right.$ ed.). Cambridge, Massachusetts: The Massachusetts Institute of Technology Press.

Wooldridge, J. M. (2006). Introductory econometrics: A modern approach (3rd ed.). Mason, Ohio: Thomson South Western College Publishing.

Xue, L. (2008). Social capital and wages: Outcome of recent immigrants to Canada. Research and Evaluation Branch, Citizenship and Immigration Canada. 


\title{
Essay III: Enhancing our Understanding of Empirical Findings on Labour Market Outcomes of Aboriginal Peoples in Canada
}

\begin{abstract}
Empirical studies in Canada consistently report strong evidence of a negative association between Aboriginal identity and economic success in the Canadian labour market. Even when all of the key measurable characteristics are taken into account, Aboriginal workers are still found to experience poorer labour market outcomes than their otherwise similar non-Aboriginal counterparts. Using qualitative methods of inquiry, as suggested in the institutional economics theory, this essay presents some new knowledge on the intangible aspects of the labour market experience of Aboriginal people gleaned from in-depth interviews with knowledgeable professionals working in the area of Aboriginal labour force development. Findings suggest that many paradoxes and puzzles that persist in the empirical literature on the topic can be better understood once the institutional arrangements related to investment in human and other capital, the legacy of historical disadvantage, and the experience of workplace discrimination are taken into account.
\end{abstract}

Key words: Aboriginal Peoples, qualitative methods, institutional arrangements, labour force development, labour market discrimination 


\section{Table of Contents}

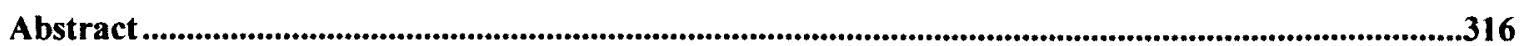

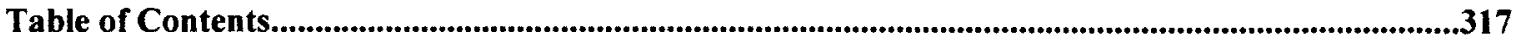

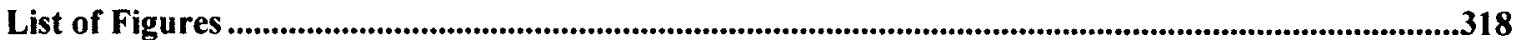

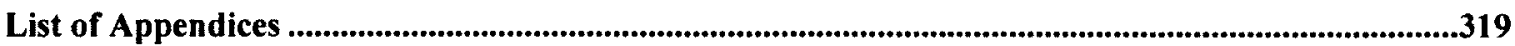

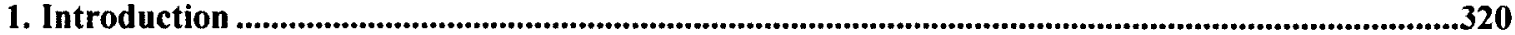

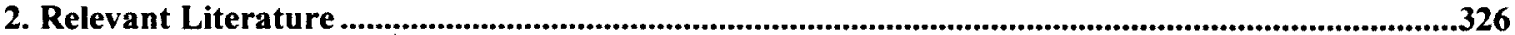

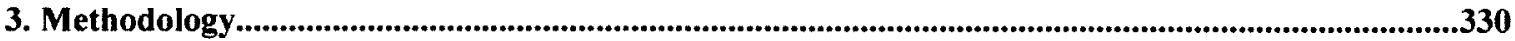

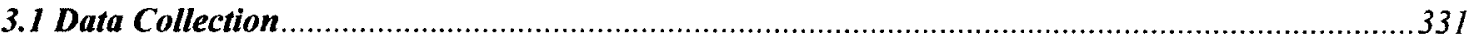

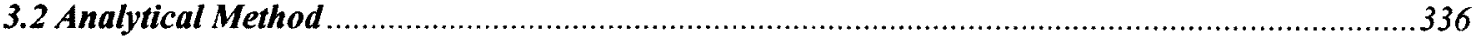

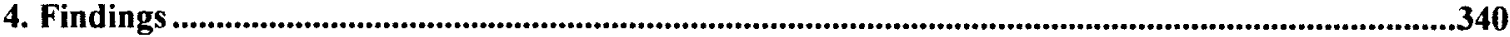

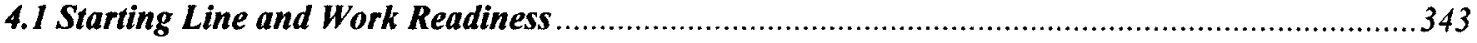

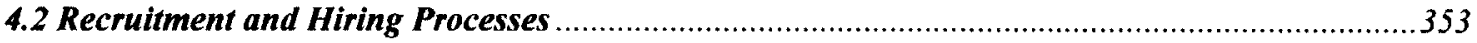

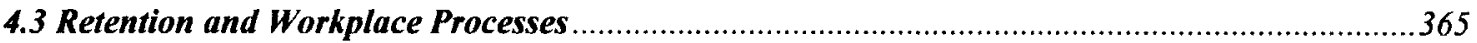

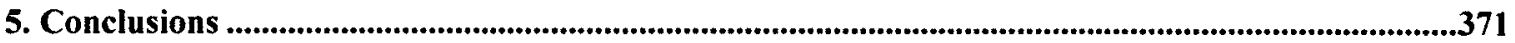

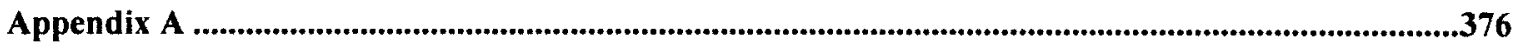

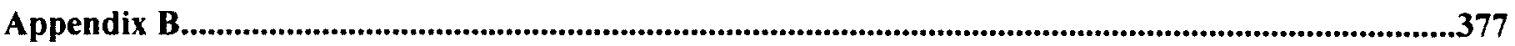

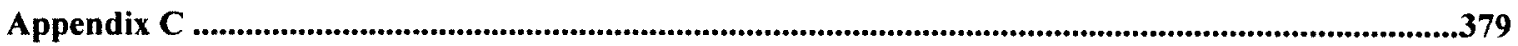

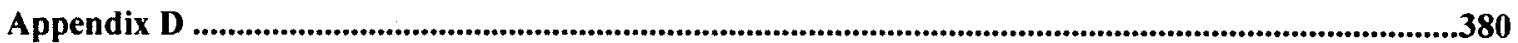

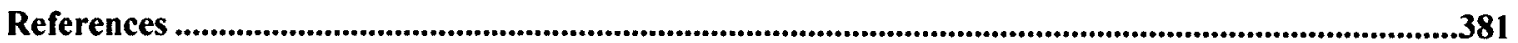




\section{List of Figures}

Figure 1: The stages and processes from potential labour pool to successful employment ...............341

Figure 2: Job application and interviewing selection processes .360 


\section{List of Appendices}

Appendix A

Appendix A 1: In-Depth Interview Theme Guide

Appendix B

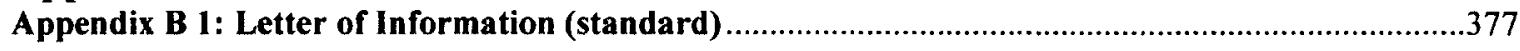

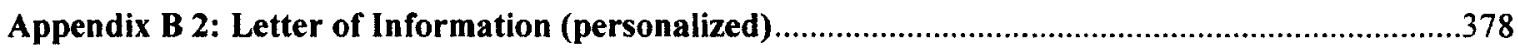

Appendix $\mathbf{C}$

Appendix C 1: A Copy of the Informed Consent Form

.379

Appendix D

Appendix D 1: List of Participating Organizations. 


\section{Introduction}

Improving the economic integration and employment prospects of Aboriginal population groups in Canada, which include North American Indian (NAI), Inuit and Métis, has long been recognized as an important step in reducing the prevalence of poverty among them and in building capacity for future economic growth (Government of Canada, 2009). ${ }^{95}$ Starting in the early 1970s, governments have introduced a range of economic development and skills training programs targeted at Aboriginal community members (Delic \& Abele, 2010). ${ }^{96}$ The challenges and the opportunities identified in the Final Report of the Royal Commission on Aboriginal Peoples (RCAP 1996) have propelled both the governments and corporate Canada, and in particular the resource extraction industries, to reconsider their old ways of dealing with Aboriginal communities and to introduce strategic and targeted policies and programs that stress greater inclusion of Aboriginal people in the Canadian labour market and greater inclusion of Aboriginal communities in decision making processes that pertain to

\footnotetext{
${ }^{95}$ Details involved in defining Aboriginal population groups in Canada were discussed earlier in Introduction to this thesis (Note 1) as well as in the preceding two Essays (Note 8 and Note 43). As defined in this Essay, Aboriginal population refers to the three groups of Aboriginal people that are recognized by the Canadian Constitution and they include North American Indian (NAI), Inuit, and Métis people (Statistics Canada, 2007). Due to the nature of the analysis, no distinction is made here between the single/single-reporting Aboriginal origin or identity and the mixed/multiple Aboriginal origin or identity.

${ }^{96}$ While many provincial and territorial government departments have some form of labour market programming targeted at Aboriginal people, the main responsibility in this area still rests with two federal government departments: the Department of Indian Affairs and Northern Development (DIAND) and Human Resource and Skills Development Canada (HRSDC). These two departments currently fund most of the programs, some more general and related to Aboriginal economic development and other more specific and related to Aboriginal labour force development. In the early years, the programs were reserved specifically for registered NAI and for Inuit people; since the early-to-mid 1980s, however, the eligibility rules were relaxed thus allowing the non-registered NAI and Métis people to have access to some of these programs (Delic \& Abele, 2009). Some details on two of the more specific programs, made available by HRSDC to all Aboriginal people, are discussed later in this essay. These programs are the Aboriginal Human Resource Development Strategy (AHRDS) and the Aboriginal Skills and Employment Partnership (ASEP), recently modified into the Aboriginal Skills and Employment Training Strategy (ASETS).
} 
education, skills development and economic development projects done on or in close vicinity to the Aboriginal land (Canada, 1996; Government of Canada, 2009; 2012).

On the legislative side, there were a number of policy initiatives put in place in the early 1980 s and in the early 1990 s, in response to the findings and recommendations of the Royal Commission on Equality in Employment (Abella, 1984), as a remedy for systemic discrimination in the workplace. ${ }^{97}$ Employment Equity policy, in particular, was promising as it required employers to take proactive steps and to identify and remove or replace workplace policies and practices that may have discriminatory effects as well as to improve the representation of under-represented or designated group members, Aboriginal people being one of the groups (Abella, 1994; Canada, 2012a). ${ }^{98}$ Prior to this policy, employers were not obliged to implement employment equity programs in their workplaces, although provisions for a voluntary implementation were given under certain

\footnotetext{
${ }^{97}$ The Canadian Human Rights Act does not contain much information needed to define the term "systemic discrimination" for in it, it is only possible to discern that race and ethnic origin are two of the prohibited grounds of discrimination (Canada, 2012). The definition provided in one of the Canadian Human Rights Commission reports is more descriptive and more precise. In relation to employment, discriminatory practices are defined to include "unfair treatment by co-workers or managers as well as by the individual employer or the company. Besides discrimination at work, it can include events that happen off the work site or outside of regular work hours if these events occur in the course of employment. It also includes discrimination that occurs in the hiring process. Unfair policies or practices by an employer or an employee organization are also discrimination when they target or have an adverse impact on individuals or groups on any ground included in the Canadian Human Rights Act." The report lists a court case (Pitawanakwat v. Canada, 19 C.H.R.R. D/110) as an example where "systemic discrimination was a factor in [a government] Department's treatment of [an Aboriginal] employee" (Canadian Human Rights Commission, 2011).

${ }^{98}$ In the Employment Equity Act, the four groups classified under the "designated groups" label are: women, aboriginal peoples, persons with disabilities and visible minorities. The term "Aboriginal Peoples" is said to refer to individuals who are "Indians, Inuit or Métis" (Canada, 2012a, p. 1-2). An interesting provision was included in this $A c t$ with respect to the employment of Aboriginal persons. In specifying the procedure to follow when creating new positions, section (d) 7. states: "Notwithstanding any other provision of this Act, where a private sector employer is engaged primarily in promoting or serving the interests of aboriginal peoples, the employer may give preference in employment to aboriginal peoples or employ only aboriginal peoples, unless that preference or employment would constitute a discriminatory practice under the Canadian Human Rights Act" (Canada, 2012, p.6).
} 
sections of the 1978 Canadian Human Rights Act and of the 1982 Constitution of Canada, Charter of Rights and Freedoms (Bakan \& Kobayashi, 2000; Agocs, 2002). ${ }^{99}$

In spite of these undertakings, the rates of successful economic integration of Aboriginal people - employment and earnings being the two key measures — have generally been poor, and for some subgroups of Aboriginal population not much has changed over the years. The gaps in labour market outcomes between Aboriginal and non-Aboriginal Canadians identified in the early academic literature on the topic (Kuo, 1976; Patrinos \& Sakellariou, 1992; George \& Kuhn, 1994; Drost, 1994; De Silva, 1999) persist (Kuhn \& Sweetman, 2002; White, Maxim \& Gyimah, 2003; Walters, White \& Maxim, 2004; Gee, Kobayashi, \& Prus, 2006; Maxim \& White, 2006; Pendakur \& Pendakur, 1998, 2002, 2007), even for Aboriginal people with high levels of education (Pendakur \& Pendakur, 2011).

Likewise, it seems that not much has been accomplished with the Employment Equity policy in place (Bakan \& Kobayashi, 2000; Agocs, 2002; Government of Canada, 2012a). In an attempt to evaluate the effectiveness of the policy in improving the representation of designated groups in the sectors covered by the Employment Equity Act, Agocs (2002), for instance, has found an obvious gap between the policy and practice, concluding that "the results of employment equity policy are disappointing because the policy is not being implemented by employers and not effectively enforced so that there are consequences for employers' failure to comply" (p. 256).

\footnotetext{
${ }^{99}$ The Employment Equity Act was first introduced in 1986 and then amended in 1995. As s a federal legislation, the provisions of the Act apply only to the designated group members working for federal public sector and for federally-regulated employers. Those working for federal contractors in the private sector (employing 100 employees or more) are covered by the Federal Contractors Program (Human Resource and Skills Development Canada, 2012a). Also, some of the employment equity issues, as well as pay equity issues, are addressed in some provinces through their own human rights legislations ( Bakkan \& Kobayashi, 2000; Agocs, 2002; Service Ontario, 2007).
} 
In relation to Aboriginal people, Agocs (2002) reports that, a decade after implementation of the Employment Equity policy, the numerical representation of Aboriginal people in the private sector reached 1.3 percent, which was only slightly over 60 percent of their availability rate, 2.1 percent. In the same decade, (1987-1998) hiring of Aboriginal people in the private sector deteriorated for four years in a row and they were subject to disproportionately higher share of terminations. In the public sector, the numerical representation of Aboriginal people reached 2.9 percent in 1999 but, almost all of that was concentrated in the Department of Indian and Northern Affairs (Agocs, 2002, p.264). ${ }^{100}$ It is interesting to note here that, in one of its recent reports, this Department asserts that "over the past 20 years, there have been significant improvements in the economic outcomes of Aboriginal Canadians. Labour force participation, selfemployment and earned income have all increased significantly" (Government of Canada, 2009, p.2). This assertion speaks directly against the existing research evidence. Applying the latest available microdata, the preceding two essays have attempted to examine the reasons behind the disparities using established quantitative methods of inquiry that are based primarily on the neoclassical economics approach to labour market analysis. Drawing on the institutional economics framework, this essay is set to examine the link between Aboriginal identity and economic success in the Canadian labour market using established qualitative methods of inquiry. The goal is to gain some insights on the effects that institutional and other constraints facing Aboriginal workers have on their labour market outcomes. Given that many of the coefficient estimates presented in the preceding two essays are dependent on the validity of the neoclassical postulations, the

${ }^{100}$ The other two federal departments where Aboriginal workers have some representation are the Correctional Service of Canada and the Human Resources and Skills Development Canada (Government of Canada, 2012a). 
attempt here is to understand the "empirical findings" such as the reported estimates of unconditional gaps in outcomes: that is, to understand the outcomes of the examination of the transition probabilities reported in Essay I, Section 3 and of the bivariate analysis reported in Essay II, Section 4.1.

The qualitative data analyzed in this essay were gathered through open-ended, indepth interviews with key informants from different Aboriginal organizations as well as other informants from other governmental, non-governmental and business organizations working either directly or indirectly in the area of Aboriginal labour force development. The central research question, derived from the paradoxes and puzzles reported in the existing empirical works, including the empirical work presented here in the preceding two essays, asks why some Aboriginal workers experience poorer labour market outcomes than others and why some of them seem to linger in lower and less stable occupational rungs of the Canadian labour market. To what extent can these outcomes be attributed to labour market discrimination, institutional biases or some other factors such as cultural differences in individuals' attitude toward paid employment? This question was examined in the preceding essay (Essay II) using econometric tools and focusing in particular on the measurable aspect of human capital (e.g., formal education); here, the focus is on trying to gain a better understanding of the influence of some unmeasured productivity-related characteristics (e.g., motivation, reliability, and work effort) of workers and on the influence of institutional arrangements.

While the interviews with the key informants of different Aboriginal and nonAboriginal organizations cannot capture the variety of 'lived experiences' of Aboriginal workers in the Canadian labour market, they do provide valuable insights which can 
assist both the design of the future quantitative research as well as the interpretation of the existing statistically-derived conclusions. The comments, provided by the key informants, on public policy implications arising from this examination also add a practical dimension to this essay. This research endeavor, however, is neither exhaustive nor comprehensive but is only a modest step in the direction of pinpointing the importance of and the need for examining labour market disadvantage facing Aboriginal workers in Canada through multiple research perspectives.

The essay is organized into five sections: a brief review of the relevant literature is presented in the following section. The methodology underlying the analysis is laid out in Section 3. Findings are presented in Section 4 while Section 5 concludes the essay. Policy implications, arising from the conclusions drawn in this thesis, are briefly discussed in a separate concluding chapter, which follows this essay. 


\section{Relevant Literature}

As pointed out earlier (Essay II, Section 2), in spite of the long-standing history of the labour market disparities between the Aboriginal and non-Aboriginal population groups in Canada, there exists relatively little research on the determinants of labour market outcomes of Aboriginal workers (George \& Kuhn, 1994; De Silva, 1999; Kuhn \& Sweetman, 2002; Pendakur \& Pendakur, 1998, 2002, 2007, 2011). What does exist focuses almost exclusively on the earnings differentials between non-Aboriginal and Aboriginal workers living off-reserve in urban parts of Canada and draws primarily on the neoclassical economics approach to labour market analysis. Comprehensive studies focusing on the labour market disadvantages of Aboriginal populations living in the North and on Indian reserves are particularly scarce (Pendakur \& Pendakur, 2011).

Overall, the evidence on the sources of wage and earnings disparities is mixed and in some instances contradictory. Using data from one local area in northern Canada, the earliest study (Kuo, 1976) found that, on average, non-Aboriginal workers were earning twice as much as Métis, three times as much as Inuit and six times as much as North American Indians. In decomposing these differences using an early version of the Blinder-Oaxaca decomposition method described in Essay I, he found that most of the difference in mean earnings was due to disparities in observable human capital characteristics such as education and work experience; only 13 to 16 percent of the gaps could be attributed to labour market discrimination and other unobservable factors (Kuo, 1976, p. 397). Over a decade later, Patrinos and Sakellariou (1992) used a national data file, the 1986 Canadian Labour Market Activity Survey, to examine earnings differentials 
between full-time non-Aboriginal men and Aboriginal men living off-reserve. They found, in contrast to Kuo, that "wage discrimination against [Aboriginal men] could account for as much as $59 \%$ of the overall earnings differential" (p.263).

Similarly, focusing on full-time, full-year, off-reserve working age population, George and Kuhn (1994) used the 1986 Census data and reported that discrimination and other unobservable factors account for about 50 percent of the wage differentials between Aboriginal and non-Aboriginal workers. They also found that, ceteris paribus, workers with exclusively Aboriginal origin had much larger wage gaps than those who reported a mixed Aboriginal origin. In examining the 1991 Census data pertaining to both full-time and part-time Aboriginal workers, living on-and off-reserve, De Silva (1999) found that discrimination played a relatively minor role in creating difference in mean outcomes. However, he found that both Aboriginal workers of mixed origin and of exclusively Aboriginal origin were equally likely to encounter labour market discrimination. Using the same data source, Pendakur and Pendakur (1998) found that two thirds of the difference in mean earnings between Aboriginal single-origin men and Canadian-born British-origin men can be explained by the differences in observable characteristics such as educational attainment. ${ }^{101}$

Notably, none of these studies included a comprehensive analysis of labour market outcomes of northern Aboriginal workers, living in the three Northern territories

\footnotetext{
${ }^{101}$ As explained in the Introduction section of Essay II (Note 43), in enumerating Aboriginal population, Statistics Canada uses different methods, one of which includes questions phrased in such a way as to accommodate multiple responses to Aboriginal origin and Aboriginal identity, thus allowing individuals to report multiple origins and to self-identify with more than one Aboriginal identity group. Literature defines those cases as "mixed-origin" or "multiple-response" Aboriginal identity while "single-origin" or "singlereporting" Aboriginal identity refers to the cases where respondents self-identified with only one of the three Aboriginal identity groups: NAI, Inuit, or Métis. The main difference with regard to the term "origin" is in the population coverage, as explained in Note 43.
} 
and four Inuit regions collectively called Inuit Nunangat. ${ }^{102}$ In their concluding remarks, however, Patrinos and Sakellariou (1992) bring up a rather intriguing discussion from the general literature on the topic that suggests that part of the unexplained difference in mean outcomes could be due to the fact that Aboriginal workers might be "traditional" persons engaged in the paid labour market for only as long as necessary to obtain a predetermined fixed sum of wages (p. 264). Although they questioned the soundness of this reasoning, particularly for their off-reserve sample, their explanation was rather problematic as it rested on a flawed premise that "many Indians have abandoned traditional pursuits and have been submerged within a dominant North American 'industrial' work ethic" (p. 264). The descriptive evidence from the Aboriginal Peoples Survey and the existing insights from field research in the northern Aboriginal communities tell quite a different story (Tait, 2008; Lautard, 1982; Stabler, 1989; Elias, 1995; Usher, Duhaime, \& Searles, 2003; Abele, 2006).

Over the past two decades, a number of researchers have emphasized that the strong presence of a 'mixed economy' in the northern Aboriginal communities is not a mere residue of an old and fading way of life but a unique aspect of the adaptation process in which subsistence economy continues to coexist with modern market economy. In this mixed economy model, as Usher et al. (2003) explain, the household functions as a "micro-enterprise" and individuals move strategically between subsistence and market activities, depending on opportunities and preferences (p. 177).

Indeed, empirically testing the dual labour market theory in the northern territories, Stabler (1989) has found that for many Indigenous people, participation in

102 The geographic coverage of the four Inuit regions was described earlier in Essay II (Note 50) and the three territories refer to Yukon, the Northwest Territories, and Nunavut. 
traditional pursuits is a way of "keeping occupied" while "waiting in the queue" for a job in the market economy (p. 830). The empirical evidence presented by Lautard (1982), however, supports other conjectures made by Patrinos and Sakellariou (1992) that relate to low educational attainment, as his findings indicate the possible existence of labour market segmentation, whereby Aboriginal workers, particularly those living on-reserve and in northern Aboriginal communities, are highly segregated in low-skill, low-wage occupations. Theoretical postulations of segmented labour markets were discussed earlier in the Introduction to this thesis.

A question that arises here is, does this evidence of labour market segregation necessarily imply that northern Aboriginal workers are trapped in such jobs? Could it be that, in the face of constraints, some of them choose this employment scenario because it permits them to sustain traditional pursuits in the mixed economy? Propagating the idea of generally weaker attachment to the paid labour market among traditional Aboriginal workers living in rural areas and Indian settlements is not new to Canada, dating back as it does to the fur trading era of the Hudson's Bay Company (Hudson's Bay Company, 1828). This speculation is also understandable when one considers that almost all of the published research on Aboriginal labour draws exclusively from the neoclassical theoretical framework where traditional pursuits are not accounted for in an obvious measurable manner. Present analysis will enrich this discourse with new evidence from key informants knowledgeable about relevant communities. 


\section{Methodology}

To address the central research question of why some Aboriginal workers are experiencing a greater disadvantage in the Canadian labour market, the essay follows the institutional economics approach to labour market analysis (Piore, 1979; 1983) by gathering, analyzing and interpreting the information from open-ended, discoveryoriented, semi-structured, in-depth interviews with key informants working in the area of Aboriginal labour force development. This method of inquiry is particularly well suited for the present analysis as it allows for gathering rich, diverse and detailed information from knowledgeable key informants about sensitive issues such as the 'lived-experience' of labour market discrimination or the seeming incongruence between traditional pursuits and paid labour market activities.

Although an attempt was made to recruit a diverse sample of participants who could offer insights from a variety of vantage points, given the purposive nature of the selection and the nature of qualitative inquiry, the findings reported in this essay are not intended to reflect a generalized depiction of all experiences that pertain to all members of the three Aboriginal identity groups (NAI, Métis and Inuit). Also, the primary criterion for selection of the participants was their area of expertise, not their Aboriginal identity. Hence, the collected information is used primarily to glean new knowledge and describe independent events which form parts of a larger whole, rather than to deduce law-like scientific statements that can be used in behavioural predictions about members of different Aboriginal identity groups (Dugger, 1979). This section describes the process of 
data collection and specifies the method of analysis of the findings that are presented in the following section.

\subsection{Data Collection}

The analysis that follows is based upon knowledge gleaned from 25 semistructured, in-depth interviews conducted with knowledgeable professionals working either directly or indirectly in the field of Aboriginal labour force development. While most institutionalists deem the "participant-observer" technique of data gathering to be the best method (Wilber \& Harrison, 1978; Peore, 1979; 1983; 2002; Wang \& Dobbs, 2008), the in-depth interviewing method is seen as the closest substitute because, after all, "the two go hand in hand, and many of the data gathered in participant observation come from informal interviewing in the field (Fontana \& Frey, 2000, p. 652). The open-ended, in-depth interviewing is regarded as a particularly effective instrument when attempting to gain a deep understanding about peoples' experiences from their own point of view (Fontana \& Frey, 2000; Quinn, 2002; Piore, 1979; 1983; 2002).

A challenge here, however, is that, although most of the participants in this study were Aboriginal workers themselves, with years of experience in the Canadian labour market, the main criterion for their recruitment was their area of expertise in helping to build Aboriginal workforce. Thus most of the interviews revolved around their professional knowledge about what the Aboriginal workers that they have served experienced in the labour market, although many of them talked about their own experience as well. Given that these participants come from diverse communities across Canada, the method still follows the institutionalists' approach to labour market analysis in which the whole idea of conducting such an inquiry is "an attempt to make sense out of 
the labour market problems as the people in these communities experienced them (or at least described their experiences) and describe the labour market as these people saw it" (Piore, 1983, p. 250).

Following this approach, the primary objective of the data collection in this study was to gain a better understanding about the role institutional and other constraints play in the labour market experience of Aboriginal workers as it pertains to their decisions to leave, remain in, or return to the paid labour market as well as their attitudes toward work experience, training, and education as one way to enhance their chances of success in the paid labour market. Central to this inquiry was hearing various perspectives on the cultural aspects and lived experience of labour market discrimination, particularly in relation to access to jobs and equal pay for equal work.

This objective was incorporated explicitly into an open-ended interview topic guide (see Appendix A) which was presented to the participants and was used to direct the interviewing process and ensure that important themes were adequately addressed. ${ }^{103}$ However, at each stage of communication, the participants were reminded that they have a significant degree of control over the process and contents of the interview and were encouraged to express their own views and elaborate on the issues they feel relevant and important, even if those issues were not listed in the interview topic guide. In other words, the participants were asked to tell 'their story' about lived experience of Aboriginal workers as they see it, feel it, and experience it (Denzin \& Lincoln, 2000). This approach was undertaken for two specific reasons: (1) to yield supplementary information that is related to the labour market issues examined in the quantitative part of

${ }^{103}$ The copy included in Appendix A is a generic sample copy that contains more or less all the themes. The actual documents presented to some participants, like the industry representatives, were tailored to their particular expertise. 
the dissertation so as to assist interpretation of the statistical findings and (2) to yield new information that can be used to amend the existing and derive new hypotheses for further research on this topic (Bauman \& Greenberg, 1992; King, Keohane \& Verba, 1994; Fontana \& Frey, 2000; Quinn, 2002; Wang \& Dobbs, 2008).

The initial recruitment targeted the leaders of the six national Aboriginal organizations that were established in the 1960s and 1970s as advocates for Aboriginal issues in general. ${ }^{104}$ An invitation letter (see Appendix B) was sent to each leader explaining the purpose of the study and inviting them to take part themselves or to recommend staff members from their organization who are knowledgeable and are actively working in the research topic area. Most of the leaders responded and provided names and contact information of the experts in the area within their organizations. Upon setting up the date and time for the meeting, the interview recruits were sent an electronic copy of the Informed Consent Form (see Appendix C) and a copy of the interview topic guide to make the interview experience less daunting and to give respondents time to think about what they might like to comment on. During the interview discussions, themes and questions surfaced which these participants could not comment on and they offered their referrals to experts in the area who worked outside of their organizations. These referrals were followed up, resulting in two additional waves of recruitment.

\footnotetext{
${ }^{104}$ These organizations include: (1) Congress of Aboriginal Peoples, which is an organization representing the rights and interests of off-reserve Aboriginal people in Canada; (2) Assembly of First Nations, which is an organization that represents all First Nations citizens in Canada, regardless of their place of residence; (3) the Métis National Council, which represents the historic Métis Nation in Canada (4) Inuit Tapiriit Kanatami, which is the national Inuit organization representing and promoting the interests of Inuit in four Inuit regions - Nunatsiavut (Labrador), Nunavik (northern Quebec), Nunavut, and the Inuvialuit Settlement Region in the Northwest Territories; (5) Pauktuutit, which is the national Inuit Women's Organization that promotes greater awareness of the needs of Inuit women; and (6) Native Women's Association of Canada, which is an organization that strives to improve the social, economic, cultural and political well-being of Aboriginal women in Canada. Pauktuutit and the Métis National Council did not respond to the invitation.
} 
The second wave of the recruitment targeted relatively recently established secondary organizations, all of whom worked directly in the area of Aboriginal labour force development. Some of these organizations were based in postsecondary institutions and some in communities; their common mandate was to deliver targeted employment and training services to Aboriginal people in partnership with governments, educational institutions, and Aboriginal communities. ${ }^{105}$ These participants, recruited using the snowball method, were particularly valuable because they possessed, and were willing to share, their first hand specialized knowledge about various issues facing Aboriginal workers in Canada. Such knowledge is not captured in the statistical microdata. As such, their insights have the greatest potential to enhance the breadth and quality of this study as they can serve as close substitutes for the insights one would receive from talking with the workers themselves.

Finally, the third wave of the recruitment targeted specific industry sectors and different union organizations. Eliciting insights from these sources of information was deemed important because of the projected labour shortages in some of those industries and the institutional aspects in the unionized workplaces. Invitation letters were sent to leaders and relevant professionals in all major union organizations and in the primary industry sectors such as mining and construction as these two sectors have traditionally

\footnotetext{
${ }^{105}$ These include organizations such as the Aboriginal Human Resource Council (AHRC) and similar organizations that use targeted programs to help create career opportunities for Aboriginal people and to facilitate their full participation in the Canadian economy. Some of these programs are funded by government and they fall broadly under the Aboriginal Human Resources Development Agreement (AHRDA) and Strategy, the latest one being the Aboriginal Skills and Employment Training Strategy (ASETS), which is designed to provide quality training and employment services to Aboriginal people. The unique aspect of the ASETS program is that it links skills training to labour market demand and is focused on end results (Human Resource and Skills Development Canada, 2012).
} 
contained largest concentration of Aboriginal workforce. ${ }^{106}$ News reports and official websites for these organizations were consulted in gathering the contact information of these representatives and the potential participants were contacted in two stages: (1) in the first stage, the representatives were provided with a brief description of the research topic and invited to participate in the study; and (2) in the second stage, those who agreed were recruited and the interviews were conducted at a predetermined time and place convenient for them.

Each recruited participant was asked to set aside about 30-45 minutes of their time for the interview. The actual interview times ranged from 40 to 150 minutes. With the respondents' permission, all interviews were fully tape recorded and some written notes were taken to describe the setting and the nonverbal features of the interaction (Fontana \& Frey, 2000). Beforehand, the respondents were given an option to decline commenting on any questions they thought unsuitable and to add any comments or themes they thought important. This provision seemed important to most participants as it allowed them to converse freely about their areas of expertise without worrying about potential consequences that could arise for their organizations from a disclosure of sensitive information. Although they all had a copy of the interview topics and the question sheet, none of them used it as a strict guideline for answering questions. It seemed that each participant had their own story to tell; as in Piore (1979), I found that the "[i]interviewees used my questions as an excuse for telling their stories" (p. 560), which is exactly what gives the added value in this type of analysis of the topic at hand.

\footnotetext{
${ }^{106}$ The unions representatives were in position to discuss issues pertaining both to private and to public sector employees and the industry representatives were experts working in the area of recruitment, employment and retention of Aboriginal workforce as well as in the area of corporate social responsibility and government and Aboriginal relations.
} 
Twenty-five open-ended in-depth interviews were conducted, either face-to-face (13 interviews) with professionals at their employment premises or over the phone (12 interviews) spanning the period of May 7, 2012 through July 12, 2012. Nineteen participants were Aboriginal identity persons (11 First Nations; 3 Inuit; and 5 Métis) and the remaining six were non-Aboriginal identity persons. A slightly larger number of participants were women (13 participants) than men (12 participants). Four participants were working in the North with Inuit and First Nations workers in remote fly-in communities and the rest were either located in the South or were serving southern Aboriginal workers in urban and rural areas. Six participants were either living onreserve or were serving the needs of the on-reserve Native workers, both in the southern and northern parts of Canada. Two representatives, one male and one female, were speaking on behalf of the national Aboriginal youth organizations. A great majority of participants had worked in the area of Aboriginal labour force development for over 20 years, in different positions and different organizations, which enabled them to reflect on a diverse series of observations and experiences.

\subsection{Analytical Method}

The analytical procedure in this essay follows the interpretative approach (Piore, 1979; King, Keohane \& Verba, 1994) in which the verbatim transcripts of the interviews were examined and used to relate the respondent's point of view, feelings and perspectives on the examined issues. The initial step involved careful organization of all of the collected information. Prior to the analysis, all of the interviews were fully transcribed into written text, verbatim, along with the description of the process by which 
the data were generated. ${ }^{107}$ As emphasized in the literature, this procedure is necessary for maximizing efficiency of data use and, more importantly, for assessing the validity of derived descriptive inferences (King, Keohane \& Verba, 1994; Silverman, 2000; Quinn, 2002).

While the analysis and interpretation of qualitative data are sometimes seen as conceptually separate processes, they both entail disciplined inspection, creative insight, and careful attention to the objectives of the study while, at the same time, upholding research ethics (Bauman \& Greenberg, 1992; McLellan, MacQueen, \& Neidig, 2003; Damianakis \& Woodford, 2012). In the analytical process, where data reduction was essential, discipline was needed to carefully organize the transcribed text description in a way that made it manageable for interpretations. This involved bringing order to the interview data and organizing what was there into patterns, categories, and basic descriptive units by considering words, tone, context, internal consistency, frequency, extensiveness, intensity, and specificity of responses and key ideas (Piore, 1979; 1983; 2002a; Silverman, 2000; Quinn, 2002).

To manage the collected verbal data effectively, two key strategies were employed concurrently. The first strategy involved conducting an inductive, thematic analysis of different respondents' responses in order to identify emergent themes. At this stage, the comments that speak to specific pre-determined themes or themes that emerge in the course of an interview were organized together, regardless of the question they relate to. The second strategy involved organizing the respondents' responses as answers to certain questions. At this stage, notes were recorded as 'answers' to specific

${ }^{107}$ The verbatim transcripts comprise 193 pages of single-spaced unformatted text. 
predetermined questions that were initially hypothesized to be important. Unprompted repetitions of certain themes and answers to specific questions were taken to signify evidence of their importance (Fontana \& Frey, 2000) and are discussed in detail in the interpretation section. The observational notes from my 'research diary' were also examined.

Following Quinn (2002), the interpretation of the extracted information entailed attaching meaning and significance to the analysis, explaining descriptive patterns, and looking for relationships and linkages among descriptive dimensions. The heterogeneity within the sample required paying close attention to the possible influence of the variations in views and priorities. For instance, the information pertaining to respondents of a particular Aboriginal identity group was examined and compared to that of the rest of the respondents. Geographic considerations were addressed in a similar manner by comparing the responses of participants serving the labour market needs of Aboriginal people living in the northern and southern parts of Canada. Atypical cases were noted and questions were set aside for further investigations.

Using direct quotations from the raw interview data, the research findings are discussed in light of the existing research and theory on the topic. Because the interviews involved open-ended conversations, these informed professionals were able not only to comment on the viability of certain concepts that have surfaced in the secondary literature but also on their applicability to the situations that these informants understand, thus making a great contribution to the existing understanding of the issues being examined. The interpretation of their contribution is based on the point of view of the target population. In other words, the interpretation is presented in a way as to convey the 
respondents' knowledge on how Aboriginal workers "see the world, how they define the situation, or what it means for them" (Quinn, 2002, p. 335).

To ensure confidentiality of the participants, an approach similar to Schmidt (2010) is adopted whereby the direct quotations are referenced with codes that are linked to the sequential interview numbers and the group identity of the respondent. ${ }^{108}$ Thus, to cite various respondents, codes were referenced indicating (a) the group identity of the respondent (FN = First Nations; $\mathrm{I}=$ Inuit; $\mathrm{M}=$ Métis; and NA = non-Aboriginal identity) and (b) the page number of the verbatim transcript where all other details of the discussion are contained. Also, unless deemed necessary, the names of workplaces, professions and any other identifying names were dropped from direct quotations and replaced with a neutral word or with a dotted line in order to preserve confidentiality of the respondents whose interview excerpts were included in the findings section.

\footnotetext{
${ }^{108}$ This does not imply that respondents' group identity is the most important characteristic about them; with respect to some aspects of the analysis, region of residence or institutional position can be just as important, if not more important, than the group identity. However, since understanding the obstacles and constraints facing different groups of Aboriginal workers (NAI, Métis and Inuit) is of primary interest here, keeping track of that perspectives can be informative. Also, in the discussions of findings, clear indications are made with respect to main indicator of place of residence (on-reserve and off-reserve) for NAI group and for the great majority of Inuit the region of residence (North) is implied in the indicator of group identity. Finally, it is important to have some idea of how opinions differ between Aboriginal identity respondents and non-Aboriginal identity respondents.
} 


\section{Findings}

The qualitative inquiry in this essay was guided by seemingly simple research questions that asked why is it that, despite the existence of a number of strategic employment and skills training programs and policies, gaps in the labour market outcomes between Aboriginal and non-Aboriginal people persist. Why is it that some Aboriginal workers continue experiencing poorer labour market outcomes than others and why is it that some of them seem to linger in lower and less stable occupational rungs of the Canadian labour market; is it the labour market discrimination, institutional biases or some other factors such as cultural differences in individuals' attitudes toward paid employment that are contributing to such a situation? To quote one of the many participants:

it's all of the above, everything you mentioned comes in the play ... you know the challenges are different from one site to another, but I think they can include everything that you've mentioned. (NA, p. 63)

Most participants gave quite elaborate responses and discussed a number of specific barriers and challenges standing in the way of full participation and employment success of different Aboriginal workers. The challenges discussed pertained both to individual and to societal conditions and they seemed intertwined thus precluding their discussion here along those two dimensions. Also, differences of opinion on some fundamental points among the respondents were obvious, thus making it even more impractical to discuss the findings in such a manner.

To capture most of the common themes and the divergences in opinion that surfaced, the presentation of findings here is organized in such a way as to illustrate 
institutional and cultural constraints, both on individual and on societal level, at three main stages: (1) entry into the labour market, (2) successful employment, and (3) retention and career progress. A simple portrayal of the processes involved along these three stages is depicted in Figure 1, which will be used to guide the presentation.

\section{Figure 1: The stages and processes from potential labour pool to successful employment}

finish line
retention and promotion

labour force
recruiting and hiring processess \{

labour force
recruiting and hiring processess \{

starting line
entry into the labour market

starting line
entry into the labour market

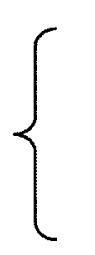

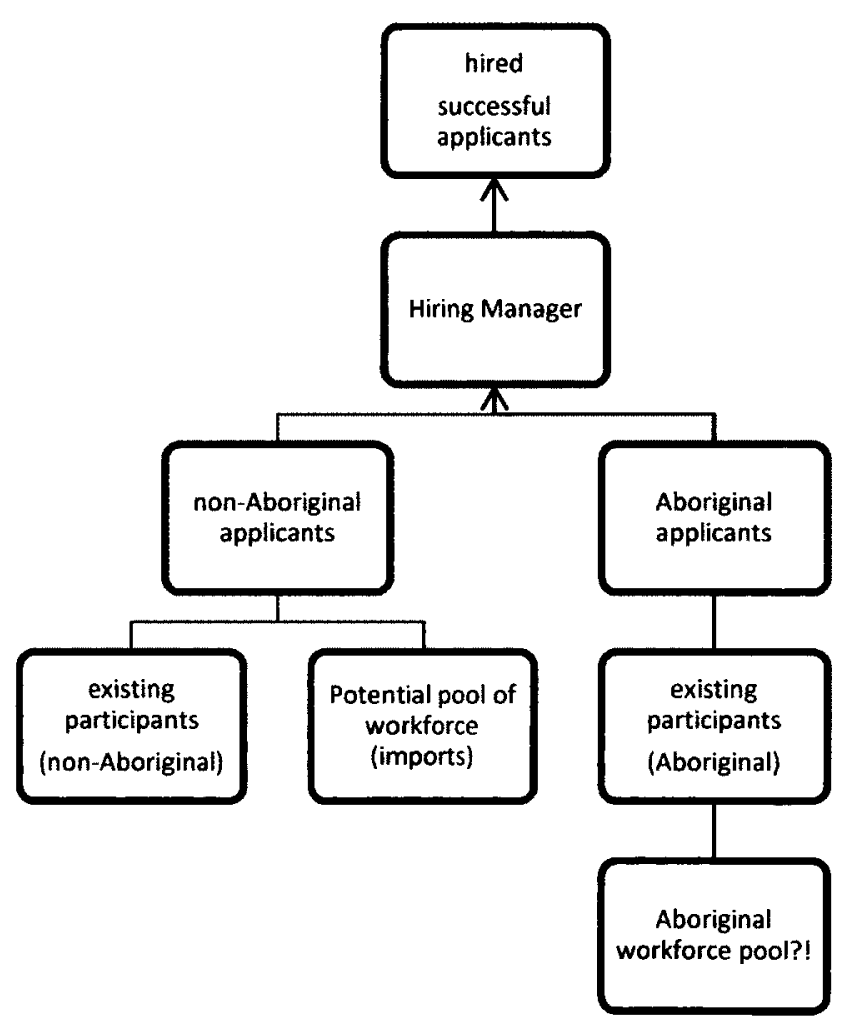

Overall, the findings suggest that many paradoxes and puzzles related to the mixed results observed in the empirical literature can be better understood once the legacy and the aftermath of colonialism and related institutional constraints, especially those pertaining to investments in human (and other) capital in Aboriginal communities, are taken into account. Educating the public, including employers and particularly 
managers, on this and assisting the current generations of Aboriginal peoples, both on an individual and on a community level, in the recovery process and in the reversal of the effects of colonialism is one of the key policy suggestion arising from this analysis.

Details on these, as well as on other issues, are discussed in the following three sections pertaining to the three stages depicted in Figure 1. Systemic discrimination, as defined earlier in the introduction to this essay (Note 95), was reportedly present at each of the three stages, although not all of the participants used that label in their expressions; some openly hesitated to call it discrimination, opting instead for "negative stereotypes" or similar labels, but a closer examination of their discussion reveals that they were in essence describing the same phenomenon.

Hence, the discussions on the labour market discrimination that follow in the three sections pertain primarily to the concept of systemic discrimination whereby Aboriginal workers are reportedly subjected to discriminatory and unfair practice from the side of their co-workers and managers as well as from the side of employers, either in public or private sector, in terms of setting up rules and procedures that consent arbitrary exclusions of Aboriginal people in relation to access to jobs, hiring and career promotions. The discussions also touch on the unequal pre-employment conditions of Aboriginal people, as compared to non-Aboriginal people, that resulted from systemic discriminatory practices from the colonial time period, which still affect virtually all aspects of economic integration of Aboriginal persons, starting with their access to education and employment opportunities to their ability to overcome barriers in the workplace. The recommendations from the Abella Report (Abella, 1984) emphasize this aspect of inequity as important in the removal of systemic barriers to employment equity. 


\subsection{Starting Line and Work Readiness}

Out of the three stages depicted in Figure 1, the insights gained on the multifaceted complexities of barriers involved at the first stage, the "starting line" stage, are perhaps the most compelling. The existing literature generally acknowledges the differences in socioeconomic background of different Aboriginal groups and highlights that the levels of impoverishment differ across Aboriginal communities but the extent to which this background has a bearing on the prospects and labour market outcomes of Aboriginal people is generally not well understood. Most participants placed strong emphases on this point, although their perspectives were not entirely similar.

An example from the discussions of the projected labour shortages can serve to illustrate this point. In discussing the challenges and opportunities in relation to the projected labour shortages, recent policy literature and government reports have coined a term, an "untapped Aboriginal labour pool," that is now being circulated and taken as granted in the media and among policy makers ("An Untapped Labour Pool," 2008). Indeed, several participants in this study have used the term as given when expressing their positive expectations in relation to the new employment opportunities that are coming along for young Aboriginal people (M, p.3; NA, p.60), and in particular for the Aboriginal youth (NA, p. 42; FN, 137). It seems that the age structure of the Aboriginal population is being taken as a sufficient and reliable indicator of the existence of the source of potential labour. As one of the industry representatives explained,

[changing demographics] is precisely why the industry is perusing quite aggressively the opportunities with Aboriginal people because they are one of the most accessible pools of labour - they are there, you don't have to look for them elsewhere. (NA, p.62, emphasis added) 
There is, however, a serious problem with this term, as most participants noted.

Some even expressed doubts about the very existence of the "untapped" pool. Upon careful calculations, one participant concluded that, in his mind, "it is not an untapped resource; it's a drop in a bucket" (FN, 50). Treating it differently, as many do, can have serious implications:

If you are dealing with [government employment programs administrators] they're gonna tell you that the Aboriginal community is fastest growing community and fastest growing youth and it's an untapped resource ... but, we as the Aboriginal community with new Census data we are roughly 4 percent of population which is little over a million people so we have 35 millions and we have million and a half so if we are growing at 3-4 times even 7 times, what's that gonna resolve in?! ... because this [non-Aboriginal] community here is growing at 1.5 percent so times 35 million - so how can this group say that this is an untapped resource, because you'll always be outnumbered, right, and they are bringing in labour, right, so even if it grows at 10 times, it's not gonna make any difference - you can't have the resource that's untapped, if you can't utilize it and the numbers are there to support it. It's like this mine that they're never sure there's resources in that mine but if it's too expensive and if there's not enough in there, they're not gonna mine it, they're gonna leave it, right, it's not profitable. (FN, 50)

Other respondents were generally more optimistic, yet while not directly questioning the term "untapped labour pool," they placed strong emphases on its restrictions. In the words of one of the participants, who was discussing the general lack of essential life skills and general struggles of the off-reserve Aboriginal population, and in particular of the recent migrants from reserves and from remote rural areas:

if we take that about 48 percent of the population is under the age of 25 , and that's your workforce, and a good deal of them are located in smaller urban areas close to major resource development projects, they're a natural labour market pool for the economy - the issue is, what do you need to get them to that place. (M, p.78)

Likewise, in discussing the population growth figures and the conditions of the on-reserve Aboriginal population, one participant acknowledges the existence of the "pool" in a similar manner and warns that serious investments are needed in order to 
actually be able to call this growing population potential labour force and to assume their willingness to work and especially their readiness to work:

you're looking at this huge potential labour force pool but you need to make sure that they have access to good housing, to good drinking water, to affordable foods, 'cause again, when you look at some of these northern communities, especially in mining areas, it costs like $\$ 5$ for a head of lettuce. (FN, p.24)

In short, there was consensus among participants on the need to be "realistic" about what is being advertized to the individuals, to the communities as well as to the employers involved and the need to avoid giving "unwarranted expectations" that could create unsatisfactory results for all involved. This, in turn, would serve to exacerbate the historic misunderstanding and perpetuate the existing stereotypes about Aboriginal people's commitment to paid labour market and their (in)ability to succeed in it.

As the conversations with different participants revealed, most of the Aboriginal communities, including many of the off-reserve (M, p.10; M, p.77; M, p.101) but especially the reserve and the northern remote communities (FN, p.94; FN, p.167; FN, p.29; I, p.115; I, p.145; NA, p.161; I, p.186) are dealing with a myriad of social and other issues that act to seriously limit participation in the labour market. Most of the issues are held to be direct outcomes of the past government forceful assimilation policies such as enfranchisement and residential and federal day school system and these facts by and large are not known to the general public, "you know, the folks that go to work every day, [so there is an urgent need for] educating workers about the historical exclusion of Aboriginal people" (M, p.8) and in particular about "how deep and severe the impacts of the residential schools have been and continue to be, and even though it's been ten years since the last one closed, there's still going to be generations that will be affected" (M, p. 
10). As this latter participant observed in his two decades of work on inclusion of

Aboriginal workforce, in general,

there is no recognition by non-Aboriginal persons of the privilege that they carry. It reminds me of the saying, you know, 'you didn't hit the double, you were born on second base' and without recognizing that you contribute to the oppression of people who don't come from that privilege. (M, p.13)

This particular point, in fact, speaks directly to the principles for achieving employment equity set forth in the Abella Report (Abella, 1984) where inequity is seen as a systemic condition which is often, but not always, based on intended discrimination. In the Abella Report, ignoring differences or refusing to accommodate them is deemed equivalent to denying equity (Bakan \& Kobayashi, 2000).

Ignoring differences that exist between Aboriginal and non-Aboriginal communities, however, is a common practice among the general public. As one participant observed, in hearing about high unemployment rates among Aboriginal populations, most non-Aboriginal Canadians are too quick to conclude,

yeah, they just want to stay home and collect welfare. Well that is not the case; it's opportunities and, you know, when you look at the reserves that have been in the news [Attawapiskat], I mean they are suffering such ills where it's the survival what keeps them busy every day. (M, p.5)

[general] population believes in the myths and the stereotypes that have been said about Aboriginal people; they just catch phrases like, you guys don't pay taxes, just 'get over it' attitude and the government counts on that, how else can they explain the crises in the Attawapiskat that was in the news for a month straight. (M, p.13-14)

The sense of frustration and despair was particularly noticeable when respondents were discussing the issues related to the delivery of education, including the basic literacy and skills training, and the chronic underfunding of the essential programs and service, especially in the on-reserve and in the remote communities. Even the respondents with 
otherwise quite optimistic views about the general progress of the current policies tending to the Aboriginal issues expressed worries and concerns about the effect this can have on the efforts in place to mend the historic relationship. In the words of one respondent,

we are convinced, we are absolutely convinced [that] their [government's] current plan, their current assimilation plan includes making life so unbearable on reserve that people just move into cities. (M, p.13-14)

Off-reserve and out-of-the-North as well, there appears to be growing bewilderment - on one hand, the underfunding on reserves and the lack of adequate financial opportunities in remote rural areas put pressure on Aboriginal people to migrate from reserves and from remote communities into urban areas yet, on the other hand, the amount of funding assigned for service delivery for this growing population is minuscule in relation to the needs. For some off-reserve service delivery centers, the amount funded is still being calculated using the "1984, 1986 dollars; it hasn't changed, there's no cost of living increase [and the rule as to who qualifies] hasn't changed" (M, p. 79).

This creates serious situations for the service delivery centers where decisions have to be made to cut down on the amount or on the quality of the offered services, which necessarily causes great confusions, with far reaching consequences, for the individuals who are dependent on these services. As one respondent, serving the needs of Inuit living in urban areas, explained, many young and enthusiastic Inuit who moved away from the North into major centers to pursue employment or postsecondary education get either turned down because of the lack of funding or their education and skills training get interrupted because of sudden cuts in the already-approved funding: 
why would you wanna cut somebody off who's already in post secondary school, I mean they have one or two more years left? What's going on here? In the application process they know it's a 3-year program so you think they'd be committed to sponsoring somebody 3-years to see them through, you know, they believed that they were gonna be funded for the 3 years when they applied, so long as they pass they will continue getting funding and they're passing so I don't understand why they're not getting funding anymore. (I, p. 190)

A number of the respondents serving the urban Aboriginal population stressed these funding issues to be essential for enabling or disabling them to effectively address the needs of the population groups they serve, including the group of urban Aboriginal youth. Perhaps this important point was overlooked in Kuhn and Sweetman (2002) whose empirical examination of the labour markets success of different Aboriginal groups led them to conclude that the best solution for northern and for on-reserve Aboriginal people lies in relocating their residence from reserves and northern territories to major urban centers (p.332). The professionals serving the needs of Aboriginal people in urban centers give no indication that the solution is that simple.

For well over a decade now, the urban Aboriginal population has been struggling with Canadian governments just to ascertain who in fact has the jurisdictional responsibility over them, and especially over First Nations people living off-reserve, and who is responsible for such underfunding (FN, p. 126; FN, p.178). As one of the national leaders explained,

for every $\$ 8$ dollars spent on reserve only $\$ 1$ dollar is spent off-reserve because the [federal] government is saying we don't have jurisdiction for those of us who live off-reserve, only those on-reserve; they are saying we are provincial jurisdiction and then provincial government says, no, you are federal jurisdiction; yeah, so we fall through the cracks. (FN, p. 126)

Living conditions of the on-reserve population, as one participant pointed out, are "the matter of public knowledge" (M, p.2), yet here too, new insights have surfaced that 
might shed some light on the observed large differences in the school completion rates at the elementary and at high school level between Aboriginal and non-Aboriginal

Canadians and among different Aboriginal identity groups. According to one participant, unlike non-Aboriginal Canadians, some Aboriginal Canadians never get a chance to go to school!. During our discussion about eligibility of First Nations youth to apply for government-sponsored skills training programs and for advertised jobs in the area, the respondent asserted the fact that many of them are not eligible because they have not completed grade 12 , which is often the key requirement. On my probing about the extent to which funding is the factor (e.g. communities not being able to offer programs and services to keep the youth in school and to graduate them), the respondent replied: well, you gotta step back and think of access to schools first before you even worry about how good the school is; just understanding that the majority of Aboriginal people on reserve live in isolated communities and [in those communities] they are not treated like a regular Canadian. Every Canadian kid doesn't even question their right to go to school, to high school, right, you just go to high school, it's just a given in grade 8 you go to grade 9 now - it's given, you don't even think of it. For First Nations children, half of them, they have to be selected by their community and screened, so if they have only eight to ten kids, they might send two of them to high school [and who's screening them?] pardon, oh well their own communities because Indian Affairs will not fund every single kid to go to high school so they reason maybe you will make it so we will sponsor you to go, because they have to be sent out of their communities. So when people, you know, have this image about why Indians are not going to school it's because some of them do not have the opportunity to go, right, it's just the way it is, so and I think that's a big eye opener because if you can't get into high school then you can't get your grade 12 and there is no way you are gonna take those next steps in terms of employment. (FN, p.95, emphases added)

Indeed, that is a big eye opener and it certainly warrants further investigation.

All of the other respondents, including the industry representatives, also urged that addressing the issues involved with the school system on reserves and in remote Aboriginal communities is the key to effectively address many of the entry barriers 
experienced by many Aboriginal labour force participants. For a variety of reasons, funding being one of the major one, schools in many of those communities have very limited resources to provide the necessary conditions to keep the kids in school and teachers in their positions. Also, in most of those communities, the great majority of kids live in extremely overcrowded homes and are often victims of various forms of abuse. Substance abuse and suicides are common occurrences in these communities.

Being faced with such harsh living conditions, for many parents the school simply is not a priory; they do not have capacities to help their children with school work and they do not even have a quiet place to give them at home to complete homework: As one respondent, speaking about Inuit in one of the four Inuit regions, put it: "it's like, how can you study if there's 15 people in a 3 bedroom house, and if your bedroom is a closet, how can you, you know, lead a normal life" (NA, p. 161). Faced with those living conditions, even the kids who stay in school do not develop the skills and abilities that are necessarily comparable to those of their non-Aboriginal peers living in urban centers.

In discussing various methods the resource extraction industries are applying in trying to reach out to local Aboriginal communities, one participant urged that there is "an important role for government [to play] in helping deliver specially that essential [literacy] and skills training and work readiness training so that [project] participants are then ready to receive the more technical training that allows them to do the work" (NA, p.61).

Respondents working in the area of labour force development in the northern Aboriginal communities expressed similar views. As one participant, working on the development of labour force for the construction sector said, "we are working really on 
their basic [math and reading] skills just to increase their employability" (I, p.146). Similarly, a respondents, working on the development of labour force for the mining sector urged that governments "do less skills training and more education upgrading" (FN, p.171) so that the mining companies can take over and train the workers into their chosen fields. In elaborating on this statement, this participant stressed that, most of the people [we serve] have only grade 10 education. Some people have completed grade 12 but they dropped out Math and English in grade 10, so when they seek employment, it's difficult for them to find and keep the job because they don't have solid grade 12. People that come from the remote [fly-in-fly-out] communities, if they have completed grade 8 in their home community are generally functioning at about Grade 5 or Grade 6 education level. (FN, p.167)

In the southern parts of Canada, respondents also stress the need to address the education system, although they speak about more general issues. While optimistic about the potential of the existing strategic skills development programs, they all expressed worries that it might just be a temporary "patch" and urged governments to address more fundamental issues pertaining to the education system. In the words of one respondent,

absolutely, that's the key, the education system, especially as it relates to First Nations people ... and I mean, there is a number of issues behind that issue, you say educational attainment, well that's just not the case of people not finishing school, I mean the teachers leave, the quality of education especially in First Nations communities, I mean there is a number of factors that influence that educational attainment; it's not just, you know, I wanna quit school; it's the quality of education, the consistency of teachers, the consistency of access to resources, you know, to support education - all those elements are part of that education gap and so it's not just a small issue, it's a huge issue. (M, p.2)

As stated at the beginning of this section, the respondents gave quite elaborative responses on a number of issues - the issues discussed in this section are only bare essentials. However, for the purpose of this essay, they provide some important insights that can be used to enlighten the discussion on the puzzles reported in the empirical studies on the topic. To many of the participants, the perplexing question of why we 
observe such disparities in the distribution of labour market disadvantage between Aboriginal and non-Aboriginal Canadians (when we observe mean differences in outcomes at the finish line in Figure 1) does not seem to be perplexing at all. In the words of one of the experienced respondents:

many of them simply can't make it [to the finish line], they can't, it's like a race. You might be too young to remember but one time long ago when they had a foot race everybody started at the same line. It didn't matter if it was a circle, everybody started at the same line. Then they realized to go in circle they had to stagger the start line because to go in circle, this person [using the rules of geometry to their advantage] is running a shorter distance than this person, all right, so they have those staggered starts so it was fair to everyone, and that's what you see [with Aboriginal people] when it comes to getting a job or getting into something [like postsecondary education programs], it's not a staggered start. (FN, p. 51) 


\subsection{Recruitment and Hiring Processes}

The second stage (the stage labeled "labour force" in Figure 1) where recruiting and hiring processes are observed is where the direct effects of systemic discrimination surface and become most obvious and most easily identified in individual cases. Findings relating to this stage suggest that entry barriers at this stage are embedded into the system in such a way that, as Piore would suggest, they are made look to serve efficiency and effectiveness but their negative effects on the prospects of Aboriginal workforce to secure employment are serious and potentially detrimental to their motivations to make repeated attempts - they are also conducive to the activation and reinforcement of the historic sense of mistrust that exists between the two population groups.

This aspect of mistrust is particularly destructive as it can affect even successful applicants who, having observed it or experienced it, carry that sense with them into often uninviting workplaces (see next section) which have their own sets of processes that challenge the job commitment of Aboriginal workers. In combination with the barriers from stage one just discussed, the barriers at this stage make the preceding quote from the last respondent ever more clear and convincing - they simply cannot make it in.

The processes at this stage are discussed separately for the job search and recruitment part and for application selection part. First, as illustrated in Figure 1, it appears that at this stage Aboriginal and non-Aboriginal workforce (and potential newcomers to Canada) are all at the same level. The findings discussed in the previous section suggest, off course, that that is not the case for most of them - they are made to appear at the same level — but new insights at this stage make it clear that the extent to which that is not the case is actually much bigger than one would have it concluded after 
discussing only the barriers at stage one. For instance, starting with the dissemination of information about jobs available and the recruitment processes, geographic location, social networks as well as 'superior' educational credentials of other-than-Aboriginal workforce put the Aboriginal workforce at a disadvantage at the very beginning.

This, of course, has not been reported by all participants in this study. As in the discussion of findings in previous section, here too differences in opinion (or experience) are present. Industry representatives, in particular, were describing a number of ways resource extraction sectors are trying to engage Aboriginal communities and to educate the community members not only about the availability of jobs but also about the variety of jobs within different fields, providing brochures and in some instances reaching out to schools and to children as young as six-graders (NA, p.45). This, however, is not necessarily a victory to celebrate as such targeted recruitment efforts may entice young Aboriginals to leave school earlier than they otherwise would. ${ }^{109}$

For the First Nations workforce living on-reserve or in remote areas, however, this certainly is the case, according to all participants. Inuit, as well, are faced with very similar challenges, as the participating on-the-site professionals report. One of these professionals, currently working on the inclusion issues of Aboriginal workforce on her own reserve in the South, has had extensive experience working in the North as well and was able to comment on the barriers at this stage that pertain both to on-reserve and to northern places of residence. Speaking first about job advertisements in the print media in the context of the on-reserve residence, this participant stated that,

\footnotetext{
${ }^{109}$ Some companies have become aware of this problem and have developed programs to encourage young people to finish high school. Hoefman (2009) provides some discussion on the experience of the Diavik Diamond Mine in the Northwest Territories. Maxim and White (2006) provide some more general evidence indicating possible "pull factors" at work in their examination of differences in the labour market outcomes between Aboriginal and non-Aboriginal school-leavers.
} 
number one concern is, and that's not just here [in my reserve community] but in a number of other First Nations communities, is that major recruitment, and by this I mean federal, provincial, and municipal organizations of government, do so in national daily newspapers - there is no First Nation in this country that gets a major national daily papers delivered in their community. So that's the first issue; you know, how you see large recruitments by companies and you open the Globe and Mail, the Post, those papers don't exist in First Nations communities - we are lucky to get any, even a local smaller daily newspapers that you can go and buy at the store; most First Nations communities don't get any at all. (FN, p.94)

Speaking on the same issue of access to print media where 'good' jobs are advertised, only in the context of northern communities, this respondent expressed similar statements - the print media is not accessible to remote community members:

I've worked in northern small isolated communities ... and in order to get it [a copy of a daily newspaper], you needed to have someone that you know to buy it and send it directly to you through the mail system because they just don't do that, and so that's the first place to look for job, the newspaper. (FN, p.94)

While important, this traditional source of job advertisements may not be as essential, particularly not to the young job searchers, because nowadays most employers make their job posts available over the Internet, and according to most respondents, Aboriginal people are "well-connected" and they use the technology on daily basis (M, p.3; FN, p.132; FN, p.180). However, according to this respondent, the "well-connected" applies only to urban off-reserve residents. Access to an alternative, the on-line job search method, is even more limited than the access to print media, both in the reserve and in the northern remote communities:

Very few First Nations community people have access to the Internet; okay, we probably have the most because we are in the Southern [part of Canada] but even in our community, you know, we just these years are starting to get access to the Internet in private family homes. Normally most First Nations communities only get access to the Internet, especially the isolated communities, through their local Band councils that might have computer at their school and someone knows how to turn it on, and that's literally, so the Internet being major place that you go to find a job is just not reliable option for our people; and so you might say, well okay go to the library, well where is the library, you know if you are in a fly-in 
community in the Northern [part of Canada], there is no library there, so for them a lot of those kind of things are difficult. (FN, p.94)

Finally, the effectiveness of the "word of mouth" as a method for job search has slowly diminished in First Nations communities, according to this participant, due to the changes that occurred in the last two decades with respect to how and where employers post their new job opportunities. This particular point speaks the most directly to what Piore was describing in his recent address of the new institutional arrangements in the information-driven economy that replaced the "old rules and regulations," and in particular in relation to the changes made to the established ways of how information flows between employers and workforce (Piore, 2002). As this participant explained it, one of the biggest issues with government is that, even if it's their internal posting like back in the day when they used to do job postings and they would send it to like every place, government place, where people work and they would say, hey there's new jobs available - they don't do that, they've gotten rid of paper so you don't get that extra thing and even if I knew someone who was looking for a job in that field and if I have seen that posting I might have made them aware of it, that doesn't happen anymore. And our people, even when they do apply for jobs are having great difficulty lacking some of the computer skills to do a good job and actually try to get into government worksites, know how to navigate, know how to create and apply for a job. (FN, p.94)

The "word of mouth" and social networks have been discussed by most

participants as by and large the most effective methods of actually securing employment.

In fact, some participants are convinced that it is the only method. As one participant, speaking about his own experience conveyed it:

[my former workplace] is heavily policy posted organization and you're suppose to post every job, and every job that gets posted you have to go through an interview, but I was invited to take a job, [in fact, in the course of my career] more than once [I was invited] to take over a job, the guy just invited me, it's an executive and he said, well would you consider come in and work for me in this job, okay I said, I was in that job for 3 years, this other guy I worked for in [another city] called and said would you consider to come and work for me in [here] - they never even posted a job, he just took me out there [right, so you 
only need the right credentials to get your foot in?] No you don't, you need a network, that's what you need, and that's the problem for being an isolated community and being away and not having a network. (FN, p.58)

Speaking about his observations of the effectiveness of social networks in his

neighbourhood, the same informant offered further details:

I know network, I got these two kids, I live in a little town, all non-Aboriginal community, right, a high school kid in the community, family's there, finishes high school, gets an apprenticeship and is working for the local carpenter guy in the town, right - that's part of the network - do you know how hard it is to get an apprenticeship in this country?! So what do you think an Aboriginal kid would have a chance to get an apprenticeship?! I know another guy, a next door neighbour, finishes high school as well, gets a job in a local shop as an apprentice - how - network, right!. (FN, p.58)

Another respondent, living on-reserve, expressed a similar degree of conviction about the effectiveness of social networks for securing employment among First Nations people:

I know a very few that have, you know, [secured a job on their own]. Most of them would get a job because they had some sort of support system inside their workplace that helped them to get there, somebody inside, and if there is nobody inside, the chances of an Aboriginal person getting inside are pretty small, even if they are working for First Nations. Even here [at my reserve] more and more nonNative people are being hired ... and it's becoming a big issue. (FN, p.99)

Another important insight that applies particularly to First Nations communities is that, not only do the First Nations communities have limited access to information on the available jobs - but local employers also have limited or no information on the available workforce in local First Nations communities. As one informant, working in the area of labour force development in northern Aboriginal communities, most of them being First Nations communities, explained:

Aboriginal communities [in the North] not only don't have the capacity but they don't have the modern technology and the skills to do proper employment counseling; so the Aboriginal communities don't know what Aboriginal workforce they have within the community. I'll give you an example of that; in 
[this local city], there's supposed to be 15,000 Aboriginal people. Trade unions wanted to hire Aboriginal people for the two big construction projects but they didn't know how to get hold of Aboriginal people living in the city; nobody has data for those Aboriginal people. So what the unions would do is they'd call us and ask us or tell us that they're looking for workers and we would refer our people to those jobs - so the 15,000 of Aboriginal people in [that city] miss out on those opportunities; nobody knows about those 15,000 Aboriginal people; where do they live? How do we contact them? What's the education level? What kind of training have they taken and what kind of work are they looking for? (FN, p.170)

Access to information on job availability, information technology and social

networks appear to be less of a problem for the off-reserve Aboriginal population and for

Métis in particular. As one participant explained:

you know I've worked in a core area in the Prairies for a very long time, the information is there, those job-search networks are available and more and more through the Aboriginal Employment Services, through the ASETS, ... there is a network of information and services, the urban Aboriginal organizations and the network of ASETS that are working hard to say to their constituents, their clients their community members here are your options here is the information you will need and, and it is a supply-demand thing; corporate Canada is working hard to say, this is what we need, these are the skills that we need, and they are not just passing those onto Aboriginal peoples, those messages they are sending them to the interlocutors, the mediators the agencies in the middle that are assisting on the ground up front line assisting Aboriginal people to get what they need, the information, the direction around employment and training, and ... you know years ago companies would say what's the greatest challenge - well Aboriginal people aren't connected - and we know that that's almost not true, I mean yes there is some communities that still rely on broadband ... but Aboriginal youth Aboriginal people I work with seniors who where, I thought okay I better sit down at the computer and help them fill out this form - no, they were way ahead of me - so you know, people are connected, so that's not a challenge. (M, p.3)

This, however, is not the case if Métis people leave in remote areas. Speaking on behalf of the same group and from the same geographic area, another respondent also talked about this "connectedness" among Métis people but emphasized more the disparities between the availability and the quality of services for urban and for rural Métis citizens: 
Living in a rural community is much different in trying to access job search tools than in an urban area. In an urban area there should be very little difficulty in accessing job search tools, connecting resource centers and job find clubs because they are very, very easily found in an urban area ... but, living in a rural community you're not going to find those sort of resources available to you; even if you find a resource centre or some sort of minimum level of service available to you, chances are there's not going to be as good of a service there, you know maybe counseling services might not be as strong in a rural area as they are in an urban; you could have difficulty trying to find a local computer, to do job search, you know, it's not a common thing for everybody to have a computer and to have access to the Internet, not at all; and there will be local newspapers, very small local newspapers that may have 1 or 2 jobs posted in them. (M, p.102)

Aside from insights on barriers related to job search and recruitment processes, the insights on barriers related to job application selection processes are quite informative in that they help to shed some light on what appears to be delusion about the power and the effectiveness of the provisions such as "inclusive workplace" or "equal opportunity employer" in helping designated groups members such as Aboriginal people obtain the applied-for job. They also help in revealing the misconceptions that employers (and hiring managers in particular) hold about individuals of an Aboriginal identity, and in particular about those with visible features of their Aboriginality such as First Nations people. Many respondents provided comments on the biases embedded in the system at this stage in the labour force.

To illustrate the key insights on the first point (delusions about 'inclusive workplace' provision), Figure 2 presents a simple diagram, depicting how the same selection process might appear (possibly to an enthusiastic designated group member) and the discussion that follows explains how it actually works in practice. 
Figure 2: Job application and interviewing selection processes

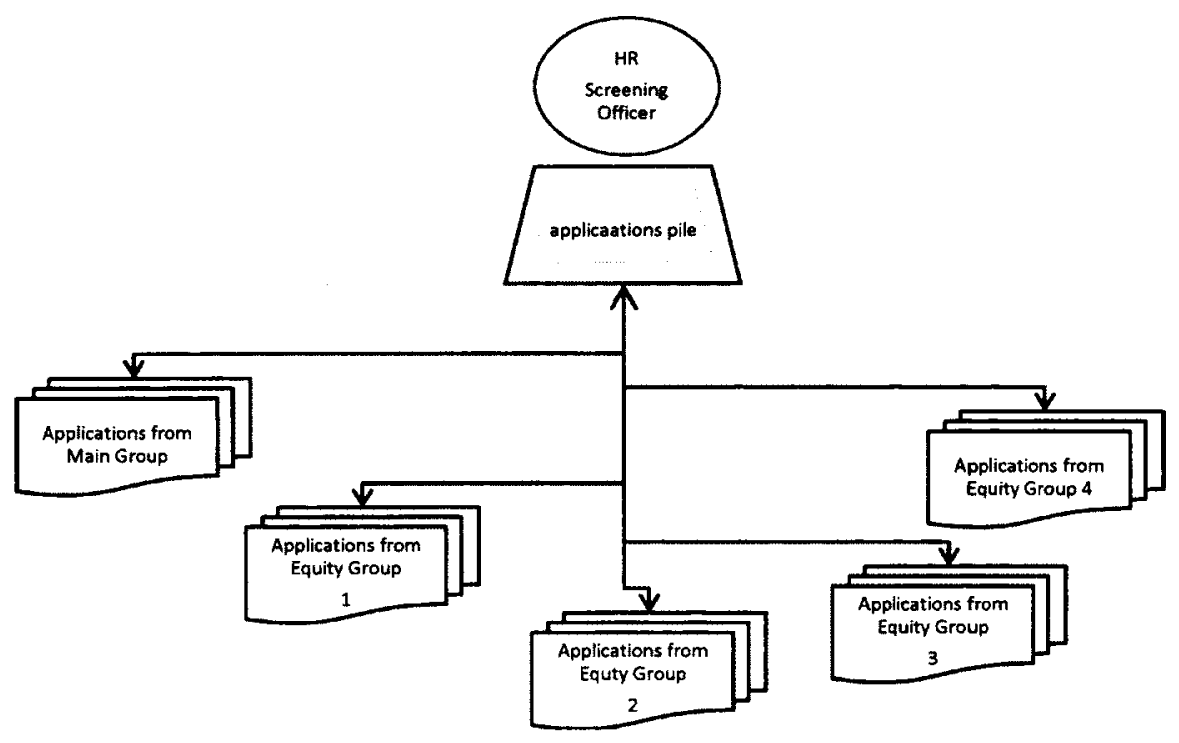

As depicted in Figure 2, when a job opening is advertised, and the employer explicitly identifies itself as an equal-opportunity employer, who welcomes diversity and encourage applications from designated group members, an uninformed designated group member such as an Aboriginal person might think that, because of that statement, his/her chances of being selected are now higher because his/her application will be treated, as that statement leads him/her to believe, - separate from the applications of the other non-designated group members. ${ }^{110}$ In other words, the impression that statement makes on designated group members is that hiring manager will have, at least, two piles of applications on his/her desk to go through and will select potential candidates for the initial job interview from those two piles - this, however, according to all participants that elaborated on this process, is not how it is done in practice; there is only one pile

${ }^{110}$ A federally-regulated employer, the Canadian Press, for example, has such a statement on its website that states:"The Canadian Press is an equal-opportunity employer. We particularly welcome applications from women, members of a visible minority, persons with a disability and aboriginal persons" (The Canadian Press, 2012). Many employers include similar statements, though not as explicit as the one presented here - as discussed earlier, some employers are obliged to adhere to the employment equity code. 
containing all applications and from that pile the hiring managers screens out applicants, generally based on the quality of job-related information that they provide on their cover letters. This essentially puts all applicants in a race where those deemed-to-be the most merited candidates for the job will get to be selected for the interview and the designated group status will not even be considered.

Given the above-discussed disadvantageous background of Aboriginal people in terms of their essential skills and training, and considering that many "don't have a good handle on resume writing or cover letter" (FN, p.169), chances of their applications being selected from that one pile would most likely be small. In these cases, the statement is misleading. Informants interviewed in this study commented that by making that statement "employers are simply making it look good" (M, p.13) and "they're all the same, all the same" (FN, p.51). When they say, we are equal opportunity employer, "all that means is that they will accept applications from many places, that's all it means, that's all - we are following the legislation" (FN, p.55). As one of the, perhaps the most experienced informants in this area stated,

without a doubt, I mean I've worked in a number of different settings ... there is an enormous amount of talk around open, inclusive, equity based workplaces, but the practices are quite shoddy in the instances where l've seen, where blatant violations of equity codes are the norm, not the anomaly. (NA, p. 89)

Several of the people who were interviewed believed that the same pretense seems to be at work with companies' famously advertised provisions such as hiring local workforce or committing to an $\mathrm{X}$ number or percentage of Aboriginal workforce - while there are "success stories" where these commitments have been fulfilled in an effective manner (NA, p.42; NA, p. 61; FN, 174), in most instances, these commitments are "primarily a public masquerade effort to show that there is some sensitivity to equity 
hiring or to local hiring" (NA, p.87) or they are sort of business tactics put forth for practical business reasons and they are not accompanied by any guarantee (NA, p.61; NA, 87; FN, p.56). Even when these companies provide skills training, the individuals from Aboriginal communities who complete it are not necessarily placed into a job position - they have to go through the regular process and "race" with the other applicants for the job (NA, p.61). Finally, not all "success stories" are a success; as one informant pointed out,

if you look closely at whom they're hiring and at what level, I think that the indications will be, the percentages are very small relative to workforce availability, the positions are very junior and they may not be dealing with underlying problems with workplace culture, hostility or xenophobia to actually ensure retention of those workers. (NA, p.87)

These insights are astounding and they merit further investigation. It would be interesting to run triangulation to check how prevalent these practices are. It would also be informative to learn more about in which sectors employers can be criticized in this way. The informants interviewed in this study were knowledgeable about different types of employment but there might be different patterns even within sectors. One indication of this was pointed out by an industry representative in discussing the difference in the ways different resource extraction companies are approaching Aboriginal communities:

what has been happening, because the industry has been working in this area for decades really, which can't be said of most industries, is that there's been a lot of lessons that have been learned over the course of history, a lot of mistakes that have been made and, you know, any mistake is a learning, something to grow from, and I think what I see happening is that new companies that are coming in, the companies who are moving from exploration into development and coming in fresh they bring in a whole new approach to this concept of Aboriginal inclusion so instead of in the past they might be coming to the site or coming to the community and telling people that here we come it's happening and, we are talking really historically, and not really consulting properly and that kind a thing; now we are seeing like years worth of consultation well before development, even in some case even before people set foot on the traditional territory. (NA, p.42-43) 
In relation to the second point (the misconceptions that employers and hiring managers hold about Aboriginal people) the general consensus is that, the more First Nations characteristics an applicant has the less likely s/he is to get a job. With the exception of two industry representatives, who thought that this would not be the case since it would be illegal - all other participants were in strong agreement on this point. Many commented on particular aspects of it and the perceived rationale for it but the excerpt that follows from the interview with one of the informants amply captures it all:

well you see the problem we have is that the Aboriginal community has been promoted as a problem community 'cause everything you read about or see is that the Aboriginal community has issues, right; they have alcohol issue, they have money management issues, they have this issue they have that issue; and, it's always been promoted that way, right - it's never the other side - and so what do you expect then, I mean that's how it's been passed on, it's always been like that for the last hundred and some years since Canada's been here because that's the approach Canada [has taken toward] Aboriginal people - to continually put them in a bad light, right, it's an industry - Aboriginal failure in this country is an industry - it's an industry for the country and you can see it; how much money, how many jobs are there in drug and alcohol, they all started the Aboriginal problem, created it right and that's why I'm saying, the Aboriginal problems are an industry in Canada. (FN, p.54)

Several respondents commented on how this awareness of negative connotations associated with Aboriginal identity often makes Aboriginal workers to choose, if possible, not to disclose their Aboriginal identity when searching for a job in hope to avoid discriminatory treatments and improve their chances of success. In the words of one of the Aboriginal youth representatives:

I guess the more Indian you look, the more chance you have of being discriminated against. That's just how it is. More chance that somebody is going to put those stereotypes on you, that you know, Native people don't work, they're not hard workers, they don't show up to work, let's not hire them, you know people don't identify themselves as being Native for probably those reasons, unless there's some sort of subsidy or something attached to their performance and then it would make sense to identify yourself as an Aboriginal person or if it's 
a job that needs to fill some sort of government mandated diversity clause hiring, you know, somebody [from designated groups], but yeah, the more Native you look, then definitely more chances are that you will be discriminated against. And that's just the way it goes and people don't mean to be racist, I don't think, I just think that's it's just usually embedded with our culture and that's gonna take a big education and awareness campaign on behalf of Aboriginal organizations as well as the government, so yeah. (FN, p. 139)

Similarly, according to one Métis informant, the conditions in urban communities where Métis people live entice them to opt for not disclosing their Aboriginality if the perceived risk of being discriminated against is attached to it. As explained by this informant:

in the urban areas you'll see the stereotype, they don't want to see an Aboriginal person working at the front desk, when they could have somebody else working at the front desk where they think maybe that person is more appealing to the consumer's eye. Or you know there's a reality around, you know, what somebody might consider to be professional and, you know, I mean Aboriginal people are, especially Métis, they're all so different you know you cannot place a stereotype on a Métis person ... a lot of Métis people may not even appear to be First Nations, you know, First Nations appearing to be Aboriginal. They're not visibly recognizable - so, if you're not visibly recognizable to be Aboriginal you may just decide not to mention that you're Aboriginal [so as to improve chances of success?] probably, because if they know you are an Aboriginal person they think oh Aboriginal people are not gonna show up to work, if they do come to work on a regular basis, they're probably going to have to miss a lot because they've got a lot of dysfunction in their family. (M, p. 103)

This arguably effective rational choice on the side of Aboriginal workers to avoid the risk of being discriminated against creates unforeseen additional complexities, and in particular in relation to the assessment of effectiveness of the employment equity programs. As one respondent pointed out:

companies can't measure their success in their [employment equity] efforts if people won't self-identify and people won't self-identify as Aboriginal if they don't feel safe and valued or they are going to, you know, be targeted as the token so it's a real issue. (M, p.8) 


\subsection{Retention and Workplace Processes}

One would imagine that at this last stage (the stage labeled "finish line" in Figure 1) at least for those who made it here, only success stories would be heard - in other words, one would expect to come to this stage and discuss lessons learned so that those at the preceding two stages can adjust their ways of "traveling this road" to successful employment - but that does not seem to be the case for many successfully-employed Aboriginal workers. In fact, this stage seems to be the most intense stage where the discriminatory practices are "lived" and where cultural clashes are common occurrences, often resulting in quits or dismissals. Thus, at this stage many of the seeminglysuccessfully-employed Aboriginal workers get squeezed out or, as some participants would imply, allow themselves to be squeezed out (FN, p.53) even from "well-paid and hard-earned jobs" (M, p.6) and thus the process becomes circular.

Getting to the finish line - successfully securing employment - is not the end of the struggle for Aboriginal workers because at this stage the attitudinal and cultural factors surface, challenging their commitment to the paid labour market. As discussed by all respondents, workplace processes are often harsh on Aboriginal workers and in great majority of instances in clash with traditional way of life. The anti-Aboriginal sentiments are amply and routinely expressed both by managers and by coworkers - steaming from general ignorance about Aboriginal people, in both cases. As one participant explained:

some of the key factors is an ongoing problem with workplace climate, and by that I mean everything from sorts of the overt to covert forms of discrimination and the phobias and hostility towards others, be they Aboriginal or any equity seeking group. That's something that persists as a problem in many workplaces. Alongside that, of course, is failure to recognize and value different world views, which in some cases Aboriginal communities and Aboriginal workers do see the world or the work very differently than sort of Eurocentric ways. So that's 
something that I think is a contributing factor affecting continued exclusion and retention for Aboriginal people in the labour force. (NA, p.87)

An interesting insight that surfaced in discussions with many of the participants is that workplace climate is the result of lack of competence, and more specifically lack of competence in relation to cultural diversity, at the managerial level; employers may well be promoting diversity but if there are no clear communication lines between the frontline managers and the top leadership then the results are not in line with what employers advertise. Many participants commented on the lack of unified vision at the leadership level in most workplaces they were familiar with. In the words of one participant:

we work with companies who would say yes we are very committed to this [hiring Aboriginal people] but then, you know, we are helping them engage, well yes your commitment is at hundred percent level, you want to do your best and do whatever it takes and you are ready to start initiatives and incubate ideas and yet your people [managers], I mean look at the employment equity report! Employers can say, oh yes look how wonderful we are, we hire, you know, now we have 15 percent Aboriginal representation but they are bleeding out the back door because they are unhappy at the workplace so it isn't a welcoming workplace. (M, p7)

we did a very small survey a couple of years ago asking Aboriginal employees why they left their job, why they took that upon themselves to leave, and we get examples every day of why Aboriginal people leave their jobs, good qualified jobs that had been hard won and with companies that worked hard to seek them and things like "well I was the token Aboriginal person" or "I faced discrimination and racism at work." So maybe that companies are committed to hiring Aboriginal people but then, you know, I've heard too often Aboriginal people saying "well when I get to work my colleagues would say to me, well yeah you get that job because you are Aboriginal not because you got your Master's in whatever but because you are Aboriginal; so, there is discrimination in the workplace, and, you know, lots of barriers when they get there, I mean there is just one of our young Indigenous professional... she worked for police force for victim services and she was told that she was too Aboriginal you know at work "Don't be so Aboriginal" and she worked in victim services for the police force so and she said you know yes I have sweetgrass braid in my office and you know she, she had some icons of her culture and she was actually told "you are too, don't be so Aboriginal." (M, p.7) 
The respondents who are helping construction companies in the North hire qualified Aboriginal labour expressed a similar sense of bewilderment. These companies have clear economic incentives to hire Aboriginal workforce, and they do that in general, but they do nothing in regard to retention of the hired workers; on the contrary, they hire managers who openly side with non-Aboriginal workers and thus, in fact, contribute to the Aboriginal workers' decision to quit their job. As one participant noted, a serious problem with the managers (foreman) "is that they have these groups and they refer to them as 'their guys' and 'those Inuit' and every foreman is like that in the construction companies (I, p.147). While the companies may be committed, the managers are not. As this respondent described it:

the construction companies who are on the contract to have Inuit on the worksite, they respond well [to hiring Inuit] and then the response from the higher level construction companies is good as well; they want to have that opportunity, they want to look good to the population, there is a political aspect where they want to be good employer and get the good working conditions on the worksite for the foreman and for the workers but there are other issues - the foreman - well between two workers, Inuit and non-Inuit on the worksite, the non-Inuit know that Inuit have a huge competitive advantage like being in the community so for sure they are way cheaper for the company; this means that they might be afraid to lose their job if someone becomes more efficient, and that's an issue they might be afraid that their brother in law or their little brother or somebody they know won't be able to come if they get too many Inuit working on the site; they see Inuit as under-qualified, people with a lot of problems that are there because they have no choice but to hire them that they are not good as non-Inuit carpenter so there is some resentment [do they express those feelings openly to the workers?] ah, no it's nothing that is verbalized but it's like with non-inclusion, they do it like leaving the Inuit on the worksite, sure the foreman would match the two guys together but if one of them doesn't work as a team at all, the Inuit who is matched with him I mean, finally he would quit, he won't stay. (I, p.145)

Language barriers in some workplaces such the construction sites in the North are reportedly a significant issue as well, resulting sometimes in voluntary severances among 
Inuit workers. The commitment is especially challenged when the managerial support is inadequate or is missing altogether. In the words of the same informant, most of the construction workers in Quebec speak French only, okay, so if they are matched with Inuit who speaks English and Inuktitut, English being the second language, it won't work unless they really, really want to understand each other, and from both sides, Inuit and non-Inuit there are some barriers that are there. Most of the Inuit who are working in construction have had bad experience with people from the South so they expect to be treated badly and when this happens and when they don't get inclusion, there you go, they, they get the validation that they are not welcome and they are quick to leave. (I, p.146)

This issue seems to be specific to the construction sector, at least for this group of Inuit people, and it seems aggravated by the lack of choice these Inuit workers feel. As this respondent explains,

for sure we have to understand that for the Inuit workers the salary being paid in construction, it's really bad, it's not good ... so to go into construction is not really interesting, it's the kind of job that they take when there's nothing else available for them so and this means that we get people who don't have any job anywhere else who get to go in construction so we get the people with social problems so the construction company, the foreman, is quick to conclude, since he is not making effort to keep them on the worksite, well they are easy quitters and for sure to solve the problem with Inuit worker nothing has been done on the worksite to go around those problems and everybody is confirming their impression when they get to work - the Inuit say, well they will treat me bad, I won't be part of the team and there you go you can see this right away they might be sensitive to jokes; the foreman will say when the guy is not showing up, well as expected, okay, so he is not going after him to see what has caused the problem - so yes they quit rapidly because they are going to work in place where they are not very welcomed, they feel unwanted and they don't really want to be there also and we get the result that we have now. (I, p.148)

Finally, cultural clashes were discussed by many respondents, particularly by those working with First Nations and Inuit workers, as being frequent sources of misunderstanding and frequent causes of voluntary severances, even among highlyeducated Aboriginal workers. As one respondent, working in the public sector, explained: 
I work with a group of fairly-educated Aboriginal workers and the turnover here is still really high [and what do you think is contributing to that?] uhm, in part, and this is something I've learned over time, a lot of it has to do with how conflict is dealt within the workplace; some of it is due to inexperienced management, a lot of it is how conflicts erupt, how conflict between coworkers and managers and all that interplay into each other but a lot of what is there is the inability to deal with the conflict because of colonial nature of what we are carrying with us, and for a lot of workers, a lot of them are direct decedents, fairly close to aftermath of residential schools ... and also there is a shortage of Indigenous managers; there is not that many Aboriginal managers in the entire country so when we are dealing with management structure versus employee there's already a lag in how people relate to each other, because there is no cultural, even with all the cultural sensitivity the fact that majority of the managers are non-Native and those that are Native have taken on a lot of the colonizing stuff themselves and so there is a huge conflict in how they deal with each other so a lot of people quit in frustration. (FN, p.16)

In regard to cultural practices, although important exceptions exist (NA, p.44-47;

FN, p.176) most employers that informants came in contact with have no strategies in

place to accommodate the differences in timing of common ceremonial practices such

Aboriginal holidays and other traditional practices. As one respondent explained:

There are certain times and certain days, like our holidays do not match [public holidays], we have our own holidays that are important to us ... and they are seasonal based, when they come they come so it's very different from December 25th so it's a little bit more haphazard, and so if you go to an employer and say you know we've got our strawberry festival or our ceremony at the longhouse, you know, can I go and they'll say WHAT they don't have a clue what it is and it's not Christmas so no you can't and it's not the $1^{\text {st }}$ of July, you know it's not Canada day or it's not Thanksgiving and that's the holidays we have so when you work there you ask for something different then they think you're trying to, you know, take advantage of or change, you know change what they have, so therefore, consequently you are a problem and you know most likely to be a candidate to be laid off. (FN, p.99)

In the North, the clashes occur in relation to timing of traditional pursuits such as hunting and trapping; however, reportedly some employers, and in particular those that are new in the resource extraction industry, make serious efforts to come up with ways to manage those and similar culturally-related issues. As one respondent explained: 
if it's caribou session, then people want to be able to take that time or they just may not show up to work because that's their priority and that is obviously a challenge for companies, so some of the things that they are doing around that is try to manage on-site / off-site time so that, especially with fly-in / fly-out, if the goose season is say two or three weeks long then you make sure how shifts work so that everybody has at least a week off who is Aboriginal and wants to hunt and a lot of that [is] managed through a community liaison. (NA, p.44) 


\section{Conclusions}

This study was designed to investigate the link between Aboriginal identity and economic success in the Canadian labour market using the less-frequently applied qualitative method of inquiry, as suggested by the institutional economics theory on labour market analysis. The study analyzed information gathered through twenty-five open-ended in-depth interviews with key informants working in the area of Aboriginal labour force development to garner some new knowledge on the role institutional and other constraints, not observable in the statistical information, play in the determination of the labour market outcomes of the members of different groups of Aboriginal population in Canada. The study was inspired by the persistence of gaps in the labour market outcomes between Aboriginal and non-Aboriginal Canadians, as reported in the existing empirical literature on the topic. The guiding research question was simple in that it asked why these gaps persist in spite of government policy intervention.

The study examined responses from knowledgeable professionals from each of the three Aboriginal identity groups, as well as from some non-Aboriginal Canadians, working either directly or indirectly on the issues facing Aboriginal workers across Canada in order to gain a better understanding of what might be contributing to the gaps in the measured outcome between them and their non-Aboriginal counterparts as well as what might be contributing to the difference in the degree of disadvantage among them as a group. More specifically, the objective here was to gain some insights about why some Aboriginal workers continue experiencing poorer labour market outcomes than others and why is it that some of them seem to linger in lower and less stable occupational rungs 
of the Canadian labour market. The study analyzed interview responses in relation to the different institutional constraints, cultural differences in individuals' attitudes toward paid employment and differences in "lived" experiences of the labour market discrimination.

The analysis was structured around three specific points that reflect the three main steps or stages of the labour force activity: (1) starting line, which involved discussions of differences in work readiness of the potential labour force pool; (2) labour force, which captured the processes involved in recruitment and hiring of the labour force participants; and (3) finish line, which focused on workplace processes related to retention and promotion issues that the employed workers experience. Overall, the findings suggest that systemic discrimination is present at each of these three stages and that workers with the most visible signs of their Aboriginality are affected the most. The specific findings pertaining to each of the three stages are summarized next.

In relation to the first stage, the findings indicate that much of the perplexities observed in the empirical literature on the topic can be explained by the fact that the most complex - and the least understood - aspect of the profile of the Aboriginal population in Canada that is reflected at this stage is not accounted for. As reported in this study, this entry stage is filled with various barriers and limitations, both at the individual and at the community level, that act to seriously limit labour force participation. What is being referred to as a fast-growing "potential labour force pool" is a group of highly diverse individuals, most of whom are caught up with dealing with basic survival issues, especially those living on reserves and in northern Aboriginal communities which are plagued with numerous and multifaceted socioeconomic issues. 
In order to be able to count on this potential labour "pool" to fill in the projected labour shortages, serious investments need to be made in terms of building their work readiness. As discussed above, due to the past government (in)action and chronic underfunding of basic services, especially those related to education at the K-12 level, many of the young Aboriginal people lack essential life skills which non-Aboriginal people obtain at a very early stage in their lives. There is even some indication of overt discrimination in terms of access to education, although this particular point warrants further investigation before it can be taken seriously into account.

In relation to the second stage, the findings indicate that institutional aspects embedded in the system of recruitment and hiring arbitrarily exclude many Aboriginal people, especially those living on reserves and in remote communities, from the opportunities made available to the rest of the population. Job postings, for example, are not being accessed as easily by Aboriginal people as they are by non-Aboriginal people because the methods of dissemination are not as available in most of the Aboriginal communities. Urban Aboriginal communities, however, are exempted. In addition, social networks, deemed to be essential for successful economic integration by most participants, are reportedly not as well developed for many Aboriginal people, with Métis living in urban areas being an exception. Finally, the findings suggest that being a part of the designated group adds no value in terms of improving prospects of Aboriginal job applicants; on the contrary, many Aboriginal people feel pressured to hide, if possible, their association with Aboriginal community in hope of avoiding discriminatory treatments in the application process and in improving their chances of success. 
Finally, in relation to the final stage, the findings indicate that many workplaces host anti-Aboriginal sentiments and they are often expressed openly in relations with managers and in relations with coworkers. Cultural clashes and conflicts in workplaces are not being handled effectively because there is a very small presence of Aboriginal people at the managerial level and non-Aboriginal managers simply do not know how to handle those conflicts. The outcomes are often voluntary severances on the side of Aboriginal people, even from well-paid and hard-earned jobs. There are some indications of companies making efforts to accommodate Aboriginal people in their needs to pursue traditional activities, although this practice is not widespread across industries.

In closing, the findings from this study suggest that non-Aboriginal Canadians have become more successful labour market participants not from a transmission of superior culture that upholds a strong attachment to the paid labour market, as some would suggest, but from continuous acquisition of knowledge and skills that have economic value, from kindergarten and on. This knowledge and skills are in large part the product of government investment and, combined with other investments, mainly account for the 'productive superiority' of the well-connected and technically advanced nonAboriginal Canadians. Being born into this privilege, non-Aboriginal Canadians have no capacity for understanding the multifaceted challenges Aboriginal workers face, which results in ultimate discriminatory treatments, both in relations with hiring managers and in relations with coworkers. As in the preceding analysis, Inuit and North American Indian here reportedly bear disproportionate share of this burden. To omit this fact when studying the distribution of the labour market disadvantage between Aboriginal and nonAboriginal Canadians and among Aboriginal groups is bound to produce the perplexities 
as reported in the empirical work to date which inspired this investigation. Some of the key policy implications are briefly discussed next in the concluding chapter of the thesis. 


\title{
Appendix A
}

\author{
Appendix A 1: In-Depth Interview Theme Guide
}

\section{Themes and Potential Open-Ended Question}

Please note that this In-depth Interview Theme Guide is only provisional and that you have a significant degree of control over the process and contents of the interview. The themes listed below serve only as discussion aid and you should feel free to phrase your own views and to elaborate more on the issues you feel relevant and important.

\section{Theme 1: Education, training, skills development and job search networks}

What key institutional and other barriers are standing in the way of successful labour market participation of Aboriginal people in urban and rural areas?

How accessible and how effective are the formal job search networks for urban Aboriginal people? What primary means do people living in small remote communities relay on to search for paid employment?

What role does the postsecondary educational attainment play in helping Aboriginal people secure paid employment? If possible, please elaborate on the different levels and types of postsecondary educational attainments by differentiating a university degree from a vocational training and the government's skill development programs.

To what extent do you think the existing gaps in the labour market participation and employment outcomes between Aboriginal and non-Aboriginal workers with similar characteristics are due to the past and current institutional arrangements in the Canadian labour market and the society at large?

\section{Theme 2: Employment, retention and career advancement}

What factors do you think contribute to a greater probability of Aboriginal workers being laid off in times of economic downturns, relative to non-Aboriginal identity workers?

What factors do you think influence the decision of Aboriginal workers to quit their job?

To what extent do you think the segregation of Aboriginal workers in less-secure occupational rungs reflects institutional and other constraints in the Canadian labour market?

What impediments do you think are preventing Aboriginal workers from making a successful transition into moresecure and better-paid employment positions?

\section{Theme 3: Other issues and policy implications}

To what extent do you think the barriers to success that Aboriginal workers are experiencing are due to negative media stereotypes and false perceptions employers in the mainstream society hold about Aboriginal workers' attitudes towards the paid labour market?

What impacts do you think the living conditions and institutional arrangements in the Aboriginal communities have on either furthering or preventing the paid labour market participation of Aboriginal workers?

To what extent do you think the attachment to traditional pursuits among Aboriginal workers is affecting their attitude towards maintaining a stable career in the paid labour market?

How common and how different do you think are the 'lived experiences' of labour market discrimination for Aboriginal male and female workers?

What key policy implications do you see arising from this discussion? 


\section{Appendix B}

Appendix B 1: Letter of Information (standard)

\section{Letter of information}

Name of Researcher: Senada Delic, Ph.D. candidate, School of Public Policy and Administration, Carleton University Supenvisor: Dr. Frances Abele, School of Public Policy and Administration, Carleton University

Title of Project: Examining the link between Aboriginal Identity and economic success in Canadian labour market

As a representative of $x$ organization, you are being asked to participate in this qualitative research study by sharing any 'specialized' knowledge you might have related to the labour market issues facing Aboriginal workers in Canada.

This Letter of Information should give you a basic idea of what the research is about and what your participation will entail. If you would like to have details about something mentioned here, or information not included here, please feel free to contact me. Please take your time to read this letter carefully and to understand any associated information.

The Carleton University Research Ethics Committee has reviewed and approved this research study.

Purpose of the Study: I am carrying out this qualitative research as a supplement to my statistical analysis in which I am using three latest data sources, the 2008-2011 Labour Force Survey, the 2006 Census and the 2006 Aboriginal Peoples Survey, to examine the link between Aboriginal identity and economic success in the Canadian labour market. This qualitative component is meant to enhance the statistical analysis by incorporating your insights on the effects of institutional and other constraints facing Aboriginal workers, which cannot be observed in the statistical information.

What Will I Be Asked to Do? Your participation would involve giving your professional reflection on the issue through an open-ended, in-depth interview, which would require about 30-45 minutes of your time. A copy of the Interview Theme Guide is attached to this document, for your convenience. Your participation is voluntary and you may withdraw from this study at any time, in which case you may either request that records of any information collected from you be destroyed or you may grant me the right to use the collected records in my study.

What Type of Personal Information Will Be Collected? Your interview responses will be considered public and your organization may be identified as a source in my Doctoral Thesis and academic papers I plan to write on this topic. With your permission (please see below), I will tape record the interviews to improve the accuracy of my work. You may decline answering any questions you deem unsuitable and you may add any comments you deem important.

Are there Risks or Benefits if I Participate? Since your interview responses will be considered public, there is a potential risk to you as a participant. You will receive no material benefit for your participation. However, all research findings from this study will be made readily available to you, at your request.

What Happens to the Information I Provide? I will keep the interview notes, drafts and audio tapes in a locked drawer at the university or my home office during the time of this study. After the study is finished, they will be archived at my home office for future research purposes. I grant permission to be audio taped: Yes: No:

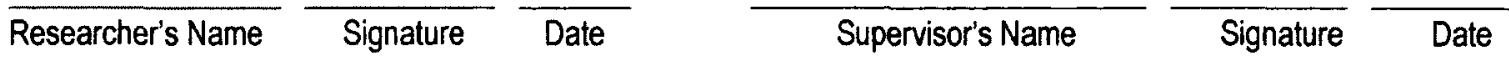

Questions/Concerns If you have further questions concerning matters related to this research, please contact: 1) Senada Delic 2) Dr. Frances Abele. If you have any concerns about the way you have been treated as a participant, please contact Prof. Antonio Gualtieri, the Chair of Carleton University Research Ethics Committee. 
Leader's Name, Position

Name of the Organization

Street Address

Province, Postal code

Month Day, 2012

Dear

I am writing to invite your organization to take part in a qualitative research study which I am undertaking as part of my doctoral dissertation examining the link between Aboriginal identity and economic success in the Canadian labour market. As a national voice representing interests of Aboriginal people, I am sure your staff members working in the area of labour force development have important knowledge about specific issues facing Aboriginal workers that can help me better understand my research question and improve the accuracy of my conclusions on this topic.

In the quantitative part of my dissertation I am using individual microdata from the national Labour Force Survey to examine the impact of latest recession on Métis and First Nations workers living off-reserve and I am comparing the outcomes to those of non-Aboriginal identity workers. I am also using individual microdata from the 2006 Census and the 2006 Aboriginal Peoples Survey to examine the factors affecting employment success for status and non-status First Nations workers living on-reserve and off-reserve, as well as for Métis and Inuit workers living in urban and rural areas across Canada.

Although informative, this statistical analysis is limited in the sense that it does not allow me to examine the issue in a preferred depth and that is why I am writing to you. Your insights on the effects of institutional and other constraints facing Aboriginal workers, which cannot be observed in the statistical information, would greatly enhance this study and allow me to discuss practical implications for the public policy in this area. If you are interested, I would be glad to share with you the results from this analysis.

The Carleton University Research Ethics Committee has reviewed and approved this research study and I have sent a copy of this letter to my supervisor for this part of the dissertation, Professor Dr. Frances Abele. I look forward to hearing from you or from your staff members regarding availability for an open-ended in-depth interview, at your convenience.

Respectfully,

Senada Delic, Ph.D. candidate, School of Public Policy and Administration, Carleton University

cc: Prof. Dr. Frances Abele, School of Public Policy and Administration, Carleton University 


\title{
Appendix C
}

Appendix C 1: A Copy of the Informed Consent Form

\author{
Informed Consent Form
}

Name of Researcher: Senada Delic, Ph.D. candidate, School of Public Policy and Administration, Carleton University Supervisor: Dr. Frances Abele, School of Public Policy and Administration, Carleton University

Title of Project: Examining the link between Aboriginal Identity and economic success in Canadian labour market

Dear

Thank you for agreeing to participate in this research study. l expect your professional insights on the specific issues facing Aboriginal workers in Canada to greatly improve the quality of this study and enable me to engage in a meaningful discussion about practical implications for public policy in this area.

This Letter of Information should give you a basic idea of what the research is about and what your participation will entail. If you would like to have details about something mentioned here, or information not included here, please feel free to contact me. Please take your time to read this letter carefully and to understand any associated information.

The Carleton University Research Ethics Committee has reviewed and approved this research study.

Purpose of the Study: I am carrying out this qualitative research as a supplement to my statistical analysis in which I am using three latest data sources, the 2008-2011 Labour Force Survey, the 2006 Census and the 2006 Aboriginal Peoples Survey, to examine the link between Aboriginal identity and economic success in the Canadian labour market. This qualitative component is meant to enhance the statistical analysis by incorporating your insights on the effects of institutional and other constraints facing Aboriginal workers, which cannot be observed in the statistical information.

What Will I Be Asked to Do? Your participation would involve giving your professional reflection on the issue through an open-ended, in-depth interview, which would require about 30-45 minutes of your time. A copy of the Interview Theme Guide is attached to this document, for your convenience. Your participation is voluntary and you may withdraw from this study at any time, in which case you may either request that records of any information collected from you be destroyed or you may grant me the right to use the collecled records in my study.

What Type of Personal Information Will Be Collected? Your interview responses will be considered public and your organization may be identified as a source in my Doctoral Thesis and academic papers I plan to write on this topic. With your permission (please see below), I will tape record the interviews to improve the accuracy of my work. You may decline answering any questions you deem unsuitable and you may add any comments you deem important.

Are there Risks or Benefits if I Participate? Since your interview responses will be considered public, there is a potential risk to you as a participant. You will receive no material benefit for your participation. However, all research findings from this study will be made readily available to you, at your request.

What Happens to the Information I Provide? I will keep the interview notes, drafts and audio tapes in a locked drawer at the university or my home office during the time of this study. After the study is finished, they will be archived at my home office for future research purposes. I grant permission to be audio taped: Yes: No:

Signatures (written consent) Your signature on this form indicates that you have understood the provided information regarding participation in this research study and that you agreed to participate voluntarily. In no way does this waive your legal rights nor release the investigators or involved institutions from their legal and professional responsibilities. You are free to withdraw from this study at any time. Your continued participation should be as informed as your initial consent, so you should feel free to ask for clarification or new information throughout your participation.

$\overline{\text { Participant Name }} \overline{\text { Signature }} \overline{\text { Date }} \overline{\text { Investigator/Delegate's Name }} \overline{\text { Signature }} \overline{\text { Date }}$

Questions/Concerns If you have further questions concerning matters related to this research, please contact: 1) Senada Delic 2) Dr. Frances Abele. If you have any concerns about the way you have been treated as a participant, please contact Prof. Antonio Gualtieri, the Chair of Carleton University Research Ethics Committee.

A copy of this consent form has been given to you to keep for your records and reference. The investigator has kept a copy of the consent form. 


\section{Appendix D}

\section{Appendix D 1: List of Participating Organizations}

\section{List of Participating Organizations}

Aboriginal Human Resource Council (AHRC)

Public Service Alliance of Canada (PSAC)

Canadian Media Guild (CMG)

Assembly of First Nations (AFN)

Mining Industry Human Resource Council (MiHRC)

Algonquin College Access program (ACAP)

Mining Association of Canada (MAC)

Canadian Union of Public Employees (CUPE)

National Association of Friendship Centres (NAFC)

Canadian Labour Congress (CLC)

National Aboriginal Peoples' Circle, Public Service Alliance of Canada (PSAC)

Manitoba Métis Federation (MMF)

Grand River Employment and Training (GREAT)

Inuit Tapiriit Kanatami (ITK)

Congress of Aboriginal Peoples (CAP)

National Youth Council, Congress of Aboriginal Peoples (CAP-NYC)

Young Indigenous Professionals, Aboriginal Human Resource Council of Canada (AHRC-YIPs)

Kativik Regional Government (KRG)

Native Women's Association of Canada (NWAC)

Kativik Municipal Housing Bureau (KMHB)

Sioux Lookout Area Management Board (SLAAMB)

Tungasuvvingat Inuit (TI)

Cameco

\section{Total number of interviews conducted: 25}

Note: For some organizations, the interview was conducted with more than one department representative. 


\section{References}

Abele, F. (2006). Education, training, employment and procurement. Paper submitted to the Joint Review Panel for the Mackenzie Gas Project, Northwest Territories. Yellowknife: Alternatives North.

Abella, R. S. (1984). Report of the Commission on Equality in Employment, Volume 1. Royal Commission on Equality in Employment, Ottawa, Minister of Supply and Services Canada.

Agocs, C. (2002). Canada's employment equity legislation and policy, 1987-2000: The gap between policy and practice. International Journal of Manpower, 23(3), 256276.

An untapped labour pool: in a graying society, low levels of education by aboriginals on and off reserve are growing concern. (2008, February 1). The Ottawa Citizen. Retrieved June 7, 2012 from: http://www.canada.com/ottawacitizen/features/ longevity/story.html?id=16a29677-1d39-4bae-bc39-a9ab822a17e7

Bakan, A. B. \& Kobayashi, A. (2000). Employment equity policy in Canada: An interprovincial comparison. Policy research paper. Queen's University.

Bauman, L. J. \& Greenberg, E. (1992). The use of ethnographic interviewing to inform questionnaire construction. Health Education Quarterly, 19(1), 9-23.

Canada (2012). Canadian Human Rights Act. R.S. C. 1985, c. H-6. Current to June 27, 2012, last amended on March 13, 2012. Published by the Minister of Justice.

Canada (2012a). Employment Equity Act. S. C. 1995, c. 44. Current to June 27, 2012, last amended on December 31, 2005. Published by the Minister of Justice. 
Canada (1996). Royal Commission on Aboriginal Peoples, Report of the Royal Commission on Aboriginal Peoples: Volume 2: Restructuring the Relationship, Part Five: Economic development. Ottawa: Canada Communications Group.

Canadian Human Rights Commission (2011). Anti-discrimination codebook: race colour, national or ethnic origin. Case summaries: Discrimination in employment. Last updated 2011-08-19.

Damianakis, T. \& Woodford, M. R. (2012). Qualitative research with small connected communities: Generating new knowledge while upholding research ethics. Qualitative Health Research, 22(5), 708-718.

Delic, S. \& Abele, F. (2010). The recession and Aboriginal workers. In G. B. Doern \& C. Stoney (Eds.), How Ottawa Spends 2010-2011: Recession, Realignment, and the New Deficit Era (pp. 187-216). Montreal \& Kingston: McGill-Queen's University Press.

Denzin, N. \& Lincoln, Y. (2000). The discipline and practice of qualitative research. In N. K. Denzin \& Y. S. Lincoln (Eds.), Handbook of Qualitative Research, $2^{\text {nd }}$ ed., (pp. 1-28). Thousand Oaks, CA: Sage Publications.

De Silva, A. (1999). Wage discrimination against natives. Canadian Public Policy, 25(1), 65-85.

Drost, H. (1994). Schooling, vocational training and unemployment: The case of Canadian Aboriginals. Canadian Public Policy, 20(1), 52-65.

Dugger, W. (1979). Methodological differences between institutional and neoclassical economics. Journal of Economic Issues, 13(4), 899-909. 
Elias, P. D. (1995). Northern economies. In Elias, P. D. (Eds.), Northern Aboriginal communities: Economies and development, (pp. 3-32). North York, Ontario: Captus Press.

Fontana, A. \& Frey, J. H. (2000). The interview: From structured questions to negotiated text. In N. K. Denzin \& Y. S. Lincoln (Eds.), Handbook of Qualitative Research, $2^{\text {nd }}$ ed., (pp. 645-672). Thousand Oaks, CA: Sage Publications.

George, P. \& Kuhn, P. (1994). The size and structure of native-white wage differentials in Canada. The Canadian Journal of Economics, 27(1), 20-42.

Gee, E., Kobayashi, K. \& Prus, S. (2006). Ethnic inequality in Canada: Economic and health dimensions. In D. A. Green \& J. R. Kesselman (Eds.), Dimensions of Inequality in Canada (pp. 249-271). Vancouver: UBC Press.

Government of Canada (2012). Proceedings of the Standing Senate Committee on Aboriginal Peoples, Issue 14 - Evidence - March 27, 2012. Ottawa: Parliament of Canada.

Government of Canada (2012a). Proceedings of the Standing Senate Committee on Human Rights, Issue 8 - Evidence - February 13, 2012. Ottawa: Parliament of Canada.

Government of Canada (2009). Federal framework for Aboriginal economic development. Published under the authority of the Minister of Indian Affairs and Northern Development and Federal Interlocutor for Métis and Non-Status Indians. Ottawa.

Hoefer, T. (2009). Diamond mining in the Northwest Territories: An industry perspective on making the most of northern resource development. In F. Abele, T. J. 
Courchene, F. L. Seidle \& F. St-Hilaire (Eds.), Northern exposure: Peoples, powers and prospects in Canada's North, the art of the state, IV (pp. 395-415). Montreal, Quebec: The Institute for Research on Public Policy. Hudson's Bay Company (1828). Osnaburgh trading post journal of occurrences for 1827/28. Hudson's Bay Company Archives, B. 155/a/39, fo. 33d-35, Hudson's Bay Company Archives Library. Winnipeg, Manitoba.

Human Resource and Skills Development Canada (2012). Aboriginal Labour Market Programs. Date Modified: 2012-07-23, Accessed July 2012: http://www.hrsdc.gc.ca/eng/employment/almp/index.shtml Human Resource and Skills Development Canada (2012a). Federal Contractors Program. Date Modified: 2012-06-15, Accessed August 2012: http://www.hrsdc.gc.ca/eng/labour/equality/fcp/index.shtml

King, G., Keohane, R. \& Verba, S. (1994). Designing social inquiry: Scientific inference in qualitative research. New Jersey: Princeton University Press.

Kuhn, P. \& Sweetman, A. (2002). Aboriginals as unwilling immigrants; Contact, assimilation and labour market outcomes. Journal of Population Economics, 15(1), 331-355.

Kuo, C. Y. (1976). The effect of education on the earnings of Indian, Eskimo, Métis, and White workers in the Mackenzie District of Northern Canada. Economic Development and Cultural Change, 24(2), 387-398.

Lautard, E. H. (1982). Occupational segregation and inequality between native and nonnative Canadians. The Canadian Journal of Native Studies, 2(2), 303-320. 
Maxim, P. \& White, J. (2006). School completion and workforce transition among urban Aboriginal youth. in White, J. P., S. Wingert, D. Beavon and P. Maxim (Eds.). Aboriginal Policy Research: Moving Forward, Making a Difference. Volume 3, (pp. 33-52). Toronto: Thompson Educational Publishing.

McLellan, E., MacQueen, K. M., \& Neidig, J. L. (2003). Beyond the qualitative interview: Data preparation and transcription. Field Methods, 15(1), 63-84.

Patrinos, H. A. \& Sakellariou, C. N. (1992). North American Indians in the Canadian labour market: A decomposition of wage differentials. Economics of Education Review, 11(3), 257-266.

Pendakur, K. \& Pendakur, R. (2011). Aboriginal income disparity in Canada. Canadian Public Policy, 37(1), 61-83.

Pendakur, K. \& Pendakur, R. (2007). Minority earnings across the distribution. Canadian Public Policy, 33(1), 41-62.

Pendakur, K. \& Pendakur, R. (2002). Colour my world: Have earnings gaps for Canadian-born ethnic minorities changed over time? Canadian Public Policy, 28(4), 489-512.

Pendakur, K. \& Pendakur, R. (1998). The colour of money: Earnings differentials among ethnic groups in Canada. Canadian Journal of Economics, 31(3), 518-548.

Piore, M. J. (1979). Qualitative research techniques in Economics. Administrative Science Quarterly, 24(4), 560-569.

Piore, M. J. (1983). Labour market segmentation: To what paradigm does it belong? American Economic Review, 73(2), 249-253. 
Piore, M. J. (2002). Thirty years later: Internal labour markets, flexibility and the new economy. Journal of Management and Governance, 6(4), 271-279.

Piore, M. J. (2002a). Economics and Sociology. Revue Économique, 53(2), 291-300.

Quinn, P. M. (2002). Qualitative research and evaluation methods, $\left(3^{\text {rd }} \mathrm{ed}.\right)$. Thousand Oaks, CA: Sage Publications.

Schmidt, C. (2010). Systemic discrimination as a barrier for immigrant teachers.

Diaspora, Indigenous, and Minority Education, 4(1), 235-252.

Service Ontario (2007). Pay Equity Act. R. S. O. 1990, CHAPTER P. 7. Last amendment: 2009, c. 33. Sched. 18, s. 24. Accessed August 2012: http://www.elaws.gov.on.ca/html/statutes/english/elaws_statutes_90p07_e.htm.

Silverman, D. (2000). Analyzing talk and text. In N. K. Denzin \& Y. S. Lincoln (Eds.), Handbook of Qualitative Research, $2^{\text {nd }}$ ed., (pp. 821-834). Thousand Oaks, CA: Sage Publications.

Stabler, J.C. (1989). Dualism and development in the Northwest Territories. Economic Development and Cultural Change, 37(4), 805-839.

Statistics Canada (2007). How Statistics Canada identifies Aboriginal Peoples. Ottawa, Minister of Industry, Catalogue No. 12-592-XIE.

Tait, H. (2008). Aboriginal Peoples Survey, 2006: Inuit health and social conditions. Statistics Canada, Social and Aboriginal Statistics Division, Analytical paper, Catalogue Number 89-637-X - No. 001.

The Canadian Press (2012). Diversity at the Canadian Press. Accessed September 2012 at: http://www.thecanadianpress.com/careers.aspx?id=106 
Usher, P., Duhaime, G. \& Searles, E. (2003). The household as an economic unit in Arctic Aboriginal communities, and its measurement by means of a comprehensive survey. Social Indicators Research, 61(1), 175-202.

Walters, D., White, J., \& Maxim, P. (2004). Does postsecondary education benefit Aboriginal Canadians? An examination of earnings and employment outcomes of recent Aboriginal graduates. Canadian Public Policy, 30(3), 283-301.

Wang, G. G. \& Dobbs, R. L. (2008). Institutional economics and human resource development. Advances in Developing Human Resources, 10(6), 770-787.

White, J., Maxim, P., \& Gyimah, S. O. (2003). Labour force activity of women in Canada: A comparative analysis of Aboriginal and non-Aboriginal women. The Canadian Review of Sociology and Anthropology, 40(4), 391-415.

Wilber, C. \& Harrison, C. K. (1978). The methodological basis of institutional economics: Pattern model, storytelling, and holism. Journal of Economic Issues, $12(1), 61-89$ 


\title{
Thesis Conclusions: Findings Summary and Policy Implications
}

\begin{abstract}
Current public policies in Canada acknowledge that the labour market disadvantage falls disproportionately on Aboriginal people and recommend programming strategically designed to accommodate different needs of Aboriginal people. Some of the major policies were based on recommendations from comprehensive and rigorous research reports such the 1984 report of the Royal Commission on Equality in Employment and the 1996 report of the Royal Commission on Aboriginal Peoples. Other policies and programs are also based on some form of analysis and there is growing consensus on the importance of research-based policy making. This concluding chapter provides a very brief summary of the key findings from the analysis presented in this thesis and offers some discussion on policy implications in relation to two important policy areas: (1) education; and (2) social inclusion. The key overall insight that this thesis offers is that the processes that determine the economic integration and distribution of the labour market disadvantage among Aboriginal populations in Canada vary substantially and policy-makers should bear that in mind when formulating strategic programming designed to address the root causes and not the mere symptoms of issues at hand.
\end{abstract}

Key words: Aboriginal people, labour market disadvantage, public policy 


\section{Table of Contents}

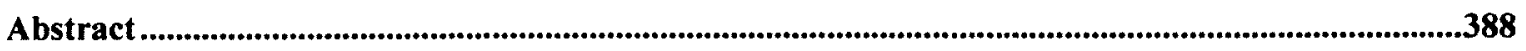

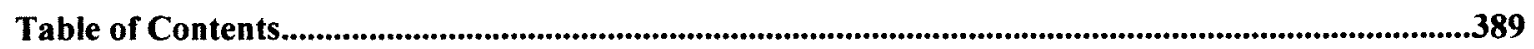

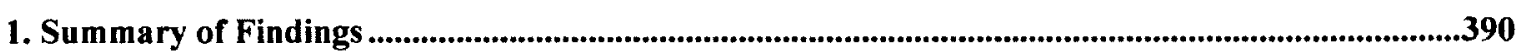

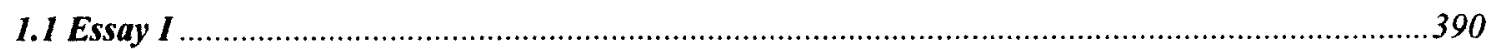

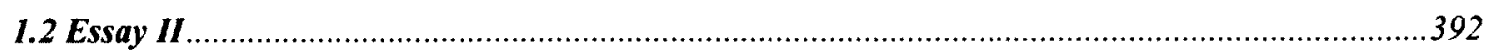

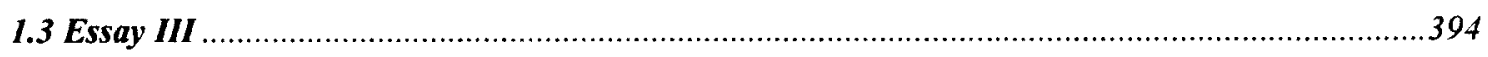

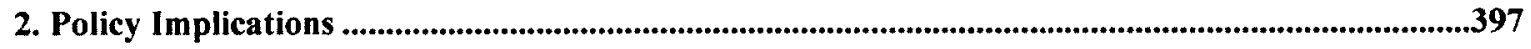

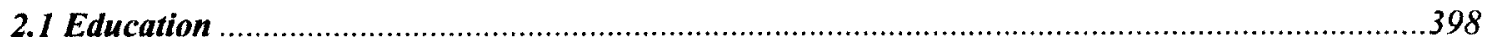

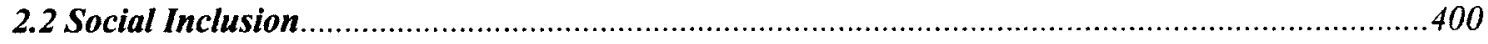

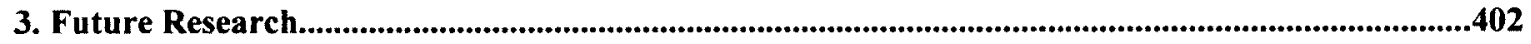




\section{Summary of Findings}

This thesis examined the relationship between Aboriginal identity and economic success in the Canadian labour market. The examination was approached from two theoretical perspectives, the neoclassical and the institutional economics perspective, and was based on both quantitative and qualitative research methods. This approach was inspired by the existing empirical evidence on the persistence of gaps in the labour market outcomes between Aboriginal and non-Aboriginal Canadians, in spite of government intervention. The thesis explored the labour market issues facing different groups of Aboriginal population living in different parts of Canada in an attempt to capture the complexities involved in factors determining their labour market outcomes. As such, the thesis made an important contribution to the existing literature on this topic. The analysis was organized into three related, yet independent essays. A brief summary of the key findings from each of the three essays is presented below.

\subsection{Essay I}

The first essay applied standard econometric techniques to monthly data from the Canadian Labour Force Survey in order to examine the link between Aboriginal identity and employment transitions in the context of an economic downturn. The primary objective of this examination was twofold: (1) to assess the difference in the impact of the latest recession on Aboriginal and non-Aboriginal identity workers; and (2) to uncover the sources that underlie the difference. The impact was measured by the probability of layoff and the probability of reemployment following the layoff. 
In regard to the measure of the impact in the form of layoff, the key finding is twofold: First, the evidence in this essay suggests that the latest recession clearly had a more negative impact on Aboriginal identity workers, and in particular on North American Indian men, than on non-Aboriginal identity workers. Second, while for one subgroup of Aboriginal workers - Métis - a large portion of the difference in the impact can be attributed to the difference in endowments for the other subgroup of Aboriginal workers - North American Indian - only a very small portion of the difference in the impact can be attributed to the difference in endowments, much remains unexplained and possibly attributable to discrimination in the labour market against this group of Aboriginal people. In the case of Métis, evidence hints possible negative sorting across layoff-prone industries and sectors and across occupational affiliations.

In regard to the measure of the impact in the form of reemployment, the key finding is somewhat unanticipated; the evidence suggests that Aboriginal identity workers, and in particular Métis men, were more successful in finding jobs following the layoff than were non-Aboriginal identity workers. In terms of sources underlying the difference in the probability of reemployment between Aboriginal identity men and Aboriginal identity women, the evidence suggests that the difference is fully explained by the differences in observable characteristics between the two gender groups. Also, in the case of Métis men, the evidence indicates possible positive sorting across industries and sectors and occupational affiliations.

A surprising finding, albeit closely in line with the existing literature examining the labour market outcomes of Aboriginal people, is that the differences in educational attainment among the examined groups play no significant role in the formation of the 
gaps in the observed outcomes. This finding is a cause for serious concern since policy discussions in this area revolve primarily around ways of improving educational attainment of disadvantaged groups such as the Aboriginal groups examined here. Further examination of this puzzlement is necessary.

\subsection{Essay II}

The second essay also applied standard econometric techniques to cross-sectional data from the 2006 Census and the 2006 Aboriginal Peoples Survey in order to examine the link between Aboriginal identity and the probability of employment, conditional on labour force participation. The primary objective of this examination was to asses the relative impact of various sources of capital - human, social and cultural - on the employment success of Aboriginal people living on-reserve, off-reserve, in urban and in rural areas across Canada. Several robustness checks were performed here in an attempt to gain a better understanding of the role different types and levels of education play in the probability of employment of different subgroups of Aboriginal identity population.

The single most important finding in this essay is that the predictions of human capital theory do not hold up when the associated empirical models are applied to the different Aboriginal groups - the essay presents strong evidence that, for Aboriginal people, more education does not lead to higher probabilities of employment - in fact, in no model is the estimated relationship between the level of education and the probability of employment strictly monotonic. On the contrary, in line with the previous research on the topic, the evidence here suggests that in some cases the estimated effects are the reverse of what the theory predicts - more education in some cases implies lower 
probability of employment. This contradiction is particularly obvious in the estimated models for North American Indian men and for North American Indian women.

If measured by the standard six-category classification of educational attainment, the estimated effects of educational attainment on the probability of employment of urban and of registered NAI men are strikingly different from those of urban and of registered NAI women — the evidence suggests that the returns to postsecondary schooling are consistently positive for urban and for registered NAI women, while for urban and for registered NAI men the returns to postsecondary schooling are positive only at the level of completed university education - at every other level of postsecondary education, the estimated average marginal effects are negative for urban and for registered NAI men.

If measured using different, and generally more-detailed, classifications of educational attainment, the estimated effects are somewhat different, suggesting that the effects from the standard six-category classification for North American Indian men are possibly biased upwards and for North American Indian women possibly biased downwards. The main reason for this lies in the hidden details of high school certification and postsecondary schooling certification.

In the assessment of high school certification and postsecondary accreditation among North American Indian groups, it becomes clear that the association between vocational schooling - when obtained without completed high school diploma - and the probability of employment is significantly negative both for men and for women. Similarly, when a college diploma is obtained after completion of high school, it has a positive effect on the probability of employment, both for men and for women. For the on-reserve North American Indian groups, completed university certificates also have 
positive effects on the probability of employment, both for men and for women, but only if obtained upon completed high school diplomas.

The evidence of the effect of educational attainment on the probability of employment for the other two groups of Aboriginal people, Inuit and Métis, is highly variable and often counterintuitive. The same overall conclusion holds for the assessed effects of the other variables - social capital and cultural factors - variation in the effects is notable across gender, residence, and Aboriginal identity sub-grouping. However, there is some indication in this evidence that for some subgroups of Aboriginal people, social capital and cultural factors are potentially important omitted variables in their equations of the probability of employment.

\subsection{Essay III}

The third essay applied qualitative methods of inquiry, as laid out in the institutional economics theory, to primary data collected through twenty five in-depth interviews with knowledgeable key informants working in the area of Aboriginal labour force development across Canada. The primary objective of this inquiry was to gather and analyze various perspectives in an attempt to gain a better understanding of the role institutional constraints and other characteristics of individuals, not observable in the statistical information, play in the labour market experience of different groups of Aboriginal people. The inquiry was motivated by the persistence of paradoxes and puzzles in the empirical evidence on the relationship between measured aspects of human capital such as educational attainment and the probability of success among Aboriginal workers and was focused on gaining an understanding of the influence of unmeasured 
productivity-related characteristics of individuals such as motivation and work ethics and of the influence of institutional arrangements in the Canadian labour market.

Findings from this inquiry suggest that many paradoxes and puzzles that persist in the empirical literature on the topic can be better understood once the institutional arrangements in relation to investments in human and other capital, the legacy of historical disadvantage, and the experience of workplace discrimination are taken into account. Systemic discrimination is reportedly present at every stage of the labour market experience of Aboriginal people. The barriers at the entry stage, however, are deemed most detrimental to their success because they involve issues such as low literacy and various social ills that are attributed to a large extent to the history of colonial policies in Canada. To effectively address these long-standing barriers requires serious commitments from the governments to assist Aboriginal people in their efforts to "heal the past" and to enable them to stand on par with the rest of the population in the competitive "race" for the available opportunities. In the absence of this, not much can be expected to change.

At present, the designated group membership provision is seen as essentially valueless in terms helping Aboriginal people get ahead because the employers are still primarily looking at merit when selecting potential employees. With all the disadvantages in place, Aboriginal people have very low chances of keeping up with their nonAboriginal counterparts who are applying for the same positions. Moreover, many Aboriginal people have no insight into jobs that are being advertised, unless their place of residence is in the major urban centers. Finally, a lack of understanding of the history of Aboriginal people on the side of managers and coworkers is creating workplace climates that are often unbearable to Aboriginal workers, especially those affected either directly 
or indirectly by the aftermath of the residential school system. Consequently, many Aboriginal people quit, even their well-paid and hard-earned job, which in return, is taken by non-Aboriginal people as evidence of weak commitment to paid labour market that stems from Aboriginal culture. 


\section{Policy Implications}

As discussed throughout this thesis, the labour market behaviour of Aboriginal Canadians and the determinants of their labour market outcomes are important empirical issues with potentially important public policy consequences. Over the past few decades, Canadian governments have come to recognize this and have introduced a range of labour market policies and programs, said to be targeted at improving the labour market prospects of Aboriginal people and at closing the long-standing gaps in the labour market outcomes between Aboriginal and non-Aboriginal Canadians. This thesis examined this issue of disparities from different angles using several related research questions, the essence of which can be summarized here in one single question - why is it that the gaps in the labour market outcomes between Aboriginal and non-Aboriginal Canadians persist, despite the existence of these targeted labour market policies and programs?

A short answer to this question that this thesis offers is - most of the policies and programs in place are targeted at addressing the symptoms and not the actual causes of the issue. The past policies from colonial days have left significant impacts on Aboriginal communities and those impacts still plague lives of many Aboriginal people, especially those living on reserves and in remote northern communities. The insights that surfaced from the analysis in this thesis, especially those from the qualitative inquiry, are too complex to be adequately addressed within the scope of this brief chapter. Many of these insights will be addressed in a separate publication, post-thesis. In this concluding chapter, two, perhaps most important, policy implications are briefly addressed: one related to education and the other related to social inclusion of Aboriginal workforce. 
The key overall insight that this thesis provides is that the processes that determine the economic integration and distribution of the labour market disadvantage among Aboriginal populations in Canada vary substantially across Aboriginal identity groups and across gender; policy-makers, thus, should bear that in mind when formulating strategic programming designed to address the root causes and not the mere symptoms of issues at hand. Among the Aboriginal subgroups, the degree of the labour market disadvantage is most acute among Inuit and among North American Indian, and in particular if they are men living on reserve and in remote northern communities. To some degree, the two policy implications addressed in this concluding chapter are relevant to all Aboriginal identity subgroups; however, they apply the most to North American Indian living on reserves and to Inuit living in northern remote communities.

\subsection{Education}

The single most important policy implication this thesis offers pertains to education of Aboriginal people, and in particular to the K-12 education of North American Indian living on reserves and of Inuit living in remote northern communities. As discussed in the empirical part of the analysis in this thesis, the estimated effects of postsecondary education at the levels below completed university education on the probability of employment success of Aboriginal people are mixed and often counterintuitive. For some groups, the robustness checks provided some clarity on some classification categories such as vocational schooling, undertaken with or without a completed high school diploma, but not for Inuit men. In fact, for Inuit men, even completed university education does not significantly increase their probability of employment. The insights obtained from the qualitative inquiry suggest that the real 
issues lies in the quality of education at the $\mathrm{K}-12$ level - it appears that for some groups of Aboriginal people, measured education does not reflect actual learning, as reflected in the cases of high school graduates with low literacy; this means that if we could actually measure "command of basic literacy skills" instead of measured educational attainment, then Aboriginal to non-Aboriginal difference in that "endowment" would be important.

As discussed in the qualitative part of the analysis in this thesis, this difference stems from the overall low quality of public education being offered in many of the pertinent Aboriginal communities and from a myriad of socioeconomic ills facing individuals in those communities. Hence, serious policy intervention with respect to these issues is crucial if any improvement in the returns to education, as observed in the labour market outcomes of Aboriginal people analyzed in this study and in the previous literature, is to be seen. The programs such as the ASETS (Aboriginal Skills and Employment Training Strategy) that are currently being administered have some potential because of the integrated approach; however, such programs are leaving out the most disadvantaged Aboriginal people because they either have no adequate high school diplomas (with Grade 11 and Grade 12 Math completed) or their levels of basic literacy are too low to enable them to follow the instruction of the program. As such, the ASETS program, like many other programs that preceded it, serves only as a temporary patch for a more fundamental problem, which must be addressed if the goal of improving the labour market prospects of Aboriginal people and closing the gaps in the labour market outcomes between Aboriginal and non-Aboriginal people is to be achieved. 


\subsection{Social Inclusion}

The second policy implication this thesis offers relates to improving social inclusion of Aboriginal people in the Canadian labour market. The case for public intervention in regard to this issue cannot be overstated; unless Aboriginal people feel (are) included in the social and economic processes, all other efforts such as offering various job training and skills development programs in an attempt to break the vicious circle of welfare dependency and unemployment are bound to be wasted efforts.

As the insights gleaned from conversations with knowledgeable informants presented in this thesis (Essay III) suggest, social exclusion of Aboriginal people is rooted in various structures and processes of Canadian labour market and it has substantial effects on their labour market behavior. The workplace processes, in particular, often play a deciding role in whether or not they opt for voluntary severances. The aspect of the institutional social exclusion in the form of residential segregation is also serious as it serves to bar them from not only viable employment opportunities but also from key information on job postings and related opportunities in surrounding areas.

Aboriginal exclusion cannot be addressed effectively by merely relying on the existence of policies such as the employment equity policy. As discussed in Essay III, as long as the implementation of this policy entails no consequences for employers' failure to comply, there will be limited if any protection from social exclusion for Aboriginal people. Better interventions need to be developed to counter Aboriginal social exclusion, especially in workplaces. 
Also, innovative policies need to be developed to deal directly with the root causes of social exclusion, which, according to all participants in this study, is a general lack of understanding of Aboriginal issues and how those issues developed over time. Programs that educate the general public on the history of social exclusion of Aboriginal people may have merit, especially if done in workplaces where presence of Aboriginal workforce is notable. Such initiatives have recently been undertaken by major union and other organizations and have reported satisfactory results. 


\section{Future Research}

In closing, this thesis addressed several important research questions related to the labour market issues of Aboriginal people. Some of the insights derived from the analysis were quite detailed and informative while others raised further research question. This section briefly discusses some of the raised issues that warrant further contemplations.

One important issue for future research is to attempt to assess the impact of social capital on the labour market outcomes of different groups of Aboriginal people using other, and possibly more precise, measures of strong and weak ties of social capital. The measures applied in this thesis (Essay II) did not provide consistent and clear evidence of the impact for all examined groups yet the discussions with respondents in the qualitative inquiry (Essay III) firmly suggested that social capital is a highly important, if not crucial, factor for successful economic integration of Aboriginal people in the Canadian labour market.

Another important issue that warrants further investigation relates to some of the insights gleaned from interviews with knowledgeable informants on the effectiveness of the employment equity policy in helping Aboriginal people gain access to jobs for which they qualify. Some of the responses on this issue reported in this thesis (Essay III) were quite surprising and they merit further exploration. It would be interesting to run triangulation to check how prevalent these practices are. It would also be informative to learn if there is any variation among different types of employers and different types of job positions. The informants interviewed in this study were knowledgeable about 
different types of employment but there might be different patterns even within sectors, as hinted in the discussion of this finding in Essay III.

Also, the insights shared in this thesis (Essay III) about some First Nations communities having a limited access to high school education for their children due to lack of funding warrants further investigation. It would be interesting to learn how prevalent this issue is. It would also be interesting to know more about those communities in regard of their size, location, and their socioeconomic profile.

Finally, it would be informative to replicate the qualitative inquire from this thesis by recruiting a diverse group of Aboriginal workers working in different fields and in different sectors. Some of the insights reported in this thesis (Essay III) involving instances of overt forms of labour market discrimination are astonishing; it would be interesting to speak with different individuals who experienced such events at their workplaces and learn more about what impacts such experience have on the individual's productivity at job. 